Mesurer les activités scientifiques, technologiques et d'innovation

\title{
Manuel de Frascati 2015
}

LIGNES DIRECTRICES POUR LE RECUEIL

ET LA COMMUNICATION DES DONNÉES

SUR LA RECHERCHE ET LE DÉVELOPPEMENT EXPÉRIMENTAL
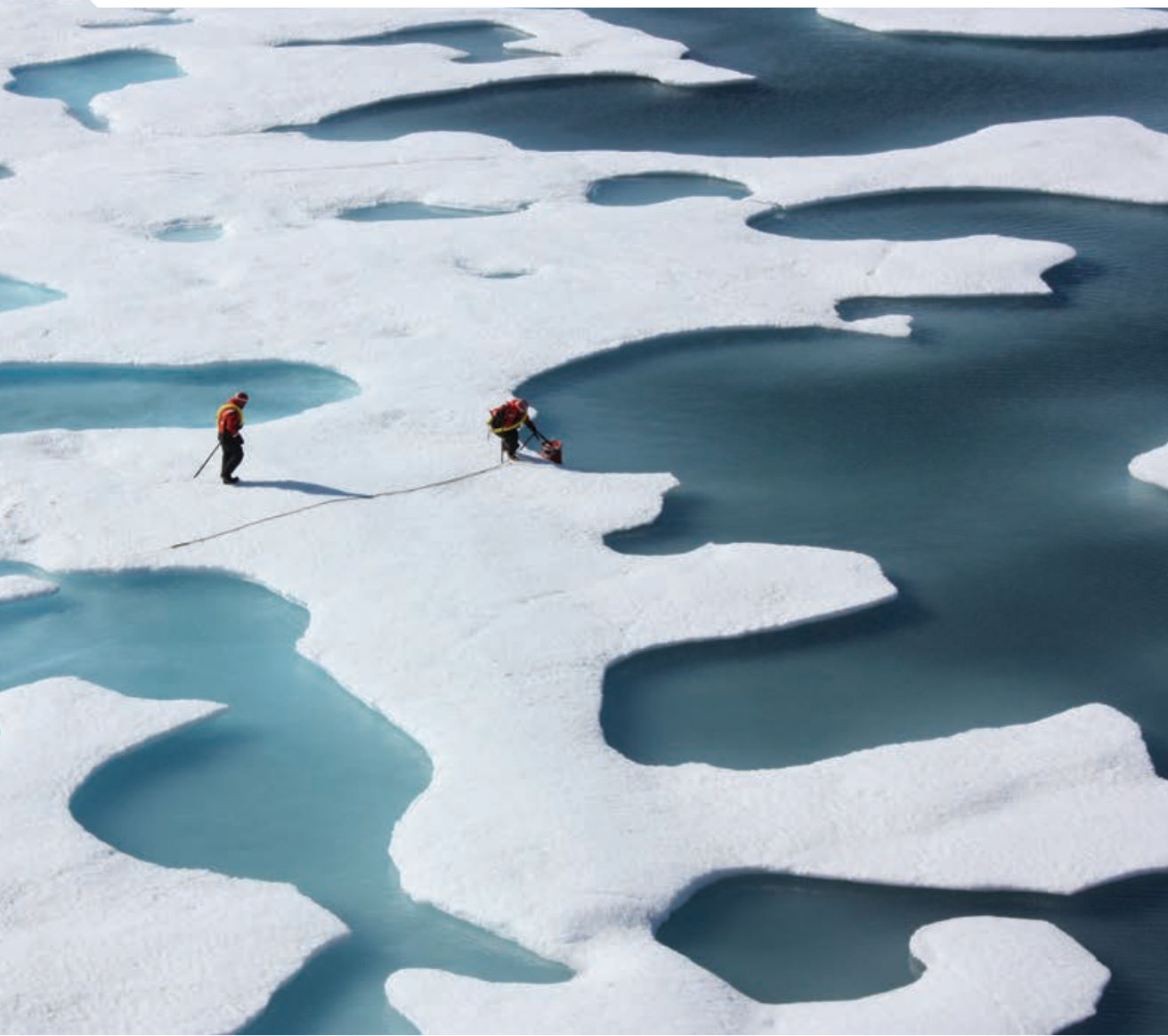

Mesurer les activités scientifiques, technologiques et d'innovation

\section{Manuel de Frascati 2015}

LIGNES DIRECTRICES POUR LE RECUEIL ET LA COMMUNICATION DES DONNÉES SUR LA RECHERCHE ET LE DÉVELOPPEMENT EXPÉRIMENTAL 
Cet ouvrage est publié sous la responsabilité du Secrétaire général de l'OCDE. Les opinions et les interprétations exprimées ne reflètent pas nécessairement les vues de l'OCDE ou des gouvernements de ses pays membres.

Ce document et toute carte qu'il peut comprendre sont sans préjudice du statut de tout territoire, de la souveraineté s'exerçant sur ce dernier, du tracé des frontières et limites internationales, et du nom de tout territoire, ville ou région.

\section{Merci de citer cet ouvrage comme suit :}

OCDE. (2016), Manuel de Frascati 2015: Lignes directrices pour le recueil et la communication des données sur la recherche et le développement expérimental, Mesurer les activités scientifiques, technologiques et d'innovation, OECD Publishing, Paris.

http://dx.doi.org/10.1787/9789264257252-fr

ISBN 978-92-6425724-5 (imprimé)

ISBN 978-92-6425725-2 (PDF)

Series: Mesurer les activités scientifiques, technologiques et d'innovation

ISSN 2414-620X (imprimé)

ISSN 2414-6137 (en ligne)

Les données statistiques concernant Israël sont fournies par et sous la responsabilité des autorités israéliennes compétentes. L'utilisation de ces données par l'OCDE est sans préjudice du statut des hauteurs du Golan, de Jérusalem Est et des colonies de peuplement israéliennes en Cisjordanie aux termes du droit international.

Ce manuel a été approuvé par le Comité de la politique scientifique et technologique et le Comité des statistiques et de la politique statistique de l'OCDE.

Crédit photographique : Photographie prise en juillet 2011 au cours de la mission ICESCAPE "Impacts of Climate on EcoSystems and Chemistry of the Arctic Pacific Environment". Photographie par la NASA/Kathryn Hansen - Flickr, https://creativecommons.org/licenses/by/2.0/

Les corrigenda des publications de l'OCDE sont disponibles sur : www.oecd.org/about/publishing/ corrigenda.htm.

(C) OCDE 2016

Vous êtes autorisés à copier, télécharger ou imprimer du contenu OCDE pour votre utilisation personnelle. Vous pouvez inclure des extraits des publications, des bases de données et produits multimédia de l'OCDE dans vos documents, présentations, blogs, sites Internet et matériel d'enseignement, sous réserve de faire mention de la source OCDE et du copyright. Les demandes pour usage public ou commercial ou de traduction devront être adressées à rights@oecd.org. Les demandes d'autorisation de photocopier une partie de ce contenu à des fins publiques ou commerciales peuvent être obtenues auprès du Copyright Clearance Center (CCC) info@copyright.com ou du Centre français d'exploitation du droit de copie (CFC) contact@cfcopies.com. 


\section{Avant-propos}

$I_{1}$

est indispensable de disposer d'une base de connaissances solide pour comprendre comment la création et la diffusion du savoir contribuent à la croissance économique et au bien-être social. À travers l'histoire, les résultats de la recherche et du développement (R-D) ont transformé à bien des égards la vie des individus et des sociétés, ainsi que le milieu naturel dans lequel nous évoluons. Les analystes des politiques et les décideurs ont constamment besoin d'être au fait du volume et de la nature des ressources humaines et financières qui sont consacrées à la $R-D$ au niveau des pays, des régions, des entreprises et autres unités statistiques, afin de trouver un moyen de les affecter à la réalisation d'objectifs donnés. Répondre à ce besoin au moyen de statistiques permettant l'établissement de comparaisons internationales et d'une terminologie commune, tel est précisément le but premier de ce manuel.

En juin 1963, l'OCDE s'est réunie avec les experts de plusieurs pays dans la ville de Frascati (Italie), à la Villa Falcioneri, pour débattre des statistiques de la recherche et du développement expérimental (R-D). De ces travaux est née la première version officielle de la "Méthode type proposée pour les enquêtes sur la recherche et le développement expérimental ", plus connue sous le nom de Manuel de Frascati. Le présent ouvrage en est la septième édition. Si le Manuel a vu le jour dans un contexte économique et géopolitique bien différent de la situation actuelle, il est toujours d'actualité après plus d'un demi-siècle, en raison de la volonté croissante de comparer les efforts de $R-D$ déployés dans différents pays et d'en recenser les caractéristiques essentielles. De plus en plus, la R-D est considérée comme un facteur d'innovation dans le cadre de l'action globale qui est menée au sein d'une économie mondiale fondée sur le savoir. De par ses spécificités, elle n'en conserve pas moins un rôle crucial et une place de choix dans les priorités des pouvoirs publics. Si la demande d'évaluations agrégées préside à l'élaboration de la présente édition, il y est également tenu compte de l'importance de dresser un tableau général plus détaillé des résultats de la R-D en cernant davantage les forces en jeu et les liens d'interdépendance qui existent à un niveau plus fin. Il convient en effet de disposer de micro-données sur la R-D afin non seulement d'établir des indicateurs agrégés, mais aussi analyser ses répercussions parmi différents types d'acteurs.

Bien qu'essentiellement à caractère technique, le Manuel forme l'un des piliers de l'action menée par l'OCDE pour aider à mieux comprendre le rôle de la science, de la technologie et de l'innovation lorsque l'on analyse les systèmes nationaux de recherche et d'innovation. En outre, en fournissant des définitions de la R-D reconnues sur le 
plan international et en fixant les nomenclatures à utiliser pour classer les activités de $R-D$, le Manuel vient enrichir la réflexion intergouvernementale sur les bonnes pratiques à suivre à l'égard des politiques scientifiques et technologiques. Cependant, les indicateurs et les données qu'il décrit n'ont pas vocation à permettre de juger de l'adéquation des objectifs publics.

La présente édition du Manuel est certainement l'une des plus remaniées par rapport à la version d'origine. Elle se caractérise par une description très détaillée des principes de base et suggestions concrètes à mettre en ouure face à la complexité grandissante du paysage de la recherche et de l'innovation dans les économies d'aujourd'hui et compte tenu des caractéristiques de chaque secteur. Entre autres nouveautés, une attention particulière est accordée au processus inexorable de la mondialisation de la R-D, ainsi qu'à la diversité croissante des modes de financement et d'exécution de la R-D rencontrés à l'échelle sectorielle et au-delà.

Ce n'est pas seulement dans les pays membres de l'OCDE que le Manuel de Frascati est la référence en matière de recueil de données de R-D. Suite aux initiatives de l'OCDE, de l'UNESCO, de l'Union européenne et d'autres organisations régionales, il sert désormais à mesurer la R-D dans le monde entier. Il fait également autorité dans d'autres domaines, comme les statistiques de l'éducation et des échanges. Plus particulièrement, c'est en se fondant sur les définitions et données décrites dans le Manuel de Frascati, que le Système de comptabilité nationale de 2008 considère pour la première fois les dépenses de R-D comme relevant de la formation de capital, autrement dit qu'elles doivent être traitées comme un investissement.

Le Manuel de Frascati s'inspire résolument de l'expérience acquise en matière de recueil de statistiques de $R-D$ dans les pays membres et non membres de l'OCDE. Il est le fruit de la collaboration des membres du Groupe de travail des experts nationaux sur les indicateurs de la science et de la technologie (GENIST) de l'OCDE. Les spécialistes qui le composent s'emploient depuis maintenant plus d'un demi-siècle, avec le concours du Secrétariat de l'OCDE, à appliquer des principes de mesure aux concepts de la science, de la technologie et de l'innovation. Ces travaux ont abouti à l'élaboration d'une série de manuels méthodologiques, connus sous le nom de "famille Frascati ", qui complète le présent ouvrage par des recommandations sur la manière de procéder pour mesurer l'innovation (Manuel d'Oslo), les ressources humaines dédiées à la science et à la technologie, les brevets et la balance des paiements technologiques et qui sert de référence à l'établissement des principaux indicateurs et données statistiques actuellement employés dans les domaines de la science et de la technologie.

Destiné à un usage pratique, le Manuel de Frascati est également disponible en ligne sur le site web de l'OCDE, à l'adresse http://oe.cd/frascati, où l'on trouvera des documents complémentaires et des liens conduisant à d'autres sources d'information sur la manière dont les pays recueillent des données de R-D ainsi que sur les base de données et les indicateurs clés. Ce site web sera régulièrement mis à jour afin de rendre compte des nouvelles ressources et lignes directrices disponibles dans les différents 
domaines de compétence du GENIST. L'application concrète des dispositions de cette nouvelle version du Manuel, qui participera également d'un effort collectif, conduira à l'apparition d'une nouvelle génération de données, d'indicateurs et d'analyses de la $R-D$ qui aideront à répondre aux besoins de l'action publique et à éclairer le débat public sur la science, la technologie et l'innovation.

Octobre 2015

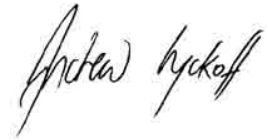

Andrew Wyckoff

Directeur de la science, de la technologie et de l'innovation, OCDE

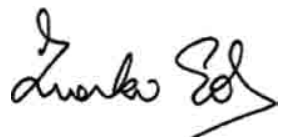

Ward Ziarko

Président du GENIST, à l'origine de la $6^{\mathrm{e}}$ révision ; Service public de programmation de la politique scientifique fédérale, Belgique

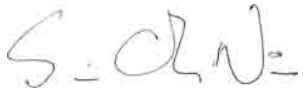

Svein Olav Nås

Actuel président du GENIST ; Conseil norvégien de la recherche, Norvège 


\section{Remerciements}

$\mathrm{L}$ a présente publication est le fruit d'un travail collectif réalisé par l'ensemble des délégués des pays membres du Groupe de travail des experts nationaux sur les indicateurs de la science et de la technologie (GENIST) et de la Division des analyses économiques et des statistiques (EAS), qui relève de la Direction de la science, de la technologie et de l'innovation (STI), au sein de l'OCDE.

Cette septième édition a été élaborée sous la direction rédactionnelle de John Jankowski (National Science Foundation, États-Unis) et de Fred Gault (ancien président du GENIST, UNU-MERIT, TUT-IERI et consultant auprès du Secrétariat), sur la base des travaux réalisés par les délégués des pays membres du GENIST et des experts de l'OCDE organisés en groupes de révision. Les membres du Bureau du GENIST, Eveline von Gässler (ministère fédéral de l'Éducation, Allemagne), Tomohiro Ijichi (National Institute of Science and Technology Policy, Japon), John Jankowski (États-Unis), Svein Olav Nås (The Research Council of Norway, Norvège), Elisabeth Pastor (Office fédéral de la statistique, Suisse), Giulio Perani (ISTAT, Italie, et Eurostat) et Ward Ziarko (Politique scientifique fédérale, Belgique), méritent des remerciements particuliers pour le temps et l'énergie considérables qu'ils ont investis dans la procédure de révision au nom du GENIST et pour leur contribution originale à l'exercice.

Il convient aussi de remercier Frank Foyn (Statistics Norway), Konstantin Fursov et Leonid Gokhberg (École des hautes études en sciences économiques de Moscou, Fédération de Russie), Daniel Ker (Office for National Statistics, Royaume-Uni), Andreas Kladroba (Stifterverband, Allemagne), Francisco Moris (National Science Foundation, États-Unis), Greg Peterson (Statistique Canada), Géraldine Seroussi (ministère de l'Enseignement supérieur et de la Recherche, France) et Susanne Sundnes (Nordic Institute for Studies in Innovation, Research and Education, Norvège), qui ont guidé les groupes de révision chargés d'enrichir le contenu de la présente édition. Roberto de Pinho (ministère de la Science, de la Technologie et de l'Innovation, Brésil) a également aidé à mettre en place un espace de collaboration en ligne dédié à la révision du Manuel, et Svein Olav Nås a repris le travail préparatoire conduit par son prédécesseur à la présidence du GENIST, Ward Ziarko. 
L'exercice de révision entrepris par le GENIST a été piloté par l'unité responsable des indicateurs de la science et de la technologie au sein de la Division EAS, sous la direction de Fernando Galindo-Rueda, avec le concours de Laudeline Auriol et de Vladimir López-Bassols. Ces travaux ont été supervisés par Alessandra Colecchia, chef de la Division EAS, et ont bénéficié des conseils et observations d'Andrew Wyckoff et de Dirk Pilat. Nadim Ahmad, Silvia Appelt, Koen de Backer, Fabienne Fortanier, Dominique Guellec, Guillaume Kpodar, Fabien Verger et Bettina Wistrom ont également fait part de commentaires utiles. La tâche n'aurait pu être menée à bien sans la contribution de plusieurs autres collaborateurs de l'OCDE, en particulier les équipes responsables de l'appui informatique, des publications et de la communication, ni le concours de Marion Barberis et de Catherine Bignon, de la Division EAS.

Les contributions fournies, à titre individuel et au nom d'institutions, à travers le mécanisme de consultation collaborative en ligne, ont été grandement appréciées, de même que les remarques formulées par les présidents du CPST et du CSSP et les délégations nationales jusqu'à ce que la déclassification du document soit approuvée. Plus précisément, la présente édition doit beaucoup à l'organisme National Science Foundation/National Center for Science and Engineering Statistics (États-Unis), dont les experts ont réalisé un considérable travail préparatoire et rédactionnel. La Direction générale des statistiques de l'éducation et de la science du Portugal a obligeamment accueilli à Lisbonne, en décembre 2014, un atelier sur la révision. La Commission européenne a financé des travaux de recherche exploratoire autour d'un certain nombre de sujets en lien avec l'exercice. Eurostat a également prêté main forte, par l'intermédiaire de ses experts qui ont participé aux travaux de révision, tandis que l'Institut de statistique de l'UNESCO a fourni des informations et observations très utiles pour faire accepter les orientations énoncées dans le Manuel et en faciliter la mise en application dans les pays, quelque soit leur stade de développement. 



\section{Table des matières}

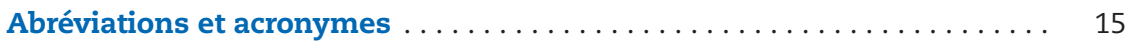

Chapitre 1. Introduction aux statistiques de R-D et au Manuel de Frascati . . 19

1.1. Objectifs et historique du Manuel de Frascati.............. 20

1.2. Aperçu général du Manuel. . . . . . . . . . . . . . . . . . . 28

1.3. Appliquer les recommandations formulées dans le Manuel . . . 40

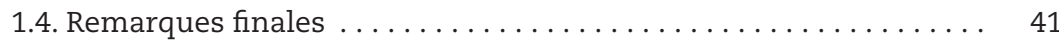

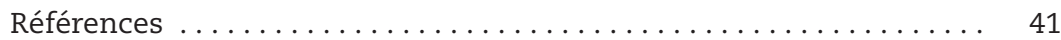

Partie I

Définir et mesurer la R-D : Orientations générales

Chapitre 2. Concepts et définitions permettant d'identifier la R-D . . . . 45

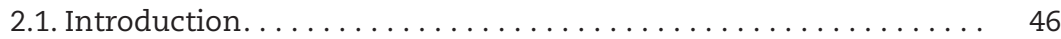

2.2. Définition de la recherche et du développement

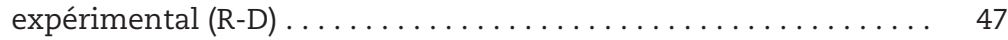

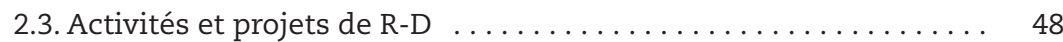

2.4. Les cinq critères permettant d'identifier la R-D . . . . . . . . 48

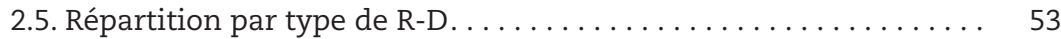

2.6. Classification et distribution par domaine de recherche et de développement $\ldots \ldots \ldots \ldots \ldots \ldots \ldots \ldots \ldots \ldots \ldots \ldots \ldots \ldots \ldots \ldots \ldots \ldots$

2.7. Exemples d'activités de R-D, de limites et d'exclusions dans différents domaines ..................... 64

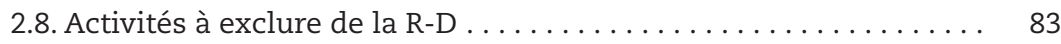

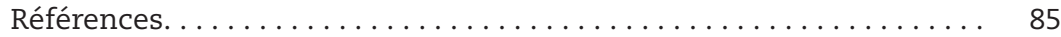

Chapitre 3. Secteurs institutionnels et classifications utilisés dans

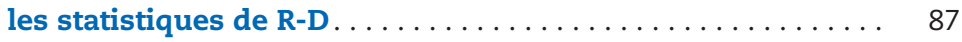

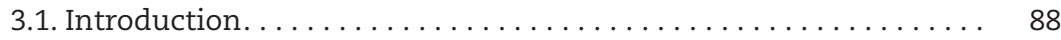

3.2. Unités institutionnelles $\ldots \ldots \ldots \ldots \ldots \ldots \ldots \ldots \ldots \ldots \ldots . \ldots \ldots$

3.3. Secteurs institutionnels. . . . . . . . . . . . . . . 92

3.4. Classifications générales applicables à toutes les unités institutionnelles .......................... 99 
3.5. Présentation succincte des principaux secteurs

du Manuel de Frascati, de leurs unités et des cas limites . . . . . . . . 105

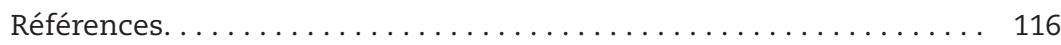

Chapitre 4. Mesurer les dépenses de R-D : Exécution et sources de financement ....................... 117

4.1. Introduction $\ldots \ldots \ldots \ldots \ldots \ldots \ldots \ldots \ldots \ldots \ldots \ldots \ldots \ldots . \ldots \ldots \ldots$

4.2. Dépenses de R-D intra-muros (exécution de travaux de R-D) . . . 120

4.3. Financement de la R-D. . . . . . . . . . . . . . . . . . . . 136

4.4. Concilier les approches fondées sur le point de vue

des exécutants et des bailleurs de fonds. . . . . . . . . . . . . . . . . 154

4.5. Compilation des valeurs totales de la R-D nationale . . . . . . . 156

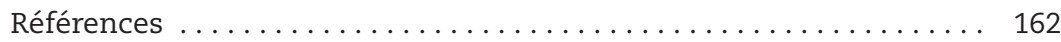

Chapitre 5. Mesurer le personnel de R-D : Personnes occupées et contributeurs externes . . . . . . . . . . . . . . 163

5.1. Introduction. . . . . . . . . . . . . . . . . . . . . . . . . . . . . . . 164

5.2. Population couverte et définitions du personnel de R-D . . . . . 165

5.3. Unités de mesure recommandées . . . . . . . . . . . . . . 181

5.4. Ventilation recommandée des données globales

du personnel de R-D . . . . . . . . . . . . . . . . . . . . . . 189

Références. . . . . . . . . . . . . . . . . . . . . . . . . . . . . . 195

Chapitre 6. Mesurer la R-D : Méthodes et procédures . . . . . . . . . . 197

6.1. Introduction. . . . . . . . . . . . . . . . . . . . . . 198

6.2. Unités . . . . . . . . . . . . . . . . . . . . . . 199

6.3. Secteurs institutionnels. . . . . . . . . . . . . . . . 201

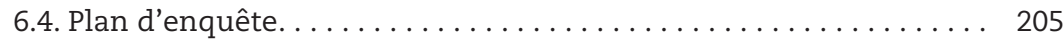

6.5. Collecte des données . . . . . . . . . . . . . . . . . . . . . . . . . . 210

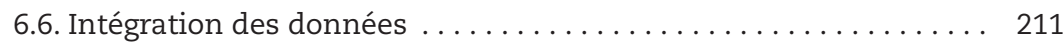

6.7. Vérification des données recueillies et imputation . . . . . . . . 211

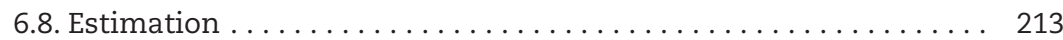

6.9. Validation des résultats $\ldots \ldots \ldots \ldots \ldots \ldots \ldots \ldots \ldots \ldots \ldots \ldots \ldots \ldots \ldots$

6.10. Communication des données à l'OCDE et à d'autres organisations internationales. . . . . . . . . . . . . . . . . . 214

6.11. Remarques finales sur la qualité des données . . . . . . . . 215

Références. . . . . . . . . . . . . . . . . . . . . . . . . . . . . . . . . . 215

Partie II

Mesurer la R-D : Directives sectorielles

Chapitre 7. R-D du secteur des entreprises . . . . . . . . . . . . 219

7.1. Introduction. . . . . . . . . . . . . . . . . . . 220

7.2. Champ couvert par le secteur des entreprises............ 220 
7.3. Unités statistiques et unités déclarantes . . . . . . . . . 222

7.4. Classifications institutionnelles des unités statistiques....... . 224

7.5. Indicateurs des activités de R-D dans le secteur des entreprises . . 228

7.6. Répartition fonctionnelle des dépenses intérieures de R-D des entreprises (DIRDE) . . . . . . . . . . . . . . . . . . . . . . . . 229

7.7. Répartition fonctionnelle de la R-D extra-muros dans le secteur

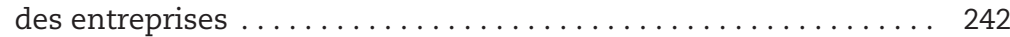

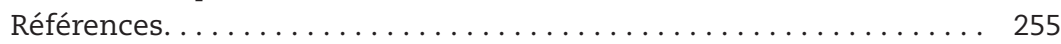

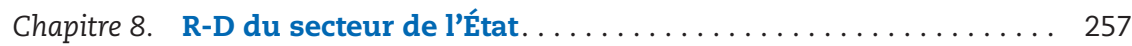

8.1. Introduction. . . . . . . . . . . . . . . 258

8.2. L'étendue du secteur de l'État à des fins de mesure de la R-D . . . . 258

8.3. Identification de la R-D dans le secteur de l'État . . . . . . . 266

8.4. Mesure des dépenses et du personnel de R-D dans le secteur

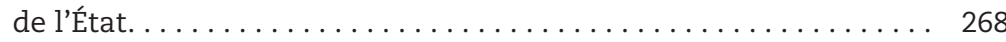

8.5. Méthodes pour compiler les dépenses et le personnel

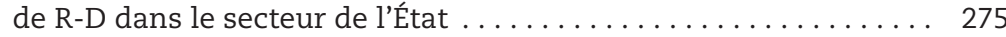

8.6. Mesure du financement public de l'exécution de la R-D . . . . . . 278

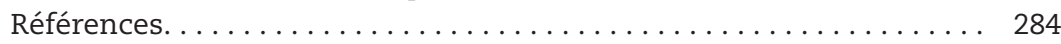

Chapitre 9. La R-D du secteur de l'enseignement supérieur . . . . . . 285

9.1. Introduction. . . . . . . . . . . . . . . . . . . . . 286

9.2. Champ couvert par le secteur de l'enseignement supérieur . . . . . 287

9.3. Identification de la R-D dans le secteur de l'enseignement supérieur. . . . . . . . . . . . . . . . . . 293

9.4. Mesure des dépenses et du personnel dans le secteur de l'enseignement supérieur. . . . . . . . . . . . . . . . . . . . . 297

9.5. Méthodes de compilation des dépenses et des données relatives au personnel de R-D dans le secteur de l'enseignement

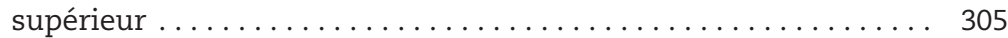

9.6. Liens avec les statistiques de l'éducation . . . . . . . . . . 315

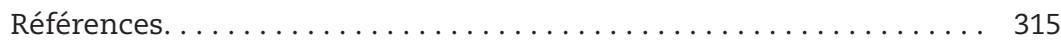

Chapitre 10. La R-D du secteur privé sans but lucratif. . . . . . . . . 317

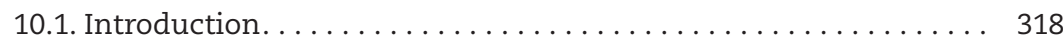

10.2. Étendue du secteur privé sans but lucratif . . . . . . . . . 318

10.3. Classifications institutionnelles recommandées pour le secteur privé sans but lucratif. . . . . . . . . . . . 321

10.4. Identification de la R-D dans le secteur privé sans

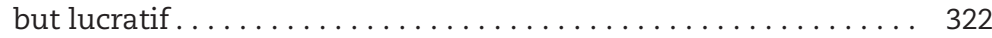

10.5. Mesure des dépenses et du personnel de R-D dans

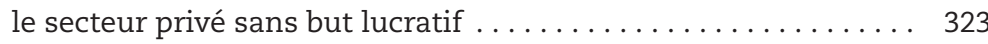

10.6. Conception de l'enquête et collecte des données dans le secteur privé sans but lucratif $\ldots \ldots \ldots \ldots \ldots \ldots \ldots . \ldots \ldots$

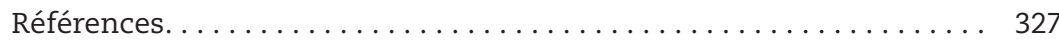


Chapitre 11. Mesurer la mondialisation de la R-D . . . . . . . . . . . . . 329

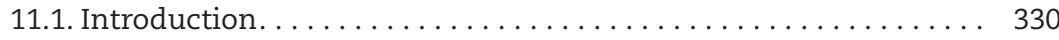

11.2. Mesure de la mondialisation de la R-D des entreprises . . . . . 331

11.3. Financement international de la R-D faisant intervenir des EMN . . . . . . . . . . . . . . . . . . . 337

11.4. Élaboration, compilation et publication des statistiques

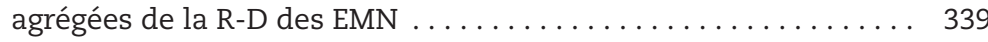

11.5. Les échanges de services de R-D . . . . . . . . . . . . 342

11.6. Mesure de la mondialisation de la R-D à l'extérieur

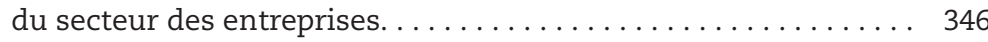

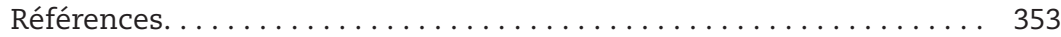

Partie III

Mesurer le soutien public en faveur de la R-D

Chapitre 12. Crédits budgétaires publics de R-D . . . . . . . . . 357

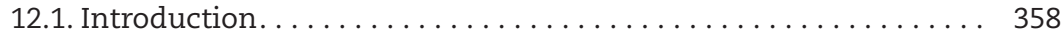

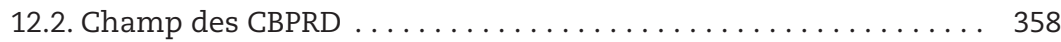

12.3. Sources des données budgétaires relatives aux CBPRD

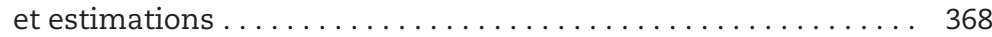

12.4. Répartition par objectif socio-économique .......... 371

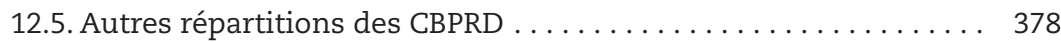

12.6. Utilisation des données de CBPRD . . . . . . . . . . . . . 379

Références............................. 382

Chapitre 13. Mesurer l'allégement fiscal en faveur de la R-D. . . . . . . 383

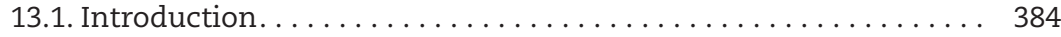

13.2. Allégement fiscal au titre des dépenses de R-D . . . . . . . . 385

13.3. Champ des statistiques relatives à l'allégement fiscal au titre des dépenses de R-D . . . . . . . . . . . . . . . . 387

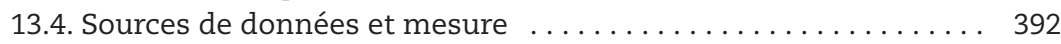

13.5. Ventilations prioritaires des statistiques relatives

à l'allégement fiscal au titre des dépenses de R-D . . . . . . . . . . 398

Références. . . . . . . . . . . . . . . . . . . . . . 399

Annexe 1 Bref historique et genese du manuel . . . . . . . . . . . . . 401

Genèse. . . . . . . . . . . . . . . . . . . . . . . . . . . . . . . . . . . . 401

Première édition. . . . . . . . . . . . . . . . . . . . . . . . . . 402

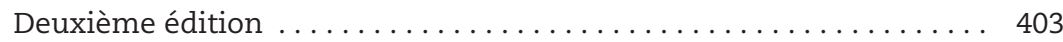

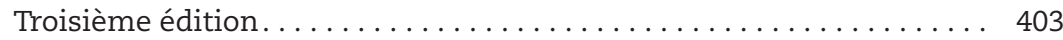

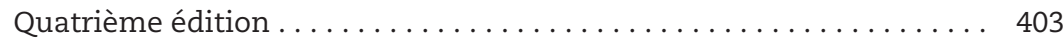

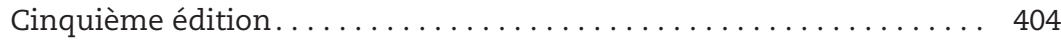

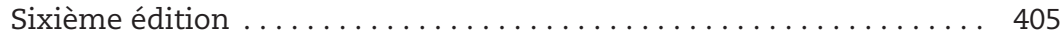


Principaux contributeurs aux éditions précédentes . . . . . . . . 406

Références. . . . . . . . . . . . . . . . . . . . . . . . . . 407

Annexe 2 Glossaire des termes . . . . . . . . . . . . . . . . . . . . 409

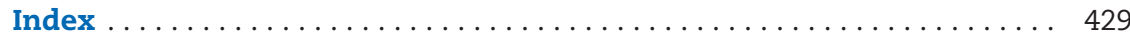

\section{Tableaux}

2.1. Exemples de questions permettant d'établir si un projet relève de la R-D............................. 52

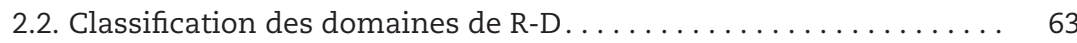

2.3. Limites de la $\mathrm{R}-\mathrm{D}$, de l'innovation et d'autres

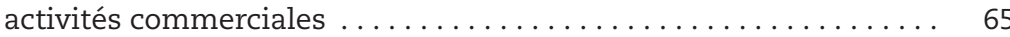

3.1. Correspondance approchée entre les secteurs institutionnels du SCN et ceux du Manuel de Frascati. . .

3.2. Exemple simplifié de structure possible pour classer les unités statistiques selon différents critères . . . . . . . . . . . . . . . . . . 104

4.1. Synthèse des catégories de dépenses de R-D intra-muros . . . . . . . . 132

4.2. Intersection entre l'exécution et le financement des travaux de R-D

4.3. Sources de financement sur lesquelles des informations doivent être recueillies lors des enquêtes auprès des exécutants de R-D . . . 144

4.4. Dépenses intérieures brutes de R-D (DIRD) . . . . . . . . . . . 158

4.5. Dépenses nationales brutes de R-D (DNRD) $\ldots \ldots \ldots \ldots \ldots \ldots \ldots 16$

5.1. Catégories de personnel à prendre en compte dans la mesure du personnel participant aux activités de R-D intra-muros d'une unité statistique . . . . . . . . . . . . . . 169

5.2. Classification et comptabilisation du personnel

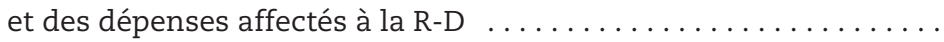

5.3. Cohérence des données sur la R-D . . . . . . . . . . . . . . . . 187

5.4.a. Total national du personnel de R-D selon le secteur et la situation au regard de l'emploi . . . . . . . . . . . . . . . . . . 193

5.4.b. Total des chercheurs selon le secteur et la situation au regard de l'emploi.

5.4.c. Total national du personnel de R-D occupé selon le secteur et la fonction au sein de la R-D. . . . . . . . . . . . . . . . . . . . . . . . 194

5.4.d. Total national des chercheurs occupés selon le secteur et l'âge . . . . 194

5.4.e. Total national des chercheurs employés selon le secteur et le niveau d'études . . . . . . . . . . . . . . . . . . . . . . . . . . 195

7.1. Identification des sources de financement de la R-D intra-muros

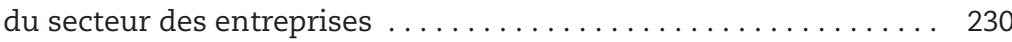

7.2. Classifications sur la base de l'activité proposées pour le secteur

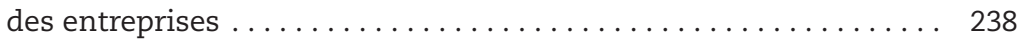

8.1. Les composantes et les limites du secteur de l'État dans le Manuel de Frascati. . . . . . . . . . . . . . . . . . . . . 
8.2. Source des financements à collecter dans les enquêtes sur l'exécution de la R-D dans le secteur de l'État. . . . . . . . . . . 271

8.3. Vue d'ensemble des transferts de fonds pour un organisme d'État, exécutant et bailleur de fonds de R-D . . . . . . . . . . 284

9.1. Profils d'établissements d'enseignement supérieur . . . . . . . . . . 292

9.2. Classification des activités des enseignants et étudiants au niveau 8 (doctorat) et au niveau 7 (master) de la CITE. . . . . . . . 295

10.1. Traitement des différentes catégories d'institutions sans but lucratif (ISBL) . . . . . . . . . . . . . . . . . . . . 319

11.1. Scénarios relatifs à la déclaration potentielle des flux de R-D des EMN par rapport aux flux effectifs dans les enquêtes sur les DIRDE et le commerce des services. . . . . . . . . . . . . 345

12.1. Classification des objectifs socio-économiques applicables aux CBPRD . . . . . . . . . . . . . . . . . . . . . . 373

12.2. Modèle indicatif de déclaration des CBPRD . . . . . . . . . . . 381

\section{Graphiques}

3.1. Arbre de décision pour l'affectation des unités institutionnelles

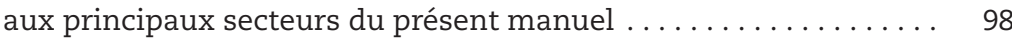

3.2. Représentation schématique des secteurs institutionnels nationaux du Manuel de Frascati et des cas frontières . . . . . . . . . . . 105

4.1. Flux de financement du point de vue d'un exécutant de travaux de R-D . . . . . . . . . . . . . . . . . . . . . . . . . . . . 140

9.1. Cadre de compilation des statistiques du secteur

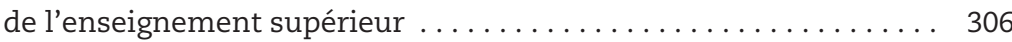

11.1. Exemple indicatif de relations de propriété entre membres d'EMN et terminologie correspondante................. 337

11.2. Agrégation des dépenses courantes1 et du personnel de R-D des EMN et des autres entreprises au sein d'un pays déclarant, et source des financements. . . . . . . . . . . . . . . 340

\section{Suivez les publications de l'OCDE sur :}

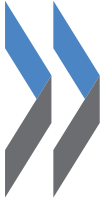

http://twitter.com/OECD_Pubs

http://www.facebook.com/OECDPublications

in. http://www.linkedin.com/groups/OECD-Publications-4645871

http://www.youtube.com/oecdilibrary

http://www.oecd.org/oecddirect/ 


\section{Abréviations et acronymes}

ADN

AEMN

AIE

AST

CBPRD

CE

CEE-ONU

CERN

CITE

CITI

CITP

CPST

CSSP

DIRD

DIRDE
Acide désoxyribonucléique

Activité des EMN

Agence internationale de l'énergie

Activités scientifiques et technologiques

Crédits budgétaires publics de R-D

Commission européenne

Commission économique des Nations Unies

pour l'Europe

Organisation européenne pour la recherche nucléaire

Classification internationale type de l'éducation

Classification internationale type, par industrie, de toutes les branches d'activité économique

Classification internationale type des professions

COFOG Classification des fonctions des administrations publiques

Nomenclature des fonctions des institutions sans but lucratif au service des ménages

Comité de la politique scientifique et technologique de l'OCDE

Comité des statistiques et de la politique statistique de l'OCDE

Dépenses intérieures brutes de R-D

Dépenses intérieures de R-D des entreprises 


\begin{tabular}{|c|c|}
\hline DIRDES & $\begin{array}{l}\text { Dépenses intérieures de R-D de } \\
\text { l'enseignement supérieur }\end{array}$ \\
\hline DIRDET & Dépenses intérieures de R-D de l'État \\
\hline DIRDISBL & $\begin{array}{l}\text { Dépenses intérieures de R-D des institutions } \\
\text { sans but lucratif }\end{array}$ \\
\hline DNRD & Dépenses nationales brutes de R-D \\
\hline EMN & Entreprise multinationale \\
\hline ETP & Équivalent temps plein \\
\hline Eurostat & $\begin{array}{l}\text { Direction générale Statistiques de la } \\
\text { Commission européenne }\end{array}$ \\
\hline FATS & Statistiques sur les filiales étrangères \\
\hline FGU & Fonds généraux des universités \\
\hline FMI & Fonds monétaire international \\
\hline GENIST & $\begin{array}{l}\text { Groupe de travail des experts nationaux } \\
\text { sur les indicateurs de la science et de la } \\
\text { technologie }\end{array}$ \\
\hline ICSU & Conseil international pour la science \\
\hline IDE & Investissement direct étranger \\
\hline ISBL & Institution sans but lucratif \\
\hline ISBLSM & $\begin{array}{l}\text { Institution sans but lucratif au service des } \\
\text { ménages }\end{array}$ \\
\hline ISU & Institut de statistique de l'UNESCO \\
\hline NABS & $\begin{array}{l}\text { Nomenclature pour l'analyse et la } \\
\text { comparaison des budgets et programmes } \\
\text { scientifiques }\end{array}$ \\
\hline OCDE & $\begin{array}{l}\text { Organisation de coopération et de } \\
\text { développement économiques }\end{array}$ \\
\hline OIT & Organisation internationale du Travail \\
\hline OMC & Organisation mondiale du commerce \\
\hline OMT & Organisation mondiale du tourisme \\
\hline ONG & Organisation non gouvernementale \\
\hline ONU & Organisation des Nations Unies \\
\hline OSE & Objectif socio-économique \\
\hline PIB & Produit intérieur brut \\
\hline
\end{tabular}


PME Petites et moyennes entreprises

PSBL Secteur privé sans but lucratif

R-D Recherche et développement expérimental

RDD Recherche, développement et démonstration (définition de l'AIE)

SCN Système de comptabilité nationale

TIC Technologies de l'information et des communications

TVA Taxe sur la valeur ajoutée

UAE Unité d'activité économique

UE Union européenne

UNESCO Organisation des Nations Unies pour l'éducation, la science et la culture 



\section{Chapitre 1}

\section{Introduction aux statistiques de R-D et au Manuel de Frascati}

Référence internationale depuis plus d'un demi-siècle, le Manuel de Frascati s'est à présent imposé dans le monde entier. Les statistiques de la recherche et du développement expérimental (R-D) conformes aux orientations qu'il contient font de plus en plus autorité et se généralisent dans un large éventail de domaines d'intervention des pouvoirs publics et dans un grand nombre de pays non membres de l'OCDE. Le Manuel pose les bases d'un langage commun pour traiter de la $R-D$ et de ses résultats. Compte tenu de sa popularité et du fait que d'autres instruments internationaux et les réglementations nationales se fondent sur sa terminologie, très peu de changements ont été apportés aux définitions de la R-D et de ses composantes formulées dans les éditions précédentes. On s'est plutôt attaché à préciser les contours de la $\mathrm{R}-\mathrm{D}$ et à répondre aux impératifs nouvellement posés par les statistiques de R-D. Ainsi, la décision de traiter les dépenses de R-D au titre des dépenses d'investissement dans le Système de comptabilité nationale (SCN) a rendu nécessaire de prêter une plus grande attention aux flux de financement de la R-D. En outre, plusieurs chapitres ont été ajoutés, dont un sur les incitations fiscales en faveur de la $R-D$, qui se généralisent, et un autre consacré à la mondialisation et à ses répercussions sur les statistiques de R-D. Afin que l'ouvrage reste d'actualité, nombre d'informations complémentaires sont accessibles en ligne. Le présent chapitre fait office d'introduction. 


\subsection{Objectifs et historique du Manuel de Frascati}

1.1 Depuis plus d'un demi-siècle le Manuel de Frascati de l'OCDE fait figure de référence mondiale en matière de recueil et de communication de statistiques comparables à l'échelle internationale sur les ressources financières et humaines de la recherche et du développement expérimental. La collaboration nouée entre les pays de l'OCDE, et d'autres, pour définir et appliquer les recommandations formulées dans le présent manuel a débouché sur la création d'une précieuse source d'informations à l'intention des responsables de l'élaboration des politiques dans les domaines de la science, de la recherche et de l'économie. Adoptées et adaptées dans un grand nombre de pays, les définitions énoncées ici constituent les rudiments du langage commun utilisé dans les débats sur l'action à mener dans divers domaines - science et technologie, développement économique, budget, fiscalité ou encore réglementation - ainsi que dans la formulation d'orientations générales traitant de comptabilité, d'investissement et de statistiques des échanges, entre autres exemples.

1.2 L'intérêt de mesurer la recherche et le développement expérimental (synonymes ici de R-D) tient aux formidables potentialités offertes en termes de croissance économique et de prospérité. Les connaissances nouvelles issues de la R-D peuvent servir à satisfaire des besoins nationaux et à relever des défis planétaires ainsi qu'à améliorer le bien-être sociétal. Que ce soit dans le monde développé ou en développement, la R-D touche à maints égards les individus, les institutions, les secteurs économiques et les pays. C'est pourquoi les indicateurs établis conformément au Manuel de Frascati orientent et éclairent l'examen de ces questions importantes.

1.3 Depuis toujours, le Manuel de Frascati a été rédigé par et pour les experts nationaux qui recueillent et diffusent des statistiques nationales relatives à la R-D et répondent aux enquêtes de l'OCDE, de l'UE, de l'UNESCO et d'autres organisations internationales. Bien que riche en exemples, il reste un document technique conçu pour servir d'ouvrage de référence. N'ayant jamais eu vocation à devenir contraignant, il vise plutôt à proposer des lignes directrices consensuelles issues d'un long travail de réflexion. Après l'adoption d'une première série de lignes directrices, en 1963, lors d'une conférence d'experts nationaux des pays membres de l'OCDE tenue à Frascati (Italie), le Manuel a ensuite été révisé à cinq reprises afin d'être ajusté aux problèmes de mesure rencontrés, aux nouveaux besoins des utilisateurs et aux meilleures pratiques établies dans le monde. Cet exercice de révision et de concertation avec les utilisateurs témoigne de la capacité des auteurs à apprendre de façon continuelle. 
1.4 Dès le début, les définitions de la R-D énoncées dans le Manuel ont été reprises dans d'autres ouvrages et le Manuel de Frascati se situe dans le cadre d'un ensemble de manuels sur les statistiques de la science, de la technologie et de l'innovation, plus connu sous le nom de "famille Frascati ». Les lignes directrices énoncées dans ces différents manuels se recoupent et évoluent constamment.

\section{Principaux objectifs de la révision}

1.5 Fruit d'une sixième révision, la présente version du Manuel de Frascati diffère grandement des précédentes en termes de présentation, de champ d'étude et de niveau de détail. Les grandes nouveautés, modifications et améliorations apportées sont exposées, pour chaque chapitre, dans la deuxième moitié de cette introduction. D'emblée, on notera que la définition de la R-D présentée dans le chapitre 2 du présent manuel cadre avec celle de l'édition précédente (OCDE, 2002) et s'applique aux mêmes activités. La différence est qu'elle a gagné en clarté et en précision. En effet, tout a été fait pour limiter au maximum la révision des grandes séries temporelles d'indicateurs de la R-D. Cela dit, la marche à suivre pour s'aligner sur les recommandations internationales étant désormais plus claires, il est possible que certains pays doivent revoir en conséquence leur façon de procéder. L'autre objectif et résultat escompté du présent manuel est que les éclaircissements apportés faciliteront la tâche des décideurs s'agissant d'évaluer et d'interpréter les statistiques officielles de R-D et les données émanant de sources connexes qu'elles soient comptables, fiscales, commerciales et autres.

1.6 À l'inverse des dernières éditions, celle-ci a été remaniée en profondeur. Un certain nombre de facteurs ont pesé dans la décision d'étendre la couverture et de compléter les orientations générales concernant les méthodes de recueil à appliquer, la nature des données à collecter et les motivations de l'exercice. Ces facteurs sont principalement les suivants :

- Depuis sa conception, le Manuel est étroitement lié au Système de comptabilité nationale (SCN). Or, dans le SCN 2008, il est explicitement recommandé de reconnaître les dépenses de R-D comme faisant partie de la formation de capital, autrement dit de la traiter comme un investissement, sur la base des définitions et données relatives à la R-D fournies dans le Manuel de Frascati. Cette nouveauté a assis le Manuel de Frascati dans l'architecture des normes des statistiques de comptabilité nationale, tout en rendant indispensable d'opérer un certain nombre d'ajustements pour aider les statisticiens nationaux à exploiter les données de R-D fondées sur Frascati. En conséquence, l'exercice de révision a notamment consisté à étudier la faisabilité de plusieurs des recommandations énoncées dans le guide intitulé OECD Handbook on Deriving Capital Measures of Intellectual Property Products (OCDE, 2009a). La correspondance avec la nomenclature du SCN et les données nécessaires sont exposées en détail dans le chapitre 3, puis précisées tout au long du Manuel. 
- À mesure que l'utilisation du Manuel à des fins statistiques et décisionnelles s'est généralisée, les demandes de clarification des concepts, définitions et méthodes de mesure se sont multipliées, témoignant généralement de priorités et d'intérêts divergents. L'objet du présent manuel n'est pas de prendre position mais de proposer des orientations claires sur les définitions et méthodes de recueil jugées préférables et recommandées, qui devraient - ou peuvent - être appliquées de façon universelle. Pour y parvenir, il a fallu élargir le champ d'étude de façon à étendre l'éventail des domaines décisionnels susceptibles d'exploiter les statistiques de R-D tout en imposant le moins de changements possible, de manière à préserver la stabilité des séries rétrospectives de base. Ainsi, l'objet du chapitre 13, consacré à l'allégement fiscal en faveur de la R-D, ne se limite plus aux traditionnels aspects de la quantification des dépenses de R-D des entreprises.

- Le Manuel de Frascati est l'ouvrage de référence sur la R-D dans des pays très divers de par le stade de développement atteint, la structure de l'économie, le système de recherche ou encore l'architecture statistique. L'OCDE voyant sa composition s'élargir et ses liens de coopération s'intensifier avec des non-membres, l'objet du présent manuel est de fournir des pistes pour repérer et recueillir les données de R-D dignes d'intérêt en fonction du profil économique du pays considéré et de son système de recherche. On s'est tout particulièrement efforcé de comprendre la ligne de conduite que certains pays semblent avoir adoptée en contradiction avec les recommandations énoncées dans le Manuel de Frascati et de mieux formuler les buts poursuivis à travers les lignes directrices existantes. Enfin, pour affiner les recommandations liées au recueil des données, la présente édition a été enrichie de chapitres par secteur (chapitres 7 à 10).

- La diversité des mutations en cours dans l'organisation des activités de R-D et les problèmes qui en découlent font l'objet d'une prise de conscience grandissante. Ces changements concernent le rôle de la R-D dans la mondialisation des chaînes de valeur ; l'adoption de nouveaux modes d'organisation qui transcendent les entités, secteurs ou pays individuels; et les nouvelles formes de soutien financier à la R-D. D'où l'apparition de nouveaux besoins du côté des utilisateurs et la nécessité de revoir et d'enrichir les méthodes de recueil de données. Compte tenu de l'ampleur du phénomène, les orientations proposées ici visent, dans la mesure du possible, à apporter des solutions aux nouveaux enjeux statistiques. Ainsi, des informations nouvelles sont fournies pour mesurer les flux de R-D (chapitre 4) et le volume de personnel de R-D (chapitre 5) selon que l'on se place du point de vue intra-muros ou extra-muros. De même, un tout nouveau chapitre (11) est consacré à différents aspects de la mondialisation de la R-D.

- Il était indispensable d'aborder les problèmes et les possibilités nouvelles rencontrés sur le plan méthodologique. S'il importe de disposer d'orientations générales sur ces questions pour pouvoir collecter tous types de statistiques économiques, les particularités de la $R-D$, tant du point de vue de ses activités 
(qui sont difficiles à définir, revêtent souvent un caractère non exclusif et prennent la forme de services immatériels) que sur le plan statistique (il s'agit d'un phénomène rare, très asymétrique et souvent occasionnel), justifient l'établissement de recommandations spéciales sur les méthodes à suivre. Il convient par ailleurs de tenir compte des nouveaux usages qu'il est fait des données de R-D, notamment pour analyser les liens de causalité entre moyens et résultats à l'aide de microdonnées mises en concordance avec les données de sources complémentaires, dans le respect des règles de confidentialité. Le chapitre 6 , qui traite de façon plus détaillée que par le passé de la méthodologie statistique, et les directives sectorielles énoncées dans les chapitres correspondants abordent des questions fondamentales, telles que les problèmes à surmonter pour maintenir les taux de réponse aux niveaux souhaités et rendre l'exercice moins contraignant pour les répondants ; l'exploitation des sources de données administratives ; et les impératifs à respecter pour que les indicateurs nationaux de R-D puissent faire l'objet de comparaisons internationales et être cohérents dans le temps. En se conformant à ces recommandations, les pays seront à même d'appliquer les meilleures pratiques statistiques et de tirer pleinement parti des microdonnées en résultant.

- Enfin, du point de vue pratique, il était nécessaire de rendre compte de l'évolution des systèmes et pratiques de classification statistique, notamment des classifications de l'ONU concernant les branches d'activité (CITI), l'enseignement (CITE) et le SCN 2008. La plupart des guides statistiques renvoient à des pratiques et concepts en partie obsolètes dans la mesure où ils ont été revus avant l'établissement de la présente édition du Manuel de Frascati. Celle-ci met davantage à profit les ressources consultables en ligne, ce qui permettra de rester au fait de l'évolution des nomenclatures.

1.7 Le reste du chapitre 1 résume le champ d'étude et le contenu du Manuel afin de faciliter l'exploitation et l'interprétation des données lors de l'application des lignes directrices. Y sont également exposées les raisons pour lesquelles certains types de données sont ou ne sont pas recueillis et les problèmes de comparabilité qui en découlent.

\section{Genèse du Manuel de Frascati}

1.8 Depuis plus d'un demi-siècle, la définition de la recherche et du développement expérimental (R-D) utilisée pour recueillir des données sur les ressources financières et humaines qui lui sont consacrées est celle donnée par le Manuel de Frascati. L'un des buts premiers du Manuel était de favoriser la compilation de données permettant de suivre l'évolution dans le temps des moyens engagés et de comparer la situation dans plusieurs pays.

1.9 À mesure que les statistiques de R-D se sont uniformisées et que leur utilisation s'est intensifiée, des questions ont surgi quant à la possibilité d'établir des comparaisons internationales et de classer les pays en fonction de leurs 
résultats de R-D. C'est ainsi que les États ont commencé à fixer des objectifs chiffrés et à prendre des initiatives en vue de soutenir la R-D et des secteurs d'importance stratégique ou encore de réaliser des ambitions stratégiques. Les statistiques de R-D se sont désormais imposées dans la politique de la science. Elles occupent également une place de choix dans la politique économique depuis que l'on s'est rendu compte que le savoir et, en particulier, les nouvelles connaissances étaient un facteur essentiel de croissance économique et de développement. Aujourd'hui encore, le Manuel donne des clés pour répondre à un certain nombre de questions même si le contexte a fortement évolué en vue de définir, en valeur absolue et en proportion, la somme des efforts de R-D à fournir au niveau national.

1.10 Pour recenser les moyens destinés à la R-D, le Manuel de Frascati propose, comme principe de base, d'étudier les activités des exécutants de la R-D, ce qui suppose entre autres de définir les sources de financement. En considérant conjointement les entités exécutantes de $\mathrm{R}-\mathrm{D}$, leurs résultats et leur financement, sans oublier leur évolution dans le temps, on obtient une vue d'ensemble du système de R-D du pays considéré ainsi que des relations qu'il entretient avec le reste du monde. Les États étant à la fois des exécutants et de grands bailleurs de fonds de R-D, le Manuel contient également des orientations sur la marche à suivre pour recueillir des renseignements utiles sur les crédits publics destinés à financer la R-D.

$1.11 \mathrm{Vu}$ l'importance stratégique des statistiques de R-D, les acteurs chargés de recueillir les données nécessaires à leur établissement sont tenus de veiller à ce qu'elles contribuent à l'élaboration et à l'évaluation des politiques tout en étant précises, d'actualité et accessibles. Le présent manuel les y aide en fournissant des définitions utiles ainsi qu'une analyse de leurs champs d'application et délimitations, fondées sur les meilleures pratiques établies par d'autres organisations et dans les pays membres et partenaires de l'OCDE. L'historique de chaque révision du Manuel est retracé en détail dans l'annexe 1, à laquelle on se reportera pour traiter les séries de données de R-D couvrant de longues périodes.

\section{Des définitions stables au service des utilisateurs}

1.12 Tout au long du vaste exercice de consultation engagé pour élaborer cette nouvelle version du Manuel, les utilisateurs ont insisté sur l'importance de définir la $R-D$ et de conserver des séries de données rétrospectives suivant une démarche cohérente. Le Manuel a été révisé en considération du fait qu'il est cité et que ses définitions sont reprises dans la législation de nombreux pays. Même s'il ne s'agissait pas de sa vocation première, il convenait d'en tenir compte. Dans un souci de stabilité, les définitions de base de la R-D ont donc été préservées autant que possible : leur révision a uniquement consisté à apporter quelques modifications mineures d'ordre linguistique et à reformuler dans des termes plus clairs la façon de procéder pour décrire le but recherché à travers de 
nouvelles applications. En conséquence, il ne devrait pas y avoir de problème du fait que le droit interne de certains pays, des classifications et autres systèmes statistiques renvoient à ces définitions.

1.13 Bien que la définition de base de la R-D soit conservée, celle de ses composantes recherche fondamentale, recherche appliquée et, surtout, développement expérimental - ont subi de légères transformations, car il était nécessaire de clarifier la distinction à établir entre la R-D et d'autres types d'activités d'innovation.

1.14 Sont présentés ici cinq critères de base au regard desquels une activité peut être considérée comme relevant de la R-D. Certes, ces critères figuraient déjà dans l'édition précédente, mais pas de façon dispersée sans pouvoir faciliter l'interprétation de la définition. Un certain nombre de pays en ont vérifié la validité tout au long de l'exercice de révision dans le cadre d'entretiens avec des exécutants potentiels de R-D.

1.15 Une autre partie du travail a consisté à mieux mettre en adéquation les informations relatives aux ressources financières et humaines consacrées à la R-D, à savoir les dépenses de R-D et le personnel de R-D. La définition de " personnel de R-D » a été quelque peu retouchée en vue de clarifier deux points : d'une part, le traitement des étudiants en doctorat et en master ; et, d'autre part, la distinction à établir entre le personnel externe et les personnes occupées au sein de l'unité statistique.

\section{Intrants et résultats de la $\mathrm{R}-\mathrm{D}$}

1.16 Dans le Manuel, la caractéristique déterminante de la R-D est qu'elle est génératrice de connaissances nouvelles indépendamment du dessein initialement poursuivi, qu'il s'agisse d'obtenir un avantage économique, de relever des défis sociétaux ou simplement d'acquérir du savoir. Cette intentionnalité sert ici à distinguer le développement expérimental de la recherche fondamentale ou appliquée, de même qu'il convient de recenser et, si possible, de mesurer les différents types de résultats de la R-D.

1.17 Cependant, cette dernière tâche est compliquée en raison des différents facteurs qui interviennent dans la répartition et l'exploitation des connaissances au sein de l'économie ou qui concernent les moyens complémentaires nécessaires à l'obtention de résultats. Les résultats et effets de la R-D, quels qu'ils soient, peuvent n'être obtenus qu'à long terme, de façon éparpillée et par d'autres acteurs que des exécutants de R-D. Seule une fraction est directement repérable et mesurable dans le cadre du recueil d'informations sur les activités de R-D et leur financement. Un certain nombre de recommandations sont formulées ici pour faciliter la démarche, en particulier en ce qui concerne la collecte de microdonnées relatives à la R-D, l'utilisation des registres liés et les classifications utiles pour analyser les flux de connaissances. 


\section{Famille Frascati de manuels}

1.18 Bien qu'intervenant dans l'ensemble de l'économie, la R-D se distingue de la grande famille des activités scientifiques mais aussi des activités économiques dont elle relève. D'emblée, il était prévu que l'OCDE élabore un ensemble de lignes directrices pour encadrer la mesure des activités scientifiques, technologiques et d'innovation. D'autres manuels sont venus au fil du temps compléter ce cadre. C'est le cas, entre autres exemples, du Manuel de l'OCDE sur les statistiques des brevets (OCDE, 2009b) et du Manuel d'Oslo (OCDE/Eurostat, 2005), qui contient des principes directeurs pour le recueil et l'interprétation des données sur l'innovation.

1.19 Un autre but de la révision était de bien circonscrire l'objet des différents ouvrages qui composent la famille de manuels à laquelle appartient le Manuel de Frascati.

1.20 Chaque manuel et les statistiques qui en découlent répondent à des objectifs distincts. Pour que ces objectifs soient atteints avec succès, il convient de se demander à quels niveaux d'agrégation et pour quels échantillons ou populations d'observations, il est possible de combiner et d'analyser conjointement les statistiques provenant de différentes sources.

1.21 L'OCDE s'emploie activement à comparer et à vérifier les expériences nationales qui ont été couronnées de succès avant de recommander leur reproduction à l'échelle internationale. La présente édition du Manuel contient une pléthore d'éléments utiles à cet égard.

\section{Nouvelles applications et nouveaux utilisateurs des statistiques de R-D}

1.22 L'ambition principale de la première édition du Manuel de Frascati était d'obtenir l'adoption de pratiques uniformes dans le pays ainsi qu'une amélioration de la qualité et de la comparabilité des statistiques de R-D. Bien que cet objectif soit toujours d'actualité, force est de constater qu'en cinquante ans, la définition de la R-D s'est glissée dans le droit interne de plusieurs pays, voire, parfois après quelques altérations, dans des dispositions législatives et réglementaires touchant des questions fiscales ou d'autres types de soutien financier. Les activités de R-D font désormais partie intégrante du champ d'étude des statistiques officielles et leur mesure constitue un élément essentiel dans l'élaboration des politiques. Par ailleurs, le Manuel de Frascati est utilisé dans le cadre de la formation des statisticiens et autres utilisateurs des données, de cours universitaires et de la recherche en science de la politique scientifique. Son rôle est donc plus étendu qu'initialement prévu, à savoir proposer des pratiques uniformes pour la conduite d'enquêtes sur la R-D.

\section{Frascati et le Système de comptabilité nationale}

1.23 En tant qu'ouvrage de référence en statistique, le Manuel de Frascati doit être en phase avec les autres instruments de normalisation en vigueur, au premier rang desquels figure le SCN. La présente édition propose des définitions de la R-D 
qui lui sont propres. En revanche, pour ce qui est des secteurs, il reprend celles du SCN, à la différence que l'enseignement supérieur est considéré à part, alors que, dans le SCN, les établissements d'enseignement supérieur peuvent relever de tous les secteurs. Cette harmonisation était d'autant plus importante que le SCN 2008 est le premier instrument dans lequel il est recommandé de traiter les dépenses de R-D, non pas au titre des dépenses, mais comme étant à l'origine de la création d'un actif fixe. Dans le SCN 2008, la R-D est donc considérée comme une activité de production et d'investissement, ce qui rejaillit sur la manière de mesurer le PIB et d'interpréter le rôle de la R-D dans la croissance économique en comptabilité de la croissance. Cette évolution du SCN tient aux comptes satellites de la R-D mentionnés en annexe à l'édition précédente du Manuel et à la décision de fonder les nouveaux indicateurs des dépenses d'investissement et du PIB sur la définition de la R-D donnée par le Manuel de Frascati et sur les données en découlant. En conséquence, le présent manuel reprend un certain nombre des recommandations énoncées dans le guide OECD Handbook on Deriving Capital Measures of Intellectual Property Products (OCDE, 2009a), dont la section consacrée à la R-D a été conjointement rédigée par des spécialistes de la comptabilité nationale et des statistiques de R-D afin d'aider les comptables nationaux à donner effet aux nouvelles dispositions du SCN.

1.24 Alors que l'engouement pour la R-D et l'utilisation des définitions et données y afférentes se généralisent et se diversifient, les chiffres communiqués au sujet des différents exécutants et objectifs de R-D peuvent être très variables. Il est extrêmement important de saisir et de comprendre ces disparités afin d'éviter toute utilisation abusive ou interprétation erronée des données. Le Manuel propose deux façons de procéder, aussi efficaces l'une que l'autre, pour recueillir les informations voulues : s'adresser (de préférence) aux exécutants de la R-D et (à titre complémentaire) à ceux qui la financent. Des différences sont également observées dans les données selon qu'elles découlent des lignes directrices de Frascati ou du SCN. Certes, les comptables nationaux s'appuient sur le Manuel de Frascati et d'autres sources et hypothèses pour obtenir des grandeurs des résultats de la $\mathrm{R}-\mathrm{D}$, des dépenses d'investissement et du stock de capital qui soient compatibles avec le SCN. Toutefois, des écarts apparaissent dans le cas de la R-D logicielle, qui relève de la R-D globale d'après Frascati et de la catégorie des actifs " logiciels » dans le SCN. Ces questions sont abordées dans le chapitre 4 du présent manuel. Un aperçu détaillé et actualisé des liens existant entre le SCN et le Manuel de Frascati est consultable en ligne, en complément au présent manuel, à l'adresse http://oe.cd/frascati.

\section{Autres normes internationales}

1.25 Il arrive aussi que les données comptables relatives à la R-D soient confondues avec celles fondées sur le Manuel de Frascati, dans la mesure où la R-D fait partie des domaines dont les entreprises rendent compte dans leurs états financiers, parfois même séparément, selon un certain nombre de règles et de stratégies. Le champ couvert est généralement variable, à l'instar de la 
définition retenue. Bien souvent, il n'est pas possible de comparer les deux séries de données. Cette question est examinée en détail dans le chapitre 7.

1.26 Vu la nécessité de placer la R-D dans un contexte plus large, tant du point de vue conceptuel qu'en ce qui concerne les bases de données, mais aussi de mettre en adéquation les données de R-D avec des informations supplémentaires, il est recouru le plus possible aux classifications de l'ONU : le Système de comptabilité nationale, 2008 (CE et al., 2009), la Classification internationale type, par industrie, de toutes les branches d'activité économique, CITI (Nations Unies, 2008) et la Classification internationale type de l'éducation, CITE (UNESCO-UIS, 2012).

1.27 Ces classifications extérieures sont régulièrement actualisées. Par conséquent, c'est le texte en vigueur au moment de la rédaction du présent manuel qui figure dans la version papier, mais les éventuelles mises à jour sont consultables en ligne, en complément au présent manuel.

\section{Un manuel d'envergure véritablement mondiale}

1.28 Mener des activités de R-D et utiliser les statistiques y afférentes dans l'élaboration des politiques n'est pas l'apanage des pays de l'OCDE ou des pays développés en général. Partout dans le monde, des enquêtes de R-D sont entreprises et leurs résultats exploités par les décideurs. C'est pourquoi la présente édition révisée du Manuel s'adresse tant aux pays développés qu'à ceux en développement, conformément à sa vocation de référence mondiale. Cela signifie également que, pour l'exercice de la révision, il a été fait appel au concours de pays émergents et en développement, ainsi qu'aux institutions qui les aident à se doter des capacités requises pour mesurer la R-D. Les recommandations relatives à la mesure de la R-D dans les pays en développement, qui étaient précédemment consultables en ligne, apparaissent désormais dans le corps du texte.

1.29 Le Manuel faisant figure de référence mondiale, il ne décrit pas en détail la procédure à suivre pour communiquer les données et indicateurs à l'OCDE. C'est à l'OCDE et aux pays déclarants qu'il incombe de le faire. Le Manuel précise en revanche le niveau de détail requis pour établir des statistiques comparables à l'échelle internationale.

\subsection{Aperçu général du Manuel}

1.30 Afin de faciliter la lecture du Manuel, le champ d'étude et le contenu de chacun des chapitres qui le composent sont décrits dans la présente section. C'est néanmoins le texte intégral qui fait foi.

\section{Organisation du Manuel}

1.31 Le chapitre 1 contient l'introduction du Manuel de Frascati et est suivi de douze chapitres thématiques dans lesquels des orientations concrètes sont formulées. L'historique de l'ouvrage est retracé dans l'annexe 1. Les chapitres 2 à 6 
fournissent des orientations générales à prendre en considération pour définir et mesurer la R-D dans l'ensemble de ses secteurs d'exécution et passent en revue les thèmes ci-après : concepts et définitions, secteurs institutionnels, dépenses de R-D, personnel de R-D, et méthodologies et procédures statistiques. Les cinq chapitres suivants (7 à 11) abordent des points de méthodologie et de classification spécifiques à chaque secteur d'exécution, à savoir : les entreprises, l'État, l'enseignement supérieur et le secteur privé sans but lucratif. Un cinquième secteur, le « reste du monde » (anciennement dénommé "Étranger "), fait l'objet du chapitre 11 sur la mondialisation de la $R-D$, qui passe en revue l'exécution et le financement de la R-D dans le reste du monde et contient autre nouveauté du Manuel des recommandations concernant la collecte de données relatives aux entreprises multinationales (EMN) et le commerce des services de R-D. Les deux derniers chapitres s'intéressent à la manière de mesurer le soutien public à la R-D du point de vue des bailleurs de fonds : le premier est consacré aux crédits publics en faveur de la R-D ; le second à l'allégement fiscal en faveur de la R-D. Des informations détaillées et des liens vers la version à jour des systèmes de classification extérieurs sont disponibles en ligne, en complément au présent manuel, à l'adresse http://oe.cd/frascati. Le Manuel comporte également un glossaire, dont la version actualisée est consultable en ligne.

\section{Concepts et définitions permettant d'identifier la $R-D$ (Chapitre 2)}

1.32 La R-D englobe les activités créatives et systématiques entreprises en vue d'accroître la somme des connaissances - y compris la connaissance de l'humanité, de la culture et de la société - et de concevoir de nouvelles applications à partir des connaissances disponibles.

1.33 Même exécutées par différents acteurs, les activités de R-D visant la réalisation d'objectifs particuliers ou généraux présentent un ensemble de caractéristiques communes. Ainsi, pour être considérée comme relevant de la $\mathrm{R}-\mathrm{D}$, une activité doit comporter un élément :

- de nouveauté

- de créativité

- d'incertitude

et être :

- systématique

- transférable et/ou reproductible.

1.34 La définition susmentionnée de la R-D cadre avec celle employée dans la précédente édition du Manuel de Frascati (OCDE, 2002) et couvre un éventail identique d'activités.

1.35 L'expression " recherche et développement » (R-D) englobe trois types d'activité : la recherche fondamentale, la recherche appliquée et le développement expérimental. La recherche fondamentale consiste en des travaux de recherche 
expérimentaux ou théoriques entrepris principalement en vue d'acquérir de nouvelles connaissances sur les fondements des phénomènes et des faits observables, sans envisager une application ou une utilisation particulière. La recherche appliquée consiste en des travaux de recherche originaux entrepris en vue d'acquérir de nouvelles connaissances et dirigés principalement vers un but ou un objectif pratique déterminé. Le développement expérimental consiste en des travaux systématiques fondés sur les connaissances tirées de la recherche et l'expérience pratique et produisant de nouvelles connaissances techniques visant à déboucher sur de nouveaux produits ou procédés ou à améliorer les produits ou procédés existants. Aux fins du présent Manuel, un " produit » désigne un bien ou un service, conformément à la convention retenue dans le SCN. De même, "procédé " s'entend de la transformation d'intrants en résultats et de la production de ceux-ci, ou des structures ou pratiques organisationnelles.

1.36 Bien souvent, il est judicieux et utile de classer les activités de R-D en fonction du domaine de connaissance dont elles relèvent, à savoir : sciences naturelles, ingénierie et technologie, sciences médicales et sciences de la santé, sciences agricoles et vétérinaires, sciences sociales, sciences humaines et arts.

\section{Secteurs institutionnels et classifications des statistiques de R-D (Chapitre 3)}

1.37 Ce chapitre traite des statistiques de R-D suivant le type d'unité institutionnelle (" approche institutionnelle »), en particulier les modalités de recueil et de présentation en fonction des caractéristiques générales des unités institutionnelles considérées. Ainsi, les ressources qu'une unité institutionnelle consacre à la R-D sont attribuées au secteur dont relève l'unité considérée. La classification des unités institutionnelles dans le contexte de la R-D a pour but d'obtenir une parfaite cohérence avec la définition de la R-D et les besoins explicites des utilisateurs habituels de statistiques de R-D, ainsi qu'avec les critères de classification utilisés par le Système de comptabilité nationale. Ce dernier prend en compte le critère de résidence, ainsi que le type d'activité économique, de structure du capital et de contrôle.

1.38 Aux fins du présent manuel, quatre grands secteurs (auxquels s'ajoute " le reste du monde ") sont retenus pour mesurer la R-D : les entreprises, l'État, l'enseignement supérieur et le secteur privé sans but lucratif. L'enseignement supérieur, dont les entités appartiennent, selon les critères de marché ou de contrôle public applicables dans chaque pays, aux catégories des sociétés, des administrations publiques ou des institutions sans but lucratif au service des ménages (ISBLSM) définies dans le SCN, constitue un secteur à part dans le cadre de l'étude des statistiques de R-D.

1.39 Un arbre de décision permettant de classer les unités institutionnelles par secteur est présenté au graphique 3.1 du chapitre 3. 


\section{Mesurer les dépenses de R-D : exécution et sources de financement (Chapitre 4)}

1.40 Le volume des fonds consacrés à la recherche et au développement expérimental (dépenses de R-D) intéresse fortement les responsables de l'élaboration des politiques nationales et internationales. Ces chiffres servent notamment à étudier, sous l'angle quantitatif, qui exécute et finance les activités de R-D, où, à quel niveau et à quelles fins celles-ci ont lieu, mais aussi les liens d'interdépendance et de collaboration existant entre unités institutionnelles et secteurs. Les dépenses de R-D intra-muros couvrent l'ensemble des dépenses courantes (main-d'œuvre et autres coûts inclus) et des dépenses brutes de capital fixe (terrains, constructions, machines et équipements) afférentes à la $\mathrm{R}-\mathrm{D}$ exécutée au sein d'une unité statistique au cours d'une période de référence donnée, quelle que soit la source des financements.

1.41 Une grande nouveauté du SCN 2008 réside dans le fait que la R-D y est explicitement reconnue comme relevant de la formation de capital, autrement dit comme un "investissement ". D'où la nécessité d'affiner la décomposition des dépenses de R-D, comme signalé tout au long du chapitre, notamment de formuler des orientations sur la façon de procéder pour recueillir des données détaillées sur les sources et les flux de financement de la R-D ainsi que sur les types d'opérations liées à la R-D. Il est nécessaire, en particulier, de disposer d'informations complémentaires plus approfondies pour quantifier les ventes et acquisitions de produits de R-D.

1.42 Aux fins du présent manuel, l'organisation du recueil des statistiques de R-D repose sur les concepts de base suivant (voir graphique 4.1, chapitre 4) :

- les dépenses de R-D intra-muros, qui correspondent à la somme des fonds consacrés aux activités de R-D exécutées au sein d'une unité déclarante ; et les dépenses (financements) de R-D extra-muros, qui correspondent à la somme des fonds consacrés aux activités de R-D exécutées à l'extérieur de l'unité déclarante

- les financements internes de R-D, qui correspondent à la somme des fonds consacrés à la R-D provenant directement des ressources internes de l'unité déclarante ; et les financements externes de R-D, qui correspondent à la somme des fonds consacrés à la R-D provenant de sources extérieures à l'unité déclarante

- les fonds d'échange pour la R-D, qui correspondent aux fonds qu'une unité statistique fournit à une autre unité en échange de l'exécution d'activités de $\mathrm{R}-\mathrm{D}$; et les fonds de transfert pour la R-D, qui correspondent aux moyens financiers circulant entre unités statistiques sans qu'une contrepartie soit exigée en termes de résultats de R-D.

1.43 Les dépenses intérieures brutes de R-D (DIRD) constituent le principal agrégat statistique utilisé pour décrire les activités de R-D d'un pays ; elles correspondent au total des dépenses intra-muros de la R-D exécutée sur le territoire 
national pendant une période de référence donnée. Les DIRD représentent le premier indicateur de comparaison internationale des activités de R-D.

\section{Personnel de R-D : personnes occupées et contributeurs externes (Chapitre 5)}

1.44 De manière générale, le personnel de R-D est composé de chercheurs hautement qualifiés, de spécialistes dotés d'un niveau élevé d'expérience et de formation techniques, et de personnel d'appui directement associé à l'exécution de projets et d'activités de R-D. Conformément à la définition de la R-D énoncée dans le présent manuel, ce concept englobe tous les domaines de connaissance.

1.45 Le personnel de R-D attaché à une unité statistique est composé des personnes qui participent directement aux activités de R-D (salariés de l'unité statistique ou contributeurs externes faisant partie intégrante des activités de R-D de l'unité statistique), et de celles qui fournissent des services directement liés aux activités de R-D (responsables de R-D, gestionnaires, techniciens et personnel de soutien).

1.46 Les individus susceptibles de contribuer aux activités de R-D d'une unité statistique peuvent appartenir à l'un des deux grands groupes ci-après, à quelques différences près selon le secteur institutionnel auquel appartient l'unité :

- les personnes occupées au sein de l'unité statistique qui participent aux activités de R-D intra-muros de celle-ci (également désignées par l'expression " personnel interne de R-D » dans le présent manuel)

- les contributeurs externes aux activités de R-D intra-muros de l'unité considérée (également désignés par l'expression " personnel externe de R-D » dans le présent manuel), eux-mêmes divisés en deux sous-groupes, selon qu'ils : (i) perçoivent un salaire/traitement d'une autre entité que l'unité statistique exécutante ou (ii), dans un certain nombre de cas particuliers, contribuent aux activités de R-D intra-muros d'une unité statistique sans être rattachés à celle-ci.

1.47 Des étudiants en doctorat ou en master peuvent être inclus dans l'un ou l'autre groupe de personnel de R-D dès lors qu'ils remplissent les critères énoncés dans le chapitre de sorte que seuls les individus qui apportent une contribution notable aux activités de R-D de l'entité étudiée soient pris en compte.

1.48 Le personnel de R-D est classé par fonction de R-D : chercheurs, techniciens et personnel de soutien.

1.49 Le personnel de R-D (personnes occupées et personnel externe de $\mathrm{R}-\mathrm{D})$ est quantifié au regard de trois types d'indicateurs :

- l'effectif en nombre de personnes physiques

- les activités de R-D exprimées en équivalent temps plein (ETP) ou en annéespersonnes

- les caractéristiques des individus (sexe, fonction de R-D, âge et qualifications). 


\section{Mesurer la R-D : méthodes et procédures (Chapitre 6)}

1.50 L'une des vocations premières des statistiques de R-D est de permettre l'établissement de comparaisons internationales, ce qui exige non seulement de disposer de définitions solides et cohérentes, mais aussi de les appliquer au stade du recueil des données. A priori, les disparités observées sur le plan méthodologique et dans l'application des définitions et recommandations empêchent de comparer les données de R-D de différents pays. C'est pourquoi un chapitre sur les problèmes méthodologiques communs à tous les secteurs a été ajouté aux précédents.

1.51 Les méthodes et procédures employées pour mesurer l'exécution de la R-D dépendent de plusieurs éléments. En règle générale, une activité de R-D concerne un nombre relativement modeste d'entités, en particulier dans le secteur des entreprises. Si l'activité de R-D est très concentrée, son champ d'intervention s'étend à l'économie. Tant la concentration que l'étendue de ce champ d'intervention influent sur les lignes directrices relatives à la stratégie d'échantillonnage. Outre ces caractéristiques, les objectifs des programmes statistiques de R-D sont pluridimensionnels : il s'agira de produire des indicateurs agrégés à l'appui de la politique scientifique ; de chiffrer les dépenses associées à un stock de capital de R-D suivant le SCN ; et d'obtenir des microdonnées à l'appui des analyses menées au niveau des unités, dans le respect des règles de protection des données. Ces objectifs parfois divergents dictent le choix des stratégies d'échantillonnage et de traitement des données.

1.52 Très diverses, les sources potentielles des données de R-D incluent, sans s'y limiter, les enquêtes et d'autres sources administratives, qu'il faut parfois compléter par des estimations. Les offices statistiques sélectionnent les sources à exploiter en fonction de la disponibilité, de la qualité, de la pertinence des données ainsi que du coût de leur collecte, la situation étant variable d'un pays à l'autre.

\section{$R-D$ du secteur des entreprises (Chapitre 7)}

1.53 Dans la plupart des pays industrialisés, le secteur des entreprises représente la plus grosse part des dépenses et du personnel de R-D. Lorsque l'on analyse ce secteur et les unités qui le composent, il importe de prendre en considération les différentes stratégies mises en œuvre par les entreprises pour gérer leurs activités de R-D. Il arrive ainsi que des entreprises apparentées se réunissent pour financer, produire, échanger et exploiter conjointement des connaissances issues dela R-D suivant des modalités diverses et complémentaires. La complexité de la structure des entreprises, en particulier des multinationales, fait obstacle à la mesure de la R-D. Par ailleurs, pour certaines entreprises, la R-D est une activité occasionnelle et non continue, ce qui la rend d'autant plus difficile à repérer et à chiffrer. Du point de vue méthodologique, recueillir des données auprès des entreprises n'est pas sans poser des difficultés d'ordre pratique, s'agissant par exemple de recenser les entreprises qui exécutent des 
activités de R-D, d'obtenir les informations sur la R-D requises dans le présent manuel, de respecter les règles de confidentialité et de réduire au minimum les contraintes pesant sur les répondants.

1.54 Le secteur des entreprises comprend :

- Toutes les sociétés résidentes, y compris et non exclusivement les entreprises légalement constituées en société, indépendamment du lieu de résidence de leurs actionnaires. En font partie les entreprises privées (qu'elles soient cotées en bourse et fassent appel à l'épargne publique, ou non) et les entreprises publiques (c'est-à-dire contrôlées par l'État).

- Les succursales d'entreprises non résidentes non constituées en société et considérées comme résidentes du fait qu'elles mènent dans la durée des activités de production sur le territoire économique.

- Toutes les institutions sans but lucratif (ISBL) qui sont des producteurs marchands de biens ou de services ou qui servent les entreprises.

1.55 Dans la présente version du manuel, ce secteur fait l'objet d'un chapitre distinct. Y sont exposés les problèmes d'ordre méthodologique à surmonter et formulées les recommandations à suivre pour recenser les unités statistiques et déclarantes, constituer les échantillons et classer les activités considérées conformément aux normes internationales en vigueur. On y trouve également une description des difficultés posées par la quantification des dépenses selon que l'on considère la R-D intra-muros ou extra-muros.

1.56 Les dépenses intérieures de R-D des entreprises (DIRDE) constituent le principal agrégat statistique utilisé pour décrire l'exécution de la R-D au sein du secteur des entreprises. Correspondant à la composante des DIRD (voir chapitre 4) engagées par les unités du secteur des entreprises, les DIRDE sont l'expression chiffrée des dépenses de R-D intra-muros de ce secteur. La compilation, la diffusion et la déclaration des DIRDE peuvent être utiles pour un certain nombre de variables.

\section{R-D du secteur de l'État (Chapitre 8)}

1.57 Ce chapitre porte essentiellement sur la manière dont les résultats et le personnel de R-D sont mesurés au sein du secteur de l'État. Il vise également à établir un lien entre l'exécutant et les modes de financement complémentaire disponibles pour mesurer le rôle de l'État dans le financement de la R-D à l'échelle de l'économie tout entière. Enfin, il lance des passerelles vers les chapitres 12 (sur la mesure des crédits publics en faveur de la R-D) et 13 (consacré à l'allégement fiscal en faveur de la R-D). Le secteur de l'État comprend :

- toutes les unités d'administration centrale (fédérale), régionale (d’États fédérés) ou locale (municipale), y compris les administrations de sécurité sociale, à l'exception des unités fournissant des services d'enseignement supérieur ou répondant à la description des établissements d'enseignement supérieur donnée dans le chapitre 3 et décrites plus en détail dans le chapitre 9 
- d'autres entités dépendantes de l'État : organismes d'exécution et/ou de financement et toutes les ISBL non marchandes contrôlées par des administrations publiques et n'appartenant pas au secteur de l'enseignement supérieur.

1.58 Dans la présente version du manuel, ce secteur fait l'objet d'un nouveau chapitre distinct. Y sont exposés des conseils sur la manière de procéder pour distinguer la R-D des autres activités connexes et, ainsi, surmonter les difficultés qui se posent lorsqu'elles sont simultanément menées au sein d'une administration publique.

1.59 Les dépenses intérieures de R-D de l'État (DIRDET) constituent le principal agrégat statistique utilisé pour décrire l'exécution de la R-D au sein du secteur de l'État. Correspondant à la composante des DIRD (voir chapitre 4) engagées par les unités du secteur de l'État, les DIRDET sont l'expression chiffrée des dépenses de R-D intra-muros de ce secteur. Des recommandations spéciales sont formulées sur la manière de traiter les flux de financement de R-D existant entre et dans les unités du secteur, notamment par l'intermédiaire d'organismes de financement, afin d'éviter une double comptabilisation des activités de R-D.

\section{R-D du secteur de l'enseignement supérieur (Chapitre 9)}

1.60 Ce secteur fait l'objet d'un nouveau chapitre distinct, qui vient remplacer et étoffer l'annexe de la précédente version du Manuel. Singularité de ce manuel, le secteur de l'enseignement supérieur n'a pas d'équivalent direct dans le SCN. Les établissements du secteur de l'enseignement supérieur peuvent relever de l'un quelconque des secteurs du SCN. Si l'enseignement supérieur est traité à part, c'est en raison de l'intérêt stratégique que revêtent les informations relatives aux établissements impliqués dans la R-D.

1.61 Le secteur de l'enseignement supérieur comprend:

- l'ensemble des universités, établissements d'enseignement post-secondaire et autres établissements proposant des programmes d'enseignement supérieur formel, indépendamment de leur source de financement ou de leur statut juridique

- l'ensemble des instituts et centres de recherche, stations d'expérimentation et centres de soins dont les activités de R-D relèvent du contrôle direct d'établissements d'enseignement supérieur ou sont administrées par ceux-ci.

1.62 Le secteur de l'enseignement supérieur est très hétérogène. De même, les systèmes et établissements d'enseignement supérieur sont organisés de différentes manières selon les pays. Tout cela vient compliquer le recueil des statistiques de $R-D$, d'autant que les méthodes statistiques varient grandement d'un pays à l'autre. Ce chapitre fournit des informations d'ordre général sur les méthodes employées pour calculer et estimer les chiffres des dépenses et du personnel de R-D de l'enseignement supérieur. Représentées sur le graphique 9.1, ces méthodes reposent notamment sur l'exploitation des 
enquêtes institutionnelles (dans leur intégralité ou en partie), des données administratives et de différentes combinaisons de ces sources, généralement complétées de coefficients de R-D tirés des enquêtes sur l'emploi du temps. Une attention particulière est accordée aux méthodes employées pour estimer la R-D financée par dotation publique globale, baptisée fonds généraux des universités (FGU) d'origine publique, dont bénéficient un grand nombre d'établissements publics d'enseignement supérieur à l'appui de l'ensemble de leurs activités.

1.63 Pour les besoins des enquêtes, la R-D doit être dissociée d'une vaste gamme d'activités connexes dotées d'un fondement scientifique et technologique. Bien qu'étroitement liées à la $\mathrm{R}-\mathrm{D}$, tant par les flux d'informations, qu'au niveau des opérations, des institutions et du personnel impliqués, ces activités ne devraient pas, dans la mesure du possible, entrer en ligne de compte dans la mesure de la R-D. Certaines activités propres au secteur de l'enseignement supérieur sont difficiles à situer par rapport à la notion de R-D. Elles concernent en particulier l'enseignement, la formation et les soins de santé spécialisés (par exemple, hôpitaux universitaires).

1.64 Les dépenses intérieures de R-D de l'enseignement supérieur (DIRDES) constituent le principal agrégat statistique utilisé pour décrire l'exécution de la R-D au sein du secteur de l'enseignement supérieur. Correspondant à la composante des DIRD (voir chapitre 4) engagées par les unités de l'enseignement supérieur, les DIRDES sont l'expression chiffrée des dépenses de R-D du secteur de ce secteur.

\section{R-D du secteur privé sans but lucratif (Chapitre 10)}

1.65 Les institutions privées sans but lucratif (ISBL) ont toujours occupé une place de choix dans la recherche et le développement, tant au stade de l'exécution qu'au niveau du financement, quoique généralement dans des proportions moindres que les autres secteurs économiques. Si ce rôle a été souligné dans les versions précédentes du Manuel contenant une définition du secteur privé sans but lucratif (PSBL), un chapitre distinct lui est à présent consacré. Y sont décrites, dans les grandes lignes, les ISBL à prendre en considération dans la mesure du PSBL et formulées des lignes directrices sur la méthode à suivre pour mesurer leurs activités de R-D, compte tenu de leurs spécificités et de l'apparition de nouvelles formes de financement de la R-D.

1.66 Le secteur privé sans but lucratif comprend:

- toutes les institutions sans but lucratif au service des ménages (ISBLSM), telles que définies dans le SCN 2008, à l'exception de celles qui relèvent du secteur de l'enseignement supérieur

- à des fins d'exhaustivité, les ménages et les particuliers qui mènent ou non des activités marchandes.

1.67 Les dépenses intérieures de R-D du secteur privé sans but lucratif (DIRDISBL) constituent le principal agrégat statistique utilisé pour décrire 
l'exécution de la R-D au sein du secteur privé sans but lucratif. Correspondant à la composante des DIRD (voir chapitre 4) engagées par les unités du secteur privé sans but lucratif, les DIRDISBL sont l'expression chiffrée des dépenses de R-D intra-muros de ce secteur.

\section{Mesurer la mondialisation de la R-D (Chapitre 11)}

1.68 Le concept de mondialisation de la R-D est abordé de façon explicite dans la présente édition du Manuel de Frascati, alors que, dans les précédentes, la dimension mondiale de la R-D était surtout associée au financement de la R-D exécutée sur le territoire national (dans le calcul des DIRD) ou à la destination des fonds de sources nationales (dans le calcul des DNRD). Il était alors question de fonds provenant ou à destination de l'étranger. Conformément à la terminologie employée dans le SCN, il a été décidé de retenir la notion de « Reste du monde » dans la présente édition du Manuel. Repérer et quantifier les sources et destinations des financements de la R-D non situées sur le territoire national restent des aspects importants de l'étude de la R-D, qui sont traités de manière exhaustive. Cependant, le champ d'étude du présent manuel dépasse le cadre des flux de financement de la R-D pour inclure une liste plus complète de questions en rapport avec la mesure de la R-D à l'échelle mondiale (OCDE, 2005 ; OCDE, 2010). On trouvera dans un chapitre distinct des recommandations sur ce point ainsi que des liens vers d'autres manuels de statistique utiles à cet égard.

1.69 D’une manière générale, la mondialisation désigne l'intégration internationale des moyens de financement, de l'offre de facteurs, de la R-D, de la production et du commerce des biens et des services. Dans le secteur à but lucratif, la mondialisation est associée aux échanges internationaux et à l'investissement direct étranger (IDE) émanant principalement des entreprises commerciales, alors que les entités des secteurs public et privé sans but lucratif (notamment les organismes de l'État et les établissements de l'enseignement supérieur) mènent elles aussi des activités d'envergure internationale en lien avec la R-D, comme le financement et la collaboration.

1.70 La mondialisation de la R-D renvoie à un sous-ensemble d'activités mondiales impliquant le financement, l'exécution, le transfert et l'exploitation de la R-D. Le chapitre à l'examen porte sur trois indicateurs de la mondialisation de la R-D d'entreprise et fait la synthèse des problèmes de mesure rencontrés en dehors du secteur des entreprises.

1.71 Les trois indicateurs statistiques de la mondialisation de la R-D d'entreprises susmentionnés sont :

- les flux de financement transnationaux de R-D

- les dépenses courantes et les dépenses de personnel liés aux activités de R-D que des composantes d'entreprises multinationales (EMN) mènent dans les pays déclarants et à l'étranger

- les échanges internationaux de services de R-D. 
1.72 Des recommandations complémentaires, intéressant les secteurs hors entreprises, sont fournies sur la manière de mesurer le phénomène de la mondialisation de la R-D. Elles concernent notamment : le rôle des organisations internationales, le financement public d'activités de R-D exécutées à l'étranger, les campus installés à l'étranger et les activités internationales de R-D des organisations non gouvernementales.

\section{Crédits budgétaires publics de R-D (Chapitre 12)}

1.73 Les montants alloués par l'État au financement des activités de R-D se calculent de différentes manières. La méthode préconisée dans le présent manuel consiste à partir du point de vue de l'exécutant pour étudier les unités résidentes qui exécutent des activités de R-D (entreprises, organismes, universités, etc.) de manière à obtenir le montant des dépenses de R-D intra-muros engagées au cours de l'année de référence. Il est ensuite possible de mettre en évidence la part de ces dépenses financée par l'État.

1.74 En complément, une autre méthode, fondée sur les données tirées des budgets, a été mise au point pour mesurer le financement public de la R-D. Partant du point de vue du bailleur de fonds, elle consiste à recenser tous les postes budgétaires susceptibles de financer des activités de R-D et à mesurer ou à estimer la part que la R-D y représente. A priori, l'un des intérêts de cette approche serait de permettre de communiquer beaucoup plus rapidement les totaux de la R-D de l'État, dans la mesure où ils seraient calculés à partir du budget, et de les rattacher à l'action des pouvoirs publics en les classant par objectif socioéconomique.

1.75 Ces données fondées sur le budget renvoient au concept officiel de " crédits budgétaires publics de R-D » (CBPRD), qui est apparu dans la troisième édition du Manuel et est décrit dans le chapitre à l'examen.

1.76 La liste des objectifs socioéconomiques qui y sont rattachés figure dans le tableau 12.1. Elle a été établie à partir de la classification de l'Union européenne adoptée par Eurostat aux fins de la Nomenclature pour l'analyse et la comparaison des budgets et programmes scientifiques (NABS).

\section{Mesurer l'allégement fiscal en faveur de la R-D (Chapitre 13)}

1.77 Les autorités de plusieurs pays ont aménagé des régimes d'aide fiscale à l'investissement dans la R-D dans le cadre desquels les dépenses de R-D éligibles, en particulier celles des entreprises, bénéficient d'un traitement fiscal préférentiel. Les dépenses fiscales sont difficiles à mesurer et les différents types de dispositif d'allégement fiscal ne transparaissent pas individuellement dans tous les systèmes statistiques. Cependant, comme l'allégement fiscal en faveur de la R-D est censé prendre la forme de subventions ou d'autres dépenses directes, il est largement admis que la notification de ces types d'aide fiscale, sous couvert de suppléments, permettrait aux comparaisons internationales de gagner en transparence et d'être plus équilibrées. 
1.78 En réponse aux utilisateurs et spécialistes qui souhaitaient voir combler cette lacune, ce nouveau chapitre du Manuel de Frascati contient des lignes directrices sur la manière de rendre compte du soutien que les pouvoirs publics apportent à la R-D sous la forme d'incitations fiscales, en vue de faciliter l'établissement d'indicateurs de l'allégement fiscal en faveur de la R-D comparables entre les pays.

1.79 Certes, les dépenses fiscales en faveur de la R-D présentent plusieurs points communs avec les crédits budgétaires publics en faveur de la R-D (CBPRD) décrits dans le chapitre 12. Cependant, comme leur notification intervient parfois dans le cadre du budget, il est proposé ici que cette catégorie soit considérée de façon distincte dans sa globalité, puis intégrée dans la présentation générale des statistiques de R-D, en particulier pour les besoins de l'établissement de comparaisons internationales.

\section{Annexes et directives complémentaires}

1.80 La version papier de la présente édition contient une annexe dans laquelle l'historique du Manuel est retracé et des remerciements sont adressés à l'intention des personnes qui ont le plus contribué à l'élaboration des éditions précédentes, permettant ainsi au Manuel d'acquérir sa réputation actuelle. S'y ajoute un glossaire des principaux termes et expressions employés dans le Manuel. La version actualisée de ce glossaire sera consultable en ligne.

1.81 Des indications complémentaires sont disponibles en ligne depuis la page web consacrée au Manuel (à l'adresse http://oe.cd/frascati). Une grande partie des informations qui figuraient dans les annexes des éditions précédentes ont été supprimées et/ou incorporées dans la version papier de la présente édition. C'est le cas, par exemple, des lignes directrices relatives à l'enseignement supérieur, qui font désormais l'objet d'un chapitre à part entière, et celles concernant la R-D des pays en développement, disséminés dans le Manuel. Les informations relatives à la régionalisation des statistiques de $\mathrm{R}-\mathrm{D}$, aux déflateurs et convertisseurs des prix de la R-D, aux méthodes à suivre pour fournir des estimations et projections actualisées concernant la $\mathrm{R}-\mathrm{D}$, à la $\mathrm{R}-\mathrm{D}$ relative à la santé, aux technologies de l'information et des communications (TIC) et aux biotechnologies sont toujours d'actualité et les utilisateurs pourraient juger utile de se reporter aux annexes de l'édition précédente en attendant que l'OCDE les révise compte tenu de l'évolution méthodologique et autre.

1.82 Les classifications et nomenclatures mentionnées dans le Manuel seront également actualisées en ligne et assorties d'hyperliens vers les normes internationales y afférentes. De même, la classification OCDE des domaines de recherche et de développement ainsi que les mises à jour consécutives feront elles aussi l'objet d'une mise en ligne, même si la classification actuelle apparaît également dans l'édition papier. La page web du Manuel de Frascati servira à diffuser le matériel d'information et les recommandations concernant les pratiques de mesure spécifiques à la R-D dont les experts nationaux travaillant 
au sein de l'OCDE conviendront à l'avenir. Comme indiqué, le Groupe de travail des experts nationaux sur les indicateurs de science et de technologie (GENIST) a, dans le cadre de l'établissement de la présente édition du Manuel, accumulé une pléthore d'éléments susceptibles de servir de base aux compléments d'information accessibles en ligne sur un certain nombre de sujets.

\subsection{Appliquer les recommandations formulées dans le Manuel}

1.83 Le présent manuel propose une terminologie commune, des principes agréés et des conventions concrètes afin d'apporter des conseils pragmatiques sur la manière de recueillir et de communiquer des données. L'objectif est de garantir la comparabilité des statistiques obtenues à l'appui de la mise en place d'une architecture d'information mondiale sur la R-D à l'intention des décideurs, des universitaires, des dirigeants d'entreprises, des journalistes et du grand public en général.

1.84 En revanche, le Manuel n'a pas vocation à couvrir l'intégralité des scénarios possibles ni à édicter quelles données soumettre à l'OCDE pour diffusion ou aux fins de l'établissement de tableaux. Le GENIST dispose en effet d'autres moyens, certes liés mais dissociés du Manuel, pour accompagner l'application de ses recommandations destinées à répondre aux besoins des utilisateurs et à servir l'intérêt général. Ces moyens sont les suivants :

- les questionnaires utilisés pour recueillir des données agrégées sur la R-D auprès des autorités nationales, comme convenu entre l'OCDE, d'autres organisations internationales et les pays sollicités

- la procédure d'assurance qualité associant l'OCDE et les experts nationaux qui fournissent les données

- la publication de bases de données et d'indicateurs sur la R-D, avec la participation des comités de l'OCDE compétents

- le recueil et la publication de métadonnées indiquant les grandes caractéristiques des sources et méthodes employées dans chaque pays

- l'analyse coordonnée des microdonnées sur la R-D issues de différents pays autour des questions que les indicateurs classiques ne permettent pas d'étudier convenablement

- et, chose nouvelle, la création d'un espace en ligne dans lequel les spécialistes peuvent échanger des questions et faire part de leur expérience, de façon à accumuler et à mettre en commun une base de précédents sur la façon d'agir dans telle ou telle situation.

1.85 L'OCDE collabore avec d'autres organisations internationales, qui apportent un soutien essentiel au renforcement des capacités et à la diffusion des données sur la $\mathrm{R}-\mathrm{D}$, de manière à réduire la charge que la communication de renseignements constitue pour les organismes nationaux. 
1.86 L'adoption de cette nouvelle version du Manuel va inciter un certain nombre de pays à revoir une partie de leurs pratiques et à se conformer aux normes acceptées dans le monde entier. Cette tâche impliquera peut-être la mise en œuvre de mesures transitoires ainsi que la communication des ajustements à opérer dans les séries de données. L'exercice a également été l'occasion de développer des compétences au sein des organisations et de former le personnel chargé de recueillir et de communiquer les données sur la R-D.

\subsection{Remarques finales}

1.87 Les renseignements recueillis en application des lignes directrices énoncées dans le Manuel de Frascati sont censés aider les décideurs et plus particulièrement les responsables de l'élaboration des politiques. Ils constituent en effet un élément important du dialogue social et politique consacré à l'exploitation et aux retombées des données de R-D, l'accès auxquelles ne cesse de s'élargir et qui occupent une place grandissante dans le débat public. Il est largement reconnu que les données de R-D, seules ou combinées à d'autres données, ne peuvent étayer qu'en partie la prise de décisions. Cependant, aussi longtemps que les administrations publiques, les entreprises et le public reconnaîtront les particularités de la R-D et conféreront à celle-ci un statut spécial, la mesure des efforts humains et financiers qui lui sont consacrés conservera une place de choix dans les données statistiques requises par les sociétés du monde entier. Faciliter cette tâche est précisément l'objet de la septième édition du Manuel de Frascati.

\section{Références}

CE, FMI, OCDE, Nations Unies et Banque mondiale (2009), Système de comptabilité nationale, Nations Unies, New York, http://unstats.un.org/unsd/nationalaccount/docs/ sna2008FR.pdf.

Nations Unies (2008), " Classification internationale type, par industrie, de toutes les branches d'activité économique (CITI), Révision 4 ", Département des affaires économiques et sociales, Division de statistique, Études statistiques, Série $M, n^{\circ} 4$, Rév. 4, Nations Unies, New York, http://unstats.un.org/unsd/cr/registry/isic-4.asp et http://unstats.un.org/unsd/publication/seriesM/seriesm_4rev4f.pdf.

OCDE (2010), Measuring Globalisation: OECD Economic Globalisation Indicators 2010, Éditions OCDE, Paris, $h t t p: / / d x . d o i . o r g / 10.1787 / 9789264084360$-en.

OCDE (2009a), Handbook on Deriving Capital Measures of Intellectual Property Products, Éditions OCDE, Paris, $h$ ttp://dx.doi.org/10.1787/9789264079205-en.

OCDE (2009b), Manuel de l'OCDE sur les statistiques des brevets, Éditions OCDE, Paris, http://dx.doi.org/10.1787/9789264056466-fr.

OCDE (2005), Manuel de l'OCDE sur les indicateurs de la mondialisation économique, Éditions OCDE, Paris, http://dx.doi.org/10.1787/9789264108110-fr.

OCDE (2002), Manuel de Frascati : Méthode type proposée pour les enquêtes sur la recherche et le développement expérimental, La mesure des activités scientifiques et technologiques, Éditions OCDE, Paris, http://dx.doi.org/10.1787/9789264299047-fr. 
OCDE/Eurostat (2005), Manuel d'Oslo : Principes directeurs pour le recueil et l'interprétation des données sur l'innovation, $3^{e}$ édition, La mesure des activités scientifiques et technologiques, Éditions OCDE, Paris, http://dx.doi.org/10.1787/9789264013124-fr.

UNESCO-ISU (2012), Classification internationale type de l'éducation (CITE) 2011, ISU, Montréal, www.uis.unesco.org/Education/Documents/isced-2011-fr.pdf. 
PARTIE I

\section{Définir et mesurer la R-D : Orientations générales}





\section{Chapitre 2}

\section{Concepts et définitions permettant d'identifier la R-D}

Dans le présent chapitre sont définis la recherche et le développement expérimental (R-D) ainsi que leurs composantes, la recherche fondamentale, la recherche appliquée et le développement expérimental. À quelques détails près, ces définitions sont identiques à celles énoncées dans les éditions antérieures. Les modifications qui y ont été apportées répondent à une évolution culturelle et linguistique. Cinq critères permettent d'établir ce qui constitue et ne constitue pas une activité de R-D : l'activité doit comporter un élément de nouveauté, de créativité et d'incertitude quant à son résultat et elle doit être systématique, transférable et/ou reproductible. Depuis la dernière édition, les dépenses de R-D au sens du Système de comptabilité nationale (SCN) sont considérées non plus comme une dépense, mais comme un investissement. Les dispositions du Manuel ont donc été rapprochées de celles du SCN et enrichies d'indicateurs des flux financiers. Si le Manuel s'est de tout temps appliqué à l'ensemble des disciplines scientifiques, la présente édition met davantage en avant les sciences sociales, les sciences humaines et les arts, en sus des sciences naturelles et de l'ingénierie. Mesurer les activités de R-D à partir d'enquêtes, de données administratives ou d'entretiens soulève des interrogations quant à la délimitation du champ d'étude. C'est pourquoi différents exemples sont présentés ici pour apporter des éléments de réponse. Alors que l'objet du Manuel est d'aider à interpréter les données de R-D dans le cadre de l'élaboration et de l'évaluation des politiques, le présent chapitre porte essentiellement sur les définitions afin de faciliter la mesure de la R-D. 


\subsection{Introduction}

2.1 Depuis plus d'un demi-siècle, le Manuel de Frascati fournit les définitions de la recherche et du développement expérimental (R-D) ainsi que de ses composantes, qui sont la recherche fondamentale, la recherche appliquée et le développement expérimental. Ces définitions ont su résister à l'épreuve du temps. Sur le fond, celles énoncées ici sont identiques à celles des éditions précédentes, si ce n'est que la définition de la R-D a évolué pour refléter un changement de type culturel et celle du développement expérimental dans sa formulation.

2.2 Depuis la précédente édition du Manuel, les dépenses de R-D au sens du Système de comptabilité nationale (SCN) sont considérées non plus comme une dépense, mais comme un investissement débouchant sur un stock de capital de connaissances issues de la R-D. Le SCN 2008 (CE et al., 2009) s'appuie d'ailleurs sur la définition de la R-D énoncée dans le Manuel. Compte tenu de ce lien avec le SCN, certaines de ses formulations sont reprises telles quelles dans le présent ouvrage, ce qui sera systématiquement signalé.

2.3 La R-D touche les sciences sociales, les sciences humaines et les arts ainsi que les sciences naturelles et l'ingénierie. Dans la présente édition du Manuel, les sciences sociales, les sciences humaines et les arts sont davantage mis en avant. Si cela n'a pas conduit à revoir les définitions et conventions, il convient en revanche de prêter une plus grande attention à la délimitation de ce qui constitue et ne constitue pas la R-D. De même, comme les pays utilisateurs du Manuel se trouvent à différents stades de développement économique, il sera question ici des besoins des uns et des autres.

2.4 Le présent chapitre fournit des définitions de la R-D et de ses composantes, ainsi qu'un ensemble de critères permettant d'identifier la R-D. Des exemples d'activités de R-D, de délimitations et d'exclusions sont également fournis afin d'illustrer la manière dont ces définitions sont appliquées. S'agissant d'un manuel à vocation statistique, le but principal de l'ouvrage est de formuler des orientations sur la manière de mesurer les activités de R-D à partir de données tirées d'enquêtes, d'entretiens et de sources administratives. Il sert également à interpréter les données de R-D dans le cadre de l'élaboration, de l'application et de l'évaluation des politiques. Ses utilisateurs ne doivent toutefois pas perdre de vue que l'objet du présent chapitre est de fournir des définitions afin de faciliter la mesure de la R-D. 


\subsection{Définition de la recherche et du développement expérimental (R-D)}

2.5 La recherche et le développement expérimental (R-D) englobent les activités créatives et systématiques entreprises en vue d'accroître la somme des connaissances - y compris la connaissance de l'humanité, de la culture et de la société - et de concevoir de nouvelles applications à partir des connaissances disponibles.

2.6 Même exécutées par différents acteurs, les activités de R-D présentent un ensemble de caractéristiques communes. Que ses objectifs soient spécifiques ou généraux, la R-D vise toujours à obtenir des résultats nouveaux à partir de concepts (et de leur interprétation) ou d'hypothèses présentant un caractère original. On ignore pour une grande part à quoi elle aboutira (ou tout au moins le temps et le volume de ressources nécessaires pour obtenir un résultat); son exécution est planifiée et les modalités de son financement établies (même quand les exécutants sont des individus) et ses résultats sont censés pouvoir être librement transférés ou négociés sur un marché. Pour être considérée comme relevant de la $\mathrm{R}-\mathrm{D}$, une activité doit remplir cinq critères de base.

2.7 L’activité considérée doit comporter un élément :

- de nouveauté

- de créativité

- d'incertitude

et être

- systématique

- transférable et/ou reproductible.

2.8 Ces cinq critères doivent être remplis, au moins en principe, chaque fois qu'une activité de R-D est entreprise, que ce soit de façon continue ou à titre occasionnel. La définition susmentionnée de la R-D cadre avec celle énoncée dans l'édition précédente du Manuel (OCDE, 2002) et couvre un éventail d'activités identique.

2.9 L'expression " recherche et développement » (R-D) englobe trois types d'activité: la recherche fondamentale, la recherche appliquée et le développement expérimental. La recherche fondamentale consiste en des travaux de recherche expérimentaux ou théoriques entrepris principalement en vue d'acquérir de nouvelles connaissances sur les fondements des phénomènes et des faits observables, sans envisager une application ou une utilisation particulière. La recherche appliquée consiste en des travaux de recherche originaux entrepris en vue d'acquérir de nouvelles connaissances et dirigés principalement vers un but ou un objectif pratique déterminé. Le développement expérimental consiste en des travaux systématiques - fondés sur les connaissances tirées de la recherche et l'expérience pratique et produisant de nouvelles connaissances techniques visant à déboucher sur de nouveaux produits ou procédés ou à améliorer les 
produits ou procédés existants. Ces trois types de R-D font l'objet d'un examen approfondi dans la section 2.5 .

2.10 Aux fins du présent Manuel, un « produit » désigne un bien ou un service conformément à la convention retenue dans le SCN (CE et al., 2009: par. 2.36). De même, " procédé » s'entend de la transformation d'intrants en résultats et de la production de ceux-ci, ou des structures ou pratiques organisationnelles.

2.11 L'ordre dans lequel ces trois types d'activité de R-D sont présentés ne signifie pas que la recherche fondamentale conduit à la recherche appliquée puis au développement expérimental. De nombreux flux d'information et de connaissances interviennent dans le système de R-D. Le développement expérimental peut éclairer la recherche fondamentale et il n'y a pas de raison que la recherche fondamentale ne puisse pas directement aboutir à de nouveaux produits ou procédés.

\subsection{Activités et projets de R-D}

2.12 Une " activité de R-D » est la somme des actions délibérément engagées par les exécutants de R-D pour produire de nouvelles connaissances. Dans la plupart des cas, il est possible de regrouper des activités de R-D en " projets de R-D ». Chaque projet de R-D est composé d'un ensemble d'activités de R-D, organisé et administré dans un but précis et assorti d'objectifs et de résultats escomptés pour la moindre de ces activités. Selon toute vraisemblance, le concept de projet de R-D, aussi utile soit-il pour comprendre la manière dont la R-D est exécutée, n'est pas appliqué uniformément dans l'ensemble des secteurs étudiés dans le Manuel.

\subsection{Les cinq critères permettant d'identifier la R-D}

2.13 Une activité doit satisfaire à cinq critères de base pour être qualifiée d'activité de R-D. Sans prétendre à l'exhaustivité, les exemples ci-après montrent comment repérer les activités ou projets concrets de R-D au regard de ces cinq critères.

\section{Viser à obtenir des résultats nouveaux (critère de nouveauté)}

2.14 Acquérir de nouvelles connaissances est un objectif escompté de tout projet de R-D ; il convient toutefois de l'adapter selon le contexte. Ainsi, les projets de recherche universitaires, de même que les projets conçus et gérés par les établissements de recherche, sont censés explorer des champs de connaissances entièrement nouveaux.

2.15 Dans le secteur des entreprises (les secteurs couverts par le Manuel de Frascati sont définis dans le chapitre 3), le potentiel de nouveauté offert par les projets de R-D doit être évalué au regard du stock de connaissances existant dans la branche d'activité considérée. L'activité de R-D engagée dans le cadre d'un 
projet doit déboucher sur des conclusions qui sont nouvelles pour l'entreprise et ne sont pas déjà exploitées dans le secteur. Sont exclues de la R-D les activités de copiage, d'imitation et de rétro-ingénierie visant à acquérir du savoir, dans la mesure où ce savoir n'est pas nouveau.

2.16 Une nouveauté peut résulter d'un projet qui amène à constater des divergences potentielles avec le résultat censé être reproduit. Il convient d'inclure dans la R-D les projets de développement expérimental qui visent à créer du savoir à l'appui de la mise au point de concepts et d'idées liés à la conception de nouveaux produits ou procédés. Dans la mesure où la R-D désigne la création structurée de connaissances, y compris de connaissances intégrées dans des produits et procédés, ce sont ces nouvelles connaissances, et non les produits ou procédés nouveaux ou fortement améliorés résultant de leur application qu'il convient de mesurer. Pourrait ainsi être considérée comme activité de R-D l'intégration du " manuel de maintenance » d'un système particulièrement complexe (comme un aéronef de transport de passagers) opérée à l'aide d'éléments nouveaux issus de l'exécution de tâches courantes d'entretien et judicieusement codifiée, sous réserve que cette intégration s'inscrive dans un projet de R-D. Un autre exemple est le fait de réaliser systématiquement des épreuves pour documenter l'utilisation potentielle d'une réaction chimique déjà exploitée dans des procédés de production (technologie existante) en vue d'aboutir à une nouvelle molécule, alors qu'un tel aboutissement est jugé improbable dans les publications scientifiques.

\section{Reposer sur des notions et hypothèses originales et non évidentes (critère de créativité)}

2.17 Appliquer des concepts nouveaux ou des idées nouvelles de nature à améliorer l'état des connaissances doit faire partie des objectifs d'un projet de R-D. Toute modification systématique de produits ou procédés étant exclue de la R-D, le facteur humain fait partie intégrante de la créativité en R-D. Par conséquent, un projet de R-D suppose la contribution d'un chercheur (dont la définition est donnée dans le chapitre 5). Le domaine des arts (section 2.6) requiert une attention particulière : certes, la créativité y est présente, mais les autres critères doivent être réunis pour que l'activité considérée puisse être qualifiée de R-D. Les tâches dites courantes sont exclues de la R-D, à la différence des nouvelles méthodes conçues pour les exécuter. Par exemple, l'activité " traitement des données " ne relève pas de la R-D, sauf si elle est menée dans le cadre d'un projet visant à mettre au point de nouvelles méthodes de traitement des données. De même, la formation professionnelle est certes exclue de la R-D, mais ce ne sera pas nécessairement le cas des méthodes imaginées pour la dispenser. Toute solution à un problème conçue dans le cadre d'un projet pourra être apparentée à la R-D dès lors que le résultat obtenu est original et qu'il est satisfait aux autres critères. 


\section{Revêtir un caractère incertain quant au résultat final (critère d'incertitude)}

2.18 La R-D comporte un élément d'incertitude à plusieurs niveaux. Au commencement d'un projet de R-D, la nature de son résultat et son coût (y compris en temps) sont impossibles à déterminer avec précision par rapport aux objectifs. Dans le cas de la recherche fondamentale, qui vise à repousser les limites du savoir formel, la possibilité de ne pas parvenir aux résultats escomptés est largement admise. Par exemple, un projet de recherche peut permettre d'éliminer un certain nombre d'hypothèses concurrentes, mais pas la totalité d'entre elles. La R-D en général se caractérise par l'incertitude qui entoure les coûts ou le temps à prévoir pour obtenir les résultats escomptés, ainsi que les objectifs susceptibles d'être atteints dans quelque mesure que ce soit. Ainsi, l'incertitude est un critère fondamental lorsqu'il s'agit de distinguer les prototypes selon qu'ils sont destinés à la R-D (modèles servant à tester des concepts techniques et des techniques avec un risque élevé d'échec en termes d'applicabilité) ou non (unités de pré-production utilisées pour obtenir des agréments techniques ou juridiques).

\section{S’inscrire dans une planification et une budgétisation (critère de systématisation)}

2.19 La R-D est une activité structurée qui est exécutée de manière systématique. En l'occurrence, "systématique " signifie que les modalités de conduite de la R-D ont été planifiées et que son déroulement et ses résultats sont consignés. Pour s'en assurer, il convient de mettre en évidence le but du projet de R-D considéré ainsi que les sources de financement. Si ces informations sont disponibles, cela signifie que le projet vise à répondre à des besoins spécifiques et que des ressources humaines et financières lui ont été allouées. S'il est plus probable de retrouver la structure de gestion et de notification susmentionnée dans les grands projets, elle peut également être mise en œuvre dans des activités d'envergure modeste, par l'intermédiaire d'un ou de plusieurs employés ou consultants (sous réserve de la présence d'un chercheur) chargés d'apporter une solution précise à un problème concret.

\section{Déboucher sur des résultats qu'il est possible de reproduire (critère de transférabilité et/ou de reproductibilité)}

2.20 Un projet de R-D devrait déboucher sur la possibilité de transférer les nouvelles connaissances acquises, en en garantissant l'utilisation et en permettant à d'autres chercheurs de reproduire les résultats obtenus dans le cadre de leurs propres activités de R-D. Cela inclut les activités de R-D qui débouchent sur des résultats négatifs, comme l'infirmation de l'hypothèse de départ ou l'impossibilité de mettre au point un produit tel qu'initialement prévu. Comme le but de la R-D est d'enrichir le stock de connaissances, ses résultats ne doivent pas rester tacites (c'est-à-dire être cantonnés dans l'esprit des chercheurs), sinon 
ils risquent d'être perdus, de même que les connaissances connexes. Codifier le savoir et le diffuser sont des pratiques courantes au sein des universités et des établissements de recherche, malgré l'apparition éventuelle de restrictions liées à la réalisation de travaux sous contrat ou dans le cadre d'une collaboration. Dans le secteur des entreprises, les résultats tomberont certes sous le coup du secret d'affaires ou d'autres règles de protection de la propriété intellectuelle, mais il est d'usage de consigner le déroulement de l'activité et ses résultats à l'intention des autres chercheurs spécialistes du domaine.

\section{Exemples}

2.21 Pour comprendre le but d'un projet, il est essentiel de mettre en évidence ce qui relève de la R-D et le contexte propre au type d'établissement dans lequel la R-D est exécutée, comme en témoignent les exemples suivants :

- Dans le domaine de la médecine, réaliser une autopsie de routine pour déterminer les causes d'un décès est une pratique médicale courante, qui ne peut pas être rattachée à la R-D ; en revanche, une enquête de mortalité destinée à établir les effets secondaires d'un traitement contre le cancer relève de la R-D (s'appliquent ici les critères de nouveauté, d'incertitude quant aux résultats de l'étude et de transférabilité des résultats en vue d'une exploitation plus large).

- De même, les tests de routine, comme les analyses de sang et les examens bactériologiques réalisés dans le cadre de bilans de santé ne relèvent pas de la $\mathrm{R}-\mathrm{D}$, contrairement aux programmes de tests sanguins visant spécifiquement les patients qui prennent un nouveau produit pharmaceutique.

- Enregistrer quotidiennement les niveaux de température ou de pression atmosphérique constitue non pas une activité de $R-D$, mais une pratique courante. En revanche, rechercher de nouvelles méthodes de mesure des températures est de la R-D, tout comme étudier et mettre au point de nouveaux modèles de prévention météorologique.

- Les activités de R-D menées dans le domaine de la construction mécanique sont souvent étroitement liées à la conception. Les petites et moyennes entreprises (PME) de cette branche d'activité comptant rarement un service spécialement dédié à la R-D, leurs activités de R-D sont généralement apparentées aux " études et projets ". Or, dès lors que la construction et le fonctionnement d'installations pilotes ou de prototypes nécessitent des calculs, modèles, dessins ou consignes d'exploitation, ils devraient être inclus dans la R-D. Ils devraient en revanche en être exclus s'ils sont menés en vue de préparer, d'exécuter ou de maintenir une production standardisée (par exemple, à l'aide de réglages de gabarit ou de machines-outils) ou afin de promouvoir la vente de produits (offres, brochures, catalogues de pièces détachées). Dans cet exemple, on retrouve plusieurs caractéristiques de la R-D : la nouveauté, qui résulte de l'étude des potentialités offertes par de nouveaux dispositifs (à travers la mise en route de prototypes) ; l'incertitude, qui tient au caractère 
imprévu des résultats de la mise à l'essai des prototypes ; la créativité, qui découle de la conception de nouveaux dispositifs destinés à la production ; la transférabilité, liée à l'établissement de documentation technique traduisant les résultats des essais en informations destinées à être exploitées au stade du développement du produit ; et l'approche systématique, dans la mesure où le détail de l'organisation du projet transparaît derrière les activités techniques susmentionnées.

2.22 Le tableau 2.1 fournit des exemples concrets de la manière d'établir si une activité relève ou non de la R-D au regard des cinq critères de base.

Tableau 2.1. Exemples de questions permettant d'établir si un projet relève de la $R-D$

\begin{tabular}{|c|c|}
\hline Question & Observation \\
\hline $\begin{array}{l}\text { a. Quels sont les objectifs } \\
\text { du projet? }\end{array}$ & $\begin{array}{l}\text { La poursuite d'objectifs originaux et ambitieux à travers la création de } \\
\text { "nouvelles connaissances » (par exemple, découvrir des phénomènes, } \\
\text { structures ou relations encore inconnus) constitue une caractéristique } \\
\text { essentielle de la R-D. On n'a pas affaire à de la R-D quand des connaissances } \\
\text { déjà disponibles sont utilisées (à des fins d'adaptation, de personnalisation, } \\
\text { etc.), sans intention d'élargir l'état des connaissances (critère de nouveauté). }\end{array}$ \\
\hline $\begin{array}{l}\text { b. Quel élément de } \\
\text { nouveauté ce projet } \\
\text { contient-il ? }\end{array}$ & $\begin{array}{l}\text { Outre le développement de «nouvelles connaissances », un projet de R-D } \\
\text { devrait suivre une démarche de création, par exemple imaginer de nouvelles } \\
\text { applications pour des connaissances scientifiques existantes ou de nouveaux } \\
\text { usages pour les techniques ou technologies disponibles (critère de créativité). }\end{array}$ \\
\hline $\begin{array}{l}\text { c. Quelles sont les } \\
\text { méthodes utilisées pour } \\
\text { le mener à bien? }\end{array}$ & $\begin{array}{l}\text { Les méthodes de recherche scientifique et technologique, ainsi que celles } \\
\text { employées en sciences sociales, en sciences humaines et dans les arts, } \\
\text { sont acceptées sous réserve qu'elles traitent de l'incertitude entourant } \\
\text { l'issue finale du projet. Cette incertitude peut concerner le temps et le } \\
\text { volume de ressources nécessaires pour atteindre l'objectif prévu. Le choix } \\
\text { méthodologique pourrait faire partie de la composante créative du projet } \\
\text { et constituer un moyen de faire face à l'incertitude (critères de créativité } \\
\text { et d'incertitude). }\end{array}$ \\
\hline $\begin{array}{l}\text { d. Quelle est l'applicabilité } \\
\text { générale des conclusions } \\
\text { ou résultats du projet? }\end{array}$ & $\begin{array}{l}\text { Pour être généralement applicables, les conclusions d'un projet de R-D } \\
\text { doivent être transférables/reproductibles, en plus de satisfaire aux quatre } \\
\text { autres critères. Par exemple, les résultats peuvent être transférés moyennant } \\
\text { leur publication dans des ouvrages ou revues scientifiques et le recours aux } \\
\text { instruments de protection de la propriété intellectuelle. }\end{array}$ \\
\hline $\begin{array}{l}\text { e. Quelles catégories de } \\
\text { personnel sont affectées } \\
\text { au projet? }\end{array}$ & $\begin{array}{l}\text { La mise en œuvre d'un projet de R-D requiert normalement la mobilisation } \\
\text { d'une panoplie de compétences (la question du personnel de R-D est } \\
\text { examinée dans le chapitre } 5 \text { du Manuel). Le personnel de recherche associé } \\
\text { à ces projets est composé de chercheurs, de techniciens et de personnel de } \\
\text { soutien, mais seule la participation de chercheurs en tant que tels doit être } \\
\text { constatée pour qualifier de R-D une activité satisfaisant implicitement aux cinq } \\
\text { critères de base. }\end{array}$ \\
\hline $\begin{array}{l}\text { f. Dans quelle } \\
\text { catégorie classer les } \\
\text { projets de recherche } \\
\text { des établissements } \\
\text { de recherche? }\end{array}$ & $\begin{array}{l}\text { Dans certains cas, on se fondera sur la nature de l'établissement. Ainsi, } \\
\text { la plupart des projets menés au sein d'établissements de recherche ou } \\
\text { d'universités de recherche peuvent être qualifiés de projets de R-D. Ceux } \\
\text { engagés par d'autres acteurs (entreprises commerciales ou établissements } \\
\text { non exclusivement dédiés à la R-D) devraient être analysés sur la base des } \\
\text { cinq critères de la R-D (voir la description des secteurs dans le chapitre 3). }\end{array}$ \\
\hline
\end{tabular}




\subsection{Répartition par type de R-D}

2.23 Il est recommandé de recourir à une ventilation par type de R-D dans les quatre secteurs considérés dans le Manuel et définis dans le chapitre 3 : les entreprises, l'enseignement supérieur, l'État et le secteur privé sans but lucratif. Pour les besoins des comparaisons internationales, cette ventilation devrait reposer sur le total des dépenses de R-D ou uniquement sur les dépenses courantes (voir chapitre 4). Elle peut s'effectuer au niveau des projets, mais certains devront peut-être faire l'objet d'une division plus fine.

2.24 Il existe trois types de R-D :

- recherche fondamentale

- recherche appliquée

- développement expérimental.

\section{Recherche fondamentale}

2.25 La recherche fondamentale consiste en des travaux de recherche expérimentaux ou théoriques entrepris en vue d'acquérir de nouvelles connaissances sur les fondements des phénomènes et des faits observables, sans envisager une application ou une utilisation particulière.

2.26 La recherche fondamentale analyse des propriétés, des structures et des relations en vue de formuler et de vérifier des hypothèses, des théories ou des lois. La présence de la mention "sans envisager une application particulière » dans la définition de la recherche fondamentale est primordiale, car l'exécutant ne connaît pas nécessairement la nature des applications potentielles au moment d'effectuer les travaux de recherche ou de répondre aux questionnaires d'enquête. En règle générale, les résultats de la recherche fondamentale sont, non pas cédés, mais publiés dans des revues scientifiques ou communiqués aux confrères intéressés. Dans certaines circonstances, la diffusion des résultats de la recherche fondamentale peut être limitée pour des raisons de sécurité nationale.

2.27 En recherche fondamentale, le chercheur est censé disposer d'une certaine latitude dans la définition des objectifs. Cette forme de recherche est habituellement exécutée dans le secteur de l'enseignement supérieur, mais aussi dans une certaine mesure dans le secteur de l'État. La recherche fondamentale peut être orientée ou dirigée vers de vastes domaines d'intérêt général, dans l'objectif explicite de déboucher à terme sur une palette d'applications. Les entreprises du secteur privé peuvent également se livrer à des activités de recherche fondamentale même si aucune application commerciale concrète n'est prévisible à court terme. Les travaux de recherche dédiés à certains types de technologies d'économie d'énergie peuvent s'apparenter à de la recherche fondamentale telle que définie ci-dessus s'ils ne visent pas une utilisation particulière. En revanche, les travaux qui suivent une direction donnée, comme accroître les économies d'énergie, relèvent, dans le Manuel, de la « recherche fondamentale orientée ". 
2.28 La recherche fondamentale orientée se distingue de la "recherche fondamentale pure " pour les raisons ci-après.

- La recherche fondamentale pure est exécutée au service de la connaissance sans intention d'en tirer des avantages économiques ou sociaux ou sans volonté d'en appliquer les résultats à des problèmes concrets ou de transmettre ces résultats aux secteurs concernés.

- La recherche fondamentale orientée est exécutée dans l'espoir qu'elle aboutira à l'établissement d'une vaste base de connaissances permettant de résoudre les problèmes ou de concrétiser les opportunités qui se présentent actuellement ou qui sont susceptibles de se présenter ultérieurement.

\section{Recherche appliquée}

2.29 La recherche appliquée consiste en des travaux de recherche originaux entrepris en vue d'acquérir de nouvelles connaissances et dirigés principalement vers un but ou un objectif pratique déterminé.

2.30 La recherche appliquée est entreprise pour déterminer les utilisations possibles des résultats de la recherche fondamentale, ou pour établir des méthodes ou modalités nouvelles permettant d'atteindre des objectifs précis et déterminés à l'avance. Elle implique de prendre en compte les connaissances existantes et de les approfondir afin de résoudre des problèmes concrets. Dans le secteur des entreprises, le passage de la recherche fondamentale à la recherche appliquée se manifeste souvent par le lancement d'un nouveau projet visant à explorer les résultats prometteurs d'un programme de recherche fondamentale (ce qui implique de passer du long au moyen terme dans l'exploitation des résultats de la $\mathrm{R}-\mathrm{D}$ intra-muros [voir glossaire]).

2.31 Les résultats de la recherche appliquée sont censés, en premier lieu, pouvoir être appliqués à des produits, opérations, méthodes ou systèmes. La recherche appliquée permet la mise en forme opérationnelle d'idées. Les applications des connaissances ainsi obtenues peuvent être protégées par les instruments de propriété intellectuelle, y compris le secret d'affaires.

\section{Développement expérimental}

2.32 Le développement expérimental consiste en des travaux systématiques - fondés sur les connaissances tirées de la recherche et l'expérience pratique et produisant de nouvelles connaissances techniques visant à déboucher sur de nouveaux produits ou procédés ou à améliorer les produits ou procédés existants.

2.33 La mise au point de nouveaux produits ou procédés est qualifiée de développement expérimental dès lors qu'elle satisfait aux critères qui caractérisent une activité de R-D. À titre d'exemple, on citera l'incertitude entourant les ressources requises pour atteindre l'objectif du projet de R-D dans 
le cadre duquel l'activité de développement considérée est exécutée. Dans le présent manuel, la lettre D de R-D signifie « développement expérimental ».

\section{« Développement expérimental » ne signifie pas « développement de produits »}

2.34 Il ne faut pas confondre le " développement expérimental » et le "développement de produits ", qui désigne le processus global allant de la formulation d'idées et de concepts à la commercialisation - engagé pour mettre un nouveau produit (bien ou service) sur le marché. Le développement expérimental ne constitue donc qu'une étape éventuelle du développement de produits, celle où l'on soumet les connaissances génériques à des tests afin d'établir la possibilité d'aboutir aux applications requises pour que ces travaux soient couronnés de succès. Cette phase se caractérise par la formation de connaissances nouvelles et prend fin au moment où les critères de la R-D (nouveauté, incertitude, créativité, caractère systématique et possibilité de transférer et/ou reproduire les résultats) ne sont plus applicables. Par exemple, lorsque l'on entreprend de mettre au point un nouveau modèle de voiture, on peut étudier la possibilité d'adopter des technologies existantes : c'est l'étape du développement expérimental, qui se rapporte aux applications nouvelles de connaissances générales débouchant sur des résultats nouveaux. Son issue est incertaine dans la mesure où les essais réalisés peuvent aboutir à des résultats négatifs ; l'activité présente un élément de créativité dans la mesure où elle consiste principalement à adapter des technologies à un nouvel usage ; elle est formalisée car elle nécessite la participation d'une main-d'œuvre spécialisée ; et elle implique une codification pour que les résultats des essais puissent être traduits en recommandations techniques en vue des étapes ultérieures du processus de développement de produits. Des cas de développement de produits sans R-D, dont traitent des ouvrages et articles économiques, sont néanmoins observés, en particulier au sein de PME.

\section{"Développement expérimental » ne signifie pas « développement préalable à la production »}

2.35 Il ne faut pas confondre le « développement expérimental » et le " développement préalable à la production ", qui désigne les travaux à but non expérimental précédant la mise en production d'un produit ou système dans les domaines de la défense, de l'aérospatiale ou d'autres secteurs. Il est difficile de définir avec précision la limite qui sépare le développement expérimental du développement préalable à la production ; la distinction entre ces deux catégories nécessite "l'appréciation d'un spécialiste " quant à la question de savoir à quel moment l'élément de nouveauté cède la place aux tâches habituelles de mise au point d'un système intégré. 
2.36 Par exemple, une fois qu'un projet de chasseur-bombardier a franchi avec succès les étapes de la recherche, de la démonstration technologique, de la conception et du développement initial pour arriver à la phase des essais en vol avant la mise en production, il peut falloir jusqu'à dix cellules supplémentaires pour garantir la pleine intégration opérationnelle de l'aéronef dans les systèmes aériens offensifs/défensifs. L'exercice se déroule en deux phases. La première consiste à mettre au point le système aérien offensif/défensif intégré, c'est-à-dire à assembler des composants et des sous-systèmes déjà existants mais encore jamais auparavant intégrés dans ce contexte. Cela passe par l'instauration d'un vaste programme d'essais en vol, potentiellement très onéreux et susceptible de constituer le plus gros poste de dépenses avant la mise en production. Si les travaux commandés lors de cette phase relèvent pour beaucoup du développement expérimental (R-D), une partie d'entre eux ne présente pas l'élément de nouveauté requis pour être classés dans la R-D et relève en fait du développement préalable à la production (hors $\mathrm{R}-\mathrm{D}$ ). La deuxième phase est celle de la mise à l'essai du système aérien offensif/défensif. Une fois établi, durant la première phase, que le système fonctionne, le projet de développement peut passer à la production à titre d'essai d'une série d'appareils destinés aux essais de fonctionnement (faible niveau de production initiale). Du succès de ces essais dépend la mise en production en bonne et due forme. Au sens du présent manuel, ces travaux ne relèvent pas de la $\mathrm{R}-\mathrm{D}$, mais du développement préalable à la production. Cependant, des problèmes risquant de se poser pendant les essais, de nouveaux travaux de développement expérimental pourraient se révéler nécessaires pour y remédier. Ces travaux, qualifiés de " R-D supplémentaire ", sont à inclure dans la R-D.

\section{Comment distinguer les différents types de R-D}

2.37 Un critère fondamental permet de classer les activités de R-D par type : l'utilisation escomptée de leurs résultats. Deux questions peuvent également aider à déterminer le type de R-D dont un projet relève :

- Dans quel délai est-il probable que le projet aboutisse à des résultats donnant lieu à des applications?

- Quelle est l'étendue du champ d'application potentiel des résultats du projet de R-D (plus la dimension fondamentale de la recherche est importante, plus le champ d'application potentiel est vaste).

2.38 La relation entre recherche fondamentale, recherche appliquée et développement expérimental doit être examinée d'un point de vue dynamique. Il est possible que la recherche appliquée et le développement expérimental parviennent à adapter les connaissances fondamentales issues de la recherche fondamentale de manière à en obtenir directement une application générale. Cependant, lorsque ces connaissances sont mises à profit pour résoudre un problème, le retour d'expérience qui intervient alors altère le caractère linéaire de l'exercice. L'interaction dynamique qui s'opère entre la création de 
connaissances et la résolution des problèmes crée des liens entre recherche fondamentale, recherche appliquée et développement expérimental.

2.39 Ces trois types de R-D sont rarement dissociés au sein des organisations exécutantes de R-D. Il arrive que tous soient confiés à la même unité, voire à la même équipe. Il arrive aussi qu'un même projet entre dans plusieurs catégories de R-D. Par exemple, la recherche d'un nouveau traitement médical d'une maladie épidémique peut toucher la recherche fondamentale et la recherche appliquée. Il est donc recommandé d'évaluer les types de R-D au niveau des projets en classant les résultats escomptés sur la base des deux "indicateurs " décrits plus haut, comme illustré dans les paragraphes qui suivent.

\section{Comment distinguer les types de R-D en sciences naturelles et en ingénierie : exemples}

2.40 Les exemples suivants illustrent les différences d'ordre général que présentent la recherche fondamentale, la recherche appliquée et le développement expérimental en sciences naturelles et en ingénierie.

- Étudier une classe de réactions de polymérisation dans diverses conditions constitue une activité de recherche fondamentale. Les efforts entrepris pour optimiser l'une de ces réactions en vue d'obtenir un polymère doté de propriétés physiques ou mécaniques données (qui lui confèrent une utilité particulière) relèvent de la recherche appliquée. Le développement expérimental consiste alors à mettre en œuvre le procédé optimisé en laboratoire à une plus grande échelle ainsi qu'à rechercher et à analyser les méthodes de fabrication éventuelles de ce polymère ainsi que les produits susceptibles d'être fabriqués dans ce matériau.

- Modéliser l'absorption du rayonnement électromagnétique par un cristal constitue une activité de recherche fondamentale. L'étude de l'absorption du rayonnement électromagnétique par ce même matériau, à des conditions expérimentales variables (température, impuretés, concentration, etc.) en vue d'obtenir certaines propriétés de détection du rayonnement (sensibilité, rapidité, etc.), appartient à la recherche appliquée. La mise à l'essai d'un dispositif utilisant ce matériau en vue de fabriquer un détecteur de rayonnement plus performant que ceux existants (dans la gamme spectrale considérée) relève du développement expérimental.

- Mettre au point une nouvelle méthode de classification des séquences d'immunoglobulines constitue une activité de recherche fondamentale. Les travaux entrepris pour distinguer les anticorps associés à différentes pathologies relève de la recherche appliquée. L'activité de développement expérimental consiste alors à concevoir une méthode permettant d'obtenir par synthèse l'anticorps associé à une pathologie donnée, à partir des connaissances acquises au sujet de sa structure, et à procéder à des essais cliniques afin de vérifier l'efficacité de cet anticorps de synthèse sur des malades ayant accepté de suivre ce traitement de pointe dans le cadre d'une expérience. 
- Étudier l'évolution possible des propriétés des fibres de carbone en fonction de leur position et orientation à l'intérieur d'une structure constitue une activité de recherche fondamentale. Des travaux de recherche appliquée pourraient déboucher sur la conceptualisation d'une méthode de transformation industrielle des fibres de carbone à l'échelle nanoscopique. Les essais d'utilisation des nouveaux matériaux composites à différentes usages relèvent du développement expérimental.

- Maîtriser les procédés matériels dans le domaine quantique est un objectif de recherche fondamentale. Mettre au point des matériaux et composants de diodes électroluminescentes organiques et non organiques à des fins d'efficience énergétique et de réduction des coûts relève de la recherche appliquée. Une activité de développement expérimental consiste à recenser les applications possibles des diodes améliorées et à les intégrer dans les appareils de consommation.

- Chercher de nouvelles méthodes de calcul, comme le calcul quantique ou la théorie de l'information quantique, constitue une activité de recherche fondamentale. Les travaux entrepris pour étudier l'application du traitement de l'information dans de nouveaux domaines ou suivant de nouveaux procédés (par exemple, élaboration d'un nouveau langage de programmation, de nouveaux systèmes d'exploitation, de générateurs de programmes, etc.) et pour mettre au point des outils comme les systèmes d'information géographique et les systèmes experts appartiennent à la recherche appliquée. Développer de nouveaux logiciels d'application et perfectionner les systèmes d'exploitation et programmes d'application sont des activités de développement expérimental.

- Étudier toutes sortes de sources (manuscrits, documents, monuments, œuvres d'art, bâtiments, etc.) pour mieux comprendre des phénomènes historiques (évolution politique, sociale et culturelle d'un pays, biographie d'un individu, etc.) constitue une activité de recherche fondamentale. Faire une analyse comparative de sites et/ou monuments archéologiques affichant des similitudes ou autres points communs (d'ordre géographique, architectural, etc.) afin de comprendre les liens potentiellement dignes d'intérêt pour le matériel d'enseignement et la présentation muséologique est de la recherche appliquée. Élaborer de nouveaux instruments et de nouvelles méthodes permettant d'étudier les artefacts et objets naturels découverts dans le cadre de travaux archéologiques (par exemple, datation d'ossements ou restes végétaux) relève du développement expérimental.

- En sciences agricoles et sylviculture :

* Recherche fondamentale : étudier les modifications génomiques et facteurs mutagènes observés dans les plantes pour en comprendre les effets sur le phénome ; procéder à une analyse génétique des espèces végétales forestières en vue de comprendre les mécanismes naturels de maîtrise des pathologies ou de résistance aux parasites. 
Recherche appliquée : étudier les génomes de la pomme de terre sauvage pour localiser les gènes responsables de sa résistance au mildiou et renforcer cette résistance chez la pomme de terre cultivée ; planter des forêts expérimentales en y modifiant l'agencement (espacement et alignement) des arbres de manière à limiter la propagation de la maladie et à atteindre ainsi un rendement optimal.

- Développement expérimental : créer un outil de traitement des séquences géniques à partir des connaissances acquises dans le séquençage d'ADN à l'aide d'enzymes ; mettre à profit les résultats de travaux antérieurs consacrés à une espèce végétale pour définir la marche à suivre afin d'améliorer le mode de plantation de forêts dans un but précis.

- En nanotechnologie :

* Recherche fondamentale : étudier les propriétés électriques du graphène au microscope à effet tunnel afin de comprendre le déplacement des électrons à l'intérieur du matériau en réaction aux variations de tension.

* Recherche appliquée : étudier les hyperfréquences et le couplage thermique impliquant des nanoparticules afin de bien aligner et classer les nanotubes de carbone.

* Développement expérimental : mettre à profit les résultats de travaux de recherche menés dans le domaine de la microfabrication dans le développement d'un système de micro-usine portative et modulaire dont les composants constituent chacun un élément clé d'une ligne d'assemblage.

- En informatique et sciences de l'information :

* Recherche fondamentale : étudier les propriétés d'algorithmes généraux permettant de traiter de gros volumes de données en temps réels.

* Recherche appliquée : trouver un moyen de réduire le volume de pourriels en cernant la structure globale ou le modèle économique impliqué, l'action des pollueurs et leurs motivations.

* Développement expérimental : à partir d'un code développé par des chercheurs, une start-up met au point un mode de commercialisation en ligne rentable d'un produit logiciel.

\section{Comment distinguer les types de R-D en sciences sociales, en sciences humaines et dans les arts : exemples}

2.41 Les sciences sociales, les sciences humaines et les arts, domaines dans lesquels, comme précédemment indiqué, la frontière entre recherche fondamentale et recherche appliquée est parfois floue, constituent une autre source d'exemples. Là encore, les activités de développement expérimental ne sont pas toujours faciles à reconnaître en raison du rôle joué par d'autres domaines en sciences naturelles et en ingénierie. Il convient de noter que tous 
ces exemples doivent également satisfaire aux critères de base de la R-D énoncés dans le présent chapitre.

- En économie et commerce :

* Recherche fondamentale : passer en revue les théories relatives aux facteurs à l'origine des disparités régionales en termes de croissance économique ; mener des travaux de recherche théorique afin d'établir l'existence ou l'absence d'un équilibre naturel dans une économie de marché ; élaborer de nouvelles théories du risque.

* Recherche appliquée : analyser un cas régional aux fins de l'élaboration de politiques gouvernementales ; étudier les propriétés d'un mécanisme d'enchères potentiellement digne d'intérêt pour l'adjudication de licences de télécommunications ; étudier de nouveaux types de contrat d'assurance censés couvrir de nouveaux risques de marché ou de nouveaux types d'instruments d'épargne.

* Développement expérimental : élaborer, à partir de données statistiques, des modèles opérationnels en vue de concevoir des moyens d'action économique permettant à une région de rattraper un retard de croissance ; définir, au sein d'une autorité nationale de réglementation des télécommunications, une méthode d'adjudication des licences de télécommunications ; définir un nouveau mode de gestion pour un fonds d'investissement, sous réserve du respect du critère de nouveauté.

- Dans l'éducation :

* Recherche fondamentale : analyser les déterminants environnementaux de la faculté d'apprentissage ; étudier les répercussions des outils pédagogiques à manipuler sur l'apprentissage des mathématiques chez les très jeunes élèves, en leur en proposant successivement de différents avant de mesurer ce que les élèves ont appris à l'aide des outils normalisés.

* Recherche appliquée : procéder à une étude comparative des programmes nationaux d'enseignement visant à combler un retard d'apprentissage au sein de groupes défavorisés ; étudier la mise en œuvre d'un programme de mathématiques donné en vue de déterminer ce que les enseignants ont besoin de savoir pour l'appliquer avec succès.

* Développement expérimental : mettre au point des tests permettant de sélectionner le programme éducatif adapté aux besoins spécifiques des enfants ; élaborer et tester (en classe) des solutions logicielles et outils de soutien, fondés sur un travail de terrain, afin d'améliorer les connaissances en mathématiques des élèves ayant des besoins éducatifs particuliers.

- En géographie sociale et économique :

* Recherche fondamentale : étudier les dynamiques fondamentales des interactions spatiales.

* Recherche appliquée : analyser les profils spatio-temporels de transmission et de diffusion d'une épidémie. 
- En histoire :

* Recherche fondamentale : étudier l'histoire et l'impact humain des inondations dues à une rupture de poche glaciaire dans un pays donné.

- Recherche appliquée : étudier, sous l'angle social, l'histoire des catastrophes naturelles (par exemple, inondations, sécheresses, épidémies) afin de comprendre la manière dont la société moderne pourrait affronter les changements climatiques.

* Développement expérimental : sur la base de travaux antérieurs, préparer une exposition sur l'adaptation des sociétés humaines aux mutations environnementales dans le passé, qui servira de prototype à d'autres musées et structures éducatives.

- En langues/linguistique :

* Recherche fondamentale : en linguistique, étudier les interactions observées entre différentes langues au contact les unes des autres.

* Recherche appliquée: en orthophonie, étudier les principes neurologiques liés aux langues et les modes d'acquisition du langage chez l'homme.

- Développement expérimental : en linguistique, mettre au point un outil de diagnostic de l'autisme chez l'enfant, fondé sur l'acquisition du langage, la poursuite de la scolarité et l'utilisation des signes.

- En musique :

* Recherche fondamentale : définir une théorie transformationnelle permettant d'appréhender les événements musicaux, non comme une collection d'objets reliés entre eux, mais comme une série de transformations du matériau de base.

* Recherche appliquée : exploiter les archives historiques et les techniques d'archéologie expérimentale pour reconstituer un instrument de musique depuis longtemps disparu et retrouver la manière dont il était fabriqué et utilisé ainsi que les sons qu'il permettait de produire.

* Développement expérimental : dans le cadre d'une collaboration entre professeurs de musique et théoriciens, mettre au point de nouveaux supports pédagogiques en se fondant sur les découvertes en neuroscience qui modifient notre compréhension du traitement des sons et des informations chez l'homme.

\subsection{Classification et distribution par domaine de recherche et de développement}

2.42 Pour un certain nombre de raisons, les spécialistes qui réalisent des enquêtes et les utilisateurs de données jugent souvent utile et pertinent de classer les unités exécutantes de R-D et de répartir les ressources qu'elles y consacrent par domaine de connaissances. À cet égard, il est proposé ici de se fonder sur la 
classification OCDE des domaines de recherche et de développement. Définie pour mesurer la R-D, cette classification repose principalement sur la notion de contenu. Les activités de R-D dont le contenu est étroitement lié sont regroupées au sein d'une catégorie générale (à un chiffre) ou plus restreinte (deux chiffres). Si cette classification peut être appliquée à un plus large éventail d'activités scientifiques et technologiques (S-T) ou autres activités intellectuelles, elle n'en est pas moins, telle que formulée par l'OCDE, axée sur la R-D au sens du présent manuel.

2.43 Le but est de répartir les efforts de R-D et de classer les unités qui les déploient. Deux projets de R-D peuvent être considérés comme appartenant au même domaine dès lors que leur contenu est identique ou suffisamment proche. L'application de la classification OCDE repose sur les critères ci-après, qui aident à évaluer le degré de similarité des activités du point de vue du contenu :

- Les sources de connaissance exploitées aux fins de l'exécution des activités de R-D : les applications obtenues dans certains domaines technologiques débouchent souvent sur de nouvelles entreprises scientifiques, de la même façon que les connaissances scientifiques servent de fondement à de nouveaux développements technologiques.

- Les objets considérés, c'est-à-dire les phénomènes à comprendre ou les problèmes à résoudre via la R-D.

- Les méthodes, techniques et profils professionnels des scientifiques et autres catégories de personnel de R-D : on distinguera parfois différents domaines selon la démarche méthodologique employée pour étudier un phénomène ou un thème donné.

- Les domaines d'application : par exemple, dans la classification OCDE des domaines de recherche et de développement, les sciences médicales et agricoles sont expressément définies par leurs applications dans le domaine de la santé humaine et les activités agricoles.

2.44 Cette classification est étroitement liée et cadre avec la Recommandation de l'UNESCO concernant la normalisation internationale des statistiques relatives à la science et à la technologie (UNESCO, 1978), dont l'OCDE s'est initialement inspirée pour classer la R-D par domaine scientifique et technologique dans les versions antérieures du Manuel. Elle est également reliée dans une certaine mesure au document Domaines d'études et de formation de la CITE (CITE-F), qui rend compte dans une large mesure de la manière dont les établissements, les facultés, etc. organisent leurs activités et décernent des diplômes. Il est admis que la classification OCDE des domaines de recherche et de développement et la CITE-F répondent à des objectifs différents et qu'il n'est pas possible d'établir des correspondances directes entre elles (UNESCO-UIS, 2014, p. 17). 
2.45 Vu l'évolution actuelle des modes d'exécution de la R-D et l'apparition progressive de nouveaux domaines, la classification OCDE des domaines de recherche et de développement fera l'objet d'un examen continu après la parution de la présente version du Manuel. Les versions actualisées seront consultables en ligne, dans un complément d'information au présent manuel qui décrira plus en détail la classification et l'usage qui en est fait.

\section{Tableau 2.2. Classification des domaines de R-D}

\begin{tabular}{|c|c|}
\hline Catégorie générale & Sous-catégorie \\
\hline 1. Sciences naturelles & $\begin{array}{l}\text { 1.1 Mathématiques } \\
\text { 1.2 Informatique et sciences de l'information } \\
1.3 \text { Sciences physiques } \\
\text { 1.4 Sciences chimiques } \\
\text { 1.5 Sciences de la terre et de l'environnement } \\
\text { 1.6 Sciences biologiques } \\
\text { 1.7 Autres sciences naturelles }\end{array}$ \\
\hline 2. Ingénierie et technologie & $\begin{array}{l}\text { 2.1 Génie civil } \\
\text { 2.2 Génie électrique, électronique, informatique } \\
\text { 2.3 Mécanique } \\
\text { 2.4 Génie chimique } \\
\text { 2.5 Génie des matériaux } \\
\text { 2.6 Génie médical } \\
\text { 2.7 Génie de l'environnement } \\
\text { 2.8 Biotechnologie environnementale } \\
\text { 2.9 Biotechnologie industrielle } \\
\text { 2.10 Nanotechnologie } \\
\text { 2.11 Autres sciences de l'ingénieur et technologies }\end{array}$ \\
\hline 3. Sciences médicales et sciences de la santé & $\begin{array}{l}\text { 3.1 Médecine fondamentale } \\
\text { 3.2 Médecine clinique } \\
\text { 3.3 Sciences de la santé } \\
\text { 3.4 Biotechnologie médicale } \\
\text { 3.5 Autres sciences médicales }\end{array}$ \\
\hline 4. Sciences agricoles et vétérinaires & $\begin{array}{l}\text { 4.1 Agriculture, sylviculture et pêches } \\
\text { 4.2 Sciences de l'animal et du lait } \\
\text { 4.3 Science vétérinaire } \\
\text { 4.4 Biotechnologie agricole } \\
\text { 4.5 Autres sciences agricoles }\end{array}$ \\
\hline 5. Sciences sociales & $\begin{array}{l}\text { 5.1 Psychologie et sciences cognitives } \\
\text { 5.2 Économie et commerce } \\
\text { 5.3 Éducation } \\
\text { 5.4 Sociologie } \\
\text { 5.5 Droit } \\
\text { 5.6 Science politique } \\
\text { 5.7 Géographie sociale et économique } \\
\text { 5.8 Médias et communications } \\
\text { 5.9 Autres sciences sociales }\end{array}$ \\
\hline 6. Sciences humaines et arts & $\begin{array}{l}\text { 6.1 Histoire et archéologie } \\
\text { 6.2 Langues et lettres } \\
\text { 6.3 Philosophie, éthique et religion } \\
\text { 6.4 Arts (arts plastiques, histoire de l'art, arts } \\
\quad \text { de la scène, musique) } \\
\text { 6.5 Autres sciences humaines }\end{array}$ \\
\hline
\end{tabular}




\subsection{Exemples d'activités de R-D, de limites et d'exclusions dans différents domaines}

\section{Activités de R-D et d'innovation et cas limites}

2.46 L'innovation est actuellement définie, pour les besoins de sa mesure, dans la troisième édition du Manuel d'Oslo (OCDE/Eurostat, 2005). Toutefois, cette définition s'applique uniquement au secteur des entreprises (défini dans le chapitre 3). En résumé, il s'agit de mettre sur le marché des produits nouveaux ou considérablement améliorés ou bien de trouver des moyens plus efficaces (grâce à des méthodes et procédés nouveaux ou considérablement améliorés) de mettre des produits sur le marché. La R-D ne relève pas nécessairement de l'innovation, mais fait partie des nombreuses activités d'innovation, parmi lesquelles figurent l'acquisition de connaissances, de machines, d'équipements et autres biens d'équipement, la formation, le marketing, la conception et le développement logiciel. Ces activités d'innovation peuvent être exécutées en interne ou par des tiers.

2.47 Il faut prendre soin d'exclure les activités qui, bien que relevant du processus d'innovation, ne satisfont pas aux critères caractérisant la R-D. Par exemple, l'activité de dépôt de brevet et de concession de licences, l'étude de marché, la mise en fabrication, l'outillage et la reconfiguration du processus de fabrication ne constituent pas des activités de R-D et ne peuvent être considérées comme s'inscrivant dans un projet de R-D. Certaines activités, comme l'outillage, le développement de procédés, la conception et la réalisation de prototypes, pouvant comporter un élément non négligeable de $\mathrm{R}-\mathrm{D}$, il est difficile de déterminer avec précision ce qui doit ou ne doit pas être considéré comme de la R-D. Cela vaut tout particulièrement pour le secteur de la défense et autres branches d'activité de grande envergure comme l'aérospatiale. Des difficultés analogues peuvent surgir lorsqu'il s'agit de distinguer certains services publics fondés sur la technologie, comme l'inspection et le contrôle des produits alimentaires et pharmaceutiques, des activités connexes de R-D.

\section{Exemples d'activités associées au processus d'innovation qui relèvent et ne relèvent pas de la $R-D$}

2.48 Le tableau 2.3 et les exemples qui suivent apportent des précisions sur ce qui devrait et ne devrait pas être comptabilisé dans la R-D.

\section{Prototypes}

2.49 Un prototype est un modèle original fabriqué pour afficher toutes les caractéristiques techniques et les performances d'un nouveau produit. Par exemple, la mise au point d'une pompe pour liquides corrosifs rend nécessaire de réaliser plusieurs prototypes pour effectuer des essais de vieillissement accéléré avec différents produits chimiques. L'existence d'une boucle de rétroaction assure qu'en cas d'essais non concluants, les résultats peuvent néanmoins être exploités dans la suite des travaux de développement de la pompe. 
2.50 La conception, la construction et la mise à l'essai des prototypes relèvent normalement de la $\mathrm{R}-\mathrm{D}$, qu'un seul ou plusieurs prototypes soient réalisés, de façon consécutive ou simultanée. Cependant, dès lors que toutes les modifications requises ont été apportées au(x) prototype(s) et que les essais ont été menés à bien, la limite de la R-D a été atteinte. Même si elle est entreprise par du personnel de R-D, la construction de plusieurs exemplaires d'un prototype préalablement testé avec succès, en réponse à un besoin ponctuel de nature commerciale, militaire ou médicale, ne constitue pas une activité de R-D. Les mêmes règles pourraient s'appliquer à la réalisation virtuelle de prototypes, qui ne relèverait donc de la R-D que si la mise à l'essai s'inscrit dans un projet de R-D et vise à recueillir des éléments factuels essentiels pour atteindre les objectifs dudit projet.

\section{Tableau 2.3. Limites de la R-D, de l'innovation et d'autres activités commerciales}

\begin{tabular}{|c|c|c|}
\hline & Traitement requis & Observations \\
\hline Prototypes & $\begin{array}{l}\text { À inclure dans la } \\
\text { R-D }\end{array}$ & $\begin{array}{l}\text { Tant que l'objectif premier est d'apporter de } \\
\text { nouvelles améliorations. }\end{array}$ \\
\hline Installation pilote & $\begin{array}{l}\text { À inclure dans la } \\
\text { R-D }\end{array}$ & $\begin{array}{l}\text { Tant que l'objectif premier est de mener des } \\
\text { activités de R-D. }\end{array}$ \\
\hline Design industriel & $\begin{array}{l}\text { À inclure en partie } \\
\text { dans la R-D }\end{array}$ & $\begin{array}{l}\text { Inclure les tâches requises au stade de la } \\
\text { R-D et exclure celles liées au processus de } \\
\text { production. }\end{array}$ \\
\hline $\begin{array}{l}\text { Ingénierie industrielle } \\
\text { et outillage }\end{array}$ & $\begin{array}{l}\text { À inclure en partie } \\
\text { dans la R-D }\end{array}$ & $\begin{array}{l}\text { Inclure la R-D « supplémentaire » ainsi que les } \\
\text { activités d'outillage et d'ingénierie industrielle } \\
\text { associées aux procédés d'innovation. Exclure } \\
\text { celles liées aux procédés de production. }\end{array}$ \\
\hline Production à titre d'essai & $\begin{array}{l}\text { À inclure en partie } \\
\text { dans la R-D }\end{array}$ & $\begin{array}{l}\text { Inclure si la production requiert des essais en } \\
\text { vraiegrandeur et donc de nouvelles études de } \\
\text { conception et d'ingénierie. Exclure toutes les } \\
\text { autres activités connexes. }\end{array}$ \\
\hline $\begin{array}{l}\text { Développement préalable } \\
\text { à la production }\end{array}$ & À exclure de la R-D & \\
\hline $\begin{array}{l}\text { Service après-vente et détection } \\
\text { de pannes }\end{array}$ & À exclure de la R-D & Sauf la R-D « supplémentaire » (à inclure). \\
\hline $\begin{array}{l}\text { Travaux relatifs aux brevets } \\
\text { et licences }\end{array}$ & À exclure de la R-D & $\begin{array}{l}\text { Toutes les formalités administratives et } \\
\text { juridiques liées au dépôt de brevets et à la } \\
\text { délivrance de licences (la documentation } \\
\text { issue des projets de R-D relève de la R-D). } \\
\text { En revanche, les travaux relatifs aux brevets } \\
\text { qui ont un lien direct avec les projets de R-D } \\
\text { relèvent de la R-D. }\end{array}$ \\
\hline Tests de routine & À exclure de la R-D & $\begin{array}{l}\text { Même s'ils sont effectués par du personnel } \\
\text { de R-D. }\end{array}$ \\
\hline Collecte de données & À exclure de la R-D & $\begin{array}{l}\text { Sauf lorsque l'exercice fait partie intégrante } \\
\text { de la R-D. }\end{array}$ \\
\hline $\begin{array}{l}\text { Respect des obligations } \\
\text { courantes liées aux services } \\
\text { publics d'inspection et de } \\
\text { contrôle, à l'application des } \\
\text { normes, aux réglementations }\end{array}$ & À exclure de la R-D & \\
\hline
\end{tabular}




\section{Installations pilotes}

2.51 La construction et l'utilisation d'une installation pilote font partie de la R-D tant que l'objectif principal est d'acquérir de l'expérience et de rassembler des données techniques ou autres qui serviront:

- à vérifier des hypothèses

- à élaborer de nouvelles formules de produits

- à établir de nouvelles spécifications de produits finis

- à concevoir les équipements et structures spéciaux nécessaires à un nouveau procédé

- à rédiger des modes opératoires ou des manuels d'exploitation du procédé.

2.52 Toutefois, si dès l'achèvement de la phase expérimentale, une installation pilote fonctionne comme une unité normale de production commerciale, son exploitation ne peut plus être considérée comme une activité de R-D, même si l'installation reste qualifiée de pilote. Tant que la vocation première d'une installation pilote est de nature non commerciale, le fait qu'une partie ou la totalité de sa production soit vendue ne change rien sur le principe. Les recettes ainsi obtenues ne devraient pas être déduites du coût des activités de R-D (chapitre 4).

\section{Projets à grande échelle}

2.53 En règle générale, les projets à grande échelle (menés dans des domaines comme la défense, l'aérospatiale ou la mégascience) couvrent une gamme d'activités allant du développement expérimental au développement préalable à la production. Dans ces conditions, l'organisme qui finance et/ou mène à bien ces projets est souvent dans l'incapacité de distinguer la R-D des autres éléments de dépenses. Or, cette distinction entre dépenses de R-D et hors R-D est particulièrement importante dans les pays où une forte proportion des dépenses publiques de R-D est consacrée à la défense.

2.54 Il importe de s'intéresser de très près à la nature des installations pilotes ou des prototypes très onéreux, comme la tête de série d'un nouveau type de centrale nucléaire ou d'un nouveau modèle de brise-glace. Ces installations et prototypes peuvent être presque entièrement réalisés à partir de matériaux existants et de technologies connues et ils sont souvent construits pour servir simultanément à l'exécution de travaux de R-D et à la prestation du service principal auquel ils sont destinés (produire de l'électricité ou briser les glaces). Leur construction ne devrait pas être entièrement attribuée à la R-D. Seuls les coûts additionnels dus à la nature expérimentale de ces produits devraient être imputés à la R-D.

\section{Production à titre d'essai}

2.55 Après qu'un prototype a fait l'objet d'une mise à l'essai concluante et de toutes les modifications requises, sa mise en fabrication peut démarrer. Cette phase est liée à la production à l'échelle industrielle et peut impliquer de 
modifier le produit ou le procédé ou encore de former le personnel aux nouvelles techniques ou à l'utilisation de nouvelles machines. Dès lors qu'elle n'exige pas de nouveaux travaux de conception et d'ingénierie, la phase de mise en fabrication ne devrait pas être comptabilisée dans la R-D, puisque l'objectif principal n'est plus de perfectionner le produit mais de mettre en route sa production. Les premières unités produites dans le cadre d'un essai en vue d'une production en grande série ne devraient pas être considérées comme des prototypes de R-D, même si, par abus de langage, on les désigne sous ce nom.

2.56 Par exemple, lorsqu'un nouveau produit doit être assemblé par soudage automatique, la recherche du réglage optimal du dispositif de soudage en vue d'obtenir la meilleure cadence de production et la meilleure efficacité possibles ne sera pas assimilée à de la R-D.

\section{Détection de pannes}

2.57 Si la détection de pannes révèle parfois la nécessité d'engager de nouveaux travaux de R-D, elle conduit plus souvent à apporter des modifications mineures dans l'équipement et les procédés standards concernés. Cette activité ne devrait donc pas être incluse dans la R-D.

\section{R-D « supplémentaire "}

2.58 Même après qu'un produit ou procédé nouveau est passé à l'unité de production, des problèmes techniques continueront de se poser, exigeant parfois la poursuite des travaux de R-D. Cette R-D « supplémentaire " devrait être incluse dans la R-D.

\section{Outillage et ingénierie industrielle}

2.59 Dans la majorité des cas, les phases d'outillage et d'ingénierie industrielle d'un projet sont considérées comme faisant partie du procédé de production et non de la R-D. L'outillage peut être divisé en trois phases :

- première utilisation des composants (y compris de ceux qui sont issus des travaux de R-D)

- mise au point initiale des équipements de production en série

- mise en place de l'équipement lié au démarrage de la production en série.

2.60 Si le processus d'outillage se traduit par de nouveaux travaux de R-D, visant notamment à améliorer les machines et outils de production, à modifier les procédures de production et de contrôle de la qualité ou à mettre au point de nouvelles méthodes et normes, ces travaux sont alors classés dans la R-D. La R-D « supplémentaire » découlant de la phase d'outillage devrait être qualifiée de R-D.

\section{Essais cliniques}

2.61 Avant de pouvoir mettre sur le marché de nouveaux médicaments, vaccins, appareils ou traitements, il y a lieu de les tester de manière systématique sur des sujets humains volontaires afin de vérifier leur innocuité et efficacité. Ces essais cliniques se divisent en quatre phases standards, dont trois précèdent 
l'autorisation de mise en fabrication. À des fins de comparaison internationale, il est admis par convention que les phases 1, 2 et 3 des essais cliniques peuvent être considérées comme de la R-D. La phase 4, qui consiste en la poursuite des essais après autorisation et mise en fabrication du médicament ou traitement concerné, ne devrait être traitée au titre de la R-D que si elle débouche sur de nouveaux progrès scientifiques ou technologiques. En outre, les activités menées avant l'autorisation de production ne sont pas toutes considérées comme de la $\mathrm{R}-\mathrm{D}$, notamment quand l'achèvement des essais de phase 3 est suivi d'un long délai pendant lequel il est possible d'engager des activités de commercialisation et de développement du procédé.

\section{R-D et design}

2.62 Les activités de R-D et de design sont difficilement dissociables. Certaines activités de design font partie intégrante des projets de R-D, de même que la R-D peut intervenir dans de nouvelles activités de design. Les deux notions présentent des similitudes et liens d'interdépendance. Cela dit, toutes les activités de conception ne remplissent pas les conditions de nouveauté fonctionnelle et d'incertitude qui sont décrites dans la section du présent chapitre consacrée aux cinq critères de base caractérisant la R-D. Le design joue un rôle clé dans le développement et le déploiement des innovations. En l'absence de définition agréée à des fins statistiques, le design peut être décrit comme une activité d'innovation potentiellement très diversifiée visant à planifier et à définir des procédures, des spécifications techniques et autres caractéristiques d'utilisation ou d'ordre fonctionnel applicables à de nouveaux produits et procédés. Il peut s'agir des préparatifs liminaires de la planification de nouveaux produits ou procédés ainsi que des travaux liés à leur mise au point et déploiement, tels que ceux consistant à apporter des ajustements et autres modifications. Cette description souligne la dimension créative du design dans le processus d'innovation, que peut également revêtir la R-D exécutée dans le même contexte. Certaines activités liées au design peuvent être considérées comme relevant de la R-D dès lors qu'elles s'inscrivent dans un processus de développement de produits qui vise à aboutir à quelque chose de " nouveau " (pas nécessairement de nouvelles connaissances), qui est créatif et original, qu'il est possible de formaliser (autrement dit, dont l'exécution est assurée par une équipe dédiée à cette tâche) et qui débouche sur un résultat codifié transmissible à l'équipe chargée du développement. La différence principale avec la R-D est qu'un projet d'innovation ne comporte normalement aucun élément d'incertitude dès lors que son exécution est confiée à des designers qualifiés. Il y a lieu d'en déduire que le design n'est pas assimilable à la R-D et qu'elle doit en être distinguée pour tout besoin d'ordre statistique.

2.63 Si tout projet de R-D comporte un élément d'incertitude quant à la possibilité d'aboutir au résultat escompté suivant le calendrier convenu, l'incertitude qui entoure un projet de design est directement liée à la clarté et à la 
faisabilité des objectifs initialement fixés. Par exemple, si le design d'un bâtiment standard ne soulève aucune incertitude majeure concernant le résultat final, plus l'ouvrage sera complexe, du fait par exemple de l'ajout des caractéristiques, plus l'incertitude sera grande quant à la durée et aux coûts de réalisation. Pour y remédier, il pourra être nécessaire d'engager une activité de R-D en complément de l'utilisation des outils de design existants.

\section{$R$-D et création artistique}

2.64 Le design se caractérise parfois par l'utilisation de méthodes artistiques, ce qui peut également donner lieu à des confusions. Il convient donc, dans toute réflexion sur la R-D et la création artistique, d'établir au préalable une distinction entre recherche à visée artistique, recherche sur l'art et expression artistique.

\section{Recherche à visée artistique}

2.65 La recherche à visée artistique consiste à mettre au point des biens et des services qui répondent aux besoins des auteurs et artistes. Il existe des entreprises spécialisées dans ce domaine qui consacrent une partie non négligeable de leurs ressources à la $\mathrm{R}-\mathrm{D}$, par exemple, à des activités de développement expérimental en vue de produire de nouveaux instruments de musique électronique adaptés aux besoins d'un groupe. D'autres types d'acteurs de la R-D (principalement des universités et établissements d'enseignement spécialisé) concourent à l'étude des nouvelles technologies dans les arts de la scène (par exemple, dans le but d'améliorer la qualité audio/vidéo). Les activités qui visent à aider les établissements à vocation artistique à adopter de nouvelles méthodes d'organisation ou de commercialisation (publicité, gestion financière, etc.) peuvent être qualifiées d'activités de R-D, mais c'est une décision à prendre avec circonspection. Ce domaine d'exécution de la R-D est déjà pris en compte dans le recueil de données existantes.

\section{Recherche sur l'art (étude de l'expression artistique)}

2.66 La recherche fondamentale ou appliquée intervient dans la majeure partie des disciplines liées à l'étude des arts (musicologie, histoire de l'art, études théâtrales, études des médias, littérature, etc.). Les établissements publics de recherche pourraient jouer un rôle dans certains domaines de recherche (car certaines des infrastructures de recherche compétentes, à l'instar des bibliothèques ou des archives, sont souvent rattachées à des lieux d'art, comme les musées, les théâtres, etc.). En ce qui concerne les activités de conservation et de restauration (dans le cas où elles ne sont pas incluses dans l'activité précédente), il est recommandé de qualifier les prestataires de tels services techniques d'exécutants de R-D (employant des chercheurs, publiant des travaux scientifiques, etc.). Ce domaine d'exécution de R-D est largement couvert par les données déjà recueillies. 


\section{L'expression artistique par opposition à la recherche}

2.67 Les prestations artistiques sont normalement exclues de la R-D. Répondant à une quête de nouvelles formes d'expression et non de connaissances, elles ne remplissent pas le critère de nouveauté. Il en va de même du critère de reproductibilité (qui renvoie au mode de transfert des connaissances supplémentaires potentiellement obtenues). Faute d'éléments supplémentaires, il n'y a donc pas lieu de supposer que les établissements d'enseignement artistique et les facultés des arts exécutent des activités de R-D. Le fait que des artistes y suivent des cours n'intervient pas dans la mesure de la R-D. Il convient néanmoins de se pencher sur les établissements d'enseignement supérieur au cas par cas afin d'établir s'ils décernent ou non des doctorats sur la base de prestations artistiques. Il est recommandé de suivre l'approche dite institutionnelle et de ne qualifier d'activité de R-D potentielle (à l'intention des acteurs chargés de recueillir des données) que les pratiques artistiques assimilées à la R-D par les établissements d'enseignement supérieur.

\section{$R-D$ et développement logiciel}

2.68 Les technologies de l'information occupent une place dominante dans la quasi-totalité des activités d'innovation et sont largement tributaires des activités de R-D. Cela dit, elles influent également sur la capacité des entreprises et des établissements à exécuter de la R-D. Le développement logiciel est une activité liée à l'innovation qui est parfois associée à la R-D et qui, lorsque certaines conditions sont réunies, comporte un élément de R-D. Pour qu'un projet de développement logiciel soit classé dans la $R-D$, son exécution doit dépendre d'un progrès scientifique et/ou technologique et son but doit être de dissiper une incertitude scientifique et/ou technologique de manière systématique.

2.69 Outre les logiciels utilisés dans le cadre d'un projet général de R-D (pour consigner et suivre le déroulement de ses différentes étapes, par exemple), l'activité associée à un logiciel qui constitue un produit final ou qui fait partie intégrante d'un produit final peut être qualifiée de R-D à partir du moment où elle satisfait aux critères applicables.

2.70 De par la nature du développement logiciel, il est difficile d'en cerner la composante de R-D, lorsqu'elle existe. Le développement logiciel fait partie intégrante de nombreux projets, eux-mêmes, dépourvus de tout élément de R-D. Cependant, la composante " développement logiciel » de ces projets peut être classée dans la R-D s’il en résulte des progrès dans le domaine informatique. En général, il s'agit davantage d'une évolution que d'une révolution. C'est pourquoi l'adoption d'une version plus puissante, l'enrichissement ou la modification d'un programme ou système existant peuvent être associés à la $R-D$, s'ils impliquent des progrès scientifiques et/ou technologiques aboutissant à un enrichissement des connaissances. Cependant le fait d'utiliser des logiciels dans une application ou finalité nouvelle ne constitue pas en soi un progrès. 
2.71 Les activités ci-après sont des exemples d'activités de R-D logicielle à inclure dans la R-D :

- développement de nouveaux systèmes d'exploitation ou langages

- conception et déploiement de nouveaux moteurs de recherche fondés sur des technologies originales

- tentative de règlement de conflits matériels ou logiciels suivant une procédure de reconfiguration du système ou du réseau

- élaboration ou perfectionnement d'algorithmes à partir de nouvelles techniques

- mise au point de nouvelles techniques originales de cryptage ou de sécurité.

2.72 Les activités logicielles courantes ne doivent pas être considérées comme relevant de la R-D. Il s'agit notamment des travaux visant à perfectionner des systèmes ou programmes librement accessibles avant le début desdits travaux. Sont également exclus les problèmes techniques surmontés dans le cadre de projets antérieurs eu égard aux mêmes systèmes d'exploitation et architectures informatiques. Les tâches courantes de maintenance informatique et logicielle ne sont pas incluses dans la R-D.

Entre autres exemples d'activités logicielles à exclure de la R-D, on peut citer :

- le développement d'applications et de systèmes d'information destinés aux entreprises, à partir de méthodes connues et d'outils logiciels déjà existants

- l'ajout de fonctionnalités propres à l'utilisateur dans des programmes d'application existants (notamment des fonctionnalités de base concernant la saisie de données)

- la création de sites web ou de logiciels à l'aide d'outils existants

- l'utilisation de méthodes types de cryptage, de contrôle de sécurité et de vérification de l'intégrité des données

- l'adaptation d'un produit à un usage particulier, sauf si l'exercice donne lieu à l'acquisition de connaissances de nature à améliorer considérablement le programme de base

- les tâches courantes de débogage de systèmes et programmes existants, sauf si elles sont effectuées avant la fin du développement expérimental.

2.73 Dans le domaine des logiciels système, les projets pris individuellement ne sont pas nécessairement rattachés à la $\mathrm{R}-\mathrm{D}$, mais leur regroupement au sein d'un projet plus vaste peut créer une incertitude technologique qui devra être levée par la R-D. À l'inverse, il est envisageable qu'un projet de grande envergure vise à mettre au point un produit commercial à l'aide des technologies disponibles et ne contienne aucun élément de R-D au stade de la planification, mais que l'intégration technologique exige finalement une activité supplémentaire de R-D à certains niveaux. 
2.74 Le SCN 1993 (CE et al., 1994) classait les dépenses totales en logiciels (y compris la R-D relative au développement logiciel) dans les dépenses d'investissement. Depuis, le SCN 2008 (CE et al., 2009) y intègre également les dépenses totales de R-D. D'après le manuel de l'OCDE intitulé Handbook on deriving capital measures of intellectual property products (OCDE, 2009), qui vient compléter les indications du SCN 2008 concernant les produits incorporels, la R-D liée aux logiciels immobilisés continue de faire partie de l'investissement en logiciels. Il importe de pouvoir distinguer clairement les dépenses de R-D consacrés aux logiciels afin que les statisticiens et les utilisateurs de la R-D et du SCN appréhendent mieux les chevauchements existants entre logiciels et R-D. Cette question est examinée plus en détail dans le chapitre 4.

\section{$R-D$, enseignement et formation}

2.75 Les établissements d'enseignement et de formation post-secondaire consacrent l'essentiel de leurs ressources à l'enseignement et sont donc peu susceptibles de participer à des projets de R-D. En revanche, dans les établissements d'enseignement supérieur, la recherche et l'enseignement sont toujours très étroitement liés dans la mesure où ces deux activités sont la plupart du temps exercées ensemble et qu'elles mobilisent une grande partie des bâtiments et des équipements.

2.76 Dans la mesure où les résultats de la recherche se répercutent sur l'enseignement et que les informations et l'expérience acquises dans le cadre de l'enseignement viennent souvent étayer la recherche, il est difficile de déterminer avec précision où commencent et se terminent les activités d'enseignement ou de formation du personnel de l'enseignement supérieur et des étudiants et les activités de R-D. La R-D se distingue de l'enseignement courant et autres activités connexes par les éléments de nouveauté qu'elle comporte. Dans le secteur de l'enseignement supérieur, les principaux critères caractérisant la R-D peuvent être complétés par la prise en compte du rôle que les acteurs ci-après jouent au sein des établissements considérés :

- les étudiants en doctorat et en master, à certaines conditions (chapitres 5 et 9)

- les superviseurs des étudiants (inclus dans le personnel universitaire)

- les prestataires de soins de santé spécialisés exerçant en hôpital universitaire.

2.77 Dans la mesure où il convient d'inclure les activités de recherche exécutées par les étudiants en doctorat dans la R-D imputable au secteur de l'enseignement supérieur, ces étudiants et le personnel universitaire dirigeant ou supervisant leurs travaux devraient être inclus dans le personnel de R-D (voir chapitre 5). Il va de soi que le temps consacré aux tâches dépourvues de lien avec la recherche est à exclure des calculs de la R-D. Tout cela s'applique à l'ensemble des disciplines scientifiques. 
2.78 De même, en hôpital universitaire, où la formation des étudiants en médecine occupe une place de choix en complément de la vocation première de prestation de soins de santé, il n'est pas rare que les activités d'enseignement, de R-D et de prestation de soins médicaux courants et de pointe soient étroitement liées. Dès lors que le personnel et les étudiants chargés des soins spécialisés peuvent être considérés, sans risque d'erreur, comme concourant à l'effort global de R-D, ils devraient être inclus dans le personnel de R-D. Toute activité courante engagée en vue de fournir des soins de santé dans le même contexte est à exclure de la R-D.

\section{La R-D dans les activités de services}

2.79 Aux termes du SCN 2008, les services sont le résultat d'une activité de production qui se traduit par un changement de l'état des unités qui les consomment ou qui facilite l'échange de produits ou d'actifs financiers. Il peut s'agir de changements apportés à l'état de biens de consommation ; à l'état physique et intellectuel de personnes (par exemple, services de santé ou de transport, d'information, d'éducation, etc.). Le SCN distingue une catégorie de produits hybride qui touche à la fois les biens et les services : les " produits basés sur la capture des connaissances ". Ils concernent la fourniture, le stockage, la communication et la diffusion d'informations, de conseils et de divertissements permettant à l'unité qui les consomme d'accéder aux connaissances de façon répétée. Les branches d'activité qui les produisent sont celles concernées par la fourniture, le stockage, la communication et la diffusion des informations, des conseils et des divertissements au sens le plus large de ces termes (CE et al., 2009).

2.80 La fourniture de services suppose un degré élevé de proximité et d'interaction avec la clientèle. Peuvent également y prendre une part active les branches d'activité spécialisées dans la production de biens. De même, les entreprises du secteur tertiaire peuvent contrôler plusieurs aspects de la production de biens, comme le développement expérimental de nouveaux biens intervenant dans la fourniture de services.

2.81 Il est donc difficile de définir les limites de la R-D dans les activités de services, pour deux raisons principales : premièrement, parce qu'il est malaisé de mettre en évidence les projets qui comportent un élément de R-D spécifique à un service et non intégré dans un bien ou un produit basé sur la capture des connaissances ; et, deuxièmement, parce que la séparation entre R-D et autres activités d'innovation n'est pas toujours clairement établie.

2.82 Ceux des nombreux projets engagés dans le secteur des services qui relèvent de la R-D débouchent sur de nouvelles connaissances ou sur la conception d'applications nouvelles grâce à l'exploitation de connaissances existantes, conformément à la définition de la R-D.

2.83 La R-D est plus difficile à repérer dans les activités de services que dans les branches d'activité marchande productrices de biens car elle ne concernera pas nécessairement un domaine de recherche donné, même si une spécialisation 
pourra exister selon le marché visé. La R-D touche plusieurs domaines : les technologies, les sciences sociales, les sciences humaines et les arts, y compris l'étude des comportements et des organisations. Ce dernier domaine est déjà inclus dans le critère " connaissance de l'homme, de la culture et de la société ", mais n'en revêt pas moins une importance particulière dans le cas des activités de services. Comme ces différents types de R-D peuvent être combinés dans un projet donné, il est important de délimiter clairement les différentes formes que prend l'activité de R-D considérée. Si l'analyse se limite à des travaux de R-D liée à la technologie, par exemple, on risque de sous-estimer le contenu de R-D. Bien souvent, les résultats de la R-D obtenus dans le secteur des services relèvent des activités de prestation de services.

2.84 En outre, dans les sociétés de services, la R-D n'est pas toujours organisée de manière aussi formelle que dans les entreprises productrices de biens (c'est-à-dire avec un service dédié à la R-D et l'emploi de chercheurs ou d'ingénieurs de recherche identifiés comme tels dans le tableau des effectifs, etc.). Le concept de R-D dans les services est encore moins précis et peut même passer inaperçu dans ces sociétés. Peut-être faudra-t-il, à mesure que l'on en saura plus sur le recueil de données sur la R-D dans les services, affiner les critères d'identification de la R-D et les exemples de R-D liée aux services.

\section{Critères d'identification de la R-D dans les services}

2.85 En complément des cinq critères de base, les éléments ci-après peuvent aider à mettre en évidence l'existence de travaux de R-D dans les activités de services :

- existence de liens avec des laboratoires publics de recherche

- participation de titulaires d'un doctorat ou d'étudiants en doctorat

- publication des résultats des travaux de recherche dans des revues scientifiques, organisation de conférences scientifiques ou contribution à des revues scientifiques.

\section{Exemples de travaux de R-D dans des activités de services}

2.86 On trouvera ci-dessous des exemples d'activités de R-D menées dans le secteur des services. Il convient par ailleurs de tenir compte des critères généraux et complémentaires de la R-D qui sont présentés à la section 2.4 .

2.87 Les contours généraux de la R-D définis plus haut s'appliquent en grande partie aux activités de services. L'élément de nouveauté est un critère essentiel pour distinguer la R-D d'activités connexes.

\section{Exemples de travaux de R-D dans les secteurs de la banque et des assurances}

- recherche mathématique liée à l'analyse des risques financiers

- mise au point de modèles de risques pour élaborer une politique du crédit 
- développement expérimental de nouveaux logiciels pour les opérations bancaires à domicile

- mise au point de techniques d'étude du comportement des consommateurs aux fins de la création de nouveaux types de comptes et de services bancaires

- recensement des nouveaux risques et nouvelles caractéristiques de risque à prendre en considération dans les contrats d'assurance

- recherche sur les phénomènes de société influant sur les nouvelles formes d'assurance (santé, retraite, etc.), comme la couverture des non-fumeurs

- R-D liée aux services de banque et d'assurance en ligne, aux services internet et aux applications de commerce électronique

- R-D liée aux services financiers nouveaux ou considérablement améliorés (nouveaux types de comptes, de prêts, de contrats d'assurance et d'instruments d'épargne).

\section{Exemples de travaux de R-D dans d'autres activités de services}

- analyse des effets de l'évolution économique et sociale sur la consommation et les activités de loisirs

- mise au point de nouvelles méthodes permettant de mesurer les attentes et les préférences des consommateurs

- mise au point de nouvelles méthodes permettant d'obtenir et de mesurer des résultats de services sociaux adaptables à différents contextes socioéconomiques ou culturels

- mise au point de nouvelles méthodes et de nouveaux instruments d'enquête

- mise au point de procédures de localisation et de suivi (logistique)

- recherche de nouveaux concepts de voyage et de vacances.

\section{$R-D$ et activités scientifiques et technologiques connexes}

2.88 Il est difficile de dissocier la R-D d'autres activités scientifiques et technologiques (AST) dès lors qu'une même unité institutionnelle mène plusieurs activités. Les critères appliqués dans le cadre du recueil de données reposent généralement sur une connaissance directe de l'unité considérée. De manière générale :

- Les établissements ou certaines de leurs unités et les entreprises dont la R-D constitue l'activité principale exercent souvent des activités secondaires hors R-D (par exemple, information scientifique et technique, réalisation d'essais, contrôle qualité, analyses). Dès lors qu'une activité secondaire est engagée dans le but premier de servir la R-D, elle devrait y être incluse ; si cette activité secondaire vise essentiellement à répondre à d'autres besoins que ceux de la $\mathrm{R}-\mathrm{D}$, elle devrait en être exclue. 
- Il n'est pas rare que les établissements principalement dédiés à une activité scientifique liée à la R-D engagent des travaux de recherche connexes. Ces travaux de recherche devraient être considérés à part et pris en compte dans la mesure de la R-D.

2.89 Dans certains secteurs, les principaux critères qui permettent de distinguer la R-D des activités scientifiques et technologiques connexes sont particulièrement difficiles à appliquer. La collecte, la vérification et la normalisation des données d'utilité générale, les projets liés aux mégadonnées, l'exploration spatiale, ou encore l'exploration et l'évaluation minières sont autant de domaines qui mobilisent d'importants volumes de ressources. Aussi, toute variation de leur traitement aura des incidences notables sur la comparabilité internationale des données de R-D qui en découlent. Il est également difficile de recenser les éléments de R-D dans les projets à grande échelle. Au moment de la rédaction du présent ouvrage, l'UNESCO avait entrepris de mettre à jour ses définitions des AST à des fins statistiques (UNESCO, 1978; UNESCO, 1984), exercice qui devrait clarifier la démarcation entre la R-D et les autres AST. Les orientations qui en résulteront seront communiquées en temps utile dans un complément au présent manuel, consultable en ligne.

\section{Recueil et enregistrement de données d'utilité générale}

2.90 Le recueil de données d'utilité générale, habituellement mené par des organismes publics, porte sur les phénomènes naturels, biologiques ou sociaux qui présentent un intérêt public ou que seuls les pouvoirs publics ont les moyens de consigner. À titre d'exemple, on peut citer les travaux courants d'établissement de cartes topographiques, de levés géologiques, hydrologiques, océanographiques et météorologiques, ainsi que les observations astronomiques. Le recueil de données exclusivement ou principalement réalisé dans le cadre de la R-D relève de la R-D (par exemple, s'il s'agit de données recueillies par un détecteur dans le cadre d'une expérience de diffusion de particules élémentaires au CERN). Ce raisonnement s'applique également au traitement et à l'interprétation des données. Les sciences sociales, en particulier, dépendent largement de la consignation exacte de faits concernant la société sous forme de recensements, d'enquêtes par échantillons, etc. Si ces données sont spécialement recueillies ou traitées aux fins de la recherche scientifique, leur coût devrait être imputé à la recherche et couvrir leur planification et systématisation. On peut également être en présence de R-D lorsqu'un projet concret porte sur l'élaboration de méthodes statistiques entièrement nouvelles (par exemple, travaux conceptuels et méthodologiques visant l'élaboration de méthodes d'enquête et de systèmes statistiques entièrement nouveaux ou profondément remaniés, travaux sur les méthodes d'échantillonnage et estimations statistiques dans des domaines limités) ou de méthodes et techniques de collecte des données. Cependant, les données recueillies à d'autres fins ou d'utilité générale, par exemple pour les enquêtes trimestrielles sur le chômage, devraient être exclues de la R-D, même si 
elles sont exploitées à des fins de recherche (sauf si l'utilisation de ces données est payante). Les études de marché sont également à exclure.

2.91 Les activités d'un service d'information scientifique et technique et d'une bibliothèque de laboratoire de recherche essentiellement réservée à l'usage des chercheurs dudit laboratoire devraient être incluses dans la R-D. Devraient en être exclues les activités du centre de documentation d'une entreprise ouvert à l'ensemble du personnel, même s'il partage les locaux de l'unité de recherche de ladite entreprise (il conviendra ici d'éviter de surévaluer les activités liées à la R-D). De même, les activités des bibliothèques centrales des universités devraient être exclues de la $\mathrm{R}-\mathrm{D}$. Ces critères, qui visent aussi les bibliothèques électroniques et référentiels de données, ne s'appliquent que s'il y a lieu de traiter dans leur intégralité les activités d'une institution ou d'un département. Le recours à des méthodes comptables plus détaillées peut permettre d'imputer une partie des coûts des activités exclues sur le poste des frais généraux de la R-D. Les frais induits par l'élaboration des publications scientifiques et techniques sont, en général, exclus ; en revanche, ceux occasionnés par l'établissement des rapports originaux exposant les résultats des recherches devraient être inclus dans la R-D.

\section{Essais et normalisation}

2.92 Bien souvent, les organismes publics et les associations de consommateurs possèdent des laboratoires qui ont pour missions principales de tester des produits et de vérifier le respect des normes établies. Outre les activités d'essais et d'analyse comparative, qui ne sont pas de la R-D, le personnel de ces laboratoires peut également s'employer à concevoir des méthodes d'essai nouvelles ou considérablement améliorées. Les activités de cette nature devraient être incluses dans la R-D.

\section{Projets relatifs aux mégadonnées}

2.93 L'apparition d'instruments et de méthodes permettant d'explorer d'importants volumes de données favorise les découvertes scientifiques et l'innovation fondée sur les données. Ces activités relèvent de la R-D, si et seulement si elles remplissent les cinq critères de base, en particulier celui qui touche au caractère systématique de la conduite de l'activité ou du projet considéré - il s'agira donc de clairement mettre en évidence un déficit de connaissance initial et de consacrer des ressources concrètes aux efforts déployés pour le combler. Un exemple en est le projet " Génome humain ", dans le cadre duquel des chercheurs et institutions de 18 pays se sont engagés à collaborer pendant 13 ans à établir le séquençage de l'ADN humain. Grâce aux technologies numériques, le critère " codification de la R-D » joue un rôle non négligeable dans les projets qui font intervenir les mégadonnées, dans la mesure où, pour être exploitables, les données issues des projets scientifiques fondés sur les "mégadonnées " doivent permettre de transmettre des connaissances sur 
les phénomènes initialement étudiés. Ces données ne sont pas nécessairement accessibles au plus grand nombre ou exploitables à des fins de recherche. Le concept de science ouverte renvoie communément aux efforts déployés pour élargir l'accès de la communauté scientifique, du secteur des entreprises et de la société en général aux résultats de la recherche financée par les fonds publics au format numérique (OCDE, 2015). Dans certains cas, l'action engagée pour que la communauté scientifique puisse librement accéder aux données de la recherche, notamment pour mettre au point des outils facilitant la reproductivité des travaux de recherche, fera partie intégrante d'un projet de R-D à condition qu'elle soit explicitement formulée comme telle dans les objectifs dudit projet et prévue au budget. Dans d'autres cas, elle sera assimilée à des efforts de diffusion distincts et ne sera donc pas comptabilisée dans la R-D.

\section{Exploration spatiale}

2.94 L'une des difficultés liées à l'exploration spatiale est qu'à certains égards, cette activité présente maintenant en grande partie un caractère de routine ; l'achat de biens et de services qui ne relèvent pas de la R-D représente assurément l'essentiel des coûts. Il peut être nécessaire de séparer les activités d'exploration spatiale (mise au point de véhicules, d'équipements, de logiciels et de techniques) de celles associées à la mise en orbite régulière de satellites ou à la mise en place de stations de communications et de guidage.

\section{Exploration et évaluation minières}

2.95 Aux termes du SCN 2008, l'exploration et l'évaluation minières constituent une catégorie d'activité conduisant à la création d'un actif de propriété intellectuelle distinct de la R-D (CE et al., 2009 ; OCDE, 2009). Ces activités viennent enrichir la connaissance du sous-sol en différents endroits pour les besoins de l'exploitation économique des gisements. Elles consistent notamment à acquérir des droits d'exploration, à effectuer des études topographiques, géologiques, géochimiques et géophysiques ainsi qu'à mener des activités d'excavation, d'échantillonnage et d'évaluation.

2.96 En outre, dans le présent manuel, la R-D est strictement dissociée de l'exploration minière, quoique des liens puissent être constatés. Par exemple, les résultats d'un certain nombre de tests géologiques entrepris dans le cadre de projets de R-D peuvent inciter les entreprises minières à poursuivre des travaux d'exploration non liés à la R-D dans un but d'exploitation. Des activités de R-D peuvent également se révéler nécessaires pour mettre au point de nouvelles techniques d'essai et de forage que le secteur minier mettra à profit au stade de l'exploration et dans les activités courantes. S'agissant des activités d'exploitation et de prospection minières, il arrive que la recherche de ressources nouvelles ou considérablement améliorées (ressources alimentaires, énergétiques, etc.) soit confondue avec la quête de réserves de ressources naturelles existantes, cette confusion terminologique estompant la distinction à établir entre, d'une 
part, la R-D et, d'autre part, les activités de levés et de prospection. En théorie, l'établissement de données précises concernant la R-D suppose de recenser, de mesurer et de regrouper les activités suivantes :

- mise au point de nouvelles méthodes et techniques topographiques

- activités topographiques faisant partie intégrante d'un projet de recherche relatif aux phénomènes géologiques

- réalisation, à titre subsidiaire, de travaux de recherche sur les phénomènes dans le cadre des programmes de levés et de prospection.

2.97 Dans la pratique, cette troisième activité pose un certain nombre de problèmes. Il est difficile de formuler une définition précise qui parlerait aux répondants des enquêtes nationales. C'est pourquoi seules les activités suivantes devraient être incluses dans la R-D :

- mise au point de méthodes et d'équipements nouveaux ou considérablement améliorés destinés à l'acquisition de données, au traitement et à l'étude des données recueillies, ainsi qu'à l'interprétation de ces données

- activités topographiques faisant partie intégrante d'un projet de recherche relatif aux phénomènes géologiques, notamment acquisition, traitement et interprétation de données à des fins principalement scientifiques.

2.98 En conséquence, les activités de levés et de prospection menées par les entreprises commerciales seront presque toutes exclues de la R-D. Ainsi, le fait de forer des puits d'exploration pour faire le point sur les services technologiques ne relève pas de la R-D.

\section{R-D et gestion des activités scientifiques et technologiques (AST)}

\section{Niveaux de maturité technologique}

2.99 La classification des grands projets de R-D est examinée dans le chapitre 8, en particulier sous l'angle des secteurs de la défense et de l'aérospatiale. Certains pays utilisent des classifications par niveau de maturité technologique dans la passation de marchés et la description de projets. Vu qu'il en existe un certain nombre, il est recommandé d'établir, le cas échéant, si celle en usage dans le pays considéré permettrait d'améliorer la collecte de statistiques de R-D.

\section{Projets de démonstration}

2.100 Deux concepts de démonstration ont déjà cours dans les statistiques de R-D : la " démonstration à l'intention des utilisateurs ", qui intervient lorsqu'un prototype est mis en œuvre en vraie grandeur ou presque dans des conditions réalistes afin de faciliter la formulation de l'action à mener ou de promouvoir son utilisation, et qui ne relève pas de la R-D ; et la « démonstration technique " (incluant notamment le développement de projets et de modèles de démonstration) qui, faisant partie intégrante d'un projet de $R-D$, constitue une activité de R-D. 
2.101 Largement employée dans la gestion de grands projets de recherche, la "démonstration technologique " est considérée comme une étape de la procédure engagée pour évaluer ex-ante ou ex-post le déploiement de nouvelles technologies. Cette définition, qui avait été initialement retenue dans le secteur de l'information et de la communication, a évolué si bien que l'expression désigne désormais l'activité menée pour montrer aux investisseurs et clients potentiels les potentialités offertes par la technologie en cours de développement. Il n'est donc pas recommandé de l'employer dans le contexte de la R-D, à moins qu'il soit possible de définir clairement le rôle conféré à une activité de démonstration dans un projet de R-D.

\section{La R-D dans les sciences sociales, les sciences humaines et les arts}

2.102 Dans la définition de la R-D énoncée dans le présent manuel, l'expression " connaissance de l'homme, de la culture et de la société » désigne les sciences sociales, les sciences humaines et les arts. Dans ces domaines aussi, il est extrêmement utile de se fonder sur des critères clairs par exemple, présenter un élément appréciable de nouveauté et résoudre une incertitude permettant de dissocier la R-D des activités scientifiques connexes (courantes) ainsi que des études non scientifiques. Il convient de tenir compte des composantes conceptuelles, méthodologiques et empiriques du projet considéré pour établir l'existence d'une activité de R-D.

2.103 En sciences sociales (par exemple, sociologie, économie ou science politique), les activités de recueil de données, comme la conduite d'enquêtes statistiques auprès de groupes de population donnés, ne peuvent être incluses dans la R-D que si elles font partie intégrante d'un projet de recherche précis ou qu'elles sont menées au profit d'un tel projet. Sont donc exclus de la recherche les projets à caractère routinier dans le cadre desquels les spécialistes appliquent à un problème concret des méthodes, principes et modèles bien établis des sciences sociales. Par exemple, si les données d'une enquête sur la population active servent à mettre en évidence les tendances du chômage à long terme dans le cadre d'un projet, l'activité " recueil des données " devrait être exclue de la composante R-D dudit projet (car ces données sont recueillies à intervalles réguliers suivant une méthode établie). En revanche, si des techniques d'entretien inédites sont employées pour effectuer une enquête dans le cadre d'une étude de cas concernant la situation du chômage dans une région particulière, le recueil de données peut être rattaché au volet R-D de l'étude. Plus généralement, dès lors qu'il est recouru à des données empiriques, ce sont les mêmes lignes directrices qui s'appliquent en sciences sociales qu'en sciences naturelles (sauf en ce qui concerne la vérification des résultats par voie expérimentale).

2.104 En sciences humaines, il est possible de procéder de la même manière que dans le domaine des arts (littérature, musique, arts visuels, théâtre, danse et autres arts de la scène). On soulignera l'aspect historique ou comparatif ainsi que le rôle joué par des universités ou autres établissements spécialisés 
dans l'élaboration de lignes directrices scientifiques spécifiques à la discipline considérée.

2.105 Le large éventail de sources utilisées en histoire, en archéologie, dans l'étude des langues et en droit, de même que la diversité des méthodes employées par les chercheurs sont autant de domaines d'activité potentiels de R-D. L'adoption des cinq critères de base de la R-D devrait être recommandée, en particulier eu égard à la nouveauté, à la créativité, à la transférabilité et/ou la reproductibilité.

2.106 Dans les domaines de la philosophie et de la théologie, par exemple, les études comparatives entreprises suivant les normes académiques actuelles sont incluses dans la R-D. Vu l'impossibilité d'édicter une règle générale, outre celle d'appliquer rigoureusement les critères d'identification de la R-D, il est également recommandé de suivre l'approche " institutionnelle » (c'est-à-dire d'exclure éventuellement les activités de recherche consacrées à des questions philosophiques et religieuses qui ne sont pas menées par des établissements de recherche agréés).

2.107 En conclusion, les travaux de recherche menés en sciences humaines et dans les arts peuvent relever de la R-D dès lors que leur nature "scientifique " est établie au regard des critères définis dans ces domaines. On appliquera par ailleurs les lignes directrices d'ordre pratique ci-après.

- Contexte dans lequel s'inscrivent les travaux (type d'établissement). Les travaux de recherche menés au sein d'une université ou d'un établissement de recherche officiellement reconnu (musées, bibliothèques inclus) peuvent être rattachés à la R-D.

- Adoption de procédures reconnues. La recherche passe par la formalisation, ce qui s'applique aussi aux sciences humaines. Les activités de recherche pourraient être recensées et leurs résultats mis à la disposition de la communauté scientifique en étant publiés dans des revues scientifiques. Dans la mesure où ces conditions sont observées et qu'une communauté scientifique œuvre activement à l'élaboration de règles permettant d'identifier ses membres, il est possible de procéder à l'identique pour recenser les résultats de la $\mathrm{R}-\mathrm{D}$.

- La recherche en sciences humaines peut porter sur l'élaboration systématique de théories ou d'interprétations de textes, d'événements, de traces matérielles ou de tout autre élément disponible. Par convention, les activités de recherche menées en dehors des disciplines de R-D énumérées dans le chapitre 3 doivent être exclues de la R-D.

\section{R-D et savoir traditionnel}

2.108 Un domaine de recherche transversal empruntant aux sciences humaines et à la médecine touche le " savoir traditionnel ", qui désigne, dans une grande mesure de façon tacite, une " accumulation de connaissances, de savoir-faire, de pratiques et de représentations conservés et étoffés par des 
peuples entretenant une relation de longue date avec leur milieu naturel [...], un ensemble culturel complexe englobant le langage, les systèmes de nomenclature et de classification, les pratiques d'utilisation des ressources, les rituels, la spiritualité et la vision du monde " (ICSU et UNESCO, 2002 : 9). La relation entre savoir traditionnel et R-D revêt une importance particulière dans les pays en développement où l'existence d'une réserve précieuse de savoir traditionnel peut fortement inciter des organisations locales ou étrangères à engager des activités de R-D.

2.109 En règle générale, si des activités associées au savoir traditionnel s'inscrivent dans un projet de R-D, l'effort engagé (sur le plan financier et en ressources humaines) devrait être comptabilisé dans la R-D. Sinon, il devrait en être exclu.

Exemples d'activités liées au savoir traditionnel à comptabiliser dans la R-D :

- Volet d'un projet de R-D consistant à déterminer le contenu d'un savoir traditionnel suivant une démarche scientifique, dans des disciplines comme l'ethnoscience (ethnobotanique, ethnopédologie, ethnosylviculture, ethnomédecine vétérinaire et ethnoécologie) ou l'anthropologie cognitive. Il est alors recouru aux méthodes de R-D en usage dans les disciplines concernées pour étudier le savoir traditionnel.

- Application de méthodes scientifiques en vue de mettre en évidence l'ingrédient actif des remèdes locaux et/ou d'en établir l'efficacité dans le traitement de certaines pathologies. Dans ce cas, les méthodes de R-D sont directement appliquées aux produits du savoir traditionnel dans le but d'élargir le stock de connaissances scientifiques.

- Activités menées par les utilisateurs de connaissances traditionnelles pour en élargir le stock moyennant l'utilisation combinée de méthodes traditionnelles et scientifiques. Ces activités doivent satisfaire aux critères de référence pour être apparentées à de la R-D ou être réalisées au sein d'universités.

2.110 Exemples d'activités liées au savoir traditionnel à exclure de la R-D :

- Utilisation régulière/continue du savoir traditionnel par des professionnels, notamment dans le traitement de maux ou la gestion des cultures agricoles.

- Tâches courantes de développement de produits fondées sur le savoir traditionnel.

- Conservation et communication de savoir traditionnel par des voies classiques (test de la nouveauté).

- Transmission traditionnelle des croyances et pratiques religieuses ou culturelles. 


\subsection{Activités à exclure de la R-D}

2.111 Pour les besoins des enquêtes, la R-D doit être dissociée d'un large éventail d'activités connexes dotées d'un fondement scientifique et technologique. Bien qu'étroitement liées à la R-D, tant par les flux d'informations qu'au niveau des opérations, des institutions et du personnel impliqués, ces activités ne devraient pas, dans la mesure du possible, entrer en ligne de compte dans la mesure de la R-D.

\section{Services d'information scientifique et technique}

2.112 Les activités spécialisées ci-après :

- collecte

- codification

- enregistrement

- classement

- diffusion

- traduction

- analyse

- évaluation

menées par :

- le personnel scientifique et technique

- les services bibliographiques

- les services de brevets

- les services de diffusion de l'information scientifique et technique et les services de conseil

- les conférences scientifiques

sont à exclure, sauf quand elles sont menées exclusivement ou principalement à l'appui de la R-D (par exemple, l'établissement du rapport original sur les résultats de la R-D fera partie des activités de R-D) ou dans le cadre de projets de R-D, tels que précédemment définis dans la présente section.

\section{Essais et normalisation}

2.113 La présente section porte sur l'administration des normes nationales, l'adaptation des normes secondaires et la réalisation d'essais et d'analyses de routine sur les matériaux, composants, produits, procédés, sols, atmosphère, etc. Il ne s'agit pas d'activités de R-D.

\section{Études de faisabilité}

2.114 L'étude de projets d’ingénierie fondée sur les techniques permettant d'obtenir des informations complémentaires avant toute décision 
de mise en œuvre ne relève pas de la R-D. En sciences sociales, les études de faisabilité consistent à examiner les caractéristiques socio-économiques et les conséquences de cas de figure particuliers (par exemple, implantation d'un complexe pétrochimique dans une région donnée). En revanche, les études de faisabilité consacrées à des projets de recherche font partie de la R-D.

\section{Soins de santé spécialisés}

2.115 La présente section couvre l'étude courante et l'application habituelle des connaissances médicales spécialisées. En temps normal, il ne s'agit pas d'activités de R-D. Elles peuvent néanmoins comporter un élément de R-D dans ce que l'on appelle communément les « soins de santé spécialisés », par exemple dès lors qu'elles sont menées dans les hôpitaux universitaires.

\section{Étude des politiques}

2.116 Le terme " politiques » désigne ici les mesures appliquées à l'échelle nationale, mais aussi régionale et locale, ainsi que l'action poursuivie par les entreprises dans la conduite de leur activité économique. Les études des politiques couvrent diverses activités, comme l'analyse et l'évaluation des programmes, mesures et activités exécutés par les ministères et autres institutions publiques, les travaux des unités chargées d'analyser et de suivre en continu des phénomènes extérieurs (par exemple, les questions de défense et de sécurité) et les travaux des commissions d'enquête parlementaires concernant les politiques et activités du gouvernement central et des ministères.

2.117 Sont à exclure de la R-D les activités visant à étayer l'action des pouvoirs publics et du législateur, telles que le conseil stratégique et les relations avec les médias, le conseil juridique, les relations publiques, voire le soutien technique aux tâches administratives (par exemple, comptabilité).

2.118 Il convient de souligner que les activités de recherche visant à renseigner les décideurs de manière approfondie sur des phénomènes sociaux, économiques ou naturels sont à inclure dans la R-D. Ces activités de R-D sont généralement exécutées par du personnel qualifié (des chercheurs), organisé en petites équipes d'experts et de consultants, et satisfont aux critères académiques des travaux scientifiques (en complément des critères définissant la R-D).

\section{Évaluations programmatiques}

2.119 Les efforts de R-D peuvent étayer la prise de décisions au sein de l'administration et autres institutions. S'il est possible de les externaliser, il arrive aussi que des équipes spécialement créées à cet effet en interne participent activement au travail d'analyse, par exemple en réalisant des évaluations et bilans ponctuels, voire formalisés. Il est possible que ces activités répondent aux critères définissant un projet de R-D, mais ce n'est pas toujours le cas, de même qu'il n'y a pas lieu de qualifier de R-D la totalité des efforts de renseignement ou d'établissement des faits associés au conseil stratégique ou programmatique. 
Il convient d'examiner, à un certain niveau de détail, les compétences techniques des personnes impliquées dans l'activité, le mode de codification des connaissances adopté au sein de l'organisation et la manière dont il est veillé au respect des normes de qualité dans les thèmes de recherche et la méthodologie appliquée. Le risque que des activités de conseil socio-économiques (internes ou externes) soient présentées à tort comme de la R-D est non négligeable.

2.120 Les conseillers scientifiques jouent un rôle important au sein de l'administration publique. Cependant, le fait d'appliquer à l'élaboration des politiques des critères décisionnels précis ne constitue pas en soi une activité de R-D. Les efforts déployés pour mettre au point de meilleures méthodes de prise de décisions fondées sur des données scientifiques peuvent être considérés comme relevant de la R-D.

\section{Activités limitées au financement de la R-D}

2.121 La levée, la gestion et la distribution de fonds assurées par les ministères, les organismes de recherche, les fondations ou organisations caritatives au profit des exécutants de la R-D ne constituent pas des activités de R-D.

\section{Activités de soutien indirect}

2.122 La présente section concerne un certain nombre d'activités qui ne relèvent pas de la $R-D$, mais y contribuent. Par convention, les données relatives au personnel de R-D portent sur la R-D proprement dite, mais pas sur les activités de soutien indirect, qui sont pourtant prises en compte dans les dépenses de R-D de ses exécutants au titre des frais généraux. Le transport, l'entreposage, le nettoyage, la réparation, l'entretien et la sécurité en sont des exemples classiques. En font également partie les activités administratives et les tâches de bureau qui ne sont pas uniquement menées à des fins de R-D, à l'instar des activités des services centraux des finances et du personnel.

\section{Références}

CE, FMI, OCDE, Nations Unies et Banque mondiale (2009), Système de comptabilité nationale, Nations Unies, New York, http://unstats.un.org/unsd/nationalaccount/docs/ sna2008FR.pdf.

CE, FMI, OCDE, Nations Unies et Banque mondiale (1994), Système de comptabilité nationale, Nations Unies, New York, http://unstats.un.org/unsd/nationalaccount/docs/1993sna.pdf.

ICSU et UNESCO (2002), " Science, traditional knowledge and sustainable development », ICSU Series on Science for Sustainable Development, $n^{\circ} 4$, UNESCO, Paris, http://unesdoc. unesco.org/images/0015/001505/150501eo.pdf.

OCDE (2015), Making Open Science a Reality, Éditions OCDE, Paris.

OCDE (2009), Handbook on Deriving Capital Measures of Intellectual Property Products, Éditions OCDE, Paris, $h t t p: / / d x . d o i . o r g / 10.1787 / 9789264079205$-en. 
OCDE/Eurostat (2005), Manuel d'Oslo: Principes directeurs pour le recueil et l'interprétation des données sur l'innovation, $3^{e}$ édition, La mesure des activités scientifiques et technologiques, Éditions OCDE, Paris, http://dx.doi.org/10.1787/9789264013124-fr.

UNESCO (1984), Manuel pour les statistiques relatives aux activités scientifiques et technologiques, Division des statistiques relatives aux sciences et la technologie Office des statistiques, ST-84/WS/12, UNESCO, Paris, http://www.uis.unesco.org/Library/ Documents/STSManual84_fr.pdf.

UNESCO (1978), Recommandation concernant la normalisation internationale des statistiques relatives à la science et à la technologie, UNESCO, Paris, http://portal.unesco.org/fr/eu.php-

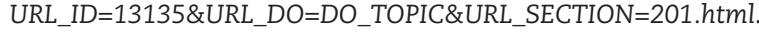

UNESCO-ISU (2014), Domaines d'études et de formation de la CITE 2013 (CITE-F 2013), UNESCO, Paris, http://unesdoc.unesco.org/images/0022/002293/229319f.pdf. 


\section{Chapitre 3}

\section{Secteurs institutionnels et classifications utilisés dans les statistiques de R-D}

Ce chapitre est consacré aux unités institutionnelles qui exécutent ou financent la recherche et le développement expérimental (R-D). Y sont exposées les caractéristiques communes sur la base desquelles ces unités sont regroupées en secteurs aux fins de l'établissement des statistiques de R-D. Ces secteurs sont au nombre de cinq : entreprises, enseignement supérieur, État, secteur privé sans but lucratif et reste du monde (anciennement "l'étranger »), ce dernier secteur n'étant concerné que par le volet financement. Leur définition repose sur le Système de comptabilité nationale (SCN). Quatre en sont directement tirés. En revanche, la définition de l'enseignement supérieur est spécifique au présent manuel en raison de l'importance stratégique de ce secteur. En effet, au sens du SCN, les entités qui le composent peuvent appartenir à l'un quelconque des autres secteurs. Sont décrites ici les particularités et délimitations des cinq secteurs étudiés qui feront ensuite chacun l'objet d'un chapitre à part. 


\subsection{Introduction}

3.1 Le but du présent chapitre est de décrire la méthode utilisée dans les statistiques de R-D pour définir et classer les entités qui exécutent et financent des activités de R-D. Les unités statistiques doivent être réparties en secteurs, en fonction de leurs qualités ou de leurs caractéristiques communes. Après avoir décrit ces unités, les objectifs de la classification, notamment au regard des besoins des utilisateurs, et les principaux critères appliqués, on s'intéressera aux secteurs institutionnels qui fournissent les bases d'une présentation homogène des statistiques de R-D et aux délimitations de ces secteurs. Le chapitre s'achève par la présentation des nomenclatures généralement applicables aux unités institutionnelles et par une brève description des principales caractéristiques de chaque secteur.

3.2 Les unités constitutives de chaque secteur et les classifications qui y sont appliquées sont définies plus en détail dans les chapitres correspondants : secteur des entreprises (chapitre 7), secteur de l'État (chapitre 8), secteur de l'enseignement supérieur (chapitre 9), secteur privé sans but lucratif (chapitre 10) et reste du monde (chapitre 11 sur la mondialisation de la R-D).

3.3 Le présent chapitre s'inspire de la classification des unités et des secteurs institutionnels utilisée dans le manuel 2008 du Système de comptabilité nationale (SCN) (CE et al., 2009), en particulier de son chapitre 4. Les termes importants sont définis dans le manuel précité ou dans le glossaire. On en trouvera un bref aperçu dans l'encadré 3.2.

\subsection{Unités institutionnelles}

3.4 La définition des unités institutionnelles intervenant dans le financement ou la réalisation des travaux de R-D est d'une importance fondamentale pour la collecte, la communication et l'interprétation des statistiques de R-D. Dans le Manuel de Frascati, ces unités sont regroupées en secteurs et sous-secteurs. Elles doivent se caractériser par un degré suffisant de cohésion interne et se distinguer facilement les unes des autres.

3.5 Une unité institutionnelle est un concept de comptabilité nationale. Elle est définie comme étant " une entité économique qui est capable, de son propre chef, de posséder des actifs, de prendre des engagements, de s'engager dans des activités économiques et de réaliser des opérations avec d'autres entités » (CE et al., 2009, p. 61, para. 4.2). Ce concept peut s'appliquer à la mesure des activités de R-D et des flux correspondants. Dans le cas de la R-D, les unités institutionnelles doivent être capables de prendre des décisions concernant 
la conduite des activités de R-D, allant de l'utilisation interne et externe des ressources financières à la gestion des projets de R-D. Ces conditions sont moins contraignantes que dans la définition d'une unité institutionnelle retenue dans le Système de comptabilité nationale, mais servent néanmoins les objectifs du présent manuel.

3.6 Il est possible de distinguer deux grands types d'unités institutionnelles, à savoir les personnes ou groupes de personnes physiques (ou ménages), et les personnes morales ou entités sociales. Les personnes morales sont des entités économiques dont l'existence est reconnue par la loi ou par la société indépendamment des personnes physiques - ou autres entités - qui en sont les propriétaires ou les gestionnaires. Ces unités sont responsables et comptables de leurs décisions économiques ou de leurs actions, même si leur autonomie peut être limitée dans une certaine mesure par d'autres unités institutionnelles, comme les actionnaires (CE et al., 2009, p. 61, para. 4.6). Pour des raisons qui sont explicitées dans le chapitre 2 (sur les définitions) et le chapitre 10, et qui seront de nouveau abordées dans le présent chapitre, les ménages sont effectivement pris en compte dans les statistiques de R-D, principalement par souci d'exhaustivité.

\section{L'approche institutionnelle des statistiques de R-D}

3.7 En principe, l'unité statistique doit être uniforme pour un même secteur dans tous les pays. Dans la pratique, cependant, cela n'est jamais totalement le cas, notamment en raison des différences terminologiques et réglementaires qui existent entre les pays en ce qui concerne l'organisation et l'information financière des entreprises et autres types d'unités. De plus, la structure des activités économiques concernées et l'interaction avec l'unité déclarante peut entraîner des disparités entre les pays (et en leur sein) et au fil du temps.

3.8 L'approche institutionnelle des statistiques de R-D a pour but de recueillir et de présenter les statistiques d'après les caractéristiques générales des unités institutionnelles. Selon cette démarche, les ressources d'une unité consacrées à la R-D sont attribuées au secteur auquel appartient l'unité, et les flux correspondent aux opérations entre l'unité statistique et les tiers. Dans l'approche fonctionnelle, les ressources d'une unité sont réparties à l'aide des informations fournies par la ou les unités déclarantes compétentes. Les critères de la répartition fonctionnelle peuvent être, par exemple, le type de R-D (recherche fondamentale, recherche appliquée, développement expérimental), le groupe de produits (ou l'activité économique concernée), le domaine de R-D (par exemple : les sciences naturelles, l'ingénierie et la technologie, les sciences sociales, les sciences humaines et les arts) et l'objectif socio-économique (développement économique, santé, environnement et éducation). Les deux approches - institutionnelle et fonctionnelle - peuvent souvent être combinées. C'est le cas, par exemple, lorsqu'une répartition fonctionnelle des activités est requise uniquement pour les grandes organisations (qui ne coïncide pas 
forcément avec la structure interne desdites organisations), tandis que, par souci de simplification, cette répartition n'est pas obligatoire pour les unités plus simples et de plus petite taille. On utilise alors une classification institutionnelle. L'approche fonctionnelle peut permettre de pallier l'éventuel décalage entre les unités déclarantes et les unités statistiques visées, lorsque les premières englobent les secondes.

3.9 Chaque fois que les pays fournissent des statistiques à des fins de comparaison internationale, les unités statistiques ainsi que l'approche utilisée - institutionnelle, fonctionnelle ou les deux combinées - doivent être précisées. On trouvera des indications plus détaillées au chapitre 6 (sur les méthodes) et aux chapitres 7 à 10 (consacrés aux différents secteurs).

\section{Les unités statistiques}

3.10 Une unité statistique est une entité au sujet de laquelle des informations sont recueillies et des statistiques compilées. En d'autres termes, il s'agit de l'unité institutionnelle sur laquelle on souhaite recueillir des statistiques de R-D. Une unité statistique peut être une unité d'observation - sur laquelle on a obtenu des informations et compilé des statistiques - ou une unité d'analyse - créée par fractionnement ou regroupement des unités d'observation à l'aide d'estimations ou d'imputations, de façon à obtenir des données plus précises ou plus homogènes qu'il ne serait possible autrement (Nations Unies, 2007).

3.11 Les unités statistiques doivent être définies en détail lorsque l'on est en présence d'entités économiques complexes et de grande envergure dont les activités se répartissent entre différentes catégories, ou dont les composantes sont situées dans différentes zones géographiques. Il existe plusieurs types ou niveaux d'unités statistiques, établis selon la structure du capital, les liens de contrôle, l'homogénéité de l'activité économique et le lieu d'implantation, à savoir : groupe d'entreprises, entreprise, établissement et unité d'activité économique (voir l'encadré 3.1). Ces concepts s'appliquent aux unités statistiques de tous les secteurs, et pas seulement à celui des entreprises, tel qu'il est défini dans le présent manuel. Le choix de l'unité statistique et de la méthode utilisée dépend dans une large mesure de la finalité des statistiques de R-D, mais aussi de l'existence ou non de données et de la capacité des répondants à fournir des informations utiles. Dans les organisations complexes et de grande taille, les décisions relatives à l'orientation stratégique et au financement des activités de R-D ont tendance à être prises à un niveau hiérarchique plus élevé que la gestion quotidienne des opérations de R-D, voire à inclure des décisions concernant les types de dépenses à effectuer et les ressources humaines à recruter pour la R-D. Ces décisions peuvent être prises dans plusieurs pays différents, ce qui crée une difficulté supplémentaire pour les autorités et les organismes nationaux, dont la tâche se limite souvent à recueillir des informations auprès des unités résidentes. 


\section{Encadré 3.1. Types d'unités statistiques}

Une entreprise est une unité institutionnelle (CE et al., 2009, para. 5.1) - pas nécessairement au sens du secteur des entreprises tel que défini dans le Manuel de Frascati - qui est considérée en tant que producteur de biens et de services. Le terme " entreprise " peut se référer à une société, une quasisociété, une institution sans but lucratif ou une entreprise non constituée en société. Il s'agit d'un agent économique qui possède une autonomie décisionnelle en matière de finance et d'investissement, ainsi que le pouvoir et la responsabilité d'allouer des ressources à la production de biens et de services. L'entreprise peut mener une ou plusieurs activités en un ou plusieurs lieux. Elle peut être constituée d'une seule unité légale.

Une unité d'activité économique (UAE) est une entreprise ou une partie d'entreprise qui exerce un seul type d'activité de production ou dans laquelle la majeure partie de la valeur ajoutée provient de l'activité de production principale. Chaque entreprise doit, par définition, comprendre une ou plusieurs unités d'activité économique.

Les entreprises exercent souvent des activités de production en plusieurs lieux et, à certaines fins, il peut être utile de les diviser en conséquence. Ainsi, une unité locale est une entreprise ou une partie d'entreprise qui exerce une activité de production en un seul lieu ou à partir d'un seul lieu.

Un établissement est une entreprise, ou une partie d'entreprise, située en un lieu unique, dans laquelle une seule activité de production est exercée ou dans laquelle la majeure partie de la valeur ajoutée provient de l'activité de production principale. Les établissements sont parfois appelés « unités d'activité économique au niveau local " (UAE locales).

Un groupe d'entreprises est un ensemble d'entreprises contrôlées par une unité légale mère qui n'est contrôlée (directement ou indirectement) par aucune autre unité légale. Il peut compter plusieurs centres décisionnels, notamment pour la politique relative à la production, à la vente et aux bénéfices, ou peut centraliser certains aspects de la gestion financière et de la fiscalité. Il s'agit d'une entité économique capable de faire des choix, particulièrement en ce qui concerne les unités qui le composent. L'unité "groupe d'entreprises" est particulièrement utile pour les analyses financières et l'étude des stratégies d'entreprise. Elle est toutefois trop hétérogène et trop instable pour être adoptée en tant qu'unité centrale d'observation et d'analyse.

Source : OCDE, d'après CE et al. (2009) et Nations Unies (2007).

\section{Les unités déclarantes}

3.12 L’unité déclarante est l'unité de laquelle émanent les données déclarées. L'institution recevant les questionnaires à remplir peut inclure plusieurs unités déclarantes. Dans le cas de données administratives, l'unité déclarante 
est celle associée à chaque déclaration. Le choix des unités déclarantes varie d'un secteur et d'un pays à l'autre et dépend des structures institutionnelles, du cadre juridique de la collecte des données, des traditions, des priorités nationales et des ressources disponibles pour les enquêtes. Si les statistiques sont recueillies dans le cadre d'une enquête, l'unité déclarante est considérée comme le répondant. Les données peuvent, dans certains pays, être collectées auprès des services de R-D ou, dans d'autres, à un niveau plus global. Le présent manuel ne peut fournir aucune recommandation générale applicable par tous les pays.

\subsection{Secteurs institutionnels}

\section{Principales justifications de la classification sectorielle}

3.13 Afin de faciliter la collecte et la production de statistiques sur le personnel, les dépenses de R-D et les flux de financement (dont il est fait état plus loin dans le présent manuel) qui soient comparables à l'échelle internationale, les unités statistiques doivent être regroupées en secteurs, en fonction de leurs qualités ou caractéristiques communes. La recommandation est de respecter le plus possible les classifications standard de ces unités, tout en répondant aux besoins déclarés des utilisateurs habituels des statistiques de R-D. La classification sectorielle des unités statistiques dans le contexte des statistiques de R-D peut servir divers objectifs.

\section{Collecte de données sur la R-D}

3.14 La classification sectorielle joue un rôle important dans l'organisation de la collecte de données car elle permet, par exemple, de déterminer l'outil d'enquête à utiliser pour des unités institutionnelles présentant un ensemble de caractéristiques communes (par exemple, le type de R-D exécutée et le domaine sur lequel elle porte, les systèmes de comptabilité utilisés ou la terminologie employée pour décrire les activités de R-D). La classification sectorielle permet également de définir des strates et de faciliter la collecte de données et les travaux d'estimation, en particulier dans les cas suivants :

- lorsqu'il s'agit de mesurer les dépenses et le personnel affectés à la R-D (respectivement les chapitres 4 et 5), l'approche sectorielle offre une méthode fiable pour constituer des agrégats nationaux

- la classification sectorielle offre un cadre permettant d'analyser les flux financiers entre les entités s'occupant du financement de la R-D et celles qui exécutent les travaux (chapitre 4). Les classifications doivent donc être relativement faciles à interpréter par les répondants, sans quoi les données déclarées au sujet des flux en provenance et à destination d'autres unités risquent d'être inexactes. 


\section{Rapprochement et mise en correspondance avec d'autres cadres statistiques et sources de données}

3.15 Dans la mesure où les unités sont classées selon des modes de classification standard, les sources d'information sur la R-D peuvent être rapprochées d'autres sources statistiques. Cela peut faciliter :

- la mise au point de registres sur les efforts de collecte de données sur la $\mathrm{R}-\mathrm{D}$, fondée sur les sources disponibles et les initiatives de classification préalablement menées dans le contexte d'autres cadres statistiques

- l'adaptation des statistiques de R-D et leur utilisation ultérieure dans d'autres cadres, comme, par exemple, le Système de comptabilité nationale (SCN) qui utilise les statistiques de R-D pour réaliser des estimations nationales et sectorielles des résultats de la R-D et de la formation de capital, reprises ensuite dans les principaux indicateurs économiques

- une meilleure compréhension du rôle de la R-D dans le développement économique et la formulation de la politique publique y afférente en permettant le rapprochement entre les données et l'étude de leurs relations.

\section{Communication des statistiques de R-D à l'échelle nationale et internationale}

3.16 L'utilisation d'une approche sectorielle normalisée permet une communication homogène et facilement comparable des statistiques de R-D, notamment pour répondre aux besoins des responsables de la politique publique et autres utilisateurs de premier plan. Il est donc recommandé d'utiliser un ensemble de grands secteurs institutionnels.

3.17 Le regroupement en secteurs permet par ailleurs d'éviter l'écueil de la nature souvent confidentielle des données sur la R-D dont la collecte est soumise aux règles du secret statistique. Les règles de confidentialité et les problèmes de qualité des données limitent le nombre de classifications différentes mais présentant des recoupements qui peuvent être publiées par les organismes produisant des statistiques sur la R-D. Lorsque des données relatives aux unités peuvent être diffusées publiquement, la classification sectorielle représente un outil utile d'organisation et de filtrage pour les utilisateurs recherchant des informations spécifiques sur des acteurs précis.

3.18 On sait qu'un système de classification unique n'est pas toujours suffisant pour remplir chacun de ces nombreux objectifs ou pour satisfaire l'éventail toujours plus large des intérêts des utilisateurs pour les statistiques de R-D. Le présent manuel propose une classification institutionnelle qui tente de trouver un équilibre entre toutes ces considérations, et la complète par une série de classifications transversales facultatives pour s'assurer de répondre largement aux besoins des utilisateurs. 


\section{Critères de classification et choix des secteurs institutionnels utilisés pour les statistiques de R-D}

3.19 Pour la présentation générale des statistiques de R-D, toute la gamme des unités institutionnelles composant une économie nationale sont prises en compte et regroupées en quatre secteurs institutionnels s'excluant mutuellement, à savoir : le secteur des entreprises, le secteur de l'enseignement supérieur, le secteur de l'État et le secteur privé sans but lucratif, auxquels s'ajoute le reste du monde afin d'incorporer, par souci d'exhaustivité, les relations avec les unités non résidentes. Le critère de base utilisé pour regrouper les unités institutionnelles en secteurs est l'homogénéité de leurs objectifs économiques, de leurs principales fonctions et de leur comportement économique.

3.20 La classification des unités institutionnelles dans le contexte de la $\mathrm{R}-\mathrm{D}$ a pour but d'obtenir une parfaite cohérence avec la définition de la R-D et les besoins explicites des utilisateurs habituels de statistiques de R-D, ainsi qu'avec les critères de classification utilisés par le Système de comptabilité nationale (SCN). Ce dernier prend en compte les critères d'exhaustivité et de résidence, ainsi que le type d'activité économique, de structure de capital et de contrôle. Le financement peut également être un critère de classification.

3.21 Comme défini dans le SCN, la résidence d'une unité institutionnelle est le territoire économique avec lequel cette unité possède la relation la plus étroite, autrement dit son centre d'intérêt économique prépondérant. Le territoire économique est composé des zones terrestres, de l'espace aérien et des eaux territoriales, et intègre les compétences concernant les droits de pêche et les droits sur les combustibles ou les minéraux. Dans le cas d'un territoire maritime, le territoire économique inclut les îles appartenant à ce territoire. Le territoire économique comporte aussi les enclaves territoriales situées dans le reste du monde. Ces dernières sont des zones terrestres clairement délimitées (ambassades, consulats, bases militaires et stations scientifiques) qui se trouvent sur d'autres territoires et que l'État qui en est propriétaire ou locataire utilise à des fins diplomatiques, militaires, scientifiques ou autres avec l'accord officiel des autorités des territoires sur lesquels ces zones terrestres sont géographiquement situées (CE et al., 2009, p. 62, para. 4.11). Le critère de résidence permet de délimiter le « reste du monde " par rapport aux autres secteurs institutionnels.

3.22 Pour les besoins des statistiques de R-D, on dit qu'une unité institutionnelle possède un centre d'intérêt économique prépondérant sur un territoire économique si elle y dispose d'un site, d'un lieu de production ou de locaux, dans lesquels ou à partir desquels elle mène ou entend mener des activités et des opérations économiques de grande envergure, pendant une période indéterminée ou déterminée mais longue. Un lieu d'implantation réel ou prévu pendant une année ou plus est utilisé comme définition opérationnelle. On trouvera des informations plus précises sur ce critère et les statistiques de R-D y afférentes dans le chapitre 11 consacré à la mondialisation de la R-D. 


\section{Encadré 3.2. La classification des secteurs institutionnels selon le SCN}

La classification sectorielle proposée dans le Manuel de Frascati est très proche de celle recommandée par le Système de comptabilité nationale (SCN). Dans celui-ci, toutes les unités institutionnelles d'une économie sont classées et regroupées en cinq secteurs s'excluant mutuellement. Les secteurs regroupent des unités institutionnelles et toute unité institutionnelle doit être classée intégralement dans l'un ou l'autre d'entre eux, à savoir : les sociétés financières, les sociétés non financières, les administrations publiques, les institutions sans but lucratif au service des ménages (ISBLSM) et enfin, les ménages. La procédure de classification recommandée par le SCN est de commencer par séparer les ménages des personnes morales, puis de se concentrer sur ces dernières qui sont les principaux acteurs de la production économique. D'autres considérations interviennent dans l'affectation sectorielle de toutes ces unités.

Tout d'abord, il convient de déterminer si une unité est un producteur marchand, autrement dit si elle fournit la majeure partie de sa production à d'autres unités à des prix économiquement significatifs (CE et al., 2009, para. 22.28).

Lorsque des unités non marchandes ne sont pas sous contrôle public, elles sont considérées comme des institutions sans but lucratif au service des ménages (ISBLSM); les autres sont classées dans le secteur des administrations publiques. Les institutions sans but lucratif (voir la définition dans CE et al., 2009, para. 4.8) peuvent être incluses dans n'importe quel secteur du SCN. Les unités marchandes sont classées dans le secteur des entreprises (privées ou publiques selon qu'elles sont contrôlées ou non par l'État).

Source : CE et al. (2009).

3.23 La classification institutionnelle du SCN sert de base à la classification recommandée des principaux secteurs dans le contexte des statistiques de R-D (autrement dit les "secteurs du Manuel de Frascati "). La relation entre les secteurs du SCN et ceux du Manuel de Frascati est résumée dans le tableau 3.1. Il en ressort deux grandes différences.

3.24 D'une part, depuis la première édition du présent manuel, les utilisateurs des statistiques de R-D n'ont cessé d'insister sur la nécessité de rendre compte de façon homogène de l'activité de R-D dans les établissements d'enseignement supérieur et les unités dont ils assurent le contrôle ou la gestion. La mise en œuvre de cette exigence supposait de définir un ensemble de critères supplémentaires permettant de recenser les unités institutionnelles d'un secteur à part entière, celui de l'enseignement supérieur - qui appartiennent, selon les critères de marché ou de contrôle public applicables dans chaque pays, aux catégories des sociétés, des administrations publiques ou des ISBLSM définies dans le SCN. Les caractéristiques particulières du secteur de l'enseignement 
supérieur au regard des statistiques de R-D sont présentées en détail au chapitre 9.

3.25 D'autre part, et principalement dans le but de rendre la présentation plus synthétique, les ménages et les institutions privées sans but lucratif qui ne sont pas affectées aux secteurs de l'enseignement supérieur, des entreprises ou de l'État (c'est-à-dire les unités du secteur des ISBLSM qui ne font pas partie du secteur de l'enseignement supérieur tel que défini dans le présent manuel) sont regroupés dans le même secteur. Le secteur qui en résulte aurait pu être appelé " autres institutions privées et sans but lucratif », afin d'inclure le groupe résiduel des ménages et les autres institutions privées et sans but lucratif, et de représenter tout l'éventail des acteurs présents dans une économie nationale. Or, dans la mesure où la contribution du groupe résiduel des ménages est faible, ce secteur est dénommé ici « secteur privé sans but lucratif » (PSBL).

3.26 Ce mode de classification sert de base pour la communication nationale et internationale des données sur la R-D. Il est important qu'il soit utilisé en priorité - en particulier lorsqu'il est question de communication internationale -, mais qu'il permette aussi l'utilisation de méthodes complémentaires plus flexibles, comme indiqué ci-après. Cinq grands secteurs ont donc été définis pour la mesure de la R-D. Ils sont brièvement présentés dans la section 3.5 et décrits plus en détail dans chacun des chapitres qui sont y consacrés :

- Secteur des entreprises

Chapitre 7

- Secteur de l'État

Chapitre 8

- Secteur de l'enseignement supérieur

Chapitre 9

- Secteur privé sans but lucratif

Chapitre 10

- Reste du monde

Chapitre 11.

\section{Mise en œuvre de la classification institutionnelle}

3.27 La classification est une activité qui peut demander des ressources importantes, en particulier lorsqu'il s'agit de concevoir de A à Z un système complet de statistiques de R-D. Elle doit être un processus continu, car les unités apparaissent, disparaissent ou doivent être reclassées. Les services statistiques ayant accès à des registres officiels auront plus de facilité à prendre des décisions en matière de classification s'ils s'inspirent de la classification du SCN qui constitue un outil de contrôle supplémentaire permettant de déterminer si une unité correspond bien, par exemple, au secteur de l'enseignement supérieur (tel que défini à la section 3.5 et décrit plus en détail au chapitre 9).

3.28 Dans certains cas, l'organisme chargé d'établir les statistiques de R-D peut avoir besoin de revoir et de réviser la classification générique qui est utilisée dans les registres standard, par exemple lorsque cette classification n'a pas été adaptée aux évolutions les plus récentes survenues dans le domaine des statistiques de R-D (notamment la modification du profil de l'unité statistique). Du fait de ses activités - le suivi du système de R-D et l'établissement de liens -, 
le statisticien est sans doute mieux placé pour constater et enregistrer les changements intervenus sur certaines unités chargées du financement ou de l'exécution de la R-D. Les constats effectués par les statisticiens de la R-D peuvent aussi être utiles aux responsables des registres statistiques généraux. Tableau 3.1. Correspondance approchée entre les secteurs institutionnels
du SCN et ceux du Manuel de Frascati

\begin{tabular}{|c|c|c|c|c|}
\hline \multirow{2}{*}{$\begin{array}{l}\text { Secteurs } \\
\text { institutionnels } \\
\text { du SCN }\end{array}$} & \multicolumn{4}{|c|}{ Secteurs institutionnels du Manuel de Frascati } \\
\hline & $\begin{array}{l}\text { Enseignement } \\
\text { supérieur }\end{array}$ & Entreprises & État & $\begin{array}{c}\text { Secteur privé sans } \\
\text { but lucratif }\end{array}$ \\
\hline $\begin{array}{l}\text { Sociétés (financières } \\
\text { et non financières) }\end{array}$ & $\begin{array}{l}\text { Établissements } \\
\text { d'enseignement } \\
\text { supérieur enregistrés } \\
\text { dans le secteur des } \\
\text { sociétés }\end{array}$ & $\begin{array}{l}\text { Même contenu que le } \\
\text { secteur des sociétés } \\
\text { du SCN, y compris } \\
\text { les entreprises } \\
\text { publiques, mais } \\
\text { hormis les } \\
\text { établissements } \\
\text { d'enseignement } \\
\text { supérieur }\end{array}$ & & \\
\hline $\begin{array}{l}\text { Administrations } \\
\text { publiques }\end{array}$ & $\begin{array}{l}\text { Établissements } \\
\text { d'enseignement } \\
\text { supérieur enregistrés } \\
\text { dans le secteur des } \\
\text { administrations } \\
\text { publiques }\end{array}$ & & $\begin{array}{l}\text { Même contenu } \\
\text { que le secteur des } \\
\text { administrations } \\
\text { publiques du } \\
\text { SCN, hormis les } \\
\text { établissements } \\
\text { d'enseignement } \\
\text { supérieur }\end{array}$ & \\
\hline $\begin{array}{l}\text { Institutions privées } \\
\text { sans but lucratif au } \\
\text { service des ménages } \\
\text { (ISBLSM) }\end{array}$ & $\begin{array}{l}\text { Établissements } \\
\text { d'enseignement } \\
\text { supérieur enregistrés } \\
\text { dans le secteur des } \\
\text { ISBLSM }\end{array}$ & & & $\begin{array}{l}\text { Même contenu que le } \\
\text { secteur des ISBLSM } \\
\text { du SCN, hormis } \\
\text { les établissements } \\
\text { d'enseignement } \\
\text { supérieur }\end{array}$ \\
\hline Ménages & & $\begin{array}{l}\text { Travailleurs } \\
\text { indépendants ayant } \\
\text { un statut proche } \\
\text { d'une entreprise } \\
\text { (très probablement } \\
\text { enregistrés en tant } \\
\text { que quasi-sociétés) }\end{array}$ & & $\begin{array}{l}\text { Par souci d'exhaustivité, } \\
\text { même contenu } \\
\text { que le secteur des } \\
\text { ménages du SCN, } \\
\text { hormis les travailleurs } \\
\text { indépendants ayant } \\
\text { un statut proche d'une } \\
\text { entreprise }\end{array}$ \\
\hline
\end{tabular}

3.29 Pour les organismes statistiques ne disposant pas d'un accès direct aux registres officiels, des efforts supplémentaires sont nécessaires pour obtenir une classification exhaustive et actualisée des unités statistiques pouvant effectuer des travaux de R-D. En l'absence de dispositifs d'échange de données, les organismes chargés d'établir des statistiques de R-D peuvent soit utiliser une classification semblable à celle du SCN (élargie avec l'ajout des établissements d'enseignement supérieur), soit mettre en place un processus de décision séquentielle plus axée sur les statistiques de R-D (voir le graphique 3.1). 


\section{Graphique 3.1. Arbre de décision pour l'affectation des unités institutionnelles aux principaux secteurs du présent manuel}

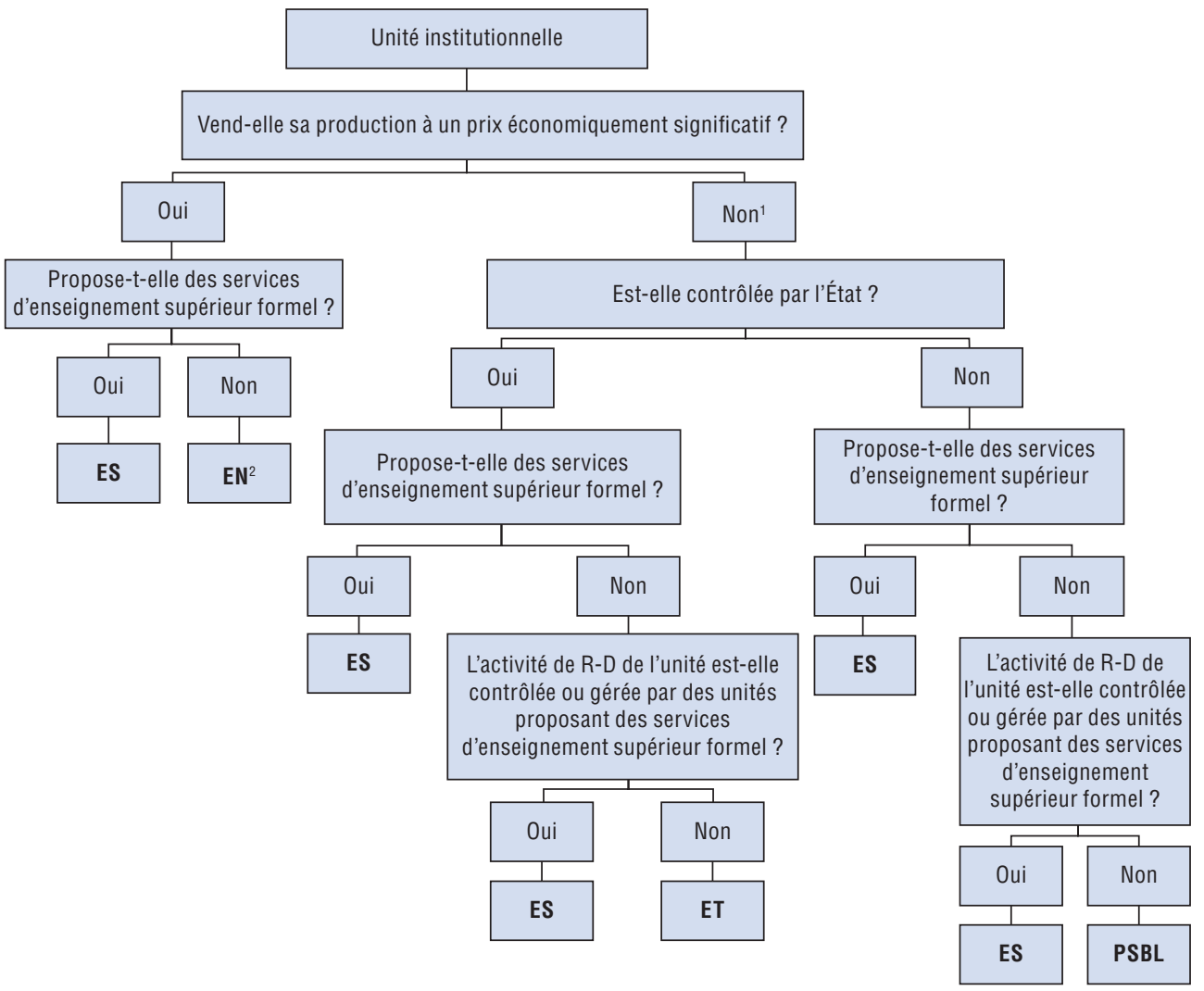

\section{Acronymes :}

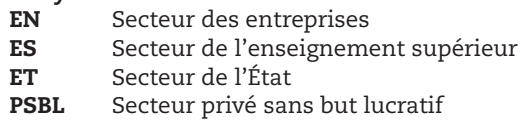

Notes :

1. Les ISBL qui sont principalement au service des entreprises (par exemple les associations commerciales) sont classées dans le secteur des entreprises, conformément à ce qui est en usage dans le SCN (où elles sont intégrées dans le secteur des sociétés).

2. Ce secteur peut être subdivisé en entreprises publiques et privées, selon que l'unité institutionnelle est contrôlée ou non par l'État. Cette subdivision équivaut à la distinction qui est faite par le SCN entre les sociétés publiques et privées.

\subsection{Si l'on applique les critères ci-dessus pour classer les unités} institutionnelles des statistiques de R-D, un nombre considérable de cas limites vont apparaître. On trouvera des orientations complémentaires concernant les principaux secteurs et les cas limites dans la section 3.5, ainsi que dans les chapitres du présent manuel consacrés aux différents secteurs. On trouvera également dans la section 3.4 ci-après des lignes directrices sur les principes généraux de classification et les types de classification globalement pertinents qui peuvent être utilisés pour compléter et éclairer la classification institutionnelle de Frascati. 


\subsection{Classifications générales applicables à toutes les unités institutionnelles}

\section{Classification des unités en fonction de leur activité économique principale}

3.31 L'activité économique, définie comme la fourniture de biens ou de services, est une caractéristique générale applicable à toutes les unités. Toutes les unités institutionnelles d'une économie peuvent être caractérisées à l'aide des biens ou des services qu'elles fournissent. La description de l'activité de R-D en fonction de l'activité économique ou de la branche d'activité (définie comme un regroupement d'établissements engagés dans des activités identiques ou similaires ; Nations Unies, 2007) peut être pertinente pour toutes sortes d'usages. Ainsi, l'activité économique qu'est la fourniture de programmes d'enseignement supérieur formel (UNESCO-ISU, 2012) est une caractéristique clé du secteur de l'enseignement supérieur tel que défini dans le présent manuel. Un autre exemple est celui des unités que sont les hôpitaux. Le fait que leur activité principale consiste à fournir des services médicaux présente des difficultés particulières qui nécessitent peut-être l'utilisation d'un type de questionnaire spécial pour recueillir des informations sur leurs activités de R-D. La classification en fonction de l'activité économique peut permettre de mieux cibler les instruments utilisés pour collecter des données sur certains types d'unités, indépendamment du secteur institutionnel auquel elles correspondent dans le Manuel de Frascati.

3.32 La présentation des statistiques de R-D peut en outre être grandement facilitée si l'on utilise une méthode de classification par branche d'activité. Étant donné que des unités exerçant la même activité économique peuvent être classées dans des secteurs institutionnels différents, la classification en fonction de la branche d'activité peut être une source d'informations complémentaires sur la structure et la dynamique des travaux de R-D effectués dans l'ensemble de l'économie, et pas seulement sur le secteur des entreprises auquel l'activité économique principale est plus systématiquement affecté. Il est par conséquent recommandé aux pays de classer les unités institutionnelles de tous les secteurs en fonction de leur principale activité économique, même s'ils font le choix de ne pas communiquer ces chiffres de façon systématique.

3.33 Une unité institutionnelle peut effectuer une ou plusieurs activités économiques. Les unités sont classées en fonction de leur activité principale. Dans la pratique, la majorité des unités de production se livrent à différents types d'activités. La Classification internationale type, par industrie (CITI) sert de référence pour classer les activités économiques (Nations Unies, 2008). Plusieurs pays et domaines l'ont adaptée en fonction de leurs besoins, tout en essayant de conserver un noyau commun qui permette d'effectuer des comparaisons internationales. Pour affecter une unité à une certaine catégorie d'activité économique de la classification de référence, il convient de déterminer quelle est l'activité principale de cette unité. Pour cela, il est nécessaire de connaître la 
part de valeur ajoutée (ou toute autre variable de classification appropriée) créée par les différentes catégories d'activités menées par l'unité en question. Dans la pratique, cependant, cette information est souvent impossible à obtenir, d'où la nécessité de déterminer l'activité à l'aide d'autres critères. Chaque fois que c'est possible et justifié, il est recommandé aux statisticiens de la R-D d'éviter de prendre des décisions de classification sans se concerter et d'utiliser les données des registres statistiques.

3.34 Un aspect particulier de la classification des unités institutionnelles - tous secteurs institutionnels confondus - en fonction de leur activité économique concerne les services de santé et d'éducation. Les unités engagées dans ces activités peuvent théoriquement être classées dans n'importe quel secteur institutionnel du Manuel de Frascati. La présentation globale des statistiques de R-D sur la base de l'activité économique peut éventuellement faire apparaître des différences entre les unités dispensant des services d'enseignement et le total des unités déclarées comme fournissant des services d'enseignement supérieur. Ces différences peuvent être dues à un certain nombre de facteurs, comme, par exemple, la distinction entre les activités principales et secondaires. S'agissant des unités travaillant dans le secteur de l'enseignement supérieur, il est important de déterminer si l'enseignement fait partie de l'activité principale ou secondaire des unités et, dans le cas des hôpitaux universitaires, de voir quel est le rôle de la R-D, voire des services de santé.

\section{Classification des unités en fonction de leur appartenance au secteur public ou au secteur privé}

3.35 La distinction entre les unités contrôlées par l'État et celles qui ne le sont pas est une étape importante du processus de classification qui permet de déterminer quelle proportion de la R-D du secteur des entreprises et du secteur de l'enseignement supérieur doit être imputée au secteur public. Une unité institutionnelle appartient au secteur public ou privé selon qu'elle se trouve ou non sous le contrôle d'une administration publique.

3.36 Dans le SCN, le secteur public comprend les administrations publiques et les sociétés publiques. Une unité qui appartient au secteur des entreprises et est contrôlée par l'État sera donc classée dans le secteur public. En revanche, une université dite " publique " mais dotée de son propre conseil d'administration habilité à prendre des décisions sur tous les aspects de son fonctionnement (y compris l'acquisition et la cession d'actifs, ainsi que la prise d'engagements) sans l'accord des pouvoirs publics, et pouvant cesser ses activités sans l'accord des autorités gouvernementales, doit être considérée comme une entité privée.

3.37 La distinction entre les établissements privés et publics peut être difficile à établir, dans la mesure où tous peuvent recevoir des aides financières importantes de l'État, directement ou indirectement, et où, même les établissements publics peuvent bénéficier d'un degré élevé d'autonomie. Le point clé est donc de déterminer si l'établissement est véritablement autonome ou s'il fait partie de l'appareil administratif de l'État. Dans certains cas, la notion 
de contrôle n'est pas facile à définir, car la capacité de décider du montant et de l'affectation des financements peut être une sorte de contrôle. Par conséquent, il peut parfois être judicieux de prendre en compte la source de financement majoritaire pour déterminer si un établissement est oui ou non contrôlé par l'État.

3.38 Bien que ce soit généralement le cas, la présentation simplifiée des statistiques de R-D ne doit pas tenter de regrouper les secteurs de l'enseignement supérieur et de l'État dans la catégorie " secteur public » (ou le secteur des entreprises et le secteur privé sans but lucratif dans la catégorie " secteur privé "), car cela ne reflète pas, par exemple, le fait que certaines entreprises publiques font partie du secteur des entreprises et que les universités privées et indépendantes relèvent du secteur de l'enseignement supérieur. Le regroupement des unités dites privées (ou publiques) - quel que soit le secteur institutionnel auquel elles appartiennent - peut se faire dans le but de fournir une présentation des statistiques qui réponde aux besoins des utilisateurs.

\section{Classification des unités en fonction de leur rattachement à un groupe plus général, national ou étranger}

3.39 La sous-classification des unités en fonction de leur indépendance ou de leur rattachement à d'autres unités - dans un secteur similaire ou différent, sur le territoire national ou à l'étranger - est clairement utile pour comprendre la nature de l'activité de R-D au sein des unités, et pour produire les statistiques de R-D. Les relations de contrôle peuvent conditionner le comportement économique et la prise de décisions d'une unité, et créer des flux entre les unités pouvant être difficiles à prendre en compte en tant qu'opérations. L'appartenance d'une unité à un groupe d'unités peut en outre lui donner accès à un large éventail de ressources pour réaliser des travaux de $\mathrm{R}-\mathrm{D}$, mais aussi influencer la façon dont cette unité gère, stocke et échange les données relatives à la R-D. L'enregistrement systématique de ces informations et leur usage sélectif dans la présentation globale des statistiques est donc important pour tous les types d'unités institutionnelles, en particulier pour le secteur des entreprises.

3.40 Les caractéristiques des unités institutionnelles qu'il peut être utile de relever sont notamment les suivantes :

- Le fait que l'unité soit contrôlée par une unité institutionnelle distincte ou qu'elle contrôle elle-même d'autres unités.

- Le secteur auquel appartient l'unité exerçant en dernier ressort les fonctions de contrôle, notamment selon qu'il s'agit d'une unité résidente ou implantée à l'étranger. Par exemple, l'unité est-elle contrôlée par une entreprise non résidente ou un établissement d'enseignement supérieur?

3.41 Comme exposé dans le chapitre 4, ces aspects sont importants pour ventiler - ainsi qu'il est recommandé - la R-D en fonction de l'origine des fonds et des destinataires des fonds affectés à la R-D par l'unité statistique. 


\section{Classification des unités en tant que sociétés, administrations publiques ou institutions sans but lucratif}

3.42 Comme indiqué au début de ce chapitre, trois types d'unités institutionnelles ayant le statut de personnes morales peuvent faire l'objet d'une collecte de données sur leurs activités de R-D. Ces unités ne coïncident pas forcément avec le secteur institutionnel auquel elles ont été affectées :

- Les sociétés sont toutes les entités capables de dégager un profit ou une autre forme de gain financier pour leur(s) propriétaire(s), reconnues par la loi comme des entités juridiques distinctes des propriétaires, qui jouissent eux-mêmes d'une responsabilité limitée, et créées dans le but de s'engager dans une production marchande (CE et al., 2009, para. 4.38). Le terme couvre en outre les coopératives, les sociétés de personnes à responsabilité limitée et les quasi-sociétés. À des fins pratiques, il peut être étendu aux ménages ou individus officiellement engagés dans une production marchande dès lors que la délimitation de la responsabilité est difficile à établir. Dans l'ensemble, les sociétés correspondent aux unités qualifiées d'entreprises (pour des informations détaillées, se reporter au chapitre 7).

- Les administrations publiques sont des types particuliers d'entités juridiques, instituées par décision politique, qui exercent un pouvoir législatif, judiciaire ou exécutif sur d'autres unités institutionnelles dans un espace donné (CE et al., 2009, para. 4.117). Elles présentent un intérêt particulier pour l'analyse des budgets de la R-D et des incitations fiscales connexes, comme cela est expliqué respectivement dans les chapitres 12 et 13 . Une analyse plus détaillée des administrations publiques et du secteur de l'État est fournie au chapitre 8.

- Les institutions sans but lucratif (ISBL) sont des entités juridiques ou sociales créées dans le but de produire des biens ou des services, dont le statut ne leur permet pas d'être une source de revenu, de profit ou d'autre forme de gain financier pour les unités qui les créent, les contrôlent ou les financent (CE et al., 2009, para. 4.83). Elles peuvent mener des activités de production marchande ou non marchande. L'intérêt de prendre en compte l'ensemble des ISBL dans les statistiques de R-D provient du caractère relativement marginal du secteur privé sans but lucratif dans la principale classification institutionnelle, comme indiqué au chapitre 10. Cette prise en compte serait cohérente avec les recommandations du SCN de créer une série de comptes satellites pour les ISBL. Celles-ci ne font pas toutes partie du secteur privé sans but lucratif. Certaines sont classées dans les secteurs de l'enseignement supérieur, des entreprises et de l'État, selon la nature des activités qu'elles mènent et le fait qu'elles sont ou non contrôlées par l'État.

3.43 Dans le SCN, les unités institutionnelles sont regroupées en secteurs. Les secteurs utilisés dans le présent manuel sont définis dans la section 3.5. Le secteur des sociétés du SCN (CE et al., 2009, paragraphes 4.94 et 4.98) équivaut dans le présent manuel au secteur des entreprises, à l'exception des unités relevant de l'enseignement supérieur (voir la section 3.5). Le secteur des 
administrations publiques (CE et al., 2009, para. 127) est l'équivalent du secteur de l'État, à l'exception des unités relevant de l'enseignement supérieur (voir la section 3.5). Le secteur des institutions privées sans but lucratif au service des ménages (ISBLSM) (CE et al., 2009, para. 4.166) correspond au secteur privé sans but lucratif (PSBL), à l'exception des unités relevant de l'enseignement supérieur (voir la section 3.5). Par souci d'exhaustivité, le secteur PSBL inclut également le secteur des ménages du SCN, à l'exception des travailleurs indépendants ayant un statut proche d'une entreprise, qui sont classés dans le secteur des entreprises.

\section{Classification des unités en fonction du domaine de R-D}

3.44 La classification des unités en fonction du domaine de R-D a été présentée dans le chapitre 2 - qui indique comment définir l'objet des activités de R-D - et est décrite en détail en ligne, en complément du présent manuel. Deux projets de R-D peuvent être considérés comme relevant du même domaine si leurs objectifs principaux sont identiques ou suffisamment similaires. Le degré de similitude peut être dû : a) aux sources de connaissances communes sur lesquelles s'appuient les activités de R-D, b) aux domaines d'application et d'intérêt communs des travaux de R-D (un phénomène à expliquer ou des problèmes à résoudre) et aux méthodes utilisées, c) aux techniques et parcours professionnels des scientifiques et autres professionnels intervenant dans les travaux de R-D.

3.45 Du fait de la grande diversité des domaines de R-D couverts par les unités institutionnelles, la pertinence de ce mode de classification se limite aux secteurs institutionnels du Manuel de Frascati dans lesquels les unités réalisant des travaux de R-D s'attachent essentiellement à produire des actifs intellectuels (en particulier dans le secteur de l'enseignement supérieur) et aux unités statistiques qui sont définies à un niveau relativement général. Dans ces types de cas, il convient d'utiliser le premier niveau de classification qui comprend les six domaines de R-D suivants :

- sciences naturelles

- ingénierie et technologie

- sciences médicales

- sciences agricoles

- sciences sociales

- sciences humaines et arts.

3.46 Des orientations particulières concernant l'utilisation de cette classification sont proposées séparément pour chaque secteur, principalement du point de vue de la répartition fonctionnelle. Une classification plus détaillée est accessible en ligne en complément du présent manuel, à l'adresse http://oe.cd/frascati. 


\section{Classification des unités en fonction de leur localisation géographique}

3.47 La localisation des unités réalisant des travaux de R-D représente certes un grand intérêt pour les utilisateurs, mais elle n'est pas facile à établir du point de vue de la collecte de statistiques car les unités statistiques définies pour les besoins de la R-D peuvent se répartir entre plusieurs sites, dans différents pays et différentes régions au sein de chaque pays. Une classification géographique détaillée est particulièrement intéressante pour les unités et les établissements locaux. Pour certains types de recherches, ces unités statistiques peuvent convenir. Toutefois, dans la mesure où il n'est pas toujours possible de trouver des données sur leurs activités de R-D, une classification géographique au niveau national n'est pas toujours faisable. Le critère prioritaire pour établir une classification géographique est la distinction entre les unités résidentes et non résidentes. L'utilisation d'une approche de répartition fonctionnelle à l'égard des unités déclarantes couvrant plusieurs sites est aussi envisageable. Ces méthodes font l'objet d'orientations complémentaires accessibles en ligne à l'adresse http://oe.cd/frascati.

\section{Pratiques d'archivage utilisées pour la classification institutionnelle}

3.48 Le tableau 3.2 fournit un exemple représentatif de la manière dont les organismes statistiques peuvent s'y prendre pour établir des registres exhaustifs d'unités et les classer selon différents critères et différentes méthodes. Des systèmes similaires peuvent leur permettre de répondre régulièrement ou au cas par cas aux besoins spécifiques des utilisateurs nationaux et internationaux.

Tableau 3.2. Exemple simplifié de structure possible pour classer les unités statistiques selon différents critères

\begin{tabular}{|c|c|c|c|c|c|c|c|}
\hline & $\begin{array}{c}\text { Secteur } \\
\text { institutionnel } \\
\text { Frascati }\end{array}$ & $\begin{array}{c}\text { Secteur } \\
\text { institutionnel } \\
\text { SCN }^{1}\end{array}$ & $\begin{array}{c}\text { Activité } \\
\text { économique } \\
\text { principale }^{1}\end{array}$ & $\begin{array}{c}\text { Activité } \\
\text { économique } \\
\text { secondaire } \\
\text { (éventuellement) }{ }^{1}\end{array}$ & $\begin{array}{c}\text { Appartenance } \\
\text { au secteur } \\
\text { public/ privé }^{1}\end{array}$ & $\begin{array}{l}\text { Institution } \\
\text { sans but } \\
\text { lucratif } \\
\text { (ISBL) ? }\end{array}$ & $\begin{array}{c}\text { Liens avec } \\
\text { d'autres } \\
\text { unités }^{1}\end{array}$ \\
\hline \multicolumn{8}{|l|}{ Unité A } \\
\hline \multicolumn{8}{|l|}{ Unité B } \\
\hline$\ldots$ & & & & & & & \\
\hline
\end{tabular}

1. Peut provenir d'autres cadres ou sources statistiques selon les accords d'échange de données qui ont été conclus, ou être attribué par l'organisme établissant les statistiques de R-D.

3.49 Afin de garantir la qualité et la comparabilité internationale des données, il est proposé que les pays fassent connaître leurs décisions en matière de classification - dans la mesure où leurs dispositions en matière de confidentialité statistique le leur permettent -, ce qui devrait grandement faciliter la compréhension des différences entre les données et favoriser une meilleure comparabilité. 


\subsection{Présentation succincte des principaux secteurs du Manuel de Frascati, de leurs unités et des cas limites}

3.50 Les secteurs du Manuel de Frascati peuvent être présentés brièvement comme quatre grands secteurs : trois d'entre eux ont un équivalent dans la classification institutionnelle du SCN (secteur des entreprises, secteur de l'État et secteur privé sans but lucratif), tandis que le quatrième a été créé pour répondre aux besoins des utilisateurs de disposer d'un secteur consacré à l'enseignement supérieur qui se superpose aux autres. Ces secteurs sont représentés dans le graphique 3.2.

\section{Graphique 3.2. Représentation schématique des secteurs institutionnels nationaux du Manuel de Frascati et des cas frontières}

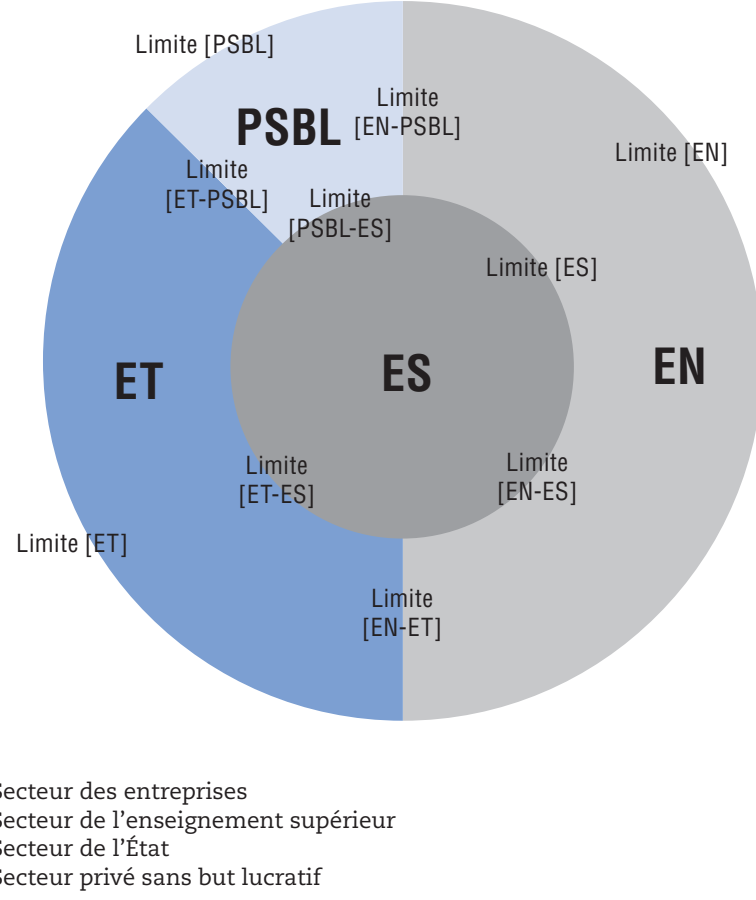

\section{Secteur des entreprises}

\section{Principales caractéristiques}

3.51 Le secteur des entreprises comprend:

- Toutes les sociétés résidentes, y compris et non exclusivement les entreprises légalement constituées en société, indépendamment du lieu de résidence de leurs actionnaires. En font également partie tous les autres types de quasisociétés, c'est-à-dire les unités capables de dégager un profit ou une autre 
forme de gain financier pour leur(s) propriétaire(s), reconnues par la loi comme des entités juridiques distinctes de leur(s) propriétaire(s) et créées dans le but de mener des activités de production marchande à des prix économiquement significatifs.

- Les succursales d'entreprises non résidentes non constituées en société et considérées comme résidentes du fait qu'elles mènent dans la durée des activités de production sur le territoire économique.

- Toutes les ISBL résidentes qui sont des producteurs marchands de biens ou de services ou qui servent les entreprises.

3.52 Il faut, pour que ces critères s'appliquent, que l'unité n'ait pas été classée dans le secteur de l'enseignement supérieur sur la base des critères présentés plus avant dans cette section. Ce secteur comprend à la fois des entreprises publiques et privées.

\section{Unités statistiques du secteur des entreprises}

3.53 Les entreprises organisent leurs activités de R-D pour réaliser plus facilement leurs objectifs. Les unités statistiques utilisées pour ce secteur sont déterminées par les données requises - qui sont spécifiées dans les chapitres 4 et 5 pour les généralités, et dans le chapitre 7 pour ce secteur en particulier. Les entreprises peuvent organiser le financement et le déroulement de leurs travaux de R-D à plusieurs niveaux. Les décisions stratégiques relatives au financement et à l'orientation des efforts de R-D peuvent être prises au niveau du groupe, indépendamment des frontières nationales. Les activités de R-D menées par les entreprises peuvent se répartir sur plusieurs pays.

3.54 L'unité statistique utilisée pour le secteur des entreprises est généralement l'entreprise, comme indiqué dans l'encadré 3.1. Lorsqu'une entreprise mène des activités économiques hétérogènes et réalise un grand nombre de travaux de R-D pour plusieurs types d'activités différents, une unité déclarante moins générale (par exemple une unité d'activité économique ou un établissement) est parfois préférable si l'on peut se procurer les informations nécessaires.

\section{Principaux cas limites}

3.55 La plupart des cas limites entre ce secteur et d'autres sont décrits plus loin dans la présente section. Des difficultés pratiques peuvent apparaître lorsqu'il faut déterminer si une unité est résidente ou non, en particulier lorsqu'il s'agit d'une succursale non constituée en société d'une entreprise sous contrôle étranger. Les universités qui vendent leur production à un prix économiquement significatif doivent être classées dans le secteur de l'enseignement supérieur, sur la base de leur activité économique principale. Les entreprises commerciales appartenant à des établissements d'enseignement supérieur (par exemple, du fait de l'existence d'accords conférant à une université une position d'actionnaire 
majoritaire dans une entreprise créée par essaimage par le personnel enseignant ou des étudiants) doivent être considérées comme des entreprises.

3.56 Des entités spéciales créées dans un but particulier par des unités institutionnelles déjà en place peuvent poser des difficultés de classification. Pour citer un exemple, de nombreuses unités publiques concluent des accords avec des entités privées ou d'autres unités publiques pour mener avec elles toute une série d'activités conjointes, notamment en matière de R-D. Comme définie dans le SCN, une coentreprise implique l'établissement d'une société, d'un partenariat ou d'une autre unité institutionnelle dans laquelle chaque partie exerce légalement un contrôle conjoint sur les activités de l'unité. Elle opère de la même façon que les autres unités, à cette exception près qu'un accord juridique entre les parties établit un contrôle conjoint sur l'unité. En tant qu'unité institutionnelle, la coentreprise peut passer des contrats en son propre nom et lever des fonds pour ses propres besoins. Une coentreprise tient sa propre comptabilité. Dès lors que des coentreprises de R-D constituent des unités à part entière, elles doivent aussi être classées en fonction des unités qu'elles servent au premier chef compte tenu, dans la mesure du possible, de la pratique établie en vertu du SCN.

3.57 Les partenariats privé-privé ou privé-public ne sont pas nécessairement des unités institutionnelles, mais souvent des contrats conclus entre deux entités de secteurs différents. Si ces partenariats ont le statut d'unités institutionnelles, leur classification dépend également de l'entité possédant l'intérêt majoritaire dans le partenariat. Dans certains pays, les partenariats de R-D ont un statut de personne morale et doivent donc être classés en fonction des unités qu'ils servent au premier chef.

3.58 Des difficultés de classification peuvent apparaître lorsqu'il faut déterminer si les ISBL se livrent ou non à une production marchande. Les établissements de recherche, les cliniques, les hôpitaux, les cabinets médicaux du secteur privé payant des droits, etc. peuvent être en mesure de trouver des fonds supplémentaires sous forme de donations ou d'avoirs propres fournissant des revenus de propriété qui leur permettent de pratiquer des tarifs inférieurs à la moyenne. De même, lorsqu'il faut déterminer si une ISBL a été créée pour servir une entreprise, la classification peut être compliquée par l'existence d'un grand nombre de parties prenantes et leur variation au fil du temps. En règle générale, les ISBL qui sont créées et gérées par des associations professionnelles dont elles sont censées promouvoir les activités (comme, par exemple, les chambres de commerce et d'agriculture, les associations commerciales et industrielles) et financées par les contributions ou les cotisations des entreprises concernées - qui assurent ainsi le financement de base ou par projet de leur R-D - doivent être classées dans le secteur des entreprises.

3.59 Les cas limites éventuels du secteur des entreprises, ainsi que les sous-classifications recommandées, sont examinés dans le chapitre 7. 


\section{Secteur de l'État}

\section{Principales caractéristiques}

3.60 Le secteur de l'État se compose des groupes d'unités institutionnelles résidentes suivantes :

- toutes les unités d'administration centrale (fédérale), régionale (d’États fédérés) ou locale (municipale), y compris les administrations de sécurité sociale, à l'exception des unités fournissant des services d'enseignement supérieur ou répondant à la description des établissements d'enseignement supérieur donnée dans la sous-section suivante

- toutes les ISBL non marchandes contrôlées par des administrations publiques et n'appartenant pas au secteur de l'enseignement supérieur.

3.61 Ce secteur ne comprend pas les entreprises publiques, même quand la totalité du capital de ces sociétés est détenue par des administrations. Les entreprises publiques sont incluses dans le secteur des entreprises ; l'élément distinctif est que les entreprises publiques sont des producteurs marchands, alors que les unités classées dans le secteur de l'État n'en sont pas.

\section{Unités statistiques du secteur de l'État}

3.62 Le secteur de l'État comprend les administrations publiques et les institutions sans but lucratif contrôlées par l'État. Les administrations publiques sont des types particuliers d'entités juridiques, instituées par décision politique, qui exercent un pouvoir législatif, judiciaire ou exécutif sur d'autres unités institutionnelles dans un espace donné. Ces entités juridiques ou succursales sont par conséquent responsables de l'exécution ou de la prestation de travaux de R-D particuliers menés à l'usage de l'administration publique et pour le bien de la société et de l'économie. Elles sont également chargées de financer, par la fiscalité ou d'autres sources de revenus, la prestation de services par leurs soins ou par des tiers. Leur participation aux activités de R-D est souvent davantage financière que concrète, mais les administrations publiques possèdent parfois des services de recherche et des laboratoires internes qui mènent certains types d'activités de R-D. Dans certains cas, des organismes peuvent être créés sous une identité juridique distincte pour mener des travaux de recherche à titre principal, secondaire ou auxiliaire. Bien que le thème central des enquêtes dépende du sujet auquel on s'intéresse - à savoir les résultats de la R-D, son financement ou les deux -, l'unité statistique est généralement l'unité institutionnelle. Cela dit, les données peuvent être collectées au niveau du ministère, de l'autorité locale, de l'agence ou de l'institution gouvernementale, même si l'unité déclarante ne présente pas toutes les caractéristiques d'une unité institutionnelle (c'est-à-dire la capacité de posséder et de gérer des actifs).

3.63 Une proportion non négligeable des travaux de R-D réalisés au sein des administrations publiques est susceptible de l'être par des institutions sans but lucratif telles que les fondations, les musées, les hôpitaux et les établissements 
contrôlés par l'État. L'unité statistique sera généralement l'entreprise, prise dans le sens énoncé plus haut dans l'encadré 3.1.

\section{Principaux cas limites}

3.64 Les cas limites entre le secteur de l'État et celui de l'enseignement supérieur sont examinés plus avant dans cette section. Le cas limite avec le secteur PSBL se présente essentiellement lorsque les administrations publiques contrôlent les opérations des unités statistiques compétentes. Dans ce cas de figure, le contrôle correspond à la capacité de définir le programme ou l'orientation générale d'une ISBL non marchande en étant habilité à en nommer les responsables ou à en orienter en dernier ressort les décisions. La notion de contrôle est parfois difficile à définir, car la capacité de décider du montant et de l'affectation des financements peut être une sorte de contrôle. Il peut donc être judicieux de prendre en compte la source de financement majoritaire pour déterminer si une institution est oui ou non contrôlée par l'État.

3.65 En ce qui concerne les unités contrôlées par l'État, le cas limite avec le secteur des entreprises se présente lorsque l'unité présente un caractère marchand, c'est-à-dire lorsque son activité principale est la production de biens ou de services marchands, et qu'elle a pour but de vendre la majorité de sa production à des prix économiquement significatifs. Un établissement de recherche public susceptible de percevoir occasionnellement des sommes importantes pour l'exploitation de certains de ses actifs intellectuels ne devrait pas être classé dans la catégorie des entreprises publiques si la majorité de ses activités de R-D sont menées dans un but non commercial. En revanche, un établissement contrôlé par l'État dont les activités consistent à fournir des services de R-D et un accès à l'infrastructure de recherche contre des honoraires correspondant au coût économique total de ces services devrait être considéré comme une entreprise publique.

3.66 Les cas limites éventuels du secteur de l'État, ainsi que les sousclassifications recommandées, sont examinés dans le chapitre 8 relatif à la R-D publique.

\section{Secteur de l'enseignement supérieur}

\section{Principales caractéristiques}

3.67 Ce secteur n'a pas d'équivalent direct dans la classification des secteurs institutionnels du SCN. On le trouve uniquement dans le présent manuel. Il a été conçu dans le but de répertorier une catégorie d'établissements réalisant des travaux de R-D qui présentent une utilité pour l'action des pouvoirs publics. Ce secteur comprend l'ensemble des universités, établissements d'enseignement post-secondaire et autres établissements proposant des programmes d'enseignement supérieur formel, indépendamment de leur source de financement ou de leur statut juridique, ainsi que l'ensemble des instituts et centres de recherche, stations d'expérimentation et centres de soins dont les 
activités de R-D relèvent du contrôle direct d'établissements d'enseignement supérieur ou sont administrées par ceux-ci. L’enseignement " formel ", défini dans la CITE (UNESCO-ISU, 2012, para. 36), est explicité dans le chapitre 9.

3.68 Dans le présent manuel, c'est l'expression « enseignement supérieur » qui est employée la plupart du temps, et non l'expression " enseignement du $3^{e}$ degré ", plus générale. En ce qui concerne le produit de l'activité des établissements d'enseignement supérieur, c'est le terme " services » qui est utilisé plutôt que " programmes ", lequel est courant en revanche dans les statistiques sur l'éducation et dans la CITE.

3.69 Les unités figurant dans ce secteur peuvent correspondre à celles classées par le SCN dans les sociétés, les administrations publiques ou les ISBLSM.

\section{Unités statistiques du secteur de l'enseignement supérieur}

3.70 La recommandation du présent manuel est que, par souci d'homogénéité, l'unité statistique utilisée pour ce secteur soit l'entreprise, ou son équivalent institutionnel. Les données peuvent toutefois être recueillies (déclarées) par la plus petite unité homogène engagée au niveau supérieur du domaine de classification de la R-D - ou de plusieurs classifications de la R-D dans le cas d'unités travaillant dans des domaines interdisciplinaires. Selon la capacité de l'unité à rendre compte de façon homogène des effectifs, des dépenses et des flux financiers, et selon la terminologie spécifique appliquée dans chaque pays, l'unité déclarante peut être un service, une faculté, un centre, un institut ou un établissement d'enseignement post-secondaire. La recommandation est que l'unité déclarante soit définie en fonction de sa capacité à fournir des statistiques homogènes.

\section{Principaux cas limites}

3.71 Ce secteur comprend toutes les unités (établissements) dont l'activité principale consiste à fournir des services d'enseignement supérieur aux niveaux 5, 6, 7 ou 8 de la CITE, quel que soit leur statut juridique (UNESCOISU, 2012, p. 83). Il peut s'agir de sociétés, de quasi-sociétés appartenant à une administration publique, d'ISBL marchandes ou d'ISBL contrôlées et financées en majorité par l'État ou par des ISBLSM (c'est-à-dire des ISBL non marchandes). Comme indiqué précédemment, le noyau du secteur se compose d'universités et d'établissements d'enseignement post-secondaire. Il convient de noter que les établissements d'enseignement supérieur n'effectuent pas tous des travaux de R-D.

3.72 Les hôpitaux et cliniques universitaires sont inclus dans le secteur de l'enseignementsupérieur lorsqu'ils fournissentce type de service (éventuellement en tant qu'activité secondaire). Pour ce qui est des autres hôpitaux et cliniques, ils ne doivent être classés dans ce secteur que lorsque la totalité de leur activité de R-D est placée sous le contrôle direct d'un établissement d'enseignement 
supérieur ou administrée par lui. Dans ce cas, en effet, l'activité de R-D peut être considérée comme faisant partie des travaux de R-D de l'établissement en question. Dans tout autre cas, l'entité hospitalière doit être classée en fonction des critères standard, à savoir son caractère marchand ou non et l'ampleur du contrôle exercé par l'État. L'application de ces lignes directrices peut nécessiter de travailler avec des unités statistiques et déclarantes situées au-dessous du niveau de l'institution médicale.

3.73 Le secteur de l'enseignement supérieur comprend les centres et les instituts de recherche dont la R-D est l'activité principale et l'enseignement supérieur une activité de premier plan, consistant, par exemple, à dispenser une formation systématique aux étudiants en doctorat. Ce secteur inclut également les centres et instituts non marchands apparentés qui n'exercent pas de fonction éducative mais dont les activités de R-D sont contrôlées par un ou des établissement(s) d'enseignement supérieur. Lorsqu'aucune de ces conditions n'est remplie, le centre de recherche doit être affecté au secteur approprié, à savoir celui des entreprises s'il présente un caractère marchand (quel que soit le contrôle exercé par l'État), celui du PSBL s'il présente un caractère non marchand et n'est pas contrôlé par l'État, ou celui de l'État s'il est non marchand et contrôlé par l'État. L'emplacement géographique n'est pas un critère déterminant.

3.74 Les cas limites éventuels du secteur de l'enseignement supérieur, ainsi que les sous-classifications recommandées, sont examinés dans le chapitre 9.

\section{Secteur privé sans but lucratif}

\section{Principales caractéristiques}

\subsection{Ce secteur comprend :}

- toutes les institutions sans but lucratif au service des ménages (ISBLSM), telles que définies dans le SCN 2008, à l'exception de celles relevant du secteur de l'enseignement supérieur

- à des fins d'exhaustivité, les ménages et les particuliers qui mènent ou non des activités marchandes (comme expliqué plus haut dans ce chapitre, dans la section intitulée Les critères de classification et le choix des secteurs institutionnels utilisés pour les statistiques de R-D).

3.76 Les unités concernées sont, par exemple, des associations professionnelles indépendantes, des sociétés savantes et des organisations caritatives qui ne sont pas administrées par des unités du secteur de l'État ou des entreprises. Ces unités fournissent des services individuels ou collectifs à des ménages, soit gratuitement, soit à des prix qui ne sont pas économiquement significatifs. Elles peuvent être créées par des associations de personnes pour fournir des biens ou, plus fréquemment, des services principalement destinés aux membres eux-mêmes ou à des fins philanthropiques générales. Leurs activités peuvent être financées par des cotisations régulières, des droits, des dons en espèces ou en nature émanant du public, de sociétés ou de l'État. 
Dans ce secteur, un plus grand nombre d'unités, dont les ménages - sont susceptibles de participer davantage au financement des activités de R-D qu'à leur réalisation.

3.77 Les lignes directrices proposées dans le présent manuel pour établir des statistiques sur la R-D se concentrent sur le rôle joué par les unités institutionnelles dans les travaux de R-D. Elles respectent ainsi la définition de la R-D qui est donnée au chapitre 2, ainsi que les critères explicatifs qui l'accompagnent pour garantir au mieux son application. Par souci d'exhaustivité et dans le but de rendre la présentation plus synthétique, et surtout de remplir certains objectifs spécifiques (par exemple, rendre compte de phénomènes tels que l'activité philanthropique de financement de la R-D par les particuliers), les ménages complètent le tableau et sont inclus dans le secteur PSBL.

- Les rôles des particuliers dans les travaux de R-D sont en fait pris en compte par le biais des unités institutionnelles pour lesquelles ils travaillent dans le cadre d'un large éventail de dispositifs. Un chercheur isolé peut parfois faire l'objet d'enquêtes consacrées à un sujet précis, notamment dans le but d'obtenir des informations complémentaires pour améliorer les processus d'estimation utilisant les données recueillies auprès des unités institutionnelles (par exemple, pour apporter des précisions sur le temps consacré à la R-D, lorsque cette information ne peut être directement recueillie auprès des unités institutionnelles).

- Les particuliers ou les ménages peuvent, dans certains cas, coïncider avec la classification institutionnelle, en particulier lorsqu'ils sont constitués en entités juridiques ou enregistrés sous une forme où leur responsabilité doit obligatoirement être assumée conjointement et où l'organisation est structurée. Les difficultés rencontrées pour respecter les critères énoncés dans le chapitre 2 et tenir compte de ces micro-unités sont abordées dans le chapitre 6 ainsi que dans les chapitres consacrés à chaque secteur, en particulier le chapitre 7 sur le secteur des entreprises.

3.78 Les particuliers - et les ménages auxquels ils appartiennent contribuent activement, et de diverses autres façons, à l'apport de savoir en matière de R-D, non seulement en tant que financeurs (à titre philanthropique), mais aussi en tant que cobayes (par exemple en participant à des tests cliniques) ou en tant que créateurs actifs de nouvelles connaissances (par exemple en tant que collecteurs de données scientifiques et inventeurs). Il existe, dans l'histoire de la science, plusieurs exemples de découvertes majeures effectuées par des individus isolés. Aujourd'hui, des initiatives individuelles d'un nouveau genre sont facilitées par les réseaux sociaux qui permettent la confrontation et la collaboration entre des groupes informels d'individus, ainsi qu'avec des unités institutionnelles formelles. La prise en compte des particuliers au titre de leur participation à une activité de recherche ou, plus généralement, d'innovation (par exemple en tant que bénévoles) s'inscrit dans le cadre du programme général "d'étude » concernant les indicateurs de la science, de la technologie et de l'innovation. Il n'est cependant pas possible à ce stade de recommander une 
méthode générale de mise en œuvre pour tous les pays. Toute initiative nationale visant, à titre expérimental, à mesurer la R-D effectuée par les particuliers ne saurait être associée à la représentation standard des statistiques de R-D.

\section{Unités statistiques du secteur privé sans but lucratif}

3.79 La recommandation du présent manuel est que l'unité statistique utilisée pour le secteur PSBL soit l'entreprise (telle que définie sommairement dans le présent chapitre). Une réflexion s'impose lorsque l'on est en présence d'institutions complexes et que la plus petite unité homogène est engagée dans un seul domaine de R-D. Un exemple est le cas des unités PSBL travaillant dans des domaines interdisciplinaires bien précis. Lorsqu'une ISBL importante exerce des activités de R-D dans plusieurs domaines, on peut s'efforcer de diviser l'unité statistique en unités plus petites et de les classer dans les domaines de R-D correspondants.

\section{Principaux cas limites}

3.80 Les cas limites avec le secteur de l'enseignement supérieur et celui de l'État ont été examinés dans les sous-sections précédentes. Les unités sans but lucratif qui dispensent des services d'enseignement supérieur ou sont administrées par des établissements d'enseignement supérieur doivent être classées dans le secteur de l'enseignement supérieur. Comme indiqué dans le présent chapitre, le type de contrôle doit être le principal critère utilisé pour la classification. Toutefois, la notion de contrôle n'est pas toujours facile à définir, car la capacité de décider du montant et de l'affectation des financements peut être une sorte de contrôle. Par conséquent, il peut parfois être judicieux de prendre en compte la source de financement majoritaire pour déterminer si un établissement est ou non contrôlé par l'État.

3.81 Conformément au SCN, les institutions sans but lucratif qui sont administrées par des entreprises ou qui les servent au premier chef (comme, par exemple, les associations commerciales, les établissements de recherche contrôlés par l'industrie, etc.) doivent être classées dans le secteur des entreprises, y compris lorsque ces institutions fonctionnent à l'aide de cotisations qui couvrent à peine leurs dépenses de fonctionnement.

3.82 Les activités marchandes des entreprises non constituées en sociétés qui appartiennent à des ménages (par exemple, les consultants indépendants entreprenant des projets de R-D pour le compte d'une autre unité à un prix économiquement significatif) doivent être incluses dans le secteur des entreprises chaque fois que c'est possible et que l'on peut démontrer, lorsque des travaux de R-D y sont menés, qu'ils satisfont aux critères définis dans le chapitre 2.

3.83 Comme précédemment indiqué, les activités menées par des particuliers sur leur temps personnel et pour satisfaire leurs propres intérêts en tant que chercheurs ou inventeurs ne rentrent pas dans le cadre de l'approche institutionnelle de mesure des statistiques de R-D décrite dans le présent manuel. 
3.84 La question du traitement des particuliers susceptibles d'appartenir à un groupe de personnes employées par une unité institutionnelle à part entière - sans en être salariées - et recevant directement de la part de tiers des fonds pour financer leurs activités de R-D est abordée dans le chapitre 4 (sur les dépenses de R-D et les sources de financement) et le chapitre 5 (sur le personnel de R-D).

3.85 Le champ couvert par le présent manuel n'inclut pas non plus le secteur informel et les activités de R-D pouvant y être menées, que ce soit par des particuliers ou des « entreprises d'employeurs informels » (CE et al., 2009, p. 475). Comme noté dans le SCN de 2008 (CE et al., 2009, p. 474), le secteur informel pose un problème non seulement pour les pays en développement, mais aussi pour l'ensemble des pays, quel que soit leur stade de développement.

3.86 Les cas limites éventuels du secteur PSBL, le traitement à réserver aux particuliers et aux ménages, ainsi que les sous-classifications recommandées, sont examinés dans le chapitre 10 (sur la R-D des institutions privées sans but lucratif).

\section{Reste du monde}

\section{Principales caractéristiques}

3.87 Ce secteur se définit sur la base de la non-résidence des unités considérées. Il inclut toutes les unités institutionnelles non résidentes qui réalisent des opérations - ou entretiennent tout autre lien économique - avec les unités résidentes. La notion de résidence a été expliquée dans la section 3.3. Le reste du monde comprend :

- l'ensemble des institutions et personnes qui ne disposent pas, sur le territoire économique considéré, de site, de lieu de production ou de locaux dans lesquels ou à partir desquels elles mènent ou entendent mener des activités économiques et des opérations économiques de grande envergure, pendant une période indéterminée ou déterminée mais longue

- l'ensemble des organisations internationales et autorités supranationales, définies ci-après, y compris les installations et activités qu'elles possèdent à l'intérieur du pays considéré.

3.88 Pour ce qui est de la compilation des statistiques de R-D, les unités non résidentes doivent être classées dans la catégorie des unités résidant à l'étranger ou dans le reste du monde. Lorsque les statistiques sont compilées pour les secteurs institutionnels, ainsi que pour l'économie tout entière, il est recommandé de rendre compte des flux de financement de la R-D qui ont lieu avec le reste du monde (voir les chapitres 4 et 11). Les opérations avec le reste du monde sont enregistrées comme s'il s'agissait d'un secteur de facto. Ce secteur englobe également les travaux de R-D effectués au sein de l'économie nationale par des unités résidentes ayant des liens avec le reste du monde. 


\section{Unités statistiques du reste du monde}

3.89 La description des unités statistiques applicables à ce secteur n'est pas pertinente, car aucune donnée statistique n'est censée être recueillie à cet égard par les statisticiens nationaux de R-D.

\section{Principaux cas limites}

3.90 Les unités résidentes peuvent mener des activités en dehors du territoire économique de leur pays, y compris en utilisant des terrains d'essai, véhicules, navires, avions et satellites gérés par des entités nationales. Les unités institutionnelles correspondantes ne sont généralement pas dissociées de l'entité nationale. Dans le SCN, les propriétaires de terrains, de bâtiments et de structures immeubles implantés sur le territoire économique d'un pays, ou encore les unités louant ces actifs sur une longue durée sont considérés comme possédant un centre d'intérêt économique dans le pays. Les terrains et les bâtiments sont donc traités comme s'ils appartenaient à des unités résidentes et des unités spéciales sont créées à cet effet.

3.91 Lorsqu'une unité possède un site, une succursale, un bureau ou un lieu de production dans un autre pays pour y réaliser des travaux de R-D sur une longue période (généralement un an ou plus), la succursale, le bureau ou le site est considéré comme une unité institutionnelle distincte de l'unité résidente. Ainsi, lorsqu'une entité du pays A entretient une présence depuis plusieurs années dans le pays $B$, cette présence sera considérée dans les statistiques comme une unité distincte, même si le personnel y séjourne pendant moins d'un an. Cette unité distincte fera alors partie du champ de collecte de données des statisticiens du pays B.

3.92 Les concepts de territoire économique et de résidence ont pour but de s'assurer que chaque unité institutionnelle réside sur un seul territoire économique. Il est donc recommandé que les services nationaux établissant les statistiques de R-D coordonnent leur évaluation du lieu de résidence des unités situées à la frontière avec ceux des autres pays qui pourraient être concernés.

3.93 Certains pays peuvent être partie prenante à un accord institutionnel qui implique des transferts monétaires depuis les pays membres vers l'autorité supranationale correspondante (voir la définition dans le glossaire) et, depuis celle-ci, vers les unités effectuant des travaux de R-D. L'autorité supranationale peut elle-même réaliser des travaux de R-D. Au niveau des différents pays, les autorités supranationales sont des unités institutionnelles non résidentes qui font partie du reste du monde et peuvent être classées dans un sous-secteur spécifique du reste du monde. Le présent manuel utilise indifféremment les expressions " autorités supranationales » et " organisations supranationales ".

3.94 Selon la définition du SCN, les organisations internationales comptent parmi leurs membres soit des États, soit d'autres organisations internationales dont les membres sont des États. Elles sont instituées par la 
conclusion, entre leurs membres, d'accords politiques formels ayant le statut de traité international ; leur existence est reconnue par la loi dans les pays qui en sont membres et elles ne sont pas assujetties aux lois ou aux règlements du ou des pays dans lesquelles elles sont présentes. Ainsi, aucun État ne peut les contraindre à fournir des renseignements statistiques sur leurs résultats en matière de R-D ou leurs activités de financement. Dans le SCN et les statistiques de $\mathrm{R}-\mathrm{D}$, les organisations internationales sont traitées comme des unités résidant à l'étranger (reste du monde), indépendamment de leur lieu d'implantation ou d'activité.

3.95 Afin d'obtenir une représentation exhaustive des activités de R-D dans une zone spécifique ou à l'échelle mondiale, les organisations statistiques compétentes au niveau international ou supranational doivent garantir une couverture intégrale des unités situées au-delà de la zone de compétence des autorités et services nationaux. Lorsque, en vertu d'accords spécifiques, les statisticiens nationaux sont habilités à recueillir des données auprès de ces organisations (par exemple pour mieux rendre compte des liens existant avec les unités nationales), la déclaration des chiffres nationaux doit être conforme aux instructions du présent manuel, afin que les unités soient classées dans le reste du monde.

3.96 Les types d'unités, leurs sous-classifications et les cas limites sont examinés dans le chapitre 11 , consacré à la mondialisation de la R-D.

\section{Références}

CE, FMI, OCDE, Nations Unies et Banque mondiale (2009), Système de comptabilité nationale, Nations Unies, New York, http://unstats.un.org/unsd/nationalaccount/docs/ sna2008FR.pdf.

Nations Unies (2008), "Classification internationale type, par industrie, de toutes les branches d'activité économique (CITI), Révision 4 ", Département des affaires économiques et sociales, Division de statistique, Études statistiques, Série $M, n^{\circ} 4$, Rév. 4, Nations Unies, New York, http://unstats.un.org/unsd/cr/registry/isic-4.asp et http://unstats.un.org/unsd/publication/seriesM/seriesm_4rev4f.pdf.

Nations Unies (2007), Statistical Units, Nations Unies, New York, http://unstats.un.org/unsd/ isdts/docs/StatisticalUnits.pdf ; UNESCO-ISU (2012), Classification Internationale Type de l'Éducation (CITE) 2011, ISU, Montréal, http://www.uis.unesco.org/Education/Documents/ isced-2011-fr.pdf. 


\section{Chapitre 4}

\section{Mesurer les dépenses de R-D : Exécution et sources de financement}

Ce chapitre porte sur la manière de mesurer les dépenses de recherche et développement expérimental (R-D), les sources de financement de la R-D et les dépenses découlant des travaux de R-D exécutés par d'autres unités statistiques. Les dépenses intérieures brutes de R-D (DIRD) constituent le principal indicateur de l'activité de R-D d'un pays ; elles correspondent au total des dépenses engagées au titre de la R-D au sein d'une économie pendant une période de référence donnée. Les DIRD et le ratio DIRD/PIB servent aux comparaisons internationales. On s'intéresse ici à la manière dont les dépenses et flux de R-D sont mesurés dans chacun des quatre secteurs d'exécution de la R-D étudiés dans le Manuel (Entreprises, État, Enseignement supérieur et secteur privé sans but lucratif), ainsi qu'au financement, par le reste du monde, de la R-D exécutée dans ces quatre secteurs. Compte tenu que les dépenses de R-D sont désormais considérées comme des dépenses d'investissement dans le Système de comptabilité nationale (SCN) et que la mondialisation de la R-D rend nécessaire de disposer de statistiques de meilleure qualité sur le phénomène, des indications sont fournies sur les données à recueillir pour répondre au besoin d'information qui en découle. Il est également question de l'usage à faire des indicateurs statistiques, à l'instar du ratio DIRD/PIB, et de l'estimation des dépenses d'investissement en R-D conformément au SCN. Il convient par ailleurs de pouvoir analyser les données au niveau de chaque unité statistique afin de mieux appréhender les forces en jeu dans l'exécution et le financement de la R-D. 


\subsection{Introduction}

\section{Pourquoi mesurer les dépenses de R-D ?}

4.1 Le volume des fonds consacrés à la recherche et au développement expérimental (dépenses de R-D) intéresse fortement les responsables de l'élaboration des politiques nationales et internationales. Ces chiffres servent notamment à déterminer qui exécute et finance les activités de R-D, où, à quel niveau et à quelles fins celles-ci ont lieu, mais aussi les liens d'interdépendance et de collaboration existant entre unités institutionnelles et secteurs. Les statistiques relatives aux dépenses servent de base à l'élaboration de mesures fiscales et financières visant à stimuler les activités de R-D et à mieux comprendre l'incidence de la R-D sur la croissance économique, la défense et le bien-être sociétal.

4.2 Comme indiqué au chapitre 1, une grande nouveauté du Système de comptabilité nationale (SCN) de 2008 réside dans le fait que la R-D y est explicitement reconnue comme relevant de la formation de capital, autrement dit comme un " investissement " (CE et al., 2009). Du fait de ce changement, il est devenu nécessaire d'affiner la décomposition des dépenses de R-D, comme signalé tout au long du présent chapitre, lequel fournit en outre des orientations sur la façon de procéder pour recueillir des données détaillées sur les sources et les flux de financement de la R-D, ainsi que sur les types d'opérations liées à la R-D. Il importe, en particulier, de disposer d'informations complémentaires plus approfondies pour quantifier les ventes et acquisitions de produits de R-D.

4.3 Bien que ce chapitre mette l'accent sur les conditions requises pour produire des statistiques à différents niveaux d'agrégation qui puissent être comparées à l'échelle internationale, il importe tout autant de s'assurer que les données relatives aux différentes unités statistiques peuvent se prêter à une analyse fine. Les lignes directrices fournies dans ce chapitre visent donc à répondre à une multiplicité de besoins et de nuances sur le plan statistique.

\section{Présentation générale de la collecte et de la compilation des données}

\section{Concepts de base}

4.4 Il peut être utile de présenter tout d'abord les concepts de base sur lesquels repose l'organisation du recueil des statistiques de R-D. Aux fins du présent manuel :

- les dépenses de R-D intra-muros correspondent à la somme des fonds consacrés aux activités de R-D exécutées au sein d'une unité déclarante ; tandis 
que les dépenses de R-D extra-muros, autrement dit les ressources financières consacrées à son financement, correspondent à la somme des fonds consacrés aux activités de R-D exécutées à l'extérieur de l'unité déclarante

- les financements internes de R-D correspondent à la somme des fonds consacrés à la R-D qui proviennent directement des ressources internes d'une unité déclarante; tandis que les financements externes de R-D correspondent à la somme des fonds consacrés à la R-D qui proviennent de sources extérieures à l'unité déclarante

- les fonds d'échange pour la R-D correspondent aux fonds qu'une unité statistique fournit à une autre unité en échange de l'exécution d'activités de R-D ; tandis que les fonds de transfert pour la R-D correspondent aux moyens financiers circulant entre unités statistiques sans qu'une contrepartie soit exigée en termes de résultats de R-D.

4.5 Ces concepts, leurs relations et les difficultés de mesure sont définis et examinés plus en détail tout au long du présent chapitre.

\section{Méthode de collecte de base}

4.6 Les dépenses d'une unité statistique peuvent être consacrées à l'exécution ou au financement de travaux de R-D. Une unité peut financer des activités de R-D sans en mener elle-même (comme c'est parfois le cas pour les entreprises qui ont occasionnellement besoin d'acheter de la R-D). Elle peut aussi exécuter des travaux de R-D mais ne pas en financer (un cas de figure pas très courant, que l'on retrouve pourtant lorsqu'une petite entreprise voit sa R-D financée intégralement par une subvention publique). Enfin, une unité statistique peut à la fois mener et financer des travaux de R-D. Le financement peut concerner des activités de R-D ayant lieu au sein de l'unité déclarante (R-D intra-muros) ou en dehors (R-D extra-muros). La méthode de mesure des dépenses comprend les étapes suivantes :

- Déterminer les sommes affectées à la R-D intra-muros exécutée au sein de chaque unité statistique (voir section 4.2).

- Déterminer l'origine des fonds utilisés pour ces dépenses de R-D intra-muros déclarées par l'exécutant (voir section 4.3).

- Déterminer les financements affectés à la R-D extra-muros par chaque unité statistique (voir section 4.3). Qu'elle exécute ou non des travaux de R-D, une unité peut financer la R-D menée par d'autres unités.

- Déterminer le montant des flux de financement existant entre les unités statistiques avec et sans contrepartie en termes de résultats de R-D de la part de l'exécutant (voir section 4.3).

- Agréger les données, par secteur d'exécution et source de financement, afin d'obtenir des totaux pour l'ensemble de l'économie. D'autres classifications et répartitions sont alors élaborées dans ce cadre (voir sections 4.3 et 4.5). 


\section{Les dépenses intérieures brutes de R-D (DIRD), principal indicateur de la R-D à l'échelle d'un pays}

4.7 Les DIRD correspondent au total des dépenses intra-muros de la R-D exécutée sur le territoire national pendant une période de référence donnée.

4.8 Il s'agit du principal agrégat statistique utilisé pour décrire les activités de R-D d'un pays ; il intègre l'ensemble des dépenses engagées pour la R-D exécutée au sein de l'économie. Les DIRD incluent par conséquent la R-D exécutée sur le territoire national mais financée par des fonds étrangers (autrement dit, le "reste du monde »), mais exclut les financements consacrés à la R-D exécutée à l'étranger. Voir au chapitre 3 la section 3.3 consacrée au concept de "résidence " et, dans le présent chapitre, la section 4.2 relative aux conventions de comptabilisation de la R-D intra-muros exécutée hors du territoire national. Les DIRD représentent le premier indicateur de comparaison internationale des activités de R-D.

4.9 Les unités statistiques d'un pays donné peuvent réaliser des travaux de R-D dans chacun des grands secteurs étudiés dans le présent manuel, à savoir : le secteur des entreprises, le secteur de l'État, le secteur de l'enseignement supérieur et le secteur privé sans but lucratif. Les définitions générales de ces secteurs sont fournies dans le chapitre 3, tandis que les définitions et caractéristiques de chacun d'eux font l'objet respectivement des chapitres 7 , 8, 9 et 10. Le total des dépenses de R-D intra-muros devrait être établi pour chacun de ces secteurs. Les DIRD correspondent à la somme des totaux de ces différents secteurs. Il convient par ailleurs, pour chaque secteur exécutant de la R-D (entreprises, État, enseignement supérieur, secteur privé sans but lucratif et reste du monde), de déterminer ses sources de financement. Les définitions et les caractéristiques du reste du monde sont abordées dans le chapitre 11. Dans la mesure du possible, et pour réduire le risque d'une double comptabilisation, le calcul des DIRD devrait s'appuyer sur les déclarations des exécutants, et non sur les informations provenant des sources de financement de la R-D. L'exécutant est le mieux placé pour indiquer :

- comment les fonds ont été effectivement utilisés (par exemple : l'affectation de certaines dépenses à une activité relevant ou non de la R-D, la nature de la $R-D$, les éléments de coût qui composent la $R-D$, etc.)

- l'année où les travaux de R-D ont effectivement eu lieu

- la source de financement immédiate utilisée pour la R-D.

\subsection{Dépenses de R-D intra-muros (exécution de travaux de R-D) Définition}

4.10 Les dépenses de R-D intra-muros couvrent l'ensemble des dépenses courantes et des dépenses brutes de capital fixe afférentes à la R-D exécutée au sein d'une unité statistique au cours d'une période de référence donnée, quelle que soit la source des financements. 
4.11 Ces dépenses correspondent à la R-D exécutée au sein d'une unité statistique. Le total des dépenses de R-D intra-muros des unités qui composent un secteur de l'économie correspond à la R-D exécutée au sein dudit secteur ; en revanche, la somme des dépenses de R-D intra-muros de la totalité des secteurs correspond à la R-D exécutée au sein de l'économie dans son ensemble (DIRD).

4.12 Les ressources financières - autrement dit, les dépenses - affectées à la R-D extra-muros (c'est-à-dire la R-D exécutée en dehors de l'unité statistique) ne sont pas incluses dans le total des travaux de R-D intra-muros. Ce financement de la R-D exécutée à l'extérieur devrait être comptabilisé séparément, afin que l'on puisse obtenir des informations exhaustives sur l'accès de chaque unité à la R-D. Dans la mesure où ces fonds vont également être comptabilisés dans la R-D intra-muros des unités récipiendaires, les dépenses de R-D extra-muros doivent être exclues afin d'éviter la double comptabilisation. Il n'est pas toujours facile de faire la distinction entre les dépenses associées à la R-D intra-muros et celles liées à la R-D extra-muros ; plusieurs exemples sont proposés dans le présent chapitre pour aider à la classification.

4.13 Les dépenses correspondant à des achats qui, ne relevant pas de la $\mathrm{R}-\mathrm{D}$, sont effectués en dehors de l'unité statistique ou du secteur mais à l'appui de la R-D intra-muros (par exemple : achat de fournitures ou de services généraux pour les travaux de R-D) sont incluses dans le total de la R-D intra-muros.

4.14 Les dépenses courantes et les dépenses en capital sont toutes deux comptabilisées dans le total de la R-D intra-muros, bien que dans des rubriques distinctes.

\section{Dépenses courantes de R-D}

4.15 Les dépenses courantes comprennent les coûts de main-d'œuvre et autres dépenses courantes imputables à la R-D. Les services et articles (équipements inclus) utilisés et consommés au cours d'une année en font partie ainsi que les redevances ou loyers annuels versés pour l'utilisation d'actifs fixes.

\section{Coûts de main-d'œuvre liés au personnel de R-D}

4.16 Les coûts de main-d'œuvre comprennent les rémunérations du personnel employé à la R-D (appelé « personnel interne de R-D » dans le présent manuel), comme les salaires et traitements annuels ainsi que l'ensemble des coûts correspondant aux avantages annexes, tels que primes, options sur titres et congés payés, ainsi que les cotisations aux fonds de pension et autres charges et cotisations sociales. Il est important de n'inclure les coûts de main-d'œuvre que si les personnes employées contribuent directement à la R-D intra-muros, en particulier lorsque ces personnes ne travaillent pas à temps plein dans un service de R-D. À titre d'exemple, le simple fait qu'une personne soit employée dans un service de R-D ne veut pas dire que tous les coûts de main-d'œuvre qui lui sont associés correspondent à des activités de R-D. Pour en savoir plus, voir chapitre 5 . 
4.17 Les coûts de main-d'œuvre représentent généralement le plus gros poste des dépenses courantes de R-D. Les pays peuvent juger utile de recueillir des données sur ces coûts ou de les évaluer en faisant la distinction entre les différentes catégories de personnel de R-D (chercheurs, techniciens et personnel assimilé, autres catégories de personnel de soutien, par exemple). Ces ventilations complémentaires peuvent être utiles pour établir des indices de coûts pour les dépenses de R-D.

4.18 Les coûts de main-d'œuvre associés aux personnes qui, employées par une unité statistique mais ne faisant pas partie du personnel de R-D, fournissent des services auxiliaires (sécurité, nettoyage, entretien et restauration ; personnel des services informatiques centraux et des bibliothèques ; services centraux des finances et du personnel contribuant directement aux efforts de R-D), devraient être comptabilisés dans les autres dépenses courantes.

4.19 Les coûts associés aux personnes qui ne sont pas employées par l'unité statistique mais fournissent des services directs faisant partie intégrante des projets ou activités de R-D de cette unité ne devraient pas être inclus dans les coûts de main-d'œuvre, mais dans les autres dépenses courantes (voir définition plus avant). Dans certains cas, la délimitation entre le personnel interne de R-D de l'unité statistique (coûts de main-d'œuvre) et les travailleurs indépendants participant aux travaux de R-D de cette même unité (autres dépenses courantes) peut être floue. De manière générale, les travailleurs indépendants ne touchent ni salaires, ni traitements, mais sont rétribués par l'unité statistique pour l'ensemble des services figurant dans leur contrat. Pour en savoir plus sur le personnel de R-D, voir chapitre 5 .

4.20 S'agissant des étudiants en doctorat ou en master, le calcul de leurs salaires et de leurs traitements peut parfois poser problème. Comme l'indique le chapitre 5, seuls les étudiants qui sont employés par l'unité statistique et participent aux projets ou activités de R-D de cette unité (par exemple, en qualité de chercheurs ou assistants de recherche) devraient être inclus dans le total des coûts. La rémunération de ces étudiants est parfois inférieure au " prix du marché ». Cela dit, seuls les salaires et les coûts de main-d'œuvre réellement associés à ces étudiants devraient être pris en compte dans les statistiques de R-D. Aucune valeur gonflée ne devrait être utilisée.

4.21 Les coûts de main-d'œuvre comprennent les cotisations - imputées ou effectives - aux fonds de pension et autres charges sociales afférentes au personnel de R-D. Ces coûts n'apparaissent pas forcément dans la comptabilité de l'unité statistique et peuvent souvent impliquer des opérations au sein des secteurs ou entre eux. Même lorsqu'aucune opération n'a eu lieu, il convient d'essayer d'évaluer ces coûts. Pour éviter une double comptabilisation, les pensions versées au personnel de R-D à la retraite n'entrent pas dans les coûts de main-d'œuvre.

4.22 Les coûts de main-d'œuvre du personnel de R-D devraient inclure les cotisations sociales et autres charges payées par l'employeur, déduction faite 
des subventions/réductions applicables au personnel en question. Cela dit, pour encourager le recrutement de personnel de R-D, certains pays mettent en place des incitations automatiques, qui se traduisent par l'instauration de divers instruments ayant trait aux cotisations sociales. Pour être sûr que le choix de l'instrument en question n'a pas d'incidence sur les dépenses de R-D qui sont déclarées, il est recommandé de ne pas prendre en compte ces incitations dans l'estimation des coûts de main-d'œuvre.

$\rightarrow$ Exemple : le pays A met en place une incitation fiscale au recrutement, à savoir une réduction des cotisations sociales (un chercheur coûte 100 unités monétaires, charges sociales comprises, moins une réduction de 10 unités correspondant à une exonération sur les cotisations sociales) ; le pays B instaure une mesure incitative équivalente, à savoir une exonération de 10 unités monétaires perçue séparément au titre du régime fiscal des sociétés. Sans la recommandation précitée, la mesure de la R-D lors de la première année d'embauche du chercheur donnerait à tort un résultat plus faible pour le pays A (90) que pour le pays B (100). Or, dans les deux cas, les coûts de main-d'œuvre comptabilisés devraient être de 100.

\section{Autres dépenses courantes de R-D}

4.23 Les autres dépenses courantes incluent les frais d'achat de matériaux, fournitures, équipements et services engagés à l'appui des activités de R-D menées par l'unité statistique au cours de l'année de référence qui n'entrent pas dans les dépenses en capital. Entre autres exemples figurent les dépenses d'eau et de combustible (gaz et électricité inclus) ; les achats d'ouvrages, de revues et de documents de référence, les abonnements de bibliothèques, les cotisations aux sociétés scientifiques, etc. ; les coûts imputés ou réels des prototypes ou modèles de petites dimensions fabriqués à l'extérieur de l'unité statistique ; et les frais d'équipement des laboratoires (par exemple, produits chimiques, animaux, etc.). Entrent également dans les dépenses courantes les redevances et licences d'exploitation des brevets et autres droits de propriété intellectuelle, la location des biens d'équipement (machines et outillage, etc.) et des bâtiments utilisés dans l'exécution de la R-D par l'unité statistique au cours de l'année de référence.

4.24 Il peut, dans certains cas, être difficile de faire la distinction entre l'achat de R-D (c'est-à-dire des travaux de R-D extra-muros à ne pas comptabiliser dans les dépenses de R-D intra-muros) et l'achat de services à l'appui de la R-D intra-muros menée dans l'unité statistique. Pour en savoir plus, voir plus avant la sous-section intitulée "Non-comptabilisation des achats de R-D ».

4.25 La rubrique des autres dépenses courantes de R-D englobe les coûts des logiciels servant à l'exécution d'activités de R-D pendant un an ou moins. Il s'agit notamment des licences ou logiciels acquis et séparément identifiables, y compris les descriptifs de programme et la documentation accompagnant les logiciels d'exploitation et d'application. Les coûts de production (par exemple, 
main-d'œuvre et matériaux) des logiciels mis au point en interne devraient être déclarés. Des logiciels peuvent être acquis auprès de vendeurs extérieurs à travers l'achat ferme des droits ou des licences d'exploitation. Les logiciels exploités ou acquis sous licence pour plus d'une année devraient être déclarés au titre des dépenses en capital (voir plus avant la sous-section intitulée « Logiciels immobilisés").

4.26 Les coûts associés aux personnes qui ne sont pas employées par l'unité statistique mais qui fournissent des services directs faisant partie intégrante des activités de R-D de cette unité devraient être inclus dans les autres dépenses courantes. Ces personnes sont notamment des consultants et des chercheurs intervenant sur site et provenant d'organisations, d'établissements de recherche, d'entreprises et autres entités extérieures à l'unité statistique, ainsi que des travailleurs indépendants. On trouve également des techniciens et du personnel assimilé employés et recrutés par des unités statistiques extérieures et contribuant directement aux travaux de R-D intra-muros de l'unité statistique. Par convention, ces personnes sont désignées dans le présent manuel sous l'appellation de " personnel externe de R-D »-c'est-à-dire des personnes qui ne perçoivent ni traitements, ni salaires de la part de l'unité statistique exécutant des travaux de R-D, par opposition au personnel interne de l'unité statistique qui, lui, perçoit des salaires et des traitements. Ces coûts devraient être comptabilisés séparément comme « Autres dépenses courantes - personnel externe de R-D ». Il peut, dans certains cas, être difficile de faire la distinction entre l'achat de R-D (c'est-à-dire la R-D extra-muros) et l'achat de services de conseil à l'appui de la R-D intra-muros menée dans l'unité statistique (imputé aux autres dépenses courantes). Pour une aide à la classification, voir plus avant la sous-section intitulée « Distinction entre les coûts de main-d'œuvre liés à la R-D intra-muros et extra-muros ".

4.27 La distinction entre l'achat de services fournis par du personnel externe à l'appui de la R-D intra-muros menée dans l'unité statistique (sousrubrique " Autres dépenses courantes - personnel externe de R-D ") et l'achat de services généraux à l'appui de la R-D intra-muros menée dans l'unité statistique (imputé aux autres dépenses courantes, mais pas dans la sousrubrique du personnel externe) peut parfois être difficile à établir. L'imputation de ces dépenses courantes dépendra principalement du degré de détail des informations figurant dans les comptes financiers de la R-D intra-muros de l'unité statistique ; quoi qu'il en soit, la comptabilisation des dépenses de R-D devrait être cohérente avec celle du personnel de R-D. Se reporter à la section 5.2 du chapitre 5 pour le traitement des coûts selon les catégories de personnel de R-D.

4.28 Comme indiqué dans le chapitre 5 , les coûts associés aux étudiants en master et en doctorat qui participent aux projets ou aux activités de R-D de l'unité statistique mais ne sont pas employés par cette dernière devraient être inclus dans la sous-rubrique " Autres dépenses courantes - personnel externe de R-D ». 
Ces coûts comprennent les subventions de recherche ou les bourses d'étude qui sont gérées par l'unité statistique ou avec son concours.

4.29 Les frais administratifs et autres frais généraux (tels que les frais de bureau, d'information et de télécommunications, des services collectifs et d'assurance) devraient aussi être inclus dans les autres dépenses courantes, au prorata si nécessaire, afin d'exclure les activités ne relevant pas de la R-D qui sont menées au sein de la même unité statistique. Les coûts - au prorata - des services indirects ou auxiliaires devraient aussi être inclus dans cette rubrique, que ces services aient été assurés au sein de l'unité statistique ou loués/achetés à l'extérieur, comme par exemple la sécurité, l'entreposage, l'utilisation, le nettoyage, la réparation et l'entretien de bâtiments ou d'équipements, les services informatiques et l'impression de rapports de R-D. Les coûts au prorata $\mathrm{du}$ personnel des services informatiques centraux et des bibliothèques, ainsi que des services centraux des finances et du personnel devraient également être inclus. Le service des intérêts ne devrait pas être pris en compte.

4.30 Les unités statistiques qui effectuent des travaux de R-D intra-muros et financent de la R-D extra-muros peuvent comptabiliser les frais administratifs liés à la préparation et au suivi des contrats de la R-D extra-muros au titre des autres dépenses courantes, et non des coûts de main-d'œuvre. La levée, la gestion et la distribution de fonds assurées par les ministères, les organismes de recherche, les fondations ou organisations caritatives au profit des exécutants de la R-D ne constituent pas des activités de R-D.

4.31 Pour la mesure de la R-D dans le cadre du SCN, les dépenses correspondant aux achats d'équipements devraient être dissociées de celles correspondant aux achats de services. Il convient cependant de veiller à la qualité des données et à ce que la charge de travail ne soit pas trop lourde pour les répondants.

\section{Imputation des coûts de la R-D au sein d'un groupe d'entreprises}

4.32 Conformément aux normes de comptabilité internationales, les entreprises faisant partie d'un groupe (en particulier les multinationales) versent aux autres membres du groupe (en particulier les sociétés-mères installées à l'étranger) des sommes importantes pour financer les travaux de R-D menés au sein du groupe, mais sans recevoir en échange des prestations de R-D. Ces " transferts " ne devraient pas être pris en compte dans le total des dépenses de R-D intra-muros de l'entreprise payante, mais être comptabilisés au titre du financement de la R-D extra-muros. S'agissant de l'entreprise qui effectue les travaux de R-D et reçoit les versements des autres membres du groupe (par exemple, la société-mère installée à l'étranger), si elle perçoit des fonds sans avoir à fournir en retour des prestations de R-D, ces sommes font partie de ses dépenses de R-D intra-muros et devraient donc être intégrées aux sources de financement externe. Or, on sait que pour des raisons pratiques, l'unité bénéficiaire des fonds ne les considère pas forcément, sur le plan comptable, 
comme des sources externes de financement de la R-D intra-muros, mais comme des sources internes (comme si elle utilisait des bénéfices réinvestis pour financer sa R-D). Voir le chapitre 11 consacré à la mondialisation de la R-D.

\section{Dépenses courantes couvertes par un financement indirect}

4.33 Les activités de R-D peuvent engendrer des coûts qui, souvent, ne sont pas couverts par le secteur lui-même mais par des unités institutionnelles appartenant à d'autres secteurs de l'économie, en général le secteur de l'État. Deux exemples de ce type de coûts sont décrits ci-après.

$\rightarrow$ Exemple $n^{\circ} 1$. Loyers des installations de recherche

4.34 Dans bon nombre de pays, la responsabilité de "l'hébergement » des organismes publics (y compris les universités) incombe à un organisme central qui n'effectue pas forcément des travaux de R-D et ne sera donc peutêtre pas soumis à une enquête. Toutefois, dans l'éventualité où il ferait l'objet d'une enquête en tant qu'unité institutionnelle du secteur de l'État, il se peut que sa comptabilité ne reflète pas la répartition fonctionnelle entre la R-D et d'autres activités. Cette remarque s'applique tout particulièrement au secteur de l'enseignement supérieur.

4.35 Dans certains cas, les installations sont mises gratuitement à la disposition des organismes et ne sont pas comptabilisées dans leurs registres comptables. Dans d'autres, les organismes versent un loyer au propriétaire des installations. Si l'on cherche à évaluer le coût véritable de la R-D, la totalité des redevances et des loyers qui relèvent de la R-D devraient en principe être inclus dans les données relatives aux dépenses. Lorsque la redevance ou le loyer est imputé à une unité, l'opération est facile. En revanche, en l'absence d'une telle imputation, il peut être souhaitable de calculer un montant représentant le coût des installations pour l'utilisateur ou leur "valeur marchande ». Ce montant peut être inclus dans les autres dépenses courantes. Il convient de veiller à éviter la double comptabilisation des coûts, à savoir du côté des prestataires et des bénéficiaires de ces services.

$\rightarrow$ Exemple $\mathrm{n}^{\circ}$ 2. Fonctionnement et entretien des installations de recherche

4.36 Il arrive que l'État soit le propriétaire d'installations spéciales réservées à des activités de R-D, et qu'il en assure l'entretien. Ces installations sont utilisées à la fois par des exécutants du secteur public (y compris par l'organisme qui en est propriétaire et par d'autres organismes publics) et du secteur privé (généralement des entreprises) dans le cadre de projets de R-D agréés. Lorsqu'elles sont utilisées par d'autres exécutants de travaux de R-D (publics ou privés), la redevance d'utilisation correspondante - qui peut inclure les frais de fonctionnement et d'entretien - qui est payée au propriétaire des installations est comptabilisée dans les dépenses courantes de l'exécutant des travaux de R-D. Afin d'éviter une double comptabilisation, ces frais de fonctionnement et d'entretien inclus dans la redevance d'utilisation ne devraient pas être déclarés par l'organisme public propriétaire des installations. 
4.37 En revanche, si les installations ne sont utilisées que quelques fois par an, leur propriétaire risque d'avoir à assumer des frais de fonctionnement et d'entretien en continu pour maintenir les installations de R-D en état. S'il n'effectue pas ces dépenses, les installations risquent de ne pas être utilisables pour un projet de R-D, que ce soit par le propriétaire ou par qui que ce soit d'autre. Si ces coûts récurrents ne sont pas répercutés sur la redevance d'utilisation (ou sur toute autre charge indirecte) payée par les exécutants publics et privés, ils pourront être comptabilisés par l'administration publique qui détient les installations au titre des dépenses intra-muros, dans la rubrique des autres dépenses courantes.

\section{Coûts d'amortissement des biens corporels et incorporels (non pris en compte dans le total des dépenses de R-D intra-muros)}

4.38 L'amortissement des biens corporels utilisés dans la R-D ne devrait pas être pris en compte dans le total des dépenses de R-D intra-muros ; il en va de même pour l'amortissement des biens incorporels. Cela dit, il est fréquent que les coûts d'amortissement des actifs fixes utilisés pour la R-D soient intégrés sur le plan comptable à la R-D intra-muros de l'unité déclarante, en général dans la rubrique des autres dépenses courantes.

4.39 Afin d'éviter les erreurs de déclaration dans les enquêtes sur la R-D, il est recommandé que les informations relatives aux coûts d'amortissement soient recueillies séparément par rapport aux coûts de la $R-D$, ou tout au moins d'indiquer clairement que ces coûts ne devraient pas être intégrés au total des dépenses de R-D intra-muros.

\section{Principe de l'estimation des dépenses de R-D : le prix d'acquisition}

4.40 La collecte et la comptabilisation des dépenses de R-D devraient se faire sur la base des prix d'acquisition, qui correspondent aux montants payés par les acquéreurs, à l'exclusion de la partie déductible de la taxe sur la valeur ajoutée (TVA) et des impôts similaires. Les prix d'acquisition représentent les coûts réellement supportés par les utilisateurs. Par conséquent, le montant estimé des dépenses consacrées aux biens et services de R-D, qu'il s'agisse de dépenses courantes ou de dépenses en capital, est le prix total réglé par l'unité déclarante. Il tient compte, le cas échéant, des taxes et subventions dont les produits acquis font l'objet et qui ont pour effet, respectivement, d'en majorer ou réduire le prix.

$\rightarrow$ Exemple : une entreprise achète pour 100 unités monétaires d'équipements utilisables pour la R-D, 60 pour l'équipement $A$ taxé à $10 \%$, et 40 pour l'équipement $\mathrm{B}$ subventionné à $4 \%$. À cela s'ajoute une TVA entièrement déductible de $15 \%$. Le coût de la R-D est donc égal à $100+\left(60^{*} 10 \%\right)-\left(40^{*} 4 \%\right)$, soit $(100+6-1.6)=104.4$. Les $15 \%$ de la TVA déductible ne sont pas pris en compte dans la mesure des coûts de la R-D. 


\section{Taxe sur la valeur ajoutée déductible (non prise en compte dans le total des dépenses de $R-D$ intra-muros)}

4.41 Les offices statistiques devraient veiller à ce que les taxes déductibles appliquées aux produits (comme certaines formes de TVA) ne soient pas prises en compte dans la déclaration des coûts. Une entreprise privée peut récupérer la TVA déductible payée sur ses achats en la déduisant du montant de la TVA due (à l'État), c'est-à-dire de la TVA facturée à ses clients. Conformément au SCN, et à des fins de comparaison internationale, il convient d'appliquer le système net d'enregistrement de la TVA. Dans ce système, la TVA est enregistrée comme devant être payée par les acheteurs et non par les vendeurs, et seulement par les acheteurs qui n'ont pas la possibilité de la déduire.

4.42 S'agissant du secteur des entreprises, conformément aux procédures comptables types, la TVA appliquée aux intrants est enregistrée séparément et est récupérable si elle vient en déduction de la TVA facturée sur les résultats. Cette règle devrait également être appliquée aux entreprises dont le chiffre d'affaires est temporairement insuffisant pour récupérer la TVA payée. Les unités statistiques devraient être informées de ces consignes à suivre lors de leur déclaration des dépenses de R-D. En ce qui concerne le secteur de l'État, la TVA appliquée aux intrants est souvent déductible et est donc enregistrée séparément.

4.43 Les choses risquent d'être plus difficiles dans le secteur de l'enseignement supérieur et le secteur privé sans but lucratif, où la TVA comprise dans le prix des biens et services achetés dans le cadre d'un projet de R-D n'est pas toujours récupérable et sera donc considérée par les répondants comme une composante légitime de leurs dépenses. Les pays devraient faire en sorte, pour ces secteurs, que la TVA déductible ne soit pas incluse dans leurs dépenses. Il est recommandé d'exclure la TVA déductible des chiffres communiqués aux fins de l'établissement de comparaisons internationales.

\section{Dépenses en capital consacrées à la R-D}

4.44 Les dépenses en capital consacrées à la R-D correspondent à la somme annuelle brute versée pour l'acquisition d'actifs fixes qui sont utilisés de façon répétée ou continue aux fins de l'exécution de travaux de R-D pendant plus d'une année. Ces dépenses devraient être déclarées dans leur intégralité au titre de la période au cours de laquelle elles ont eu lieu, qu'il s'agisse d'actifs acquis ou mis au point en interne, et ne devraient pas être enregistrées comme élément d'amortissement.

4.45 Ces dépenses, qui comprennent les achats et autres frais associés à l'acquisition ou à la formation d'actifs fixes, sont appelées "dépenses en capital ». En règle générale, les actifs fixes incluent à la fois des actifs matériels - ou corporels - (tels que bâtiments et structures, matériels de transport, machines et équipements divers, etc.) et des actifs immatériels (comme des logiciels ou des droits de prospection minière). 
4.46 La mesure des dépenses en capital consacrées à la R-D tient davantage compte des opérations de transfert de capital utilisé pour la R-D - pour lesquelles un traçage est possible - que du coût économique lié à la possession et l'utilisation d'actifs dans le cadre de la R-D. Si le coût de l'utilisation d'actifs appartenant à des tiers dans le cadre de la R-D intra-muros doit être comptabilisé au titre des " autres dépenses courantes ", en revanche, les dépenses en capital consacrées à la R-D doivent être enregistrées séparément au coup par coup. Afin d'éviter la double comptabilisation des dépenses en capital, toutes les dotations aux amortissements d'actifs immatériels (bâtiments, usines et équipements), qu'elles soient réelles ou imputées, doivent être exclues de la mesure des dépenses de R-D intra-muros (voir plus haut la sous-section intitulée " Coûts d'amortissement des biens corporels et incorporels ").

\section{Types d'actifs fixes utilisés pour la R-D}

4.47 Les types d'actifs exploités en R-D pour lesquels il conviendrait en premier lieu de collecter des données sur les dépenses en capital consacrées à la R-D sont les suivants :

- terrains et constructions

- machines et équipements

- logiciels immobilisés

- autres produits de la propriété intellectuelle.

\section{Terrains et constructions}

4.48 Cette catégorie comprend les terrains acquis à des fins de R-D (par exemple, réalisation d'essais, aménagement de laboratoires et d'usines pilotes) ainsi que les bâtiments construits ou acquis à des fins de $R-D$, grands travaux d'aménagement, de modification et de réparation inclus. Comme les constructions sont des actifs produits et les terrains des actifs non produits au regard du Système de comptabilité nationale, il convient de distinguer les dépenses de R-D selon qu'elles concernent les premiers ou les seconds.

4.49 Lorsqu'un nouveau bâtiment est acquis ou construit pour un usage mixte, la part des dépenses en capital consacrée à la R-D est souvent difficile à déterminer, raison pour laquelle elle est souvent laissée de côté dans les compilations statistiques des dépenses de R-D. Voir plus bas la sous-section intitulée « Détermination de la part des dépenses en capital consacrée à la R-D » pour savoir comment procéder pour évaluer le pourcentage d'utilisation des dépenses en capital à des fins de R-D.

4.50 Il arrive souvent que l'achat de nouveaux équipements de recherche soit inclus dans le coût des nouveaux bâtiments, et qu'il n'apparaisse pas séparément dans les registres comptables de l'unité déclarante. Cela peut entraîner une sous-estimation de la rubrique des " machines et équipements » dans le total des dépenses en capital consacrées à la R-D. Les unités déclarantes 
devraient être explicitement encouragées à recenser et comptabiliser séparément ces achats d'équipements dans la catégorie des actifs fixes qui convient.

\section{Machines et équipements}

4.51 Cette catégorie comprend les machines et équipements immobilisés qui ont été acquis pour l'exécution d'activités de R-D. Aux fins du Système de comptabilité nationale, les dépenses afférentes devraient être ventilées de façon plus fine, et inclure notamment les postes " matériel d'information et de communication " et " matériels de transport ». Il convient cependant de veiller à la qualité des données et à ce que la charge de travail ne soit pas trop lourde pour les répondants.

\section{Logiciels immobilisés}

4.52 Cette catégorie comprend les logiciels servant à l'exécution d'activités de R-D pendant plus d'une année. Il s'agit notamment des licences ou logiciels acquis pour une longue durée et séparément identifiables, y compris les descriptifs de programme et la documentation accompagnant les logiciels d'exploitation et d'application. Les coûts de production (par exemple, main-d'œuvre et matériaux) des logiciels mis au point en interne devraient être déclarés. Des logiciels peuvent être acquis auprès de vendeurs extérieurs à travers l'achat ferme des droits ou des licences d'exploitation. Les logiciels exploités ou acquis sous licence pour un an ou moins devraient être déclarés au titre des dépenses courantes (voir plus haut la sous-section intitulée « Autres dépenses courantes de R-D »).

\section{Autres produits de la propriété intellectuelle}

4.53 Cette catégorie comprend les brevets achetés, les licences d'exploitation à long terme et autres actifs incorporels destinés à la R-D et utilisés pendant plus d'une année. N'en font pas partie les éléments incorporels susceptibles d'être déclarés dans les comptes financiers internes d'une unité, tels que les fonds de commerce et autres actifs commerciaux (voir la sous-section intitulée «Comparaison avec les dépenses en capital déclarées dans le SCN »).

\section{Conventions concernant la distinction entre les dépenses courantes et les dépenses en capital}

4.54 L'évaluation des dépenses en capital réellement effectuées ne comprend généralement ni le petit matériel, l'outillage ou les réparations/ améliorations mineures apportées aux bâtiments existants puisque, dans la plupart des systèmes comptables, ces dépenses sont généralement affectées au poste des dépenses courantes. La délimitation entre les dépenses " mineures » et les dépenses "importantes " varie légèrement d'un pays à l'autre en fonction des pratiques fiscales, voire d'une entreprise ou d'un organisme à l'autre au sein d'un même pays en fonction des pratiques comptables. Ces différences étant rarement très grandes, il n'est ni nécessaire, ni pratique de fixer une norme stricte. 
L'imputation des frais au poste des dépenses courantes ou à celui des dépenses en capital dépendra donc des pratiques en usage dans chaque pays. Cependant, dans les pays où les dépenses afférentes à la mise au point de prototypes très coûteux (d'avions, par exemple) ou de matériel à durée de vie limitée (des fusées de lancement, par exemple) sont considérées comme des dépenses courantes, il convient d'indiquer toujours clairement ces conventions.

4.55 Bien que le paiement des achats courants de livres, de périodiques et de revues annuelles doive être imputé aux autres dépenses courantes, les dépenses correspondant à l'achat de bibliothèques complètes ou de grandes collections de livres, de périodiques, de spécimens, etc., sont à inscrire dans les dépenses d'équipement lourd, en particulier lorsqu'elles sont engagées pour équiper un nouvel établissement.

\section{Détermination de la part des dépenses en capital consacrée à la $R-D$}

4.56 Il est parfois possible de savoir, dès l'achat, quelle part d'un actif fixe sera utilisée pour des travaux de R-D. Dans ce cas, seule la fraction correspondante des coûts devra être imputée aux dépenses en capital de R-D. La plupart du temps, quand on ne sait pas quelle part de l'actif fixe sera consacrée à la R-D et que cet actif est destiné à plusieurs activités sans que l'on puisse dire s'il servira davantage à la R-D ou à d'autres usages (c'est le cas, par exemple, des ordinateurs et des installations connexes, ou des laboratoires qui servent à la fois à la R-D, à des essais et au contrôle de la qualité), il convient en principe de répartir les dépenses au prorata entre la R-D et les autres activités. Le pourcentage affecté à la R-D peut être défini en fonction du nombre de personnes employées à la R-D qui utilisent l'installation par rapport à l'effectif total, ou sur la base de calculs administratifs effectués au préalable (une fraction des dépenses en capital pourra, par exemple, être imputée au budget de R-D ou une certaine proportion du temps de travail ou des locaux pourra être attribuée à la R-D).

4.57 Il peut arriver qu'une unité statistique (généralement un organisme public ou une grande entreprise) réalise de gros investissements dans des actifs fixes de grande ampleur, considérés comme " les meilleurs de leur catégorie » ou comme offrant des capacités auparavant inexistantes. Du fait de leur contribution éventuelle à des activités innovantes, les unités déclarantes peuvent être enclines à comptabiliser toutes ces dépenses de construction au titre de la R-D. Or, pour les comparaisons internationales, seules les dépenses spécifiquement identifiées comme jouant un rôle capital pour la R-D sont à inclure dans les dépenses de R-D intra-muros. En règle générale, ces dépenses ne devraient pas être comptabilisées comme des dépenses de R-D courantes, mais comme des dépenses en capital consacrées à la R-D.

\section{Vente de biens de capital utilisés pour la R-D}

4.58 La vente ou le transfert d'actifs fixes acquis à l'origine pour des activités de R-D est un cas de figure problématique. La cession de ces actifs peut être 
considérée comme un désengagement par rapport à la R-D. Or, aucune modification ne doit être apportée à l'enregistrement des dépenses en capital : les dépenses en capital consacrées à la R-D par l'unité statistique ne doivent pas être réduites en conséquence, que ce soit pour l'exercice en cours ou rétrospectivement (c'est-à-dire pour les exercices antérieurs où les dépenses en capital avaient été enregistrées). Les révisions sur l'exercice en cours peuvent générer des anomalies telles que des dépenses de R-D intra-muros négatives. Les modifications rétrospectives sont difficiles à réaliser et sources de confusion. Dans l'idéal, l'achat de biens d'équipement d'occasion par un autre exécutant de R-D installé sur le territoire national ne devrait pas être pris en compte dans la mesure des dépenses de R-D, de manière à éviter une double comptabilisation de ces biens dans le temps. Il arrive que les actifs vendus soient affectés à d'autres usages ou transférés à un exécutant de R-D à l'étranger, auquel cas la double comptabilisation n'existe pas.

4.59 Le tableau 4.1 représente les différentes catégories de dépenses décrites précédemment. Les circonstances propres à chaque pays détermineront le niveau de détail des données devant éventuellement être recueillies.

\section{Tableau 4.1. Synthèse des catégories de dépenses de R-D intra-muros}

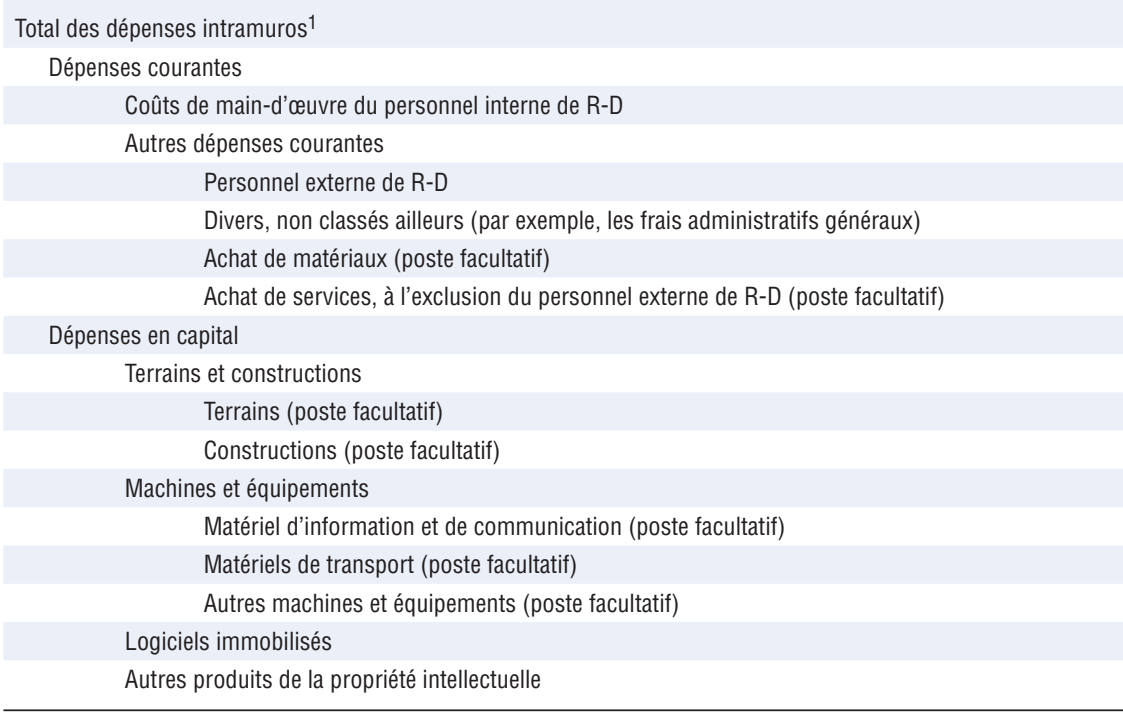

1.Les dotations aux amortissements ne doivent pas être incluses dans le total des dépenses intra-muros mais être enregistrées séparément.

\section{Axes de réflexion et problèmes concernant le calcul du total des dépenses de R-D intra-muros}

\section{Non-comptabilisation des achats de R-D}

4.60 Les coûts d'achat de R-D auprès d'autres unités ou secteurs ne devraient pas être inclus dans les dépenses de R-D intra-muros de l'unité statistique ou du secteur déclarant(e). Lorsqu'il s'agit de l'achat de services étroitement liés aux 
activités de R-D intra-muros, il peut être difficile, sur le plan conceptuel, de faire la distinction entre les coûts de la R-D intra-muros et ceux de la R-D extra-muros. Si ces services sont fournis pour des projets de R-D distincts faisant l'objet d'un contrat, en l'absence de cahier des charges détaillé fourni par le responsable du projet de l'unité qui finance le projet, les dépenses correspondantes peuvent dans la plupart des cas être affectées à la R-D extra-muros. S'il s'agit de tâches (ne relevant pas nécessairement de la R-D) qui sont indispensables pour la R-D intra-muros de l'unité mais qui ont été sous-traitées, les coûts correspondants peuvent généralement être comptabilisés dans les dépenses de R-D intra-muros (autres dépenses courantes).

4.61 Dans le cas des grandes entreprises, il est parfois très difficile de faire la distinction entre les coûts de la R-D intra-muros et ceux de la R-D extra-muros. Conformément aux normes comptables internationales, il est fréquent que les rapports financiers annuels de ces entreprises fassent état des dépenses internes consacrées à la fois à la R-D intra-muros et extra-muros, si tant est que les travaux de R-D qui ont été menés « bénéficient » à l'entreprise déclarante. Il incombe aux pays de fournir aux unités déclarantes des orientations claires afin de minimiser le risque que les dépenses de R-D intra-muros et de R-D extra-muros ne soient pas comptabilisées séparément, comme le recommande le présent manuel.

\section{Distinction entre les coûts de main-d'œuvre liés à la R-D intra-muros et extra-muros}

4.62 Lorsque le personnel externe (travailleurs indépendants ou personnes employées par d'autres unités statistiques) est totalement intégré aux activités de R-D de l'unité statistique déclarante, et que ses tâches sont gérées par l'unité en question, les coûts de main-d'œuvre correspondants devraient être inclus dans les dépenses de R-D intra-muros au titre des autres dépenses courantes, de préférence dans une sous-rubrique relative au personnel externe de R-D. La recommandation est de comptabiliser séparément le nombre de personnes physiques et l'équivalent temps plein (ETP) de ces "non-salariés » et de ne pas ajouter ces valeurs aux effectifs de l'unité exécutante (voir chapitre 5). Pour éviter une double comptabilisation, il ne faut pas enregistrer ces valeurs dans les dépenses de R-D ou les coûts de main-d'œuvre liés à la R-D de l'unité statistique exécutante où ce personnel externe est salarié.

4.63 Les coûts associés aux consultants qui fournissent des services de R-D dans le cadre d'une commande spécifique (achat) - et non dans le cadre des projets de R-D de l'unité déclarante - sont à comptabiliser par l'unité déclarante bénéficiant de ces services dans les dépenses de R-D extra-muros. En revanche, l'unité statistique qui emploie ces consultants devrait déclarer ces coûts dans les dépenses de R-D intra-muros.

4.64 Les personnes travaillant à distance depuis l'étranger pour une longue durée constituent un cas particulier. Les coûts correspondants devraient généralement être comptabilisés par l'unité déclarante installée sur le territoire 
national dans la rubrique des dépenses de R-D extra-muros (pour des travaux effectués dans le reste du monde). Du côté de l'unité installée à l'étranger, ces coûts doivent être comptabilisés dans les dépenses de R-D intra-muros. Aucune définition précise de ce qu'est un engagement de "longue durée " ne peut être fournie dans le présent manuel, car cette notion peut dépendre des arrangements administratifs et contractuels qui ont été convenus avec l'établissement hôte.

\section{Prise en compte de la R-D intra-muros exécutée en dehors du territoire national}

4.65 Les dépenses de R-D intra-muros mesurent les activités de R-D qui sont menées dans les unités statistiques résidant sur le territoire du pays déclarant. Certaines de ces dépenses peuvent cependant être liées à des activités qui ont eu lieu hors site, voire à l'étranger. Les dépenses de R-D intra-muros peuvent donc inclure :

- les coûts liés à l'utilisation d'un navire océanographique effectuant des études sur la vie aquatique

- les dépenses associées à la mise à disposition par la société-mère d'une multinationale de son personnel technique pour une mission de courte durée dans les locaux de l'une de ses filiales à l'étranger, la société-mère continuant de payer les salaires et autres coûts de main-d'œuvre

- les coûts du maintien et du fonctionnement d'une présence permanente de chercheurs dans l'Antarctique

- les dépenses liées à l'envoi d'un chercheur de l'enseignement supérieur hors du pays déclarant pour y réaliser des travaux pratiques.

4.66 Pour classer la R-D « intra-muros » exécutée hors du territoire du pays déclarant, la priorité est de s'intéresser non pas littéralement à l'emplacement géographique de l'activité en question, mais à sa structure organisationnelle. Bien qu'il soit difficile de fournir des lignes directrices précises à cet égard, la consigne de base est que la R-D intra-muros effectuée à l'étranger devrait inclure uniquement les travaux réalisés par une unité statistique pour remplir ses propres objectifs, et en y ayant investi ses propres ressources financières et affecté son propre personnel de R-D. Les travaux doivent avoir eu lieu sous la responsabilité de l'unité déclarante, et cette dernière doit répondre au critère de résidence économique décrit au chapitre 3 (Secteurs institutionnels et classifications).

\section{Comptabilisation incomplète ou erronée de la R-D intra-muros}

4.67 Toutes les activités de R-D menées au sein de l'unité statistique devraient être déclarées. Il existe toutefois de nombreuses raisons pour lesquelles cela n'est pas toujours le cas. Les plus courantes sont les suivantes :

- La R-D a bien été exécutée au sein de l'unité statistique, mais dans des services spécifiques situés en dehors (par exemple pour des essais ou la préparation de la phase initiale de production) ou lors d'activités générales de développement de technologie. 
- La R-D fait partie intégrante du contrat de développement d'un produit bien précis (par exemple, un montant relativement faible de R-D fait partie d'un très gros contrat d'achat d'avions de chasse).

- La R-D est financée par les clients (travaux sous contrat) mais n'est pas reconnue comme de la $\mathrm{R}-\mathrm{D}$ dans les comptes financiers des unités exécutantes (plutôt comme la vente de services techniques).

4.68 Ces problèmes de sous-comptabilisation peuvent être difficiles à résoudre efficacement. Une solution à laquelle ont couramment recours les offices statistiques consiste à assurer un suivi actif avec les répondants lors de la collecte et la validation des données. Cela dit, un facteur de difficulté pour les répondants comme pour les offices statistiques est que l'on ne trouve pas toujours dans les comptes financiers des unités statistiques les informations détaillées dont on a besoin sur les dépenses de R-D, ou qu'il est trop contraignant pour les répondants de consacrer les ressources nécessaires pour fournir ces informations détaillées.

4.69 Il peut par ailleurs y avoir surévaluation des dépenses de R-D, pour différentes raisons. Ainsi, il peut arriver que les unités statistiques enregistrent dans leurs comptes financiers des dépenses d'innovation qui vont au-delà de l'activité de R-D ; elles pourraient par exemple déclarer comme relevant de la $\mathrm{R}-\mathrm{D}$ des dépenses relatives aux technologies de l'information ou à la formation qui ne sont pas forcément liées à des travaux de R-D.

4.70 Pour ce qui est des groupes d'entreprises, et en particulier des multinationales, les causes de déclaration erronée des dépenses de R-D sont nombreuses. Les transferts interentreprises et les règles d'imputation des coûts propres à chaque entreprise compliquent la collecte de données précises sur les dépenses de R-D (pour en savoir plus sur la mesure des dépenses de R-D dans les multinationales, voir le chapitre 11 sur la mondialisation).

\section{Déclaration des dépenses en capital à titre de service}

4.71 La R-D est une activité de service et le résultat qu'elle produit est un service. Comme indiqué plus haut, on mesure l'activité de R-D en effectuant la somme des coûts de R-D (dépenses intra-muros) d'une unité statistique. Ces dépenses correspondent aux sommes consacrées, au cours d'une période de référence donnée, à la main-d'œuvre et à d'autres postes ne constituant pas des immobilisations, auxquelles s'ajoute le montant brut payé durant cette même période pour l'achat d'actifs fixes utilisés de manière répétée pour des travaux de R-D. Dans le cas - inhabituel - où la seule activité ayant un lien avec la R-D qui est menée par l'unité statistique au cours de la période de référence est l'achat d'un nouveau bâtiment qui sera utilisé pour des activités de R-D futures, ces immobilisations sont effectivement déclarées pour la période en cours comme une activité de service (en référence aux travaux). L'utilisation future du bâtiment à des fins de R-D ne sera pas enregistrée, lors des périodes futures, dans la R-D intra-muros. La compréhension de ces conventions de déclaration 
aidera à interpréter les valeurs totales des dépenses de R-D et à établir des comparaisons avec d'autres sources de données agrégées sur les dépenses de R-D compilées différemment, notamment la R-D effectuée au sein des multinationales et enregistrée dans la comptabilité nationale (voir le chapitre 11 sur la mondialisation).

\section{Comparaison avec les dépenses en capital déclarées dans le SCN}

4.72 Le présent manuel ne propose pas la même comptabilisation des dépenses en capital consacrées à la R-D que le Système de comptabilité nationale. Dans l'édition 2008 du SCN, la catégorie " actifs fixes incorporels " a été renommée "droits de propriété intellectuelle ", et le champ des actifs fixes a été étendu pour y inclure la R-D. La R-D est donc considérée comme un bien de capital dans le SCN, dont les mesures de la production et de l'investissement dans la R-D correspondent à des adaptations des données de la R-D intra-muros du Manuel de Frascati. À titre de comparaison, la notion de « dépenses en capital consacrées à la $\mathrm{R}-\mathrm{D}$ » employée dans le présent manuel fait référence à la somme annuelle brute versée pour l'acquisition d'actifs corporels et incorporels qui sont utilisés pour l'exécution de travaux de R-D. La notion de travaux de R-D utilisée dans le présent manuel est proche, sur le plan théorique, du concept de production brute de R-D employé dans le SCN, mais les deux mesures diffèrent - entre autres - de par le traitement qu'elles réservent au coût du capital utilisé pour la R-D (OCDE, 2009).

4.73 Alors que les dépenses en capital sont, dans le présent manuel, intégrées au total des dépenses intra-muros (et que les coûts d'amortissement en sont exclus), le SCN comptabilise séparément les dépenses en capital (un compte de capital pour les différents actifs), mais intègre les mesures du coût d'usage du capital existant. Le coût d'usage du capital existant comprend la dépréciation pour obsolescence et usure, ainsi que la mesure du coût d'opportunité du financement du bien de capital. Les offices statistiques qui mesurent les travaux de R-D (dépenses de R-D intra-muros) ne procèdent pas à de tels ajustements. Des informations complémentaires sur les liens entre ces deux cadres de comptabilisation sont accessibles en ligne en complément du présent manuel, à

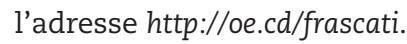

\subsection{Financement de la R-D}

4.74 La R-D et les ressources nécessaires pour la financer donnent lieu à d'importants flux entre les unités, les secteurs et les pays. Pour répondre aux besoins des responsables de l'action publique, des analystes et des comptables nationaux chargés de financer la $R-D$, tout doit être fait pour assurer le suivi des différents flux de financement et catégories de fonds consacrés à la R-D, depuis leur origine jusqu'à leur destination finale.

4.75 S'agissant des travaux de R-D, ils sont effectués par l'unité statistique avec des fonds provenant soit de sources internes à cette unité, soit de sources 
externes. Ces notions sont explicitées plus avant dans la sous-section intitulée "Sources de financement internes et externes ".

4.76 S'agissant du financement, les sommes utilisées pour la R-D permettent de financer les coûts des travaux qui sont menés soit au sein de l'unité statistique (R-D intra-muros), soit en dehors (R-D extra-muros).

4.77 Des moyens financiers peuvent circuler entre les unités statistiques, que ce soit avec ou sans contrepartie en termes de résultats de R-D de la part de l'exécutant ; on parle alors respectivement de fonds d'échange et de fonds de transfert. Ces notions sont explicitées plus avant dans la sous-section intitulée "Fonds de transfert et d'échange pour la R-D ».

4.78 Il est fréquent que ces volets distincts de l'exécution et du financement des travaux de R-D se chevauchent, et il est donc parfois difficile de distinguer les exécutants et les sources de financement de la R-D. À titre d'exemple, un laboratoire de recherche public fournit des fonds pour la R-D dans le cadre d'un contrat avec une société aérospatiale. Cette dernière peut utiliser une partie de ces fonds pour acheter de la R-D auprès d'une autre entreprise spécialisée dans les services de recherche. La société aérospatiale peut aussi utiliser une partie de ses propres ressources pour acheter de la R-D personnalisée à la société de services de recherche en vue de l'intégrer à un autre projet de R-D intra-muros. L'identification des bailleurs de fonds et des exécutants n'est pas une tâche facile. Une autre difficulté tient au fait que la R-D extra-muros de l'unité qui fournit le financement correspond à la R-D intra-muros de l'unité exécutante. Il est important, par conséquent, de définir clairement ce que recouvre chaque rubrique de la déclaration, afin que les dépenses de R-D ne soient pas comptabilisées en double ou en triple, ni au contraire qu'elles ne soient pas comptabilisées du tout.

\section{Méthodes de mesure des flux de financement de la R-D}

4.79 Les flux de financement de la R-D peuvent être mesurés de deux manières. La première méthode consiste à utiliser les données communiquées du point de vue des exécutants concernant les montants reçus par une unité statistique ou un secteur de la part d'une autre unité ou d'un autre secteur pour exécuter de la R-D intra-muros pendant une période donnée. Les fonds reçus pour des travaux de R-D qui ont été exécutés au cours d'une période antérieure ou n'ont pas encore commencé ne doivent pas être comptabilisés pour la période en cours. La seconde méthode s'appuie sur la déclaration qui est faite par le bailleur de fonds des sommes qu'une unité statistique ou un secteur indique avoir payées - ou s'être engagé(e) à payer - à une autre unité ou à un autre secteur pour l'exécution de la R-D pendant une période donnée.

4.80 Il est vivement recommandé de collecter les données sur la R-D en se plaçant du point de vue de l'exécutant. Ce dernier est en effet le mieux placé pour savoir si des fonds ont été effectivement employés pour les travaux de R-D ; il connaît en outre leur montant, les sources de financement utilisées et l'année 
où l'activité a eu lieu. Cela dit, les données communiquées par les bailleurs de fonds peuvent permettre d'en savoir plus sur les intentions des unités qui financent la R-D.

4.81 Le tableau 4.2 représente de façon synthétique l'intersection entre les unités exécutant des travaux de R-D et les sources de financement de la R-D. Les montants figurant dans la cellule (i) plus ceux de la cellule (ii) représentent le total des dépenses de R-D intra-muros et les sources de ces fonds (telles que déclarées par l'exécutant). La cellule (iii) représente les sommes versées/ transférées à d'autres unités pour des travaux de R-D extra-muros (telles que déclarées par le bailleur de fonds).

\section{Tableau 4.2. Intersection entre l'exécution et le financement des travaux de R-D}

\begin{tabular}{lll}
\hline & $\begin{array}{l}\text { Exécution de travaux de R-D au sein } \\
\text { de l'unité }\end{array}$ & $\begin{array}{l}\text { Exécution de travaux de R-D en dehors } \\
\text { de l'unité }\end{array}$ \\
\hline Fonds d'origine interne & $\begin{array}{l}\text { (i) R-D intra-muros financée avec des } \\
\text { fonds d'origine interne }\end{array}$ & $\begin{array}{l}\text { (iii) Financement de la R-D extra-muros } \\
\text { avec des fonds d'origine interne }\end{array}$ \\
Fonds d'origine externe & $\begin{array}{l}\text { (ii) R-D intra-muros financée avec des } \\
\text { fonds d'origine externe }\end{array}$ & $\begin{array}{l}\text { (iv) Financement de la R-D extra-muros } \\
\text { avec des fonds d'origine externe }\end{array}$ \\
\hline
\end{tabular}

4.82 La cellule (iv) représente les sommes que reçoit une unité (le bénéficiaire) de la part d'une deuxième unité (le bailleur de fonds), et qui sont ensuite versées/transférées (c'est-à-dire sous-traitées/sous-attribuées) à une troisième unité pour effectuer des travaux de R-D extra-muros. Afin d'éviter une double comptabilisation, la troisième unité doit déclarer les sommes (iv) comme de la R-D intra-muros financée avec des fonds d'origine externe, provenant de la deuxième unité. Le bénéficiaire initial des financements externes ne doit en principe pas comptabiliser ces sommes dans ses dépenses de R-D. Les offices statistiques nationaux peuvent avoir besoin d'inclure dans leurs enquêtes de $\mathrm{R}-\mathrm{D}$ des questions visant explicitement à repérer et éliminer ces sommes qui ne doivent pas figurer dans le total des dépenses.

\section{Présentation schématique des flux de R-D et des fonds utilisés pour son financement}

4.83 Le graphique 4.1 représente les différents flux possibles en ce qui concerne la R-D et ses moyens de financement du point de vue d'un exécutant de R-D. S'ils sont généralisés, ces flux permettent de mesurer l'activité de R-D d'une unité statistique, d'un secteur ou d'un pays. Il convient de préciser que les flux de R-D ne doivent pas être confondus avec les flux de financement de la R-D. Les fonds de transfert n'impliquent aucune contrepartie en termes de résultats de R-D.

4.84 Du point de vue de l'unité statistique qui exécute des travaux de $\mathrm{R}-\mathrm{D}$, l'enquête porte sur les champs 1 et 2 du graphique 4.1. Une fois que l'on a déterminé le volume de R-D exécutée par l'unité, la principale question qui 
est posée concerne la provenance des moyens de financement. Les sources de financement de la R-D sont de deux types : internes (champ 1 du graphique) ou externes (champ 2). Les financements externes - c'est-à-dire provenant d'entités autres que l'unité statistique - doivent être comptabilisés par le secteur qui finance la R-D (voir le tableau 4.3 sur les sources de financement par secteur institutionnel).

4.85 Du point de vue de l'unité statistique qui finance des travaux de R-D, l'enquête porte sur les champs 1 et 3 du graphique 4.1. La principale question qui est posée concerne la destination des fonds. Les bénéficiaires des fonds consacrés à la R-D sont de deux types : l'unité bailleur de fonds (champ 1 du graphique) ou les bénéficiaires externes (champ 3). Les fonds destinés à des bénéficiaires externes - c'est-à-dire servant à financer la R-D extra-muros - doivent être comptabilisés par le secteur qui exécute la R-D. Voir dans la présente section 4.3 les sous-sections "Mesure des sommes consacrées à la R-D extra-muros » et «Ventes et achats de R-D ».

4.86 L'interprétation des éléments du graphique est la suivante :

R-D exécutée par l'unité (le total des dépenses de R-D intra-muros correspond aux travaux de R-D exécutés) :

(1) = R-D exécutée par l'unité et financée par elle (avec des fonds d'origine interne).

(2) = R-D exécutée par l'unité avec des fonds externes

(4) = Échange (par exemple : exécution de R-D en vue de la vendre)

(7) = Transfert (par exemple : l'unité reçoit des subventions)

R-D financée par l'unité :

(1) = Financement par l'unité de travaux de R-D intra-muros (c'est-àdire effectués par l'unité)

(3) = Financement par l'unité de travaux de R-D extra-muros (c'est-àdire effectués par d'autres unités)

(5) = Échange (par exemple : achat de R-D)

(6) = Transfert (par exemple : l'unité verse des subventions)

4.87 Pour éviter une double comptabilisation (que ce soit des travaux de R-D ou des sommes totales engagées), il est important de déterminer si le financement par une unité des travaux de R-D exécutés par d'autres (R-D extra-muros) repose uniquement sur des fonds émanant de cette unité. Les financements externes reçus par l'unité puis transmis ou sous-traités à d'autres ne doivent pas être inclus dans les sommes consacrées à la R-D intra-muros par cette unité. C'est un point qu'il peut être difficile d'apprécier.

4.88 Par souci d'exhaustivité, précisons qu'un exécutant de R-D peut fournir des services de R-D à d'autres unités sans contrepartie financière. C'est le cas par exemple lorsqu'un exécutant met gratuitement et librement ses travaux de R-D à la disposition de la collectivité. 


\section{Graphique 4.1. Flux de financement du point de vue d'un exécutant de travaux de R-D}

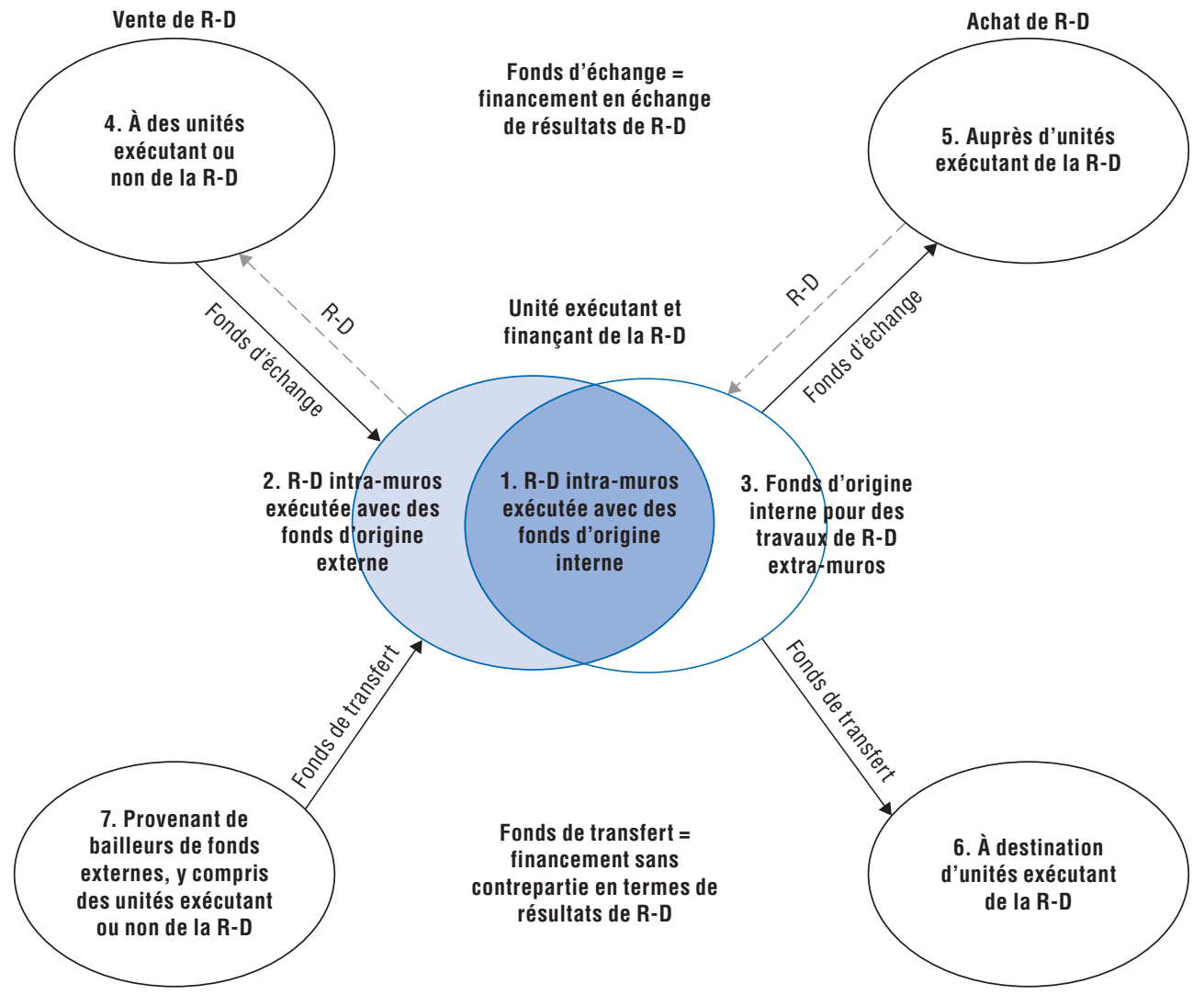

\section{Sources de financement internes et externes}

4.89 Les fonds utilisés pour financer les travaux de R-D peuvent provenir de l'unité statistique (financements internes) ou de l'extérieur (financements externes).

\section{Financements internes}

4.90 Les financements internes de R-D peuvent se définir différemment selon le secteur auquel appartient l'unité déclarante et, dans une certaine mesure, selon les circonstances propres au pays. Les financements internes de R-D correspondent à la somme des fonds consacrés à la R-D qui proviennent directement des ressources internes d'une unité déclarante et que celle-ci emploie, à sa discrétion, à des activités de R-D. Ils n'incluent pas les financements que l'unité statistique considérée reçoit d'autres unités statistiques explicitement pour les activités de R-D exécutée en son sein. L'expression « financements internes » 
est préférée à " fonds propres », qui peut être interprétée de façon plus large par certains répondants. À titre d'exemple, les bénéficiaires d'une prime de recherche peuvent considérer à tort (eu égard aux conventions énoncées dans le présent manuel), après avoir reçu leur prime, que les fonds publics sont des "fonds propres ".

4.91 Dans le secteur des entreprises, les financements internes comprennent par exemple les provisions ou les bénéfices réinvestis (c'est-àdire les bénéfices qui n'ont pas été distribués sous forme de dividendes), les ventes de produits ordinaires de l'unité (autres que la R-D), la levée de fonds sous forme de prises de participation, de prêts ou autres instruments hybrides (par exemple : levée de fonds sur les marchés financiers, prêts bancaires, capital-risque, etc.). Les déductions de l'impôt sur le revenu résultant des incitations gouvernementales à la R-D et correspondant à des travaux passés sont également considérées comme des financements internes, car elles ne sont pas nécessaires pour financer des travaux pendant la période en cours (voir dans la présente section 4.3 la sous-section intitulée « Incitations fiscales à la R-D ").

4.92 Dans le secteur de l'enseignement supérieur, les financements internes incluent notamment les versements des frais d'inscription des étudiants, les dotations ainsi que les revenus provenant de la formation tout au long de la vie et de la fourniture d'autres services. La convention au regard des comparaisons internationales est que les fonds généraux des universités (FGU) d'origine publique - qui sont une forme de dotation publique aux établissements d'enseignement supérieur - ne sont pas classés dans les financements internes (voir chapitre 9).

4.93 Autre exemple, un établissement de recherche peut financer une partie de ses travaux sur les redevances qu'il perçoit et sur les bénéfices tirés de la vente de biens et services. Même si ces fonds proviennent d'autres unités et d'autres secteurs, ils ne devraient pas être considérés comme des transferts à des fins de $\mathrm{R}-\mathrm{D}$, mais comme des "recettes non réparties " et donc comme des sources internes de financement de l'unité exécutante elle-même, puisque les acheteurs des biens et services fournis par l'établissement n'avaient pas l'intention de fournir des fonds pour la R-D).

4.94 Il convient de noter que " intra-muros " n'est pas synonyme de " interne ». La dichotomie se comprend mieux lorsque l'on indique que tous les travaux de R-D intra-muros ne sont pas financés avec des fonds d'origine interne (voir graphique 4.1).

\section{Financements externes}

4.95 Les financements externes de R-D correspondent à la somme des fonds consacrés à la R-D qui proviennent de sources extérieures à l'unité déclarante. 
4.96 Les fonds reçus d'autres unités statistiques pour financer des travaux de R-D exécutés au cours d'une période antérieure ou n'ayant pas encore commencé ne devraient pas être comptabilisés pour la période en cours mais pour la période pendant laquelle la R-D considérée a été/sera exécutée.

4.97 Les fonds fournis sous la forme de subventions, de cadeaux et de dons dans le but explicite de financer des activités de R-D sont des financements externes. Bien que l'unité statistique bénéficiaire jouisse parfois d'une très grande latitude pour décider comment et quand utiliser cet argent pour des activités de R-D intra-muros, l'origine de ces fonds est toujours extérieure à l'unité qui les reçoit. Ces fonds devraient être comptabilisés pour la période au cours de laquelle la R-D sera effectuée (d'où une déclaration possible sur plusieurs périodes) et non pour la période pendant laquelle les subventions/cadeaux/dons ont été reçus. Les dons pour la R-D provenant de particuliers - notamment pour développer la recherche sur une maladie bien précise - constituent un phénomène qui prend de plus en plus d'ampleur. Comme indiqué aux chapitres 3 et 10, l'origine déclarée des financements externes est le secteur privé sans but lucratif - dans lequel se classent les particuliers. Les cadeaux et les dons effectués sans que leurs auteurs n'exigent ou n'attendent qu'ils soient utilisés pour des travaux de R-D sont assimilés à des financements internes s'ils sont consacrés, à la discrétion de l'unité qui les reçoit, à des activités de R-D.

4.98 La convention, en ce qui concerne les comparaisons internationales, est que les fonds généraux des universités sont à comptabiliser dans les fonds publics externes (voir le chapitre 9).

4.99 Autre convention, les fonds reçus pour la R-D par un membre d'un groupe d'entreprises de la part d'autres membres du même groupe devraient être enregistrés dans les financements externes (voir la section 7.7 du chapitre 7).

\section{Incitations fiscales à la R-D}

4.100 Dans certains pays, l'administration - principalement centrale/ fédérale, mais aussi parfois régionale/locale - met en place des mesures spéciales d'allégement fiscal destinées à encourager le financement ou l'exécution de travaux de R-D, en particulier dans les entreprises. Bien que ce type d'allègement fiscal soit une forme de soutien public à la R-D, il ne devrait pas être pris en compte dans les sources publiques de financement des activités de R-D. On trouvera au chapitre 13 des conseils concernant la comptabilisation séparée de ce type d'aide financière ; quant à la question de l'incidence éventuelle des incitations fiscales à la R-D sur la déclaration des coûts de main-d'œuvre du personnel de R-D, elle est abordée dans la section 4.2 du présent chapitre.

4.101 Une difficulté de taille est que les allégements fiscaux ou les éventuels transferts directs de fonds vers l'entreprise ou l'établissement sollicitant une aide pendant une période donnée correspondent généralement à une activité de R-D qui a déjà eu lieu. Lorsque la décision d'investir dans la R-D est prise, le montant des éventuels allégements fiscaux n’est en général pas connu précisément. 
Compte tenu des difficultés pratiques à établir un lien clair entre l'aide fiscale et l'exécution des travaux de R-D, il est recommandé que le coût des travaux de R-D qui sont « financés » en anticipation des recettes ou des allégements fiscaux futurs - ou les demandes d'allégement effectuées pendant la période en cours pour des travaux passés - soient assimilés à des financements internes.

4.102 Mis à part le total des dépenses de R-D intra-muros déclarées pour l'année de référence, il peut être utile de connaître à la fois : i) le montant de l'allégement fiscal ou de l'indemnité futur(e) qui est attendu(e) du dispositif d'incitation fiscale à la R-D ; et ii) le montant du remboursement d'impôts ou des exonérations fiscales pendant la période couverte par l'enquête. Les pays souhaitant inclure dans leurs enquêtes des questions sur les incitations fiscales devraient s'assurer que cela ne fausse pas les déclarations relatives à la R-D intra-muros ni à la ventilation des sources de financement.

4.103 Dans les pays où les incitations fiscales font clairement partie de la politique budgétaire mise en œuvre par l'État pour soutenir la R-D et où elles correspondent à des structures prévisibles de financement des travaux de R-D, les enquêtes peuvent inclure une rubrique pour recueillir des informations sur le niveau d'aide fiscale qui est reçue pour les travaux de R-D exécutés pendant la période de référence. Pour les besoins de la comptabilité nationale, certains pays peuvent enregistrer ces sommes au titre des fonds publics. Or, pour ce qui est des comparaisons internationales, la convention est d'exclure ces sommes du total des financements publics et de les assimiler aux financements internes. Lorsque ces avantages fiscaux sont inscrits dans les fonds publics, les sommes correspondantes devraient être enregistrées séparément pour garantir la cohérence des déclarations au niveau international et éviter la double comptabilisation avec les dépenses fiscales (voir le chapitre 13).

\section{Sources de financement de la R-D}

4.104 Le tableau 4.3 répertorie les sources de financement sur lesquelles des informations doivent être recueillies - dans la mesure où cela est utile et possible - lors des enquêtes sur la R-D qui sont menées auprès des entités des quatre grands secteurs exécutant des travaux de R-D, à savoir : les entreprises, l'État, les établissements d'enseignement supérieur et le secteur privé sans but lucratif. De manière générale, il existe cinq grandes sources de financement de la R-D : les entreprises, l'État, l'enseignement supérieur, le secteur privé sans but lucratif et le reste du monde. Au sein de chaque secteur, des informations plus précises sur le financement peuvent en outre être recueillies. L'importance relative des diverses sources de financement (et donc du degré de détail des informations à collecter) diffère selon chaque secteur et peut varier en fonction des circonstances propres à chaque pays.

4.105 Il convient de classer les financements internes utilisés pour les travaux de R-D intra-muros selon le secteur auquel appartient l'unité statistique exécutant les travaux de R-D. 
Tableau 4.3. Sources de financement sur lesquelles des informations doivent être recueillies lors des enquêtes auprès des exécutants de R-D

\begin{tabular}{|c|c|c|c|c|}
\hline \multirow[b]{2}{*}{ Sources de financement } & \multicolumn{4}{|c|}{ Unité statistique faisant l'objet de l'enquête } \\
\hline & $\begin{array}{l}\text { Secteur des } \\
\text { entreprises }\end{array}$ & $\begin{array}{l}\text { Secteur } \\
\text { de l'État }\end{array}$ & $\begin{array}{c}\text { Secteur de } \\
\text { l'enseignement } \\
\text { supérieur }\end{array}$ & $\begin{array}{c}\text { Secteur privé } \\
\text { sans but } \\
\text { lucratif }\end{array}$ \\
\hline Secteur des entreprises & $X$ & $x$ & $x$ & $x$ \\
\hline $\begin{array}{l}\text { Entreprise considérée (fonds } \\
\text { internes) }\end{array}$ & $x$ & - & - & - \\
\hline Entreprises du même groupe & $x$ & - & - & - \\
\hline Entreprises non affiliées & $X$ & - & - & - \\
\hline Secteur de l'État & $x$ & $x$ & $x$ & $x$ \\
\hline $\begin{array}{l}\text { Organisme/ministère/institution } \\
\text { considéré(e) (fonds internes) }\end{array}$ & - & $x$ & - & - \\
\hline $\begin{array}{l}\text { Administration centrale ou fédérale } \\
\text { (hors FGU) }\end{array}$ & $x$ & $x$ & $x$ & $x$ \\
\hline $\begin{array}{l}\text { Administration de province ou d'État } \\
\text { (hors FGU) }\end{array}$ & $x$ & $X$ & $x$ & $x$ \\
\hline FGU d'origine publique & - & - & $X$ & - \\
\hline Autres organismes publics & $x$ & $x$ & $x$ & $x$ \\
\hline Secteur de l'enseignement supérieur & $x$ & $x$ & $x$ & $x$ \\
\hline $\begin{array}{l}\text { Établissement considéré (fonds } \\
\text { propres) }\end{array}$ & - & - & $x$ & - \\
\hline $\begin{array}{l}\text { Autres établissements de } \\
\text { l'enseignement supérieur }\end{array}$ & - & - & $x$ & - \\
\hline Secteur privé sans but lucratif & $x$ & $x$ & $x$ & $X$ \\
\hline $\begin{array}{l}\text { Institution considérée (fonds } \\
\text { internes) }\end{array}$ & - & - & - & $x$ \\
\hline $\begin{array}{l}\text { Autres institutions privées sans but } \\
\text { lucratif }\end{array}$ & - & - & - & $x$ \\
\hline Reste du monde & $x$ & $x$ & $x$ & $x$ \\
\hline Secteur des entreprises & $x$ & $x$ & $x$ & - \\
\hline Entreprises du même groupe & $X$ & - & - & - \\
\hline Entreprises non affiliées & $x$ & - & - & - \\
\hline Secteur de l'État & $x$ & $x$ & $x$ & $x$ \\
\hline Secteur de l'enseignement supérieur & $X$ & $x$ & $X$ & $x$ \\
\hline Campus à l'étranger & - & - & $x$ & - \\
\hline $\begin{array}{l}\text { Autres établissements de } \\
\text { l'enseignement supérieur }\end{array}$ & - & - & $x$ & - \\
\hline Secteur privé sans but lucratif & $x$ & $x$ & $x$ & $x$ \\
\hline Organisations internationales & $x$ & $x$ & $x$ & $x$ \\
\hline Total des dépenses par exécutant & $x$ & $x$ & $x$ & $x$ \\
\hline
\end{tabular}

Note : $\mathrm{X}=$ Le recueil et la déclaration sont nécessaires pour cette source de financement ; - = le recueil de données auprès de cet exécutant n'est pas utile.

4.106 Les fonds généraux des universités (FGU) utilisés pour financer la R-D intra-muros devraient être différenciés des autres sources publiques servant au financement direct des travaux de R-D de l'enseignement supérieur (voir au chapitre 9 la section 9.4). 
4.107 En ce qui concerne le secteur de l'État, il peut être utile de concevoir des enquêtes personnalisées distinctes pour les exécutants de travaux de R-D qui relèvent des administrations centrales/fédérales et ceux relevant des administrations de province/d'État.

4.108 Le financement provenant du reste du monde comprend la catégorie des " organisations internationales ", qui inclut les organisations supranationales. Des organisations internationales différentes seront spécifiées selon les pays répondant à l'enquête. Dans le cas, par exemple, des pays membres de l'Union européenne, la catégorie indiquée pourra être celle des « institutions et organes divers de l’Union européenne ».

\section{Fonds de transfert et d'échange pour la R-D}

4.109 Il peut exister des flux financiers entre les unités statistiques pour financer la R-D, que ce soit avec ou sans contrepartie - réelle ou attendue - en termes de résultats de R-D de la part de l'exécutant ; on parle alors respectivement de fonds d'échange et de fonds de transfert. Afin de mieux répondre aux demandes des utilisateurs institutionnels et aux besoins statistiques de la comptabilité nationale, il est recommandé que les travaux de R-D intra-muros qui sont financés par des sources externes soient classés dans ces deux catégories de fonds. Des sous-catégories peuvent être créées pour chaque source de financements externes si cela est possible du point de vue de la charge de travail pour les répondants et de la qualité des données.

4.110 S'agissant des enquêtes soumises aux exécutants de R-D, il y aura moins d'erreurs d'interprétation et de compréhension si l'on illustre ce que sont les fonds de transfert et les fonds d'échange par des exemples (respectivement des subventions et des contrats) que si l'on utilise les termes " transfert " et "échange ".

\section{Fonds de transfert pour la R-D}

4.111 Les fonds de transfert pour la R-D sont des fonds qu'une unité statistique fournit à une autre unité afin que cette dernière exécute des travaux de R-D. L'unité qui fournit les ressources financières n'exige aucun bien ou service en contrepartie et ne jouit pas de droits importants sur les résultats des activités de R-D qu'elle finance. Elle peut imposer certaines conditions à l'exécutant, comme celles d'établir des rapports à intervalles réguliers, de mener les activités ou projets décrits dans l'accord conclu, voire de rendre publics les résultats des travaux de recherche. Entre autres exemples de fonds de transfert figurent le subventionnement, la remise de dettes, la philanthropie, le financement participatif (à moins qu'il n'entraîne un rabais sur le prix dans le cas d'un nouveau produit), les transferts effectués à titre personnel, comme les dons, et les fonds généraux des universités (par convention pour faciliter les comparaisons internationales). Pour que l'opération soit qualifiée de " transfert de fonds destinés à la R-D ", les fonds en question devraient être destinés à des activités de R-D par l'entité qui les 
fournit à l'origine. Cette forme de financement de la R-D est qualifiée de transfert dans la mesure où, en règle générale, l'exécutant de la R-D conserve la plupart des droits attachés aux résultats des travaux.

4.112 Il existe des cas dans lesquels l'équipement ou le matériel de laboratoire utilisé pour la R-D est transféré d'une unité institutionnelle à une autre, que ce soit au sein du même secteur ou dans un autre secteur, dans le pays déclarant ou à l'étranger. Un exemple courant est celui d'une administration ou d'une institution privée sans but lucratif fournissant de l'équipement de R-D dans le cadre d'initiatives d'assistance internationale. Ces transferts " en nature » n'entraînent pas de flux monétaires et ne doivent donc pas être comptabilisés dans le total des dépenses de R-D (intra-muros ou extra-muros). Toutefois, pour les besoins de la comptabilité nationale et de l'analyse, les pays peuvent juger utile de classer et de déclarer séparément la "valeur marchande " de ces dons en nature ; en revanche, comme cela a déjà été indiqué, pour les besoins de la comparaison internationale, ces dons ne peuvent être comptabilisés dans le total des dépenses de R-D.

\section{Fonds d'échange pour la R-D}

4.113 Les fonds d'échange pour la R-D sont des fonds qu'une unité statistique fournit à une autre unité en échange de l'exécution d'activités de R-D et de l'obtention de résultats de R-D pertinents. L'unité qui finance les travaux encourt un risque de non-réalisation associé au caractère incertain du projet. Entre autres exemples figurent les achats de R-D (ventes du point de vue de l'exécutant), l'externalisation de travaux de R-D et les contributions fournies en vertu d'accords de collaboration en matière de R-D.

4.114 L'achat de R-D est l'une des formes les plus courantes de fourniture de fonds d'échange pour la R-D. Cela inclut les fonds versés à des prestataires de services de recherche ou à d'autres établissements effectuant des travaux de R-D sous contrat. Pour l'unité qui reçoit les fonds, ceux-ci sont comptabilisés dans les travaux intra-muros financés par des fonds d'origine externe. Il peut arriver que dans le cadre d'un contrat ou d'une procédure d'achat, l'unité qui finance la R-D renonce à certains de ses droits au regard des résultats des travaux de recherche. Un organisme public peut, par exemple, accorder à l'exécutant des droits de propriété intellectuelle exclusifs sur les résultats de la recherche à condition que l'État conserve le droit d'utiliser ces résultats en interne. Malgré son volet implicite de transfert, cet exemple relève de l'échange de fonds pour la R-D.

4.115 Un organisme public finançant un projet de R-D dans le cadre d'un contrat d'achat peut décider de renoncer à tous ses droits au regard des résultats des travaux de R-D qui ont été confiés à un exécutant. Cette décision étant à l'initiative du bailleur de fonds, l'opération de financement de l'activité de R-D est toujours considérée comme un échange de fonds pour la R-D. Le transfert des connaissances issues de la R-D à la société est un processus distinct qui ne rentre pas dans le cadre de la classification de la R-D intra-muros. 
4.116 Le suivi des flux des fonds d'échange peut permettre de renseigner les analystes et les économistes sur les activités de sous-traitance de la R-D, c'està-dire le processus par lequel des fonctions de base ou de soutien actuellement assurées en interne sont confiées - en tout ou partie - à des fournisseurs extérieurs indépendants ou à des entreprises affiliées implantées sur le territoire national ou à l'étranger. La sous-traitance internationale est parfois appelée " délocalisation".

4.117 En principe, les fonds d'origine interne servant à financer la participation active d'une unité à des projets de R-D collaboratifs doivent être comptabilisés au titre des travaux de R-D intra-muros de l'unité. Chaque participant déclare les fonds qu'il a affectés au projet commun. Lorsque l'une des unités joue un rôle prépondérant dans le projet, les sommes totales consacrées à la R-D peuvent être intégralement imputées aux dépenses de R-D intra-muros de cette unité ; quant aux fonds apportés par les autres participants (comme par exemple les paiements intermédiaires et les versements effectués dans le cadre d'accords de partage des coûts), ils sont enregistrés en tant que soutien fourni à l'unité dominante pour la R-D extra-muros. Toutefois, si le consortium ou le partenariat responsable du projet collaboratif est une personne morale distincte et indépendante, l'unité statistique sera une entité séparée et les contributions effectuées par l'ensemble des partenaires devront être considérées comme des sources de financement de la R-D extra-muros.

\section{Mesure des sommes consacrées à la R-D extra-muros}

\section{Importance de la mesure de la R-D extra-muros}

4.118 Afin de mieux comprendre les flux financiers s'opérant entre les unités qui financent et celles qui exécutent les travaux de R-D, il est suggéré que les pays recueillent auprès de toutes les unités qui effectuent de la R-D intramuros des informations sur les sommes consacrées à la R-D extra-muros. Les pays doivent également, dans la mesure du possible, s'enquérir de la répartition entre les fonds d'échange et les fonds de transfert, ainsi que des secteurs bénéficiaires de ces fonds. Cela peut se faire en incluant dans les enquêtes s'adressant aux exécutants de R-D une ou plusieurs questions sur le type de financement utilisé. Les réponses à ces questions correspondraient au champ 3 du graphique 4.1 .

4.119 La somme des dépenses de R-D intra-muros de l'unité statistique et du financement affecté à la R-D extra-muros (champs $1+2+3$ du graphique 4.1) permet d'obtenir un tableau plus complet de l'ensemble des activités de R-D menées par une unité. En revanche, du fait de la double comptabilisation, il n'est pas pertinent de faire la somme du total des fonds consacrés à la R-D intramuros et du total des fonds consacrés à la R-D extra-muros dans un secteur ou un pays donné. La R-D extra-muros d'une unité statistique correspond, en principe, à la R-D intra-muros d'une autre unité statistique. 


\section{Problèmes liés à la mesure de la R-D extra-muros}

4.120 Comme c'est le cas en ce qui concerne les fonds d'échange et de transfert, le risque d'erreur de compréhension et d'interprétation de la part des répondants aux enquêtes est moins grand si l'on utilise pour ces concepts une terminologie plus familière.

- Du point de vue de l'unité qui achète de la R-D, les fonds d'échange sont généralement considérés comme des " dépenses de R-D extra-muros ».

- Du point de vue de l'unité qui finance de la R-D, les fonds de transfert sont généralement considérés comme le « financement de travaux de R-D extramuros".

4.121 Pour le secteur des entreprises, la plupart des fonds consacrés à la R-D extra-muros sont censés être des fonds d'échange, c'est-à-dire permettant l'achat et la vente de R-D. Pour le secteur de l'État, l'importance relative d'une catégorie de R-D par rapport à l'autre dépendra de la mission de l'administration publique/l'organisme public considéré(e) et du type d'instrument d'action dont il/elle dispose.

4.122 La délimitation entre les fonds affectés à la R-D intra-muros et à la R-D extra-muros n'est pas toujours claire ; les sommes consacrées à la R-D extramuros sont donc parfois imputées à tort à la R-D intra-muros. Les enquêtes doivent indiquer clairement et explicitement aux répondants que pour éviter la double comptabilisation, le financement des travaux de R-D extra-muros ne doit pas être inclus dans les sommes déclarées pour la R-D intra-muros.

4.123 L'unité qui finance la R-D sait vraisemblablement si les sommes qu'elle fournit sont destinées à un échange ou à un transfert (aucune contrepartie n'est attendue en termes de R-D). Elle connaît aussi certainement l'unité ou le secteur (exécutant extérieur) auquel sont versés directement les fonds. En revanche, elle n'a sans doute pas connaissance des types de coûts que ces fonds permettront de financer (par exemple : main-d'œuvre, matériaux), ni du pourcentage de ces fonds susceptible d'être sous-traité ou sous-attribué à d'autres unités/secteurs.

4.124 Dans le cas d'un achat de R-D, il ne devrait pas être nécessaire de préciser avec quels fonds est financée l'opération (dans le cadre d'un échange), car ce sont habituellement des fonds d'origine interne qui sont utilisés. Toutefois, le recours fréquent - et croissant - à la sous-traitance, en particulier dans le secteur des entreprises, entraîne un certain nombre de difficultés. Lorsque les fonds versés par une entreprise à un sous-traitant étaient à l'origine des fonds publics, le sous-traitant ne sait pas forcément que ces fonds proviennent d'une source publique. Il peut donc les déclarer comme provenant du maître d'œuvre. De la même manière, le maître d'œuvre aura peut-être du mal à indiquer si ce sont des financements internes ou externes qui ont été utilisés pour payer le sous-traitant. 
4.125 Les financements externes consacrés à l'achat de résultats de R-D auprès d'une autre unité statistique ne doivent pas être inclus dans la R-D intra-muros.

4.126 L'achat de R-D ne doit pas être confondu avec l'acquisition de licences ou de droits de propriété intellectuelle liés à des travaux de R-D passés, car cette acquisition n'équivaut pas au financement d'une activité de R-D.

4.127 La comptabilisation des flux de R-D et de moyens de financement de la R-D entre les unités faisant partie d'un groupe d'entreprises est une tâche particulièrement difficile. La recherche d'exactitude est compliquée par le fait que le coût de la R-D ne reflète pas nécessairement le " prix du marché ", c'està-dire les coûts intra-muros effectifs. Aucun conseil spécifique ne peut être donné pour pallier les erreurs de comptabilisation, hormis l'encouragement des répondants à déclarer les coûts effectifs.

\section{Mesure du financement de la R-D extra-muros provenant d'unités n'effectuant pas de la R-D}

4.128 Un problème de sous-estimation des flux de financement apparaît lorsque les questions y afférentes sont soumises uniquement aux unités dont on sait/suppose qu'elles mènent des activités de R-D. Les enquêtes conçues pour les exécutants de la R-D ne ciblent souvent que les unités menant des activités de R-D. Or, dans chaque secteur, il existe des unités qui financent de la R-D extra-muros sans exécuter elles-mêmes des travaux de recherche. En l'absence d'enquêtes spécifiques, ces unités sont peu susceptibles d'être interrogées, ce qui signifie que les informations sur le financement extra-muros recueillies auprès des exécutants de la R-D seront incomplètes.

4.129 Avant de tirer des conclusions sur l'importance d'un secteur en tant que bailleur de fonds pour la R-D et, éventuellement, de soumettre des enquêtes aux unités concernées, une procédure d'investigation préalable est recommandée. Il est ainsi souhaitable que les offices statistiques nationaux commencent par évaluer (en partant peut-être d'un échantillon ciblé) le nombre d'unités statistiques qui, dans un secteur donné, financent de la R-D extra-muros sans en mener elles-mêmes. Ces offices devraient également évaluer le volume relatif de ce financement. Si le nombre d'unités et les sommes totales investies sont relativement faibles, le fait d'ajouter aux enquêtes existantes destinées aux exécutants de la R-D des questions sur le financement (sans modifier le cadre de l'enquête ni l'échantillon) devrait donner des résultats acceptables car les financements "manqués " provenant des unités n'effectuant pas de la R-D seraient sans doute peu élevés. Dans le cas contraire, pour obtenir une estimation exhaustive du financement de la R-D extra-muros, les unités n'exécutant pas de la R-D doivent être incluses dans l'échantillon. 


\section{Ventes et achats de R-D}

4.130 L'objectif du présent chapitre est de fournir des conseils concernant la collecte de statistiques sur les activités de R-D et les fonds utilisés pour financer ces activités. Toutefois, comme cela est indiqué au début du chapitre ("Mesure des sommes consacrées à la R-D extra-muros "), il peut aussi être utile de recueillir des informations complémentaires sur les ventes et les achats de $\mathrm{R}-\mathrm{D}$, en particulier ceux effectués par des unités statistiques appartenant au secteur des entreprises.

4.131 Le présent manuel ne peut fournir aucune recommandation particulière concernant la définition précise des "ventes " et des " achats " de $R-D$, si ce n'est que les premières représentent les recettes émanant de la fourniture de R-D à d'autres unités, et les seconds les versements effectués pour la R-D fournie par d'autres unités. Lors de la collecte de ces statistiques, il convient de préciser très clairement que le total des achats et des ventes de R-D déclarés n'est pas forcément égal aux dépenses de R-D intra-muros correspondantes. Pour citer un exemple, les dépenses intra-muros engagées pour de la R-D qui sera vendue comprennent les coûts de main-d'œuvre et " d'autres dépenses courantes ». En revanche, lorsque l'unité exécutante vend la $\mathrm{R}-\mathrm{D}$, le prix de vente a tendance à inclure le coût de l'amortissement du capital fixe et une certaine marge bénéficiaire. Le coût pour l'acheteur inclut donc le coût total de la R-D, dont les coûts d'amortissement, la marge bénéficiaire de l'exécutant, les frais de fourniture, etc.

4.132 De surcroît, la période utilisée comme référence pour l'exécution des travaux de R-D n'est pas forcément la même que celle à laquelle la R-D est vendue et achetée. La source de financement apparente de la R-D peut aussi être différente selon que l'on considère les activités de R-D ou leur achat/vente. Pour citer un exemple, une unité peut exécuter une activité de R-D financée par des fonds d'origine interne, puis ensuite en vendre les résultats - alors que ce n'était initialement pas son intention - à une autre unité. Pour ce qui est de la déclaration des travaux de R-D intra-muros, la source de financement est la première unité ; s'agissant en revanche de l'échange de R-D extra-muros, la source de financement est la seconde unité.

\section{Ventilation des fonds extra-muros selon leurs fournisseurs et leurs bénéficiaires}

4.133 Pour ventiler les fonds d'échange de la R-D (ainsi que les financements des achats et ventes de R-D), la classification sectorielle recommandée est la suivante. Cette classification peut également être utilisée pour ventiler les fonds de transfert pour la R-D.

Territoire national :

- Secteur des entreprises :

* Entreprises du même groupe

* Entreprises non affiliées 
- Secteur de l'État

- Secteur de l'enseignement supérieur

- Secteur privé sans but lucratif

Reste du monde :

- Secteur des entreprises :

* Entreprises du même groupe

* Entreprises non affiliées

- Secteur de l'État

- Secteur de l'enseignement supérieur

* Campus à l'étranger

* Autres établissements de l'enseignement supérieur

- Secteur privé sans but lucratif

- Organisations internationales

4.134 L'importance relative des différents secteurs en tant que bénéficiaires des financements de la R-D extra-muros peut varier en fonction des circonstances nationales. Par ailleurs, seules des entreprises peuvent déclarer des financements de R-D (ou des achats de R-D) à des entreprises du même groupe ; de même, seuls des établissements d'enseignement supérieur peuvent déclarer des financements de R-D à des campus affiliés à l'étranger. Pour une définition de ces établissements, se reporter à la section $9.4 \mathrm{du}$ chapitre 9.

\section{Problèmes d'identification du secteur auquel doivent être imputés les fonds}

4.135 Les concepts " intra-muros ", « extra-muros », " interne » et " externe " peuvent aussi être utilisés à des niveaux d'agrégation différents de ceux recommandés dans le présent manuel pour les unités statistiques. Ainsi, le concept de "travaux de R-D extra-muros " n'est pas le même selon que l'on se place du point de vue d'une entreprise, d'un groupe d'entreprises, d'un secteur institutionnel ou d'un pays. Dans le présent chapitre, ce sont les caractéristiques de l'unité institutionnelle (et du secteur auquel elle appartient) qui déterminent si les fonds doivent être considérés comme étant d'origine interne ou externe, et si le financement est destiné à des travaux intra-muros ou extra-muros. Comme indiqué à la section 3.2, il existe différents types d'unités statistiques, dont les entreprises, les groupes d'entreprises et les établissements ; pour les statistiques de R-D, l'unité présentant de l'intérêt est l'entreprise. Dans le présent manuel (voir la section 6.2), l'expression « unité institutionnelle » est préférée à " entreprise ", ce terme étant réservé au secteur des entreprises. 
4.136 Il n'est parfois pas facile de déterminer comment imputer les flux financiers circulant d'une unité à l'autre, et ce, quel que soit le secteur auquel appartiennent l'une et l'autre. Les recommandations en matière de traitement et de déclaration des fonds de R-D sont présentées dans les exemples sectoriels ci-après.

4.137 S'agissant du secteur des entreprises, l'unité institutionnelle pertinente est l'entreprise. Par conséquent, il convient d'enregistrer les fonds pour la R-D reçus par l'un des membres d'un groupe d'entreprises de la part d'autres membres du même groupe dans les financements externes de la R-D intra-muros. Du point de vue de l'entreprise du groupe qui fournit le financement, les fonds devraient être imputés aux travaux de R-D extra-muros.

4.138 En ce qui concerne le secteur de l'État, l'unité institutionnelle retenue est celle correspondant à un sous-secteur de l'administration (autrement dit l'administration centrale/fédérale, régionale/d'État, municipale/locale). Ainsi, les fonds reçus par le ministère $\mathrm{Y}$ de l'administration centrale de la part du ministère $\mathrm{X}$ au titre du financement de la R-D devraient être comptabilisés par le ministère $Y$ comme des fonds d'origine interne consacrés à la R-D intra-muros. $\mathrm{Du}$ point de vue du ministère $\mathrm{X}$ qui fournit les fonds, ces financements ne devraient pas être déclarés, que ce soit au titre des financements internes ou des financements de la R-D extra-muros. La raison est que ces opérations ont lieu entre des subdivisions de la même unité institutionnelle, à savoir l'administration centrale (même si les unités statistiques sont des entités plus petites, comme des ministères).

4.139 Les opérations ayant lieu entre des unités extrabudgétaires et des institutions publiques sans but lucratif (ISBL) du secteur de l'État, ainsi qu'entre l'administration centrale et les administrations d'États fédérés sont à comptabiliser au titre du financement extra-muros, car ce sont des unités institutionnelles indépendantes possédant leur propre comptabilité.

4.140 Pour citer un exemple, les fonds pour la R-D reçus par l'organisme Z d'une administration d'État de la part du ministère $\mathrm{X}$ de l'administration centrale devraient être comptabilisés dans les financements externes de la R-D intra-muros de l'organisme $\mathrm{Z}$. Du point de vue du ministère $\mathrm{X}$ qui fournit le financement, les fonds sont à imputer aux travaux de R-D extra-muros.

4.141 En ce qui concerne le secteur de l'enseignement supérieur, l'unité institutionnelle retenue est une catégorie d'établissements (universités, instituts de recherche, hôpitaux universitaires, etc.). Par conséquent, les fonds pour la R-D reçus par exemple par la faculté d'économie de l'université A de la part de la faculté d'ingénierie de la même université devraient être déclarés au titre de la R-D intra-muros de la faculté d'économie ; en revanche, ils ne doivent pas être déclarés au titre de la R-D extra-muros de la faculté d'ingénierie. Les fonds fournis par cette dernière provenaient de l'université A. 


\section{Problèmes de suivi des flux de financement de la R-D}

4.142 Un certain nombre de difficultés peuvent apparaître lorsqu'il s'agit de comptabiliser avec précision les flux de financement de la R-D, que ce soit dans le cadre de la R-D intra-muros d'une unité ou du financement total consacré aux travaux de R-D extra-muros.

4.143 Des problèmes peuvent survenir lorsque les fonds transitent par plusieurs unités avant d'atteindre l'exécutant. C'est le cas lorsque la R-D est sous-traitée, comme cela arrive en particulier dans le secteur des entreprises. C'est le cas également lorsqu'une subvention pour la R-D est sous-attribuée et que les fonds correspondants transitent par des intermédiaires, notamment dans le secteur de l'enseignement supérieur. L'exécutant devrait déclarer uniquement les coûts se rapportant à des projets de R-D effectivement mis en œuvre, et non ses contributions à la R-D d'une autre unité ; il doit en outre, dans la mesure du possible, spécifier la provenance initiale du financement de la R-D.

- Exemple : une grande compagnie pharmaceutique $(\mathrm{X})$ fait appel à une société privée de recherche biomédicale (Y) pour réaliser la phase 3 des essais cliniques d'un nouveau médicament développé par ses soins. La société Y rémunère plusieurs hôpitaux pour recruter des patients et procéder aux tests afin de confirmer l'efficacité du médicament et étudier ses effets secondaires. Bien que chaque hôpital puisse fournir à la société Y des rapports techniques sur les résultats des essais cliniques, c'est la société Y qui mesure l'efficacité du médicament et rend compte des résultats à la compagnie X. Dans cet exemple, les fonds fournis par la compagnie $\mathrm{X}$ servent à financer des services de R-D extra-muros payés à la société Y. Tous les coûts de la phase 3 d'expérimentation relèvent de la R-D intra-muros de la société Y et sont financés par des fonds d'origine externe (fournis par la compagnie $\mathrm{X}$ ). Les sommes versées aux hôpitaux devraient être déclarées par la société Y au titre des autres dépenses courantes (et non en tant qu'achat de R-D). S'agissant des hôpitaux, les versements qu'ils ont reçus pour participer aux essais cliniques ne doivent en aucun cas être comptabilisés, que ce soit dans la R-D intra-muros ou extra-muros.

- Des problèmes de suivi/déclaration similaires se présentent dans le contexte de l'Union européenne, où les financements sont versés dans un premier temps au maître d'œuvre, puis répartis entre les différents participants (sous-traitants). Dans certains pays, des entités intermédiaires n'exécutant pas de travaux de R-D jouent un rôle important dans le financement de la R-D en distribuant aux exécutants des subventions provenant de différentes sources mais non "réservées " à des usages spécifiques. Dans ce type de cas, bien qu'il soit acceptable de considérer ces entités comme les sources de financement, il est préférable d'essayer d'identifier les bailleurs de fonds d'origine au sein de l'UE. 
4.144 Lorsque l'achat de R-D est étroitement lié à des activités de R-D intra-muros, la délimitation entre le financement des travaux intra-muros et extra-muros n'est pas toujours claire :

- Il peut y avoir sous-déclaration lorsque l'exécutant de R-D sous-traite partiellement un projet de R-D. Si l'activité de sous-traitance est un projet de R-D à part entière, le bailleur de fonds peut - à raison - la déclarer au titre de la R-D extra-muros. En revanche, si l'unité responsable de la partie sous-traitée estime qu'il ne s'agit pas d'un projet de R-D mais d'une contribution - sous forme de service - au projet du bailleur de fonds, elle ne l'inclura pas dans sa propre R-D intra-muros financée avec des fonds d'origine externe.

- Inversement, il peut y avoir surdéclaration lorsque le bailleur de fonds et l'exécutant comptabilisent tous les deux les fonds et les dépenses de R-D au titre de leur R-D intra-muros. Si l'activité de sous-traitance est un projet à part entière, la R-D intra-muros devra être déclarée par l'unité à laquelle ont été confiés les travaux. En revanche, si l'activité de sous-traitance ne répond pas aux critères de la R-D intra-muros, c'est l'unité qui finance les travaux qui déclarera ces sommes dans la rubrique des autres dépenses courantes de R-D intra-muros, les services concernés étant considérés comme ayant été achetés pour mener sa propre activité de R-D.

\subsection{Concilier les approches fondées sur le point de vue des exécutants et des bailleurs de fonds}

4.145 L'estimation des dépenses totales de R-D à partir des déclarations des exécutants sera probablement différente de celle établie d'après les déclarations des bailleurs de fonds, à la fois en raison des difficultés pour établir un échantillon et des différences en matière de suivi/déclaration.

4.146 Lorsque les estimations des DIRD reposent sur des enquêtes réalisées auprès d'un échantillon de personnes et non auprès de l'ensemble de la population, l'échantillonnage est une source d'erreurs. Les valeurs obtenues seront donc certainement différentes selon qu'elles s'appuient sur les déclarations des exécutants ou des bailleurs de fonds.

4.147 Lorsque les estimations des DIRD sont obtenues à partir de dossiers administratifs, il peut y avoir un manque d'homogénéité selon que les unités visées dans les documents étudiés sont des exécutants ou des bailleurs de fonds. Voir la section 6.3 du chapitre 6.

4.148 Le financement peut être assuré par un intermédiaire, d'où la difficulté de l'exécutant à identifier l'origine des fonds. À cette difficulté s'ajoute celle de mesurer les fonds qui sortent du secteur de financement mais qui lui reviennent sous forme de R-D financée par des fonds d'origine externe. 
4.149 Il peut arriver que des particuliers reçoivent des fonds de la part d'une tierce partie (sous la forme de salaires ou de subventions) pour réaliser des travaux de R-D dans des unités d'accueil, mais où ces unités n'ont pas la capacité (parce qu'elles n'ont pas accès aux informations) d'assurer le suivi des flux de financement en question. C'est ce qui peut arriver par exemple lorsque des fonds publics sont versés à des étudiants ou des universitaires pour effectuer des travaux de R-D dans d'autres établissements, sans que ces derniers n'exercent un contrôle direct sur les fonds qui sont versés, de sorte que les individus peuvent naviguer librement d'un établissement à un autre. Bien que les pays ne doivent ni imputer eux-mêmes - ni demander à l'unité statistique d'imputer - les coûts des travaux de R-D effectués par ces individus au niveau de l'unité statistique, il est possible d'utiliser les données agrégées du bailleur de fonds pour pallier les insuffisances des déclarations dans ces dispositifs triangulaires. Lorsque les pays utilisent les données des établissements de financement pour réaliser des statistiques sur les travaux de R-D menés par des particuliers dans tel ou tel secteur, il est possible d'obtenir une comptabilisation plus complète de l'activité globale en matière de R-D. Si elle doit être comptabilisée, cette activité globale sera enregistrée non pas dans la rubrique "Autres dépenses courantes - personnel externe de R-D » mais dans les " autres dépenses courantes ". La prudence est de mise, afin d'éviter de comptabiliser deux fois les sommes déclarées par l'unité d'accueil pour ses activités de R-D intra-muros.

4.150 Les coûts pris en compte peuvent varier selon que l'on s'intéresse aux travaux de R-D sous l'angle de leur financement ou de leur exécution, en particulier dans le calcul des fonds d'échange. Du point de vue des exécutants, les dépenses de R-D intra-muros comprennent les coûts de main-d'œuvre et " d'autres dépenses courantes ". Or, lorsqu'un exécutant cède des résultats de R-D, le prix de vente est susceptible d'inclure le coût comptable de l'amortissement du capital fixe et une certaine marge bénéficiaire. Quant au bailleur de fonds, il est susceptible d'inclure le prix global de la R-D, y compris les coûts d'amortissement et la marge bénéficiaire de l'exécutant.

4.151 Les registres comptables des exécutants et des bailleurs de fonds peuvent traduire des interprétations différentes de l'appartenance des travaux à la R-D. Dans le secteur de la défense, par exemple, le financement de la R-D dans le cadre de vastes contrats de production et de livraison (couvrant par exemple la R-D et la construction d'avions en vue d'essais) se traduit parfois par des estimations plus élevées des sommes versées pour la R-D par l'État que de celles déclarées par les entreprises exécutantes au titre des travaux de R-D.

4.152 Les contrats de recherche s'étendent souvent sur plus d'un an, et des décalages peuvent intervenir entre la déclaration de l'exécutant et celle du bailleur de fonds. 
4.153 Des différences peuvent aussi apparaître entre les données déclarées par l'exécutant et les crédits budgétaires publics de R-D (CBPRD), qui sont en substance les données déclarées par le bailleur de fonds qu'est l'État (même si elles correspondent à des dotations et non à des dépenses). Dans ce type de cas, l'impossibilité de comparer peut être due au fait que le montant de la R-D effectivement réalisée n'est pas celui qui était prévu au stade de l'affectation des fonds. Des divergences peuvent aussi intervenir du fait de l'imprécision des crédits budgétaires, qui ne permet pas de mettre en évidence les dotations spécialement consacrées à la R-D. Pour en savoir plus sur l'affectation des CBPRD, voir le chapitre 12.

4.154 Une autre source de divergence possible est le fait que les bailleurs de fonds et les exécutants répondant aux enquêtes sélectionnent des secteurs différents (entre celui auquel sont affectés des fonds pour la R-D extra-muros et celui qui reçoit des financements externes pour la R-D intra-muros). Pour citer un exemple, si une unité statistique reçoit des fonds de la part d'une institution sans but lucratif contrôlée par l'État, il est possible qu'elle déclare les fonds comme provenant du secteur privé sans but lucratif (ce qui est incorrect du point de vue du présent manuel). En revanche, dans sa déclaration, le bailleur de fonds classerait la source du financement comme relevant du secteur de l'État (voir le chapitre 3).

4.155 Il est recommandé, dans la mesure du possible, que les différences entre l'estimation des dépenses totales de R-D qui est faite, d'une part, par le bailleur de fonds et, d'autre part, par l'exécutant soient signalées, et que les causes de ces différences soient, si elles sont connues, mises en évidence. Il convient de noter que ces divergences ne résultent pas nécessairement de mesures incorrectes ou imprécises, et que le fait de fournir ces informations contribuera à l'exactitude de l'analyse et des statistiques.

\subsection{Compilation des valeurs totales de la $R-D$ nationale Dépenses intérieures brutes de R-D (DIRD)}

4.156 Il est recommandé, pour obtenir des valeurs totales des DIRD qui puissent être comparées à l'échelle internationale, d'établir des agrégats généraux des travaux de R-D en fonction du secteur d'exécution et de la source de financement. Les DIRD correspondent à la somme des dépenses totales de R-D intra-muros des quatre grands secteurs étudiés dans le présent manuel, à savoir : le secteur des entreprises, le secteur de l'État, le secteur de l'enseignement supérieur et le secteur privé sans but lucratif. Dans les déclarations des DIRD, tous les niveaux d'administration sont souvent combinés. Pour chaque secteur d'exécution, les principales sources de financement doivent être regroupées : secteur des entreprises, secteur de l'État, secteur de l'enseignement supérieur, secteur privé sans but lucratif et reste du monde.

4.157 Dans le cadre du présent manuel, qui fournit des orientations pour la définition, la mesure et la déclaration des activités de R-D, le présent chapitre énonce des conventions et des recommandations pour compiler les données 
relatives aux dépenses de R-D. L'addition - recommandée - des dépenses de R-D intra-muros déclarées par l'exécutant donne une vision d'ensemble de l'activité de R-D menée à l'échelle d'un pays. D'autres compilations de la R-D peuvent toutefois être confondues avec le total des DIRD recommandé ici. On notera en particulier que les valeurs totales de la R-D sont désormais disponibles à titre indicatif dans les comptes nationaux. Comme indiqué au chapitre 1 et dans le complément en ligne au présent manuel (disponible à l'adresse http://oe.cd/frascati), les valeurs totales de la R-D figurant dans les comptes nationaux peuvent être sensiblement différentes du total des DIRD, entre autres du fait du traitement différent réservé à la R-D logicielle. Il peut donc être utile, pour les besoins de l'analyse, de mettre en évidence le pourcentage de R-D logicielle inclus dans le total des DIRD, ou tout au moins la part de la R-D des entreprises qui correspond à de la R-D logicielle (voir encadré 4.1).

\section{Encadré 4.1. R-D logicielle}

Comme indiqué au chapitre 1, une grande nouveauté du Système de comptabilité nationale ( $\mathrm{SCN}$ ) de 2008 réside dans le fait que la $\mathrm{R}-\mathrm{D}$ y est explicitement reconnue comme relevant de la formation de capital, autrement dit comme un " investissement ». Pour certains pays, les valeurs totales de la R-D obtenues en respectant les consignes du SCN peuvent être très différentes des DIRD. Une importante source de divergence est liée au traitement de la R-D logicielle (c'est-à-dire la R-D consacrée aux applications logicielles, que ce soit les produits logiciels ou les logiciels intégrés dans d'autres produits ou projets, à la fois pour un usage interne ou pour la vente). La révision du SCN de 1993 avait déjà considéré le logiciel comme une formation de capital. Par conséquent, pour appliquer la révision de 2008 et éviter la double comptabilisation, les estimations de l'investissement dans la R-D n'ont jusqu'ici pas tenu compte de la R-D logicielle. En effet, selon la nomenclature du SCN, la R-D logicielle était déjà comptabilisée dans les investissements logiciels.

Dans les pays où la R-D logicielle représente une part relativement élevée de la R-D totale, la différence entre les valeurs totales de la R-D obtenues par l'application des conventions du SCN ou des recommandations du présent manuel peut être non négligeable. Il est donc recommandé que les enquêtes sur la R-D (ou tout au moins celles portant sur le secteur des entreprises) incluent des questions sur les sommes consacrées à la R-D logicielle. Ces valeurs peuvent être extrêmement utiles pour comprendre les éventuelles différences entre les déclarations de R-D effectuées selon les recommandations du présent manuel et la R-D totale obtenue selon le SCN.

4.158 Les DIRD sont généralement représentées sous forme de tableau où l'on peut voir les principaux secteurs exécutant des travaux de R-D ainsi que leurs sources de financement classées par secteur (voir tableau 4.4). 


\section{Tableau 4.4. Dépenses intérieures brutes de R-D (DIRD)}

\begin{tabular}{|c|c|c|c|c|c|}
\hline \multirow[b]{2}{*}{ Secteur d'exécution } & \multicolumn{5}{|c|}{ Secteur d'exécution des activités de R-D } \\
\hline & \multirow{2}{*}{$\begin{array}{l}\text { Secteur des } \\
\text { entreprises }\end{array}$} & \multirow[b]{2}{*}{ Secteur de l'État } & \multirow{2}{*}{$\begin{array}{l}\text { Secteur de } \\
\text { l'enseignement } \\
\text { supérieur }\end{array}$} & \multirow{2}{*}{$\begin{array}{c}\text { Secteur privé } \\
\text { sans but lucratif }\end{array}$} & \multirow[b]{2}{*}{ TOTAL } \\
\hline Secteur de financement & & & & & \\
\hline Secteur des entreprises & & & & & $\begin{array}{l}\text { 1. Total exécuté sur } \\
\text { le territoire national } \\
\text { financé par le secteur } \\
\text { des entreprises }\end{array}$ \\
\hline Secteur de l'État & & & & & $\begin{array}{l}\text { 2. Total exécuté sur } \\
\text { le territoire national } \\
\text { financé par le secteur } \\
\text { de l'État }\end{array}$ \\
\hline \multicolumn{6}{|l|}{$\begin{array}{l}\text { Fonds généraux des } \\
\text { universités (FGU) }\end{array}$} \\
\hline \multicolumn{6}{|l|}{ Autres que les FGU } \\
\hline $\begin{array}{l}\text { Secteur de l'enseignement } \\
\text { supérieur }\end{array}$ & & & & & $\begin{array}{l}\text { 3. Total exécuté sur } \\
\text { le territoire national } \\
\text { financé par le secteur } \\
\text { de l'enseignement } \\
\text { supérieur }\end{array}$ \\
\hline $\begin{array}{l}\text { Secteur privé sans but } \\
\text { lucratif }\end{array}$ & & & & & $\begin{array}{l}\text { 4. Total exécuté sur } \\
\text { le territoire national } \\
\text { financé par le secteur } \\
\text { privé sans but lucratif }\end{array}$ \\
\hline Reste du monde & & & & & $\begin{array}{l}\text { 5. Total exécuté sur } \\
\text { le territoire national } \\
\text { financé par le reste } \\
\text { du monde }\end{array}$ \\
\hline \multicolumn{6}{|l|}{ Secteur des entreprises } \\
\hline \multicolumn{6}{|l|}{$\begin{array}{l}\text { Entreprises du même } \\
\text { groupe }\end{array}$} \\
\hline \multicolumn{6}{|l|}{ Entreprises non affiliées } \\
\hline \multicolumn{6}{|l|}{ Secteur de l'État } \\
\hline \multicolumn{6}{|l|}{$\begin{array}{l}\text { Secteur de } \\
\text { l'enseignement } \\
\text { supérieur }\end{array}$} \\
\hline \multicolumn{6}{|l|}{$\begin{array}{l}\text { Secteur privé sans but } \\
\text { lucratif }\end{array}$} \\
\hline \multirow[t]{2}{*}{$\begin{array}{l}\text { Organisations } \\
\text { internationales }\end{array}$} & & & & & \\
\hline & $\begin{array}{l}\text { Total exécuté } \\
\text { sur le territoire } \\
\text { national par } \\
\text { le secteur des } \\
\text { entreprises } \\
\text { (DIRDE) }\end{array}$ & $\begin{array}{l}\text { Total exécuté } \\
\text { sur le territoire } \\
\text { national par le } \\
\text { secteur de l'État } \\
\text { (DIRDET) }\end{array}$ & $\begin{array}{l}\text { Total exécuté } \\
\text { sur le territoire } \\
\text { national par } \\
\text { le secteur de } \\
\text { l'enseignement } \\
\text { supérieur } \\
\text { (DIRDES) }\end{array}$ & $\begin{array}{l}\text { Total exécuté } \\
\text { sur le territoire } \\
\text { national par le } \\
\text { secteur privé } \\
\text { sans but lucratif } \\
\text { (DIRDISBL) }\end{array}$ & $\begin{array}{l}\text { DIRD (somme de } \\
1 \text { à } 5)\end{array}$ \\
\hline
\end{tabular}


4.159 Pour les besoins du présent manuel, les organisations internationales (y compris les organisations supranationales comme l'Union européenne ; voir la définition à la section 3.5 du chapitre 3) doivent être traitées comme des unités du " reste du monde ", indépendamment de leur lieu d'implantation ou d'activité.

4.160 S'agissant des pays qui recueillent et déclarent des statistiques concernant les travaux de R-D des organisations internationales installées sur leur territoire, la recommandation - pour permettre les comparaisons internationales - est que ces données ne soient pas incluses dans les DIRD, mais comptabilisées à part.

4.161 Il serait utile d'établir séparément le total des DIRD à usage civil et le total des DIRD à usage militaire, car cela permettrait de déterminer dans quelle mesure les tendances dans ces domaines influent sur le niveau et la structure du total des DIRD. Cela se vérifie en particulier dans les pays ayant mis en place de vastes programmes de R-D dans le domaine militaire. Cette différenciation est également encouragée pour les autres pays, car elle faciliterait les comparaisons internationales des données sur la R-D civile.

4.162 En tant qu'indicateur de l'intensité de la R-D au sein de l'économie nationale - à la fois au fil du temps et en comparaison avec d'autres pays -, les DIRD sont souvent présentées sous forme de ratio, c'est-à-dire divisées par le produit intérieur brut (PIB). Le ratio DIRD/PIB permet ainsi d'aplanir les profondes divergences qui existent entre les valeurs globales de la R-D des différents pays divergences qui ne font peut-être que refléter, en partie, les différences entre la taille des économies de ces pays.

\section{Répartition de la $R-D$ au niveau régional}

4.163 Il peut être utile pour les pays de calculer les valeurs totales des DIRD région par région. Le choix de ce mode de calcul dépendra des besoins nationaux et internationaux. Des conseils sur le calcul de la répartition régionale de la $\mathrm{R}-\mathrm{D}$ sont accessibles dans le complément en ligne au présent manuel, à l'adresse http://oe.cd/frascati.

4.164 Pour classer la R-D « intra-muros » menée en dehors des locaux de l'unité statistique, la priorité doit généralement être de s'intéresser non pas littéralement à l'emplacement géographique de l'activité en question, mais à sa structure organisationnelle. Il est difficile de fournir des lignes directrices précises à cet égard. Le minimum à savoir est que la R-D intra-muros qui est effectuée en dehors des locaux de l'unité statistique mais est incluse dans le total géographique (par exemple régional) de cette unité ne doit concerner que la R-D qui permet à l'unité de remplir ses propres objectifs, en ayant investi ses propres ressources et son propre personnel de R-D dans l'activité.

$\rightarrow$ Exemple : un chercheur universitaire fait partie de l'unité A située dans la région $\mathrm{X}$ d'un pays donné. Il effectue occasionnellement des missions de terrain de courte durée (dans le cadre d'un projet de R-D de l'unité A) en se 
rendant physiquement dans la région $Y$ du même pays. Sauf raisons contraires (par exemple, des accords de financement avec une unité B située dans une autre région), toutes les dépenses intra-muros engagées pour ce projet de R-D peuvent être déclarées pour la région $\mathrm{X}$.

\section{Dépenses nationales brutes de R-D (DNRD)}

4.165 D'autres tableaux récapitulatifs des travaux de R-D et des sommes totales consacrées à cet usage peuvent fournir des informations complémentaires sur les liens et les interactions entre les unités statistiques de différents secteurs et différents pays. Les dépenses nationales brutes de R-D (DNRD) comprennent la totalité des dépenses de R-D financées par les unités institutionnelles d'un pays, indépendamment du lieu d'exécution de la R-D. Est ainsi incluse la R-D exécutée dans le "reste du monde » et financée par des unités institutionnelles nationales ou résidentes ; en revanche, elle n'inclut pas la R-D qui est exécutée dans le pays mais est financée par des unités institutionnelles implantées hors du territoire national (c'est-à-dire faisant partie du " reste du monde "). Le montant des DNRD est égal à la somme des dépenses intra-muros de chaque secteur d'exécution qui sont financées par des sources implantées sur le territoire national et du montant de la R-D exécutée dans le "reste du monde » qui est financée par des sources implantées sur le territoire national (voir le tableau 4.5). L'ajout de cette deuxième catégorie de R-D suppose que les offices statistiques nationaux ont collecté des données sur le financement par les unités installées sur le territoire national de la R-D extra-muros exécutée à l'étranger (autrement dit, dans le " reste du monde "). 


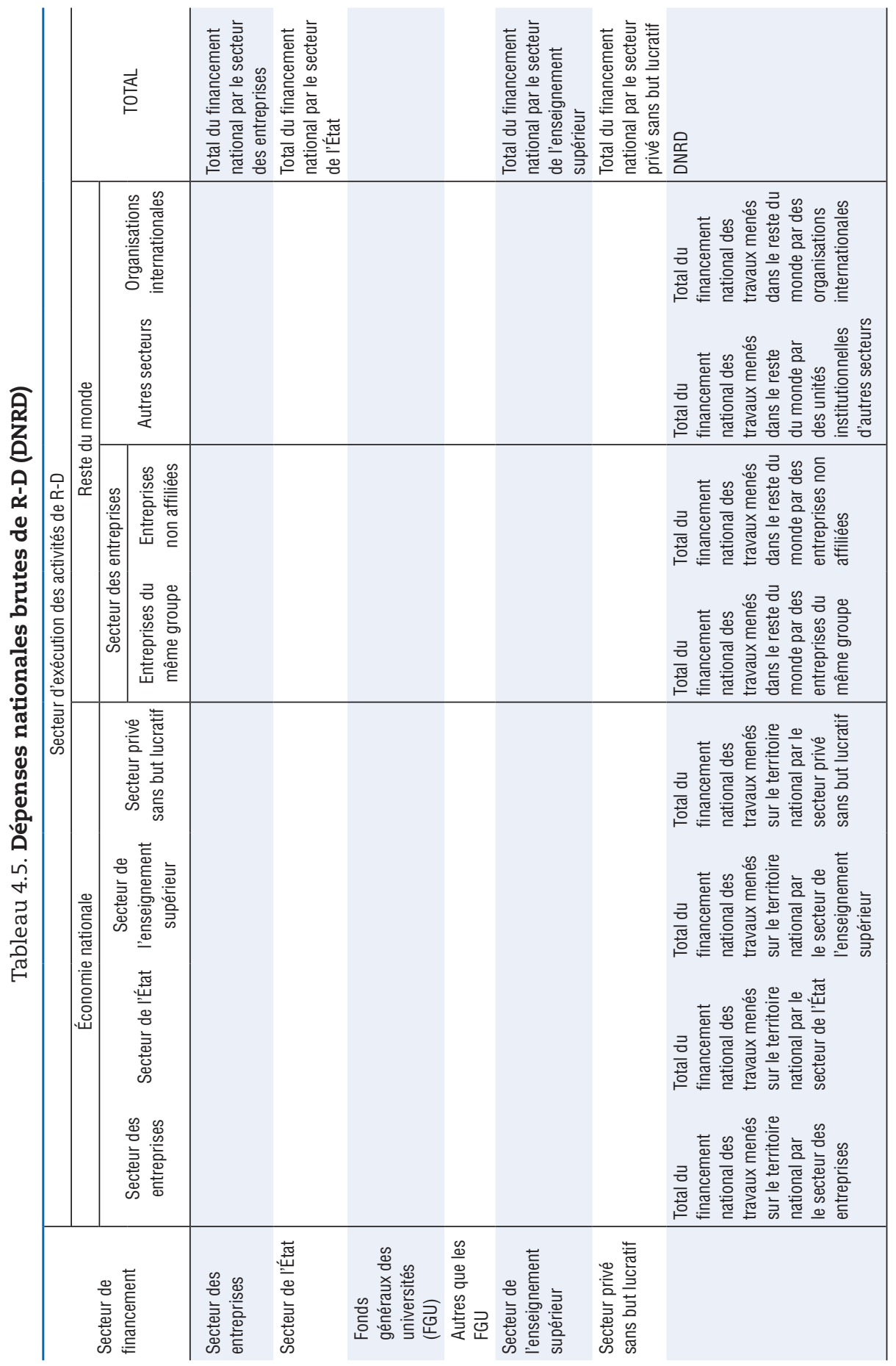




\section{Références}

CE, FMI, OCDE, Nations Unies et Banque mondiale (2009), Système de comptabilité nationale 2008, Nations Unies, New York, http://unstats.un.org/unsd/nationalaccount/ docs/SNA2008FR.pdf.

OCDE (2009), Handbook on Deriving Capital Measures of Intellectual Property Products, Éditions OCDE, Paris, DOI : http://dx.doi.org/10.1787/9789264079205-en. 


\section{Chapitre 5}

\section{Mesurer le personnel de R-D : Personnes occupées et contributeurs externes}

Ce chapitre propose des orientations sur la façon de procéder pour définir, recenser et quantifier le personnel chargé d'exécuter les activités de recherche et de développement expérimental (R-D). Le personnel de R-D est composé de scientifiques et d'ingénieurs hautement qualifiés (chercheurs), de spécialistes dotés d'un niveau élevé d'expérience et de formation techniques et du personnel d'appui directement associé à l'exécution de projets et d'activités de R-D au sein d'unités statistiques qui mènent des travaux de R-D. On distingue le personnel interne, qui regroupe les personnes occupées par l'unité statistique, du personnel externe. Sont ici présentées les activités menées par le personnel de R-D et définis les termes suivants : personnel de R-D, chercheurs, techniciens et personnel assimilé, personnel de soutien, équivalent temps plein de personnel de R-D et effectifs de personnel de R-D. Il est également question de la place des étudiants en doctorat et en master dans le personnel de R-D. Enfin, différents exemples sont fournis pour aider à déterminer qui inclure dans le personnel de $R-D$. Les statistiques ainsi obtenues sur les effectifs, la disponibilité et la démographie des ressources humaines intervenant dans les activités de $R$-D sont exploitées par les chercheurs et les responsables de l'élaboration des politiques soucieux d'assurer la pérennité de la R-D. 


\subsection{Introduction}

5.1 Les responsables de l'élaboration des politiques et les chercheurs ont clairement besoin d'avoir accès à des informations sur l'ampleur, la disponibilité et la démographie des ressources humaines qui participent directement aux activités de R-D des institutions, des secteurs économiques et des pays dans leur ensemble. Ces acteurs de la R-D sont des chercheurs hautement qualifiés, des spécialistes dotés d'un niveau élevé de formation et d'expérience techniques et le personnel de soutien directement associé à l'exécution de projets et d'activités de R-D au sein d'unités statistiques qui mènent des travaux de R-D. Les personnes travaillant sur la R-D peuvent être soit directement employées par l'unité statistique (on parle alors de personnel interne de R-D), soit employées par d'autres unités (on parle de personnel externe de R-D) participant directement aux activités de R-D intra-muros de l'unité statistique au sujet de laquelle des informations sont recueillies. Il existe un certain nombre de cas dans lesquels le personnel de R-D ne perçoit aucune contrepartie ni rémunération pour sa participation aux activités de R-D intra-muros de l'unité statistique. Le présent chapitre fournit des conseils pour définir, recenser et mesurer avec précision le personnel de R-D. Les mesures du personnel de R-D apportent un complément aux données relatives aux dépenses de R-D (abordées dans le chapitre $4 \mathrm{du}$ présent manuel), qui évaluent le coût total des activités de R-D, en y incluant la rémunération du personnel qui y est affecté.

\section{Concepts de base pour caractériser le personnel de R-D}

5.2 Pour identifier les personnes affectées à la R-D au sein d'une unité statistique menant des travaux de R-D et les distinguer du reste du personnel, on peut utiliser comme référence la liste des tâches clés effectuées par le personnel de R-D, à savoir :

- réalisation de travaux scientifiques et techniques dans le cadre d'un projet de R-D (organisation et exécution d'expériences ou d'enquêtes, construction de prototypes, etc.)

- programmation et gestion de projets de R-D

- préparation de rapports d'étape et de rapports finals sur les projets de R-D

- prestation, au plan interne, de services destinés aux projets de R-D (par exemple, travaux informatiques ou services de bibliothèque et de documentation)

- contribution aux tâches administratives liées au financement et à la gestion du personnel des projets de R-D. 
5.3 Toute personne effectuant une au moins des tâches ci-dessus contribue aux activités de R-D intra-muros de l'unité statistique et doit être prise en compte dans le total du personnel de R-D, quelle que soit sa fonction (rôle officiel) ou sa situation au regard de l'emploi au sein de l'unité statistique.

5.4 Cela dit, toutes les personnes apportant leur contribution ou leur aide à l'exécution des activités de R-D ne doivent pas être comptabilisées dans le total du personnel de R-D. Seules celles qui participent directement à ces activités (voir la liste des tâches ci-dessus) sont prises en compte dans la mesure statistique du personnel de R-D. Sont exclues de la comptabilisation les personnes effectuant des tâches auxiliaires ou de soutien indirect dans les unités menant des travaux de R-D. Ces tâches sont notamment les suivantes :

- prestations spécifiques fournies par des services informatiques centraux et par des bibliothèques pour les besoins de la R-D

- activités des services centraux des finances et du personnel concernant les projets et le personnel de R-D

- prestation de services de sécurité, de nettoyage, d'entretien, de restauration, etc., à des unités effectuant des travaux de R-D.

5.5 Si les personnes fournissant ces types de services ne sont pas prises en compte dans le total du personnel de R-D de l'unité statistique, en revanche, les coûts correspondants (notamment la rémunération de ces personnes) doivent être incorporés aux dépenses de R-D de l'unité statistique et comptabilisés dans les « autres dépenses courantes ". Ces coûts peuvent être calculés au prorata si l'on ne veut pas inclure les activités hors R-D de l'unité statistique (voir la section 4.2 du chapitre 4).

\subsection{Population couverte et définitions du personnel de $R-D$}

\section{Population initialement prise en compte : personnes occupées et contributeurs externes}

5.6 Le personnel de R-D attaché à une unité statistique est composé des personnes qui participent directement aux activités de R-D (salariés de l'unité statistique ou contributeurs externes faisant partie intégrante des projets de R-D de l'unité statistique) et de celles qui fournissent des services directement liés aux activités de R-D (responsables de R-D, gestionnaires, techniciens et personnel de soutien).

5.7 Les personnes qui fournissent des services auxiliaires et de soutien indirect (par exemple dans les domaines de la restauration, de l'entretien, de l'administration et de la sécurité) devraient être exclues, même si leurs traitements et salaires sont comptabilisés dans les dépenses de R-D au titre des autres dépenses courantes.

5.8 Les unités de R-D peuvent être organisées chacune à leur manière et utiliser des types de contrat différents pour leur personnel. Par conséquent, 
lorsque l'on comptabilise les ressources humaines travaillant pour la R-D, il convient d'inclure l'ensemble du personnel de R-D de l'unité statistique.

5.9 Les individus susceptibles de contribuer aux activités de R-D d'une unité statistique peuvent appartenir à l'un des deux grands groupes ci-après (avec quelques variantes selon le secteur institutionnel auquel appartient l'unité) :

- les personnes occupées par l'unité statistique qui participent aux activités de R-D intra-muros de celle-ci (également désignées par l'expression " personnel interne de R-D » dans le présent manuel)

- les contributeurs externes aux activités de R-D intra-muros de l'unité considérée (également désignés par l'expression " personnel externe de R-D » dans le présent manuel), eux-mêmes divisés en deux sous-groupes, selon qu'ils : i) perçoivent un salaire/traitement d'une autre entité que l'unité statistique exécutante ou ii), dans un certain nombre de cas particuliers, contribuent aux activités de R-D intra-muros d'une unité statistique sans être rattachés à celle-ci.

5.10 De manière générale, le personnel de R-D comprend toutes les personnes qui travaillent au sein ou pour le compte d'une unité statistique à temps plein ou à temps partiel - et contribuent à la R-D intra-muros. Il peut s'agir de personnes employées par l'unité statistique ou par d'autres unités participant aux activités de R-D intra-muros de ladite unité. Cela inclut à la fois des travailleurs indépendants et des salariés. Le tableau 5.1 représente les différentes catégories de personnes à prendre en compte dans la mesure du personnel de R-D. Ces catégories s'appuient sur la terminologie et les concepts établis dans la publication des Nations Unies intitulée Recommandations internationales concernant les statistiques industrielles (Nations Unies, 2009).

5.11 Il est essentiel, pour mesurer le personnel de R-D, d'identifier les personnes (internes et externes) fournissant des services pour l'exécution de la R-D intra-muros. Sur le plan pratique, une unité menant des travaux de R-D ne peut rendre compte des caractéristiques de son personnel de R-D (sexe, âge, niveau de qualification, etc. ; voir la section 5.4) lors des enquêtes statistiques que si elle participe à la sélection de ces personnes. Par conséquent, si un contrat de prestation de service identifie les personnes qui fourniront ce service, ces dernières (ainsi que leurs caractéristiques socio-économiques) pourront être comptabilisées au titre du personnel externe de R-D par l'unité bénéficiant de leurs services. Dans le cas contraire, on supposera que les services seront fournis par des prestataires extérieurs faisant appel à des personnes non identifiées. Si cette distinction est importante pour mesurer le personnel de R-D (c'est-àdire pour déterminer si les personnes fournissant des services de R-D doivent être comptabilisées dans le total du personnel de R-D), elle n'a en revanche pas d'incidence sur la mesure des dépenses de R-D, car les deux types d'activités sont regroupées dans la même catégorie des " autres dépenses courantes " (même si les coûts associés aux personnes identifiées comme du personnel externe de 
R-D doivent, si possible, être enregistrés dans une sous-rubrique). Pour en savoir plus sur la comptabilisation du personnel et des dépenses de R-D, se reporter à la section 5.2 du présent chapitre et à la section 4.2 du chapitre 4.

$\rightarrow$ Si une personne est engagée par une agence d'intérim pour effectuer sur site des travaux administratifs en rapport avec la $\mathrm{R}-\mathrm{D}$, mais est ensuite remplacée par l'agence en question sans l'intervention directe/l'accord de l'unité statistique menant des activités de R-D, cette personne ne sera pas considérée comme du personnel de R-D, mais les coûts qui y sont associés (en fait, le total des frais payés à l'agence d'intérim) seront intégrés dans les " autres dépenses courantes » (et pas uniquement dans la sous-catégorie du " personnel externe »).

\section{Personnes occupées (personnel interne) participant aux activités de R-D intra-muros de l'unité statistique}

5.12 Dans la plupart des unités statistiques, la R-D intra-muros est effectuée principalement par des personnes employées par les unités en question. Les "personnes occupées " comprennent les salariés et certaines catégories de travailleurs indépendants. Les salariés sont toutes les personnes qui travaillent dans ou pour une unité statistique, en vertu d'un contrat de travail conclu avec celle-ci, et qui reçoivent une rémunération en espèces ou en nature à intervalles réguliers. Sont inclus les salariés qui exécutent une activité auxiliaire à l'activité principale de l'unité, de même que les groupes de personnes suivants : les personnes en congé de courte durée (congé de maladie, congé pour accident ou congé annuel), les personnes en congés payés spéciaux (congé d'études ou de formation, congé de maternité ou congé parental), les grévistes, les travailleurs à temps partiel, les travailleurs saisonniers et les apprentis lorsqu'ils sont comptabilisés dans la masse salariale. Font également partie des salariés les personnes qui sont rémunérées par l'unité statistique, se trouvent sous son contrôle mais travaillent à l'extérieur (travailleurs à domicile), par exemple, les agents technico-commerciaux ou encore le personnel d'entretien et de réparation. Lorsque ces personnes participent aux activités de R-D intramuros de l'unité statistique, elles sont comptabilisées dans le personnel interne de R-D.

5.13 Dans le secteur des entreprises, les «personnes occupées 》 comprennent, dans la catégorie des " travailleurs indépendants », les travailleurs familiaux non rémunérés et les propriétaires exploitants (c'est-à-dire les propriétaires individuels ou associés). En sont exclus les associés passifs ou inactifs dont l'activité principale est menée en dehors de l'unité statistique.

5.14 Dans le secteur de l'État, le secteur de l'enseignement supérieur et le secteur privé sans but lucratif, on part de l'hypothèse que les " personnes occupées " ne comprennent pas de travailleurs indépendants (à l'exception des ménages qui, par convention, sont inclus dans le secteur PSBL). Dans ces secteurs, la quasi-totalité des «personnes occupées » sont des salariés. 


\section{Personnel externe participant aux activités de R-D intra-muros d'une unité statistique}

5.15 Les unités statistiques qui effectuent des travaux de R-D font de plus en plus appel à du personnel externe pour améliorer l'efficacité de leur personnel interne de R-D ou acquérir des connaissances et des compétences qu'elles ne possèdent pas. Dans ces cas de figure, le personnel externe est totalement intégré aux activités de R-D intra-muros de l'unité statistique déclarante, et ses tâches sont gérées par l'unité en question. Les services fournis par ce personnel externe de R-D ne sauraient être confondus avec les activités de R-D extra-muros de l'unité, c'est-à-dire l'achat de R-D auprès d'unités externes pour répondre à des commandes spécifiques en dehors des projets de R-D intra-muros de l'unité déclarante (voir la section 4.2 du chapitre 4 consacrée aux autres dépenses courantes et à la distinction entre les dépenses intra-muros et extra-muros liées à la main-d'œuvre). Il convient également de ne pas confondre les contributions du personnel externe de R-D avec l'achat de services fournis par des unités externes à l'appui des travaux de R-D intra-muros sans qu'un accord n'ait été conclu concernant la/les personne(s) chargée(s) d'exécuter lesdits services.

5.16 Le « personnel externe de R-D » comprend les personnes qui ne sont pas employées par l'unité statistique déclarante mais qui fournissent des services directs faisant partie intégrante des projets ou activités de R-D de l'unité statistique. Autrement dit, ce sont des personnes qui contribuent directement à la R-D intra-muros de l'unité statistique déclarante. Cette catégorie inclut à la fois les travailleurs indépendants intervenant en qualité de consultants dans la R-D intra-muros et les personnes assurant la prestation de services scientifiques ou techniques au nom de leur(s) employeur(s) à l'unité statistique déclarant des travaux de R-D intra-muros. Les employés intérimaires sont inclus dans cette catégorie. Ils sont mis à la disposition d'entreprises clientes en échange d'honoraires. Ils sont comptabilisés dans la masse salariale de l'agence d'emploi (ou de placement) temporaire et non dans celle de l'unité statistique qui verse les honoraires. Cette mise à disposition de ressources humaines s'effectue généralement pour une courte durée (voir les cas spécifiques d'exclusion dans les notes du tableau 5.1).

5.17 Pour les besoins du présent manuel, une définition concise du personnel externe de R-D peut être proposée en s'appuyant sur les principales caractéristiques du personnel de R-D n'appartenant pas à la catégorie des " personnes occupées ».

5.18 Le personnel externe de $R-D$ regroupe les travailleurs indépendants et les salariés qui font partie intégrante des projets de R-D d'une unité statistique sans être officiellement employés par ladite unité statistique exécutante. 


\section{Tableau 5.1. Catégories de personnel à prendre en compte dans la mesure du personnel participant aux activités de R-D intra-muros d'une unité statistique}

\begin{tabular}{|c|c|c|c|c|}
\hline & \multicolumn{4}{|c|}{ Secteurs institutionnels } \\
\hline & Entreprises & État & Enseignement supérieur & Privé sans but lucratif \\
\hline \multicolumn{5}{|c|}{ Personnes occupées (personnel interne) participant aux activités de R-D intra-muros d'une unité statistique } \\
\hline Salariés & Salariés ${ }^{1}$ & Salariés ${ }^{1}$ & Salariés ${ }^{1}$ & Salariés ${ }^{1}$ \\
\hline $\begin{array}{l}\text { Travailleurs } \\
\text { indépendants }\end{array}$ & $\begin{array}{l}\text { Propriétaires } \\
\text { exploitants }^{2} \\
\text { Travailleurs familiaux } \\
\text { non rémunérés }{ }^{3}\end{array}$ & Non applicable & Non applicable & $\begin{array}{l}\text { Applicable } \\
\text { uniquement aux } \\
\text { ménages }\end{array}$ \\
\hline \multicolumn{5}{|c|}{ Cas particuliers de personnel externe participant aux activités de R-D intra-muros d'une unité statistique ${ }^{4}$} \\
\hline $\begin{array}{l}\text { Salariés ou } \\
\text { travailleurs } \\
\text { indépendants }\end{array}$ & & $\begin{array}{l}\text { Bénéficiaires de } \\
\text { subventions pour la } \\
\text { R-D ; étudiants en } \\
\text { doctorat/master }{ }^{5}\end{array}$ & $\begin{array}{l}\text { Étudiants en doctorat/ } \\
\text { master }{ }^{5} \text {; bénéficiaires de } \\
\text { subventions pour la R-D } \\
\text {; professeurs émérites }\end{array}$ & Bénévoles 6 \\
\hline \multicolumn{5}{|c|}{ Personnel externe participant aux activités de R-D intra-muros d'une unité statistique } \\
\hline Salariés & \multicolumn{4}{|c|}{$\begin{array}{l}\text { Spécialistes et techniciens intervenant en tant que consultants pour fournir des services scientifiques ou } \\
\text { techniques au nom de leur(s) employeur(s) à l'unité statistique déclarant des travaux de R-D intra-muros. } \\
\text { Les employés intérimaires }{ }^{7} \text { sont inclus dans cette catégorie. }\end{array}$} \\
\hline $\begin{array}{l}\text { Travailleurs } \\
\text { indépendants }\end{array}$ & \multicolumn{4}{|c|}{ Spécialistes indépendants intervenant comme consultants pour des travaux de R-D intra-muros. } \\
\hline
\end{tabular}

1. La catégorie "Salariés " comprend toutes les personnes participant à l'activité économique de l'unité statistique, autres que les propriétaires exploitants et les travailleurs familiaux non rémunérés. Elle inclut les travailleurs à domicile qui sont rémunérés par l'unité statistique et se trouvent sous son contrôle. Sont également inclus les salariés qui exécutent une activité auxiliaire à l'activité principale de l'unité, de même que les groupes de personnes suivants : les personnes en congé de courte durée (congé de maladie, congé pour accident ou congé annuel), les personnes en congés payés spéciaux (congé d'études ou de formation, congé de maternité ou congé parental), les grévistes, les travailleurs à temps partiel, les travailleurs saisonniers et les apprentis lorsqu'ils sont comptabilisés dans la masse salariale.

2. Comprend les propriétaires individuels ou associés. En sont exclus les associés passifs ou inactifs dont l'activité principale est menée en dehors de l'unité statistique.

3. Les travailleurs familiaux rémunérés pour le travail effectué doivent être classés dans la catégorie des salariés.

4. Ces catégories concernent plusieurs secteurs. Sont indiqués ceux dans lesquels leur incidence devrait être la plus grande.

5. Les étudiants ne devraient être pris en compte que s'ils participent formellement à l'activité de R-D de l'établissement qui les accueille.

6. Les bénévoles qui participent aux activités de R-D intra-muros d'une institution sans but lucratif doivent être clairement identifiés par l'unité statistique déclarant des activités de R-D, à la fois en ce qui concerne leur qualification et les tâches effectuées.

7. Les employés intérimaires sont mis à la disposition d'entreprises clientes en échange d'honoraires. Ils sont comptabilisés dans la masse salariale de l'agence d'emploi temporaire et non dans celle de l'unité statistique qui verse les honoraires. Cette mise à disposition de ressources humaines s'effectue généralement pour une courte durée. Sont exclus de cette catégorie : les services achetés ou gérés, par exemple en matière de conciergerie, de sécurité et de paysagisme ; les services techniques ou spécialisés achetés auprès d'une autre entreprise, comme par exemple les services de conseil et de programmation informatique, d'ingénierie et de comptabilité. Est exclu de cette catégorie le personnel temporaire envoyé par une agence de placement, des prestataires extérieurs, des sous-traitants ou des prestataires indépendants lorsque ce personnel ne participe pas directement à la R-D intra-muros de l'unité statistique.

\subsection{Le personnel externe de R-D se compose généralement de spécialistes} ou de techniciens dotés d'un niveau élevé de compétences et de spécialisation dans les activités de R-D. Le personnel externe de R-D étant, par définition, censé posséder les mêmes compétences que le personnel interne équivalent, une unité statistique de n'importe quel secteur économique peut théoriquement effectuer 
ses travaux de R-D intra-muros uniquement en faisant appel à du personnel externe, sans employer de personnel interne.

5.20 Le personnel externe de R-D fait souvent référence à des travailleurs indépendants - lesquels devraient, à ce titre, être classés dans la catégorie des entreprises individuelles. Dans certains cas, ces personnes sont employées par des organisations externes, des établissements de recherche ou des entreprises qui commercialisent des services techniques ou scientifiques fournis par des personnes qualifiées. Dans d'autres, les agences qui emploient ne vendent pas au client un " service de R-D " particulier, mais lui proposent de mettre à sa disposition pendant une période donnée des travailleurs qualifiés pour répondre à un besoin spécifique. Il peut également arriver que les travailleurs externes soient salariés d'une institution publique (par exemple, dans certains pays, des professeurs d'université ou des chercheurs employés par l'État), mais autorisés par leur employeur à réaliser des travaux de R-D dans le secteur privé tout en continuant de remplir leurs obligations professionnelles dans le secteur public. Dans ce type de situation, il n'est pas rare qu'une personne ait plusieurs affectations, ce qui signifie qu'elle peut être déclarée par au moins deux entités.

5.21 Outre les travailleurs indépendants et les employés d'autres unités statistiques engagés comme contributeurs externes pour les travaux de R-D, plusieurs autres catégories de personnes effectuent des tâches similaires et devraient donc être incluses dans le total du personnel externe de R-D.

5.22 Dans le secteur de l'enseignement supérieur, les étudiants en master et en doctorat (pour en savoir plus, voir plus bas la sous-section " Traitement des étudiants en master et en doctorat ") ainsi que les individus bénéficiant de subventions pour la R-D peuvent faire l'objet d'un traitement différent selon leur situation au regard de l'emploi. Ainsi, lorsque ces personnes ont un statut de salarié au sein d'un établissement d'enseignement supérieur (ou, inversement, lorsque les salariés de l'établissement sont inscrits en tant qu'étudiants en doctorat) - autrement dit, lorsqu'elles sont comptabilisées dans la masse salariale de l'université -, ces personnes devraient être incluses dans le total du personnel de R-D en tant que " personnes occupées » (personnel interne de R-D) par l'université (ou par toute autre unité statistique du secteur de l'enseignement supérieur). En revanche, si ces personnes ne sont pas comptabilisées dans la masse salariale de l'université, les étudiants en doctorat et bénéficiaires de subventions pour la R-D devraient être enregistrés dans le total du personnel de $R-D$ en tant que personnel externe de R-D s'ils reçoivent une rémunération pour le travail de R-D qu'ils effectuent - quels que soient la source et le canal de financement utilisés. Par ailleurs, les étudiants en doctorat qui ne reçoivent pas de financement peuvent malgré tout être comptabilisés dans le total du personnel de R-D en tant que personnel externe. Cette consigne applicable aux étudiants ne recevant pas de financement vaut également pour les étudiants en master, à condition qu'ils soient inscrits dans un programme de recherche au niveau du master et que l'équivalent temps plein (ETP) des travaux de recherche puisse être clairement mis en évidence et dissocié des frais de scolarité. 
5.23 Le secteur de l'enseignement supérieur comporte une catégorie spécifique (presque exclusive) de personnel externe de R-D, celle des « professeurs émérites ". Il s'agit des professeurs à la retraite qui continuent de mener des travaux de recherche et de collaborer aux activités académiques de leur ancien employeur, habituellement une université, sans recevoir la moindre rémunération (mais éventuellement une forme de soutien logistique). Pour être considéré comme un contributeur externe aux activités de R-D intra-muros d'une unité statistique de l'enseignement supérieur, le professeur émérite doit avoir été comptabilisé dans la masse salariale d'une université avant de prendre sa retraite, et être encore très actif dans le domaine de la recherche (mais plus beaucoup, en général, dans l'enseignement). Dans la mesure où les professeurs émérites peuvent contribuer de façon non négligeable à la R-D d'un établissement, il est justifié qu'ils soient inclus dans le total du personnel externe de R-D.

5.24 Enfin, il existe une dernière catégorie de personnes pouvant être incluse (à des fins de mesure) dans le personnel externe de R-D, à savoir les personnes intervenant dans la R-D intra-muros à titre bénévole. Les bénévoles sont des travailleurs non rémunérés qui apportent une contribution active et précise en matière de R-D à une unité statistique qui en assure la responsabilité. C'est dans le secteur privé sans but lucratif que cette catégorie de personnes peut faire le plus de différence dans les estimations. Les bénévoles ne peuvent être inclus dans le total du personnel externe de R-D que s'ils satisfont à des critères très stricts :

- Ils participent aux activités de R-D intra-muros d'institutions (privées sans but lucratif).

- Leurs compétences en matière de recherche sont comparables à celles des salariés. Par conséquent, les individus qui se portent volontaires pour participer en tant que cobayes à des essais cliniques, fournir un appui informatique à des projets de R-D, etc., ne devraient pas être inclus dans le personnel externe de R-D.

- Leurs activités de R-D sont systématiquement planifiées en fonction de leurs besoins et de ceux de l'institution.

- Leur contribution doit être suffisante et être une condition essentielle pour permettre à l'institution d'entreprendre une activité ou un projet de R-D intra-muros.

\section{Concordance entre les catégories du personnel de R-D et des dépenses de R-D}

5.25 Comme expliqué ci-après, le traitement du personnel de R-D et des coûts y afférents peut varier selon la situation au regard de l'emploi des différentes personnes (voir le tableau 5.2). Ainsi que nous l'avons déjà vu, deux grandes catégories de personnes peuvent être prises en compte dans la comptabilisation du personnel de R-D et de leurs coûts : les personnes employées par l'unité - c'est-à-dire le personnel interne de R-D -, et le personnel externe de R-D. Cette 
seconde catégorie comprend les personnes employées par d'autres unités, ainsi que plusieurs cas particuliers de personnes extérieures à l'unité qui ne sont pas salariées.

\section{Tableau 5.2. Classification et comptabilisation du personnel et des dépenses affectés à la R-D}

\begin{tabular}{|c|c|c|c|c|}
\hline Classification & $\begin{array}{l}\text { Situation des personnes } \\
\text { au regard de l'emploi }\end{array}$ & Description & Secteur institutionnel & $\begin{array}{l}\text { Imputation des } \\
\text { dépenses de } R-D\end{array}$ \\
\hline \multirow[b]{2}{*}{$\begin{array}{l}\text { Personnel interne } \\
\text { de R-D participant } \\
\text { à la R-D intra- } \\
\text { muros d'une unité }\end{array}$} & $\begin{array}{l}\text { Personnes occupées } \\
\text { (salariés) }\end{array}$ & Salariés ${ }^{1}$ & & $\begin{array}{l}\text { Coûts de main- } \\
\text { d'œuvre (masse } \\
\text { salariale) }\end{array}$ \\
\hline & $\begin{array}{l}\text { Personnes occupées } \\
\text { (travailleurs } \\
\text { indépendants) }\end{array}$ & $\begin{array}{l}\text { Propriétaires } \\
\text { exploitants, } \\
\text { travailleurs familiaux } \\
\text { non rémunérés, etc. }\end{array}$ & $\begin{array}{l}\text { Catégorie non } \\
\text { applicable pour les } \\
\text { secteurs de l'État et } \\
\text { de l'enseignement } \\
\text { supérieur, ni pour } \\
\text { la plupart des } \\
\text { institutions privées } \\
\text { sans but lucratif }\end{array}$ & $\begin{array}{l}\text { Personnes } \\
\text { généralement non } \\
\text { comptabilisées car } \\
\text { ne recevant pas de } \\
\text { rémunération }\end{array}$ \\
\hline \multirow{2}{*}{$\begin{array}{l}\text { Personnel externe } \\
\text { de R-D rémunéré }\end{array}$} & $\begin{array}{l}\text { Consultants } \\
\text { indépendants }\end{array}$ & $\begin{array}{l}\text { Contribuent à la R-D } \\
\text { intra-muros de leurs } \\
\text { clients sur une base } \\
\text { contractuelle }\end{array}$ & & $\begin{array}{l}\text { Autres dépenses } \\
\text { courantes - personnel } \\
\text { externe de R-D }\end{array}$ \\
\hline & $\begin{array}{l}\text { Salariés d'autres unités } \\
\text { intervenant comme } \\
\text { consultants en matière } \\
\text { de R-D }\end{array}$ & $\begin{array}{l}\text { Contribuent à la } \\
\text { R-D intra-muros } \\
\text { des clients de leur } \\
\text { employeur contre } \\
\text { rémunération }\end{array}$ & & $\begin{array}{l}\text { Autres dépenses } \\
\text { courantes - personnel } \\
\text { externe de R-D }\end{array}$ \\
\hline \multirow{4}{*}{$\begin{array}{l}\text { Personnel externe } \\
\text { de R-D : cas } \\
\text { particuliers }\end{array}$} & $\begin{array}{l}\text { Étudiants en doctorat/ } \\
\text { master }\end{array}$ & & $\begin{array}{l}\text { Principalement } \\
\text { dans le secteur } \\
\text { de l'enseignement } \\
\text { supérieur, mais aussi } \\
\text { dans d'autres secteurs } \\
\text { institutionnels }\end{array}$ & $\begin{array}{l}\text { Autres dépenses } \\
\text { courantes - personnel } \\
\text { externe de R-D (dans } \\
\text { la mesure où les } \\
\text { étudiants reçoivent } \\
\text { des subventions pour } \\
\text { la R-D ou des salaires/ } \\
\text { traitements extérieurs) }\end{array}$ \\
\hline & $\begin{array}{l}\text { Bénéficiaires de } \\
\text { subventions pour } \\
\text { la R-D }\end{array}$ & & $\begin{array}{l}\text { Principalement } \\
\text { dans les secteurs } \\
\text { de l'enseignement } \\
\text { supérieur et de l'État }\end{array}$ & $\begin{array}{l}\text { Autres dépenses } \\
\text { courantes - personnel } \\
\text { externe de R-D } \\
\text { (dans la mesure } \\
\text { où les subventions } \\
\text { pour la R-D sont } \\
\text { comptabilisées) }\end{array}$ \\
\hline & Bénévoles & & $\begin{array}{l}\text { En particulier dans le } \\
\text { secteur privé sans but } \\
\text { lucratif }\end{array}$ & $\begin{array}{l}\text { Généralement non } \\
\text { comptabilisés car ils } \\
\text { ne reçoivent pas de } \\
\text { rémunération }\end{array}$ \\
\hline & $\begin{array}{l}\text { Professeurs émérites } \\
\text { (situation semblable } \\
\text { aux bénévoles) }\end{array}$ & & $\begin{array}{l}\text { Cas particulier existant } \\
\text { presque exclusivement } \\
\text { dans le secteur de } \\
\text { l'enseignement } \\
\text { supérieur }\end{array}$ & $\begin{array}{l}\text { Généralement non } \\
\text { comptabilisés car ils } \\
\text { ne reçoivent pas de } \\
\text { rémunération }\end{array}$ \\
\hline
\end{tabular}

1. Comprend les étudiants en doctorat/master comptabilisés dans la masse salariale de l'unité effectuant des travaux de R-D. Il peut être utile de comptabiliser séparément les étudiants inclus dans la masse salariale des établissements d'enseignement supérieur. 
Groupe 1. Personnel interne de R-D (c'est-à-dire les personnes employées par l'unité statistique et participant à ses activités de R-D intra-muros) :

- Les salariés travaillant sur la R-D sont considérés comme faisant partie intégrante de l'unité statistique, et leurs salaires/traitements doivent être inclus dans les coûts de main-d'œuvre des dépenses de R-D intra-muros comptabilisées. Les étudiants en doctorat/master sont inclus dans le total du personnel interne de R-D s'ils figurent dans la masse salariale de l'unité statistique pour laquelle ils effectuent des travaux de R-D (pour des orientations plus détaillées, voir plus bas la sous-section « Traitement des étudiants en master et en doctorat ").

- Les propriétaires exploitants et autres personnes occupées travaillant à leur compte ne sont généralement pas directement rémunérés pour leur travail.

Groupe 2 (i). Le personnel externe de R-D comprenant des personnes qui ne sont pas employées par l'unité statistique déclarante mais par d'autres unités, et qui fournissent des services directs faisant partie intégrante des projets ou activités de R-D de l'unité statistique, dans le cadre d'un contrat spécifique, ne devrait pas être inclus dans les coûts de main-d'œuvre. Les coûts s'y rapportant doivent en revanche être comptabilisés dans la rubrique des autres dépenses courantes (de préférence dans une sous-rubrique «Autres dépenses courantes personnel externe de R-D ») des dépenses de R-D intra-muros de l'unité. Ce groupe comprend :

- Les salariés d'autres unités, engagés en qualité de consultants pour contribuer à la R-D intra-muros des clients de leurs employeurs contre rémunération.

- Les consultants indépendants contribuant à la R-D intra-muros de leurs clients sur une base contractuelle. On les appelle souvent des « prestataires extérieurs de R-D ».

Groupe 2 (ii). Le personnel externe qui n'est pas employé par l'unité mais qui accomplit des tâches de R-D similaires à celles effectuées par le personnel interne de R-D :

- Les étudiants en doctorat/master sont inclus dans le total du personnel externe de R-D s'ils sont rétribués pour leur contribution à la R-D autrement que par le versement d'un traitement/salaire par l'unité statistique exécutante. S'ils reçoivent des « subventions pour la R-D » ou des traitements/salaires externes, ils seront comptabilisés dans la sous-rubrique "Autres dépenses courantes personnel externe de R-D » (pour des orientations plus détaillées, voir plus bas la sous-section "Traitement des étudiants en master et en doctorat »).

- Les bénéficiaires de subventions pour la R-D ne perçoivent pas de salaire. Les coûts associés à ces subventions peuvent être comptabilisés, le cas échéant, dans la rubrique des autres dépenses courantes.

- Les bénévoles contribuant à la R-D intra-muros ne sont généralement pas directement rémunérés pour leur travail. 
- Les professeurs émérites contribuant à la R-D intra-muros - principalement dans des établissements d'enseignement supérieur - ne sont généralement pas directement rémunérés pour leur travail.

Comme indiqué ci-dessus, le total du personnel de R-D se compose de deux grands groupes de personnes : les personnes occupées exécutant des travaux de R-D (c'est-à-dire le personnel interne de R-D, un sous-groupe du total des personnes employées par l'unité statistique) et les personnes exécutant des travaux de R-D mais non employées par l'unité statistique (c'est-à-dire le personnel externe de R-D). Il est recommandé, dans la mesure du possible, que les données se rapportant à chacun de ces deux groupes soient recensées, recueillies et comptabilisées séparément, que ce soit au titre du personnel ou au titre des dépenses. Cette recommandation se justifie par le besoin de cohérence entre les données relatives au personnel de R-D et celles relatives aux dépenses de R-D, ainsi que par la nécessité de rendre compte avec précision de la composition de la main-d'œuvre de R-D.

5.26 S'agissant des unités qui mettent du personnel de R-D intérimaire à disposition d'autres unités menant des travaux de R-D, il est important que ces prestataires n'intègrent pas ce personnel externe de R-D et les coûts y afférents dans leur R-D intra-muros, car cela équivaudrait à une double comptabilisation. Il peut en effet arriver que ces fournisseurs de personnel intérimaire effectuent eux-mêmes de la R-D intra-muros (pour leur propre usage ou à titre marchand), auquel cas il risque d'être difficile pour eux de faire la différence entre le personnel et les dépenses affectés à la R-D intra-muros et ceux aux activités de $\mathrm{R}-\mathrm{D}$ extra-muros.

\section{Traitement des étudiants en master et en doctorat}

5.27 Les étudiants en master et en doctorat sont identifiables par leur niveau d'études : ils ont achevé leurs études universitaires au niveau licence (niveau 6 de la CITE) et les poursuivent au niveau master (niveau 7 de la CITE) ou doctorat (niveau 8 de la CITE). Pour en savoir plus sur les niveaux de la CITE, se reporter, dans la section 5.4 , à la ventilation du personnel de R-D et des chercheurs selon le niveau d'études.

5.28 Les étudiants en doctorat suivent des « programmes d'enseignement supérieur qui conduisent à l'obtention d'un titre de chercheur hautement qualifié [et] sont donc consacrés à des études approfondies et à des travaux de recherche originaux et ne sont pas fondés uniquement sur des cours ". En règle générale, ces étudiants sont tenus de soutenir une thèse d'une qualité suffisante pour en permettre la publication, cette thèse devant être le produit d'un travail original de recherche et représenter une contribution appréciable à la connaissance. Les étudiants en doctorat (niveau 8 de la CITE) qui sont engagés en tant que chercheurs devraient par conséquent être inclus dans la mesure du personnel de R-D et des dépenses correspondantes des 
établissements d'enseignement supérieur. Les raisons pour lesquelles les limites entre activités de R-D et de formation de ces étudiants (et de leurs enseignants/mentors) sont difficiles à définir font l'objet d'un examen général à la section 9.2 du chapitre 9 .

5.29 En principe, tous les étudiants en doctorat participent aux activités de R-D de l'université dans laquelle ils sont inscrits. Il est en outre fréquent que les universités possèdent des droits sur les résultats des travaux de recherche en fonction du temps de supervision qui y est consacré et de l'accès aux installations. Cela dit, il n'existe pas forcément d'obligation formelle pour les étudiants de consacrer du temps à la R-D intra-muros de l'université et, même si elle existe, elle n'est pas toujours respectée. Par convention, une distinction est faite entre les étudiants en doctorat qui reçoivent une rémunération ou un autre type de soutien financier de la part de l'université (ou de toute autre source) pour leur activité de R-D, et ceux qui ne reçoivent rien. Dans certains cas, les étudiants perçoivent un salaire (ils sont alors comptabilisés dans la masse salariale de l'université) ; dans d'autres, ils reçoivent uniquement une subvention, généralement une prime de recherche ou une aide comportant un volet axé sur la recherche. Pour des raisons pratiques, on considère que les étudiants sans salaires/subventions ne peuvent contribuer de façon substantielle aux activités de R-D de l'université, contrairement à ceux qui sont aidés financièrement. Seuls les étudiants de la seconde catégorie doivent être pris en compte dans le calcul du total du personnel de R-D (interne ou externe, selon les modalités de financement) ; ceux de la première catégorie peuvent être, selon les circonstances, comptabilisés dans le personnel externe de R-D, comme expliqué ci-après.

5.30 Les étudiants en master peuvent, dans certains cas, être comptabilisés en qualité de chercheurs. Cela concerne, en particulier, les étudiants du niveau 7 de la CITE, qui suivent un programme d'enseignement conduisant à l'obtention d'un titre de chercheur et consacré à des travaux de recherche originaux, mais au-dessous du niveau de doctorat. Néanmoins, il est important de n'inclure dans le total du personnel de R-D que les étudiants en master percevant une forme de rétribution pour leur activité en matière de $\mathrm{R}-\mathrm{D}$ ou pour lesquels le montant de l'équivalent temps plein (ETP) peut être clairement mis en évidence et dissocié de la composante éducative.

5.31 Afin de faciliter la comparaison internationale des données, la méthode heuristique suivante de classification et de traitement des étudiants contribuant aux activités de R-D est recommandée. Comme indiqué plus haut, la première étape consiste à identifier les étudiants participant aux activités de R-D.

- Cas $\mathrm{n}^{\circ} 1$ : Les étudiants en master/doctorat reçoivent un financement - sous forme de salaire ou de subvention - de la part de l'unité exécutante pour effectuer des travaux de recherche. Ils sont considérés comme du personnel 
interne de R-D et les dépenses qui y sont associées sont incluses dans les coûts de main-d'œuvre. Ils ne sont pas comptabilisés séparément comme des étudiants.

- Cas $n^{\circ} 2$ : Les étudiants en doctorat reçoivent un financement externe ou pas de financement pour effectuer des travaux de recherche dans l'unité exécutante. Ils sont considérés comme du personnel externe de R-D et les dépenses qui y sont associées (lorsqu'ils reçoivent un financement) sont incluses dans le poste "Autres dépenses courantes - personnel externe de R-D ». Ces dépenses peuvent être enregistrées et comptabilisées par l'unité exécutante, ou évaluées au niveau du secteur à l'aide d'une méthode triangulaire (voir au chapitre 4 la section 4.4) ; il convient de prendre des dispositions pour éviter le risque d'une double comptabilisation. Cette manière de procéder s'applique également aux étudiants en master si le financement qu'ils reçoivent est explicitement dédié à la R-D ou si un montant significatif d'équivalent temps plein (ETP) peut être clairement mis en évidence et dissocié de la composante éducative. Il est recommandé, dans la mesure du possible, que le nombre d'étudiants en master/doctorat soit comptabilisé, en particulier pour le secteur de l'enseignement supérieur. Dans certains pays, il peut en outre être utile de comptabiliser séparément les étudiants en doctorat et ceux en master.

- Cas $n^{\circ} 3$ : Les étudiants en doctorat mènent des travaux de recherche indépendants, avec ou sans l'aide d'un financement. Ils ne sont pas comptabilisés dans le personnel de $\mathrm{R}-\mathrm{D}$, mais le financement qu'ils reçoivent peut être inclus dans les " autres dépenses courantes " s'il s'agit d'une subvention externe (laquelle peut être évaluée au niveau du secteur à l'aide d'une méthode triangulaire ; voir la section $4.4 \mathrm{du}$ chapitre 4). Cette manière de procéder s'applique également aux étudiants en master si le financement qu'ils reçoivent est explicitement dédié à la R-D.

\section{Classification du personnel de R-D par fonction}

5.32 Une fois que l'on a identifié les personnes pouvant participer aux activités de R-D intra-muros d'une unité statistique, il est nécessaire de mettre en évidence, à l'aide de critères bien précis, les personnes ayant effectivement réalisé des travaux de $\mathrm{R}-\mathrm{D}$ au sein de l'unité statistique déclarante pendant la période de référence :

- Pour ce qui est du personnel externe de R-D, il n'existe pas de différence entre les travaux de R-D réels et potentiels, ce personnel étant identifié sur la base d'éléments précis démontrant qu'ils contribuent à des activités de R-D intramuros.

- Pour ce qui est des " personnes occupées » au sein de l'unité statistique - notamment les propriétaires exploitants, les salariés et autres catégories de personnes -, il convient d'examiner les tâches accomplies par chacune de ces 
personnes dans les activités de R-D de l'unité statistique afin de déterminer lesquelles ont participé « directement » à la R-D intra-muros pendant l'année de référence. Par convention, n'importe quel type ou niveau (intensité) de contribution directe à la R-D est suffisant pour que les personnes soient classées dans le personnel interne de R-D. Il est toutefois recommandé de n'inclure dans le total du personnel de R-D que les personnes occupées ayant contribué de façon suffisante à la R-D intra-muros en pourcentage de leur temps de travail (sur la base d'une année). Pour en savoir plus sur ce que peut être une contribution " suffisante ", se reporter à la sous-section intitulée "Équivalent temps plein (ETP) du personnel de R-D ».

5.33 Les personnes faisant partie des deux groupes précités seront classées d'après la fonction qu'ils exercent au regard de la R-D : chercheur, technicien ou personnel de soutien. Ce classement donne souvent lieu à des erreurs d'interprétation en raison de l'utilisation qui est faite des mêmes termes dans des contextes différents. Il convient, à cet égard, de préciser que dans le présent manuel, ce mode de classification du personnel de R-D fait référence à la fonction réelle (c'est-à-dire les tâches) des personnes participant aux activités de R-D intra-muros de l'unité statistique. Sur le plan pratique, on sait que les unités déclarantes (voire les offices statistiques compilant les données de R-D) peuvent parfois utiliser des critères existants et facilement accessibles pour classer le personnel de R-D. Il peut donc être utile de mettre en évidence les critères qui ne deuraient pas être utilisés comme seules références pour classer le personnel de R-D dans les catégories " chercheurs ", " techniciens " ou " personnel de soutien ", même si ces critères peuvent faciliter la démarche :

- Le poste occupé. Bien que certaines personnes occupées aient un contrat de " chercheur ", cela ne veut pas nécessairement dire qu'elles effectuent des tâches de « chercheur » dans l'ensemble des activités de R-D de leur employeur. Il arrive que dans un projet de R-D particulier, un " chercheur » accomplisse des tâches de " technicien ". C'est cet aspect qu'il convient de prendre en compte dans les statistiques de R-D. Inversement, les personnes occupant un poste de " technicien " peuvent avoir à effectuer des tâches similaires à celles d'un " chercheur » sur un projet particulier : là encore, c'est cet aspect (c'est-àdire les tâches réellement accomplies par la personne) qui devrait être déclaré dans les enquêtes sur la R-D.

- Les qualifications ou le niveau d'études. Si les personnes titulaires d'un doctorat ont toutes les chances de participer à des projets de R-D en qualité de " chercheurs ", il ne faut pas croire que les tâches de R-D accomplies par les personnes sont toujours cohérentes avec leurs qualifications. À titre d'exemple, un technicien possédant un diplôme d'enseignement secondaire mais plusieurs années d'expérience professionnelle est susceptible, dans un contexte donné, d'accomplir des tâches similaires à celles d'un chercheur. 
- L'ancienneté de l'expérience. Il n'est pas rare que de jeunes « chercheurs " gèrent des projets de R-D complexes, alors que leurs collègues (ou des consultants) possédant plus d'expérience qu'eux remplissent des rôles différents (appui technique ou administratif, par exemple).

- Les liens au regard de l'emploi avec l'unité menant des travaux de R-D. Bien que la plupart des activités de R-D intra-muros soient gérées par le personnel interne (ou les propriétaires exploitants dans le cas des entreprises de petite taille), il peut aussi arriver que le personnel externe accomplisse les mêmes tâches de R-D que le personnel interne. Une unité statistique peut donc effectuer des travaux de R-D en s'appuyant uniquement sur les services du personnel externe.

5.34 Il peut parfois être nécessaire, pour les besoins de l'analyse, d'établir un lien entre les catégories de personnel de R-D définies ci-après et les données sur l'emploi et la main-d'œuvre provenant de classifications internationales types telles que la Classification internationale type des professions (CITP) (OIT, 2012) et la Classification internationale type de l'éducation (CITE) 2011 (UNESCO-ISU, 2012). Pour en savoir plus sur la ventilation du personnel de R-D et des chercheurs selon le niveau d'études, voir plus avant. Pour savoir dans quels grands groupes professionnels se classent les travailleurs de R-D, le document de référence à utiliser est le CITP-08 : les chercheurs sont classés dans le grand groupe 2, "Professions intellectuelles et scientifiques ", et dans "Directeurs et cadres de direction, recherche-développement » (CITP-08, 1223) ; les techniciens et le personnel assimilé sont classés dans le grand groupe 3, "Professions intermédiaires "; quant au personnel de soutien, il se trouve essentiellement dans les grands groupes 4, "Employés de type administratif ", 6, "Agriculteurs et ouvriers qualifiés de l'agriculture, de la sylviculture et de la pêche ", et 8, "Conducteurs d'installations et de machines, et ouvriers de l'assemblage ». Par convention, le personnel de R-D travaillant dans le domaine de la défense est classé dans le grand groupe 0, "Professions militaires ».

\section{Les chercheurs}

5.35 Les chercheurs sont les spécialistes travaillant à la conception ou à la création de nouveaux savoirs. Ils mènent des travaux de recherche en vue d'améliorer ou de mettre au point des concepts, théories, modèles, techniques, instruments, logiciels ou modes opératoires.

5.36 Les chercheurs peuvent être totalement ou partiellement engagés dans différents types d'activités (par exemple : recherche fondamentale ou appliquée, développement expérimental, maniement d'équipement de recherche, gestion de projet, etc.), et ce dans n'importe quel secteur de l'économie. Ils identifient des pistes pour de nouvelles activités de R-D et assurent leur planification et leur gestion en utilisant des compétences et des connaissances de haut niveau résultant d'une formation ou d'un enseignement structuré, ou de l'expérience 
concrète dans le domaine de la recherche. Les chercheurs jouent un rôle capital dans la conduite d'un projet ou d'une activité de R-D. Les projets de R-D sont généralement dirigés par des chercheurs (le reste du personnel de R-D pouvant être aux commandes de certains volets de ces projets). Par voie de conséquence, toute unité statistique effectuant des travaux de R-D possède au moins un chercheur ; ce dernier peut faire partie du personnel interne ou externe de l'unité, mais il ne doit pas forcément être occupé à temps plein par les activités de R-D.

5.37 En règle générale, les tâches assurées par les chercheurs dans le cadre de projets spécifiques ou d'activités générales de R-D sont les suivantes :

- réalisation de travaux de recherche, d'expériences, d'essais et d'analyses

- mise au point de concepts, théories, modèles, techniques, instruments, logiciels et modes opératoires

- collecte, traitement, évaluation, analyse et interprétation des données issues de la recherche

- évaluation des résultats des enquêtes et des expériences, et émission de conclusions en s'appuyant sur différents types de techniques et de modèles

- application de principes, de techniques et de processus pour mettre au point ou améliorer les applications concrètes

- émission d'avis sur la conception, la planification et l'organisation de l'expérimentation, la construction, l'installation et l'entretien des structures, des machines, des systèmes et de leurs composants

- conseils et services de soutien aux pouvoirs publics, aux organisations et aux entreprises pour l'application des résultats de la recherche

- planification, orientation et coordination des activités de R-D des organisations fournissant des services connexes à d'autres organisations

- élaboration de documents et rapports scientifiques.

5.38 Les cadres de direction et les administrateurs intervenant dans la gestion et la planification des aspects techniques et scientifiques des travaux d'un chercheur sont également classés dans la catégorie des « chercheurs ". Leur position hiérarchique au sein de l'unité est généralement égale ou supérieure à celle des personnes employées en tant que chercheurs, et ils occupent parfois un poste de chercheur à temps partiel.

5.39 Pour des raisons pratiques, les étudiants en doctorat participant à la R-D doivent être comptabilisés en tant que " chercheurs ". Ils possèdent généralement un diplôme universitaire de base (niveau 7 de la CITE) et effectuent des travaux de recherche tout en préparant leur thèse de doctorat (niveau 8 de la CITE). Lorsqu'ils ne peuvent être identifiés clairement, ces étudiants peuvent être comptabilisés soit avec les techniciens, soit avec les chercheurs, ce qui peut donner lieu à des incohérences dans les séries de données relatives aux chercheurs. 


\section{Les techniciens et le personnel assimilé}

5.40 Les techniciens et le personnel assimilé sont les personnes dont les tâches principales requièrent des connaissances et une expérience techniques dans un ou plusieurs domaines de l'ingénierie, des sciences physiques, des sciences de la vie, des sciences sociales, des sciences humaines et des arts. Ils participent à la R-D en exécutant des tâches scientifiques et techniques faisant intervenir l'application de concepts et de modes opératoires ou encore l'utilisation de matériel de recherche, habituellement sous la supervision de chercheurs.

5.41 Dans la plupart des cas, les techniciens et le personnel assimilé interviennent dans les projets de R-D sous la supervision des chercheurs. Leur rôle consiste essentiellement à mettre en œuvre - en fonction de leur expérience et de leurs qualifications - les instructions qui leur sont données par les chercheurs responsables de la gestion des projets.

5.42 Les techniciens et le personnel assimilé ont néanmoins tendance à jouir d'un degré élevé d'autonomie dans l'accomplissement de leurs tâches, car ce sont généralement des travailleurs hautement qualifiés. En règle générale, les tâches assurées par les techniciens et le personnel assimilé sont les suivantes :

- effectuer des recherches bibliographiques et trouver des éléments d'information appropriés dans les archives et les bibliothèques

- élaborer des programmes informatiques

- procéder à des expériences, des essais et des analyses

- fournir une assistance et un appui techniques à la R-D, ou tester des prototypes

- assurer le fonctionnement, l'entretien et la réparation de l'équipement de recherche

- préparer les matériaux et l'équipement nécessaires à la réalisation d'expériences, d'essais et d'analyses

- relever des mesures, faire des calculs et préparer des tableaux et des graphiques

- recueillir des informations en utilisant des méthodes scientifiques agréées

- aider à l'analyse des données, à la réalisation de dossiers et à l'élaboration de rapports

- mener des enquêtes statistiques et des interviews.

Le personnel de soutien

5.43 Le personnel de soutien comprend les travailleurs, qualifiés et non qualifiés, ainsi que le personnel administratif et les employés de bureau participant ou directement associés à des projets de R-D. 
5.44 Le personnel de soutien englobe toutes sortes de métiers et de compétences. En principe, toute activité qui contribue directement à la réalisation des travaux de R-D intra-muros mais n'est pas menée par des chercheurs ou des techniciens est le fait du personnel de soutien. Il est donc pratiquement impossible de fournir une liste exhaustive ou une description des activités potentielles de cette catégorie de personnel. Ces activités vont du travail administratif et de secrétariat à la fourniture ou à la gestion des matériaux et des appareils nécessaires à la mise en œuvre d'un projet de R-D. Cette catégorie de personnel assure généralement les fonctions de soutien en rapport avec la R-D telles que : planification, information et soutien financier, services juridiques et en matière de brevets, ou encore aide à l'assemblage, au réglage, à l'entretien et à la réparation des équipements et instruments scientifiques. Les cadres de direction et les administrateurs qui s'occupent principalement des questions financières, de la gestion du personnel et de l'administration générale - pour autant toutefois que leurs activités aient un rapport direct avec la R-D - sont classés dans la catégorie du personnel de soutien.

5.45 Un aspect important est que seuls les " services de soutien direct » sont pris en compte dans les statistiques du personnel de R-D. Ainsi, si le budget d'un projet de R-D de grande envergure est géré par un comptable salarié travaillant uniquement pour une équipe de recherche bien précise, on considère qu'il s'agit d'un service " direct » : le comptable est alors comptabilisé dans le " personnel de soutien » et les dépenses correspondantes liées à sa rémunération sont enregistrées dans les " coûts de main-d'œuvre " de l'unité statistique effectuant de la R-D. Dans un autre cas de figure, à savoir le service de comptabilité d'une grande entreprise chargé de gérer les budgets de plusieurs projets de R-D intra-muros menés par des équipes différentes, les activités en question sont considérées comme un service administratif "indirect " : aucun personnel de R-D n'est comptabilisé, et les frais administratifs générés par les activités de R-D sont inscrits, pour les besoins des enquêtes sur la R-D, dans les " autres dépenses courantes".

\subsection{Unités de mesure recommandées}

5.46 La mesure du personnel (interne et externe) de R-D comporte trois volets :

1. mesure des effectifs exprimés en nombre de personnes physiques

2. mesure des activités de R-D exprimées en équivalent temps plein ou en années-personnes

3. mesure des caractéristiques des individus.

5.47 L'intérêt de mesurer à la fois les effectifs en termes de personnes physiques et les activités en équivalent temps plein tient au constat que la R-D peut être la fonction principale de certaines personnes (par exemple, les chercheurs d'un laboratoire de R-D), mais une fonction secondaire pour d'autres 
(par exemple, les employés d'un bureau d'études et d'essais). La R-D peut aussi être une activité de premier plan exercée à temps partiel (par exemple, par les professeurs d'université, les étudiants en master/doctorat, les consultants et autres experts indépendants), sans nécessairement impliquer la participation à temps plein d'un personnel spécialement affecté à cette tâche. Le fait de ne prendre en compte que les personnes dont la R-D est la fonction principale entraînerait une sous-estimation des efforts consacrés à cette activité ; à l'opposé, le fait de comptabiliser toutes les personnes consacrant telle ou telle durée à des activités de R-D donnerait lieu à une surestimation. La comptabilisation des personnes engagées dans des travaux de R-D doit donc être exprimée à la fois en nombre de personnes physiques et en équivalent temps plein (ETP), ces deux types de données fournissant des informations complémentaires.

5.48 Afin d'assurer la compatibilité entre les deux séries de données (personnes physiques et ETP), une approche intégrée de la mesure du personnel de R-D est proposée. Ses principes sont les suivants :

- L'ETP est la principale donnée statistique utilisée dans les comparaisons internationales du personnel de R-D.

- Les données relatives aux personnes physiques sont surtout recommandées pour étudier - généralement en termes de pourcentage - les caractéristiques du personnel de R-D.

- Le recueil direct de données sur le personnel de R-D est la méthode recommandée pour produire des séries de données à la fois sur les effectifs et sur l'ETP.

- Les données sur les effectifs et l'ETP doivent être cohérentes, même si elles n'ont pas été recueillies lors d'un même exercice statistique ou ne résultent pas d'un effort de collecte coordonné de la part de sources statistiques et/ou administratives différentes.

- Lorsque le recueil direct de données n'est pas possible, un processus d'estimation peut être engagé pour obtenir à partir des données administratives des indicateurs sur les personnes physiques et l'ETP.

- Qu'elles soient obtenues ex ante ou ex post, les données relatives au personnel de R-D doivent être cohérentes avec les données relatives aux dépenses de $\mathrm{R}-\mathrm{D}$, principalement avec les rubriques « Coûts de main-d'œuvre » et " Autres dépenses courantes - personnel externe de R-D ».

\section{Équivalent temps plein (ETP) de personnel de R-D}

5.49 L'équivalent temps plein (ETP) de personnel de R-D est défini par le nombre d'heures de travail réellement consacrées à la $R-D$ au cours d'une période de référence donnée (habituellement une année civile) rapporté au nombre total d'heures qu'une personne ou un groupe de personnes est censé travailler au cours de la même période. 
5.50 Les offices statistiques nationaux compilant des données sur la R-D devraient accorder une attention particulière à la mesure du temps de travail total, car il sert de base au calcul de l'équivalent temps plein (ETP) de personnel de R-D. Bien que l'évaluation du temps de travail total et du temps consacré à la R-D ne soit pas nécessairement facile (en particulier pour ce qui concerne le personnel externe de R-D), on sait qu'une personne ne peut représenter plus d'un ETP au cours d'une seule année, et donc qu'elle ne peut consacrer plus d'un ETP à la R-D pendant une année.

5.51 Dans la pratique, cependant, ce principe n'est pas toujours facile à appliquer. Certains chercheurs peuvent, par exemple, avoir des activités dans plusieurs unités de R-D. C'est en effet une situation courante pour les universitaires qui interviennent également en tant que consultants extérieurs pour la R-D auprès des entreprises. Dans ce type de cas, il peut être nécessaire de ramener l'ETP d'un individu à un, si l'on dispose d'informations concernant ses contributions à la R-D dans plusieurs unités statistiques. Le même principe est à adopter lorsque l'ETP total consacré à la R-D est évalué à partir des données administratives.

5.52 Pour être comptabilisé dans le total du personnel de R-D, un individu doit contribuer de façon suffisante aux travaux de R-D. Par conséquent, qu'il s'agisse du personnel interne ou externe, il est recommandé d'exprimer l'ETP en valeurs décimales et d'évaluer l'importance de la contribution aux travaux de R-D d'une unité par les personnes consacrant moins de $\mathbf{0 . 1}$ ETP à la R-D sur une base annuelle (c'est-à-dire $10 \%$ du temps de travail total, soit une vingtaine de jours par an).

5.53 Il convient de noter que lorsque de très faibles contributions à la R-D, en termes de temps de travail, sont comptabilisées dans le total du personnel de R-D (quelques jours par an, par exemple), il risque d'être difficile - à la fois au niveau de l'unité et au niveau global - de rendre correctement compte des caractéristiques du personnel de R-D (leur fonction au regard de la R-D, par exemple), comme indiqué dans la section 5.2 et explicité plus avant dans la section 5.4 .

5.54 L'ETP de personnel de R-D correspond aux travaux de R-D exécutés pendant un an par l'ensemble des personnes (personnel interne et personnel externe de R-D, y compris les bénévoles) ayant participé aux activités de R-D intra-muros d'une unité statistique, d'un secteur institutionnel ou d'un pays.

5.55 L'ETP du total du personnel interne de R-D correspond aux travaux de R-D exécutés pendant un an par l'ensemble du personnel interne ayant participé aux activités de R-D intra-muros d'une unité statistique, d'un secteur institutionnel ou d'un pays.

5.56 Il convient d'indiquer explicitement aux unités déclarantes d'inclure dans l'ETP total les contributions à la R-D (en termes d'heures de travail) des personnes travaillant à temps plein et à temps partiel, y compris le personnel 
permanent et temporaire. La section consacrée à l'estimation de l'ETP fournit des exemples de la manière dont les unités déclarantes peuvent procéder pour évaluer en équivalent temps plein les contributions à la R-D des différentes catégories de personnel si elles ne conservent pas un suivi détaillé des activités de R-D de leur personnel.

5.57 Bien qu'il soit recommandé, pour obtenir l'ETP du total du personnel de R-D, que cet ETP soit calculé pour chacune des personnes ayant participé à la $\mathrm{R}-\mathrm{D}$ intra-muros pendant la période de référence, certaines unités statistiques répondant aux enquêtes de R-D décident parfois de prendre pour base de calcul une " moyenne " de ces individus. Lorsque c'est le cas, il est fortement recommandé de vérifier la cohérence entre l'ETP et les effectifs qui sont déclarés pour l'ensemble du personnel de R-D. Par convention, toute mesure du personnel de R-D exprimée en ETP devrait être inférieure ou égale à la même mesure exprimée en nombre de personnes physiques, quel que soit le niveau d'agrégation.

\section{Effectifs du personnel de R-D}

5.58 L'effectif de R-D en nombre de personnes physiques correspond au nombre total des individus qui participent à la R-D intra-muros, au niveau d'une unité statistique ou à un niveau agrégé, au cours d'une période de référence donnée (habituellement une année civile). Il peut être exprimé de diverses manières :

- par le nombre de personnes affectées à des travaux de R-D à une date donnée (par exemple, fin de la période)

- par le nombre moyen de personnes affectées à des travaux de R-D pendant l'année (civile)

- par le nombre total de personnes affectées à des travaux de R-D pendant l'année (civile).

Ces trois options peuvent produire des résultats différents, la troisième risquant de donner lieu à une double comptabilisation. L'option à privilégier pour calculer les effectifs de R-D est la première. La date retenue pour le calcul devrait, de préférence, être la même pour l'ensemble des unités déclarantes de tous les secteurs du pays déclarant. Le choix de cette date doit tenir compte d'une éventuelle variation saisonnière et d'autres facteurs pouvant biaiser les valeurs totales sur l'année ; il est donc laissé à chaque pays le soin de la fixer, car le caractère saisonnier peut varier considérablement selon les pays. Dans la mesure du possible, la date choisie devrait être la même que celle utilisée pour recueillir d'autres données statistiques relatives aux personnes physiques (par exemple, sur l'emploi ou l'éducation), susceptibles d'être comparées avec les données relatives à la $\mathrm{R}-\mathrm{D}$.

5.59 Lorsque l'on produit des données sur le personnel de R-D, la cohérence entre les valeurs totales de l'ETP et des effectifs est une priorité. Cela signifie que 
tous les individus inclus dans le total de l'ETP doivent aussi être pris en compte dans le nombre total de personnes physiques, autrement dit que toute personne ayant participé à la R-D intra-muros pendant la période de référence doit être comptabilisée de façon homogène au titre de l'ETP et du nombre de personnes physiques. De la même manière, les personnes qui participent à la R-D mais ne sont pas prises en compte dans le total de l'ETP (c'est-à-dire les personnes consacrant moins de 0.1 ETP à la R-D sur une base annuelle) ne doivent pas non plus être comptabilisées dans l'effectif total de R-D. Il convient de noter que la comptabilisation de tous les individus, y compris ceux contribuant de façon très minime aux activités de $R-D$, risque de gonfler considérablement le total du personnel de R-D, et donc de rendre les comparaisons internationales problématiques et l'analyse des caractéristiques du personnel de R-D difficile (voir la section 5.4).

5.60 Lorsque l'on calcule le nombre de personnes physiques, le risque est que les individus qui participent à la R-D d'au moins deux unités statistiques (des entreprises ou autres entités) soient comptabilisés deux fois. L'indicateur peut alors être interprété comme la somme de plusieurs emplois. L'utilisation $\mathrm{du}$ total de l'ETP fournit une estimation plus fiable des ressources humaines engagées dans la R-D.

5.61 Lorsque les données dont on dispose sur les caractéristiques du personnel externe de R-D sont incomplètes, il est préférable, plutôt que de ne pas en tenir compte pour calculer le total des effectifs, de les compléter en recueillant le plus d'informations possible à cet égard et de comptabiliser le personnel externe séparément du personnel interne. Dans l'idéal, il conviendrait de mesurer les indicateurs de base du personnel de R-D - aussi bien l'ETP que le nombre de personnes physiques -en tenant compte de la situation au regard de l'emploi des individus, comme indiqué à la section 5.2, à savoir : les personnes occupées (dont la rémunération est comptabilisée dans les " coûts de main-d'œuvre ") ; le personnel externe rémunéré (dont la rémunération est comptabilisée dans la sous-rubrique " Autres dépenses courantes - personnel externe de R-D ") ; toutes les autres personnes (qui contribuent à la R-D intramuros sans être rémunérées). En résumé, pour calculer les effectifs de R-D en s'appuyant sur le recueil direct de données (les enquêtes), les recommandations sont les suivantes :

- Recenser le total du personnel de R-D, comprenant le personnel interne participant à la R-D et l'ensemble du personnel externe contribuant aux activités de R-D intra-muros. (En ce qui concerne le personnel interne, il est recommandé d'utiliser comme références des registres administratifs actualisés et, pour le secteur des entreprises, des registres officiels du commerce, le cas échéant.)

- Recueillir et comptabiliser séparément les données relatives au personnel interne de R-D et celles relatives au personnel externe (qui comprend à la fois des personnes rémunérées et d'autres qui ne le sont pas). Les étudiants 
travaillant dans le domaine de la recherche et faisant partie du personnel externe de R-D sont à comptabiliser séparément.

- En ce qui concerne le calcul des effectifs, établir des séries chronologiques distinctes pour les deux groupes composant le personnel de R-D. L'unité statistique doit disposer d'un certain nombre de caractéristiques de base sur ces groupes de travailleurs, qui comprennent à la fois le personnel interne travaillant dans l'unité et participant à la R-D, et le personnel externe ayant participé "sur site ", ou tout au moins à proximité, aux activités de R-D de l'unité. Si ce n'est pas possible, recueillir au minimum des données sur le personnel interne de R-D ayant participé à la R-D intra-muros pendant la période de référence.

\section{Recueil de données coordonné sur l'ETP et le nombre de personnes physiques}

5.62 Le processus de recueil de données sur l'ETP et le nombre de personnes physiques est présenté de façon synthétique dans l'encadré 5.1.

\section{Encadré 5.1. Recueil et comptabilisation des données}

1. Nombre total de personnes occupées au sein de l'unité statistique ayant effectué des travaux de R-D intra-muros pendant la période de référence (données provenant habituellement des registres d'entreprises). Toutes ces personnes sont " potentiellement » des contributeurs à la R-D $\left(\mathrm{N}_{\mathrm{t}}\right)$.

2. Personnes occupées au sein de l'unité statistique (personnes physiques, ou PP) et ayant réellement contribué aux activités de R-D intra-muros pendant l'année de référence $\left(\mathrm{PP}_{\text {int }}\right)$.

3. Personnes occupées au sein de l'unité statistique et ayant réellement contribué aux activités de R-D intra-muros pendant l'année de référence, pondérées par la part du temps de travail réellement consacrée à la R-D ; $100 \%=1\left(\right.$ ETP $\left._{\text {int }}\right)$.

4. Personnel externe de R-D (y compris les personnes non rémunérées) ayant réellement contribué aux activités de $\mathrm{R}-\mathrm{D}$ intra-muros pendant l'année de référence $\left(\mathrm{PP}_{\text {ext }}\right)$.

5. Personnel externe de R-D (y compris les personnes non rémunérées) ayant réellement contribué aux activités de R-D intra-muros pendant l'année de référence, pondéré par la part du temps de travail réellement consacrée à la R-D ; $100 \%=1\left(\right.$ ETP $\left._{\text {ext }}\right)$.

Total du personnel de R-D $(\mathrm{PP})=\mathrm{PP}_{\text {int }}+\mathrm{PP}_{\text {ext }}$

Total du personnel de R-D (ETP) $=\mathrm{ETP}_{\text {int }}+\mathrm{ETP}_{\text {ext }}$

Ratio Personnes occupées à des activités de R-D/Nombre total de personnes occupées $=\mathrm{ETP}_{\text {int }} / \mathrm{N}_{\mathrm{t}}$ 
5.63 Il est vivement recommandé que les enquêtes sur la $\mathrm{R}-\mathrm{D}$ recueillent sur un même formulaire, directement auprès des unités menant des travaux de $\mathrm{R}-\mathrm{D}$, l'ensemble des données disponibles concernant à la fois les dépenses en matière de R-D et le personnel de R-D (personnes physiques), ainsi que le niveau de participation de ces personnes (représenté par l'ETP). Cette méthode limite au minimum les coûts de la collecte de données et assure une cohérence maximale entre les différents indicateurs de la R-D. La vérification de la cohérence entre les dépenses et le personnel de R-D devrait faire partie du processus de recueil des données. Par convention, la valeur de l'ETP devrait être inférieure ou égale au nombre de personnes physiques (PP).

5.64 Les liens entre les dépenses de R-D et les valeurs relatives au personnel de R-D (effectifs et ETP) sont présentés de façon synthétique dans le tableau 5.3.

Tableau 5.3. Cohérence des données sur la R-D

\begin{tabular}{|c|c|c|c|c|}
\hline Dépenses de R-D & & ETP total de personnel de R-D & & $\begin{array}{l}\text { Effectif total } \\
\text { du personnel de R-D }\end{array}$ \\
\hline Coûts de main-d'œuvre & $\leftrightarrow$ & $\begin{array}{l}\text { Personnel interne de R-D } \\
\text { (personnes occupées) }\end{array}$ & $\leq$ & $\begin{array}{l}\text { Personnel interne } \\
\text { de R-D (personnes } \\
\text { occupées) }\end{array}$ \\
\hline $\begin{array}{l}\text { Autres dépenses } \\
\text { courantes - personnel } \\
\text { externe de R-D }\end{array}$ & $\leftrightarrow$ & Personnel externe de R-D & $\leq$ & $\begin{array}{l}\text { Personnel externe } \\
\text { de R-D }\end{array}$ \\
\hline Non applicable & & Personnel de R-D non rémunéré & $\leq$ & $\begin{array}{l}\text { Personnel de R-D } \\
\text { non rémunéré }\end{array}$ \\
\hline
\end{tabular}

\section{Estimation des indicateurs du personnel de R-D : l'ETP et les effectifs Estimation de l'ETP}

5.65 La collecte directe de données sur l'ETP et les effectifs de R-D n'est pas toujours possible. Il peut alors être nécessaire d'estimer ces indicateurs à partir des données disponibles auprès de sources autres que les enquêtes directes. Lorsque c'est le cas, il est vivement recommandé aux offices de statistiques nationaux de vérifier la cohérence entre le montant déclaré des dépenses de R-D et l'estimation du total du personnel de R-D.

5.66 L'estimation de l'ETP de personnel de R-D devrait être effectuée par l'unité déclarante, au moyen des informations recueillies au niveau de l'entité (par exemple des données administratives) ou, parfois, des individus (comme par exemple les enquêtes d'emploi du temps). En l'absence d'autres sources de données fiables, les enquêtes d'emploi du temps peuvent procurer des informations utiles; elles sont couramment utilisées pour estimer l'ETP de personnel de R-D dans le secteur de l'enseignement supérieur. Pour en savoir plus à ce sujet, voir le chapitre 9.

5.67 La première étape pour estimer l'ETP de personnel de R-D consiste à recueillir des informations détaillées (auprès de sources administratives si l'on ne dispose pas de données d'enquêtes) sur la participation réelle ou contractuelle 
(normative/statutaire) du personnel de R-D à la R-D intra-muros. Cette étape peut être très simple dans le cas des établissements publics de recherche (ou des universités), car ce sont des secteurs où les rôles professionnels et les situations au regard de l'emploi sont souvent définis de façon formelle.

5.68 Les données concernant le personnel affecté aux travaux de R-D à temps plein ou à temps partiel sont à recueillir et comptabiliser séparément selon qu'il s'agit du « personnel interne » ou du « personnel externe » de R-D. Une personne travaillant à temps plein sera donc identifiée sur la base de sa situation au regard de l'emploi, de son type de contrat (en l'occurrence à temps plein) et de son degré de participation à la R-D.

5.69 Dans ces conditions, 1 ETP correspond à un individu se consacrant à temps plein à la R-D. Cet indicateur s'obtient à l'aide de deux variables : la participation effective aux activités de R-D et la participation théorique sur la base du temps de travail normatif/statutaire. Lorsque l'on ne dispose pas d'informations sur l'une de ces deux variables, le calcul peut s'appuyer sur l'une des deux seulement.

5.70 L'estimation de l'ETP implique en outre de :

- comptabiliser le temps consacré par un individu ou un groupe de contributeurs à la R-D, par rapport au temps consacré à d'autres activités

- prendre en compte les différentes situations au regard de l'emploi du personnel de R-D considéré, à savoir emploi à temps plein, à temps partiel ou temporaire

- sélectionner les sources de données et les méthodes appropriées pour établir les valeurs totales de l'ETP.

5.71 Le processus d'estimation peut se résumer par la formule suivante :

$$
\mathrm{ETP}=\mathrm{RDtp}+(\mathrm{nhtRD} / \mathrm{nnsh} \text { ) }
$$

où

RDtp = nombre de personnes travaillant sur la R-D à temps plein nhtRD = nombre d'heures de travail consacrées à la R-D par d'autres catégories de personnel

nnsht $=$ nombre normatif/statutaire d'heures de travail pour le personnel à temps plein dans un secteur/pays donné.

5.72 S'agissant du personnel ne travaillant pas à temps plein sur la R-D, différents cas de figure peuvent être recensés :

- Les personnes occupées travaillent à temps partiel sur la R-D, que ce soit en raison d'horaires de travail réduits ou d'une participation limitée aux activités de R-D.

- Les personnes occupées (ou le personnel externe de R-D) ont un contrat temporaire pendant la période utilisée comme référence pour le calcul de l'ETP (par exemple, une année civile). 
5.73 Les exemples suivants montrent comment la formule peut être utilisée pour calculer l'ETP :

- un salarié à temps plein qui consacre $100 \%$ de son temps à la R-D au cours d'une année = 1 ETP

- un salarié à temps plein qui consacre $30 \%$ de son temps à la R-D au cours d'une année $=0.3 \mathrm{ETP}$

- un salarié à temps plein d'un établissement de R-D sur une période de six mois, qui consacre $100 \%$ de son temps à la R-D = 0.5 ETP

- un salarié à temps plein qui consacre $40 \%$ de son temps à la R-D pendant la moitié de l'année (cette personne n'est active que six mois par an) =0.2 ETP

- un salarié à temps partiel (40\%) qui consacre $100 \%$ de son temps à la R-D au cours d'une année $=0.4$ ETP

- un salarié à temps partiel (40\%) qui consacre $60 \%$ de son temps à la R-D pendant la moitié de l'année (cette personne n'est active que six mois par an) = 0.12 ETP.

\section{Estimation des effectifs}

5.74 Lors du recueil d'informations sur la taille et la composition du personnel de R-D en termes de personnes physiques, il est recommandé d'utiliser au maximum les registres administratifs et du personnel (y compris, le cas échéant, les données relatives à la masse salariale), ainsi que les registres d'enterprises, lorsqu'ils existent. Dans la mesure où les offices de statistiques nationaux ne peuvent produire des effectifs cohérents pour tous les groupes composant le personnel de R-D (le personnel interne, le personnel externe rémunéré et le personnel externe non rémunéré/bénévole), il leur est recommandé de se concentrer sur les effectifs du personnel interne de R-D (c'est-à-dire les personnes employées par l'unité statistique menant des travaux de R-D intra-muros).

\subsection{Ventilation recommandée des données globales du personnel de R-D}

\section{Caractéristiques du personnel de R-D}

5.75 Pour répondre aux besoins des utilisateurs des données, les valeurs globales de l'ETP et des effectifs du personnel de R-D doivent être ventilées, dans la mesure du possible, sur la base de différents critères tels que : le sexe, la fonction, la situation au regard de l'emploi, l'âge et le niveau d'études, mais aussi le grade d'ancienneté, l'origine géographique et les flux de personnel.

\section{Le sexe}

5.76 Conformément à la Déclaration de Beijing diffusée à l'issue de la Conférence mondiale sur les femmes de 1995 (Nations Unies, 1995), il convient 
de prendre des dispositions pour que les données statistiques relatives au personnel de R-D soient ventilées par sexe. Ce principe s'applique à la fois aux valeurs globales de l'ETP et des effectifs, et suppose l'utilisation d'informations fiables sur les individus appartenant aux deux groupes du personnel de R-D : le personnel interne et le personnel externe.

\section{La fonction au sein de la R-D}

5.77 Un critère de ventilation essentiel est celui de la fonction occupée par le personnel de R-D (chercheurs, techniciens et personnel assimilé, et personnel de soutien). Même lorsque la collecte directe de données sur le personnel pose problème, le fait d'identifier « qui fait quoi » dans les projets de R-D intra-muros permet de vérifier l'exactitude des données déclarées par les unités statistiques en ce qui concerne les travaux de R-D. De surcroît, la collecte et la comptabilisation de ces données par fonction met en évidence la contribution des chercheurs à l'effort global de R-D au sein d'une unité statistique, d'un secteur économique ou de l'économie tout entière. Cette ventilation est recommandée à la fois pour les valeurs de l'ETP et des effectifs du personnel de R-D (interne et externe).

\section{La situation au regard de l'emploi}

5.78 Il est recommandé que tout individu comptabilisé dans le total du personnel de R-D soit identifié comme faisant partie soit du personnel interne (c'est-à-dire comme une " personne occupée » dont la rémunération est comptabilisée dans les " coûts de main-d’œuvre »), soit du personnel externe rémunéré (dont la rémunération est incluse dans une sous-rubrique spécifique, celle des " autres dépenses courantes"), ou du personnel externe composé de bénévoles ou personnes au statut équivalent (qui contribuent à l'activité de R-D intra-muros sans être rémunérés). Cette ventilation est recommandée à la fois pour les valeurs de l'ETP et les effectifs du personnel de R-D.

Il peut également être utile, lorsque c'est possible, de ventiler les données entre le personnel permanent et le personnel temporaire. Certains indicateurs (comme par exemple le salaire annuel moyen d'un chercheur) peuvent n'être disponibles que pour les personnes employées (on peut supposer que ce type de donnée figure dans les dossiers de leurs employeurs).

\section{L'âge}

5.79 Pour ventiler le personnel de R-D, et en particulier les " chercheurs ", d'après le critère de l'âge, il est recommandé d'utiliser les six catégories ci-dessous, qui sont conformes aux Directives provisoires concernant les classifications internationales types par âge des Nations Unies (Nations Unies, 1982) :

- moins de 25 ans

- de 25 à 34 ans

- de 35 à 44 ans 
- de 45 à 54 ans

- de 55 à 64 ans

- 65 ans et plus.

5.80 Pour ce qui est du personnel externe de R-D, il est rare de disposer de données sur l'âge car cette donnée n'est généralement pas (ou ne peut pas être) un critère déterminant pour recruter du personnel appelé à contribuer à la R-D intra-muros d'une unité statistique. Néanmoins, dans la mesure où les données sur l'âge suscitent un vif intérêt, la collecte de cette information - pour le personnel interne et le personnel externe de R-D - ne sera privilégiée que si elle est fiable.

\section{Le niveau d'études}

5.81 S'agissant de la ventilation du personnel de R-D, et en particulier des " chercheurs ", selon le niveau d'études, le document de référence est la Classification internationale type de l'éducation (CITE) 2011 (UNESCO-ISU, 2012). Cinq catégories sont recommandées comme faisant l'objet des statistiques de R-D : les niveaux 5, 6, 7 et 8 de la CITE pris individuellement, et les niveaux 1 à 4 pris collectivement. Une ventilation entre ces cinq catégories devrait permettre une comparaison approfondie avec les autres statistiques économiques et sociales.

5.82 Les niveaux de la CITE sont définis exclusivement sur la base du niveau d'études, sans considération du domaine de qualification.

- Titulaires de diplômes universitaires au niveau du doctorat ou équivalent (niveau 8 de la CITE). Cela inclut les diplômes délivrés par des universités proprement dites ainsi que par des établissements spécialisés ayant statut d'université.

- Titulaires de diplômes universitaires au niveau du master ou équivalent (niveau 7 de la CITE). Cela inclut les diplômes délivrés par des universités proprement dites ainsi que par des établissements d'enseignement supérieur équivalents.

- Titulaires de diplômes universitaires au niveau de la licence ou équivalent (niveau 6 de la CITE). Cela inclut les diplômes délivrés par des universités proprement dites ainsi que par des établissements d'enseignement supérieur équivalents.

- Titulaires de diplômes de l'enseignement supérieur de cycle court (niveau 5 de la CITE). Les études débouchent en général sur une spécialisation dans un domaine précis et à un niveau qui nécessite d'avoir reçu, au préalable, un enseignement complet de niveau secondaire ou son équivalent. L'enseignement dispensé est de nature plus pratique et prépare à un métier plus précis ; il peut en outre préparer à d'autres programmes de l'enseignement supérieur.

- Titulaires de diplômes de l'enseignement post-secondaire non supérieur (niveau 4 de la CITE). Cela inclut les diplômes fournissant aux étudiants ayant achevé le niveau 3 les qualifications requises pour poursuivre des études supérieures ou obtenir un emploi, perspectives que ne leur permettent pas les qualifications du niveau 3. 
- Titulaires de diplômes du deuxième cycle de l'enseignement secondaire (niveau 3 de la CITE). Cela inclut non seulement les diplômes du niveau 3 de la CITE, obtenus à l'issue d'études dans un établissement d'enseignement secondaire, mais aussi les diplômes de formation professionnelle de niveau équivalent au niveau 3, obtenus dans un autre type d'établissement d'enseignement.

- Autres qualifications. Cela inclut les diplômes inférieurs au niveau 3 de la CITE ou les études ne pouvant être classées dans aucun des six niveaux ci-dessus.

5.83 On sait cependant qu'il n'est pas facile de recueillir des informations fiables sur le niveau d'études du personnel interne (et encore moins lorsqu'il s'agit du personnel externe), et que les employeurs ne conservent pas nécessairement des données actualisées concernant le niveau d'études de leurs employés. À cet égard, pour ce qui est de la ventilation du personnel de R-D et des chercheurs d'après le niveau d'études, la priorité en matière de collecte d'informations doit aller au personnel interne de R-D.

\section{Le niveau de grade}

5.84 Les données relatives au niveau de grade peuvent permettre d'en savoir plus sur les pratiques de gestion de la R-D et fournir de précieux renseignements sur les parcours professionnels des chercheurs. La priorité, à cet égard, est de recueillir cette information pour le personnel interne de R-D travaillant dans les secteurs de l'État (voir le chapitre 8) et de l'enseignement supérieur (voir le chapitre 9).

\section{L'origine géographique}

5.85 Les utilisateurs des statistiques sont également intéressés par l'origine géographique du personnel de R-D, principalement pour analyser la mobilité internationale de ce personnel en général et des chercheurs en particulier. L'origine géographique peut se définir à l'aide de différents critères : la nationalité, la citoyenneté ou le pays de naissance. D'autres encore peuvent présenter un intérêt, comme par exemple le pays où se trouvait la résidence ou l'emploi précédent(e), ou celui où ont été menées les études les plus poussées. Tous présentent des avantages et des inconvénients et fournissent des informations de type différent. La combinaison d'au moins deux de ces critères permet d'obtenir une richesse d'informations beaucoup plus importante en vue de l'analyse. Néanmoins, il n'est pas facile de trouver des sources d'informations fiables lorsque l'on collecte ces données auprès des employeurs. La priorité doit être de recueillir cette information pour le personnel interne de R-D.

\section{Les flux de personnel}

5.86 Pour finir, les indicateurs relatifs aux flux du personnel de R-D (les nouvelles recrues, d'une part ;les personnes qui partent ou prennent leur retraite, d'autre part) sont souvent demandés par les utilisateurs de statistiques 
pour compléter les informations disponibles - qui concernent principalement le stock de personnel de R-D. Ce type d'information permet aux employeurs, aux analystes et aux responsables de l'action publique d'anticiper les besoins et les éventuelles pénuries de personnel de R-D. Ces indicateurs sont particulièrement utiles au niveau sectoriel, raison pour laquelle la priorité doit être de collecter ces informations uniquement pour le personnel interne de R-D.

\section{Tableaux recommandés pour la comptabilisation du personnel de R-D et des chercheurs (ETP et effectifs)}

5.87 Dans la mesure du possible, les pays devraient recueillir les informations sur les caractéristiques socio-économiques en dissociant le personnel interne (les personnes occupées) et le personnel externe de R-D. Au fil du temps, ces ensembles de données permettront d'obtenir un tableau plus complet de toutes les personnes participant à la R-D, et d'améliorer les comparaisons internationales. Il est cependant indéniable que le recueil de ces informations risque d'être difficile, voire impossible, pour certains pays, et que les comparaisons internationales risquent de conduire à des conclusions erronées si les pays produisent des agrégats différents pour le total du personnel de R-D. Il est donc recommandé que pour les variables socio-économiques suivantes, la priorité soit de fournir des agrégats nationaux pour le personnel interne de R-D, et en particulier pour les chercheurs. Pour les besoins de la présentation, et pour différencier ces valeurs globales de celles incluant le personnel externe de R-D, ces agrégats sont appelés « Total national du personnel de R-D occupé». En revanche, les agrégats regroupant le personnel interne et le personnel externe de R-D sont appelés « Total national du personnel de R-D ».

5.88 Il est recommandé d'utiliser les exemples de tableaux ci-après pour présenter les totaux du personnel de R-D.

\section{Tableau 5.4.a. Total national du personnel de R-D selon le secteur} et la situation au regard de l'emploi

(ETP et effectifs, par sexe)

\begin{tabular}{|c|c|c|c|c|c|}
\hline & \multicolumn{5}{|c|}{ Secteur } \\
\hline & Entreprises & État & $\begin{array}{l}\text { Enseignement } \\
\text { supérieur }\end{array}$ & $\begin{array}{l}\text { Privé sans } \\
\text { but lucratif }\end{array}$ & Total \\
\hline \multicolumn{6}{|l|}{$\begin{array}{l}\text { a) Personnel interne de R-D } \\
\text { (c.-à-d. personnes occupées) }\end{array}$} \\
\hline \multicolumn{6}{|l|}{$\begin{array}{l}\text { b) Personnel externe de R-D (dont les } \\
\text { coûts sont comptabilisés dans la rubrique } \\
\text { "Autres dépenses courantes - } \\
\text { personnel externe de R-D ») }\end{array}$} \\
\hline \multicolumn{6}{|l|}{$\begin{array}{l}\text { c) Personnel externe de R-D non rémunéré } \\
\text { (y compris les bénévoles et les professeurs } \\
\text { émérites) }\end{array}$} \\
\hline Total & & & & & \\
\hline
\end{tabular}




\section{Tableau 5.4.b. Total des chercheurs selon le secteur et la situation au regard de l'emploi}

(ETP et effectifs, par sexe)

\begin{tabular}{|c|c|c|c|c|c|}
\hline & \multicolumn{5}{|c|}{ Secteur } \\
\hline & Entreprises & État & $\begin{array}{l}\text { Enseignement } \\
\text { supérieur }\end{array}$ & $\begin{array}{l}\text { Privé sans but } \\
\text { lucratif }\end{array}$ & Total \\
\hline $\begin{array}{l}\text { a) Chercheurs internes } \\
\text { (c.-à-d. personnes occupées) }\end{array}$ & & & & & \\
\hline $\begin{array}{l}\text { b) Chercheurs externes (dont les } \\
\text { coûts sont comptabilisés dans } \\
\text { la rubrique «Autres dépenses } \\
\text { courantes - personnel externe } \\
\text { de R-D») }\end{array}$ & & & & & \\
\hline $\begin{array}{l}\text { c) Chercheurs externes non } \\
\text { rémunérés (y compris les bénévoles } \\
\text { et les professeurs émérites) }\end{array}$ & & & & & \\
\hline Total & & & & & \\
\hline
\end{tabular}

Tableau 5.4.c. Total national du personnel de R-D occupé selon le secteur et la fonction au sein de la R-D

(ETP et effectifs, par sexe)

\begin{tabular}{|c|c|c|c|c|c|}
\hline & \multicolumn{5}{|c|}{ Secteur } \\
\hline & Entreprises & État & $\begin{array}{c}\text { Enseignement } \\
\text { supérieur }\end{array}$ & $\begin{array}{l}\text { Privé sans } \\
\text { but lucratif }\end{array}$ & Total \\
\hline \multicolumn{6}{|l|}{ Chercheurs } \\
\hline \multicolumn{6}{|l|}{$\begin{array}{l}\text { Techniciens et personnel } \\
\text { assimilé }\end{array}$} \\
\hline \multicolumn{6}{|l|}{ Personnel de soutien } \\
\hline Total & & & & & \\
\hline
\end{tabular}

Tableau 5.4.d. Total national des chercheurs occupés selon le secteur et l'âge

(effectifs, par sexe)

\begin{tabular}{|c|c|c|c|c|c|}
\hline & \multicolumn{5}{|c|}{ Secteur } \\
\hline & Entreprises & État & $\begin{array}{c}\text { Enseignement } \\
\text { supérieur }\end{array}$ & $\begin{array}{l}\text { Privé sans but } \\
\text { lucratif }\end{array}$ & Total \\
\hline \multicolumn{6}{|l|}{ Moins de 25 ans } \\
\hline \multicolumn{6}{|l|}{ De 25 à 34 ans } \\
\hline \multicolumn{6}{|l|}{ De 35 à 44 ans } \\
\hline \multicolumn{6}{|l|}{ De 45 à 54 ans } \\
\hline \multicolumn{6}{|l|}{ De 55 à 64 ans } \\
\hline \multicolumn{6}{|l|}{65 ans et plus } \\
\hline Total & & & & & \\
\hline
\end{tabular}




\section{Tableau 5.4.e. Total national des chercheurs employés selon le secteur et le niveau d'études}

(ETP, par sexe)

\begin{tabular}{|c|c|c|c|c|c|}
\hline & \multicolumn{5}{|c|}{ Secteur } \\
\hline & Entreprises & État & $\begin{array}{l}\text { Enseignement } \\
\text { supérieur }\end{array}$ & $\begin{array}{l}\text { Privé sans but } \\
\text { lucratif }\end{array}$ & Total \\
\hline \multicolumn{6}{|l|}{ Diplômes de l'enseignement supérieur } \\
\hline \multicolumn{6}{|l|}{$\begin{array}{l}\text { Doctorat ou équivalent (niveau } 8 \\
\text { de la CITE) }\end{array}$} \\
\hline \multicolumn{6}{|l|}{$\begin{array}{l}\text { Master ou équivalent (niveau } 7 \\
\text { de la CITE) }\end{array}$} \\
\hline \multicolumn{6}{|l|}{$\begin{array}{l}\text { Licence ou équivalent (niveau } 6 \\
\text { de la CITE) }\end{array}$} \\
\hline \multicolumn{6}{|l|}{$\begin{array}{l}\text { Diplômes de l'enseignement supérieur } \\
\text { de cycle court (niveau } 5 \text { de la CITE) }\end{array}$} \\
\hline \multicolumn{6}{|l|}{$\begin{array}{l}\text { Autres diplômes } \\
\text { (niveaux } 1 \text { à } 4 \text { de la CITE) }\end{array}$} \\
\hline Total & & & & & \\
\hline
\end{tabular}

\section{Références}

Nations Unies (2009), " Recommandations internationales concernant les statistiques industrielles, 2008 ", Études statistiques, Série M, n 90, Nations Unies, New York, http://unstats.un.org/unsd/publication/seriesM/seriesm_90f.pdf.

Nations Unies (1995), Déclaration et Programme d'action de Beijing, Déclaration diffusée à l'issue de la Conférence mondiale sur les femmes de 1995, Nations Unies, New York, http://www.un.org/womenwatch/daw/beijing/pdf/BDPfA\%20F.pdf.

Nations Unies (1982), « Directives provisoires concernant les classifications internationales types par âge ", Études statistiques, Série $M, n^{\circ} 74$, Nations Unies, New York, http://unstats.un.org/unsd/pubs/gesgrid.asp?id=134.

OIT (2012), Classification internationale type des professions (CITP), OIT, Genève, http://www. ilo.org/public/french/bureau/stat/isco/index.htm.

UNESCO-ISU (2012), Classification internationale type de l'éducation (CITE) 2011, ISU, Montréal, http://www.uis.unesco.org/Education/Documents/isced-2011-fr.pdf. 



\section{Chapitre 6}

\section{Mesurer la R-D : Méthodes et procédures}

Ce chapitre porte sur la façon de mesurer les activités de recherche et de développement expérimental (R-D), sachant que les activités de R-D sont fortement concentrées, en particulier dans le secteur des entreprises, et largement disséminées dans l'ensemble de l'économie. Des difficultés particulières résultent de ces caractéristiques, ainsi que des diverses formes d'utilisation des statistiques de R-D, notamment l'utilisation de statistiques agrégées aux fins de l'analyse des politiques, de leur évaluation et de la fixation d'objectifs, l'utilisation des dépenses de R-D comme élément d'évaluation du stock de capital de R-D dans le Système de comptabilité nationale (SCN) et, l'analyse des activités de R-D au niveau des unités. Le travail de mesure, qu'il soit effectué via des enquêtes, l'exploitation de données administratives ou une combinaison de ces deux méthodes, exige une infrastructure statistique, en particulier des registres d'institutions, une base méthodologique, les moyens de relier les ensembles de données pour accroître les capacités d'analyse, ainsi que des normes d'assurance qualité. Ces différents aspects sont abordés ici, mais comme l'infrastructure et les problèmes de mesure rencontrés sont assez différents selon les pays, on ne trouvera pas dans ce chapitre de recommandation formelle, mais diverses sources utiles pour le développement de la mesure statistique de la R-D. 


\subsection{Introduction}

6.1 Les méthodes et procédures employées pour mesurer l'exécution de la R-D dépendent de plusieurs éléments. En règle générale, une activité de R-D concerne un nombre relativement modeste d'entités, en particulier dans le secteur des entreprises. Si l'activité de R-D est très concentrée, son champ d'intervention s'étend à l'économie et ses exécutants changent avec le temps. Tant la concentration que l'étendue de champ d'intervention influent sur les lignes directrices relatives à la stratégie d'échantillonnage. Outre ces caractéristiques, les objectifs des programmes statistiques de R-D sont pluridimensionnels : il s'agira de produire des indicateurs agrégés à l'appui de la politique scientifique, de chiffrer les dépenses associées à un stock de capital de R-D conformément au Système de comptabilité nationale et, d'obtenir des micro-données à l'appui des analyses menées au niveau des unités (par exemple, entreprises, administrations publiques, établissements d'enseignement supérieur et institutions privées sans but lucratif) dans le respect des règles de protection des données. Ces objectifs, parfois divergents, dictent le choix des stratégies d'échantillonnage et de traitement des données.

6.2 La mesure de l'exécution des activités de R-D dépend de l'infrastructure statistique en place - registres d'unités statistiques, statisticiens expérimentés et de l'existence ou non d'une législation permettant des enquêtes obligatoires et de mettre en rapport les données d'enquête avec d'autres sources de données pour produire des analyses appropriées aux fins de l'action publique. Le chapitre présente des méthodes et procédures qui s'appuient sur des infrastructures statistiques et propose des orientations relatives à la mesure de la R-D, la qualité des données et la communication de celles-ci aux organisations internationales. Il n'entre pas dans le but du présent manuel de recommander telle ou telle méthode d'enquête et d'analyse des données, car les différences de situation entre pays sont trop grandes pour qu'il soit possible de proposer des normes sur la manière d'aborder les répondants, les questionnaires ou les techniques d'échantillonnage-types.

6.3 Très diverses, les sources potentielles des données de R-D incluent, sans s'y limiter, les enquêtes et d'autres sources administratives. Les données administratives englobent à la fois les données financières recueillies par les administrations fiscales et les données d'autres sources administratives comme les registres comptables des entreprises. L'utilisation des données administratives est examinée dans la section 6.4. Dans certains cas, des estimations sont nécessaires pour compléter les enquêtes et les sources de données administratives. La modélisation (ou estimation indirecte) des dépenses de R-D dans le secteur de l'enseignement supérieur en est un exemple. Les offices 
statistiques sélectionnent les sources à exploiter en fonction de la disponibilité, de la qualité, de la pertinence des données ainsi que du coût de leur collecte, la situation étant variable d'un pays à l'autre.

6.4 La collecte directe de données présente un net avantage dans la mesure où elle peut être réalisée en complète harmonie avec les concepts et les définitions retenus dans le présent manuel. Cependant, elle a un double coût : le coût direct de la collecte elle-même et le coût indirect supporté par les répondants qui doivent remplir les questionnaires d'enquête.

6.5 Dans le présent chapitre, les " enquêtes sur la R-D » désignent la collecte directe de données au moyen d'une enquête statistique, l'obtention de données à partir de sources administratives ou la combinaison de ces deux méthodes.

\subsection{Unités}

6.6 La population cible des enquêtes sur la R-D est constituée par l'ensemble des unités institutionnelles qui exécutent (ou financent) la R-D. Cette population peut être classée par secteur institutionnel d'exécution ou de financement de la R-D. Ces secteurs sont les suivants : secteur des entreprises, secteur de l'État, secteur de l'enseignement supérieur et secteur privé sans but lucratif. Le présent manuel est axé sur la collecte de données auprès des exécutants de la R-D. Il est également nécessaire de recueillir des données sur le financement de la R-D répondant aux critères du Système de comptabilité nationale (SCN), en distinguant fonds d'échange et fonds de transfert pour la R-D (voir chapitre 4). La population cible d'exécutants n'est pas toujours suffisante pour permettre d'établir des statistiques sur les sources de financement. Inversement, le groupe cible de bailleurs de fonds ne permet pas toujours l'établissement des statistiques sur les exécutants de la R-D.

6.7 Il est recommandé d'utiliser l'unité institutionnelle comme unité d'échantillonnage dans les enquêtes sur la R-D.

\section{Unité institutionnelle}

6.8 L'unité institutionnelle est un concept de comptabilité nationale désignant " une entité économique qui est capable, de son propre chef, de posséder des actifs, de prendre des engagements, de s'engager dans des activités économiques et de réaliser des opérations avec d'autres entités " (chapitre 3 et CE et al., 2009, p. 61, par. 4.2).

\section{Unité statistique}

6.9 Une unité statistique est une unité au sujet de laquelle des informations sont recueillies et des statistiques compilées. Une base de sondage se compose d'unités statistiques. Les unités statistiques sélectionnées dans un échantillon doivent avoir un poids initial (l'inverse de la probabilité de sélection) qui sera utilisé pour produire des estimations de la population dont est tiré l'échantillon. 
6.10 Une unité statistique peut avoir un certain nombre d'attributs. Par exemple :

- l'appartenance à un secteur du Manuel de Frascati (entreprises, État, enseignement supérieur ou secteur privé sans but lucratif)

- l'appartenance à l'un des secteurs définis dans le SCN (par exemple, sociétés, administrations publiques, institutions sans but lucratif)

- une activité économique principale qui permet de classer l'unité en se servant, par exemple, de la Classification internationale type, par industrie, de toutes les branches d'activité économique, Révision 4 (Nations Unies, 2008)

- la situation géographique

- la taille (nombre de salariés, chiffre d'affaires, etc.).

6.11 Plusieurs types possibles d'unités statistiques sont examinés au chapitre 3, notamment les groupes d'entreprises, les entreprises et les établissements. Cette nomenclature a été développée pour le secteur des entreprises mais elle peut être appliquée à d'autres secteurs institutionnels.

6.12 Dans le présent chapitre, c'est le terme " unité institutionnelle » qui est privilégié ; le terme " entreprise " n'est utilisé de façon interchangeable avec « unité institutionnelle » que dans le secteur des entreprises.

\section{Unité déclarante}

6.13 Dans ce manuel, l'unité déclarante désigne l'entité dont émanent les données. Au sein d'une unité statistique donnée, il peut exister plusieurs unités au niveau desquelles les données recherchées sont disponibles et peuvent être déclarées. Par exemple, des données sur la distribution géographique des activités de R-D des entreprises peuvent être obtenues à partir des unités déclarantes au niveau de l'établissement, des données sur les domaines de R-D des établissements d'enseignement supérieur peuvent être obtenues au niveau de chaque département d'enseignement. Dans le cas des données administratives, l'unité déclarante est l'unité associée à chaque déclaration. Un point de collecte unique peut être utilisé pour recueillir des données à partir de plusieurs unités déclarantes.

\section{Relation entre les unités}

6.14 Les unités déclarantes correspondent en général aux unités institutionnelles individuelles ou à des regroupements de ces unités. Il peut toutefois y avoir des exceptions, en particulier lorsqu'il est plus facile pour les répondants de communiquer des données à un échelon différent : groupe d'unités institutionnelles, dans le cas d'opérations de portée internationale, ministère, département d'université (lorsque ces unités ne remplissent pas tous les critères requis pour être traitées comme des unités institutionnelles).

6.15 Des unités institutionnelles apparentées peuvent être rassemblées dans un groupe d'unités institutionnelles (par exemple, un groupe d'entreprises dans le secteur des entreprises). À ce niveau, il doit être possible 
d'obtenir des états financiers consolidés pour l'ensemble du groupe des unités institutionnelles.

6.16 Dans le cas des structures simples, une entité légale peut constituer une unité institutionnelle. Dans les structures plus complexes, la correspondance entre entités légales et entreprises est du type de "plusieursàune " ou de "plusieursàplusieurs".

6.17 Les modalités de traitement de ces structures complexes diffèreront d'un pays à l'autre et l'on ne cherche pas ici à prescrire de méthode particulière à ce sujet.

\subsection{Secteurs institutionnels}

\section{Secteur des entreprises}

6.18 Le secteur des entreprises est défini dans la section $3.5 \mathrm{du}$ chapitre 3. Les exécutants de la R-D appartenant à ce secteur présentent deux caractéristiques distinctes. Premièrement, ils constituent un sous-ensemble rare de l'ensemble de la population du secteur des entreprises. Deuxièmement, ils n'exécutent pas toujours des activités de R-D de manière continue. Cela est source de difficultés dans la définition et le maintien des bases de sondage (chapitre 7).

6.19 Certaines entreprises ne mènent qu'occasionnellement des activités de R-D, tandis que d'autres le font de manière continue. Les deux types d'entreprises doivent être pris en compte dans les bases de sondage des enquêtes sur la R-D. Il est recommandé d'inclure dans ceux-ci l'ensemble des entreprises susceptibles d'exécuter des activités de R-D de façon soit continue, soit occasionnelle (chapitre 7, section 7.3).

6.20 L'unité d'échantillonnage pour le secteur des entreprises est généralement l'unité institutionnelle ou l'entreprise. Les attributs recherchés de l'unité d'échantillonnage sont les suivants : activité économique principale, taille, situation géographique et structure du capital et de contrôle. L'unité déclarante sera l'entité la mieux à même de fournir des données. Cela pourra impliquer une combinaison d'entreprises ou d'établissements (chapitre 3, encadré 3.1).

6.21 L'échantillon pourra être tiré d'une base coordonnée contenant des informations à la fois sur les secteurs SCN et les secteurs Frascati, comme on l'a vu au chapitre 3. L'emploi d'une base de ce type aide à s'assurer que la répartition des unités de R-D par secteur SCN s'effectue de manière cohérente, tout en permettant la sélection d'unités sur la base des secteurs Frascati. Dans le secteur des entreprises, un exemple serait une université offrant des programmes d'enseignement institutionnalisé qui vendrait ses résultats de R-D à un prix économiquement significatif. Cette université serait classée dans le secteur de l'enseignement supérieur de la classification Frascati et dans le secteur des sociétés de la classification SCN. Si le lien avec un registre des entreprises est possible, on pourra aussi intégrer les données d'enquête sur la R-D à d'autres types de données afin de permettre des analyses au niveau micro-économique. 
6.22 Le développement d'une base de sondage d'unités d'entreprises peut se faire de plusieurs façons. Lorsque des informations ou des données administratives actuelles et historiques sont disponibles, les unités d'exécution et de financement de la R-D doivent être identifiées dans la base de sondage. Si ces indicateurs ne sont pas disponibles, d'autres méthodes d'identification de ces unités pourront constituer la première étape d'un processus en deux temps de définition d'un échantillon pour les enquêtes sur la $\mathrm{R}-\mathrm{D}$. Une autre manière de tester le champ couvert est de prendre un premier contact avec les unités qui sont susceptibles d'exécuter ou de financer des activités de R-D dans les secteurs d'activité ciblés. Les pays qui commencent des enquêtes sur la R-D trouveront des informations plus détaillées dans UNESCO-ISU (2014).

6.23 D'autres sources de données peuvent être utilisées pour améliorer le champ couvert par l'enquête. Ces sources extérieures doivent faire l'objet d'une évaluation afin de vérifier qu'elles sont d'un niveau de qualité suffisant pour pouvoir être utilisées en complément de la base de sondage. Si tel est le cas, elles devraient être utilisées pour détecter les entreprises qui exécutent ou financent des activités de R-D.

\subsection{Il est recommandé :}

- d'inclure dans les enquêtes sur la R-D visant le secteur des entreprises toutes les entreprises dont on sait qu'elles mènent, ou financent, des activités de R-D, ou qui sont fortement susceptibles de le faire

- d'identifier les exécutants de la R-D non connus ou incertains en réalisant une enquête sur un échantillon de toutes les autres entreprises.

6.25 La base de sondage devrait inclure toutes les entreprises menant, ou finançant, des activités de R-D. Les contraintes budgétaires ou liées à la charge de travail des répondants qui pèsent sur l'échantillonnage et la stratification doivent être documentées dans le cadre de l'évaluation de la qualité des données.

\section{Secteur de l’État}

6.26 Le secteur de l'État est défini dans la section 3.5 du chapitre 3.

6.27 L'unité statistique recommandée dans le présent manuel est l'entreprise ou un équivalent mais, dans le cas du secteur de l'État, à des fins pratiques, l'unité d'échantillonnage retenue sera généralement une administration, un ministère ou un organisme public, même si l'unité ne présente pas toutes les caractéristiques d'une unité institutionnelle, c'est-à-dire la capacité de détenir et de contrôler des actifs. Ce choix d'unité d'échantillonnage ne signifie pas que les flux financiers entre ministères doivent être traités comme des financements externes (voir Glossaire et chapitre 4, section 4.3, sur l'identification des flux financiers entre secteurs). Les attributs recherchés de l'unité d'échantillonnage dans le secteur de l'État comprennent : l'activité économique principale, la situation géographique et le niveau d'administration. L'unité déclarante sera l'entité la mieux à même 
de fournir des données. Celle-ci pourra inclure l'ensemble d'une administration, infranationale ou municipale, par exemple.

6.28 Les listes d'unités d'exécution et de financement de la R-D peuvent être actualisées de différentes façons, par exemple : législation, mesures budgétaires ou mesures de financement connexes, registres existants, annuaires des unités menant des activités de R-D, associations de recherche, sources bibliométriques ou encore, demandes de mise à jour émanant d'organes administratifs.

6.29 La base de sondage devrait, si possible, être relié au registre central des entreprises. Cela facilitera l'intégration de données de sources diverses, en réduisant aussi le risque de double compte.

6.30 Il convient de prêter une attention particulière à l'utilisation des données administratives dans l'identification des unités institutionnelles qui exécutent ou financent des activités de R-D.

6.31 L'identification des activités de R-D au niveau des collectivités locales (municipalités) est particulièrement difficile à cause du grand nombre d'unités, du petit nombre d'exécutants probables de la R-D et de difficultés d'interprétation du concept de R-D. En général, les listes d'exécutants de la R-D n'incluent pas ces unités. S'il apparaît que les collectivités locales entreprennent un volume important d'activités de R-D, on pourra envisager d'inclure les exécutants de la R-D à l'intérieur de grandes collectivités locales. Vu la nature de nombreuses formes d'administration infranationale, les ministères ou organismes publics n'ont normalement pas pour mission de conduire des activités de R-D mais ils peuvent avoir à résoudre des problèmes spécifiques identifiés par l'organe législatif ou par eux-mêmes. Certaines activités de R-D, par conséquent, sont seulement occasionnelles. Le secteur de l'État est analysé plus en détail au chapitre 8.

\section{Enseignement supérieur}

6.32 Le secteur de l'enseignement supérieur est défini dans la section $3.5 \mathrm{du}$ chapitre 3. Les enquêtes et procédures d'estimation doivent couvrir l'ensemble des universités, des établissements d'enseignement post-secondaire et autres établissements proposant des programmes d'enseignement supérieur formel, indépendamment de leur source de financement ou de leur statut juridique, et l'ensemble des instituts et centres de recherche, stations expérimentales et centres de soins dont les activités de R-D relèvent du contrôle direct d'établissements d'enseignement supérieur ou sont administrées par ceux-ci.

6.33 Ce secteur n'a pas d'équivalent direct dans la nomenclature SCN des secteurs institutionnels. On trouvera au tableau 3.1 du chapitre 3 des indications sur les recoupements du secteur de l'enseignement supérieur avec les secteurs de la classification SCN. Des établissements appartenant au secteur de l'enseignement supérieur apparaissent dans tous les secteurs SCN et sont traitées séparément en raison de la grande utilité que revêtent pour l'action publique les activités de R-D de ces établissements. 
6.34 Dans le secteur de l'enseignement supérieur, l'unité d'échantillonnage est l'établissement d'enseignement supérieur (qui correspond à l'unité institutionnelle). Les attributs recherchés comprennent : la localisation géographique et le secteur économique. L'unité déclarante peut être une faculté ou un département. Il s'agit en effet, dans un établissement d'enseignement supérieur, du niveau le mieux apte à fournir des données sur les dépenses et les flux de financement. Il convient de prêter une attention particulière aux hôpitaux qui mènent des activités de R-D et font partie du secteur de l'enseignement supérieur.

6.35 Dans les enquêtes visant les secteurs des entreprises, de l'enseignement supérieur, de l'État ou des institutions privées sans but lucratif, on s'assurera que les hôpitaux de recherche sont bien inclus dans la base de sondage de l'enquête et assignés correctement à un secteur (chapitre 3).

6.36 Il peut être nécessaire, dans certains pays ou territoires, de distinguer les hôpitaux de recherche qui conduisent la R-D et les fondations philanthropiques associées à ces hôpitaux qui financent la R-D. Lorsque les hôpitaux universitaires sont financièrement intégrés à un établissement d'enseignement supérieur, ils peuvent être traités conjointement comme une seule unité d'échantillonnage. Par contre, lorsqu'ils constituent des unités séparées avec une administration et une comptabilité distinctes, ils doivent être traités comme deux unités d'échantillonnage distinctes.

6.37 On veillera aussi soigneusement à traiter de manière cohérente les unités de R-D gérées conjointement par deux ou plusieurs entités, par des personnes rémunérées par des entités différentes ou par des personnes employées par d'autres institutions. Cette remarque vaut pour les unités de tous les secteurs, mais s'applique tout particulièrement aux unités de R-D des hôpitaux qui posent un problème spécifique.

6.38 Le chapitre 9 fournit des indications supplémentaires sur la mesure des activités de R-D dans le secteur de l'enseignement supérieur.

\section{Secteur privé sans but lucratif}

6.39 Ce secteur est défini dans la section 3.5 du chapitre 3. Les sources supplémentaires à utiliser pour identifier les répondants possibles à l'enquête sont essentiellement les mêmes que celles décrites pour le secteur de l'État. Si l'information cadre est moins détaillée, elle pourra être complétée par des informations obtenues auprès des autorités fiscales, des chercheurs et des administrations qui s'occupent de la recherche. Ce secteur est sans doute particulièrement pertinent pour la collecte de données sur le financement de la R-D.

6.40 L'unité d'échantillonnage dans le secteur privé sans but lucratif est généralement l'unité institutionnelle. Les attributs recherchés de l'unité d'échantillonnage sont les suivants : activité économique principale, taille, localisation géographique et structure de contrôle. L'unité déclarante sera l'entité 
la mieux à même de fournir des données. Il pourra s'agir de combinaisons d'unités. On trouvera au chapitre 10 des précisions sur la mesure des activités de R-D dans le secteur privé sans but lucratif.

\subsection{Plan d'enquête Plan d'échantillonnage}

6.41 Comparées à d'autres activités, les activités de R-D sont en fait des activités rares regroupées dans un petit nombre d'unités institutionnelles. Dans les populations où une part importante de l'estimation globale correspond à de grandes unités, une strate d'échantillonnage distincte devra être créée. Il s'agira d'une "strate à tirage exhaustif " au niveau de laquelle les grandes unités seront échantillonnées avec certitude. Le poids d'échantillonnage de ces unités sera de un. Des unités plus petites pourront être sélectionnées avec une probabilité de sélection inférieure à un. Le plan d'échantillonnage présenté dans cette section n'a pas un caractère prescriptif, car le secteur des entreprises et le secteur privé sans but lucratif diffèrent fortement des secteurs de l'État et de l'enseignement supérieur. Le plan d'enquête et le plan d'échantillonnage devront tenir compte du contexte et des pratiques nationales.

6.42 Pour stratifier l'échantillon, il est important de choisir la variable de taille qui représente au mieux la population. Les données de l'année précédente, ou d'autres données indirectes sur la R-D, sont préférables à d'autres variables de taille (par exemple, le chiffre d'affaires, le montant global des crédits budgétaires ou le nombre de personnes occupées) lorsque la corrélation est faible entre ces variables et les dépenses ou le financement de la R-D. Les données de l'année précédente sont préférables dans le cas des unités qui mènent de façon continue des activités de R-D.

6.43 Pour déterminer la taille optimale des échantillons stratifiés, il est nécessaire de prendre en compte le degré de précision recherché pour les estimations. La taille de l'échantillon devra aussi être adaptée en fonction du taux de non-réponse prévu, du taux prévu de classification incorrecte des unités et d'autres insuffisances de la base de sondage de l'enquête ayant servie à l'échantillonnage.

6.44 Un échantillon stratifié peut contenir des strates « à tirage exhaustif » et " à tirage obligatoire " dans lesquelles les unités sont sélectionnées avec une probabilité de 100\%. Les unités sélectionnées avec certitude sont celles dont on prévoit qu'elles seront les plus importantes unités d'exécution et de financement de la R-D. Une strate " à tirage obligatoire » est utilisée pour les répondants qui sont des entités complexes relevant de plusieurs classifications sectorielles, situées en plusieurs localités ou relevant de plusieurs compétences. Un échantillon stratifié peut également contenir une strate "à tirage partiel » avec une probabilité inférieure à un. Un échantillon aléatoire est préférable pour ces strates, car il permet de calculer le taux d'erreur de l'échantillon, en tant que mesure de la qualité, et aide à réduire le risque de biais. 
6.45 Lorsqu'il existe des données auxiliaires suffisantes pour identifier les unités d'un registre existant comme exécutantes de la R-D, on peut construire une base de sondage en s'appuyant sur l'identification des exécutants de R-D connus. Si l'information auxiliaire n'est pas suffisante ou inexistante, il peut être nécessaire de définir l'échantillon en deux temps. En pareil cas, on commence d'abord par sélectionner un échantillon étendu afin d'identifier les entités statistiques impliquées dans des activités de R-D puis, dans un deuxième temps, on sélectionne un sous-échantillon à partir du premier échantillon.

6.46 Étant donné la ventilation sectorielle des activités de R-D, il est possible d'utiliser plusieurs bases de sondage. Dans ce cas, il importe de bien contrôler l'appartenance de chaque unité à une base de sondage. Si l'on combine plusieurs bases de sondage, les unités institutionnelles ne doivent apparaître qu'une seule fois dans la base de sondage obtenue. Qualifier les unités institutionnelles de R-D d'un registre centralisé des entreprises est un moyen de réduire les possibilités de duplication et les risques de double compte des activités de R-D.

\section{Définition de la méthode de collecte des données}

6.47 La collecte directe de données peut être effectuée par plusieurs moyens : questionnaire papier, entretien téléphonique, collecte via internet, à condition que soient mises en place des mesures de sécurité suffisantes pour protéger les données sensibles, ou encore entretien en face-à-face dans les pays où ordinateurs, téléphones et services postaux ne sont pas suffisamment répandus. Les données peuvent aussi être recueillies à partir de sources administratives.

6.48 En cas de collecte directe de données, il est nécessaire d'examiner au préalable le coût de l'enquête et la charge de travail qui en résulte pour les répondants.

6.49 Quelles que soient les modalités de l'enquête, le questionnaire devrait inclure un nombre minimum de questions clés pour obtenir les données requises sur la R-D. Il devrait être aussi bref que possible, sans que cela nuise à la collecte des données essentielles, présenté de manière claire et ordonnée, avec des renvois aux définitions et aux instructions qui l'accompagnent. On examinera la possibilité d'utiliser un questionnaire électronique permettant d'intégrer ces informations à chaque question. Les questionnaires électroniques peuvent inclure des contrôles de validation pour vérifier que les données fournies par les répondants sont complètes et cohérentes. La vérification des données permet de détecter et de corriger les erreurs (incohérences logiques) pouvant se glisser dans les données fournies. L'insertion d'une lettre dans un champ numérique peut, par exemple, être détectée lors du processus de vérification et, dans ce cas, le questionnaire est déclaré non valide.

6.50 La méthode de collecte des données doit prendre en compte le type de personne qui remplit le questionnaire. Le répondant peut en effet être la personne qui gère les activités de R-D ou, ce qui est plus fréquent, un membre du 
service financier ou comptable de l'unité de R-D. Ces deux situations présentent des avantages et des inconvénients. Le gestionnaire de la R-D sera sans doute mieux à même d'identifier les activités de R-D et de comprendre les concepts présentés dans le présent manuel. Cependant, le chef du service financier pourra être mieux placé pour fournir des informations financières détaillées. Le chef du service des ressources humaines sera peut-être, quant à lui, mieux apte à répondre aux questions sur le personnel de $\mathrm{R}-\mathrm{D}$, si l'enquête inclut ce type de questions. La fourniture des données requiert parfois des compétences dans tous ces domaines.

6.51 La stratégie de collecte doit donc envisager la possibilité que le questionnaire soit obligé de circuler à l'intérieur d'une organisation pour obtenir des réponses complètes. Il est préférable, cependant, d'identifier un point de contact unique dans chaque entreprise ou institution. Si ce point de contact n'est pas connu, un entretien préalable peut être nécessaire pour identifier la personne la plus indiquée pour assurer la coordination des réponses au questionnaire. Cela est particulièrement important dans le cas des unités institutionnelles complexes.

6.52 Outre la collecte de données sur les dépenses et le personnel de R-D pendant la période de référence qui vient de s'achever (t), il est recommandé aux statisticiens qui s'occupent de R-D de recueillir des données sur les dépenses budgétisées pour l'année suivante, c'est-à-dire l'année pendant laquelle l'exercice de collecte a lieu $(t+1)$. Ces statisticiens pourront aussi recueillir des informations sur les dépenses budgétisées pour l'année qui suit celle de la collecte $(t+2)$. La prudence s'impose, cependant, dans l'interprétation de ces données budgétaires, car elles ne font qu'exprimer les prévisions des entreprises et il y a parfois au final un écart important entre les données prévues et les données effectives. La recommandation de recueillir des données sur les dépenses budgétisées pour l'année suivant celle de la collecte - et non sur le personnel - se fonde sur l'expérience du manque de fiabilité des données relatives au personnel. S'agissant des dépenses, la collecte de données sur les dépenses budgétisées est préférable à l'estimation des dépenses sur la base des performances antérieures de R-D ou d'autres mesures des résultats économiques comme les chiffres de ventes.

\section{Données administratives et plan d'enquête}

6.53 Si les concepts et définitions retenus dans les sources de données administratives ainsi que le champ qu'elles couvrent sont suffisamment proches de ceux du présent manuel, les données administratives pourront être utilisées comme source première d'informations. Si des écarts existent entre les concepts utilisés par une source de données administratives et le présent manuel, l'ensemble de données administratives pourra néanmoins servir de source d'informations auxiliaires en vue de l'imputation des informations manquantes ou des données aberrantes. L'imputation est la procédure qui consiste à attribuer 
une valeur à un élément de données spécifique lorsqu'une réponse manque ou se révèle inutilisable. L'utilisation des données administratives variera d'un pays à l'autre en fonction de leur disponibilité et de leur qualité.

6.54 L'existence de données administratives a aussi des incidences sur la conception de l'exercice de collecte de données. Par exemple, lorsqu'il existe un registre des entités ayant déposé une demande de crédits d'impôt au titre de la R-D, ces données administratives peuvent être utilisées pour évaluer les performances de R-D des exécutants de petits volumes de R-D, en réduisant le poids de ces unités. Cette option ne se présente évidemment pas dans les pays où n'existe aucune mesure d'allégement fiscal en relation avec les activités de R-D.

6.55 Les données administratives peuvent être utilisées de plusieurs façons en vue de parvenir à des estimations. Lorsque les concepts appliqués dans le programme statistique de l'administration sont suffisamment proches de ceux présentés dans le présent manuel, l'ensemble de données administratives peut être utilisé pour remplacer directement les données. Le remplacement de données intervient soit en cas de remplacement prévu à l'avance, soit en cas de non-réponse partielle ou totale du répondant. Les données administratives peuvent être utilisées non seulement à des fins de remplacement mais aussi pour le maintien de la base d'échantillonnage. Elles peuvent également servir à la validation des données en permettant de vérifier, par exemple, que les tendances évolutives révélées par l'enquête recoupent les tendances déduites des données administratives. S'il existe un degré de corrélation suffisant entre une variable de l'ensemble de données administratives et une variable présente dans les données de l'enquête, les données administratives pourront être utilisées comme variable auxiliaire dans un estimateur par calage (voir Australian Bureau of Statistics (2005) pour une description de plusieurs utilisations de ce type).

6.56 Pour évaluer l'utilité des données administratives, un certain nombre d'éléments doivent être pris en compte. Le champ couvert par les données administratives devrait, en premier lieu, recouper la population des bailleurs de fonds et des exécutants de la R-D. Il convient également de déterminer le degré d'actualité des données administratives en évaluant le temps nécessaire à l'autorité fiscale ou de régulation pour mener à bien son travail sur les données saisies. Les définitions et les concepts qui organisent les données administratives devraient être identiques à ceux retenus dans le présent manuel. La qualité des données administratives doit être mise en regard des normes et des résultats escomptés de l'enquête. Si l'on constate, par exemple, un taux de rejet élevé lors des contrôles de validation, il se peut que la qualité des données administratives soit insuffisante. Si le programme statistique qui génère les données administratives fait l'objet de modifications fréquentes, il est probable que l'homologie entre les concepts qui organisent les données administratives et les concepts présentés dans ce manuel ne se maintienne 
pas dans le temps. L'utilité d'une source de données administratives peut s'en trouver réduite. La stabilité d'une source de données administratives dans le temps est importante. Pour cela, il est nécessaire qu'une autorité légale ou de régulation compétente utilise effectivement les données à des fins statistiques. Enfin, les données administratives doivent être suffisamment documentées pour pouvoir être utilisées.

\section{Remarques sur l'élaboration du questionnaire}

6.57 L'impact d'un questionnaire - qu'il soit imprimé ou sous forme électronique - sur le comportement des répondants, les relations avec eux et la qualité des données ne doit pas être sous-estimé. Un questionnaire doit s'efforcer par conséquent de réduire au minimum la charge de travail du répondant, en lui facilitant les choses autant que possible. Un questionnaire bien conçu aide à réduire la quantité de vérifications et d'imputations à effectuer, en simplifiant ainsi les tâches de traitement après la collecte.

6.58 Les termes et concepts utilisés dans un questionnaire devraient, dans la mesure du possible, avoir le même sens pour l'organisme d'enquête que pour les répondants. Ces derniers ne devraient pas se trouver dans l'obligation d'interpréter les besoins d'informations de l'enquêteur. Les questions doivent au contraire être formulées de façon à pouvoir être immédiatement comprises des répondants, même si une transformation des variables est nécessaire après la collecte afin de les aligner sur les concepts décrits dans le présent manuel. Il est donc judicieux de ne pas utiliser les mêmes questions pour les entreprises et pour les établissements du secteur public si elles emploient des normes de comptabilité et une terminologie différentes.

6.59 Les instructions fournies aux répondants doivent être brèves, claires et faciles à trouver. La définition des concepts doit aussi être d'accès facile. Les éléments à inclure et à exclure doivent être expliqués clairement dans le matériel d'enquête. Un certain nombre de vérifications de bornes et de cohérences doivent être intégrées aux questionnaires électroniques pour aider le répondant à remplir correctement le questionnaire. Quelles que soient les modalités de l'enquête, les formulations utilisées et les options permettant de sauter certaines questions doivent être identiques tout au long du questionnaire.

6.60 Pour assurer la bonne compréhension du questionnaire par les répondants, il est recommandé de le tester avant de commencer à recueillir des données. On se servira pour cela de tests qualitatifs (groupes de consultation, tests cognitifs) ou d'enquêtes pilotes, par exemple. Dans le cas de la collecte de données par voie électronique, il est important de tester l'application finale devant servir à recueillir les données avec divers usagers et systèmes d'exploitation pour s'assurer que le contenu est bien compris et que l'application est fonctionnelle. On trouvera des exemples de méthodes pour tester les questionnaires dans Couper et al. (2004). 
6.61 Les enquêtes sur la R-D sont généralement conçues comme des outils autonomes de collecte de données. Lorsqu'une enquête sur la R-D est associée à d'autres outils, on veillera soigneusement à ce que le champ couvert par les outils utilisés conjointement demeure inclusive et représentative de l'ensemble de l'activité de R-D et que la qualité des données ne s'en trouve pas modifiée. Il existe des exemples d'enquêtes sur la R-D combinées à des enquêtes sur l'innovation, et la discussion se poursuit sur la possibilité de les combiner à des enquêtes sur les dépenses d'investissement, mais il est recommandé de mener les enquêtes sur la R-D séparément.

\subsection{Collecte des données}

6.62 La charge de travail imposée au répondant devrait se limiter au minimum. Le maintien de la confidentialité des données est particulièrement important pour les répondants du secteur des entreprises, dans lequel les dépenses de R-D représentent de véritables décisions stratégiques.

6.63 Le processus de collecte des données peut générer des paradonnées ou des informations sur le processus d'enquête lui-même. Le fait de savoir si une unité fait ou non partie de l'échantillon, le suivi d'une réponse et le mode de collecte des données sont des exemples de paradonnées. L'utilisation des paradonnées à l'issue d'une campagne peut servir à améliorer l'outil d'enquête pour l'avenir.

6.64 Le processus de collecte devrait être conçu de manière à limiter au minimum la charge de travail et le coût de l'enquête, et d'en renforcer au maximum l'actualité, le taux de réponse et la précision. La collecte de données via internet semble de plus en plus le mode d'enquête privilégié. Cependant, il importe de maintenir différents modes de collecte, en particulier dans les pays où la collecte de données par voie électronique ou postale fait problème. Les stratégies de collecte doivent être suffisamment souples pour permettre de changer de mode de collecte (par exemple, en recueillant l'information par téléphone lorsqu'un répondant n'a pas rempli en temps voulu un questionnaire imprimé ou en ligne). En cas d'utilisation de plusieurs modes de collecte des données dans une enquête, il sera sans doute indiqué d'examiner après la collecte si tel ou tel mode peut être source de biais.

6.65 Un contact préliminaire est un moyen utile de confirmer l'information de référence avant la collecte. Ce contact préliminaire pourra porter sur : les coordonnées du répondant (y compris une adresse électronique), la classification de l'activité économique ou de la branche d'activité d'une unité institutionnelle et, la confirmation qu'elle mène des activités de R-D.

6.66 Dans le cas des organisations complexes de grande taille, on peut envisager d'établir des relations spéciales pour aider les répondants à remplir le questionnaire. Cela inclut, par exemple, la désignation d'un point de contact unique pour la collecte des données, la définition ad hoc de l'unité déclarante et la modification de l'outil de collecte des données pour que les répondants 
voient plus facilement comment les questions de l'enquête se rapportent à leurs systèmes de gestion financière et de gestion des ressources humaines. Un tel traitement doit sans doute être réservé aux unités institutionnelles qui entrent pour une part importante dans l'estimation globale.

6.67 Étant donné le caractère complexe de la mesure des dépenses de R-D, il convient d'examiner la possibilité de produire un manuel destiné aux enquêteurs et d'organiser des sessions de formation pour préparer ceux-ci à répondre aux questions des répondants. Certains outils élaborés dans ce contexte pourront être communiqués directement aux répondants, en particulier lorsque les données sont recueillies par voie électronique. Ces outils pourront inclure des notes explicatives, des exemples hypothétiques, ainsi qu'une documentation sur le traitement de différents types de cas.

6.68 À la réception des données, il convient d'effectuer une série préliminaire de vérifications et, en cas de résultats négatifs, un suivi auprès des répondants. Dans le cas des questionnaires électroniques, ces vérifications peuvent être intégrées au questionnaire lui-même, ce qui réduit le besoin de suivi par les enquêteurs.

6.69 Le taux de réponse devrait être surveillé tout au long du processus de collecte des données. Lorsqu'il est faible, il peut être souhaitable, dans un but d'assurance qualité, de contacter, après la collecte, un sous-échantillon des unités qui n'ont pas répondu, afin de déterminer s'il y a ou non un biais de non-réponse dans les estimations (c'est-à-dire, si les répondants sont plus ou moins susceptibles de mener des activités de R-D). Cette information pourra être obtenue directement (appels téléphoniques de suivi, par exemple) ou indirectement à partir des données administratives.

6.70 De plus, au terme du processus d'enquête, les paradonnées pourront être utilisées pour évaluer l'efficacité et le rapport coût-efficacité de la campagne de collecte de données. Ce type d'analyse est utile pour rationaliser et améliorer les activités d'enquête.

\subsection{Intégration des données}

6.71 Pour intégrer des ensembles de données (par exemple, dans le cas d'une analyse au niveau de l'entreprise), il est important de disposer d'une clé d'appariement permettant de combiner les données. Des clés d'appariement existent généralement dans le registre central des entreprises. Lorsque l'on procède ainsi, il convient, dans un but d'assurance qualité, de surveiller le taux d'appariement entre les ensembles de données. Un taux de non-appariement trop élevé signale que les données combinées ne sont pas utilisables.

\subsection{Vérification des données recueillies et imputation}

6.72 Les données recueillies sont soumises à des contrôles, qui visent à détecter les erreurs éventuelles, valider des fichiers ou des variables et corriger des erreurs ou des incohérences. Il importe de veiller à ce que ces contrôles 
ne soient pas source de biais dans les estimations globales. Il existe un risque d'intervention excessive sur les données lorsque l'impact des contrôles sur les estimations finales d'une enquête est négligeable ; Il faut donc veiller à éviter ce risque dans la méthode utilisée.

6.73 Les contrôles doivent être automatisés et reproductibles. Un processus automatisé permet d'en accroître la portée et l'exactitude. On se gardera soigneusement, dans la mise au point de systèmes automatisés, de tout contrôle trop poussée des données. S'agissant de l'examen et de la correction consécutive aux rejets, on pourra opter pour un contrôle sélectif des données, le suivi devant se focaliser sur les unités qui entrent pour une part importante dans l'estimation d'un domaine particulier. Des taux de rejet élevés doivent être pris en compte car ils fournissent des indications utiles sur les moyens d'améliorer le questionnaire et d'autres aspects de la collecte des données. Leur suivi doit inclure les corrections manuelles, lorsque celles-ci sont nécessaires.

6.74 L'imputation sert à assigner des valeurs valides lorsque des données manquent ou sont incohérentes. Ce travail a lieu généralement après la collecte des données (y compris le suivi auprès des répondants) et l'examen manuel initial des questionnaires. L'imputation peut être utilisée en cas d'absence complète de réponse à un questionnaire (non-réponse totale) ou de non-réponse à propos d'une variable particulière (non-réponse partielle). Une fois l'imputation effectuée, le fichier de microdonnées doit contenir uniquement des données complètes et intrinsèquement cohérentes. Les processus d'imputation doivent être automatisés, objectifs et reproductibles.

6.75 Plusieurs méthodes d'imputation permettent de remplacer les informations manquantes ou incohérentes. Les méthodes d'imputation déterministes comprennent :

- l'imputation déductive

- l'imputation par la moyenne

- l'imputation par le ratio

- l'imputation par le plus proche voisin.

6.76 D'autres méthodes existent et leur utilisation dépend des pratiques de l'institution qui effectue la collecte des données.

6.77 Lorsqu'il existe une relation suffisante entre les données administratives disponibles et les données manquantes ou incohérentes, les données administratives peuvent être utilisées pour remplacer directement l'information manquante ou incohérente.

6.78 Étant donné la variabilité de la R-D en tant qu'activité d'investissement, il est préférable de recourir aux méthodes d'imputation qui privilégient l'utilisation des données obtenues du répondant (directement ou par l'intermédiaire de données auxiliaires) plutôt que l'utilisation d'informations provenant d'autres unités répondantes, comme l'imputation par le plus proche voisin, par exemple. 
6.79 Il convient de conserver les métadonnées afin de pouvoir identifier les éléments et variables qui ont été imputés, ainsi que les méthodes d'imputation utilisées. Le taux d'imputation est un indicateur important de la qualité des données et les éléments qui présentent un taux pondéré d'imputation élevé doivent être traités avec prudence. La variance d'échantillonnage liée à l'imputation est nécessaire pour toute décision à ce sujet.

\subsection{Estimation}

6.80 Toutes les unités de l'échantillon ont un poids initial déterminé par la probabilité de leur sélection dans l'échantillon. Le poids initial est égal à l'inverse de la probabilité de sélection. Dans le cas d'un échantillonnage en deux temps, il est égal au produit des poids correspondant à chaque phase.

6.81 Les poids initiaux peuvent être ajustés afin de tenir compte du nombre d'unités ayant effectivement répondu. Cette intervention n'est justifiée que si l'on pense que répondants et non-répondants présentent des caractéristiques identiques. Plusieurs méthodes d'estimation sont examinées dans Lundström et Särndal (2005).

6.82 Lorsque l'on dispose d'un ensemble de données administratives contenant des données auxiliaires qui présentent une forte corrélation avec l'une des variables du questionnaire (par exemple, le total des dépenses de R-D), le poids initial peut être ajusté par calage des poids d'échantillonnage. Dans un estimateur par calage, les poids sont ajustés de manière à obtenir des totaux de contrôle connus. Les méthodes spécifiques existant à ce sujet incluent les estimateurs par régression, les estimateurs par quotient et les estimateurs par la méthode itérative du quotient.

6.83 L'utilisation d'un estimateur par calage est sans doute préférable car elle permet des estimations plus précises. Elle peut aussi contribuer à une meilleure cohérence des différentes sources de données.

6.84 Pour déterminer la précision d'une estimation, on se servira de l'estimation de l'écart-type.

\subsection{Validation des résultats}

6.85 Un certain nombre d'étapes doivent être suivies pour valider et certifier les estimations issues d'une enquête sur la R-D.

6.86 Il importe que les unités déclarantes de l'échantillon restent représentatives de la population d'exécutants de la R-D. L'examen des taux de réponse permettra de vérifier la population couverte par l'enquête. Ensuite, la pondération des taux de réponse par la valeur des dépenses de R-D fournira une indication sur la part de l'estimation qui est effectivement fondée sur les données.

6.87 Pour assurer la continuité des estimations, il convient de comparer les données des enquêtes sur la R-D à celles recueillies lors des campagnes précédentes, en expliquant les différences. 
6.88 Les données devraient aussi être comparées à d'autres sources de données comparables. Il faut examiner les écarts éventuels afin d'améliorer les estimations ou d'expliquer les différences.

6.89 Dans un but de validation, les estimations finales devraient être confrontées aux résultats escomptés et aux connaissances disponibles sur le secteur concerné. Ici encore, il convient d'examiner les écarts éventuels afin d'améliorer les estimations ou de trouver une explication aux différences.

\subsection{Communication des données à l'OCDE et à d'autres organisations internationales}

6.90 Les autorités nationales réalisent, dans le cadre des dispositifs institutionnels existants, des enquêtes sur la R-D pour recueillir des données appropriées au regard des préoccupations du pays. Les pratiques de chaque pays s'éloignent parfois des normes internationales décrites dans le présent manuel ou d'autres documents de référence. Cependant, aucun effort ne doit être épargné pour réduire l'impact de ces différences lors de la communication de données à l'OCDE ou à d'autres organisations internationales, notamment à l'aide d'ajustements ou d'estimations, même s'il en résulte des écarts entre les données de R-D apparaissant dans les sources internationales et celles qui sont publiées dans les études nationales. Si les autorités nationales ne sont pas en mesure de procéder à de tels ajustements de leur propre chef, elles peuvent aider les organisations appropriées à produire des estimations fondées. Lorsque ces ajustements ne sont pas possibles, il convient de présenter des notes techniques détaillées. Les différences sont généralement de deux types, à savoir :

- des différences explicites de méthode entre les enquêtes nationales de R-D et celle recommandée dans le présent manuel

- des différences "implicites » entre les classifications des branches d'activité économique ou du secteur de l'éducation utilisées dans les enquêtes nationales et les classifications internationales recommandées dans le présent manuel.

6.91 Il est important d'identifier et de notifier les deux types de différences, ainsi que toute rupture dans les séries de données. Lorsque la modification d'un aspect quelconque du processus d'enquête (par exemple, la constitution de l'échantillon et la classification des unités, la méthode de collecte des données, la conception de l'outil d'enquête, l'élaboration des questions et les définitions) se traduit par une rupture connue des séries chronologiques, cette rupture doit être clairement identifiée et signalée aux utilisateurs des données. Et s'il est possible de quantifier l'impact probable d'une rupture des données (par exemple, en pourcentage des totaux fournis) ou de rapporter continûment les estimations actuelles ou futures à des estimations passées, il convient de le faire.

6.92 Les autorités nationales devraient fournir des indicateurs de la qualité des agrégats publiés. Ces indicateurs devraient s'appuyer à la fois sur les écartstypes et sur les taux de réponse. Ils devraient porter sur l'ensemble des données 
et sur chacun des domaines d'estimation. Les indications concernant le champ couvert devraient préciser la manière dont la population cible a été définie, maintenue et évaluée. Les divers taux d'imputation devraient être indiqués. Ces taux sont utiles pour déterminer la qualité des données et examiner la construction des questions. Certains concepts peuvent être peu familiers aux répondants, ou difficiles à comprendre, et la conception du questionnaire doit être régulièrement réexaminée afin de remédier à ces défauts. La suppression de certaines cellules pour des raisons de confidentialité est un moyen d'assurer l'anonymat d'une population trop peu nombreuse ou trop fortement concentrée pour permettre le niveau de détail requis. Des aspects tels que les délais de transmission et les taux de révision doivent aussi être pris en compte.

\subsection{Remarques finales sur la qualité des données}

6.93 Le présent chapitre a présenté plusieurs méthodes pour mesurer la $\mathrm{R}-\mathrm{D}$ dans les principaux secteurs institutionnels pris en compte dans le présent manuel et obtenir des résultats de qualité reproductible, en s'appuyant sur les normes de qualité généralement reconnues. Pour ce qui concerne la qualité des données, les praticiens sont invités à se reporter à Snijkers et al. (2013) et Lyberg et al. (1997), ainsi qu'aux documents suivants : www.oecd.org/std/ qualityframeworkforoecdstatisticalactivities.htm et http://unstats.un.org/unsd/dnss/ QualityNQAF/nqaf.aspx. Les approches décrites visent à la fois à proposer plusieurs options de collecte des données aux pays dont l'infrastructure statistique est encore limitée et à fournir des thèmes de réflexion à ceux qui disposent d'un système statistique très développé. Cependant, les méthodes statistiques, ainsi que les technologies et pratiques correspondantes, évoluent avec le temps et les utilisateurs du présent manuel sont donc invités à suivre - et mettre en œuvre les pratiques les mieux adaptées à leur environnement statistique.

\section{Références}

Australian Bureau of Statistics (2005), " The Experience of ABS with Reducing Respondent Burden Through the Use of Administrative Data and Through the Use of Smarter Statistical Methodology ", 35e réunion plénière de la Conférence des statisticiens européens de la CEE-ONU, CES/2005/18.

CE, FMI, OCDE, Nations Unies et Banque mondiale (2009), Système de comptabilité nationale 2008, Nations unies, New York, http://unstats.un.org/unsd/nationalaccount/docs/ SNA2008FR.pdf.

Couper, M. P., J.T. Lessler, E.A. Martin, J. Martin, J.M. Rothgeb et E. Singer (2004), Methods for Testing and Evaluating Survey Questionnaires, John Wiley and Sons, Hoboken (NJ), États-Unis.

Lundström, S. et C.-E. Särndal (2005), Estimation in Surveys with Nonresponse, John Wiley and Sons, Hoboken (NJ), États-Unis.

Lyberg, L., P. Biemer, M. Collins, E. de Leeuw, C. Dippo, N. Schwarz et D. Trewin (dir. pub.) (1997), Survey Measurement and Process Quality, John Wiley and Sons, Hoboken (NJ), États-Unis. 
Nations Unies (2008), " Classification internationale type, par industrie, de toutes les branches d'activité économique (CITI), Révision 4 ", Département des affaires économiques et sociales, Division de statistique, Études statistiques, Série $M, n^{\circ} 4$, Rév. 4, Nations Unies, New York, https://unstats.un.org/unsd/cr/registry/isic-4.asp et http://unstats.un.org/unsd/publication/seriesM/seriesm_4rev4f.pdf.

Snijkers, G., G. Haraldsen, J. Jones et D. Willimack (2013), Designing and Conducting Business Surveys, John Wiley and Sons, Hoboken (NJ), États-Unis.

UNESCO-ISU (2014), “ Guide pour la conduite d'une enquête de R-D : À destination des pays commençant à mesurer la recherche et le développement expérimental ", Document technique, $\mathrm{n}^{\circ}$ 11, ISU, Montréal, http://www.uis.unesco.org/Library/Documents/ tp11-guide-research-experimental-development-r-d-survey-countries-starting-measuring2014-fre.pdf. 
PARTIE II

\section{Mesurer la R-D : Directives sectorielles}





\section{Chapitre 7}

\section{R-D du secteur des entreprises}

Le présent chapitre propose des orientations sur la mesure des activités de recherche et de développement expérimental (R-D) conduites dans le secteur des entreprises, les sources de financement et la ventilation des statistiques par activité économique principale, taille de l'entreprise en termes d'emploi et localisation géographique. Les indicateurs d'activités de $R$-D pour ce secteur comprennent les dépenses de $R-D$, les effectifs de $R-D$, les sources de financement de la $R-D$ et la répartition de la $R-D$ entre recherche fondamentale, recherche appliquée et développement expérimental. La possibilité de ventiler les statistiques par branche d'activité (domaine de produit ou secteur desservi) est aussi examinée, ainsi que les raisons qui militent en ce sens. D'autres répartitions - par domaine de recherche et de développement, objectif socioéconomique, localisation géographique et domaine technologique spécifique - sont également abordées. Le chapitre examine pour finir la conception des enquêtes, la collecte des données et les estimations dans ce secteur. Les statistiques ainsi obtenues étayent le débat d'orientation sur des questions comme les secteurs d'activité dominants et émergents, la concentration de la $R-D$ dans certaines régions, branches d'activité ou entreprises et les secteurs desservis par la R-D d'entreprise. 


\subsection{Introduction}

7.1 Dans la plupart des pays industrialisés, le secteur des entreprises représente la plus grosse part des dépenses et du personnel de R-D. Lorsque l'on analyse ce secteur et les unités qui le composent, il importe de prendre en considération les différentes stratégiques mises en œuvre par les entreprises pour gérer leurs activités de R-D. Il arrive ainsi que des entreprises apparentées se réunissent pour financer, produire, échanger et exploiter conjointement des connaissances de R-D selon des modalités diverses et complémentaires. La complexité de la structure des entreprises, en particulier des entreprises multinationales (EMN), fait obstacle à la mesure de la R-D. En outre, pour certaines entreprises, la R-D est une activité occasionnelle et non continue, ce qui la rend d'autant plus difficile à repérer et à chiffrer. Néanmoins, il est particulièrement utile pour l'action publique de recueillir auprès des entreprises des informations sur le rôle des pouvoirs publics dans le soutien financier de la R-D et sur les interactions entre la R-D des entreprises et la base scientifique et de la recherche publique. $\mathrm{Du}$ point de vue méthodologique, la collecte de données auprès des entreprises n'est pas sans poser des difficultés d'ordre pratique, s'agissant par exemple de recenser les entreprises qui exécutent des activités de R-D, d'obtenir les informations sur la $\mathrm{R}-\mathrm{D}$ requises dans le présent manuel, de respecter les règles de confidentialité et de réduire au minimum les contraintes pesant sur les répondants.

\subsection{Champ couvert par le secteur des entreprises}

7.2 Comme indiqué au chapitre 3, le secteur des entreprises comprend :

- Toutes les sociétés résidentes, y compris et non exclusivement les entreprises légalement constituées en société, indépendamment du lieu de résidence de leurs actionnaires. En font également partie tous les autres types de quasisociétés, c'est-à-dire les unités capables de dégager un profit ou une autre forme de gain financier pour leur(s) propriétaire(s), reconnues par la loi comme des entités juridiques distinctes de ceux-ci et créées dans le but de mener des activités de production marchande à des prix économiquement significatifs. Cela inclut les sociétés financières et non financières.

- Les succursales d'entreprises non résidentes non constituées en société et considérées comme résidentes du fait qu'elles mènent dans la durée des activités de production sur le territoire économique.

- Toutes les institutions sans but lucratif (ISBL) résidentes qui sont des producteurs marchands de biens ou de services ou qui servent les entreprises. Les premières regroupent les instituts de recherche indépendants, les centres 
de soins et autres établissements dont l'activité principale consiste à produire des biens ou des services vendus à des prix fixés de manière à couvrir la totalité du coût économique. Les secondes, qui sont au service des entreprises, sont composées d'entités contrôlées par des associations professionnelles et financées par des contributions et cotisations.

- En sont expressément exclues les unités du secteur de l'enseignement supérieur telles que décrites dans la section 3.5 du chapitre 3, et au chapitre 9 . Cependant, les entreprises commerciales appartenant à des institutions de l'enseignement supérieur, par exemple, sous l'effet d'un contrat accordant à une université des parts importantes au sein d'une entreprise dérivée créée par des membres de son personnel ou des étudiants, doivent être traitées comme des entreprises.

7.3 Le secteur des entreprises comprend à la fois les entreprises privées (cotées ou non en bourse) et les entreprises contrôlées par l'État (voir chapitre 3, section 3.5) qui, dans ce manuel, sont appelées " entreprises publiques". L'assignation d'une entreprise publique au secteur des entreprises ou au secteur de l'État dépend de son degré d'intégration au marché, c'est-à-dire du fait de savoir si elle a pour activité principale la production marchande de biens ou de services à des prix économiquement significatifs. Un institut de recherche public qui tire occasionnellement des revenus très importants de la vente ou de l'octroi de licences sur sa propriété intellectuelle ne peut être considéré comme une entreprise publique si la majorité de ses activités de R-D sont conduites dans un but non commercial. En revanche, un institut contrôlé par l'État dont le fonctionnement repose sur la perception de redevances en échange de la fourniture de services de R-D et de l'accès à une infrastructure de recherche doit être classé comme une entreprise publique si les redevances perçues reflètent pleinement le coût économique de ce type de services. La notion de " secteur public » est plus étendue que celle de " secteur de l'État ».

7.4 Conformément au Système de comptabilité nationale (SCN), les institutions sans but lucratif contrôlées par des entreprises ou ayant comme activité principale de fournir des services aux entreprises, comme les associations professionnelles ou les instituts de recherche contrôlés par l'industrie, par exemple, doivent être classées dans le secteur des entreprises, même si ces institutions fonctionnent sur la base de cotisations couvrant à peine leurs dépenses de fonctionnement et équilibrent en grande partie leur budget avec des subventions de l'État. D'une manière générale, les ISBL créées et gérées par des associations professionnelles pour promouvoir leurs activités - par exemple, les chambres de commerce, les chambres d'agriculture et les associations manufacturières ou commerciales - et qui sont financées au moyen de contributions ou de cotisations des entreprises couvrant les ressources de base ou le financement par projets de leurs activités de R-D sont à classer dans le secteur des entreprises.

7.5 Les activités marchandes des entreprises non constituées en société, qui appartiennent à des ménages (par exemple, certaines formes de partenariats, 
ou même les consultants ou sous-traitants indépendants qui conduisent des projets de R-D pour une autre unité à des prix économiquement significatifs) doivent aussi, dans la mesure du possible, être incluses dans le secteur des entreprises.

7.6 Comme indiqué au chapitre 3, les activités de recherche ou d'invention des individus qui poursuivent de telles activités par intérêt personnel sur leur temps personnel et à leurs frais ne sont pas actuellement prises en compte dans l'approche institutionnelle des statistiques de R-D décrite dans le présent manuel.

7.7 Une coentreprise, telle que définie dans le Système de comptabilité nationale, implique l'établissement d'une société, d'un partenariat ou d'une autre unité institutionnelle au sein de laquelle chaque partie exerce légalement un contrôle conjoint sur les activités de l'unité. Elle opère de la même façon que les autres unités, à cette exception près qu'un accord juridique entre les parties établit un contrôle conjoint sur l'unité. Les coentreprises doivent être classées en fonction des unités qu'elles servent au premier chef, en tenant compte, dans la mesure du possible, des pratiques établies sur la base du SCN.

7.8 Une unité institutionnelle distincte créée pour gérer une coentreprise doit faire l'objet d'une classification sectorielle identique à celle de l'unité institutionnelle dont les intérêts prédominent dans ce partenariat. Les partenariats de R-D ont parfois un statut formel indépendant. Dans ce cas, ils doivent être classés en fonction des unités qu'ils servent au premier chef.

\subsection{Unités statistiques et unités déclarantes}

7.9 Les entreprises gèrent leurs activités de financement et d'exécution de la R-D à différents niveaux pour atteindre au mieux leurs objectifs propres. Les décisions stratégiques relatives au financement et à l'orientation des efforts de R-D sont parfois prises au niveau du groupe, au-delà des frontières nationales, tandis que la gestion au jour le jour des activités de R-D, y compris éventuellement les décisions concernant le type de financement à allouer à l'exécution de la R-D et au recrutement des ressources humaines correspondantes, peut avoir lieu à un niveau moins élevé de l'organisation. Les EMN mènent parfois des activités de R-D dans plusieurs pays à la fois, ce qui rend difficile le repérage des décideurs et la réalisation d'enquête auprès d'eux. Ces facteurs peuvent avoir des incidences sur la classification assignée à une unité statistique et peser sur le choix de l'unité déclarante (pour la définition de ces deux types d'unité, voir chapitre 6).

\section{Unités statistiques}

7.10 L'unité statistique du secteur des entreprises sera généralement l'entreprise, telle que définie au chapitre 6.

7.11 En règle générale, toutes les unités statistiques incluses dans la population d'une enquête de R-D doivent être identifiées de manière adéquate 
sur la base d'un certain nombre de variables descriptives qu'il est normalement possible d'extraire des registres statistiques des entreprises. Les variables d'identification (ou de qualification, comme indiqué au chapitre 3) doivent inclure : un code d'identification, une variable géographique, une variable portant sur le type d'activité économique et une variable de taille. Toute information supplémentaire sur le statut juridique et l'organisation économique d'une unité statistique, ainsi que sur la structure de son capital, sera extrêmement utile pour renforcer l'efficacité et l'efficience du processus d'enquête.

\section{Unités déclarantes}

7.12 La sélection des unités déclarantes du secteur des entreprises variera d'un pays à l'autre en fonction des structures institutionnelles, du cadre juridique dans lequel s'inscrit la collecte des données, des traditions et priorités nationales, du montant des ressources allouées à l'enquête et des accords ad hoc établis avec les entreprises. Lorsqu'une entreprise mène des activités économiques hétérogènes et exécute un volume important de R-D en relation avec plusieurs types d'activités, il est conseillé de recueillir (ou de faire déclarer) les données à un niveau statistique plus fin, par exemple sur la base du type d'activité ou même de l'établissement si la situation régionale est un facteur important. Le présent manuel ne formule aucune recommandation générale sur le choix des unités déclarantes à interroger dans chaque pays. Les offices statistiques nationaux doivent veiller à l'additivité des données sur l'exécution de la R-D et le personnel qui y est affecté, et traiter de manière appropriée les données concernant les flux de financement, quelle que soit la démarche de collecte adoptée. La sélection des unités déclarantes doit être effectuée conformément aux orientations générales formulées au chapitre 6 sur l'identification des unités statistiques et des unités déclarantes, sans oublier la recommandation d'éviter de recueillir des données à partir d'une unité déclarante non soumise à l'obligation de produire des documents comptables officiels.

7.13 Certaines informations ne pouvant parfois être obtenues des entreprises qu'à un niveau d'agrégation plus élevé, les offices statistiques nationaux devront peut-être entrer en contact avec les unités pertinentes pour s'assurer que les statistiques sur la R-D du secteur des entreprises sont conformes aux principes régissant les statistiques nationales, en ventilant leurs activités par juridiction et par domaine d'activité spécifique. Le profilage des groupes d'entreprises est un travail important qui doit, dans la mesure du possible, être effectué en coordination avec les entités qui gèrent les registres d'entreprises et leurs représentants. Dans certains cas, le compilateur des statistiques de R-D pourra juger approprié, à des fins de synergie ou d'exhaustivité, de retenir dans l'échantillon la totalité des entreprises faisant partie de l'ensemble consolidé des entreprises résidant dans le pays.

7.14 Le niveau du groupe joue parfois un rôle clé en tant qu'unité déclarante, les questionnaires étant remplis ou les réponses approuvées par un 
bureau de l'administration centrale. Dans le cas des holdings, plusieurs méthodes différentes pourront être envisagées : par exemple, leur demander de déclarer les activités de R-D des entreprises qu'ils regroupent dans tel ou tel secteur, ou bien transmettre le questionnaire à l'entreprise qui exécute effectivement de la R-D sur la demande du holding.

\subsection{Classifications institutionnelles des unités statistiques Codes d'identification}

7.15 Un code d'identification est un numéro unique assigné à une unité statistique incluse dans la population cible d'une enquête. L'existence de codes d'identification est extrêmement importante pour les statisticiens de la R-D qui ont en général à traiter des exécutants potentiels de R-D identifiés à partir de sources statistiques et administratives différentes. Les codes d'identification permettent d'éviter la duplication des unités de la population ainsi que les chevauchements partiels entre unités (dans la mesure où les unités statistiques renvoient à des niveaux organisationnels différents : établissements, entreprises, groupes). Ils sont essentiels aux fins de l'efficacité du processus d'échantillonnage et, le cas échéant, de la collecte de données administratives (lorsque des codes identiques sont utilisés aussi dans un but administratif). Du point de vue des utilisateurs des statistiques de R-D, les codes d'identification rendent possible l'appariement de micro-données provenant de sources différentes, notamment des enquêtes de R-D, d'autres enquêtes sur les entreprises ou des campagnes de collecte de données administratives et ils facilitent les analyses longitudinales lorsque la structure d'une unité évolue avec le temps. Lorsque des codes d'identification sont déjà présents dans les registres statistiques sur les entreprises, il est préférable de se servir de ces codes pour la compilation des données de R-D.

\section{Classification sur la base de l'activité économique principale}

7.16 Une entreprise peut poursuivre plusieurs activités économiques. En tant qu'unités (statistiques) institutionnelles, les entreprises sont classées sur la base de leur activité principale. En pratique, la majorité des unités de production mènent des activités de type mixte. La Classification internationale type par industrie (CITI) (Nations Unies, 2008a) est le document de référence pertinent pour la classification internationale des activités économiques ou des branches d'activité. Les entreprises peuvent être actives dans de nombreux secteurs économiques : agriculture, mines, secteur manufacturier et services.

7.17 Les pays qui utilisent des systèmes de classification nationaux (ou régionaux) d'activités économiques doivent appliquer des tables de concordance pour convertir au format CITI leurs données classées par branche d'activité, aux fins de déclaration et de comparaison au niveau international. Le mode de déclaration de l'activité économique principale d'une unité statistique doit permettre de préférence l'identification au niveau des " classes » 
(quatre chiffres) et donc des " groupes " (trois chiffres) de la CITI. Toutefois, il ne doit pas se rapporter à un niveau supérieur à celui des " divisions " (deux chiffres). L'adoption de classifications par activité économique différentes - comme le Système de classification des industries de l'Amérique du Nord (NAICS) ou la Nomenclature des activités économiques dans la Communauté européenne (NACE) - est sans incidence sur la production de statistiques de R-D dès lors que la définition des activités économiques est compatible avec celle de la CITI (une correspondance directe peut normalement être établie au niveau des codes à un et deux chiffres et une correspondance indirecte au niveau des codes à trois et quatre chiffres).

7.18 Aucune activité économique ne doit être exemptée de la déclaration des activités de R-D. Tous les secteurs d'activité économique inclus dans la classification CITI mènent potentiellement des activités de R-D, bien qu'avec des degrés de probabilité variables. Il est possible, par conséquent, d'assigner à chaque unité de chaque activité économique un niveau de probabilité en tant qu'exécutant de R-D. Il conviendra à cet égard de mettre au point et d'appliquer des méthodes adaptées pour traiter les secteurs d'activité où les entreprises ont en moyenne peu de chances d'être identifiées comme des exécutants de R-D (par exemple, l'agriculture ou les services aux ménages). Il est recommandé en pareil cas d'effectuer une sélection préliminaire avant d'inclure des entreprises de ces secteurs dans une enquête régulière de R-D.

7.19 L'identification de l'activité principale est nécessaire pour classer une unité statistique dans une catégorie d'“ activité économique principale ". Pour déterminer l'activité principale d'une unité, il faut connaître la part de valeur ajoutée correspondant à ses différentes activités économiques. En pratique, cependant, il est souvent difficile d'avoir accès à une information aussi détaillée, sauf lorsque celle-ci peut être obtenue à partir d'un registre central, et la classification de l'activité doit donc être établie à l'aide d'autres critères. Les offices statistiques nationaux qui compilent les données de R-D doivent éviter, dans la mesure du possible, de faire des choix de classification séparés et s'appuyer sur l'information conservée dans les registres d'entreprises ou sur d'autres sources administratives de qualité comparable. Dans les enquêtes par échantillonnage, cette information est essentielle pour constituer un échantillon représentatif des entreprises.

7.20 Les grandes entreprises dont la structure est complexe et qui mènent de nombreuses activités économiques différentes posent en pratique des problèmes particuliers. Ces entreprises représentent parfois une part très importante de la R-D. La pertinence des classifications par activité économique pour mesurer les totaux de R-D est examinée plus en détail dans la section 7.6. Les offices statistiques nationaux doivent s'efforcer de trouver un équilibre adéquat entre le maintien d'un degré minimum d'homogénéité quant à l'activité économique de l'entreprise et la prise en compte de l'aptitude de l'entreprise à fournir l'information requise sur ses activités. 


\section{Classification sur la base de l'appartenance au secteur public ou privé}

7.21 Conformément aux indications fournies dans la section $3.4 \mathrm{du}$ chapitre 3, il est recommandé d'utiliser la classification des entreprises suivante :

- entreprises privés contrôlées par des intérêts nationaux (qui ne sont pas contrôlées par l'État ou par des unités institutionnelles non résidentes)

- entreprises publiques (soumises au contrôle d'organes de l'État)

- entreprises apparentées ou membres d'un groupe national ou étranger

- entreprises étrangères (contrôlées par des unités institutionnelles non résidentes). L'intérêt de cette catégorie est précisé au chapitre 11 sur la mondialisation de la R-D.

7.22 Les compilateurs de données de R-D pourront aussi souhaiter se servir de classifications prenant en compte le statut juridique des entreprises (par exemple, les entreprises cotées en bourse, les entreprises non constituées en société anonyme) pour répondre à certains besoins spécifiques des utilisateurs des données à l'intérieur de leur pays.

\section{Classification par taille de l'entreprise}

7.23 Les unités peuvent être classées par taille en fonction de l'emploi, des revenus ou d'autres attributs économiques et financiers. L'emploi est souvent une mesure moins ambiguë et donc préférable, mais certains pays préfèrent utiliser le nombre de personnes occupées par l'unité statistique (comme le recommande le présent manuel), tandis que d'autres optent pour le nombre de salariés, la différence correspondant au nombre de propriétaires exploitants et de travailleurs non rémunérés.

7.24 La classification par taille est pertinente aux fins de la stratification, de la définition de l'échantillon, des modalités de ciblage au moyen du formulaire d'enquête et de la présentation des résultats statistiques. Dans certains pays, la réglementation statistique restreint les possibilités de sondage des entreprises de très petite taille. L'exécution de la R-D étant en général une activité fortement concentrée, la sous-représentation des exécutants de R-D de petite taille n'a sans doute guère d'impact substantiel sur les totaux agrégés obtenus mais elle pourrait fausser de manière non négligeable d'autres types de statistiques et d'analyses basées sur la R-D. C'est la raison pour laquelle tous les moyens doivent être utilisés pour assurer la couverture la plus complète possible.

7.25 La taille de l'entreprise est une variable d'identification essentielle pour la définition de l'échantillon et l'estimation des données, ainsi que pour la bonne gestion des activités de collecte des données. Il est recommandé de définir la taille sur la base du nombre moyen de personnes occupées par l'unité statistique. Une telle définition est préférable en raison de sa simplicité, de son applicabilité générale et de son utilité, et aussi parce qu'elle permet les comparaisons au niveau international. Il est possible d'utiliser une variable de taille pour exclure délibérément certaines unités de la population cible 
(les entreprises en dessous d'un seuil donné, le cas échéant) ou d'adapter les méthodes de collecte des données à la taille et à l'organisation des unités ciblées.

7.26 Il est recommandé de considérer toutes les unités appartenant au secteur des entreprises, quelles que soient leur taille et leur activité économique principale, comme des exécutants potentiels de R-D. Dans les pays où les petites ou microentreprises sont exclues des enquêtes régulières de R-D en vertu de la réglementation statistique ou en raison de contraintes pratiques et techniques, il faudrait s'efforcer d'identifier la contribution globale de ces entreprises au total de la R-D des entreprises.

7.27 Les catégories de taille suivantes (basées sur le nombre de personnes occupées par l'unité statistique) sont proposées pour le classement des entreprises :

\section{1 à 4}

5 à 9

10 à 19

20 à 49

50 à 99

100 à 249

250 à 499

500 à 999

1000 à 4999

5000 et plus

Pour des raisons pratiques, étant donné le très grand nombre d'entreprises sans effectifs dans les registres et l'impossibilité pour ces entreprises de mener des activités de R-D, il est recommandé de les exclure du champ des enquêtes de R-D.

7.28 Ces catégories ont été choisies pour diverses raisons, en particulier dans un but de cohérence avec la classification par taille généralement utilisée pour les microentreprises (à savoir les entreprises comptant moins de 5, 10 ou 20 personnes occupées, selon les pratiques propres à chaque pays) et pour les petites et moyennes entreprises (à savoir les entreprises de moins de 250 ou 500 personnes occupées, selon les pratiques propres à chaque pays). Ces dix catégories, par conséquent, ne sont pas proposées dans un but d'utilisation commune, mais en vue de fournir une structure de classification sur laquelle pourront s'appuyer les pratiques particulières de chaque pays. Néanmoins, il est recommandé à tous les pays de maintenir des catégories allant jusqu'à 9, 49 et 249 personnes occupées, afin d'assurer la comparabilité internationale des statistiques concernant les petites, moyennes et grandes entreprises. Dans les économies de grande taille, il est également recommandé de maintenir une catégorie allant jusqu'à 999 personnes occupées. 


\section{Classification sur la base de la situation géographique}

7.29 La situation géographique des unités est aussi une variable de classification essentielle. Cette situation est généralement définie comme le " pays de résidence " mais différents niveaux de détail peuvent être envisagés : l'état ou la région (en fonction de l'organisation administrative du pays), la localité (ville ou municipalité) ou même l'adresse. Dans le traitement des unités déclarantes qui diffèrent des unités statistiques - ou de plusieurs unités déclarantes appartenant à une seule entreprise -, un soin particulier doit être consacré à l'identification de la situation géographique pertinente en vue de la compilation des données.

\subsection{Indicateurs des activités de R-D dans le secteur des entreprises}

7.30 Les activités de R-D menées par les unités du secteur des entreprises sont mesurées à la fois en termes de dépenses de R-D et de coût des effectifs de R-D. Ces deux types d'indicateurs peuvent souvent être obtenus conformément aux recommandations formulées respectivement aux chapitres 4 et 5, et les dépenses de R-D des entreprises peuvent être réparties fonctionnellement selon les modalités décrites dans la section 7.6.

\section{Dépenses de R-D}

7.31 Pour assurer la cohérence entre les informations portant sur la R-D et les autres informations fournies par les répondants, les offices statistiques nationaux qui traitent les données de R-D doivent, lorsque cela est possible, vérifier certaines relations comptables. Par exemple, les coûts de main-d'œuvre de la R-D doivent être moins élevés que le total des coûts de main d'œuvre (ou identiques si toutes les personnes occupées travaillent à temps plein à la R-D). Les dépenses de capital consacrées à la R-D doivent aussi être comprises dans les dépenses de capital globales de l'entreprise et ne peuvent donc être supérieures à ces dernières. Les coûts totaux de R-D ne doivent généralement pas excéder la valeur ajoutée dégagée par l'entreprise pendant une année de référence donnée. Dans le cas des entreprises qui mènent continûment des activités de $R-D$, la R-D peut représenter une part stable de la valeur ajoutée sur une période de plusieurs années. Le contrôle de ces relations comptables permet de réduire les erreurs de déclaration des données sur la R-D et contribue ainsi à améliorer la qualité globale des données.

\section{Personnel de R-D}

7.32 Il est également nécessaire de veiller à la cohérence entre le total des effectifs des entreprises déclarantes et les données de R-D recueillies. La mesure de la force de travail de la R-D dans les entreprises, ainsi que de la participation de personnel extérieur aux activités de R-D intra-muros (voir chapitre 5), peuvent poser des difficultés. Il est suggéré que l'unité déclarante, dans un premier 
temps, détermine les activités de R-D menées pendant la période de référence par les personnes occupées dans l'entreprise. La prise en compte complète de leur contribution à la R-D - à la fois en termes de temps (équivalent temps plein) et de coûts de main d'œuvre - sera plus facile si l'unité se sert directement des données de paie, en incluant ainsi les travailleurs à temps partiel et les stagiaires. S'il est possible d'obtenir ces données à partir des registres d'entreprises ou de sources administratives, les offices statistiques nationaux doivent vérifier la cohérence des totaux relatifs aux effectifs : le total du personnel interne de R-D ne doit pas, par exemple, être plus élevé que celui de l'ensemble des effectifs.

7.33 L'étape suivante consistera à identifier tous les contributeurs externes à la R-D intra-muros, qui comprennent parfois des postes et des profils très variés : consultants indépendants, employés de sous-traitants agissant comme consultants intra-muros, personnel intérimaire, etc. Un effort important est requis des répondants pour identifier le personnel contribuant de façon appréciable (en termes de temps, voir chapitre 5, section 5.3) à la R-D intra-muros de l'unité.

7.34 La ventilation du personnel de R-D par fonction (chercheurs, techniciens et personnel assimilé, autre personnel de soutien) est examinée en détail au chapitre 5 et les recommandations figurant dans ce chapitre sont tout à fait pertinentes pour le secteur des entreprises. Il est nécessaire d'obtenir à la fois des données en équivalent temps plein et en chiffres absolus, ainsi que la répartition démographique de ces totaux au regard de caractéristiques telles que le sexe, l'âge, le niveau de qualification, etc. (voir section 5.4). La répartition fonctionnelle du personnel de R-D doit s'appuyer sur l'observation directe du rôle que jouent effectivement les individus concernés dans la R-D intra-muros, quelles que soient leurs fonctions officielles dans l'entreprise. Les pratiques courantes exigent de ne présupposer aucune relation directe entre le niveau de compétences formel (ou contractuel) - même décrit en termes de profession d'une personne et son type de contribution à la R-D intra-muros. Il est fréquent de constater, par exemple, que des personnes participant comme " chercheurs " aux activités de R-D intra-muros d'une entreprise occupent officiellement un poste de " technicien » ou de " gestionnaire » et non de « chercheur ».

\subsection{Répartition fonctionnelle des dépenses intérieures de R-D des entreprises (DIRDE)}

7.35 Les dépenses intérieures de R-D des entreprises (DIRDE) constituent le principal agrégat statistique utilisé pour décrire l'exécution de la R-D au sein du secteur des entreprises. Les DIRDE correspondent à la composante des dépenses intérieures brutes de R-D (DIRD) (voir chapitre 4) engagées par les unités du secteur des entreprises. Il s'agit des dépenses de R-D intra-muros engagées dans ce secteur au cours d'une période de référence donnée. La compilation, la diffusion et la déclaration des DIRDE peuvent être utiles pour plusieurs variables. Certaines répartitions présentent un très grand intérêt et une forte applicabilité au niveau international ; d'autres n'ont de pertinence qu'au niveau national, 
aux fins de l'analyse et de l'action publique. On trouvera ci-dessous une liste des répartitions recommandées aux offices statistiques nationaux afin de prendre au mieux en compte les besoins de la comparabilité internationale. La plupart des répartitions fonctionnelles recommandées ne sont possibles qu'à condition d'identifier séparément l'activité de chaque unité statistique, puis d'additionner les informations distribuées par unité dans l'ensemble du secteur (par ex. R-D par source de financement). D'autres répartitions signalées des DIRDE découlent évidemment de l'accumulation de la R-D totale des unités statistiques au regard des critères de classification institutionnels initiaux (par ex., R-D par activité économique principale et par taille des entreprises). Il est conseillé de ne pas perdre de vue ces recommandations lors des enquêtes et d'autres activités de collecte de données.

\section{Répartition des DIRDE par source de financement de la R-D}

7.36 Comme on l'a vu dans la section 4.3 du chapitre 4, il est recommandé de prendre en compte cinq sources majeures lors de la collecte des données et de l'analyse des sources de financement des DIRDE, selon l'origine des fonds : le secteur des entreprises (qui inclut à la fois le financement interne et le financement externe reçu d'autres entreprises), le secteur de l'État, le secteur de l'enseignement supérieur, le secteur privé sans but lucratif et le reste du monde (tableau 7.1).

\section{Tableau 7.1. Identification des sources de financement de la R-D intra-muros du secteur des entreprises}

\begin{tabular}{l}
\hline Sources de financement \\
\hline Secteur des entreprises \\
Entreprise individuelle (fonds d'origine interne) \\
Autres entreprises du même groupe \\
Autres entreprises non affiliées \\
Secteur de l'État ${ }^{1}$ \\
Administration centrale ou fédérale \\
Administration provinciale ou de l'État \\
Autres organes du secteur de l'État \\
Secteur de l'enseignement supérieur \\
Secteur privé sans but lucratif \\
Reste du monde \\
Secteur des entreprises \\
Entreprises du même groupe \\
Autres entreprises non affiliées \\
Secteur de l'État \\
Secteur de l'enseignement supérieur \\
Secteur privé sans but lucratif \\
Organisations internationales (y compris supranationales)
\end{tabular}

1. Il est recommandé de distinguer les fonds d'échange pour la R-D des fonds de transfert pour la R-D. 


\section{Financement provenant du secteur des entreprises}

7.37 Le présent manuel ne recommande pas une méthode spécifique de collecte de données sur les sources de financement des DIRDE. Certains pays choisiront d'estimer d'abord le total de la R-D intra-muros, puis de déterminer les sources individuelles de financement. Dans ce cas, le financement interne des entreprises pourra être mesuré comme un montant résiduel, après identification de toutes les sources extérieures de financement qui contribuent aux activités de R-D intra-muros. D'autres pays demanderont aux répondants d'indiquer séparément la $R-D$ intra-muros financée sur fonds d'origine interne et la R-D intra-muros financée par d'autres afin de permettre l'extraction de données des comptes financiers. Cette seconde approche facilitera sans doute le travail de déclaration des entreprises qui maintiennent une séparation physique et financière entre la R-D intra-muros financée sur fonds d'origine interne et la R-D intra-muros effectuée sur une base contractuelle (par exemple, les activités liées à la défense, pour des questions de sécurité).

7.38 Dans le secteur des entreprises, les fonds d'origine interne comprennent les réserves ou bénéfices non distribués (par exemple sous forme de dividendes), les ventes des produits ordinaires de l'unité (autres que la R-D), les capitaux mobilisés sous la forme de levée de fonds propres, d'emprunt ou d'autres instruments hybrides (fonds levés sur les marchés financiers, prêts de banques, capital-risque, etc.). Les déductions de l'impôt sur le revenu dues à des incitations publiques en faveur d'activités de R-D menées dans le passé font aussi partie des fonds d'origine interne, car elles ne servent pas nécessairement à financer la R-D dans la période de référence choisie. Étant donné la grande importance de cette catégorie de financement, les pays pourront décider d'inclure dans leurs enquêtes des questions sur certaines sources internes particulières afin de déterminer, par exemple, l'impact de politiques spécifiques de $R-D$, mais le présent manuel ne recommande aucune ventilation particulière de ces sources de financement.

7.39 Dans certains cas, une entreprise se voit obligée de contracter un prêt pour financer ses activités de R-D. Un prêt constitue, d'une manière générale, une dette avec intérêt encourue par une unité (institution ou ménage) à l'égard d'une autre unité. Par conséquent, lorsqu'une entreprise menant des activités de R-D reçoit, en tant qu'emprunteur, une somme d'argent d'un prêteur pour financer ses activités de R-D, elle s'engage à rembourser ultérieurement au prêteur un montant identique auquel s'ajoute une rémunération pour le service fourni sous forme d'intérêt de la dette. Le montant du prêt doit être inclus dans les fonds d'origine interne puisque la source de financement extérieure escompte que la somme prêtée lui sera remboursée. Le coût de l'emprunt n'est pas inclus dans la R-D. Le même raisonnement s'applique lorsqu'une unité externe fournit les garanties nécessaires à l'obtention d'un prêt ou lorsque les intérêts sont couverts en totalité ou en partie par d'autres sources de financement (comme cela est 
parfois le cas pour les prêts de R-D subventionnés par l'État). Ces formes d'aide sont à inclure dans les fonds d'origine interne.

7.40 Il est recommandé de distinguer les fonds reçus d'autres entreprises nationales non affiliées et d'entreprises affiliées faisant partie du même groupe national. Les deux catégories d'entreprises sont considérées comme des sources de financement extérieures. Dans la plupart des statistiques internationales sur les DIRDE, la source de financement représente la somme du financement interne des entreprises, du financement des entreprises nationales non affiliées et du financement des entreprises affiliées faisant partie d'un même groupe national (voir aussi la section 7.7 sur la déclaration de la R-D extra-muros).

7.41 Les données relatives aux entreprises affiliées et non affiliées situées à l'étranger doivent être obtenues séparément et présentées comme faisant partie du « reste du monde ».

\section{Financement provenant du secteur de l'État}

7.42 S'agissant du financement de la R-D intra-muros reçu de l'État, il importe de veiller à ce que les répondants distinguent les fonds versés sans attente de résultats de $\mathrm{R}-\mathrm{D}$ en contrepartie (par exemple, les aides ou les subventions) et les fonds fournis en échange de résultats de $R-D$, généralement dans le cadre de marchés publics avec une institution de l'État. Certaines entreprises ont du mal à différencier les contrats impliquant la fourniture de $\mathrm{R}-\mathrm{D}$ à une entreprise publique des contrats de R-D établis avec un organe de l'administration publique. Il est aussi parfois difficile de distinguer en pratique fonds d'échange et fonds de transfert, car cela dépend de l'allocation des risques et des droits sur le résultat incertain d'activités de R-D exécutées par les entreprises et financées par l'État. En définitive, le but est de réussir à distinguer les deux catégories décrites dans la section 4.3 du chapitre 4. Les entreprises emploient fréquemment, par exemple, le terme de " contrat " pour désigner un accord de subvention, mais il faut s'efforcer de classer avec exactitude ces différentes formes de financement.

7.43 Certains pays souhaitent recueillir des informations sur le niveau d'administration qui fournit les fonds de R-D et, parfois même sur les institutions ou programmes concernés. En pratique, le plus souvent, le " financement ayant pour origine l'administration centrale ou fédérale » est distingué du " financement ayant pour origine les régions ou États fédérés " (conjointement, en général, avec le partage entre fonds de transfert, comme les subventions et fonds d'échange, comme les revenus tirés de marchés publics).

7.44 Dans la mesure du possible, la source originale de financement doit être identifiée aux fins de la déclaration des données, même lorsqu'une institution publique ou privée sert d'intermédiaire et effectue les transferts de fonds. Dans bien des cas, l'entreprise bénéficiaire ne peut fournir d'informations que sur l'institution intermédiaire, c'est-à-dire la source de financement la plus proche. 
7.45 Certains pays accordent des formes spécifiques d'allégement fiscal dans le but d'encourager le financement ou l'exécution d'activités de R-D. On trouvera des instructions sur les moyens de mesurer séparément ce type de soutien au chapitre 12. Comme indiqué au chapitre 4 (section 4.3), le présent manuel recommande de traiter les coûts d'exécution de la R-D qui sont " financés " en escomptant des revenus ou des allègements fiscaux futurs ou les remises d'impôt obtenues dans la période en cours au titre d'activités antérieures, comme un financement d'origine interne et non comme des aides de l'État.

\section{Financement provenant du reste du monde}

7.46 Lors de la collecte de données sur le financement reçu de l'étranger, il est souvent pertinent d'identifier le secteur d'où provient le financement, tout comme pour les sources de financement nationales. Comme indiqué précédemment, il importe particulièrement de distinguer les fonds reçus d'entreprises affiliées situées à l'étranger de ceux qui proviennent d'entreprises non affiliées non résidentes. Les pays choisiront de classer diverses organisations et agences de financement internationales et supranationales comme des sources de financement pertinentes. Dans le cas des pays membres de l'Union européenne (UE), les « institutions et autres organes de l'UE » pourront constituer une telle source de financement.

\section{Répartition des DIRDE par type de R-D}

7.47 Comme dans tous les autres secteurs, il est recommandé de recueillir auprès des entreprises des données sur la ventilation des dépenses de R-D par type de R-D, comme indiqué au chapitre 2 et en tenant compte des précisions ci-dessous :

- recherche fondamentale : les entreprises mènent effectivement des activités de "recherche pure ". Il est clair, cependant, qu'elles entreprennent plus fréquemment des recherches en vue de préparer une nouvelle génération de technologies, même lorsqu'elles ne visent pas encore une application ou une utilisation commerciale immédiate. Ce type de recherche entre dans la définition de la recherche fondamentale, puisqu'il ne vise pas une utilisation spécifique mais est réalisé dans l'espoir qu'il en découlera un nombre indéterminé d'applications potentielles à l'avenir. C'est ce qu'on appelle couramment la " recherche fondamentale orientée ". Comme on présuppose que seule une petite partie de la R-D des entreprises relève de la recherche fondamentale, il est recommandé aux offices statistiques nationaux de vérifier soigneusement si les répondants qui déclarent des dépenses intra-muros de recherche fondamentale assez élevées ont bien compris ce que recouvre la recherche fondamentale, telle que définie dans le présent manuel ;

- recherche appliquée : ce type d'activité vise à résoudre un problème particulier ou à atteindre un objectif commercial spécifique. Le passage de la recherche 
fondamentale à la recherche appliquée se manifeste souvent par le lancement d'un nouveau projet visant à explorer les résultats prometteurs d'un programme de recherche fondamentale (ce qui implique aussi souvent le passage d'une perspective à long terme à une perspective à court ou moyen terme). Les entreprises ont aussi fréquemment besoin, pour soutenir leurs activités de " développement de produit ", de s'appuyer sur les connaissances supplémentaires issues d'activités de recherche appliquée, dont les résultats ouvrent souvent la voie à une large gamme d'applications potentielles ;

- développement expérimental : composante généralement la plus importante de la R-D des entreprises, le développement expérimental a pour but la mise au point de plans ou de modèles devant déboucher sur des produits ou procédés nouveaux ou nettement améliorés, que ce soit pour la vente ou l'utilisation propre de l'entreprise. S'appuyant sur des recherches antérieures ou sur l'expérience pratique, il comprend la formulation d'un concept, la conception et la mise à l'essai de produits de substitution, et peut aussi englober la construction de prototypes et le lancement d'une installation-pilote (voir chapitre 2, section 2.7). Il n'inclut pas les tests de routine, la résolution de problèmes ou les modifications périodiques de produits, de chaînes de production, de procédés existants ou d'opérations en cours. Les premières unités produites dans le cadre d'un essai en vue d'une production en grande série ne doivent pas être considérées comme des prototypes de R-D. De telles activités, en effet, ne satisfont pas explicitement aux critères de nouveauté et d'incertitude. Pour être comptabilisées comme relevant du développement expérimental, des activités doivent requérir le savoir et les compétences d'un " chercheur ». Les statisticiens doivent en outre aider les répondants à distinguer le « développement expérimental » du développement de produit - qui est plus vaste et englobe les activités de mise sur le marché - ainsi que du stade de la pré-production, terme fréquemment utilisé dans les projets publics de grande ampleur concernant la défense ou l'aérospatiale et qui englobe le travail non expérimental sur les produits ou les systèmes comme la conception technique ultime, l'outillage et l'ingénierie industrielle, les démonstrations à l'intention des utilisateurs et même parfois, les premières activités limitées de production. La frontière n'est cependant pas toujours tout à fait claire.

\section{Répartition des DIRDE par orientation sectorielle ou par classification de l'activité économique}

\section{$R-D$ par activité économique principale des entreprises}

7.48 Comme indiqué plus haut, les variables de classification institutionnelle peuvent être utilisées pour répartir les DIRDE. Des indicateurs des dépenses de R-D et des effectifs de R-D sont, par exemple, régulièrement produits pour un grand nombre de secteurs. Une variable de la classification CITI des activités économiques (Nations Unies, 2008a) devrait exister pour l'ensemble 
des entreprises incluses dans la population cible d'une enquête de R-D (voir section 7.4). En agrégeant la R-D intra-muros des entreprises, il est possible de déterminer le niveau de la R-D mise en œuvre par toutes les unités d'un secteur particulier. L'intérêt de ce type d'indicateur reposant sur les unités est qu'il peut facilement être mis en relation avec d'autres statistiques économiques sectorielles établies sur la base de l'activité principale, à condition que les critères de définition des entreprises, en tant qu'unités statistiques et leur assignation sectorielle soient mutuellement compatibles.

7.49 L'activité économique principale d'une entreprise désigne généralement l'activité grâce à laquelle celle-ci obtient les résultats économiques les plus importants. Ce mode de classification des entreprises est pertinent aux fins de la mesure de la R-D. Les ressources de R-D des entreprises classées dans la division 72 de la CITI Révision 4 doivent, par exemple, être comptabilisées comme telles. Les approches fondées sur le contenu de la R-D recoupent la répartition fonctionnelle par domaine de produit ou secteur desservi par la R-D présentée plus bas.

7.50 Le présent manuel reconnaît que dans certains pays, la répartition fonctionnelle de la R-D par orientation sectorielle est considérée comme une méthode particulièrement pertinente pour comptabiliser la R-D des entreprises, et que le recoupement complet avec la classification des unités qui exécutent de la R-D sur la base de l'activité économique principale n'est pas certain. Diverses priorités doivent être prises en compte avant de définir une stratégie nationale en matière de déclaration de R-D et de diffusion de cette information. Néanmoins, les pays sont explicitement incités à se servir aussi des classifications internationales standard des activités économiques dans le domaine de la R-D.

\section{R-D par orientation sectorielle (domaine de produit ou secteur desservi)}

7.51 L'orientation sectorielle de la R-D exécutée par des unités du secteur des entreprises ne peut être déterminée simplement sur la base de leur activité économique principale. Cela tient à deux raisons principales :

- Premièrement, une entreprise peut travailler activement à la fois sur plusieurs lignes de produit courantes ou en projet. Elle peut développer un nouveau produit afin d'entrer sur un nouveau marché qui ne fait pas encore partie de son portefeuille de spécialisation. En outre, les écarts existant entre les pratiques nationales de classification des entreprises peuvent être cause de différences quant au niveau de détail des données collectées sur l'activité économique principale. Utilisé à diverses fins, le tableau agrégé risque d'être faussé si l'on ne prend pas en compte la répartition fonctionnelle interne de la R-D, qui ne correspond pas nécessairement à la composition de la valeur ajoutée ou du chiffre d'affaires en termes de biens et de services produits.

- Deuxièmement, pour beaucoup d'entreprises, le présupposé implicite selon lequel l'activité principale de R-D est réalisée entièrement en interne et sert uniquement les activités propres de l'entreprise n'est pas valable. 
Certaines entreprises se spécialisent dans la fourniture de services de R-D à d'autres entreprises qui utilisent la R-D à l'appui de leur activité économique ; d'autres exécutent la R-D à des fins spéculatives sur la base de leurs ressources internes et préfèrent ne pas utiliser la R-D elles-mêmes mais laissent à d'autres le soin de la commercialiser contre paiement de redevances ou de droits de licence, ou vendent directement les droits de propriété intellectuelle issus de la R-D. De telles pratiques affaiblissent le lien entre l'activité économique principale, l'exécution de la R-D et son orientation sectorielle.

7.52 Sur un plan plus pratique, le fait est que la classification par activité économique principale ne reflète pas toujours le domaine principal des activités de R-D d'une entreprise. Par exemple, une entreprise classée comme entreprise de gros peut néanmoins vendre des produits qu'elle fabrique et ses activités de R-D peuvent être axées entièrement sur l'amélioration de ses procédés de fabrication et de production. Il est probable également que les pratiques générales de classification vont évoluer à l'avenir car les offices statistiques nationaux sont chargés d'appliquer les directives spécifiques concernant le traitement de différents types de producteurs de marchandises sans usine (CEEONU, 2014). Ces directives mettent très fortement l'accent sur l'importance des produits de propriété intellectuelle, y compris les actifs basés sur la R-D.

7.53 Un moyen de minimiser les écarts potentiels de mesure dus à ces différents modèles de financement, d'exécution et d'utilisation de la R-D consiste à demander aux exécutants de la R-D de préciser l'orientation sectorielle de leurs activités de R-D. Cela doit en principe faciliter certains types de comparaison avec les statistiques de production, dans la mesure où il est possible de mettre en relation l'apport de connaissances et l'activité économique à laquelle servent ces connaissances.

7.54 Plusieurs concepts sont potentiellement pertinents au regard de la notion d'orientation sectorielle de la R-D, et les enquêtes de R-D pourront utiliser différents moyens pour obtenir cette information. L'orientation sectorielle peut être identifiée à partir :

- du produit (bien ou service) dont on escompte qu'il intègrera les résultats de la $\mathrm{R}-\mathrm{D}$, quel que soit le secteur qui le produit ou

- du secteur qui utilisera probablement les résultats escomptés de la R-D (sous forme de R-D codifiée comme un brevet ou intégrée à de nouveaux biens ou services).

7.55 Les deux approches se recoupent fortement et peuvent être difficiles à distinguer par les répondants. La R-D, en outre, peut concerner un produit qui est une sous-composante d'un système plus complexe, ou un processus qui sera commercialisé ou intégré à la production d'autres biens et services. La R-D peut être à usage interne dans un secteur donné ou servir en définitive aux branches d'activité auxquelles elle est verticalement intégrée. 
7.56 Pour résoudre ces difficultés, des approches pragmatiques s'imposent. Une contrainte majeure tient au fait que les répondants ne savent pas toujours quel est le secteur qui a le plus de chances de bénéficier des futurs biens et services issus de la R-D, en particulier dans le cas de la recherche fondamentale et de la recherche appliquée. L'utilisation de biens et de services intégrant les résultats de la R-D peut évoluer avec le temps en fonction de la conjoncture et des débouchés commerciaux. La ventilation des données fournies résultera donc parfois d'une approche heuristique s'appuyant sur les activités antérieures de $\mathrm{R}-\mathrm{D}$ et la documentation interne, en particulier les applications commerciales de projets de R-D. Dans le cas de la recherche fondamentale non orientée ou des activités de recherche ayant donné lieu à plusieurs applications connues, les répondants pourront envisager de ventiler les données sur la base des domaines d'activité de l'entreprise.

7.57 En ce qui concerne le choix d'un système de classification, les classifications internationales types, par activité économique et par produit, constituent des outils possibles. La CITI n'est pas conçue pour mesurer les données relatives aux produits à un niveau détaillé. Il existe à cette fin une classification distincte de l'ONU : la Classification centrale des produits (CCP, Nations Unies, 2008b). Chacune des catégories de la CCP est accompagnée d'une référence à l'activité économique de la classification CITI où sont produits pour l'essentiel des biens ou services (critère d'origine sectorielle), mais cela ne veut pas dire que toutes les unités produisant ces biens ou services y soient répertoriées. La classification des produits repose sur les caractéristiques intrinsèques des biens ou la nature des services fournis et, par conséquent, la structure de cette classification diffère de celle de la CITI. Dans le cas de la R-D, l'utilisation des classifications existantes des produits ou des marchandises pose plusieurs difficultés, étant donné que ces classifications englobent des éléments comme les licences d'exploitation de produits à forte intensité de savoir. De telles catégories reflètent surtout le modèle économique correspondant à la mise en œuvre de l'activité de R-D et à l'exploitation de ses résultats, mais non le contenu de la R-D. C'est la raison pour laquelle il n'est pas possible de recommander l'utilisation générale de la CCP, mais les pays qui le souhaitent pourront utiliser certaines catégories de la CCP sur une base ad hoc pour répondre à des besoins spécifiques d'utilisation des statistiques.

7.58 En l'absence de recommandation spécifique (afin de permettre l'adoption des méthodes les mieux adaptées à la situation de chaque pays), il est suggéré, aux fins de la répartition de la R-D des entreprises par orientation sectorielle, d'utiliser une liste simplifiée des d'activités économiques (basée sur la CITI ou une classification équivalente) en privilégiant le secteur desservi ou le domaine de produit. Le présent manuel reconnaît que, pour diverses raisons pratiques, certains pays pourront être amenés à utiliser des approches hybrides (tableau 7.2) mais cela doit être évité dans la mesure du possible. 


\section{Tableau 7.2. Classifications sur la base de l'activité proposées pour le secteur des entreprises}

\begin{tabular}{|c|c|c|c|c|}
\hline Classification & $\begin{array}{c}\text { Base } \\
\text { de classification }\end{array}$ & $\begin{array}{c}\text { Critère } \\
\text { et classification }\end{array}$ & $\begin{array}{c}\text { Application du critère } \\
\text { de classification }\end{array}$ & $\begin{array}{l}\text { Autres caractéristiques } \\
\text { et limites potentielles }\end{array}$ \\
\hline $\begin{array}{l}\text { Principale } \\
\text { activité } \\
\text { économique } \\
\text { (recommandée } \\
\text { pour toutes les } \\
\text { unités de tous } \\
\text { les secteurs } \\
\text { institutionnels) }\end{array}$ & $\begin{array}{l}\text { Institutionnelle : } \\
\text { les dépenses et } \\
\text { les effectifs de } \\
\text { R-D déclarés par } \\
\text { l'unité statistique } \\
\text { sont entièrement } \\
\text { alloués au } \\
\text { secteur d'activité } \\
\text { correspondant à } \\
\text { la classification } \\
\text { de l'unité. }\end{array}$ & $\begin{array}{l}\text { Principale } \\
\text { activité de l'unité } \\
\text { statistique, sur } \\
\text { la base de la CITI } \\
\text { ou de la version } \\
\text { nationale/ } \\
\text { régionale de cette } \\
\text { classification, } \\
\text { paractivités } \\
\text { économique, } \\
\text { branches } \\
\text { d'activité. }\end{array}$ & $\begin{array}{l}\text { Chiffre d'affaires, valeur } \\
\text { ajoutée brute ou autre critère } \\
\text { suffisamment proche. Les } \\
\text { statisticiens de la R-D pourront } \\
\text { s'appuyer sur les classifications } \\
\text { utilisées pour d'autres } \\
\text { statistiques sur les entreprises, } \\
\text { telles qu'elles apparaissent dans } \\
\text { les registres d'entreprises. En } \\
\text { pareil cas, aucune autre question } \\
\text { n'est requise. }\end{array}$ & $\begin{array}{l}\text { Cette classification permet } \\
\text { dans la plupart des cas } \\
\text { d'améliorer la cohérence avec } \\
\text { les statistiques économiques } \\
\text { sur la production et l'emploi. } \\
\text { Cependant, elle peut } \\
\text { entraîner une surallocation } \\
\text { des unités et ressources de } \\
\text { R-D à certaines branches } \\
\text { d'activité (principalement le } \\
\text { commerce de gros) dans le } \\
\text { cas des entreprises menant } \\
\text { de nombreuses activités } \\
\text { économiques différentes et } \\
\text { lorsque la composition du } \\
\text { chiffre d'affaires ou de la } \\
\text { valeur ajoutée (ou d'autres } \\
\text { critères de classification) } \\
\text { ne correspond pas à la } \\
\text { répartition des activités de } \\
\text { R-D au sein de l'entreprise. }\end{array}$ \\
\hline $\begin{array}{l}\text { Orientation } \\
\text { sectorielle } \\
\text { (secteur desservi/ } \\
\text { domaine de } \\
\text { produit) } \\
\text { (recommandée } \\
\text { pour les } \\
\text { entreprises, } \\
\text { en sus de la } \\
\text { classification } \\
\text { sur la base } \\
\text { de l'activité } \\
\text { économique } \\
\text { principale) }\end{array}$ & $\begin{array}{l}\text { Répartition } \\
\text { fonctionnelle: } \\
\text { l'unité statistique } \\
\text { distribue ses } \\
\text { ressources } \\
\text { de R-D entre } \\
\text { les différents } \\
\text { domaines } \\
\text { d'activité pour } \\
\text { lesquels la R-D } \\
\text { est pertinente. }\end{array}$ & $\begin{array}{l}\text { Orientation } \\
\text { sectorielle de la } \\
\text { R-D, sur la base } \\
\text { soit du secteur } \\
\text { desservi ou du } \\
\text { type de produit } \\
\text { intégrant les } \\
\text { résultats de la R-D } \\
\text { (éventuellement } \\
\text { en le reclassant } \\
\text { conformément à } \\
\text { la CITI). }\end{array}$ & $\begin{array}{l}\text { L'application passe } \\
\text { nécessairement par une ou } \\
\text { plusieurs questions spécifiques } \\
\text { dans le questionnaire d'enquête. } \\
\text { Différentes formulations sont } \\
\text { possibles afin de bien exprimer } \\
\text { le concept voulu. }\end{array}$ & $\begin{array}{l}\text { Forte pertinence du point } \\
\text { de vue des politiques et des } \\
\text { utilisateurs au sujet des } \\
\text { activités économiques qui } \\
\text { bénéficient potentiellement } \\
\text { de la R-D. } \\
\text { Entraîne une charge de } \\
\text { réponse supplémentaire pour } \\
\text { les entreprises. Certaines } \\
\text { d'entre elles, en outre, n'ont } \\
\text { qu'une connaissance limitée } \\
\text { de l'utilisation finale de leur } \\
\text { R-D, en particulier dans le cas } \\
\text { de la recherche fondamentale } \\
\text { et de la recherche appliquée. }\end{array}$ \\
\hline $\begin{array}{l}\text { Approches } \\
\text { hybrides } \\
\text { (non } \\
\text { recommandées, } \\
\text { sauf lorsqu'il } \\
\text { n'existe aucune } \\
\text { autre possibilité) }\end{array}$ & $\begin{array}{l}\text { Fonctionnelle et } \\
\text { institutionnelle } \\
\text { à la fois : les } \\
\text { ressources de } \\
\text { R-D de certaines } \\
\text { entreprises } \\
\text { sont réparties } \\
\text { sur une base } \\
\text { fonctionnelle, } \\
\text { alors que } \\
\text { pour d'autres, } \\
\text { l'ensemble de la } \\
\text { R-D est alloué à } \\
\text { un secteur donné. }\end{array}$ & $\begin{array}{l}\text { Application d'une } \\
\text { approche fondée } \\
\text { sur la répartition } \\
\text { fonctionnelle } \\
\text { à un groupe } \\
\text { d'entreprises } \\
\text { et du critère } \\
\text { d'activité } \\
\text { économique } \\
\text { principale à un } \\
\text { autre. }\end{array}$ & $\begin{array}{l}\text { Plusieurs approches: } \\
\text { - simple extension de la } \\
\text { classification sur la base de } \\
\text { l'activité principale, en appliquant } \\
\text { la classification distributionnelle } \\
\text { uniquement au secteur de la R-D; } \\
\text { - répartition fonctionnelle limitée } \\
\text { aux grandes entreprises, la } \\
\text { question de la répartition des } \\
\text { produits n'étant pas posée aux } \\
\text { petites entreprises afin de réduire } \\
\text { la charge de réponse; } \\
\text { - application de la répartition } \\
\text { fonctionnelle comme alternative à } \\
\text { l'activité économique principale. }\end{array}$ & $\begin{array}{l}\text { Cette approche n'est justifiée } \\
\text { qu'en l'absence d'information } \\
\text { fiable sur l'activité } \\
\text { économique principale des } \\
\text { unités de R-D (à partir des } \\
\text { registres d'entreprises, par } \\
\text { exemple) ou lorsque certaines } \\
\text { considérations relatives à la } \\
\text { charge de réponse empêchent } \\
\text { d'interroger les entreprises } \\
\text { sur l'orientation sectorielle. } \\
\text { Ses résultats sont plus } \\
\text { difficilement comparables au } \\
\text { niveau international à cause } \\
\text { des différentes combinaisons } \\
\text { de critères. }\end{array}$ \\
\hline
\end{tabular}


7.59 Dans la CITI de 2008 (CITI Révision 4), la division 72 « recherche scientifique et développement " inclut les activités relevant de deux types de recherche et développement, tels que définis dans ce manuel : la recherche et le développement expérimental en sciences physiques et naturelles et en ingénierie et la recherche et le développement expérimental en sciences sociales et humanités (la division 72, comme ce manuel, exclut les études de marché ; voir classe 7320, CITI Révision 4). La division 72 de la CITI englobe les unités impliquées principalement dans la fourniture de services de R-D à des entreprises affiliées ou à des tiers. Certaines de ces unités fournissent des services indifférenciés à l'industrie ; il pourra s'agir, dans certains cas, d'entreprises développant de nouveaux produits ou fournissant à leurs clients un savoir technologique ou organisationnel nouveau. D’une manière générale, il est recommandé que, dans la répartition par orientation sectorielle, la R-D exécutée par des entreprises dont l'activité économique principale correspond à la division 72 de la CITI soit attribuée aux secteurs pertinents desservis (généralement les branches d'activité CITI auxquelles appartiennent la plupart de leurs clients). Il en va de même pour les entreprises spécialisées dans la location de produits de propriété intellectuelle et d'autres produits (groupe 774, CITI Révision 4).

7.60 Bien qu'il soit recommandé de classer toutes les entreprises par activité économique principale, il est vivement suggéré de distribuer les dépenses de R-D intra-muros sur la base de l'orientation sectorielle, quelles que soient leur ampleur et les activités concernées. En principe, les dépenses en capital consacrées à la R-D doivent être exclues de la classification par domaine de produit ou secteur desservi et, par conséquent, seules les dépenses courantes de R-D doivent normalement être ventilées sur la base de ces critères. La raison en est que seules les activités courantes de R-D d'une unité peuvent être mises en relation avec des résultats escomptés et avec les utilisateurs potentiels de ces résultats. En pratique, il est parfois plus facile aux entreprises de déclarer leurs dépenses totales de R-D. Pour assurer la cohérence entre les séries de données fournies par différents pays, il est recommandé d'indiquer clairement dans les métadonnées sur la R-D l'approche qui a été utilisée pour recueillir et distribuer les données. Dans le cas des pays qui déclarent l'ensemble des DIRDE, il sera utile, le cas échéant, d'indiquer l'écart entre celles-ci et une répartition qui s'appliquerait uniquement aux dépenses courantes de R-D, en se basant sur la part des dépenses en capital de la R-D totale dans un secteur donné.

\section{Utilisation conjointe des informations sur l'activité principale et sur l'orientation sectorielle}

7.61 Il peut être utile, aux fins de certaines analyses, d'établir des tableaux permettant de mettre en relation la ventilation par orientation sectorielle et par activité principale des entreprises qui exécutent la R-D. Ces tableaux pourront servir à construire des matrices ad hoc des ressources et des utilisations de la R-D montrant la relation entre les exécutants de la R-D et ses utilisations probables. 
De telles matrices pourront ensuite être utilisées dans les analyses d'impact de la R-D. Les pays sont incités à produire, si possible, de tels états analytiques qui serviront aussi à évaluer la qualité des données fournies par les répondants.

\section{Répartition des DIRDE par domaine de R-D}

7.62 La répartition des DIRDE par domaine de R-D n'est couramment employée que dans un petit nombre de pays. Il devrait être possible de ventiler la plupart des activités précédemment identifiées comme " recherche fondamentale " ou " recherche appliquée » par domaine de R-D, mais la répartition du développement expérimental en se servant des catégories de domaine de R-D peut poser des difficultés dans le cas des entreprises. Dans beaucoup de pays, il est rare que les entreprises classent la documentation sur les projets et activités de R-D en se servant de telles catégories, et il est probable que les activités de développement expérimental qu'elles mènent portent sur des domaines technologiques interdisciplinaires et sur la combinaison de plusieurs domaines difficiles à distinguer les uns des autres. En raison de ces difficultés, le présent manuel ne peut recommander spécifiquement de distribuer les DIRDE par domaine de R-D. Néanmoins, si certains pays décident d'utiliser une répartition par domaine de $\mathrm{R}-\mathrm{D}$, il leur est recommandé d'adopter les catégories de domaine de R-D identifiées dans la section 3.4 du chapitre 3. On trouvera une classification plus détaillée dans le complément du présent manuel consultable

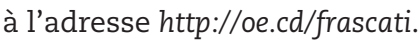

\section{Répartition des DIRDE par objectif socioéconomique}

7.63 Peu de pays tentent actuellement de distribuer leurs DIRDE sur la base de catégories correspondant à des objectifs socioéconomiques. Il est possible qu'une grande partie des activités de R-D puissent être classées sur une base pragmatique selon des catégories d'objectifs sociétaux, mais il est peu probable que les entreprises envisagent leurs crédits budgétaires de R-D en ces termes. C'est pourquoi le présent manuel ne recommande pas explicitement ce mode de répartition. Néanmoins, dans les pays où ont été mises en place des politiques visant spécifiquement à agir sur l'orientation fonctionnelle de la R-D du secteur des entreprises (santé, environnement, énergie ou défense, par exemple), il peut être pertinent de recueillir des informations sur la contribution de la R-D des entreprises à la réalisation d'objectifs particuliers d'importance politique ou sociale. Toutefois, comme il est probable que les données ainsi recueillies reflètent de fortes spécificités nationales, la prudence devra s'imposer dans les comparaisons au niveau international.

\section{Répartition des DIRDE par situation géographique}

7.64 Les pays peuvent juger utile d'établir des totaux distincts de répartition des DIRDE par localité ou région. Cependant, assigner entièrement les DIRDE d'une unité à un lieu ou centre d'opérations principal ne permet pas de voir où la R-D est effectivement exécutée. Les entreprises mènent fréquemment 
des activités en plusieurs lieux à la fois. Comme on l'a vu dans la section 7.4, une variable de classification géographique doit être attribuée à chaque unité statistique. Cette variable n'est pas toujours pertinente pour identifier où a lieu la R-D d'une entreprise. Celle-ci peut en effet disposer d'une division ou d'un département spécifique de R-D situé en un autre lieu que les équipements de production ayant déterminé la variable de classification géographique qui lui a été attribuée. En outre, une entreprise peut mener des activités de R-D (y compris des activités de R-D occasionnelles) en plusieurs sites ou établissements, dans de nombreux emplacements géographiques différents. Le choix de la répartition géographique doit être effectué en tenant compte des besoins nationaux et internationaux. On trouvera des indications supplémentaires sur la répartition de la R-D par région dans les orientations publiées en ligne, en complément du présent manuel à l'adresse http://oe.cd/frascati.

\section{Répartition des DIRDE par domaine technologique spécifique}

7.65 Pour compléter les recommandations spécifiques présentées dans le présent chapitre, on trouvera des indications supplémentaires au sujet de la collecte et de la compilation des données sur la R-D des entreprises dans les orientations publiées en ligne, en complément au présent manuel à l'adresse http://oe.cd/frascati. Y sont abordées, par exemple, la question des technologies qui sous-tendent la R-D et des technologies génériques. Les utilisateurs de statistiques s'intéressent notamment à des aspects comme les processus de mise au point de nouvelles technologies, ainsi que leurs modes de diffusion et d'application.

7.66 La plupart des travaux statistiques de l'OCDE consacrés à la mesure des technologies s'appuient sur des méthodes, procédures et classifications conçues pour mesurer les phénomènes liés aux TIC et aux biotechnologies. La relation entre les statistiques des TIC et les statistiques de R-D a été résolue grâce à la mise au point d'indicateurs pour les TIC qui reposent sur la classification de la R-D par activité économique principale et domaine de produit. Plus récemment, des efforts ont été engagés dans le domaine des nanotechnologies, en appliquant le modèle général conçu pour les biotechnologies, et un vif intérêt se porte également sur les moyens de saisir l'impact de la R-D sur le développement de logiciels dans toute une gamme de secteurs et de domaines de recherche (pour les méthodes proposées de collecte des données sur la R-D logicielle, voir l'encadré 4.1 du chapitre 4). Plusieurs pays ont inclus des questions sur ces domaines technologiques dans leurs enquêtes sur la R-D des entreprises mais leurs méthodes diffèrent, notamment en ce qui concerne l'attribution potentielle d'une même ressource de R-D à des technologies différentes, compte tenu des risques de chevauchement (par exemple dans le cas des activités de R-D concernant les bio-nanotechnologies).

7.67 L'OCDE a adopté en 2005 un Cadre pour les statistiques de biotechnologie (OCDE, 2005) et, a lancé plus récemment un projet statistique de collecte de données sur la R-D concernant les nanotechnologies à l'échelon international. 
Plusieurs pays ont d'ores et déjà adapté leurs questionnaires de R-D pour recueillir ce type d'informations auprès des entreprises, et l'OCDE publie régulièrement un recueil statistique à ce sujet.

7.68 Les utilisateurs de statistiques manifestent un vif intérêt pour les domaines d'application des technologies (par exemple, les activités de R-D concernant la santé, l'énergie, l'agro-bio et les technologies vertes ou bas carbone). Ces catégories se rapportent en général à des objectifs socioéconomiques spécifiques, mais souvent de manière transversale. Les utilisateurs cherchent aussi à obtenir un degré de granularité beaucoup plus élevé que celui qu'il est généralement possible d'atteindre de manière systématique dans les enquêtes. Les offices statistiques nationaux qui s'occupent des statistiques de R-D doivent bien entendu réfléchir aux méthodes les mieux adaptées pour recueillir des informations sur les activités des entreprises qui sont pertinentes au regard de certains défis sociétaux, mais il n'est pas possible de formuler ici des orientations ou recommandations générales à ce sujet. Les méthodes et stratégies de collecte des données de R-D portant sur un domaine technologique particulier doivent être définies au moyen de consultations intensives entre statisticiens, responsables de l'élaboration des politiques, utilisateurs des données et spécialistes du domaine concerné.

\subsection{Répartition fonctionnelle de la R-D extra-muros dans le secteur des entreprises}

7.69 Les entreprises peuvent fournir un financement à d'autres entreprises pour l'exécution d'activités de R-D extra-muros ; elles peuvent aussi acheter ou vendre de la R-D à d'autres entreprises. Ces cas de figure, qui concernent les unités statistiques de tous les secteurs économiques, sont abordés en détail au chapitre 4 (notamment dans la section 4.3 sur la mesure du financement de la R-D extra-muros, et la vente et l'achat de R-D). L'entreprise étant l'unité qui présente de l'intérêt pour les statistiques de R-D, le financement de la R-D versé par une entreprise “ $A$ » faisant partie d'un groupe d'entreprises à une autre entreprise " $B$ » appartenant au même groupe doit être déclaré comme le financement par " A » de la R-D extra-muros exécutée par « B ». Conformément aux indications présentées au chapitre 4 , la répartition succincte suivante est recommandée pour les entreprises qui financent des activités de R-D réalisées extra-muros et pour le suivi des achats et des ventes de R-D :

Échelon national :

- secteur des entreprises :

* entreprises d'un même groupe

* autres entreprises non affiliées

- secteur de l'État

- secteur de l'enseignement supérieur

- secteur privé sans but lucratif. 
Reste du monde :

- secteur des entreprises:

* entreprises d'un même groupe

* autres entreprises non affiliées

- secteur de l'État

- secteur de l'enseignement supérieur

- secteur privé sans but lucratif

- organisations internationales.

\section{Plan d'enquête : cadrage de la population de R-D}

7.70 La détermination du groupe de référence (population cible) est la première étape de tout projet statistique. La population cible aux fins des opérations de collecte de données sur la R-D des entreprises est constituée de toutes les entreprises qui exécutent de la R-D (ou qui financent la R-D, comme indiqué dans la section 4.3 du chapitre 4, sur la mesure du financement de la $\mathrm{R}-\mathrm{D}$ extra-muros) et qui sont situées sur un territoire donné (généralement un pays). Comme recommandé dans la section 6.3 du chapitre 6 , les enquêtes de R-D visant le secteur des entreprises doivent recenser et inclure dans la population cible l'ensemble des entreprises situées sur un territoire donné dont on sait ou dont il est fort probable qu'elles exécutent (ou financent) des activités de R-D pendant une période de temps donnée. La population des entreprises dont on sait ou dont il est probable qu'elles exécutent des activités de R-D constitue à son tour une sous-population de l'ensemble des entreprises en activité, dont chacune peut potentiellement devenir un exécutant de R-D. Par conséquent, il est aussi recommandé de sonder un échantillon de toutes les autres entreprises afin d'identifier celles qui exécutent des activités de R-D parmi celles dont on ignore a priori si elles mènent effectivement des activités de R-D ou si elles sont fortement susceptibles de le faire. Dans la plupart des pays, il est raisonnable de considérer qu'il est très peu probable que la plupart des microentreprises - qui constituent souvent la majorité des entreprises - exécutent (ou financent) des activités de R-D. C'est pourquoi, en pratique, les microentreprises sont souvent traitées (à des fins statistiques) comme n'entrant pas dans le champ des exécutants " potentiels" de R-D. Les offices statistiques nationaux utilisent couramment, pour toutes leurs enquêtes sur les entreprises, un " registre des entreprises " unique incluant l'ensemble des entreprises en activité pendant une année donnée.

\section{Registres des entreprises}

7.71 Les registres des entreprises, bien qu'étant des outils essentiels pour la compilation des statistiques de R-D, ne permettent pas toujours d'identifier la population pertinente à inclure dans une enquête sur la R-D. Ils constituent une source d'informations indispensable sur les caractéristiques clés - taille, secteur d'activité, structure du capital ou âge, par exemple - des entreprises 
à inclure potentiellement dans un échantillon d'enquête sur la R-D mais ne contiennent fréquemment aucune indication sur leurs activités effectives de R-D ou sur la probabilité de telles activités. C'est pourquoi, en pratique, on se contente couramment d'étudier la population totale des entreprises (ou un sousensemble de celle-ci sélectionné éventuellement sur la base de la taille ou du secteur d'activité) à des fins exploratoires seulement, c'est-à-dire pour isoler ou détecter les entreprises qui présentent un potentiel de R-D. Lors de la collecte des données sur la R-D, il est recommandé de cibler uniquement les entreprises pour lesquelles certains éléments indiquent au moins la probabilité d'activités de R-D, afin de réduire le coût de la collecte des données et la charge de travail des répondants.

7.72 L'exécution d'activités de R-D dans le secteur des entreprises étant une occurrence rare, puisque seul un pourcentage réduit d'entreprises mènent généralement de telles activités, il faut s'efforcer d'identifier et de suivre les entreprises qui présentent un fort potentiel de R-D. Un simple échantillonnage aléatoire ne constitue sans doute pas à cet égard le moyen le plus fiable de déterminer le niveau des activités de R-D : en effet, des entreprises en apparence similaires du point de vue des produits qu'elles offrent sur le marché peuvent poursuivre des stratégies de R-D très différentes et les estimations obtenues à partir d'un échantillon aléatoire peuvent donc se révéler insuffisamment fiables.

7.73 Cependant, nombre de pays ne disposent ni d'un registre des entreprises détaillé et à jour, ni d'un annuaire des entreprises qui exécutent des activités de R-D. Et même lorsqu'un registre des entreprises existe, il est important - avant de lancer une enquête - de s'assurer que celui-ci a été mis à jour de façon adéquate, qu'il inclut uniquement les entreprises en activité et qu'il exclut les sociétés fictives ou dormantes. En l'absence d'un registre complet des entreprises (ou de listes similaires), il n'est pas possible de produire des estimations de population ou d'échantillon fiables, ni même de réaliser une enquête de présélection exacte sur la R-D.

7.74 Lorsqu'on peut disposer d'informations suffisamment complètes sur la population totale d'entreprises en activité (à partir d'un registre des entreprises ou d'autres sources), il est assez simple de réaliser une enquête finalisée pour, d'abord, recenser délibérément les exécutants de R-D, puis obtenir directement d'eux les données requises. Cela implique d'interroger toutes les entreprises dont on sait ou dont il est fort probable qu'elles exécutent des activités de R-D. Pour mener à bien une enquête finalisée, il faut constituer un registre ad hoc (ou un annuaire) des entreprises qui exécutent des activités de R-D. L'obtention et la compilation de cette information demandent du temps mais ce travail représente un investissement important aux fins des enquêtes futures.

\section{Compilation d'annuaires des exécutants potentiels de R-D}

7.75 Lorsqu'il n'existe pas au préalable d'annuaire des exécutants de R-D du secteur des entreprises, des efforts importants doivent être consacrés, 
avant le lancement d'une enquête de R-D, à l'établissement d'une liste ou au recensement des entreprises qui ont de fortes chances d'être des exécutants de R-D. Les sources d'informations suivantes sur le comportement des firmes peuvent être utiles pour identifier ces entreprises :

- Chambre de commerce et d'industrie, associations professionnelles, associations d'entreprises exécutant des activités de R-D. Il est bon de commencer par contacter ces diverses associations et d'interroger leurs services de relations publiques sur les informations dont elles disposent au sujet des activités de R-D de leurs membres (si elles sont autorisées à divulguer des informations sur chaque entreprise). Les associations d'entreprises exécutant des activités de R-D peuvent dans certains cas communiquer les annuaires de leurs membres et d'autres informations pertinentes.

- Listes de sociétés cotées en bourse, par exemple sur les marchés boursiers nationaux. Il est nécessaire de passer en revue la liste des entreprises cotées à la bourse principale.

- Rapports annuels des sociétés, revues professionnelles, annuaires des laboratoires de R-D. Une liste de référence des entreprises qui mènent des activités de R-D peut être établie en passant en revue les dépenses de R-D mentionnées dans les rapports financiers ou les systèmes comptables habituels. Ces sources doivent aussi être exploitées afin de recueillir des informations spécifiques sur les activités de R-D, en particulier la construction de prototypes, la création d'installations pilotes, etc.

- Registres des subventions et contrats de recherche sur fonds publics pour la R-D. Dans les environnements plus élaborés, les ministères de la science et de la technologie ou de la recherche - qui sont généralement les instances nationales jouant le rôle le plus important dans le financement des subventions de recherche - disposent parfois d'une liste des entreprises bénéficiaires d'aides à la recherche ou à l'innovation. Les listes des contributeurs aux programmes de recherche internationaux sont aussi d'accès facile.

- Listes des entreprises demandant à bénéficier d'allégements fiscaux en relation avec des activités et des projets de R-D. Les liens de coopération étroite établis entre les responsables des enquêtes sur les entreprises et les services de l'administration publique s'occupant des incitations fiscales en faveur de la R-D et des mesures de facilitation des importations, de promotion des exportations et de contrôle des prix sont aussi utiles pour identifier les exécutants de R-D.

- Listes des entreprises ayant déclaré des activités de R-D dans les enquêtes précédentes sur la R-D, dans les enquêtes sur l'innovation ou d'autres enquêtes structurelles sur les entreprises.

- Listes des entreprises ayant déposé une demande de brevet au cours des dernières années. Il s'agit, là encore, d'un indicateur d'une possible activité de R-D.

- Registres des essais cliniques autorisés ou registres administratifs similaires. 
7.76 Il est possible d'établir un cadre d'enquête sur les entreprises menant des activités de R-D en consultant ces sources et en contactant directement les entreprises connues pour mener des activités de R-D. Le travail d'identification des exécutants effectifs de R-D doit privilégier en premier lieu les grandes entreprises appartenant à des secteurs affichant généralement une forte intensité de R-D. Pour identifier les exécutants de la R-D parmi plusieurs centaines de grandes entreprises, on adoptera un processus séquentiel, en donnant d'abord la priorité aux secteurs où les activités de R-D sont les plus probables, puis en examinant les liens entre les exécutants de R-D identifiés et d'autres entreprises auxquelles ils sont liés dans les chaînes d'approvisionnement, leurs concurrents, etc. En l'absence d'informations publiées confirmant l'existence d'activités de R-D, l'établissement de contacts directs avec les entreprises sera nécessaire pour vérifier l'existence de telles activités. Une enquête en deux étapes pourra aussi être envisagée : la première pour identifier les exécutants de la R-D au moyen d'un questionnaire très court, intégré éventuellement à d'autres enquêtes sur les entreprises, et la seconde pour cibler les entreprises ayant déclaré des activités de R-D, à l'aide d'un questionnaire plus détaillé.

\section{Stratégies d'enquête}

7.77 Les pratiques nationales d'enquête sur la R-D des entreprises sont très diverses. Néanmoins, dans tous les cas, l'identification de la population des entreprises dont on sait qu'elles mènent des activités de R-D ou qui sont fortement susceptibles de le faire constitue une première étape essentielle du processus de collecte des données. Contrairement à ce qui se passe dans d'autres secteurs où, par exemple, les établissements d'enseignement supérieur et les administrations publiques sont répertoriés dans des annuaires connus, les enquêtes sur la R-D des entreprises dépendent fortement de la qualité du cadre existant et de sa fiabilité pour éviter la sous-estimation ou la surestimation des activités de R-D.

7.78 S'ils peuvent compter sur un cadre très fiable, les offices statistiques nationaux peuvent réaliser soit un recensement, soit une enquête par sondage. Compte tenu de la forte concentration des activités de R-D (à la fois en termes de dépenses et d'effectifs) dans un nombre assez restreint de catégories de taille et de secteurs d'activité, un recensement est généralement recommandé dans cette cohorte d'entreprises puisqu'elles présentent une plus forte probabilité d'avoir engagé des activités de R-D pendant l'année de référence. Les exécutants connus de R-D de très grande taille doivent être inclus dans cette strate « à tirage exhaustif ».

7.79 Les entreprises moins susceptibles d'avoir mené des activités de R-D pendant l'année de référence pourront faire l'objet soit d'un recensement, soit d'une enquête par sondage. Cette approche part du présupposé que tous les exécutants potentiels de R-D sont effectivement pris en compte dans le cadre d'enquête et que la probabilité de découvrir des exécutants supplémentaires 
hors du cadre est négligeable ou ne concerne que des petites entreprises ou des microentreprises.

7.80 Lorsqu'il n'existe pas de cadre d'enquête fiable, à savoir un annuaire des entreprises qui sont des exécutants potentiels de R-D, une approche différente est requise. En pareil cas, il se peut, en effet, qu'un nombre non négligeable d'unités de R-D n'aient pas encore été identifiées ou que certains exécutants de R-D de grande taille ne soient pas encore inclus dans le cadre. Dans ces conditions, une enquête sur les exécutants connus de grande taille (de préférence une enquête de recensement) doit être complétée par une enquête par sondage portant sur une sous-population du registre général des entreprises (ou d'un registre similaire), en présupposant que la plupart des unités manquantes seront prises en compte avec un degré de probabilité donné (essentiellement sur la base d'une classification croisée par taille et secteur d'activité). Une enquête à deux étapes - identification des exécutants de la R-D, puis collecte de données est également recommandée dans ce cas afin de réduire le coût de collecte des données et la charge statistique pour les entreprises.

7.81 Il est inutile d'introduire un seuil de taille minimum dans le cas d'une enquête sur la R-D des entreprises basée sur un cadre fiable regroupant les entreprises qui répondent à des critères nationaux d'activité probable de R-D. En revanche, lorsqu'on établit un échantillon à partir d'un registre général des entreprises dans le but d'identifier de nouveaux exécutants potentiels de R-D, il est recommandé d'exclure les microentreprises de l'échantillon, sauf si l'on dispose de ressources suffisantes pour passer au crible les exécutants potentiels de R-D. Cette recommandation se fonde sur la nécessité d'éviter le risque de surestimation du nombre d'exécutants que peut induire le nombre élevé de microentreprises dans l'ensemble de la population. Elle doit aussi permettre de réduire les coûts d'enquête et la charge de travail globale des répondants.

\section{Conception du questionnaire}

7.82 Le questionnaire est un outil conçu pour recueillir des données. Il doit être facile à comprendre et à utiliser, efficace et adaptable. Les questionnaires d'enquête auprès des entreprises, qui sont généralement auto-administrés, doivent être remplis par une large gamme d'individus travaillant pour des entreprises de types très divers. De ce fait, les questionnaires doivent pouvoir s'adapter à des conditions d'utilisation et à des besoins très différents.

7.83 Les questionnaires électroniques offrent la possibilité de prétraiter les données recueillies au moyen de questions filtres permettant aux répondants de sauter les modules non pertinents et de dispositifs de vérification intégrés. Ils permettent une interaction effective avec les répondants en vue de prévenir les erreurs ou les incohérences dans les données fournies. Il est parfois nécessaire de recourir à plusieurs modes de collecte des données lorsqu'un nombre assez important d'entreprises n'ont pas facilement accès à l'internet ou n'y ont pas accès à un coût abordable. La nécessité qu'un questionnaire puisse être géré 
facilement par plusieurs correspondants à l'intérieur d'une entreprise qui peuvent avoir des connaissances et des compétences différentes au regard des dépenses, des contrats et des effectifs de R-D de l'entreprise, doit aussi être prise en compte.

7.84 Plusieurs pays recourent à des « enquêtes combinées », principalement en fusionnant les enquêtes sur la R-D des entreprises et les enquêtes sur l'innovation des entreprises, comme décrit dans le Manuel d'Oslo (OCDE/ Eurostat, 2005). Cette méthode, bien que reconnue par le présent manuel, n'est pas recommandée car elle peut nuire à la comparabilité internationale des résultats de R-D : en présence de questions portant à la fois sur la R-D et sur l'innovation dans un même questionnaire, les répondants risquent d'avoir $d u$ mal à différencier la R-D de diverses autres activités touchant à l'innovation (voir chapitre 2). Il est donc recommandé aux pays qui décident de réaliser une enquête combinée sur l'innovation et la R-D de : (a) fournir aux répondants deux questionnaires ou au moins un questionnaire comportant deux parties distinctes, en indiquant clairement que les deux notions statistiques ne sont pas complémentaires mais se recoupent, (b) réduire autant que possible la taille du questionnaire combiné, afin d'en faciliter la compréhension, (c) présenter de manière systématique la méthode de collecte des données adoptée (principalement aux fins de la comparaison des résultats de R-D avec ceux d'autres pays qui ne recourent pas à des enquêtes combinées) et, (d) utiliser le même registre des entreprises comme cadre statistique pour enquêter à la fois sur l'innovation et sur la R-D des entreprises (en respectant les procédures décrites plus haut). Ces mesures permettront aussi d'assurer la cohérence avec les instructions et recommandations figurant dans le Manuel d'Oslo (OCDE/ Eurostat, 2005).

\section{Pratiques de collecte des données}

7.85 L'obtention d'un taux de réponse élevé est l'ambition de toute enquête statistique mais cela est particulièrement important dans le cas des enquêtes de $\mathrm{R}-\mathrm{D}$, car la R-D des entreprises est une occurrence rare (un nombre assez peu élevé d'unités de l'ensemble du secteur des entreprises mènent des activités de $\mathrm{R}-\mathrm{D}$ et elles sont donc difficiles à identifier). Cette remarque vaut à la fois pour les enquêtes de recensement (puisqu'il est extrêmement difficile de formuler des hypothèses sur les activités effectives de R-D des non-répondants) et les enquêtes par sondage, car un taux de réponse peu élevé peut conduire à des biais de mesure importants (en sus d'une erreur d'échantillonnage plus grande). Dans l'idéal, aucun effort ne doit être épargné pour réduire au minimum le taux de nonréponse. Pour maintenir le niveau de qualité des données, les offices statistiques doivent définir un taux de réponse minimum acceptable En-deçà duquel il n'est pas possible de produire des estimations sur une population. Lorsque le taux de réponse tombe au-dessous du niveau acceptable, une analyse de suivi du biais de non-réponse doit être effectuée. Le présent manuel ne peut recommander de 
seuils de tolérance spécifiques à cet égard. Toutefois, il est clair qu'une enquête obligatoire a plus de chance d'obtenir un taux de réponse élevé qu'une enquête volontaire.

7.86 Néanmoins, en pratique, le taux de réponse moyen non pondéré n'est sans doute pas l'indicateur le plus adapté pour mesurer le taux de couverture des activités de R-D atteint dans une enquête sur les entreprises. En effet, l'hétérogénéité très marquée que l'on observe systématiquement parmi les entreprises qui mènent des activités de R-D laisse à penser que, dans la plupart des pays, un petit groupe de grands exécutants de R-D, s'ils sont pleinement pris en compte, représentent une part très élevée des dépenses totales de R-D des entreprises (et aussi, mais à un degré moindre, des effectifs de R-D).

7.87 Il convient par conséquent d'appliquer des méthodes spécifiques pour atteindre le double objectif d'une amélioration du taux de réponse global et d'une couverture quasi-exhaustive des grands exécutants de R-D. La nécessité d'exploiter toutes les sources d'informations disponibles à l'appui du travail de collecte des données s'en trouve renforcée. L'accès aux données sur les allégements fiscaux de R-D est particulièrement important dans ce contexte, car cette information peut être utile pour identifier les exécutants essentiels qui devront être spécifiquement ciblés lors du travail de collecte des données.

7.88 Outre l'amélioration du cadre d'enquête, diverses autres mesures doivent être prises pour améliorer la qualité du travail de collecte des données de R-D. À toutes les étapes, les répondants doivent avoir conscience du fait qu'ils participent à une enquête statistique : ils doivent être informés de l'objet et de la nature de l'enquête, de l'organisme qui en est responsable et de la possibilité ou non pour eux de choisir le mode de transmission des données demandées (ou même de la possibilité pour eux d'être exclus de l'enquête). D'une manière générale, l'équipe chargée de la collecte des données doit être en permanence disponible pour répondre aux questions des répondants et leur apporter l'aide technique et les conseils dont ils ont besoin. Il s'agit là aujourd'hui d'une norme reconnue par la plupart des organismes officiels de statistique et qui doit être respectée par toute institution cherchant à produire des données sur la R-D des entreprises comparables au niveau international.

7.89 Pour évaluer la mise en œuvre d'une enquête et la couverture de la population concernée, les offices statistiques sont invités à calculer le taux de réponse (TR), le taux de réponse pondéré (TRP) et le taux de couverture (TC). Ces trois mesures de la qualité fournissent chacune un point de vue différent, selon l'aspect étudié. Dans le cas des populations assez homogènes du point de vue des variables étudiées, la différence entre ces trois mesures sera probablement très faible. Mais la répartition des activités de R-D étant asymétrique, puisque celles-ci sont fortement concentrées dans le secteur des entreprises, les trois mesures constituent des outils pertinents (voir l'encadré 7.1). 


\section{Encadré 7.1. Nécessité de plusieurs mesures de la qualité des données d'enquête}

Il existe plusieurs moyens de mesurer le taux de couverture et le taux de réponse. Lors de la collecte de données sur la R-D intra-muros dans le secteur des entreprises, plusieurs méthodes de mesure de la qualité de l'enquête peuvent être indiquées.

Imaginons, par exemple, le cas d'une population de 1000 unités d'un secteur CITI avec des mesures de R-D de :

- 1000000 pour 1 unité, 1000 pour 1 unité et 1 pour chacune des 998 unités restantes

- l'office statistique ayant sélectionné un échantillon de 10 unités, en retenant intentionnellement les unités avec la mesure 1000000 et la mesure 1000 et un échantillon aléatoire de 8 unités avec chacune la mesure 1.

On trouvera ci-dessous quatre scénarios de collecte de données aboutissant chacun à un taux de réponse (TR) de 70 \% (c'est-à-dire que 7 unités de l'échantillon sur 10 ont répondu à l'enquête). Les résultats, cependant, se présentent sous un jour différent selon le type d'unités qui ont répondu (en supposant que 7 unités sur 10 ont répondu) dans les quatre scénarios :

\begin{tabular}{cccc}
\hline \multicolumn{4}{c}{$\begin{array}{c}\text { Mesures de la R-D des unités } \\
\text { et nombre ayant répondu }\end{array}$} \\
\hline Scénario & 1000000 & 1000 & 1 \\
1 & 0 & 0 & 7 \\
2 & 0 & 1 & 6 \\
3 & 1 & 0 & 6 \\
4 & 1 & 1 & 5 \\
\hline
\end{tabular}

\begin{tabular}{ccc}
\hline \multicolumn{3}{c}{ Analyse des réponses } \\
\hline TR & TRP & TC \\
$70,0 \%$ & $87,3 \%$ & $0,0 \%$ \\
$70,0 \%$ & $75,0 \%$ & $0,1 \%$ \\
$70,0 \%$ & $75,0 \%$ & $99,8 \%$ \\
$70,0 \%$ & $62,6 \%$ & $99,9 \%$ \\
\hline
\end{tabular}

Dans cet exemple hypothétique, le scénario 3 semble se distinguer favorablement des autres en termes de taux de réponse pondéré (TRP) de la population d'enquête, bien que l'unité venant en deuxième place pour les dépenses de R-D dans le secteur n'ait pas répondu. Du point de vue du taux de couverture global (TC), le scénario 4 aboutit à la meilleure couverture des DIRDE totales du secteur.

\section{Pondération et estimation}

7.90 L'étape finale du traitement des données recueillies sur la R-D des entreprises est la production de résultats statistiques sur le niveau des activités de R-D menées par les entreprises dans le pays déclarant (en termes de dépenses de R-D et d'effectifs de R-D). Dans le cas des enquêtes sur la R-D des entreprises, le processus d'estimation dépend fortement des procédures utilisées pour identifier la population de référence. Quelques cas particuliers et certaines questions connexes sont examinés ici. 
7.91 En guise de préliminaire, le présent manuel déconseille l'utilisation de coefficients extérieurs (par exemple, l'application de ratios sectoriels antérieurs R-D/ventes aux ventes totales des entreprises) pour estimer les totaux de la R-D des entreprises à l'échelle d'un secteur d'activité. Sous certaines conditions particulières, les coefficients peuvent être utiles pour estimer les activités de R-D des établissements d'autres secteurs économiques (principalement le secteur de l'enseignement supérieur, voir chapitre 9) mais tel n'est pas le cas dans le secteur des entreprises. Les entreprises ont constamment à décider d'engager ou non - et dans quelle mesure - des activités de R-D. La R-D intra-muros est risquée et coûteuse et une entreprise peut décider à tout moment d'abandonner ses projets de R-D internes pour acheter à la place des services de R-D externes ou acquérir des connaissances déjà codifiées sous forme de propriété intellectuelle. L'extrême diversité des stratégies des entreprises, en particulier de leurs stratégies de R-D, selon la branche d'activité et la catégorie de taille à laquelle elles appartiennent est la raison fondamentale pour laquelle le présent manuel déconseille l'utilisation de coefficients dans la production de statistiques sur la R-D des entreprises.

7.92 Avant d'engager les procédures d'estimation, les données d'enquête doivent être vérifiées avec précision et validées. Les valeurs aberrantes doivent être identifiées et corrigées. Pour remédier à la non-réponse de grands exécutants de R-D, l'imputation - sur la base d'informations auxiliaires tirées, par exemple, des rapports des sociétés ou de réponses antérieures - est préférable à la repondération des données d'autres répondants.

7.93 Une remarque supplémentaire s'impose quant à l'extrapolation des résultats d'une enquête de R-D portant sur une population indifférenciée d'entreprises (par exemple un échantillon tiré d'un registre d'entreprises). La sous-population des entreprises susceptibles de mener des activités de R-D étant d'un point de vue statistique une population rare, il est recommandé, avant d'extrapoler les résultats d'une enquête, de procéder d'abord au filtrage des unités non éligibles. Des méthodes statistiques peuvent aussi être utilisées pour réduire au minimum la possibilité d'introduire un biais en surestimant l'activité totale de R-D des entreprises (voir chapitre 6).

\section{Contrôle de la qualité des réponses sur la $R-D$ des entreprises}

\section{Remarques préalables sur la comptabilité financière des entreprises}

7.94 Comme indiqué plus haut, les rapports annuels des entreprises constituent généralement une bonne source d'informations pour identifier les entreprises susceptibles de mener des activités de R-D. Les totaux figurant dans ces rapports sont aussi utiles pour vérifier l'exactitude des données déclarées dans l'enquête et remédier aux lacunes en cas de non-réponse partielle (voir chapitre 6). Toutefois, il convient d'insister sur le fait que les chiffres relatifs aux activités de R-D publiés conformément aux normes et directives comptables nationales et internationales diffèrent parfois des données de R-D recueillies sur la base des 
recommandations présentées dans le présent manuel. Certaines dépenses de R-D sont portées au bilan des entreprises, alors que d'autres sont comptabilisées sous forme de charges (avec amortissement, voir le chapitre 4) dans leurs comptes de résultats. Certaines entreprises incluent aussi, à des fins de déclaration publique, les « services techniques » fournis par le personnel de R-D dans la R-D intra-muros (voir le chapitre 5 sur les fonctions et titres du personnel).

7.95 Même lorsque la R-D est définie exactement de la manière recommandée au chapitre 2, la teneur des totaux tirés de la comptabilité n'est pas toujours identique à celle des totaux de R-D obtenus selon les préconisations du présent manuel. Par exemple, lorsque la R-D n'est pas " significative » au regard du total des coûts de l'entreprise, les dépenses correspondantes ne sont pas toujours explicitement mentionnées. De plus, la R-D payée par d'autres n'est pas nécessairement déclarée séparément de la R-D intra-muros sur fonds internes. En effet, les activités de R-D réalisées sous contrat ne peuvent pas être comptabilisées ou perçues comme relevant de la R-D dans les états financiers (chapitre 4). Dans les comptes des grandes entreprises, en particulier, les coûts de la R-D intra-muros ne sont pas toujours distingués de ceux de la R-D extra-muros. En conformité avec la plupart des normes comptables, leurs états financiers annuels sur les dépenses de R-D combinent parfois le financement interne de la R-D intra-muros et de la R-D extra-muros, dès lors que les activités de R-D correspondantes ont été menées " au profit » de l'entreprise concernée. Dans le cas des EMN, tout particulièrement, les totaux de R-D publiés peuvent inclure les dépenses de R-D du groupe mondial (voir chapitre 12) et non de chacune des entreprises qui en font partie.

\section{Différenciation de la R-D intra-muros et de la R-D extra-muros}

7.96 Le recensement exact des flux de financement de la R-D, en tant qu'élément de la R-D intra-muros d'une unité ou en tant qu'élément du financement total des activités de R-D extra-muros, peut poser un certain nombre de difficultés.

7.97 Des problèmes se posent, en particulier, lorsque le financement passe directement et indirectement par plusieurs unités avant de parvenir à l'exécutant de R-D. Cela se produit en cas de sous-traitance de la R-D, ce qui est particulièrement fréquent dans le secteur des entreprises. Chaque exécutant doit donc déclarer uniquement le coût des projets de R-D qu'il a effectivement conduits et non sa contribution à la R-D d'une autre unité et préciser, dans la mesure du possible, la source originale de financement de la R-D. Pour plus de précisions sur les moyens de différencier le financement de la R-D intra-muros de celui de la R-D extra-muros, voir chapitre 4.3.

\section{Surestimation ou sous-estimation possible des activités de R-D des entreprises}

7.98 Produire des statistiques sur la R-D des entreprises, compte tenu en particulier des différences de pratiques entre pays, est un processus assez complexe. Même lorsque l'on parvient à recueillir des données de qualité, des 
questions subsistent quant à la sous-déclaration ou la sur-déclaration potentielle des activités de R-D des entreprises. En se basant sur l'expérience des pays, il est possible de suggérer - en sus des recommandations formelles présentées dans le présent manuel - certaines bonnes pratiques pour réduire le risque d'erreur dans le travail de mesure de la R-D des entreprises. Deux points sont particulièrement pertinents à cet égard : (i) l'identification d'une population de référence adéquate pour une enquête sur la R-D des entreprises (en évitant la sous-couverture ou la sur couverture des exécutants), (ii) l'identification des activités effectives de R-D menées par les répondants à l'enquête (en évitant la sous-couverture ou la surcouverture des activités de R-D).

7.99 La sous-couverture des unités de R-D résulte le plus souvent d'une connaissance insuffisante du secteur des entreprises. La sous-couverture des grandes entreprises est par définition exclue puisque ces entreprises ne constituent qu'une petite partie du secteur et sont faciles à identifier. Le risque de sous-couverture se pose en revanche dans le cas des exécutants de petite taille. Comme le criblage de la population d'entreprises au moyen d'enquêtes statistiques ne peut aboutir au mieux qu'à des approximations, il est recommandé d'exploiter systématiquement les sources de données administratives (financement public de la $R-D$, incitations fiscales en faveur de la $R-D$, participation à des projets publics de R-D, dépôt de brevets, etc.) afin d'identifier une part importante des exécutants potentiels de R-D parmi les petites et microentreprises. Toutefois, même après ce travail, il est fort probable que certains exécutants de R-D ne seront pas pris en compte et il convient donc de reconnaître la possibilité d'une sous-couverture (réduite au minimum) des petits exécutants dans l'interprétation des données sur la R-D. Toutefois, ses incidences au regard des dépenses totales de R-D ou des effectifs de R-D sont considérées comme négligeables dans la plupart des pays.

7.100 La sous-estimation des activités de R-D des entreprises est toujours une source de préoccupations. L'adoption de certaines des recommandations méthodologiques présentées dans le présent chapitre doit aider à limiter ce risque au maximum (par exemple, en identifiant le bon contact à l'intérieur des entreprises visées par l'enquête). Les bonnes pratiques de collecte des données doivent aussi inciter les répondants à :

- prendre en compte l'ensemble des activités de R-D menées à l'intérieur de l'unité statistique, y compris celles exécutées en dehors des départements spécialisés de R-D, par exemple, les activités d'essai pilote, de préparation en vue de la production ou de développement technologique général

- inclure les activités de R-D « non évidentes » qui sont entièrement intégrées à des contrats de développement (généralement importants) de produits ou de systèmes particuliers (connaissances incorporées à des produits)

- inclure les activités de R-D financées par des clients sur la base de projets.

7.101 La surcouverture des entreprises qui exécutent des activités de R-D est due le plus souvent à une interprétation erronée de l'information recueillie à partir d'autres enquêtes sur les entreprises ou de bases de données administratives. 
Les sources de ce type, qui sont essentielles pour sélectionner la population d'une enquête de R-D, doivent toutes être maniées avec prudence : il y a en effet peu de chances que nombre des sources de référence s'appuient exactement sur les concepts de la R-D définis dans le présent manuel. Tel est le cas, par exemple, des listes d'entreprises ayant demandé des allégements fiscaux au titre de dépenses de R-D, car la notion d'“ activité de R-D » qu'emploie l'administration fiscale peut englober les activités internes de R-D et le financement d'activités de R-D réalisées par d'autres unités. C'est pourquoi, dans une enquête statistique, il est nécessaire de fournir aux répondants des définitions claires de la R-D (et de vérifier l'exactitude des réponses), afin de réduire au minimum le risque d'erreur des répondants dans l'interprétation des activités de R-D.

7.102 La surestimation des activités de R-D des entreprises peut être due à divers facteurs :

- une connaissance insuffisante des activités à déclarer

- la difficulté objective à distinguer les activités de R-D d'autres activités axées sur l'innovation ou sur les technologies

- l'inclusion de la R-D achetée à d'autres unités dans le total de la R-D exécutée intra-muros (avec le risque supplémentaire de double déclaration).

7.103 On ne saurait trop insister sur le risque de sur-déclaration car les entreprises sondées sont souvent réticentes à adapter la conception qu'elles se font des activités de R-D (qui est souvent le produit de normes comptables, fiscales et réglementaires) pour prendre en compte les définitions à usage statistique présentées dans le présent manuel. La vérification de l'exactitude des données obtenues auprès des répondants (en s'efforçant idéalement d'identifier tout écart par rapport au comportement attendu d'une entreprise au vu de sa taille et de son activité économique principale) et le traitement adéquat des valeurs aberrantes font partie des bonnes pratiques pour résoudre les problèmes susmentionnés.

\section{Contrôle de la qualité des totaux de R-D des entreprises}

7.104 Comme on l'a vu au chapitre 6, l'application de normes de contrôle de la qualité dans l'établissement des statistiques de R-D est fortement recommandée. Il en va de même à cet égard pour la R-D des entreprises que pour la R-D exécutée dans d'autres secteurs. Toutefois, la diversité des méthodes nationales utilisées dans les enquêtes réalisées sur la $\mathrm{R}-\mathrm{D}$ des entreprises montre la nécessité de critères communs de contrôle de la qualité des données et des enquêtes.

7.105 Pour compléter les recommandations sur la qualité des statistiques émises par l'OCDE (2011) ou les directives sur les cadres d'assurance-qualité émanant, par exemple, des Nations Unies (2012), on trouvera ci-dessous quelques recommandations pratiques en vue d'améliorer la compréhension et la comparabilité internationale des statistiques sur la R-D des entreprises. Ces recommandations portent plus particulièrement sur les critères de précision des 
estimations réalisées à partir des données sur la R-D des entreprises et sur la cohérence entre ces estimations et d'autres statistiques sur les entreprises.

7.106 Étant donné l'incidence rare des activités de R-D et le caractère fortement asymétrique de la population des exécutants effectifs de R-D, disposer d'un cadre exact et actualisé constitue l'un des moyens les plus sûrs de garantir la réussite d'une enquête de R-D et la qualité de ses résultats. La qualité des statistiques recueillies sur la R-D des entreprises dépend, dans une large mesure, de l'identification de la population d'entreprises dont on sait qu'elles exécutent des activités de R-D ou qui sont fortement susceptibles de le faire, mais cette identification reste, dans bien des cas, largement approximative. L'incertitude sur le nombre d'entreprises susceptible de mener effectivement des activités de $\mathrm{R}-\mathrm{D}$, où dont on estime, au sens statistique, qu'elles pourraient le faire, est la raison principale pour laquelle on ne produit pas généralement de statistiques sur le nombre ou la proportion des exécutants de R-D par rapport à la population totale des entreprises.

7.107 Lors de la publication de données sur les DIRDE, des indications détaillées sur la méthode utilisée pour produire les données doivent également être fournies. Plus précisément, il est recommandé que la diffusion des statistiques sur la R-D des entreprises au niveau national s'accompagne conjointement de la publication de métadonnées : par exemple, le nombre d'unités incluses dans la population de référence (exécutants potentiels de R-D), identifiées éventuellement sur la base de leur activité économique principale, le nombre d'unités sondées au cours de l'enquête de recensement, avec le taux de réponse et, le nombre d'unités retenues dans l'échantillon, avec le taux de réponse également.

7.108 Les statistiques sur la R-D des entreprises présentent l'intérêt essentiel de pouvoir être intégrées à d'autres statistiques sur les entreprises, notamment lorsque les critères d'échantillonnage et de classification utilisés dans les enquêtes de R-D sont identiques à ceux qui ont servi à la collecte de données sur d'autres variables économiques des entreprises. Les métadonnées suivantes doivent donc être mises à la disposition des utilisateurs, parallèlement à la publication des données nationales, en tant qu'indicateurs partiels du degré de cohérence entre les estimations des dépenses et des effectifs de R-D et d'autres indicateurs statistiques : le ratio dépenses de $R-D /$ valeur ajoutée des entreprises, par activité économique principale, et le ratio effectifs totaux de R-D (équivalent temps plein)/ total des personnes occupées, par activité économique principale (pour tous les secteurs).

\section{Références}

CEE-ONU (2015), Guide to measuring global production, CEE-ONU, Genève, https://www. unece.org/fileadmin/DAM/stats/documents/ece/ces/bur/2015/February/16Add.1-Guide_to_ Measuring_Global_Production.pdf. 
Nations Unies (2012), National Quality Assurance Frameworks, Nations Unies, New York, http://unstats.un.org/unsd/dnss/QualityNQAF/nqaf.aspx.

Nations Unies (2008a), "Classification internationale type, par industrie, de toutes les branches d'activité économique (CITI), Révision 4 ", Département des affaires économiques et sociales, Division de statistique, Études statistiques, Série $M, n^{\circ} 4$, Rév. 4, Nations Unies, New York, https://unstats.un.org/unsd/cr/registry/isic-4.asp et http://unstats.un.org/unsd/publication/seriesM/seriesm_4rev4f.pdf.

Nations Unies (2008b), Central Product Classification (CPC Ver. 2), Nations Unies, New York, http://unstats.un.org/unsd/cr/registry/cpc-2.asp.

OCDE (2011), Quality Framework and Guidelines for OECD Statistical Activities, version 2011/1, Éditions OCDE, Paris, www.oecd.org/statistics/qualityframework.

OCDE (2005), Cadre pour les statistiques de biotechnologie, Éditions OCDE, Paris, http://www. oecd.org/fr/sti/sci-tech/35878269.pdf.

OCDE/Eurostat (2005), Manuel d'Oslo : Principes directeurs pour le recueil et l'interprétation des données sur l'innovation, $3^{e}$ édition, La mesure des activités scientifiques et technologiques, Éditions OCDE, Paris, $h t t p: / / d x . d o i . o r g / 10.1787 / 9789264013124-f r$. 


\section{Chapitre 8}

\section{R-D du secteur de l'État}

On trouvera dans ce chapitre des orientations sur la façon de procéder pour quantifier les ressources financières et humaines consacrées aux activités de recherche et de développement expérimental (R-D) exécutées dans le secteur de l'État. Il y est également question du secteur de l'État en tant que financeur de R-D, en lien avec le chapitre 12 sur les crédits budgétaires publics de R-D (CBPRD) et le chapitre 13 sur les allégements fiscaux en faveur de la R-D. Le secteur de l'État, qui regroupe les administrations publiques et les institutions sans but lucratif contrôlées par l'État, est décrit sur la base du Système de comptabilité nationale (SCN). Sont présentées les approches à adopter pour mesurer les dépenses intérieures de R-D de l'État (DIRDET). La répartition fonctionnelle de la R-D par type de coûts suit les indications du chapitre 4, et attire l'attention sur des cas particuliers. Il est également question de la répartition des DIRDET par source de financement, type de R-D, domaine de R-D, secteur technologique, objectif socio-économique, fonction administrative et situation géographique. Des indications sont données sur la méthode à suivre pour mesurer le personnel de R-D dans le secteur de l'État. Le chapitre se termine par un tour d'horizon des problèmes suscités par la mesure du financement public de la R-D du point de vue des bailleurs de fonds. 


\subsection{Introduction}

8.1 L'intérêt porté à la mesure du rôle du secteur de l'État dans la R-D est une caractéristique constante du présent manuel depuis sa première édition, en 1963. Les pouvoirs publics jouent un rôle important à la fois comme exécutants et bailleurs de fonds d'activités de R-D, à l'échelle nationale et dans le reste du monde. Le présent chapitre se concentre principalement sur la mesure des résultats et du personnel de R-D au sein du secteur de l'État, conformément à la méthode recommandée par le présent manuel pour mesurer les ressources consacrées à la R-D. Il vise également à établir un lien entre l'approche fondée sur le point de vue de l'exécutant, qui est celle préconisée ici, et celle, complémentaire, qui est fondée sur le point de vue du bailleur de fonds pour mesurer le poids des pouvoirs publics dans le financement de la R-D dans l'ensemble de l'économie. $\mathrm{Au}$ fil du temps, les pays ont évolué dans leur utilisation des instruments de politique de R-D, si bien que les offices statistiques nationaux qui compilent les données de la R-D ont dû réfléchir à la meilleure façon de refléter ces pratiques dans ce cadre statistique. Le présent chapitre donne des indications de base à ce sujet. Il établit également des liens avec les chapitres 12 et 13, consacrés respectivement à la mesure des crédits budgétaires publics de R-D (CBPRD) et des allégements fiscaux au titre des dépenses de R-D.

\subsection{L'étendue du secteur de l'État à des fins de mesure de la R-D Définition et étendue du secteur de l'État}

8.2 À toutes fins statistiques, le Système de comptabilité nationale (SCN) relève que les principales fonctions des administrations publiques sont d'assumer la responsabilité de la fourniture de biens et de services à la collectivité ou aux ménages et de financer cela par l'impôt ou d'autres recettes, de redistribuer les revenus et la richesse au moyen de transferts, et de mener des activités de production non marchande.

8.3 Telles que définies dans le SCN (CE et al., 2009 : para. 4.117) et tel qu'il ressort de ce manuel (chapitre 3, section 3.4), les administrations publiques sont des types particuliers d'entités juridiques, instituées par décision politique, qui exercent un pouvoir législatif, judiciaire ou exécutif sur d'autres unités institutionnelles dans un espace donné. Conformément au SCN toutefois, le secteur de l'État est une entité plus large qui comprend non seulement ces administrations publiques " de base ", mais également les institutions sans but lucratif (ISBL) qu'elles contrôlent. Dans le reste de ce chapitre, l'expression " administrations publiques » est utilisée au sens large comme un synonyme 
de "secteur de l'État ". Cependant, comme expliqué au chapitre 3, la définition du secteur de l'État utilisée dans le présent manuel - et dans la déclaration des statistiques de R-D - diffère de celle du SCN (" Administrations publiques "), car le secteur de l'État (ou administrations publiques) tels que définis dans le présent manuel n'incluent pas les établissements d'enseignement supérieur qui correspondent aux éléments constitutifs des institutions gouvernementales d'après les critères du SCN. À cette seule exception près, les définitions sont censées correspondre entièrement.

8.4 Conformément au SCN, les entreprises contrôlées par l'État (nommées " entreprises publiques " dans le présent manuel) sont exclues du secteur de l'État ; ou plutôt, les entreprises publiques sont incluses dans le secteur des entreprises (voir le chapitre 7 et les indications ci-dessous concernant la limite entre le secteur de l'État et celui des entreprises).

8.5 Le secteur de l'État comprend ainsi toutes les unités des administration centrale (fédérale), régionale (d’États fédérés) ou locale (municipale), y compris les administrations de sécurité sociale, à l'exception des unités fournissant des services d'enseignement supérieur ou répondant à la description des établissements d'enseignement supérieur donnée au chapitre 3 et décrites plus en détail au chapitre 9, ainsi que toutes les institutions sans but lucratif non marchandes contrôlées par des administrations publiques et n'appartenant pas au secteur de l'enseignement supérieur.

8.6 L’administration centrale (ou fédérale) se compose en général d'un noyau central de services ou de ministères qui constituent une seule unité institutionnelle - souvent désignée par l'expression " administration publique nationale " ou encore " État » et couverte par le compte budgétaire principal et, dans beaucoup de pays, elle comprend d'autres unités institutionnelles. Bien qu'ils puissent être responsables d'importantes dépenses de R-D (intramuros et extra-muros) au titre du budget général de l'État, les ministères ne constituent généralement pas des unités institutionnelles distinctes habilitées à détenir des actifs, contracter des engagements, effectuer des opérations, etc., indépendamment de l'administration centrale prise dans son ensemble. En règle générale, leurs recettes et dépenses sont réglementées et contrôlées par le ministère des Finances ou par un organe fonctionnel équivalent au moyen d'un budget général approuvé par le parlement.

8.7 Outre les ministères et services apparentés, le secteur de l'État comprend également d'autres organes administratifs comme les agences, qui ont une identité juridique distincte et une autonomie substantielle, notamment une discrétion quant au volume et à la composition de leurs dépenses et de leurs frais, et peuvent également avoir des sources directes de revenus. Ces administrations publiques sont souvent désignées sous le nom "d'unités extrabudgétaires " car elles ont des budgets distincts, et tous les transferts en provenance du compte budgétaire principal sont complétés par leurs propres sources de revenu, comme les impôts d'affectation spéciale ou les frais de service. 
Ces unités peuvent avoir été constituées pour remplir des fonctions spécifiques, par exemple le financement de la R-D, son exécution, ou les deux. Dans certains pays, ces organisations, centres et instituts spécialisés peuvent représenter une proportion très importante de l'exécution de la R-D dans l'administration publique, voire dans l'ensemble de l'économie, et font également partie du secteur de l'État.

8.8 Sont incluses dans " autres organes administratifs " les institutions sans but lucratif (ISBL) non marchandes contrôlées par une administration publique, que leur statut juridique les décrive ou non comme créées indépendamment de l'administration publique. Un certain nombre d'institutions exécutant de la R-D comme les centres de recherche, les musées, etc., peuvent entrer dans cette catégorie. Comme indiqué au chapitre 3, le contrôle économique de ces entités peut être difficile à définir ; il donne lieu à des nuances considérables qui peuvent se traduire par des différences internationales en termes de mise en œuvre pratique. Dans de nombreux cas, les pouvoirs publics peuvent être à même d'exercer un contrôle par le biais de décisions de financement, mais cela ne doit pas être le seul critère utilisé pour déterminer si une institution de la sorte est effectivement contrôlée par l'État. Il est possible pour une ISBL d'être majoritairement financée par l'État, sans que ce dernier ait nécessairement le pouvoir de diriger ses activités de recherche.

8.9 Le sous-secteur des administrations régionales (ou d’États fédérés) comprend les administrations régionales ou d'États fédérés qui sont des unités institutionnelles distinctes, ainsi que les organisations et les ISBL non marchandes contrôlées par les administrations régionales (ou d’États fédérés). Ce sous-secteur exerce certaines fonctions d'administration à un niveau inférieur à celui de l'administration centrale/fédérale et supérieur à celui des unités institutionnelles d'administration qui se situent au niveau local. Il réunit les unités institutionnelles dont le pouvoir fiscal, législatif et exécutif ne s'étend qu'aux « États » qui peuvent composer le pays. Ces "États » peuvent être désignés de différentes manières selon les pays, par exemple, "régions " ou " provinces ".

8.10 Le sous-secteur des administrations locales (ou municipales) comprend les administrations locales (ou municipales) qui sont des unités institutionnelles distinctes, ainsi que les organisations et institutions sans but lucratif non marchandes contrôlées par les administrations locales. En principe, les administrations locales (ou municipales) sont des unités institutionnelles dont le pouvoir fiscal, législatif et exécutif s'étend sur les plus petits territoires géographiques distingués à des fins administratives et politiques. Leur pouvoir est généralement d'une étendue beaucoup plus limitée que celui de l'administration centrale ou des administrations d'États fédérés.

\section{Identification et limites des unités du secteur de l'État}

8.11 Les unités du secteur de l'État peuvent être associées dans un certain nombre d'activités économiques, comme l'administration publique, la santé et l'action sociale, la défense, l'enseignement (sauf le secteur de l'enseignement supérieur), ainsi que d'autres services publics pouvant inclure des institutions 
8.13 Les administrations publiques de base peuvent adopter un large éventail de désignations juridiques dont la terminologie et l'application varient d'un pays ou territoire à l'autre. Elles peuvent inclure des ministères ou des services aux prérogatives similaires à celles d'un ministère, des organisations, des organismes publics non ministériels et des institutions dotées de chartes spéciales. Ces termes sont donnés à titre indicatif.

\section{La limite entre unités dans le secteur de l'État et le secteur sans but lucratif privé}

8.14 Le contrôle est le critère fondamental pour déterminer si une ISBL est explicitement autonome ou si elle fait partie du système administratif de l'État. Il est parfois délicat d'établir si un centre de recherche, un musée, un institut, etc. donné est contrôlé ou non par l'État, en particulier s'il n'est pas possible de s'appuyer sur les nomenclatures autorisées intégrées dans les registres statistiques. À l'exception des chevauchements avec l'enseignement supérieur, qui n'est pas identifié distinctement comme un secteur institutionnel dans le SCN, les classifications du SCN constituent a fortiori des critères appropriés pour attribuer des unités au secteur de l'État. Des indications générales sur la façon d'appliquer des critères de contrôle sont données aux chapitres 3 et 10 .

8.15 En général, la prédominance d'un financement public sur d'autres sources n'est pas suffisante pour définir un contrôle par l'État, mais elle peut être un facteur important à prendre en compte, en combinaison avec d'autres informations sur la nature de ce financement (par ex., adjudication par appel d'offres ou non, participation au conseil d'administration, actions spécifiques, etc.), pour décider si l'État a le pouvoir de décision (c.-à-d. le contrôle) sur l'unité exécutant la R-D (voir encadré 8.1).

8.16 Des académies nationales des sciences existent dans plusieurs pays. Leurs rôles et leurs responsabilités peuvent varier. Elles peuvent parfois englober des instituts de recherche scientifique, mais leur rôle peut aussi être davantage lié à la diffusion du savoir et à la promotion générale de la recherche scientifique. Le statut de ces organisations peut changer au fil du temps, en particulier dans les économies en transition, où elles peuvent passer du secteur de l'État au secteur sans but lucratif privé, voire au secteur des entreprises, qui englobe les entreprises privées et publiques.

\section{La limite entre unités dans le secteur de l'État et le secteur des entreprises}

8.17 Comme noté précédemment ainsi que dans le tableau 8.1, il est important de ne pas confondre le concept de "secteur de l'État " et celui de " secteur public ». Il est possible de produire des indicateurs pour le secteur public dans sa totalité en faisant la somme du secteur de l'État et des composantes contrôlées par l'État des secteurs de l'enseignement supérieur 
et des entreprises. Les sociétés et autres types d'entreprises contrôlées par l'État n'entrent pas dans la définition du secteur de l'État. Comme indiqué aux chapitres 3 et 7 et conformément aux indications normalisées du SCN, la ligne de partage entre ces entreprises publiques et les unités du secteur de l'État est que les premières nommées visent à vendre la majorité de leur production à des prix économiquement significatifs (voir Glossaire), en incluant une marge bénéficiaire pour leurs activités.

\section{Encadré 8.1. Contrôle public des institutions sans but lucratif (ISBL)}

Le contrôle d'une ISBL est défini comme étant la possibilité de définir la politique ou le programme général de l'ISBL. En règle générale, on détermine si une ISBL est contrôlée par l'État au regard des cinq critères suivants :

1. Possibilité de nommer les responsables ou le conseil de direction.

2. Possibilité de prendre d'autres dispositions permettant à l'administration publique de déterminer des aspects importants de la politique générale ou du programme de l'ISBL, comme relever de ses fonctions du personnel clé ou opposer un veto à une nomination proposée, imposer que les budgets ou opérations financières soient préalablement soumis à son autorisation, ou encore empêcher l'ISBL de modifier ses statuts ou de se dissoudre.

3. Existence d'accords contractuels, autorisant l'administration publique à imposer les conditions susmentionnées.

4. Degré et type de financement assuré par les pouvoirs publics, qui peuvent empêcher l'ISBL de définir sa propre politique ou son propre programme

5. Existence d'une exposition aux risques, si une administration publique s'expose ouvertement à la totalité, ou à une grande partie, des risques financiers associés aux activités d'une ISBL.

Source : FMI (2014), Manuel de statistiques de finances publiques, www.imf.org/external/np/ sta/gfsm.

8.18 Il peut exister des institutions exécutant de la R-D, comme des centres de recherche, des musées ou des académies des sciences qui génèrent des revenus commerciaux significatifs, grâce par exemple à la concession de licences de propriété industrielle provenant d'activités de R-D antérieures ou à la fourniture de services de recherche et de conseil aux conditions du marché. Dans la mesure du possible, il est important que les décisions de classification ne s'appuient pas sur des circonstances inhabituelles, par exemple une occurrence unique telle que la réalisation de revenus commerciaux extraordinaires résultant de la cession d'un actif. 


\section{La limite entre unités dans le secteur de l'État et le secteur de l'enseignement supérieur}

8.19 Il est difficile à plus d'un titre de distinguer les activités du secteur de l'État de celles du secteur de l'enseignement supérieur. Les chapitres 3 et 9 traitent en détail de ces questions de limites. Il peut exister toutes sortes de liens et de chevauchements institutionnels et de personnel entre les établissements d'enseignement supérieur telles que définis dans le présent manuel et le secteur des administrations publiques tel que défini par le SCN.

8.20 Dans de nombreux pays, les administrations publiques peuvent être habilitées à diriger et contrôler les activités d'une partie, voire de la totalité, des établissements d'enseignement supérieur. Cette forme de contrôle n'implique pas que ces établissements font partie du secteur de l'État tel que défini dans le présent manuel, mais elle leur confère les qualités requises pour faire partie du secteur public.

8.21 Les administrations publiques peuvent employer du personnel de recherche affilié à d'autres institutions, en particulier des établissements d'enseignement supérieur. Il peut s'avérer parfois difficile de distinguer leurs activités liées à l'administration de celles exercées pour le compte de leurs employeurs de l'enseignement supérieur. La double affiliation de personnes à un établissement d'enseignement supérieur ne doit pas conduire à la reclassification de l'administration publique, à moins que d'autres mécanismes de contrôle institutionnels autorisent la classification de l'administration publique comme établissement d'enseignement supérieur.

8.22 Une question pouvant poser problème est le traitement des hôpitaux publics et des établissements de santé apparentées présentant un type de lien formel avec les établissements d'enseignement supérieur, où la fourniture formelle de programmes d'enseignement tertiaire et autres critères importants de relations de contrôle pourraient orienter la classification de ces institutions gouvernementales vers le secteur de l'enseignement supérieur. Cependant, il est possible pour un hôpital universitaire (décrit comme tel sur la base de son histoire et de ses liens avec un établissement d'enseignement supérieur, qu'il autorise par exemple à soutenir/héberger la formation des étudiants en médecine) d'être classé dans le secteur de l'État. Ce sera le cas s'il se trouve que l'hôpital est contrôlé et majoritairement financé par les pouvoirs publics, qu'il fonctionne sur une base non lucrative, et qu'il est suffisamment indépendant de l'institution d'enseignement supérieur par rapport aux activités de R-D qu'il mène.

\section{Autres cas particuliers}

8.23 Les administrations publiques peuvent nouer des partenariats avec d'autres organismes dans le secteur de l'État ou d'autres secteurs pour créer des 
entités engagées dans l'exécution de la R-D. Ces entités, si elles prennent le statut d'unités institutionnelles, sont soumises aux mêmes principes de classification générale que ceux exposés au chapitre 3.

\section{Classifications possibles des unités du secteur de l'État Classification par activité économique principale}

8.24 La pertinence transversale de cette classification institutionnelle pour ventiler l'exécution (ainsi que le personnel) de la R-D a été soulignée au chapitre 3. Cela s'applique également au secteur de l'État à la lumière des diverses activités économiques possibles, en particulier les services, réalisées par les institutions gouvernementales. Il est recommandé de classer toutes les administrations publiques comprenant des ISBL contrôlées par l'État dans leur activité économique principale telle qu'identifiée selon les catégories par divisions (à deux chiffres) de la CITI Rév. 4 (Nations Unies, 2008), dont la classification détaillée est consultable en ligne, en complément du présent manuel à l'adresse http://oe.cd/frascati. Si cela n'est pas pratique, il est recommandé au minimum d'identifier les unités qui font partie de la division 72 de la CITI : Recherche et développement scientifiques, afin d'identifier les établissements publics de recherche. Il est également recommandé d'identifier de façon distincte les hôpitaux et cliniques contrôlés par l'État (généralement classés dans la division 86 : Activités relatives à la santé), pour les raisons expliquées ci-dessus.

\section{Classification des fonctions des administrations publiques}

8.25 La Classification des fonctions des administrations publiques (COFOG) est une classification générique des fonctions, ou objectifs socioéconomiques, que les administrations publiques visent à remplir en engageant différentes sortes de dépenses. La COFOG a été créée par l'OCDE et publiée conjointement avec trois autres classifications (Nations Unies, 2000). Elle fournit une nomenclature des entités et des dépenses financières des administrations publiques par fonction d'intérêt général (pour les catégories de la COFOG, voir le complément du présent manuel consultable en ligne à l'adresse http://oe.cd/ frascati). Les intitulés du premier niveau présentent de nombreuses similitudes avec les nomenclatures des objectifs socio-économiques utilisés en R-D (voir la section 8.4 sur la répartition des DIRDET par objectif socio-économique et la section $12.5 \mathrm{du}$ chapitre 12). Dans le présent manuel, il est déconseillé aux institutions gouvernementales d'employer cette classification dans le contexte des statistiques de la R-D car les catégories qui la composent ne se prêtent pas à une description optimale des dépenses de R-D, même si une table de correspondance provisoire serait utile au cas où la COFOG venait à être adoptée plus largement dans les systèmes généraux de statistiques nationales. 


\subsection{Identification de la R-D dans le secteur de l'État}

8.26 L'identification de ce qu'est la R-D devrait suivre les principes exposés $\mathrm{au}$ chapitre 2. Les unités de base des administrations centrales/fédérales, régionales/d'États fédérés et municipales/locales peuvent exécuter elles-mêmes de la R-D. À titre d'exemple, on peut citer des unités de recherche spéciales de taille variable dans des ministères, mais également des organismes plus importants comme les forces armées.

8.27 Il est difficile de dissocier la R-D d'autres activités connexes dès lors que ces activités sont menées simultanément au sein d'une entité. Les administrations publiques exécutant de la R-D ne se consacrent pas toutes entièrement à ces activités, mais elles réalisent souvent de la R-D pour servir les principaux objectifs de l'administration. Les administrations publiques peuvent prendre part à des activités comme les prestations de santé, la collecte de données d'utilité générale pour la surveillance des systèmes naturels ou sociaux, ou le développement d'infrastructures à grande échelle, dont la mise en œuvre pourrait profiter d'une exécution interne ou externe de la R-D. Les actifs intellectuels et corporels émanant des activités centrales des administrations publiques peuvent également être utilisés comme ressources principales pour des projets de R-D pouvant être menés à l'intérieur de ces organismes. Le caractère systématique du traitement de ces activités dans la collecte de statistiques de R-D peut avoir des incidences notables sur la comparabilité internationale des données de R-D qui en découlent.

\section{Activités scientifiques et technologiques apparentées}

8.28 Outre les travaux de recherche fondamentale ou appliquée et de développement expérimental, les activités courantes liées à la R-D dans le secteur de l'État comprennent la fourniture de services technologiques comme la normalisation et les essais techniques, le transfert de technologie (par ex. le transfert physique de technologie, de prototypes et de processus et/ou de "savoir-faire "), le développement de nouvelles instrumentations, la préservation, le stockage et l'accès à des collections scientifiques et de connaissances par le biais de bibliothèques, de bases de données et d'archives, la fourniture d'installations et d'infrastructures scientifiques de grande envergure (par ex. des réacteurs nucléaires, des satellites, de grands télescopes, des navires océanographiques, etc.). Ces activités scientifiques et technologiques apparentées ne devraient pas être incluses dans la R-D.

\section{Démonstration et développement de systèmes}

8.29 Les administrations publiques peuvent parfois réaliser des investissements très importants dans des actifs fixes de grande ampleur considérés comme " premiers du genre » ou porteurs de capacités précédemment inexistantes. Elles peuvent avoir tendance à inclure ces coûts de construction dans la R-D en raison de leur contribution potentielle à l'innovation. À des fins 
de comparabilité internationale cependant, seuls les coûts spécifiquement identifiés comme capital affecté à la R-D devraient être inclus dans la R-D intra-muros. Généralement, ces coûts ne devraient pas être comptabilisés comme dépenses courantes de R-D, mais comme dépenses en capital consacrées à la R-D (voir chapitre 4).

8.30 Certains pays utilisent des classifications par niveaux de maturité technologique dans la description et la gestion de projets dans la défense, l'aérospatiale et autres secteurs nécessitant de l'ingénierie de systèmes. Différents modèles ont été élaborés pour aider à évaluer la maturité des éléments technologiques de ces programmes, mais ils demeurent en grande partie non testés dans d'autres domaines. Étant donné le degré d'engagement de l'État dans les domaines où sont utilisés des modèles de niveaux de maturité technologique, ils peuvent figurer dans la description de la R-D intra-muros d'État ainsi que dans les marchés publics spécifiant l'exécution de la R-D par un tiers. Comme indiqué au chapitre 2, il est conseillé, en cas d'utilisation de ces modèles, de les évaluer afin de déterminer s'ils peuvent contribuer d'une manière quelconque à l'amélioration de la collecte de statistiques sur l'exécution de la R-D d'un État ou sur le financement public de la R-D (section 8.6).

8.31 En raison de la multiplicité des systèmes de classification par niveaux de maturité technologique et de leur description générique, il n'est pas possible de fournir une cartographie concrète et globalement applicable des niveaux - ou plus spécifiquement, des travaux entrepris pour mener le programme à un niveau de maturité supérieur - pour les types de R-D (recherche fondamentale, recherche appliquée et développement expérimental) tels que définis dans le présent manuel. L'application de niveaux de maturité technologique à la R-D peut être extrêmement compliquée au vu des différentes étapes qui impliquent la démonstration des projets/systèmes dans des environnements d'utilisation variés, plus réalistes, qui peuvent déboucher sur de nouvelles exigences de spécifications pour les projets/systèmes. Le chapitre 2 indique que lorsque la performance d'un prototype est évaluée par son usage opérationnel réel, cette évaluation constituera rarement de la R-D. Les efforts entrepris pour remédier aux principaux défauts identifiés lors de l'exploitation ou en conséquence de nouvelles exigences peuvent toutefois représenter de la R-D, à condition que les critères formulés au chapitre 2 soient remplis.

\section{Études utiles pour l'action des pouvoirs publics}

8.32 Les efforts de R-D peuvent étayer la prise de décision au sein des administrations publiques. S'il est possible de les externaliser, il arrive aussi que les administrations publiques disposent en interne d'équipes qui participent activement au travail d'analyse, par exemple en réalisant des évaluations et bilans. Il est possible que ces activités répondent aux critères définissant un projet de R-D, mais ce n'est pas toujours le cas, de même qu'il n'y a pas lieu de qualifier de R-D la totalité des efforts d'établissement des faits associés au conseil 
stratégique. Il convient d'examiner, à un certain niveau de détail, les compétences techniques des personnes associées à l'activité, le mode de codification des connaissances adopté au sein de l'organisation et les moyens pris pour veiller au respect des normes de qualité dans les thèmes de recherche et la méthodologie appliquée. Le risque que des activités de conseil socio-économique (internes ou externes) soient présentées à tort comme de la R-D n'est pas négligeable.

8.33 Les conseillers scientifiques jouent un rôle important au sein de l'administration publique. Cependant, le fait d'appliquer à l'élaboration des politiques des critères décisionnels précis ne constitue pas en soi une activité de R-D. Les efforts déployés pour mettre au point de meilleures méthodes de prise de décision fondées sur des données scientifiques peuvent être considérés comme relevant de la R-D.

\section{Soins de santé et R-D dans les hôpitaux «publics »}

8.34 Comme noté plus haut, dans beaucoup de pays une part substantielle des hôpitaux et autres établissements de santé sont contrôlés par l'État et ne remplissent pas les critères qui leur permettraient d'être classés dans le secteur de l'enseignement supérieur. De ce fait, la R-D consacrée à la santé peut être une composante importante de la R-D exécutée au sein de l'État. La combinaison des activités de soin, de recherche et de formation peut rendre difficile l'identification de la part de la R-D dans les activités de ces institutions. La R-D peut se faire en partenariat avec des établissements d'enseignement supérieur, des institutions sans but lucratif publiques ou privées ou des entreprises, par exemple dans le contexte d'essais cliniques. Des indications pertinentes sont données aux chapitres 2, 4 et 9 .

\section{Le financement de la R-D et son administration}

8.35 Comme indiqué au chapitre 4, la levée, la gestion et la distribution de fonds assurées par les ministères, les agences de financement, les instituts de recherche et autres administrations publiques au profit des exécutants de la R-D ne devraient pas être considérées comme des activités de R-D. Dans le cas des administrations publiques qui à la fois exécutent de la R-D intra-muros et financent de la R-D extra-muros, les coûts administratifs liés à la préparation et au suivi des contrats de $\mathrm{R}-\mathrm{D}$ extra-muros peuvent être assimilés à des dépenses de R-D intra-muros.

\subsection{Mesure des dépenses et du personnel de R-D dans le secteur de l'État}

\section{Dépenses intérieures de R-D de l'État (DIRDET)}

8.36 Les dépenses intérieures de R-D de l'État (DIRDET) constituent le principal agrégat statistique utilisé pour décrire l'exécution de la R-D au sein du secteur de l'État. Elles correspondent à la composante des dépenses intérieures brutes de R-D (DIRD) (voir chapitre 4) engagées par les unités du secteur de l'État 
et sont l'expression chiffrée des dépenses de R-D intra-muros de ce secteur au cours d'une période de référence donnée.

8.37 Les administrations publiques hébergent souvent une part significative des projets de R-D menés sous la responsabilité d'institutions non gouvernementales. Par exemple, un établissement public peut autoriser l'utilisation de ses installations à des fins d'essais par diverses entreprises dans le cadre de leur projet de R-D pour développer de nouveaux produits. En l'espèce, et indépendamment du fait que cette $R-D$ a été exécutée en partie dans des installations publiques, cela peut ne pas suffire à décrire l'administration publique concernée comme un exécutant des travaux de R-D. L'État fournit un service à l'entreprise, laquelle est l'unité exécutante de la R-D. L'administration publique peut toutefois être une exécutante de la R-D si elle conduit ses propres projets dans les installations.

\section{Répartitions fonctionnelles des DIRDET Répartition des DIRDET par type de dépenses}

8.38 Les règles qui s'appliquent à la répartition des DIRDET par type de coûts sont détaillées au chapitre 4 (tableau 4.1). Ces recommandations incluent une ventilation entre les coûts salariaux du personnel de R-D et les autres charges courantes (dépenses courantes) et dépenses d'équipement (par type d'actifs), avec des coûts d'amortissement des équipements détaillés séparément pour les biens d'équipement propres. En raison de la spécificité des différentes administrations publiques dans un pays, cette information devrait, si possible, être demandée directement aux unités interrogées et non imputée à partir d'autres unités.

8.39 Parmi ces orientations générales, certains cas bien précis méritent une attention particulière :

- Les coûts salariaux comprennent des cotisations réelles ou imputées à des caisses de retraite et autres charges de sécurité sociale pour le personnel de R-D. Il n'est pas nécessaire de les faire apparaître dans la comptabilité de l'unité statistique ; elles peuvent souvent comporter des opérations vers d'autres entités du secteur de l'État, comme les caisses de sécurité sociale. Même si aucune opération n'intervient, une tentative d'estimation de ces coûts du point de vue de l'unité déclarante doit être effectuée.

- La taxe sur la valeur ajoutée (TVA) pour les biens et les services peut ne pas être récupérable pour des unités du secteur de l'État, auquel cas elle doit être comptabilisée au titre des autres charges courantes.

8.40 La quantification des dépenses de R-D engagées dans le contexte des équipements utilisés pour la R-D est l'un des aspects potentiellement les plus délicats de la mesure de la R-D dans le secteur de l'État. Les exemples analysés au chapitre 4 montrent la nécessité d'identifier le coût économique des équipements utilisés sans verser de rémunération réelle, et d'éviter une 
possible double comptabilisation des coûts de construction ou d'acquisition d'équipements et des coûts pour les utilisateurs de ces installations.

8.41 Il existe des exemples où l'État est propriétaire et responsable de l'entretien d'installations spéciales utilisées par des chercheurs employés par le service correspondant et par des visiteurs provenant d'autres organismes et entreprises pour des projets de R-D approuvés. Quand ces équipements sont utilisés par d'autres exécutants publics ou non publics, les frais d'utilisation - qui peuvent comporter des coûts d'exploitation et d'entretien - réglés aux propriétaires de ces équipements font partie des charges courantes déclarées par les exécutants de la R-D utilisant ces installations. Ces coûts d'exploitation et d'entretien récupérés dans les frais d'utilisation devraient être exclus des déclarations de l'organisme d'État propriétaire des installations, afin d'éviter la double comptabilisation de ces dépenses. Étant donné que l'utilisation de ces équipements peut être rare ou son coût d'utilisation trop faible pour couvrir les dépenses nécessaires à maintenir les installations de R-D en état d'exploitation, une composante dûment justifiée des coûts d'exploitation et d'entretien peut être comptabilisée comme dépenses intra-muros, au titre des autres charges courantes, par l'administration publique propriétaire de l'équipement.

\section{Répartition des DIRDET par source de financement}

8.42 De longue date, les financements provenant de sources privées étaient pour la plupart supposés avoir une pertinence limitée dans le cas de l'exécution de la R-D dans le secteur de l'État, étant donné le rôle dominant des sources budgétaires internes. Cependant, dans les circonstances actuelles, l'absence de ces informations pourrait donner une image assez déformée de la réalité. L'utilisation généralisée de partenariats public-privé et de formules mixtes, la recherche d'autres sources de financement possibles au sein des administrations publiques extrabudgétaires et des ISBL contrôlées par l'État, et l'existence d'accords internationaux entre pays et organisations supranationales exigent une collecte d'informations minutieuse sur l'origine des fonds utilisés pour la R-D de l'État, à la fois de sources internes et du reste du monde.

8.43 Les sources de financement des institutions gouvernementales et de leurs activités de R-D peuvent provenir de revenus spécialement affectés (par ex. quand elles sont définies comme un pourcentage du total des recettes publiques ou correspondent à des cotisations de sécurité sociale ou des taxes spécifiques), de transferts budgétaires, de ventes générales de biens et/ou de services ou de frais d'utilisation, de ventes d'actifs financiers et non financiers, d'emprunts, ou de fonds généraux de donateurs (FMI, 2014). Les sources extrabudgétaires désignent des opérations des administrations publiques, souvent avec des dispositions institutionnelles et bancaires distinctes qui ne sont pas incluses dans la loi de finances annuelle de l'administration centrale ni dans les budgets des administrations publiques infranationales. 
8.44 La structure de déclaration présentée dans le tableau 8.2 est conseillée pour la collecte d'informations sur la source des fonds. La ventilation entre l'échange et les fonds provenant de transferts est plus pertinente pour les administrations publiques extrabudgétaires ainsi que pour les ISBL contrôlées par l'État, qui peuvent dépendre dans une plus grande mesure de sources non budgétaires et sont plus susceptibles d'entreprendre de la R-D comme un service rendu à d'autres organismes ou entreprises moyennant des compensations financières.

\section{Répartition des DIRDET par type de R-D}

8.45 Comme pour tous les autres secteurs, il est recommandé de collecter les données des administrations publiques concernant la ventilation des dépenses de R-D par type de R-D, recherche fondamentale, recherche appliquée et développement expérimental, tel que défini au chapitre 2.

\section{Répartition des DIRDET par domaine de R-D}

8.46 Il est conseillé, autant que possible, de répartir l'exécution de la R-D dans les administrations publiques selon le domaine de recherche et développement du niveau le plus élevé.

\section{Tableau 8.2. Source des financements à collecter dans les enquêtes} sur l'exécution de la R-D dans le secteur de l'État

\begin{tabular}{|c|c|c|c|}
\hline $\begin{array}{l}\text { Source des financements pour la R-D exécutée } \\
\text { dans une institution gouvernementale }\end{array}$ & $\begin{array}{c}\text { Financement } \\
\text { en échange de } \\
\text { travaux de R-D }\end{array}$ & $\begin{array}{c}\text { Financement } \\
\text { sous forme de } \\
\text { transfert pour } \\
\text { des travaux } \\
\text { de } \text { R-D }^{1} \\
\end{array}$ & $\begin{array}{c}\text { Financement total } \\
\text { pour l'exécution } \\
\text { de R-D intra- } \\
\text { muros }\end{array}$ \\
\hline Secteur de l'État & $x$ & $x$ & $\checkmark$ \\
\hline $\begin{array}{l}\text { - Organisme/institution propre (financement } \\
\text { interne) }\end{array}$ & $x$ & $x$ & $\checkmark$ \\
\hline - Autre - central ou fédéral & $x$ & $x$ & $\checkmark$ \\
\hline - Autre - régional ou d'États fédérés ou local & $x$ & $x$ & $\checkmark$ \\
\hline Secteur des entreprises & $\checkmark$ & $\checkmark$ & $\checkmark$ \\
\hline Secteur de l'enseignement supérieur & $\checkmark$ & $\checkmark$ & $\checkmark$ \\
\hline Secteur privé sans but lucratif & $\checkmark$ & $\checkmark$ & $\checkmark$ \\
\hline Reste du monde & $\checkmark$ & $\checkmark$ & $\checkmark$ \\
\hline - Secteur de l'État & $\checkmark$ & $\checkmark$ & $\checkmark$ \\
\hline $\begin{array}{l}\text { - Organisations internationales (y compris } \\
\text { organisations supranationales) }\end{array}$ & $\checkmark$ & $\checkmark$ & $\checkmark$ \\
\hline - Secteur des entreprises & $\checkmark$ & $\checkmark$ & $\checkmark$ \\
\hline - Secteur de l'enseignement supérieur & $\checkmark$ & $\checkmark$ & $\checkmark$ \\
\hline - Secteur privé sans but lucratif & $\checkmark$ & $\checkmark$ & $\checkmark$ \\
\hline Toutes les sources & & & = DIRDET \\
\hline
\end{tabular}

Notes : adapté du tableau 4.1 du présent manuel.

$\mathrm{x}=$ sans objet ; collecte non nécessaire.

1. Ventilation échange/transfert plus pertinente pour les administrations publiques extrabudgétaires et les ISBL contrôlées par l'État. 
8.47 Au moins dans le cas des institutions gouvernementales classées dans la division 72 de la CITI Rév. 4 (Recherche scientifique et développement), il peut être utile de classer ces dernières d'après le domaine de recherche et de développement principal, en utilisant les intitulés du niveau le plus élevé. La classification détaillée des domaines est consultable dans le complément en ligne du présent manuel à l'adresse http://oe.cd/frascati. Étant donné l'existence de centres pluridisciplinaires au sein de l'État, il peut être utile d'indiquer un champ de recherche secondaire ou d'utiliser une catégorie pluridisciplinaire complémentaire.

\section{Répartition des DIRDET par domaine technologique}

8.48 Les pays pourront trouver utile de déclarer leurs DIRDET par domaine technologique. La biotechnologie, les nanotechnologies et les technologies de l'information et des communications (TIC) comptent parmi les plus importants.

\section{Répartition des DIRDET par objectif socio-économique}

8.49 Il est en principe possible, pour les institutions du secteur de l'État, de communiquer les DIRDET du point de vue des exécutants par objectif socioéconomique. Cette approche ne doit pas être confondue avec l'analyse des crédits budgétaires publics de R-D (CBPRD) par objectif socio-économique (voir le chapitre 12 pour des informations complètes sur ces répartitions).

8.50 La liste de répartition recommandée s'appuie sur les catégories de la Nomenclature pour l'analyse et la comparaison des budgets et des programmes scientifiques (NABS) (Eurostat, 2008) et autres adaptations nationales présentant une correspondance directe avec celle-ci. Cette liste est la même que celle suggérée pour le financement public de la R-D, à l'exception de la recherche financée par des fonds universitaires généraux, qui ne constitue pas une catégorie appropriée dans le cas présent. La R-D devrait être répartie par unités de déclaration selon les objectifs de projet primaires et l'agrégation du portefeuille de recherche de ces derniers.

8.51 Dans le cas du secteur de l'État et conformément aux indications du chapitre 4 , il est particulièrement important de compiler des totaux distincts pour les DIRDET civiles et les DIRDET militaires et de faire état de toute souscouverture potentielle de la R-D militaire. Cela est particulièrement valable pour des pays ayant des programmes importants de R-D militaire, qui peuvent en partie être réalisés dans les administrations publiques. En outre, les informations concernant ces projets de R-D militaire peuvent être hautement sensibles et classées de façon à ce que les dépenses de R-D ne puissent pas être distinguées des programmes hors R-D. Comme indiqué au chapitre 4, il est important de garantir la comparabilité internationale au moins pour les données sur la R-D civile. Il est également important que les métadonnées auxiliaires sur les DIRDET donnent des informations claires sur les marges d'incertitude liées à la R-D non mesurée au sein de l'État. 


\section{Répartition des DIRDET par fonctions des administrations publiques}

8.52 Certains pays peuvent trouver utile de répartir les DIRDET selon les catégories de la COFOG (voir la section 8.2 sur les classifications institutionnelles). Cependant, pour les raisons décrites précédemment, le présent manuel déconseille l'utilisation des catégories de la COFOG dans le contexte des statistiques de R-D.

\section{Répartition des DIRDET par situation géographique}

8.53 Des pays peuvent trouver utile de compiler des totaux distincts pour la répartition des DIRDET par lieu/région. Le choix de la répartition géographique est déterminé en fonction de besoins nationaux et internationaux. Des renseignements supplémentaires sont accessibles en ligne, en complément du présent manuel à l'adresse http://oe.cd/frascati.

\section{DIRDET par opposition au financement public de l'exécution de R-D extra-muros}

8.54 La section 8.6 examinera de façon plus détaillée la façon dont les administrations publiques déclarent le financement de la R-D extra-muros. La présente sous-section s'intéresse principalement aux limites entre la R-D intra-muros et la R-D exécutée extra-muros pour le secteur de l'État. S'agissant des exécutants de R-D dans ce secteur, certaines dépenses peuvent avoir été engagées pour des activités réalisées hors établissement voire à l'étranger, par exemple dans l'espace, en Antarctique, dans les représentations consulaires ou diplomatiques de l'institution gouvernementale à l'étranger, ou à court terme, par ex. comme travail sur le terrain, dans un autre pays. Quand cette R-D a lieu sous la responsabilité de l'institution gouvernementale observée, cela correspond à sa R-D intra-muros. Les paiements pour des services à des tiers qui contribuent aux efforts de R-D correspondent également à sa propre R-D intra-muros.

8.55 Le paiement de consultants qui fournissent un service de R-D en exécution d'une commande (acquisition) spécifique, sans toutefois être partie intégrante des projets de R-D de l'administration publique, devrait être traité comme financement de R-D extra-muros par l'unité statistique faisant l'acquisition de la R-D. L'unité statistique exécutante, qui emploie ces consultants, devrait déclarer cette activité comme dépenses de R-D intra-muros. La ventilation des dépenses pour les dépenses de R-D extra-muros devrait se faire comme indiqué au chapitre 4.

8.56 Pour décider s'il convient de classer le financement accordé par une entité administrative à une autre entité administrative comme un financement correspondant à une exécution intra-muros ou extra-muros, le niveau d'administration (c.-à-d. central/fédéral, régional/d'États fédérés, local/ municipal ; voir tableau 8.1) est l'unité institutionnelle pertinente et fournit le critère déterminant. Par exemple, des fonds pour la R-D reçus par un ministère $Y$ de l'administration centrale de la part du ministère $\mathrm{X}$ d'une autre administration centrale devraient être déclarés comme fonds propres du ministère Y pour de la 
R-D intra-muros. Pour le ministère $\mathrm{X}$ qui fournit (est la source de) ces fonds de $\mathrm{R}-\mathrm{D}$, ces derniers ne devraient pas être déclarés comme fonds propre ni comme fonds destinés à des travaux extra-muros. Dans un agrégat de secteur, ces fonds sont simplement des fonds propres de l'administration centrale pour l'exécution de la R-D intra-muros de l'administration centrale. La raison en est que ces opérations constituent différentes parties de la même unité institutionnelle de l'administration centrale, même si les unités statistiques sont des entités plus petites, comme des ministères.

8.57 Il faut bien veiller à éviter la possibilité de comptabiliser doublement les financements pour la R-D provenant d'organisations " intermédiaires " qui reçoivent des fonds de ministères et d'organismes, puis réaffectent et transmettent ces fonds à d'autres institutions exécutantes. Dans l'exemple précédent, si les fonds fournis par le ministère $\mathrm{X}$ au ministère $\mathrm{Y}$ sont transmis par le ministère intermédiaire $Y$ à un exécutant de R-D externe au secteur de l'État, l'institution gouvernementale n'est pas l'exécutante - c.-à-d. qu'il n'y a pas de DIRDET), et les fonds sont déclarés comme fonds publics pour une exécution non gouvernementale extra-muros, émanant du ministère $\mathrm{X}$, si ce type de renseignement est collecté (voir section 8.5 et tableau 8.3).

8.58 Les opérations entre entités à l'intérieur des administrations centrales et régionales ou de différentes unités extrabudgétaires et d'autres ISBL contrôlées par l'État dans le secteur de l'État devraient être déclarées comme financements extra-muros lorsqu'il s'agit d'institutions distinctes possédant leurs propres comptes. Par exemple, des fonds destinés à la R-D reçus par l'organisme $Z$ d'une administration régionale de la part d'un ministère $\mathrm{X}$ de l'administration centrale devraient être déclarés comme fonds (publics) externes pour la R-D intra-muros de l'organisme $\mathrm{Z}$. Pour le ministère $\mathrm{X}$ de l'administration centrale qui fournit (est la source de) ces fonds de R-D, ces derniers doivent être déclarés comme fonds destinés à une exécution extra-muros par l'administration régionale.

\section{Personnel de R-D dans le secteur de l'État}

8.59 Les catégories de personnel de R-D qui doivent être déclarées pour le secteur de l'État sont les mêmes que celles des autres secteurs exécutants de R-D ; elles sont définies au chapitre 5 du présent manuel. Les répartitions conseillées ci-dessus pour les dépenses devraient être appliquées autant que possible au personnel de R-D.

8.60 Les agents des institutions gouvernementales employés uniquement à l'administration et à l'évaluation des projets de financement de R-D, par ex. dans le contexte de l'octroi de subventions ou de marchés publics, ne devraient pas être considérés comme du personnel de R-D. Leurs activités ne constituent pas de la R-D. Comme indiqué au chapitre 4, les institutions gouvernementales qui combinent des rôles d'exécution et de financement de R-D peuvent inclure les coûts de leur personnel travaillant sur les aspects concrets, financiers ou administratifs des contrats de R-D dans " autres charges courantes ", mais ce personnel ne devrait pas être classifié comme personnel de R-D. 
8.61 Étant donné la présence de personnel externe de R-D dans les installations publiques de R-D, il est conseillé - comme indiqué au chapitre 5 - de déclarer ces personnes dans la catégorie appropriée, distinctement du personnel interne de R-D. Cela s'applique également aux stagiaires comme les étudiants en doctorat ou en master, s'ils exécutent réellement de la R-D conformément aux critères exposés aux chapitres 2 et 5 .

8.62 En général, dans les établissements publics de recherche, les catégories proposées au chapitre 5 peuvent facilement être déclarées, ce qui n'est pas forcément le cas pour certaines administrations publiques de base. Autant que possible, une classification des chercheurs par grade hiérarchique similaire à celle proposée au chapitre 9 peut être utile à des fins de documentation de l'organisation de la R-D dans les institutions gouvernementales. Les catégories incluent les postes types pour chaque groupe (CE, $2013: 87)$ :

- Catégorie A : grade/poste le plus élevé auquel des travaux de recherche sont normalement menés.

* Exemple : « directeur de recherche ».

- Catégorie $B$ : chercheurs travaillant à un niveau moins élevé que le niveau supérieur (A), mais plus élevé que celui des titulaires de doctorat nouvellement diplômés (niveau 8 de la CITE).

* Exemples : " chargé de recherche principal " ou « chercheur principal ».

- Catégorie C : premier grade/poste auquel un titulaire de doctorat nouvellement diplômé sera normalement recruté.

* Exemples : " chercheur » ou « boursier de recherches postdoctorales ».

- Catégorie D : Étudiants en doctorat (niveau 8 de la CITE) travaillant comme chercheurs, ou chercheurs travaillant à des postes ne nécessitant normalement pas un diplôme de doctorat.

* Exemples : « doctorant » ou " assistant de recherche » (non titulaire d'un doctorat).

\subsection{Méthodes pour compiler les dépenses et le personnel de R-D dans le secteur de l'État}

\section{Les unités déclarantes et statistiques dans le secteur de l'État}

8.63 Les administrations publiques qui devraient être couvertes par les enquêtes incluent :

- les instituts, laboratoires et centres de R-D

- dans le cadre des administrations centrales/fédérales, régionales/d’États fédérés ou municipales/locales, les activités de R-D de l'administration générale les services statistiques, météorologiques, géologiques et autres services publics, les musées et les hôpitaux

- les activités de R-D à tous les niveaux d'administration (le cas échéant : central/fédéral, régional/d’États fédérés, et municipal/local). 
8.64 L'unité statistique sera généralement le ministère ou service central apparenté, ou l'organisme, même si l'unité n'a pas toutes les caractéristiques d'une unité institutionnelle (par ex. souvent les ministères n'ont pas la capacité de détenir et de contrôler des actifs séparément de l'ensemble de l'administration centrale/fédérale [ou régionale/d'États fédérés]). Les attributs souhaités de l'unité d'échantillonnage pour le secteur de l'État incluent : branche d'activité, situation géographique et niveau d'administration. L'unité déclarante dépendra de l'entité la mieux à même de déclarer. Cela peut inclure l'administration dans son ensemble dans le cas des administrations régionales/d'États fédérés ou municipales/locales.

8.65 Le cadre de l'enquête devrait être lié à un registre statistique central s'il en existe un. Cela faciliterait l'intégration de données provenant de sources diverses et simplifierait les décisions de classification. Cela réduirait également le risque de double comptabilisation des unités traitées à partir de perspectives différentes.

8.66 Une attention particulière devrait être portée à l'utilisation des données administratives dans l'identification des institutions de financement et d'exécution de la R-D. Dans certains pays, cela inclurait les institutions qui pourraient identifier les dépenses de R-D comme une COFOG de deuxième niveau.

8.67 Il est particulièrement difficile d'identifier les activités de R-D au niveau des administrations locales (et dans certains pays, des administrations régionales/d'États fédérés) étant donné la multitude d'unités, le petit nombre d'exécutants de R-D possibles et les difficultés dans l'interprétation du concept de R-D. Si les administrations locales se chargent d'une proportion significative de l'activité de R-D, il est conseillé de faire l'effort d'inclure les exécutants de R-D dans les grandes administrations locales. Étant donné la nature de nombreuses formes d'administration régionales/d'États fédérés, elles peuvent exécuter de la R-D de manière occasionnelle : il n'est peut-être pas au cœur de la mission du ministère ou de l'organisme d'État de faire de la R-D proprement dite, mais cette dernière doit porter sur un problème spécifique identifié par le pouvoir législatif ou le ministère concerné. Par conséquent, certaines activités de R-D peuvent être temporaires.

\section{Collecte de données d'enquête}

8.68 La pratique courante est de mener un recensement des unités et des organes administratifs qui sont réputés ou présumés effectuer de la R-D. Pour des raisons liées à la charge de travail pratique, ces derniers représenteront une liste relativement courte de toutes les administrations publiques connues. Les registres/annuaires des administrations publiques, des instituts de recherche et des organes officiels, ainsi qu'une analyse de la législation en vigueur et des mesures budgétaires, peut aider à identifier les exécutants potentiels de 
R-D dans le secteur de l'État. D'autres sources d'information pourraient être de nature universitaire ou professionnelle et provenir de sociétés savantes ; d'association de recherches; de listes d'institutions de services scientifiques et technologiques ; de registres ou de bases de données de scientifiques et d'ingénieurs ; et de bases de données de publications scientifiques, de brevets et autres documents de propriété intellectuelle, ainsi que de demandes de mise à jour adressées à des organes publics.

8.69 Les agents chargés de compiler les données de R-D ne devraient pas sous-estimer les difficultés que peut présenter la collecte de ces données auprès d'institutions gouvernementales. Un manque d'informations sousjacentes dans le format demandé et une adhésion limitée peuvent altérer significativement l'exhaustivité et la qualité des données collectées. Dans le cas des instituts de recherche dont le personnel a le statut de fonctionnaire, il est conseillé de faire des démarches préalables pour s'assurer de l'adhésion du haut fonctionnaire responsable de l'institution déclarante. En général, il est conseillé d'utiliser un programme "d'approche » approprié pour étayer la collecte de données, comprenant notamment la fourniture de mallettes pédagogiques aux répondants, la réalisation d'études approfondies afin de fournir une terminologie de R-D connue du personnel des administrations infranationales, et un retour d'informations direct sur les résultats des données recueillies.

8.70 Certaines institutions dans le secteur de l'État peuvent déclarer que l'intégralité de leur personnel exécute de la recherche et que l'ETP de leur personnel de R-D est égal à ou proche de 100 \% (UNESCO-ISU, 2014). Les activités qui ne sont pas de la R-D devraient être exclues, mais cela peut être difficile à appliquer dans la pratique. Étant donné que les institutions gouvernementales diffèrent dans leur orientation et leur culture institutionnelle, le présent manuel déconseille l'application de "règles empiriques " générales en vertu desquelles un pourcentage fixe de travailleurs intellectuels dans ces institutions serait considéré comme des chercheurs. Un comptage systématique sera attendu de la part des répondants. Quand des institutions gouvernementales principalement actives dans les services scientifiques et technologiques réalisent de la recherche en relation avec cette activité, ces activités de recherche devraient être clairement identifiées et systématiquement prises en compte dans l'enquête de R-D.

\section{Estimation des dépenses et du personnel de R-D}

8.71 L'estimation des dépenses et du personnel de R-D dans le secteur de l'État devrait représenter une difficulté a priori moindre que pour d'autres secteurs où l'énumération exhaustive n'est pas la norme. Cependant, étant donné la possibilité de non-réponse et les limites concernant le type d'informations pouvant être demandées aux institutions gouvernementales, il sera peut-être nécessaire d'utiliser un certain nombre de stratégies. 
8.72 Dans certains cas, les systèmes d'information des administrations visant à améliorer la coordination et à garantir une transparence accrue peuvent fournir une base appropriée pour collecter des informations sur les travaux de recherche financés ou exécutés par l'administration centrale. Ces systèmes peuvent intégrer tous les projets de recherche et développement financés ou exécutés par une part considérable de l'administration et permettre la production de statistiques sur l'exécution de la R-D par les administrations publiques. Dans d'autres cas, l'utilisation des informations budgétaires peut être nécessaire pour combler des lacunes statistiques et assurer en qualité la cohérence entre les enquêtes et les totaux.

8.73 L'utilisation de coefficients pour évaluer la structure des dépenses et du personnel de R-D à l'intérieur des organismes est en général déconseillée, en raison de la grande hétérogénéité entre des unités exécutantes de R-D.

8.74 Autant que possible et quand la qualité des données collectées le permet, il est recommandé de publier des données désagrégées sélectionnées au niveau des institutions gouvernementales désignées individuellement, car cela peut répondre à plusieurs besoins d'autres utilisateurs.

\subsection{Mesure du financement public de l'exécution de la R-D}

8.75 Comme noté au chapitre 4, deux approches principales existent pour mesurer le coût des ressources que les États consacrent au financement de la R-D. La première consiste à se placer du point de vue des exécutants, en comptabilisant les sommes qu'un secteur ou une unité statistique a reçu des administrations publiques pour exécuter de la R-D intra-muros durant une période de référence spécifique. La seconde consiste à se placer du point de vue des bailleurs de fonds et à comptabiliser les sommes que les administrations publiques déclarent avoir versées ou s'être engagées à allouer à d'autres secteurs ou unités statistiques pour l'exécution de R-D durant une période de référence spécifique. L'approche fondée sur le point de vue des bailleurs de fonds repose sur les déclarations faites par les administrations publiques de financement, y compris pour la R-D exécutée au sein de l'administration, ainsi que les fonds pour la R-D devant être exécutée en dehors de l'administration.

\section{Approche du point de vue des exécutants (recommandée)}

8.76 L'approche recommandée pour compiler les chiffres du financement public de la R-D est l'approche du point de vue des exécutants, qui s'appuie sur la combinaison des niveaux de financement déclarés par les unités dans tous les secteurs, y compris celui de l'État. Pour un pays donné, le total agrégé représente l'exécution de la $R-D$ intérieure totale financée par le secteur de l'État. Cet indicateur, les DIRD financées par le secteur de l'État ne doit pas être confondu avec les DIRDET, qui représentent l'exécution de R-D intra-muros total du secteur de l'État. Le chevauchement entre ces deux totaux est la part de la R-D 
exécutée au sein du secteur de l'État qui est financée de façon interne par l'État avec ses propres ressources.

8.77 La mesure du financement par le secteur de l'État des DIRD est tributaire d'une mesure rigoureuse des sources de financement dans tous les secteurs. La plupart des grandes difficultés ont été examinées de façon détaillée au chapitre 4 et dans les chapitres consacrés aux secteurs concernés.

8.78 Il est vivement recommandé que les enquêtes sur les exécutants de $\mathrm{R}-\mathrm{D}$ dans tous les secteurs non gouvernementaux fournissent une ventilation des fonds reçus de l'État selon qu'il s'agit de fonds accordés en échange de services de R-D ou de fonds obtenus sous la forme d'un transfert. Cette information est particulièrement importante pour les décideurs et pour parvenir à une meilleure compréhension des moyens d'action utilisés pour soutenir la R-D. Cette information est également requise pour la production des chiffres de l'investissement dans la comptabilité nationale.

8.79 La mise en œuvre de la méthode de déclaration du point de vue des exécutants peut toutefois présenter des difficultés pour certaines formes de soutien financier de l'État à la R-D, comme par exemple :

- L'utilisation de formes spéciales d'allégements fiscaux ayant comme objectif d'encourager le financement ou l'exécution de R-D a été abordée au chapitre 4 et fait l'objet d'indications spécifiques au chapitre 13. À quelques exceptions près, la majorité des formules d'aide fiscale à la R-D s'alignent difficilement, dans la forme comme dans la pratique, sur la conception de l'exécution de la R-D selon le présent manuel. Pour cette raison, cette forme de soutien est prise en compte principalement du point de vue de la source et, dans les comparaisons internationales, elle est généralement exclue des analyses des statistiques sur les DIRD financées par l'État.

- Les prêts à la R-D consentis par l'État et autres investissements financiers visant à fournir des ressources financières à la R-D dans d'autres secteurs devraient être traités comme financements des exécutants internes (chapitre 4). Les investissements financiers représentent un échange d'actifs financiers (par ex. des subsides soumis à un remboursement futur à un taux d'intérêt convenu, ou un droit sur les bénéfices d'une institution). Il est possible qu'un investissement de ce type ne soit pas remboursé ou que l'intérêt convenu comporte une subvention, et il serait irréaliste de demander aux exécutants d'estimer et de révéler sa valeur implicite.

- Utilisation gratuite ou subventionnée d'installations publiques pour de la R-D. Pour des raisons pratiques, il est impossible d'obtenir des estimations fiables de la part des exécutants de R-D concernant la valeur économique des services obtenus ou de la subvention implicite équivalente. Dans certains cas et comme relevé précédemment, les frais de service qui ne sont pas facturés aux utilisateurs peuvent être alloués à l'exécution de R-D du prestataire de service pour une meilleure représentation agrégée de l'intégralité de l'activité de R-D. 


\section{Approche du point de vue des bailleurs de fonds (complémentaire)}

8.80 Si le présent manuel insiste sur l'importance de garantir une approche commune et cohérente concernant la déclaration de la R-D de la part des exécutants (par le biais d'enquêtes et d'autres méthodes annexes possibles), il admet également un certain nombre de pratiques complémentaires destinées à améliorer la qualité, l'opportunité et la pertinence des statistiques de R-D. La référence à ces pratiques s'appuie sur l'expérience acquise dans plusieurs pays qui ont déjà développé des statistiques fondées sur les sources concernant la R-D financée par les pouvoirs publics, ventilée selon qu'elle est exécutée à l'intérieur ou à l'extérieur de l'administration.

8.81 Souvent, les données sur le financement de la R-D extra-muros par les administrations publiques peuvent être utilisées pour combler les lacunes dans les déclarations des exécutants, et améliorer ainsi la qualité des statistiques d'exécution de la R-D. Cela peut s'appliquer notamment dans le cas de financements accordés pour que des particuliers, par exemple des étudiants ou des chercheurs, prennent part à des activités de R-D dans d'autres unités, sans que ces dernières aient un contrôle direct sur le financement (voir section 4.4). Ce système peut avoir comme objectif de permettre à des particuliers de passer librement d'un organisme à un autre. Les données des sources de financement public peuvent ainsi permettre une représentation plus complète de l'ensemble de l'exécution de la R-D. Il est toutefois nécessaire que les organismes hôtes conservent une trace formelle de la présence et de la contribution de ces particuliers car sinon, il pourrait être impossible de démontrer leur conformité avec les critères pour la R-D exposés au chapitre 2 .

8.82 Un autre exemple de l'application des statistiques liées aux bailleurs de fonds est l'utilisation de mesures des crédits budgétaires pour aider à estimer le financement général destiné aux universités, à l'aide de coefficients sur l'utilisation de ces ressources générales (voir chapitre 9).

\section{Crédits budgétaires publics de R-D (recommandé)}

8.83 Le présent manuel fournit des indications concernant la collecte de données sur les crédits budgétaires publics de R-D (CBPRD) au chapitre 12. La principale raison d'être de cette approche s'appuyant sur les crédits budgétaires est la plus grande actualité des données (y compris les prévisions budgétaires) et la capacité de fournir une approximation de premier ordre de la répartition des niveaux de financement public de R-D par objectif socio-économique.

\section{Enquêtes statistiques sur le financement public de la R-D (facultatif)}

8.84 Comme indiqué plus haut, il est conseillé d'inclure dans les enquêtes R-D du secteur de l'État des questions sur le financement de la R-D extra-muros, par les unités de ce secteur. En général, ces enquêtes ne peuvent pas être utilisées pour construire des agrégats de financement public, à moins que leur champ ne 
soit étendu afin de couvrir non seulement les unités exécutantes de R-D dans le secteur de l'État, mais également les autres unités qui ont seulement un rôle de financement de la R-D.

8.85 La pertinence potentielle de ces données provient en outre des éléments d'informations supplémentaires qui peuvent être collectés par le biais de questions spécifiquement ciblées sur le financement de la R-D exécutée intramuros et extra-muros, que les informations budgétaires standard ne peuvent pas fournir de façon suffisamment détaillée. Un certain nombre d'exemples peuvent être donnés, indiquant plusieurs domaines de développement au sein des pays pour lesquels il n'est pas possible, à l'heure actuelle, de fournir des indications communes.

\section{Informations plus détaillées sur les différentes sources de financement public de la R-D}

8.86 Les enquêtes menées du point de vue des bailleurs de fonds peuvent avoir pour avantage de permettre une comptabilisation plus détaillée des institutions gouvernementales qui fournissent des fonds pour la R-D exécutée par les unités dans chacun des secteurs économiques. Bien que les enquêtes sur les exécutants de R-D dans les secteurs des entreprises, de l'enseignement supérieur, et du secteur privé sans but lucratif requièrent des données sur le financement public de la R-D, il peut être particulièrement fastidieux de demander aux répondants d'énumérer les administrations publiques spécifiques à la source de ces financements. Les enquêtes sur les bailleurs de fonds publics de la R-D, identifiés individuellement, qui visent à obtenir les totaux de financement de R-D accordés à des exécutants de R-D extra-muros, par secteur, ne connaissent pas cette limitation.

\section{Financement par l'État de R-D exécutée à l'étranger}

8.87 Les indicateurs de financement de la R-D par l'État au bénéfice d'exécutants œuvrant à l'étranger et dans des organisations internationales (les deux appartenant à la catégorie " Reste du monde ") ne peuvent pas être obtenus à partir des enquêtes menées auprès des exécutants nationaux. De même, les informations sur les contributions publiques aux programmes et aux institutions développés en partenariat avec les administrations d'autres pays ou des organisations supranationales peuvent présenter un intérêt significatif pour l'action publique au point de rendre possible le suivi de la collaboration internationale en matière de R-D et de la mesure dans laquelle les accords bilatéraux ou multilatéraux sont réellement financés par des fonds publics.

\section{Informations sur les modes de financement du point de vue des bailleurs de fonds}

8.88 Les informations pourraient être collectées en distinguant les financements alloués sous forme de transfert (par exemple une subvention ou un accord de contribution) de ceux accordés en échange de services de R-D (comme c'est le cas pour de nombreuses formes de marchés publics de R-D) 
(voir chapitre 4). Pour un certain nombre de raisons, la perspective fournie par les bailleurs de fonds peut différer sensiblement de celle des exécutants, ces derniers pouvant parfois déclarer un financement externe comme interne et ainsi sous-évaluer le volume réel des financements publics.

8.89 Les informations pourraient être collectées au sujet de plusieurs autres aspects des modes de financement utiles pour l'action publique, comme le fait de savoir si un financement est alloué par voie d'appel à la concurrence (plutôt que selon d'autres critères) ou s'il est accordé en fonction d'un programme ou d'un projet plutôt que sous la forme d'une subvention institutionnelle. Dans le cas d'un mode de financement institutionnel, les organisations bénéficiant d'un financement ont toute liberté d'action concernant le type d'activités et de projets de R-D qu'elles peuvent entreprendre, tandis que pour des fonds accordés sur la base d'un projet ou d'un programme, leur marge de décision est plus limitée. Les fonds généraux des universités d'origine publique (FGU) représentent un cas particulier de financement institutionnel de la R-D. Ils sont destinés aux établissements d'enseignement supérieur, auxquels le présent manuel attribue un statut spécial (voir chapitres 4, 9 et 12). Il est important de noter que lorsque les bénéficiaires de dotations globales sont libres de décider d'utiliser les fonds pour la R-D ou à d'autres fins, il est peu probable que ce que les bailleurs de fonds déclarent comme financement alloué selon des critères de R-D - par exemple, en fonction des publications scientifiques publiées auparavant - coïncide rigoureusement avec ce que les exécutants déclarent comme étant utilisé à des fins de R-D.

\section{Difficultés qui se posent dans les enquêtes statistiques sur le financement public de la R-D}

8.90 La collecte exhaustive de données sur le financement public de la R-D présente un certain nombre de difficultés pratiques à prendre en compte :

- La disponibilité de données supplémentaires exige un surcroît d'efforts pour " harmoniser » les différences entre les données fondées sur les budgets et les sources de financement public déclarées par tous les secteurs exécutants nationaux. Si les informations sont recueillies en fonction de l'affiliation sectorielle des exécutants probables, cela peut donner lieu à une matrice exécution-bailleurs de fonds différente de celle tirée des enquêtes s'appuyant sur les exécutants. En l'absence de définitions et d'explications adéquates, cela donne lieu à un risque de confusion non négligeable chez les utilisateurs de données.

- La méthode nécessite également d'étendre la couverture des enquêtes R-D du secteur de l'État aux unités gouvernementales non exécutantes de R-D, ce qui peut porter à conséquence en termes de charge de travail et de ressources. La charge de travail imposée aux organismes publics dépendra du fait de savoir dans quelle mesure les informations sont déjà disponibles, au moins de 
manière interne à d'autres fins administratives, et si celles-ci cadrent avec les notions statistiques recherchées.

- Afin de suivre la démarche fondée sur les bailleurs de fonds, il est nécessaire de prendre en compte la double comptabilisation potentielle des financements de la R-D provenant d'organisations « intermédiaires » qui reçoivent des fonds de ministères et d'organismes, puis réaffectent et transmettent ces fonds à d'autres institutions exécutantes. Cela suppose également l'élaboration de critères clairs concernant la répartition de ces fonds entre les diverses catégories fonctionnelles. Par exemple, les fonds alloués par un ministère à une importante agence de financement peuvent coïncider avec l'objectif " avancement général de la connaissance ", tandis que le financement propre de l'organisme pour la R-D au niveau des projets ou des programmes peut être enregistré de façon plus granuleuse.

8.91 Le tableau 8.3 donne une représentation schématique des diverses situations dans lesquelles un organisme du secteur de l'État à la fois exécutant et bailleur de fonds d'activités de R-D peut se trouver lorsqu'il est face à un questionnaire sur ses activités d'exécution de R-D et de financement de R-D avec des tiers. Ce tableau montre que pour compiler des estimations agrégées de financement de la R-D par le secteur de l'État, il serait nécessaire de mettre l'accent soit sur les fonds fournis en dernier ressort aux exécutants de R-D, soit sur la première allocation - chronologiquement parlant - de fonds. En général, les répondants peuvent se voir demander d'inclure les sommes transférées à d'autres organismes en soutien à la recherche et au développement, mais dans ce cas, les organismes auxquels ces fonds ont été transférés ne devraient pas les déclarer. De même, une subdivision d'un organisme qui transfère des fonds à une autre subdivision au sein de ce même organisme pourrait déclarer ces fonds comme des dépenses propres. Pour éviter toute distorsion excessive concernant les fonds destinés à l'exécution de R-D intra-muros, l'organisme transférant les fonds devrait s'efforcer, dans la mesure du possible, de déterminer si l'exécutant de dernier ressort est intra-muros ou extra-muros, et le déclarer en conséquence. Le transfert de fonds à un autre organisme d'État ne devrait pas être la seule base pour déclarer que l'exécution de R-D est intra-muros.

8.92 Des divergences peuvent survenir entre les données fondées sur les budgets et les données sur les dépenses pour l'exécution de la $R-D$, selon que l'on demande aux administrations publiques de déclarer leurs dépenses d'après leur comptabilité de caisse ou d'exercice. Les paiements en espèces ou assimilés peuvent être effectués au cours d'une autre période que l'année où l'utilisation des fonds par l'organisme a été approuvée, laquelle peut à son tour être différente de celle où l'engagement est pris de payer un exécutant donné, et également de celle où l'exécution de la R-D est considérée comme ayant été menée à bien. 


\section{Tableau 8.3. Vue d'ensemble des transferts de fonds pour un organisme d'État, exécutant et bailleur de fonds de R-D}

\begin{tabular}{|c|c|c|c|}
\hline $\begin{array}{l}\text { Fonds à disposition de } \\
\text { l'organisme }\end{array}$ & $\begin{array}{l}\text { Utilisation des fonds par } \\
\text { l'organisme }\end{array}$ & Utilisation finale possible des fonds & $\begin{array}{l}\text { Exécution de R-D } \\
\text { intra/extra/muros }\end{array}$ \\
\hline \multirow{5}{*}{$\begin{array}{l}\text { Sources internes } \\
\text { ou autres sources } \\
\text { publiques, y } \\
\text { compris le budget, } \\
\text { et les fonds non } \\
\text { utilisés les années } \\
\text { précédentes }\end{array}$} & \multirow[t]{2}{*}{ Fonds non utilisés } & $\underline{\mathrm{R}-\mathrm{D} \text { intra-muros au sein de l'organisme d'État }}$ & Intra-muros \\
\hline & & Décision de dépense différée & Sans objet \\
\hline & \multirow[t]{3}{*}{ Fonds transférés } & $\begin{array}{l}\text { L'organisme délègue la décision d'allocation } \\
\text { des fonds de R-D à un autre organisme }\end{array}$ & $\begin{array}{l}\text { Risque de double } \\
\text { comptabilisation }\end{array}$ \\
\hline & & \multirow{2}{*}{$\begin{array}{l}\text { Fonds alloués aux exécutants par le biais } \\
\text { de subventions, d'achats publics de R-D, } \\
\text { de sous-traitance de la R-D, etc. }\end{array}$} & Extra-muros \\
\hline & & & $\begin{array}{l}\text { Risque de double } \\
\text { comptabilisation }\end{array}$ \\
\hline \multirow{5}{*}{$\begin{array}{l}\text { Autres sources } \\
\text { externes }\end{array}$} & \multirow[t]{2}{*}{ Fonds non utilisés } & 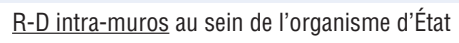 & Intra-muros \\
\hline & & Décision de dépense différée & Sans objet \\
\hline & \multirow[t]{3}{*}{ Fonds transférés } & $\begin{array}{l}\text { L'organisme délègue la décision d'allocation } \\
\text { des fonds de R-D à un autre organisme }\end{array}$ & $\begin{array}{l}\text { Risque de double } \\
\text { comptabilisation }\end{array}$ \\
\hline & & \multirow{2}{*}{$\begin{array}{l}\text { Fonds alloués aux exécutants par le biais } \\
\text { de subventions, d'achats publics de R-D, } \\
\text { de sous-traitance de la R-D, etc. }\end{array}$} & Extra-muros \\
\hline & & & $\begin{array}{l}\text { Risque de double } \\
\text { comptabilisation }\end{array}$ \\
\hline
\end{tabular}

8.93 Un certain nombre de pays recensent déjà de façon systématique le financement et l'exécution de la R-D au sein du secteur de l'État tout entier. Les pays qui souhaitent expérimenter cette méthode sont encouragés à le faire. Cependant, des travaux plus poussés sont nécessaires afin de converger vers une norme permettant de mener des enquêtes exhaustives sur le financement public de la R-D.

\section{Références}

CE (2013), She Figures 2012: Statistics and Indicators - Gender in Research and Innovation, Commission européenne, Bruxelles, http://ec.europa.eu/research/sciencesociety/document_library/pdf_06/she-figures-2012_en.pdf.

CE, FMI, OCDE, Nations Unies et Banque mondiale (2009), Système de comptabilité nationale 2008, Nations unies, New York, http://unstats.un.org/unsd/nationalaccount/ docs/SNA2008FR.pdf.

Eurostat (2008), Nomenclature pour l'analyse et la comparaison des budgets et programmes scientifiques (NASB).

FMI (2014), Government Finance Statistics Manual, FMI, Washington, D.C., www.imf.org/ external/np/sta/gfsm/.

Nations Unies (2008), " Classification internationale type, par industrie, de toutes les branches d'activité économique (CITI), Révision 4 ", Département des affaires économiques et sociales, Division de statistique, Études statistiques, Série $M, n^{\circ} 4$, Rév. 4, Nations Unies, New York, http://unstats.un.org/unsd/class/default.asp?Lg=2.

Nations Unies (2000), Nomenclature des dépenses par fonction: Classification des fonctions des administrations publiques, Nations Unies, New York, http://unstats.un.org/unsd/ EconStatKB/Attachment221.aspx.

UNESCO-ISU (2014), « Guide pour la conduite d'une enquête de R-D : À destination des pays commençant à mesurer la recherche et le développement expérimental ", Document technique, $\mathrm{n}^{\circ}$ 11, ISU, Montréal, http://www.uis.unesco.org/Library/Documents/ tp11-guide-research-experimental-development-r-d-survey-countries-starting-measuring2014-fre.pdf. 


\section{Chapitre 9}

\section{La R-D du secteur de l'enseignement supérieur}

De par l'importance stratégique de l'enseignement supérieur, la définition de ce secteur est spécifique au Manuel et n'a pas d'équivalent dans le Système de comptabilité nationale (SCN). Cette définition, qui fait l'objet du présent chapitre, repose sur celles déjà existantes des programmes d'enseignement supérieur et de l'enseignement formel. Afin de couvrir l'ensemble des activités de recherche et de développement expérimental (R-D) menées dans ce secteur, elle englobe tous les instituts et centres de recherche, stations expérimentales et centres de soins dont les activités de $R-D$ relèvent du contrôle direct d'établissements d'enseignement supérieur ou sont administrées par ceux-ci. Le secteur de l'enseignement supérieur revêtant différentes formes selon les pays, la première démarche consiste à recenser les unités qui le composent puis à recueillir et communiquer des statistiques de R-D concernant celles-ci, de manière à permettre l'établissement de comparaisons internationales. Ce chapitre fournit des orientations sur la marche à suiure pour y parvenir ainsi que pour mesurer les dépenses de R-D du secteur, ses flux de R-D internes et externes et les ressources humaines qu'il consacre à la $R-D$. 


\subsection{Introduction}

9.1 La définition du secteur de l'enseignement supérieur est spécifique à ce manuel et n'a pas d'équivalent dans le Système de comptabilité nationale (SCN) (CE et al., 2009). Les établissements qu'il regroupe peuvent aussi être classés dans n'importe quel secteur du SCN, en fonction de leurs caractéristiques. Si ce secteur fait l'objet d'une définition particulière, c'est en raison de l'importance stratégique que revêtent les informations relatives aux établissements prenant part à la R-D.

9.2 Les statistiques de l'enseignement sont bien établies et conformes à la Classification internationale type de l'éducation (CITE) ainsi qu'au manuel de l'UNESCO, de l'OCDE et d'Eurostat sur les concepts, définitions et classifications pour la collecte des données sur l'enseignement formel (UOE, 2014). Le manuel de l'UOE utilise la même définition de la R-D que le présent manuel.

9.3 Dans les statistiques de l'enseignement, les programmes sont classés conformément à la CITE, et l'enseignement supérieur correspond aux niveaux 5, 6,7 et 8 de cette classification. Dans le Manuel, les établissements sont classés dans le secteur de l'enseignement supérieur lorsqu'ils correspondent à la définition adoptée pour ce secteur. Il s'agit donc là de deux processus assez différents, et il apparaîtra clairement qu'aux fins du Manuel, l'enseignement supérieur est défini de façon plus large que dans la CITE.

9.4 Comme le montre la définition du secteur de l'enseignement supérieur donnée à la section 9.2, les établissements qui sont rattachés à ce secteur comprennent non seulement ceux qui proposent des programmes d'enseignement supérieur formel, mais aussi les instituts et centres de recherche, les stations d'expérimentation et les centres de soins qui, sans nécessairement proposer des programmes d'enseignement, remplissent l'une des conditions énoncées au chapitre 3 et expliquées plus en détail dans la section qui suit.

9.5 Étant donné que le secteur de l'enseignement supérieur diffère selon les pays, il faut commencer par déterminer les établissements qui en relèvent, puis recueillir et communiquer les statistiques de R-D sous une forme qui permette les comparaisons internationales. Ce point est particulièrement important pour les statistiques sur l'exécution des travaux de R-D dans le secteur de l'enseignement supérieur, et le présent chapitre explique comment il faut procéder. 


\subsection{Champ couvert par le secteur de l'enseignement supérieur}

9.6 Ce secteur comprend :

- l'ensemble des universités, établissements d'enseignement post-secondaire et autres établissements proposant des programmes d'enseignement supérieur formel, indépendamment de leur source de financement ou de leur statut juridique

- l'ensemble des instituts et centres de recherche, stations d'expérimentation et centres de soins dont les activités de R-D relèvent du contrôle direct d'établissements d'enseignement supérieur ou sont administrées par ceux-ci.

9.7 Pour être plus précis, le secteur comprend toutes les unités institutionnelles (établissements) qui ont pour activité principale d'exécuter des programmes d'enseignement supérieur formel correspondant aux niveaux 5, 6, 7 ou 8 de la CITE, quel que soit leur statut juridique (UNESCO-ISU, 2012, p. 86). L'enseignement formel est défini dans la CITE (UNESCO-ISU 2012, para. 36 à 42), et il figure dans la définition du secteur de l'enseignement supérieur, où il sert à inclure les programmes d'enseignement reconnus par les autorités nationales compétentes de l'éducation ou leur équivalent, et à exclure les programmes qui ne le sont pas. Le Manuel utilise le terme "services d'enseignement " plutôt que "programmes d'enseignement ", mais les deux termes sont considérés comme équivalents. Comme il est indiqué dans la définition, le champ de ce secteur est plus étendu dans le Manuel, afin de tenir compte des travaux de R-D exécutés par des prestataires de programmes d'enseignement supérieur d'autres établissements non commerciaux, tels que certains types d'instituts de recherche et de centres de soins où toutes les activités de R-D sont menées sous le contrôle direct d'établissements d'enseignement supérieur et peuvent donc être considérées, pour des raisons pratiques, comme faisant partie de leur R-D intra-muros.

9.8 La définition ci-dessus délimite le champ de ce secteur (voir aussi chapitre 3, section 3.5). L'arbre de décision représenté au graphique 3.1 du chapitre 3 indique les secteurs utilisés dans le Manuel dans lesquels les établissements du secteur de l'enseignement supérieur seraient classés si ce secteur n'existait pas. Étant donné que le secteur des entreprises, le secteur de l'État et le secteur privé sans but lucratif s'apparentent aux secteurs du SCN, le graphique 3.1 donne également des informations sur la façon dont les établissements du secteur de l'enseignement supérieur seraient regroupés dans les secteurs correspondants du SCN.

9.9 Il est recommandé de distinguer clairement les établissements du secteur de l'enseignement supérieur selon qu'ils sont publics ou privés. Dans ce dernier cas, il est important également, pour permettre le lien avec le SCN, que l'on puisse déterminer si, dans ce cadre, l'établissement d'enseignement supérieur doit être rattaché au secteur des sociétés, au secteur des administrations 
publiques ou au secteur des institutions sans but lucratif au service des ménages (ISBLSM). Ce point est examiné plus en détail à la section 9.2 ci-après, consacrée aux établissements publics et privés et aux comparaisons internationales.

9.10 Comme indiqué au chapitre 3 (section 3.4) et au chapitre 8, un établissement est classé comme public ou privé selon qu'il fonctionne ou non sous le contrôle ultime d'une entité publique. Pour juger de ce contrôle ultime, tel qu'il est défini dans les chapitres cités, il faut apprécier quelle unité institutionnelle a le pouvoir de déterminer les politiques et les activités générales de l'établissement considéré et de nommer les responsables chargés de sa gestion. Sachant que de nombreux établissements opèrent sous le contrôle opérationnel d'un organe directeur, la constitution de celui-ci aura également une incidence sur la classification.

9.11 Dans tous les pays, le noyau central du secteur de l'enseignement supérieur est constitué des universités et des établissements d'enseignement post-secondaire. Là où le traitement des unités institutionnelles varie, c'est lorsqu'on aborde la question d'autres établissements d'enseignement supérieur et surtout de plusieurs types d'unités liées aux universités et aux établissements d'enseignement post-secondaire. Trois catégories sont examinées ci-dessous :

- les établissements d'enseignement supérieur

- les hôpitaux et les centres de soins universitaires

- les établissements de recherche situés à la frontière de l'enseignement supérieur.

\section{Établissements d'enseignement supérieur}

9.12 Le secteur comprend tous les établissements qui ont pour activité principale de dispenser un enseignement supérieur formel, quel que soit leur statut juridique. Il peut s'agir de sociétés ou de quasi-sociétés - privées ou appartenant à une administration publique -, d'institutions sans but lucratif (ISBL) marchandes, ou d'ISBL contrôlées et principalement financées par l'État ou par des ISBLSM (institutions sans but lucratif au service des ménages). Comme il a été indiqué plus haut, le noyau central est essentiellement constitué des universités et des établissements d'enseignement post-secondaire. Tous les établissements d'enseignement supérieur n'ont pas d'activités de R-D, mais il arrive que certains établissements du deuxième cycle du secondaire ou de l'enseignement post-secondaire non supérieur (niveau 3 ou 4 de la CITE) en aient. Selon leur gouvernance et leur financement, ces derniers peuvent être inclus dans le secteur de l'enseignement supérieur, à condition que cette prise en compte soit clairement indiquée lors de la présentation des données. Dans certains pays, il existe des établissements d'enseignement supérieur à vocation professionnelle, dont le but est d'enseigner, et qui n'exécutent pas de travaux de R-D. Ces établissements peuvent être exclus des enquêtes sur le secteur. 


\section{Hôpitaux et centres de soins universitaires}

9.13 Bien qu'il n'existe pas de définition formelle, la notion d'" hôpital universitaire " s'applique normalement aux hôpitaux qui sont affiliés à une université, même si de nombreux autres types de liens et d'arrangements sont souvent qualifiés ainsi. La classification de ces établissements peut soulever un certain nombre de difficultés conceptuelles et pratiques, parce qu'ils combinent des activités liées à la santé, à l'éducation et à la recherche et que leur gouvernance prend des formes différentes.

9.14 La prise en compte de la plupart des types d'hôpitaux et de centres de soins universitaires dans le secteur de l'enseignement supérieur se justifie parce qu'il s'agit à la fois d'établissements d'enseignement supérieur (hôpitaux de formation) à part entière et d'unités de recherche " associées " à des établissements d'enseignement supérieur (soins médicaux de pointe dispensés dans des centres de soins universitaires, par exemple).

9.15 Le financement de la R-D effectuée dans ce type d'établissements peut provenir de nombreuses sources : de la " dotation forfaitaire " globale des universités, c'est-à-dire des fonds généraux des universités (FGU) ; des " fonds propres " des hôpitaux (recettes tirées des traitements administrés aux patients ou dotations forfaitaires globales destinées aux prestations de soins de santé, par exemple) ; de fonds publics directs pour la R-D (de la part d'un conseil de la recherche médicale, par exemple) ; ainsi que de fonds privés (dons ou aides d'entreprises pour des essais cliniques, entre autres).

9.16 Lorsque l'ensemble ou la quasi-totalité des activités d'un établissement hospitalier ou médical comporte une composante d'enseignement ou de formation, l'établissement doit être intégralement compris dans le secteur de l'enseignement supérieur. En revanche, si les activités d'enseignement ou de formation ne concernent que quelques-uns des centres de soins ou des services d'un établissement hospitalier ou médical, ce sont ces centres ou services d'enseignement ou de formation, et eux seuls, qui doivent être classés dans le secteur de l'enseignement supérieur. Les autres centres de soins ou services, qui ne dispensent aucun enseignement ni aucune formation, doivent normalement être inclus dans le secteur approprié (secteur des entreprises, secteur de l'État ou secteur privé sans but lucratif). La classification institutionnelle correspondante du SCN devra aussi être notée, autant que possible, pour faire le lien avec ce système. Il faut veiller à éviter le double comptage des activités de R-D entre les divers secteurs concernés.

9.17 Il peut être difficile d'établir une distinction entre les universités et les hôpitaux et centres de soins universitaires. Néanmoins, il est recommandé de séparer les deux groupes d'établissements dans la déclaration des dépenses et du personnel de R-D. Dans la logique de la recommandation, formulée au chapitre 3, qui invite à étiqueter les unités institutionnelles par activité économique (Nations Unies, 2008), l'étiquetage des établissements de santé à 
l'intérieur du système de l'enseignement supérieur peut faciliter la production de statistiques pour les hôpitaux et les centres de soins universitaires.

\section{Établissements de recherche situés à la frontière de l'enseignement supérieur}

9.18 Certains établissements se situent à la frontière entre le secteur de l'enseignement supérieur et d'autres secteurs institutionnels, d'où des problèmes de classification particuliers, qui peuvent être résolus de différentes façons (voir l'arbre de décision présenté au chapitre 3). Généralement, le fait que ces établissements dispensent un enseignement supérieur est considéré comme un critère décisif en faveur de leur rattachement au secteur de l'enseignement supérieur. On peut également s'appuyer sur leur financement, leur administration, leur contrôle et leur situation géographique ainsi que sur leur intégration éventuelle dans des budgets universitaires pour décider de leur classement. Dans les pays qui tiennent des registres institutionnels détaillés, l'utilisation de la classe CITI (Nations Unies, 2008) peut aussi s'avérer pratique.

9.19 On trouvera ci-après quelques exemples courants d'établissements situés à la frontière de l'enseignement supérieur.

\section{Institutions intervenant dans le financement de l'enseignement supérieur}

9.20 Certaines institutions jouant un rôle important dans le financement de l'enseignement supérieur, comme les conseils de l'enseignement supérieur ou des entités similaires, peuvent être incluses dans ce secteur si elles assurent aussi des services d'enseignement supérieur formel, ou si elles sont contrôlées ou administrées par des universités ou qu'elles leur fournissent des services.

\section{Instituts de recherche " chargés de missions ou spécialisés 》}

9.21 Les universités sont de grands centres de recherche. Aussi les pays ont-ils souvent jugé opportun d'y implanter des instituts et des unités de recherche lorsqu'ils voulaient accroître leurs activités de R-D dans certains domaines. La plupart de ces unités sont financées, pour l'essentiel, par l'État et peuvent même être chargées de missions précises, cependant que d'autres sont financées par le secteur privé sans but lucratif ou par le secteur des entreprises. On peut citer comme exemples les unités créées pour répondre à des priorités nationales dans le domaine de l'environnement, des sciences du vivant, de la médecine ou des sciences exactes et sciences de l'ingénieur ; ces unités sont souvent d'une durée limitée dans le temps. Lorsqu'elles sont constituées pour être gérées par des universités ou des départements universitaires, on peut considérer qu'elles appartiennent au secteur de l'enseignement supérieur. Quelle que soit l'option choisie, il est important de rendre compte des activités des institutions incluses dans le secteur. 


\section{Établissements liés à des universités}

9.22 Un établissement d'enseignement supérieur peut avoir des « liens » avec d'autres instituts de recherche qui ne s'intéressent pas directement à l'enseignement ou qui ont des fonctions autres que la R-D, telles que le conseil. Ces liens peuvent prendre la forme, par exemple, de détachements de personnel des établissements d'enseignement vers les instituts de recherche considérés ou vice-versa, ou d'une utilisation partagée d'équipements et d'installations par des instituts classés dans des secteurs différents. Ces instituts peuvent être classés selon d'autres critères portant, par exemple, sur le contrôle, le financement ou les services rendus.

9.23 Dans certains pays, les établissements qui se situent à la frontière de l'enseignement supérieur peuvent avoir un statut juridique privé et effectuer de la recherche contractuelle pour d'autres secteurs ; il peut aussi s'agir d'établissements de recherche financés par l'État. Il est alors difficile de décider si les liens entre les unités sont suffisamment étroits pour justifier la prise en compte de l'unité « extérieure » dans le secteur de l'enseignement supérieur.

\section{Instituts employant des chercheurs rattachés à des universités}

9.24 Certains instituts habituellement financés et contrôlés par des administrations publiques, tels que les académies des sciences ou les conseils nationaux de la recherche, emploient aussi des chercheurs rattachés à des universités. En règle générale, ces instituts sont inclus dans le secteur de l'État, surtout lorsqu'ils sont indépendants de l'université et qu'ils ne sont pas intégrés dans des budgets universitaires. Il peut arriver, toutefois, qu'ils soient considérés comme faisant partie du secteur de l'enseignement supérieur s'ils participent avec leurs chercheurs à des activités d'enseignement.

\section{Autres cas}

9.25 «Les parcs de recherche et les parcs scientifiques ou technologiques " situés dans l'enceinte ou à proximité des universités et des établissements d'enseignement post-secondaire accueillent diverses entités qui produisent des biens et services et exécutent des travaux de R-D. Pour ce type de groupements, il est recommandé de ne pas se servir des critères de la localisation géographique et de l'utilisation de ressources communes pour les classer dans le secteur de l'enseignement supérieur. Les unités contrôlées et accueillies dans ces parcs et financées principalement par l'État doivent être classées dans le secteur de l'État, celles qui sont contrôlées et principalement financées par le secteur privé sans but lucratif doivent être classées dans ce secteur, tandis que les entreprises et autres unités au service des entreprises doivent être classées dans le secteur des entreprises.

9.26 Les unités contrôlées ou administrées par des unités d'enseignement supérieur (notamment les hôpitaux de formation), telles qu'elles sont définies ci-dessus, et qui ne sont pas principalement des producteurs marchands seront, 
quant à elles, incluses dans le secteur de l'enseignement supérieur. Si ces unités sont essentiellement des producteurs marchands, elles doivent être incluses dans le secteur des entreprises, quels que soient leurs liens avec des unités relevant du secteur de l'enseignement supérieur (chapitre 3, graphique 3.1).

9.27 Conformément aux indications fournies au chapitre 3, les unités institutionnelles qui font partie des producteurs non marchands et qui sont affiliées à des établissements d'enseignement supérieur ou dont l'ensemble des activités de R-D se fait sous le contrôle de ces derniers doivent être considérées comme appartenant au secteur de l'enseignement supérieur, alors que les unités issues de la recherche universitaire et qui font appel à du personnel universitaire, mais qui sont des producteurs marchands, doivent être classées dans le secteur des entreprises.

\section{Établissements publics et privés et comparaisons internationales}

9.28 Comme il est recommandé au chapitre 3, le classement des unités institutionnelles en unités publiques et privées donne des informations utiles pour l'action publique et facilite la comparaison avec les secteurs et soussecteurs du SCN. Il est donc recommandé d'opérer cette distinction dans le cas des établissements d'enseignement supérieur.

9.29 Outre cette différenciation, il est utile, dans la perspective de comparaisons internationales, de connaître la décomposition en universités proprement dites, hôpitaux universitaires et autres établissements d'enseignement supérieur.

9.30 Toutes les unités statistiques de ce secteur doivent par conséquent être classées, en fonction du profil qui leur correspond le mieux, comme il est indiqué au tableau 9.1. Les éventuels problèmes d'affectation doivent être signalés ainsi que leurs répercussions.

Tableau 9.1. Profils d'établissements d'enseignement supérieur

\begin{tabular}{l}
\hline Type d'établissement \\
\hline A. Établissements d'enseignement supérieur
\end{tabular}

9.31 Il est donc recommandé de faire état des dépenses et du personnel de R-D du secteur de l'enseignement supérieur par type d'établissements, conformément aux catégories figurant dans le tableau ci-dessus. 


\subsection{Identification de la R-D dans le secteur de l'enseignement supérieur}

9.32 Pour les besoins des enquêtes, la R-D doit être dissociée d'un large éventail d'activités connexes dotées d'un fondement scientifique et technologique. Bien que parfois étroitement liées à la $R-D$, tant par les flux d'informations et de financement qu'au niveau des opérations, des institutions et du personnel qui y prennent part, ces activités ne devraient pas, dans la mesure du possible, entrer en ligne de compte dans la mesure de la R-D. Certaines activités propres au secteur de l'enseignement supérieur sont difficiles à situer par rapport à la notion de R-D. Elles concernent en particulier l'enseignement, la formation et les soins de santé spécialisés (par exemple, hôpitaux universitaires).

\section{Limites entre R-D, enseignement et formation}

9.33 Dans les établissements d'enseignement supérieur, la recherche et l'enseignement sont toujours très étroitement liés, dans la mesure où ces deux activités sont la plupart du temps exercées ensemble et mobilisent une grande partie des bâtiments et des équipements.

9.34 Conformément aux orientations fournies au chapitre 2, la règle générale est d'exclure de la R-D toutes les activités d'enseignement et de formation assurées par le personnel des universités et des établissements spécialisés d'enseignement supérieur dans les domaines suivants : sciences naturelles, sciences de l'ingénieur, médecine, agriculture, sciences sociales, sciences humaines et arts. En revanche, les recherches effectuées par des étudiants de niveau doctorat dans les universités devraient être prises en compte, autant que possible, dans le personnel et les dépenses de R-D. Dans certains cas, les étudiants en master de recherche (niveau 7 de la CITE, section 9.4) ainsi que les dépenses de R-D qui leur sont associées peuvent aussi être comptées dans la catégorie appropriée (coûts de main-d'œuvre / autres dépenses courantes ; personnel de R-D interne ou externe) conformément aux indications fournies aux chapitres 4 et 5 .

9.35 Dans la mesure où les résultats de la recherche se répercutent sur l'enseignement, et que les informations et l'expérience acquises dans le cadre de l'enseignement viennent souvent étayer la recherche, il est difficile de déterminer avec précision où commencent et se terminent les activités d'enseignement ou de formation du personnel de l'enseignement supérieur et des étudiants et les activités de R-D. La R-D se distingue de l'enseignement courant et autres activités connexes du fait des cinq critères énoncés dans sa définition. Il est toutefois difficile de savoir s'il faut ou non inclure dans la R-D les activités scientifiques qui sont elles-mêmes les sous-produits d'une activité d'enseignement ou de formation.

9.36 Les cas suivants sont envisagés :

- les étudiants en doctorat (niveau 8 de la CITE), les étudiants en master (niveau 7 de la CITE), et leurs activités 
- la supervision des étudiants par le personnel universitaire

- l'approfondissement des connaissances personnelles des membres du personnel universitaire (lectures personnelles).

\section{Étudiants en doctorat (niveau 8 de la CITE) et étudiants en master (niveau 7 de la CITE)}

9.37 Pour les étudiants en doctorat, il est particulièrement difficile de distinguer les activités d'enseignement et de formation, des travaux de R-D. Les activités des étudiants ainsi que celles de leurs enseignants et directeurs de travaux doivent être examinées.

9.38 Certaines parties des cursus de niveau 8 de la CITE sont très structurées et comprennent, par exemple, des plans d'étude, des cours déterminés et des travaux obligatoires en laboratoire. Dans ce cas, le professeur transmet des connaissances et dispense une formation en méthodologie de la recherche. À ce titre, les activités types des étudiants consistent à assister aux cours obligatoires, à étudier les travaux publiés sur le sujet considéré et à acquérir les méthodes de recherche. Ces activités ne répondent pas au critère de nouveauté spécifié dans la définition de la R-D.

9.39 Par ailleurs, pour obtenir une qualification finale de niveau 8 de la CITE (niveau 7 pour les étudiants en master de recherche), les étudiants doivent également faire la preuve de leur compétence en réalisant une étude relativement indépendante dont ils doivent ensuite exposer les résultats. En général, ces études présentent les éléments de nouveauté requis pour se classer parmi les projets de R-D. Les activités ainsi menées par les étudiants devraient donc être classées dans la R-D, tout comme la supervision exercée par le professeur. Il est aussi possible, à la fois aux professeurs et aux étudiants, d'entreprendre d'autres projets de R-D, indépendamment des travaux de ce type exécutés dans le cadre de l'enseignement du $3^{\mathrm{e}}$ cycle.

9.40 Autre point, les étudiants de ce niveau dépendent souvent de l'établissement concerné ou sont directement employés par celui-ci et sont liés par un contrat ou un engagement similaire qui leur fait obligation de dispenser certains cours à des niveaux inférieurs ou d'exécuter d'autres activités, telles que la fourniture de soins de santé spécialisés, tout en leur permettant de poursuivre leurs études et d'effectuer des recherches.

9.41 On trouvera au tableau 9.2 des exemples illustrant la délimitation entre R-D et enseignement aux niveaux 8 et 7 de la CITE. Les problèmes plus pratiques d'application de ces concepts sont développés au chapitre 5 (personnel de R-D), en particulier à la section 5.2 sur le traitement des étudiants en doctorat ou en master.

\section{Supervision des étudiants par le personnel universitaire}

9.42 L'un des problèmes étroitement liés à la détermination de la part de $\mathrm{R}-\mathrm{D}$ dans le travail des étudiants de $3^{\mathrm{e}}$ cycle est d'extraire la composante R-D du temps consacré par les directeurs des travaux à superviser ces étudiants et leurs 
projets de recherche. Le problème se pose aussi sur une période plus courte pour les étudiants en master (niveau 7 de la CITE).

\section{Tableau 9.2. Classification des activités des enseignants et étudiants au niveau 8 (doctorat) et au niveau 7 (master) de la CITE}

\begin{tabular}{|c|c|c|c|}
\hline & $\begin{array}{l}\text { Enseignement et formation aux } \\
\text { niveaux } 7 \text { et } 8\end{array}$ & $R-D$ & Autres activités \\
\hline \multirow[t]{2}{*}{$\begin{array}{l}\text { Personnel } \\
\text { enseignant non } \\
\text { étudiant }\end{array}$} & $\begin{array}{l}\text { Enseignement aux étudiants de } \\
\text { niveaux } 7 \text { et } 8\end{array}$ & $\begin{array}{l}\text { Supervision des projets de R-D } \\
\text { nécessaires à la qualification des } \\
\text { étudiants de niveaux } 7 \text { et } 8\end{array}$ & $\begin{array}{l}\text { Enseignement aux } \\
\text { niveaux inférieurs } \\
\text { au niveau } 7\end{array}$ \\
\hline & $\begin{array}{l}\text { Formation des étudiants de niveaux } 7 \\
\text { et } 8 \text { à la méthodologie de la R-D, aux } \\
\text { travaux de laboratoire, etc. }\end{array}$ & $\begin{array}{l}\text { Supervision d'autres projets de } \\
\text { R-D et exécution de projets de R-D } \\
\text { personnels }\end{array}$ & Autres activités \\
\hline \multirow[t]{2}{*}{$\begin{array}{l}\text { Étudiants } \\
\text { en master } \\
\text { et doctorat } \\
\text { (niveau } 7 \text { et } 8 \\
\text { de la CITE) }\end{array}$} & $\begin{array}{l}\text { Réalisation de travaux dans le } \\
\text { cadre de cours pour obtenir une } \\
\text { qualification formelle }\end{array}$ & $\begin{array}{l}\text { Exécution et rédaction d'études } \\
\text { menées de manière indépendante } \\
\text { (projets de R-D), nécessaires pour } \\
\text { obtenir une qualification formelle }\end{array}$ & $\begin{array}{l}\text { Enseignement } \\
\text { aux étudiants de } \\
\text { niveaux inférieurs }\end{array}$ \\
\hline & & Toutes autres activités de R-D & Autres activités \\
\hline
\end{tabular}

9.43 Ces activités de supervision ne devraient être classées dans la R-D que si elles sont équivalentes à la direction et à la gestion d'un projet de R-D particulier contenant un élément de nouveauté suffisant et ayant pour objectif de produire des connaissances nouvelles. Si ces conditions sont remplies, aussi bien la supervision par les membres du personnel universitaire que le travail de l'étudiant devraient être considérés comme de la R-D. Si cette supervision consiste uniquement à enseigner les méthodes de R-D ou à lire et corriger les thèses, les mémoires ou les travaux d'étudiants non diplômés, elle doit être exclue de la R-D.

\section{Approfondissement des connaissances personnelles des membres du personnel universitaire (lectures personnelles)}

9.44 Cette activité correspond au temps consacré à la formation permanente (lectures personnelles), à la formation liée à la recherche (sur les équipements, par exemple) et à la fréquentation des conférences et séminaires.

9.45 En dissociant la R-D des activités connexes, on est souvent amené à se demander si les « lectures personnelles » devraient être considérées comme des activités de R-D. Ces lectures font indiscutablement partie du perfectionnement professionnel du personnel de recherche et, à terme, les connaissances et l'expérience ainsi acquises finissent par s'intégrer à la réflexion du chercheur sur la $\mathrm{R}-\mathrm{D}$, voire à la mise en œuvre de celle-ci. De fait, les « lectures personnelles » s'inscrivent dans une démarche cumulative et, lorsque l'information tirée de ces activités débouche sur des travaux de recherche, elle doit être mesurée en tant que R-D.

9.46 Cependant, seul l'approfondissement des connaissances personnelles ( lectures personnelles » comprises) entrepris spécifiquement en vue d'un projet de recherche est à considérer comme une activité de R-D. De façon générale, 
assister à des conférences ne peut pas être considérée comme de la R-D, mais présenter des travaux de recherche personnels peut l'être.

\section{Soins de santé spécialisés}

9.47 En hôpital universitaire, où la formation des étudiants en médecine occupe une place de choix en complément de la vocation première de prestation de soins de santé, il n'est pas rare que les activités d'enseignement, de R-D et de prestation de soins médicaux courants et de pointe soient étroitement liées. Les " soins de santé spécialisés " constituent une activité qui doit habituellement être exclue de la R-D. Cependant, il peut y avoir une part de R-D dans ces soins, lorsqu'ils sont dispensés dans des hôpitaux universitaires, par exemple. Il est difficile pour les professeurs et leurs assistants de définir la part de leurs activités correspondant exclusivement à de la R-D. Pourtant, si le temps et l'argent consacrés aux soins médicaux courants sont pris en compte dans les statistiques de R-D, on aboutira à une surestimation des ressources de R-D dans les sciences médicales. En général, les soins de santé spécialisés ne sont pas considérés comme de la R-D et tout soin médical qui n'est pas directement lié à un projet spécifique de R-D est à exclure des statistiques de R-D.

9.48 D'après ces critères, toutefois, un projet particulier peut ou non être considéré comme de la R-D selon qu'il est entrepris dans un but ou un autre, comme l'illustre l'exemple suivant : dans le domaine de la médecine, réaliser une autopsie de routine pour déterminer les causes d'un décès est une pratique médicale courante, qui ne peut pas être rattachée à la R-D ; en revanche, une enquête de mortalité destinée à établir les effets secondaires d'un traitement contre le cancer relève de la $\mathrm{R}-\mathrm{D}$. De même, les tests de routine, comme les analyses de sang et les examens bactériologiques réalisés dans le cadre de bilans de santé ne relèvent pas de la $R-D$, contrairement aux programmes de tests sanguins visant spécifiquement les patients qui prennent un nouveau produit pharmaceutique.

9.49 Les hôpitaux universitaires peuvent aussi participer à des essais cliniques. On trouvera au chapitre 2 des indications supplémentaires sur la détermination de la R-D dans les essais cliniques.

\section{La R-D dans les sciences sociales, les sciences humaines et les arts}

9.50 Une grande partie de la R-D en sciences sociales et humaines est exécutée dans le secteur de l'enseignement supérieur. On trouvera au chapitre 2 des lignes directrices précisant comment déterminer la part de la R-D dans ces domaines et ce qu'il faut en exclure.

9.51 La majeure partie des travaux de recherche sur, pour et dans le domaine des arts sont également exécutés dans le secteur de l'enseignement supérieur. Le chapitre 2 donne des lignes directrices sur les éléments à considérer comme de la R-D dans ce domaine. 


\subsection{Mesure des dépenses et du personnel dans le secteur de l'enseignement supérieur}

9.52 La présente section a pour objet de donner des lignes directrices sur les principales variables et ventilations à recueillir, en tenant compte des spécificités propres au secteur de l'enseignement supérieur. La section 9.5 complétera le tableau en décrivant les méthodes couramment utilisées (enquêtes directes, exploitation des données administratives et coefficients de R-D, par exemple) pour recueillir et évaluer ces variables et ventilations.

9.53 Les dépenses intérieures de R-D de l'enseignement supérieur (DIRDES) constituent le principal agrégat statistique utilisé pour décrire l'exécution de la R-D au sein du secteur de l'enseignement supérieur. Correspondant à la composante des dépenses intérieures brutes de R-D (DIRD) engagées par les unités de l'enseignement supérieur (voir chapitre 4), les DIRDES sont l'expression chiffrée des dépenses de R-D intra-muros du secteur de l'enseignement supérieur pour une période de référence donnée.

\section{Dépenses intérieures de R-D de l'enseignement supérieur (DIRDES) par type de coûts}

9.54 Conformément aux indications du chapitre 4, les DIRDES doivent être décomposées en dépenses courantes et en dépenses en capital. Les premières se composent des coûts de main-d'œuvre et des autres dépenses courantes, et les secondes, des dépenses afférentes aux actifs fixes utilisés pour la $R-D$, tels que le machines et équipements, et les terrains et constructions.

9.55 Si les données relatives à chacun de ces éléments ne sont pas directement disponibles pour une unité, il faut procéder à une estimation fondée sur le montant total des dépenses.

9.56 Les coûts de main-d'œuvre (salaires et dépenses associées) constituent une part importante des dépenses de R-D totales dans le secteur de l'enseignement supérieur. En principe, les coûts de main-d'œuvre de la R-D doivent être liés au temps consacré à cette activité, lui-même exprimé en équivalent temps plein (ETP). Les informations sur les coûts totaux de maind'œuvre sont généralement disponibles ou peuvent être calculées sur la base d'une ou de plusieurs des sources de données suivantes :

- barème salarial et position de chaque chercheur, technicien ou autre membre du personnel dans ce barème

- coûts de main-d'œuvre par catégorie de personnel

- coûts de main-d'œuvre par catégorie de personnel, domaine de R-D et, le cas échéant, département.

9.57 Les coûts de main-d'œuvre comprennent les cotisations réelles ou imputées aux fonds de pension et à d'autres organismes de sécurité sociale correspondant au personnel de R-D. Il n'est pas nécessaire qu'ils apparaissent distinctement dans la comptabilité de l'unité statistique. Même en l'absence 
de transaction, il convient d'essayer d'estimer ces coûts. Pour éviter le double comptage, les coûts de main-d'œuvre ne comprennent pas les prestations de retraite aux anciens salariés de la R-D.

9.58 Les informations sur les autres dépenses courantes sont généralement disponibles par département ou par unité équivalente et concernent souvent les ressources dont ces unités disposent pour acheter des articles tels que des documents ou du petit matériel, s'abonner à des revues scientifiques ou payer des frais de voyage. Il est habituellement demandé aux unités déclarantes d'estimer la part de la R-D dans ces dépenses sur la base de l'« utilisation prévue ». La partie des dépenses pour laquelle il n'est pas possible d'obtenir des informations au niveau du département (frais généraux tels que l'eau, l'électricité, les loyers, l'entretien, l'administration générale, etc.) doit être obtenue par ventilation de ces frais sur les unités institutionnelles concernées. Si le critère d'« utilisation prévue » n'est pas possible, on peut utiliser les mêmes coefficients de répartition que pour les coûts de main-d'œuvre (pour une analyse des « coefficients de R-D ", voir la section 9.5 ci-après). Les parts de R-D peuvent aussi être déterminées sur la base de conventions ou d'une estimation de valeur par les unités déclarantes.

9.59 L'imputation des dépenses des établissements d'enseignement supérieur afférentes à la gestion des biens immobiliers et des installations diffère selon les pays. En effet, les bâtiments et les terrains utilisés à des fins d'enseignement ou de recherche peuvent être soit détenus, soit utilisés à titre gratuit, soit loués par les établissements. De même, les coûts de l'énergie peuvent être imputés selon diverses méthodes. Ces différences de traitement des coûts selon les pays auront donc une incidence sur les comparaisons internationales des dépenses courantes et des dépenses en capital. Pour assurer la comparabilité internationale et obtenir des coûts réalistes, il peut être souhaitable de faire figurer un montant théorique en lieu et place d'un paiement réel. Il pourrait s'agir, par exemple, d'une "valeur marchande " estimée, à inclure dans les " autres dépenses courantes".

9.60 Les informations sur le total des dépenses en capital afférentes aux machines et équipements sont généralement disponibles au niveau des institutions. Dans de nombreuses enquêtes, ce sont les établissements qui estiment la part des activités de R-D d'après l'« utilisation prévue " des équipements. Les coefficients de R-D (voir section 9.5) servent moins souvent à estimer la part de la R-D dans les machines et équipements que dans le cas des divers types de dépenses courantes. La part de la R-D dans ces investissements peut aussi être établie sur la base de conventions ou d'estimations de valeur, comme pour certaines catégories de dépenses courantes examinées ci-dessus.

9.61 Les informations sur le total des dépenses en capital afférentes aux terrains et constructions ne sont généralement disponibles qu'au niveau de l'établissement ou de l'université. Les coefficients de R-D servent rarement à estimer la part de la R-D dans ces dépenses. Là encore, les données relatives à la R-D sont souvent estimées d'après l'utilisation prévue des lieux. 


\section{DIRDES par source de financement}

\section{Généralités}

9.62 Comme il est indiqué au chapitre 4, le financement de la R-D exécutée dans le secteur de l'enseignement supérieur provient de différentes sources.

- Dans de nombreux pays, traditionnellement, la source principale est une partie de la dotation forfaitaire de fonds publics désignée sous l'appellation de " fonds généraux des universités " (FGU), que les établissements publics d'enseignement supérieur reçoivent pour financer l'ensemble de leurs activités. Le plus souvent, les différentes activités du personnel des établissements d'enseignement supérieur - enseignement, R-D, administration, soins de santé, etc. - ne sont pas isolées dans le but d'être rémunérées séparément à partir de ces fonds qui, d'une façon générale, couvrent le paiement d'un large éventail d'activités professionnelles.

- Parmi les autres sources de financement de la R-D figurent les subventions ou les contrats émanant de ministères, de départements et d'autres institutions publiques, y compris les conseils de recherche, ainsi que d'institutions privées sans but lucratif, de l'industrie et du reste du monde.

- En outre, certaines universités peuvent aussi avoir des fonds internes (tels que les revenus tirés de dotations ou des frais d'inscription des étudiants) qu'elles utilisent au final pour payer l'exécution de travaux de R-D.

9.63 Aux fins du présent manuel, les FGU correspondent à la part des ressources financières destinées à la R-D qui sont issues de la dotation globale allouée aux universités, à l'appui de l'ensemble de leurs activités de recherche et d'enseignement, par le ministère de l'Éducation de l'administration centrale (fédérale) ou par les autorités régionales (d’États fédérés) ou locales (municipales) compétentes.

9.64 Les enquêtes sur l'emploi du temps et autres méthodes visant à isoler la part de R-D dans l'ensemble des activités des universités ne portent généralement que sur les FGU. Les fonds externes sont généralement destinés à la R-D, mais peuvent aussi être utilisés à d'autres fins. Le répondant doit donc souvent déterminer, pour chaque projet alimenté par des sources externes, si les crédits financent sa recherche dès lors que cette information ne figure pas dans les registres des administrations centrales.

9.65 Certains de ces fonds externes (en particulier ceux qui émanent de fondations et de conseils de recherche) ne sont pas toujours intégralement enregistrés dans la comptabilité centrale des universités. Les fonds de certains contrats de recherche peuvent d'ailleurs être versés directement à un institut universitaire ou à des professeurs. Pour que le champ couvert soit aussi complet que possible, il faut donc parfois - bien que le Manuel recommande d'appliquer le principe d'une déclaration des dépenses par l'organisme qui exécute la R-D - se reporter à la comptabilité des bailleurs de fonds pour se procurer les données sur les fonds externes des instituts ou, tout au moins, pour recouper 
les informations déclarées par ailleurs. Les éléments fournis par les bailleurs de fonds ne permettent généralement de connaître que les dépenses, ce qui ne résout en rien la difficulté d'obtenir les données relatives au personnel de R-D.

9.66 Ce sont donc principalement les procédures comptables qui déterminent dans quelle mesure les sources de financement de la R-D peuvent être définies et identifiées séparément. Les statisticiens de la R-D sont tributaires du degré de détail offert par la comptabilité. Un autre aspect vient encore compliquer la détermination des sources de financement de la R-D : le fait que, très souvent, les organisations extérieures ne paient pas le " coût marchand intégral " (quelle que soit la définition que l'on en donne) de la R-D effectuée pour leur compte dans les établissements d'enseignement supérieur.

9.67 Tous les pays rencontrent régulièrement des problèmes pour retracer avec exactitude les sources de financement de la R-D, mais le point sur lequel la comparabilité internationale achoppe le plus est l'établissement d'une distinction entre les FGU et les autres sources de revenus de la R-D d'origine publique.

\section{Distinction entre les fonds généraux des universités (FGU) et les autres sources de financement}

9.68 Certains des problèmes que pose la détermination de la part de ces subventions attribuable à la R-D ont déjà été évoqués précédemment. Cette détermination fait partie intégrante de la méthode appliquée dans chaque pays. Les incohérences résultent de ce que les pays ne classent pas de la même façon la composante R-D des FGU.

9.69 Pour le secteur de l'enseignement supérieur, on a défini une catégorie distincte, les FGU, afin de tenir compte de la particularité des mécanismes de financement de la R-D dans ce secteur par rapport aux autres. La plupart des pays estiment que, comme la R-D fait partie intégrante des activités des établissements d'enseignement supérieur, tous les fonds affectés à un établissement de ce type comportent systématiquement une composante R-D. Suivant cette interprétation, ces fonds sont classés dans les FGU.

9.70 Lorsqu'on établit les totaux nationaux, ces données sont en général incluses dans les sous-totaux de la source de financement État au motif que celui-ci est la source originelle et prévoit qu'une part importante de ces fonds généraux sera consacrée à la R-D.

9.71 Or, il appartient aux universités de décider du montant à prélever sur leur réserve générale de ressources pour la $R-D$, et cette réserve comprend à la fois les fonds généraux des universités et les ressources propres des établissements. Ainsi, certains pays estiment que les sommes en question pourraient être d'abord attribuées à l'enseignement supérieur en tant que source de financement. Certains pays adoptent cette convention lors de la communication des données à l'échelle nationale. 
9.72 Par convention, la part de R-D financée au moyen de ces FGU d'origine publique devrait être attribuée à l'État en tant que source de financement, et c'est la méthode recommandée aux fins de comparaison internationale. Dans tous les cas, les FGU doivent être déclarés séparément, comme indiqué au chapitre 4. Les coûts de sécurité sociale, les provisions liées à la retraite et les autres coûts pertinents (réels ou imputés) doivent être pris en compte et enregistrés dans les FGU. Dans un souci de clarté, les DIRD financées par l'État sont divisées en deux sous-catégories : les fonds publics directs et les FGU (pour le calcul des FGU, voir section 9.5 ci-après).

\section{Autres fonds d'origine interne}

9.73 Les revenus provenant de dotations, de portefeuilles d'actions et de biens immobiliers, ainsi que les recettes tirées de la vente de services hors $R-D$, tels que les frais d'inscription et les droits de scolarité des étudiants, les abonnements aux revues, la vente de sérums ou de produits agricoles, doivent être considérés comme des fonds d'origine interne. Bien que ce soient les pratiques comptables nationales qui déterminent la facilité avec laquelle il est possible de les isoler, ces revenus de la R-D ("recettes retenues ") peuvent, notamment dans le cas des universités privées, constituer une source de revenus très importante et doivent être classés dans les fonds d'origine interne.

\section{Fonds d'origine externe}

9.74 En plus des FGU, la R-D de l'enseignement supérieur reçoit des crédits d'unités institutionnelles du secteur de l'État, du secteur des entreprises et du secteur privé sans but lucratif, sous la forme de subventions ou de contrats destinés spécifiquement à la recherche. Ces fonds peuvent aussi provenir du reste du monde. Ces ressources sont plus faciles à isoler et ne posent en général pas de problème majeur aux statisticiens, qui peuvent les classer dans les sources de financement direct.

\section{Recommandations}

9.75 Pour améliorer la comparabilité internationale des statistiques de R-D du secteur de l'enseignement supérieur, il est préférable de désagréger les sources de financement le plus possible, ce qui dépend, dans une large mesure, des informations fournies par les systèmes de comptabilité centrale des établissements d'enseignement supérieur.

9.76 La comparabilité internationale devient difficile lorsque les données relatives aux FGU ne sont pas communiquées séparément et sont classées par certains pays soit dans les fonds d'origine interne du secteur de l'enseignement supérieur, soit dans les fonds en provenance du secteur de l'État.

9.77 Lorsque ce type de financement existe, les FGU doivent être communiqués séparément sous la rubrique des fonds en provenance du secteur de l'État, et non du secteur de l'enseignement supérieur. 


\section{Dépenses de R-D extra-muros}

9.78 La complexité croissante de l'organisation des activités de R-D pose problème dans le secteur de l'enseignement supérieur comme dans les autres. Dans les grands projets de R-D menés en coopération, les universités peuvent recevoir une subvention de l'État ou d'autres organisations et en transmettre une partie à d'autres partenaires du projet. Il est donc souhaitable de collecter le montant des fonds de R-D transmis (au moyen de contrats de sous-traitance et de subventions en cascade) aux exécutants de la R-D extra-muros dans le secteur de l'enseignement supérieur, afin d'éviter le double comptage (voir chapitre 4, section 4.3). Comme il a été noté au chapitre 4, les flux financiers transférés à d'autres départements d'un même établissement d'enseignement supérieur ne doivent pas être considérés comme de la R-D extra-muros, car les différents départements font partie de la même unité statistique.

\section{$R-D$ en lien avec le reste du monde}

9.79 Le secteur de l'enseignement supérieur participe aux activités de mondialisation de la R-D, telles que définies au chapitre 11. La présente section fournit des informations sur quatre aspects internationaux de ce secteur : le financement de la R-D à destination/en provenance du secteur représentant le reste du monde ; les campus satellites sous contrôle étranger ; les campus satellites à l'étranger; et les étudiants étrangers. Les statistiques de R-D relatives à ces activités peuvent être utiles pour comprendre la mondialisation de la recherche scientifique dans certains domaines de la $\mathrm{R}-\mathrm{D}$, à des fins d'analyse ou d'élaboration des politiques dans les marchés émergents ou au sujet de ces marchés (surtout en ce qui concerne les campus sous contrôle étranger) et à des fins de politiques ou de recherches dans le domaine de l'éducation.

9.80 Les établissements d'enseignement supérieur doivent fournir des informations sur tous les types de financement de R-D fournis à des organisations implantées en dehors du pays déclarant ou reçus d'organisations de ce type.

9.81 Le champ d'application comprend toutes les activités d'enseignement intérieures du pays déclarant (exercées sur son territoire national), indépendamment de qui détient ou parraine les établissements concernés et indépendamment du mécanisme utilisé pour dispenser les enseignements. Les établissements d'enseignement supérieur ont mis en place des antennes ou des campus hors des frontières du pays où ils se trouvent. Dans la mesure où les campus satellites sous contrôle étranger qui se trouvent dans le pays déclarant et les campus satellites à l'étranger (c'est-à-dire dans le secteur représentant le reste du monde) sous contrôle d'établissements d'enseignement nationaux exécutent des travaux de R-D, les enquêtes sur les DIRDES peuvent comprendre des informations supplémentaires sur ces campus (pour en savoir plus sur la délimitation entre la R-D et l'enseignement ou la formation, voir la section 9.3).

9.82 Aux fins du présent manuel, un campus satellite étranger est un établissement d'enseignement supérieur qui se trouve sur le territoire du 
pays déclarant ; qui est détenu, du moins en partie, par une entité implantée (ou résidant) en dehors du pays déclarant (appelée « prestataire d'enseignement étranger ") ; dont l'activité est régie par cette entité ; qui consacre au moins une partie de ses activités à l'enseignement direct ; et qui donne accès à un programme universitaire complet sanctionné par un diplôme du prestataire étranger. Dans le cadre des informations d'identification (pour une éventuelle tabulation des données de R-D lorsqu'elle est faisable), les enquêtes relatives aux DIRDES peuvent chercher à établir si un répondant qui entre dans le champ d'investigation est un campus satellite sous contrôle étranger.

9.83 Aux fins du présent manuel, un campus satellite à l'étranger est un établissement d'enseignement supérieur qui est détenu, du moins en partie, par un établissement local d'enseignement supérieur (c'est-à-dire résidant sur le territoire du pays déclarant), mais est situé dans le reste du monde (résidant en dehors du pays déclarant) ; dont l'activité est régie par l'établissement local ; qui consacre au moins une partie de ses activités à l'enseignement direct ; et qui donne accès à un programme universitaire complet sanctionné par un diplôme de l'établissement local. Les enquêtes sur les DIRDES peuvent demander a) quel est le pays d'implantation (appelé " pays hôte ") des campus satellites situés à l'étranger ; b) si ces campus ont exécuté des travaux de R-D dans le pays hôte (question binaire appelant une réponse par oui ou par non) et c) quel est le montant des dépenses correspondant à ces travaux dans la monnaie du pays déclarant. Quand une institution possède plusieurs campus de ce type dans un pays donné, les informations de R-D peuvent être communiquées après consolidation au niveau du pays hôte, si cela facilite les réponses. Les informations détaillées par domaine de R-D peuvent être à des niveaux d'agrégation supérieurs pour ces campus.

9.84 Les dépenses correspondant à la R-D exécutée par les campus satellites sous contrôle étranger font partie des DIRDES totales du pays déclarant. En revanche, celles correspondant à la R-D exécutée par les campus satellites à l'étranger ne peuvent pas être incluses dans les DIRDES totales du pays déclarant, et pourraient, au lieu de cela, être isolées et tabulées comme de la R-D exécutée dans le secteur représentant le reste du monde par des établissements d'enseignement supérieur se trouvant en dehors des établissements d'enseignement du pays déclarant.

9.85 Outre le fait de recenser séparément ces campus à la lumière des définitions ci-dessus, il est recommandé de recueillir les dépenses de R-D et les statistiques relatives aux ressources humaines de ces unités en suivant les indications fournies ailleurs dans le présent chapitre.

9.86 Les campus satellites à l'étranger ne se trouvant pas, par définition, sur le territoire du pays déclarant, la collecte ou la modification des informations les concernant peuvent se révéler particulièrement difficiles. L'obtention de ces informations est donc considérée comme une priorité de moindre importance, même si elle est encouragée. En effet, les informations sur les activités mondiales 
extérieures du secteur de l'enseignement supérieur, telles que celles des campus à l'étranger exécutant des travaux de R-D, peuvent être particulièrement intéressantes pour les unités institutionnelles répondantes.

9.87 Le nombre d'étudiants étrangers inscrits est un autre aspect de la mondialisation des établissements d'enseignement supérieur. Les étudiants étrangers (parfois appelés "étudiants internationaux ") sont définis comme les individus non-ressortissants du pays dans lequel ils étudient (voir volume 1, section 4.6.1 du Manuel de l'UOE). Les travaux de recherche menés dans les universités par tous les étudiants de niveau doctorat et master (niveau 7 de la CITE) doivent être pris en compte dans les dépenses de R-D, quels que soient le statut au regard de la citoyenneté des étudiants ou la nationalité de parrainage.

\section{Catégories de personnel de R-D}

9.88 Les catégories de personnel de R-D dont il faut faire état pour le secteur de l'enseignement supérieur sont identiques à celles des autres secteurs exécutant des travaux de R-D et sont définies au chapitre 5 . En particulier, la classification de référence utilisée pour présenter ces données par niveau d'études est la Classification internationale type de l'éducation (CITE 2011).

9.89 La notion de "personnel de R-D », voire de " chercheur », peut toutefois ne pas être employée ni comprise de la même façon dans les établissements d'enseignement supérieur, et donc nécessiter le recours à des titres universitaires apparentés. Il peut aussi être utile de communiquer les données sur les chercheurs par grade universitaire de manière à renseigner sur le niveau d'ancienneté dans la recherche/dans la carrière universitaire.

9.90 Chaque fois que possible, il est proposé d'appliquer la classification par niveau de grade ci-après pour présenter les données sur les chercheurs du secteur de l'enseignement supérieur (CE, 2013) lorsqu'il existe des titres universitaires. Les catégories comprennent des postes types pour chaque groupe :

- Catégorie A : grade/poste le plus élevé auquel des travaux de recherche sont normalement réalisés.

* Exemple : " professeur titulaire " ou « directeur de recherche ».

- Catégorie $B$ : chercheurs travaillant à un niveau moins élevé que le niveau supérieur (A), mais plus élevé que celui des titulaires de doctorat nouvellement diplômés (niveau 8 de la CITE).

* Exemples : «maître de conférences » ou « chargé de recherche principal ».

- Catégorie C : premier grade/poste auquel un titulaire de doctorat nouvellement diplômé sera normalement recruté.

* Exemples : «chargé de cours » ou « boursier de recherches postdoctorales ». 
- Catégorie D : étudiants en doctorat (niveau 8 de la CITE) travaillant comme chercheurs, ou chercheurs travaillant à des postes ne nécessitant pas normalement un diplôme de doctorat.

* Exemples : « doctorant » ou « assistant de recherche » (non titulaire d'un doctorat).

9.91 Les étudiants en master peuvent être comptés comme des chercheurs (voir chapitre 5) s'ils participent à un programme de master de recherche du niveau 7 de la CITE 2011, c'est-à-dire " menant à l'obtention de certifications de chercheur conçues explicitement pour former les participants à la conduite de projets de recherche originaux, mais dont le niveau est inférieur à celui d'un doctorat ". Aux termes de cette définition, " ces programmes remplissent souvent plusieurs critères identiques à ceux d'un programme de niveau 8 de la CITE, même s'ils tendent à être plus courts (durée cumulée de cinq à six ans depuis le début de l'enseignement supérieur), ils ne donnent généralement pas le niveau d'indépendance exigé des étudiants poursuivant une certification de chercheur spécialisée et préparent à accéder à des programmes de niveau 8 de la CITE ». Les étudiants en master comptés comme chercheurs entrent normalement dans la catégorie D susmentionnée.

9.92 Il importe néanmoins que seuls les étudiants en master rémunérés, directement ou indirectement, pour leur activité de R-D soient comptabilisés avec le personnel de R-D (voir chapitre 5, section 5.2).

\subsection{Méthodes de compilation des dépenses et des données relatives au personnel de R-D dans le secteur de l'enseignement supérieur}

9.93 Cette section donne des informations d'ordre général sur les méthodes employées pour calculer et estimer les chiffres des dépenses et du personnel de R-D de l'enseignement supérieur. Y sont également représentées les différentes approches utilisées dans le cadre de compilation des statistiques sur les DIRDES (graphique 9.1). Une attention particulière est accordée aux méthodes employées pour estimer la R-D, en particulier les fonds généraux des universités (FGU), qui peuvent être une composante essentielle du financement dans ce secteur. Normalement, ces fonds constituent une part importante du financement de la R-D, mais la proportion des FGU consacrée à la R-D est souvent méconnue des universités elles-mêmes.

\section{Méthode générale}

9.94 Le secteur de l'enseignement supérieur est très hétérogène. De même, les systèmes et établissements d'enseignement supérieur sont organisés de différentes manières selon les pays. Tout cela vient compliquer le recueil des statistiques de R-D, d'autant que les méthodes statistiques varient grandement d'un pays à l'autre. Dans la pratique, cela signifie que plusieurs méthodes peuvent être utilisées pour obtenir des statistiques de R-D de bonne qualité. 
9.95 Le cadre de compilation des statistiques sur les DIRDES donne aux statisticiens plusieurs options parmi lesquelles ils peuvent choisir la méthode la mieux adaptée à leur institution pour produire les statistiques de R-D du secteur de l'enseignement supérieur, en tenant compte des ressources dont ils disposent (utilisation ou non d'une enquête), de la qualité et de la disponibilité des données administratives relatives aux établissements d'enseignement supérieur, et de la disponibilité des données au niveau du type souhaité d'unité statistique, d'institut ou de département. Les enquêtes sur l'emploi du temps sont des éléments importants des statistiques de R-D du secteur de l'enseignement supérieur dans de nombreux pays, et peuvent être associées à une enquête institutionnelle sur la R-D (complète ou partielle) ou à des données administratives uniquement, ou encore à une combinaison de données d'enquête et de données administratives.

9.96 Les différentes méthodes d'obtention des données sont indiquées dans le cadre illustré au graphique 9.1. L'un des prérequis importants pour utiliser des données administratives est que ces données soient de bonne qualité, c'est-àdire qu'elles soient disponibles, fiables et actualisées (voir ci-après). Les méthodes vont des enquêtes institutionnelles (complètes ou partielles) à l'exploitation de données administratives, en passant par les différentes combinaisons de ces sources, souvent conjuguées à l'utilisation de coefficients de R-D établis à partir d'enquêtes sur l'emploi du temps.

\section{Graphique 9.1. Cadre de compilation des statistiques du secteur de l'enseignement supérieur}

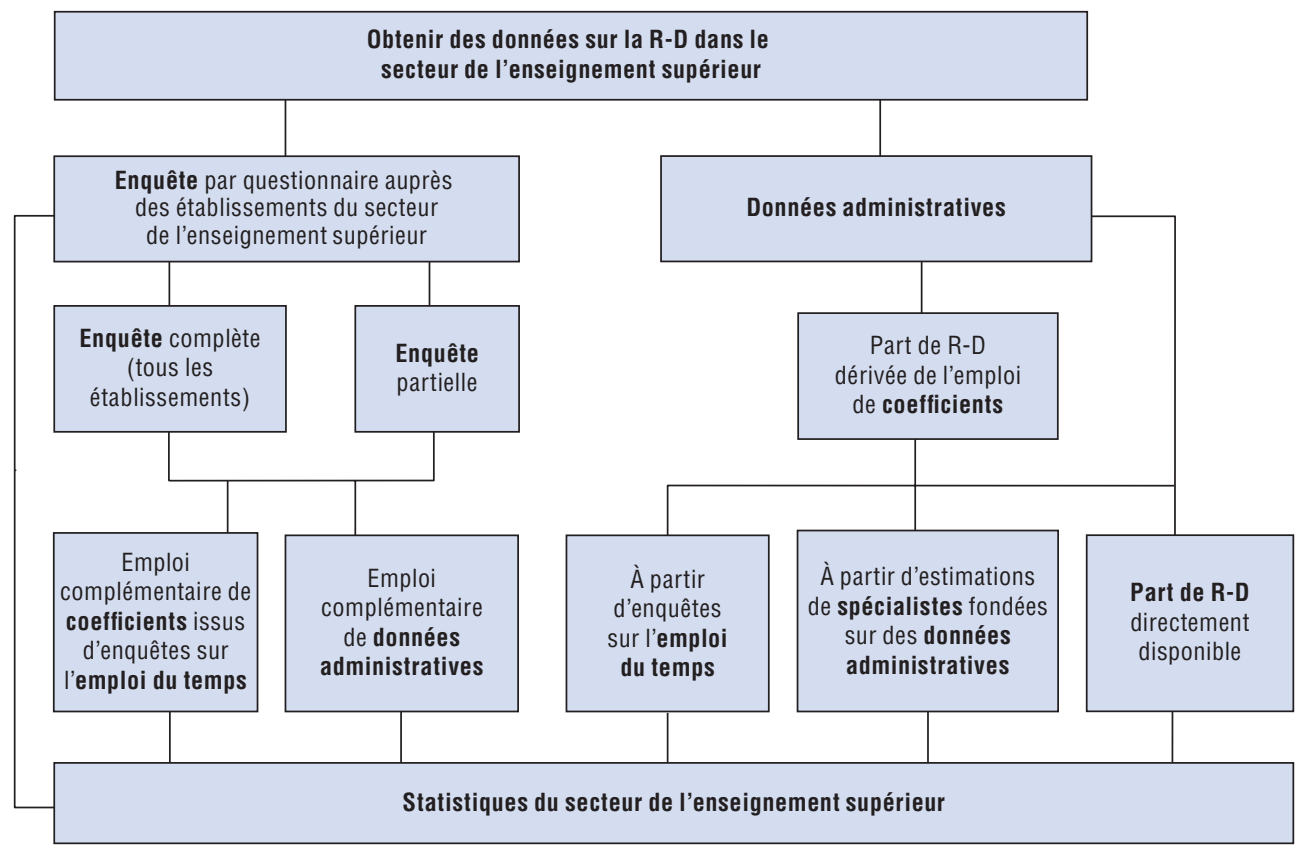




\section{L'unité statistique}

9.97 Les unités institutionnelles du secteur de l'enseignement supérieur sont à peu près clairement définies (voir section 9.3, et chapitre 3, section 3.2 ), mais il est plus délicat de définir les unités statistiques, c'est-à-dire les entités sur lesquelles on cherche à recueillir l'information. On ne peut pas donner de règle universelle ici, car les systèmes éducatifs varient grandement selon les pays.

9.98 Autant que possible, les unités statistiques du secteur de l'enseignement supérieur sont classées en six grands domaines de recherche et de développement, comme suit :

- sciences naturelles

- sciences de l'ingénieur et technologie

- sciences médicales et sciences de la santé

- sciences agricoles et vétérinaires

- sciences sociales

- sciences humaines et arts.

9.99 Les principaux domaines et sous-domaines de la R-D sont présentés au chapitre 2.

9.100 Si les grands domaines sont clairement définis, le niveau de désagrégation de chacun d'eux est laissé à la discrétion des pays. Dans le secteur de l'enseignement supérieur, lorsqu'on dispose d'informations administratives circonstanciées, une nomenclature détaillée des domaines de recherche et de développement peut être utilisée comme classification institutionnelle.

9.101 Les établissements d'enseignement supérieur menant souvent des activités dans plusieurs des six grands domaines de R-D, ce type d'informations peut être disponible à des niveaux plus détaillés des unités déclarantes, tels que les départements, les instituts ou les " centres " de recherche, les facultés, les hôpitaux ou les établissements d'enseignement post-secondaire.

9.102 Dans certains pays, les informations sur les établissements d'enseignement supérieur peuvent être fournies par le ministère de l'Éducation. Elles peuvent aussi être communiquées par les autorités régionales. Les enquêteurs s'adressent souvent aux établissements directement. Bien souvent, il sera nécessaire de décomposer les données jusqu'au niveau des départements universitaires. Le choix des unités déclarantes est crucial si l'on veut pouvoir extraire les données de R-D à partir des données générales.

\section{Données d'enquête}

9.103 La conduite d'enquêtes spéciales régulières, méthodiques et harmonisées est le moyen à privilégier pour recueillir des données sur la R-D. Néanmoins, lorsqu'on dispose de données administratives satisfaisantes et 
que les enquêtes statistiques sont jugées trop lourdes, d'autres approches conviennent aussi, en particulier dans le secteur de l'enseignement supérieur.

9.104 Il est possible de réunir les informations sur la R-D dans le secteur de l'enseignement supérieur à partir de deux grandes sources : les enquêtes et les données administratives. Les deux méthodes sont souvent combinées. La méthode fondée sur les enquêtes présente de nombreux avantages, par exemple pour isoler les activités de R-D et leur associer un domaine ou un type de R-D.

9.105 Pour améliorer la comparabilité internationale des données rassemblées, la présente section propose quelques lignes directrices méthodologiques concernant la réalisation d'enquêtes sur la R-D. Comme les méthodes et procédures d'enquête sur la R-D sont bien établies dans de nombreux pays, ces lignes directrices demeurent relativement générales de façon à être aussi largement applicables que possible. Ces méthodes complètent celles examinées au chapitre 6 .

\section{Champ des enquêtes de R-D}

9.106 Théoriquement, ce type d'enquêtes doit permettre de connaître et de mesurer l'ensemble des ressources financières et humaines consacrées aux activités de R-D dans toutes les unités du secteur de l'enseignement supérieur exécutant des travaux de R-D. Elles s'adressent essentiellement aux unités exécutantes, lesquelles peuvent aussi financer des activités de R-D réalisées dans d'autres unités.

\section{Détermination de la population cible et des répondants aux enquêtes}

9.107 Les statisticiens de la R-D n'ont pas toujours les moyens de mener une enquête exhaustive et fiable auprès de toutes les unités exécutantes du secteur. Le plus souvent, leur champ d'investigation est restreint par de nombreuses contraintes. Il leur faut, par exemple, limiter le nombre de répondants pour respecter un budget serré ; ou associer l'enquête sur la R-D à une autre, ce qui impose un choix de répondants acceptable, mais pas toujours idéal ; parfois aussi, les enquêtes auprès de certains groupes exigent la participation d'autres organismes ayant d'autres besoins d'information, ce qui implique des questions différentes à poser aux répondants. Il n'est donc pas possible de formuler, sur les méthodes d'enquête, des recommandations détaillées qui soient pareillement pertinentes dans tous les pays, d'autant que les capacités nationales de R-D varient considérablement par leur importance et leur structure.

9.108 Dans le secteur de l'enseignement supérieur, les enquêtes et les techniques d'estimation devraient couvrir toutes les universités et tous les établissements correspondants, en particulier ceux qui décernent des diplômes de niveau doctorat. Les autres établissements de ce secteur dont on sait ou suppose qu'ils exécutent des travaux de R-D devraient aussi être pris en compte (voir section 9.2). Dans la mesure du possible, il est souvent préférable d'utiliser 
comme unités déclarantes des sous-unités telles que les départements ou les instituts composant l'université.

\section{Hôpitaux et centres de soins universitaires}

9.109 Les hôpitaux et autres établissements de santé constituent une catégorie spéciale. Certains pays peuvent juger satisfaisant d'inclure les centres hospitaliers et autres établissements de santé dans les enquêtes régulières de R-D, en utilisant pour ce faire le questionnaire type établi pour le secteur concerné. Des indications supplémentaires sur la délimitation entre activités de recherche et de soins de santé et sur le traitement des essais cliniques peuvent être jointes aux questionnaires, conformément aux définitions et critères fournis au chapitre 2 .

9.110 Lorsque les hôpitaux universitaires sont très étroitement intégrés aux établissements d'enseignement, sur le plan administratif et financier, ils peuvent être traités avec ces derniers pour ce qui concerne les enquêtes et la compilation des données sur la R-D. En revanche, lorsqu'ils constituent des unités séparées possédant leur propre comptabilité et leur propre administration, ils peuvent recevoir soit un questionnaire spécifique, si celui-ci est plus approprié, soit un questionnaire standard sur la R-D. Pour les hôpitaux universitaires (ou certaines parties de ces établissements) qui ne sont pas intégrés à des établissements d'enseignement, il peut être utile de procéder à une enquête spéciale. Si ce n'est pas possible, le questionnaire standard sur la R-D peut être employé.

9.111 Quelle que soit la démarche adoptée pour réaliser les enquêtes, il convient de veiller à traiter de façon cohérente les unités / projets de R-D gérés conjointement par plusieurs entités, par des personnes rémunérées par plusieurs entités et par des personnes travaillant dans des centres hospitaliers, mais employées par d'autres établissements.

9.112 Le questionnaire utilisé pour l'enquête doit comprendre un nombre minimal de questions fondamentales sur l'activité de R-D, afin de permettre la production de statistiques harmonisées et comparables à transmettre aux organisations internationales. Compte tenu de la charge de travail qu'il représente pour les répondants, le questionnaire doit se présenter de façon logique, être aussi simple et aussi succinct que possible et être accompagné de définitions et d'instructions claires. En général, plus le questionnaire est long, plus le taux de réponse est faible. La plupart des pays utilisent des questionnaires électroniques, disponibles en ligne (pour plus d'informations sur les méthodes d'enquête, voir chapitre 6).

\section{Données administratives}

9.113 Les données administratives sont une source courante de statistiques de R-D dans le secteur de l'enseignement supérieur (voir l'examen du cadre précédent et le graphique 9.1). Entre autres exemples de données 
administratives, on citera les enregistrements comptables et les registres du personnel des établissements d'enseignement supérieur ou encore les données fournies par les organismes de financement.

9.114 La majorité des pays établissent leurs statistiques de R-D à partir d'une enquête (complète ou partielle), mais, pour le secteur de l'enseignement supérieur, certains pays s'appuient uniquement sur des données administratives. Beaucoup aussi combinent ces deux méthodes. En général, l'exploitation des registres et des données administratives à des fins statistiques demandera moins de ressources qu'une enquête et allégera la charge de travail pour les répondants. D'où l'importance qu'il peut y avoir à améliorer la disponibilité et la qualité des données administratives de façon à en étendre l'utilisation et à rationaliser la production de statistiques de $\mathrm{R}-\mathrm{D}$.

9.115 Il ne faudrait toutefois pas sous-estimer les avantages évidents qu'offrent les enquêtes, en particulier pour déterminer la part de la R-D dans différentes activités, sa répartition par domaine ou son type. Données d'enquête et données administratives sont souvent combinées pour fournir les statistiques de R-D dans le secteur de l'enseignement supérieur, et conjuguées à des enquêtes sur l'emploi du temps ou à d'autres méthodes d'estimation de la composante R-D.

9.116 Les données administratives peuvent être exploitées de différentes façons pour compiler les statistiques de R-D dans le secteur de l'enseignement supérieur. Si les concepts et définitions utilisés et le champ couvert par les sources de données administratives sont suffisamment proches de ceux donnés dans le présent manuel, ces sources peuvent servir de sources d'information primaires. Plus souvent, les données administratives sont utilisables conjointement avec des coefficients de R-D dérivés d'enquêtes sur l'emploi du temps (voir section 9.5.5) lorsqu'on cherche à estimer la part de la R-D. Les données administratives peuvent aussi être utilisées pour suppléer des données d'enquête manquantes ou incohérentes et à des fins de contrôle après l'étape de correction des données (voir chapitre 6).

9.117 Dans de nombreux cas, les données sont dérivées de multiples sources administratives. Le rôle des administrations centrales varie d'un pays à l'autre et d'un niveau à l'autre - nationalement au ministère de l'éducation, régionalement, localement ou à l'intérieur de l'établissement d'enseignement supérieur lui-même. Quel que soit le niveau d'administration, ces sources disposent en général d'une information abondante résultant des activités menées à cet échelon. Les informations détenues dans les registres des administrations centrales varient suivant les fonctions exercées par chacune de ces administrations. Les ministères de l'Éducation peuvent disposer d'éléments très généraux, tandis que les responsables financiers des établissements d'enseignement supérieur ont des données de recettes et de dépenses individualisées par chercheur et autre membre du personnel. Cependant, ces informations ne sont pas nécessairement conformes aux 
définitions du Manuel, ce qui limite la possibilité de les exploiter directement (même si elles demeurent utiles pour dériver des coefficients d'estimation voir ci-après).

9.118 Pour identifier la R-D par discipline ou par domaine, il faut parfois s'adresser aux chercheurs eux-mêmes ou aux instituts ou départements des grands établissements qui mènent des recherches dans de nombreuses disciplines. Les informations disponibles au niveau de l'unité institutionnelle suffisent si celle-ci n'aborde qu'un domaine de R-D.

\section{Méthodes d'estimation}

9.119 Les enquêtes et les données administratives (si elles sont compilées en respectant les définitions et les consignes du présent manuel) sont les meilleurs moyens de recueillir des informations sur le secteur de l'enseignement supérieur. Cependant, elles ne sont pas toujours adaptées aux ressources, au cadre juridique ou aux besoins des différents pays. Si, pour une raison ou une autre, il n'est pas possible de conduire une enquête complète ou d'utiliser les données administratives pour déterminer les dépenses ou le personnel de R-D dans le secteur de l'enseignement supérieur, on peut associer des techniques d'estimation à des données d'enquête ou à des données administratives.

\section{Coefficients de R-D}

\section{Objet des coefficients}

9.120 Les coefficients de R-D permettent de calculer ou d'estimer la part du personnel et des dépenses affectée à la R-D. Ils servent en particulier à répartir l'ensemble des ressources entre les activités de recherche, d'enseignement et autres (notamment administratives). Ils peuvent servir à estimer le total des DIRDES, ou certaines composantes de ces dépenses, comme celle financée par les FGU, ou uniquement les données totales relatives au personnel de R-D.

\section{Concepts}

9.121 Les coefficients peuvent être obtenus de différentes façons pour remplacer des enquêtes à grande échelle, plus coûteuses, ou pour compléter des enquêtes. La méthode retenue dépend du contexte national, aussi n'existe-t-il pas de solution universelle. Différentes méthodes sont décrites ci-dessous :

- exploitation directe de données administratives (de registres administratifs) : pertinente dans certains cas, mais difficile à mettre en pratique dans la plupart des pays

- estimations établies par des spécialistes à partir de données administratives

- calculs effectués à partir d'enquêtes sur l'emploi du temps (voir instructions ci-après).

9.122 Aux fins de contrôle de qualité, il doit être envisagé de communiquer des métadonnées sur les méthodes de calcul des coefficients. 


\section{Méthodes}

- Les coefficients de R-D sont appliqués directement au niveau approprié (individu, institut, département, université) pour estimer la part de R-D dans le montant total des coûts de main-d'œuvre ; le cas échéant, on procède à des ajustements pour tenir compte du coût des divers régimes de sécurité sociale ou de retraite.

- On peut s'attendre à ce que les coefficients de R-D varient suivant la discipline d'enseignement ou de recherche, la catégorie professionnelle du personnel qui participe directement à la R-D et le type d'établissement dans lequel l'activité se déroule. Au plus haut degré de détail, les coefficients peuvent s'appliquer aux données relatives au financement et au personnel d'un établissement donné.

\section{Les coefficients sont généralement appliqués par étapes}

- L'application des coefficients de R-D aux différentes catégories de personnel, si possible par discipline et par établissement, permet d'obtenir des estimations du personnel en équivalent temps plein (ETP).

- Ces estimations, converties elles-mêmes en coefficient, peuvent ensuite s'appliquer aux données financières pour fournir une estimation des dépenses de R-D.

9.123 En l'absence de données d'enquête directement exploitables, les coefficients sont le seul moyen d'estimer la part de la R-D dans les coûts de main-d'œuvre. Ils jouent un rôle important pour évaluer cette part dans d'autres dépenses courantes, mais présentent un intérêt mineur pour calculer la part d'utilisation du matériel et des équipements ou des terrains et des constructions dans des activités de R-D.

9.124 Lors de la communication des données aux fins de comparaisons internationales, les statisticiens de la R-D sont encouragés à indiquer à quels ensembles de dépenses et de données de personnel ils ont appliqué des coefficients pour calculer les données de R-D et à préciser les coefficients utilisés. Ces métadonnées pourraient être recueillies tous les deux ans environ, parallèlement à la collecte régulière des données, et mises à disposition en ligne.

\section{Enquêtes sur l'emploi du temps}

9.125 Si l'on ne peut pas établir les coefficients nécessaires à partir d'autres enquêtes ou données administratives, il est recommandé d'utiliser des enquêtes sur l'emploi du temps pour obtenir les informations nécessaires à l'estimation de la part de R-D dans les équivalents temps plein et les dépenses. Dans le but de réduire les écarts dus aux diverses méthodes possibles de conduite de ce type d'enquêtes, il est proposé ci-après, à titre facultatif, des lignes directrices permettant de concevoir des enquêtes plus uniformes. 


\section{Recensement ou enquête par sondage}

9.126 Du fait de la très grande hétérogénéité des situations nationales (cadre juridique, taille du pays, etc.), il n'est pas possible de recommander un recensement pour tous les pays. Lorsqu'on procède par sondage, l'échantillon doit être représentatif des catégories de salariés considérées au cours d'une année universitaire normale, et stratifié par domaine de recherche et de développement.

\section{Unité déclarante}

9.127 Dans une enquête sur l'emploi du temps, l'unité déclarante à privilégier doit être le chercheur, et non l'administration de l'université.

\section{Catégories de salariés considérées}

9.128 Les enquêtes sur l'emploi du temps doivent prendre en considération, au minimum, les chercheurs salariés (c'est-à-dire le personnel interne ; voir le chapitre 5) participant aux activités de R-D au sein des établissements d'enseignement supérieur, et y ajouter, si possible, d'autres membres du personnel de R-D, tels que les chercheurs sous contrat (personnel externe de R-D), les techniciens et le personnel de soutien.

\section{Types d'activités}

9.129 Les enquêtes doivent porter principalement sur le temps consacré à l'exécution de la R-D. Elles doivent présenter une liste standard et compréhensible d'activités, autour de trois activités principales :

1. R-D

- R-D

- Administration de la R-D

2. Enseignement

- Enseignement

- Administration de l'enseignement

3. Autres travaux : tous les autres travaux

9.130 Pour répondre à leurs besoins spécifiques, la plupart des pays recueillent des informations plus précises sur une liste plus détaillées d'activités. Il est recommandé de prévoir le regroupement de celles-ci dans l'une ou l'autre des trois activités principales mentionnées ci-dessus.

\section{Période}

9.131 La période de référence utilisée dans les questionnaires sur l'emploi du temps peut être très différente selon les pays (d'une année entière à une ou deux semaines, en passant par des enquêtes organisées sur le modèle des inventaires tournants). S'il n'est pas possible de conduire une enquête complète, il faut s'assurer que les estimations couvrent les divers types d'activités menées tout au long de l'année. Toutes les périodes caractéristiques sur une année doivent être traitées. L'une des approches possibles consiste à prendre en 
considération une première semaine type pendant la période de cours et une seconde hors période de cours. Compte tenu des différences dans l'organisation de l'enseignement supérieur, la période de référence doit être choisie par chaque pays.

\section{Périodicité des enquêtes sur l'emploi du temps}

9.132 Il est préférable de mener les enquêtes à intervalles réguliers, mais la périodicité dépendra de la taille du pays, de son cadre juridique et des ressources dont il dispose pour procéder à ces enquêtes. Il est néanmoins proposé, dans la mesure du possible, de ne pas laisser passer plus de cinq ans entre chaque enquête.

\section{Procédure à suivre pendant les années intermédiaires}

9.133 Si les enquêtes sont espacées de plus de deux ans, on peut envisager de réaliser des prévisions en temps réel de l'évolution potentielle des coefficients, en se fondant, par exemple, sur les changements intervenus dans la structure du personnel universitaire.

\section{Heures de travail contractuelles}

9.134 En introduction de l'enquête sur l'emploi du temps, il est recommandé de recueillir le nombre d'heures de travail contractuel pendant la semaine (ou les semaines) de référence, puis de communiquer la distribution relative des différentes activités en pourcentage (pour la définition des heures de travail, voir le chapitre 5).

\section{Calcul des FGU}

9.135 Les données sur les fonds généraux des universités figurent généralement dans les registres des établissements. Un nombre croissant de pays recueillent les données sur les FGU au moyen d'enquêtes sur la R-D. Dans certains cas, c'est le montant total de la " dotation forfaitaire " qui est collecté à l'aide de l'enquête, et la part de la R-D (FGU consacrés à la R-D) est ensuite estimée à l'aide de coefficients dérivés d'enquêtes sur l'emploi du temps.

9.136 Dans les pays où il n'y a pas d'enquête sur les DIRDES, les données sont compilées en combinant différentes sources et en appliquant des coefficients qui sont le plus souvent dérivés des enquêtes sur l'emploi du temps. Dans certains cas, les FGU sont calculés en déduisant les autres sources de financement du montant des DIRDES.

9.137 Les enquêtes sur l'emploi du temps et d'autres méthodes visant à isoler la part de R-D dans l'ensemble des activités des universités servent principalement à calculer les FGU, qui financent la majorité des dépenses de R-D de l'enseignement supérieur dans de nombreux pays (voir section 9.4). La dotation forfaitaire publique des établissements couvre toutes les activités de base : enseignement, R-D, supervision, administration, loyers et autres frais généraux. Étant donné que les universités elles-mêmes ne connaissent généralement pas la part de la R-D dans cette dotation, l'application de coefficients est la méthode la plus pratique pour la déterminer. Différentes méthodes sont utilisées à cet effet. 


\subsection{Liens avec les statistiques de l'éducation}

9.138 Les données sur les dépenses de R-D dans le secteur de l'enseignement supérieur sont aussi recueillies dans le cadre de la collecte de données de l'UNESCO / OCDE / Eurostat (UEO) sur les statistiques de l'éducation. Un manuel méthodologique conjoint de l'UEO (UEO, 2014), élaboré par des statisticiens de l'éducation, expose les concepts, les définitions et les classifications à utiliser pour communiquer les données au niveau international. Les indications fournies dans ce manuel pour faire état des données de R-D s'inspirent du Manuel de Frascati. Des statisticiens de l'éducation et de la R-D ont collaboré pendant plusieurs dizaines d'années, et continueront à collaborer, dans le but d'harmoniser les lignes directrices fournies dans les deux manuels. Il semble inévitable que certaines disparités dans les données persistent du fait la nature différente des deux collectes, mais l'expérience a montré que la coordination entre les pourvoyeurs de données des deux côtés contribuait à réduire ces disparités.

\section{Références}

CE (2013), She Figures 2012: Statistics and Indicators - Gender in Research and Innovation, Commission européenne, Bruxelles, http://ec.europa.eu/research/sciencesociety/document_library/pdf_06/she-figures-2012_en.pdf.

CE, FMI, OCDE, Nations Unies et Banque mondiale (2009), Système de comptabilité nationale 2008, Nations unies, New York, http://unstats.un.org/unsd/nationalaccount/ docs/SNA2008FR.pdf.

Nations Unies (2008), "Classification internationale type, par industrie, de toutes les branches d'activité économique (CITI), Révision 4 ", Département des affaires économiques et sociales, Division de statistique, Études statistiques, Série $M, n^{\circ} 4$, Rév. 4, Nations Unies, New York, https://unstats.un.org/unsd/cr/registry/isic-4.asp et http://unstats.un.org/unsd/publication/seriesM/seriesm_4rev4f.pdf.

UNESCO-ISU (2012), Classification internationale type de l'éducation (CITE) 2011, ISU, Montréal, http://www.uis.unesco.org/Education/Documents/isced-2011-fr.pdf.

UNESCO-ISU/OCDE/Eurostat (2014), UOE data collection on formal education: Manual on concepts, definitions and classifications, version du 5 septembre 2014, ISU, Montréal, Éditions OCDE, Paris, Eurostat, Luxembourg, https://circabc.europa.eu/sd/a/38b873d64694-459f-ae56-d5025f3d7cf3/UOE2014manual.pdf. 



\section{Chapitre 10}

\section{La R-D du secteur privé sans but lucratif}

Les unités institutionnelles du secteur privé sans but lucratif jouent depuis longtemps un rôle important dans les activités de R-D de nombreux pays. Des institutions sans but lucratif (ISBL) peuvent être recensées et classées dans tous les secteurs ; il peut s'agir de producteurs marchands ou non, qui comprennent à la fois des exécutants de la R-D et des bailleurs de fonds. On trouvera dans ce chapitre une description des ISBL à prendre en considération dans la mesure du secteur privé sans but lucratif, ainsi que des lignes directrices sur la méthode à appliquer pour mesurer leurs activités de R-D, compte tenu de leurs spécificités et de l'apparition de nouvelles formes de financement de la $R-D$. Le secteur est résiduel, en ce sens qu'il regroupe les ISBL qui n'ont pas été classées dans le secteur des entreprises, le secteur de l'État ou celui de l'enseignement supérieur. Dans un but d'exhaustivité, il inclut aussi les ménages et les individus engagés ou non dans des activités de production marchande. Ce chapitre fournit aussi des indications utiles sur les classifications institutionnelles par activité économique principale, la mesure des dépenses et du personnel de R-D dans le secteur privé sans but lucratif, ainsi que la conception des enquêtes et la collecte des données dans ce secteur. Enfin - nouveauté dans la présente édition du Manuel -, il aborde brièvement le rôle des philanthropes et du financement participatif et ses implications pour le travail de mesure. 


\subsection{Introduction}

10.1 Les unités institutionnelles appartenant au secteur privé sans but lucratif jouent depuis longtemps un rôle significatif dans les activités de R-D de nombreux pays. Leur importance a été reconnue dans les versions antérieures du Manuel. Comme indiqué au chapitre 3, des institutions sans but lucratif (ISBL) peuvent être recensées et classées dans tous les secteurs : il peut s'agir de producteurs marchands ou non, qui comprennent à la fois des exécutants et des bailleurs de fonds de la R-D. On s'intéressera ici aux ISBL à prendre en considération dans la mesure du dans le secteur privé sans but lucratif, et on trouvera des lignes directrices sur la méthode à appliquer pour mesurer leurs activités de R-D, compte tenu de leurs spécificités, de l'émergence de nouvelles tendances et de nouvelles formes de financement de la R-D.

\section{2. Étendue du secteur privé sans but lucratif}

\section{Définition du secteur privé sans but lucratif aux fins de la mesure de la R-D}

10.2 Ce secteur comprend:

- toutes les institutions sans but lucratif au service des ménages (ISBLSM), telles que définies dans le SCN 2008, à l'exception de celles relevant du secteur de l'enseignement supérieur

- et, à des fins d'exhaustivité, les ménages et les particuliers qui mènent ou non des activités marchandes.

10.3 Comme exemples d'unités dans ce secteur, on peut citer : les professionnels indépendants, les sociétés savantes et les organisations caritatives qui ne dépendent pas du secteur de l'État ou du secteur des entreprises. Les ISBL fournissent, individuellement ou collectivement, des services aux ménages de manière gratuite ou à des prix économiquement non significatifs. En pratique, les institutions de ce secteur sont désignées par l'un des noms suivants : fondation, association, consortium, coentreprise, organisation philanthropique ou organisation non gouvernementale (ONG). Néanmoins, les institutions, les individus et les ménages doivent être assignés aux secteurs pertinents conformément aux indications du présent Manuel, quel que soit leur intitulé générique.

\section{Aspect résiduel du secteur}

10.4 Le secteur privé sans but lucratif, tel que défini ci-dessus, est un secteur résiduel. En effet, aux termes des définitions présentées ailleurs dans 
le Manuel (voir les chapitres 6 et 9), les unités privées sans but lucratif qui fournissent des services d'enseignement supérieur ou qui sont contrôlées par un établissement d'enseignement supérieur sont à classer dans le secteur de l'enseignement supérieur. De même, les unités privées sans but lucratif que l'État possède ou contrôle doivent être classées dans le secteur de l'État si elles n'ont pas d'activités marchandes. Les unités sans but lucratif contrôlées par des entreprises ou fournissant principalement des services à celles-ci sont à classer dans le secteur des entreprises. Enfin, les activités marchandes des entreprises non constituées en sociétés qui appartiennent à un individu ou un ménage, par exemple les consultants indépendants qui réalisent des projets de R-D pour une autre unité à un prix économiquement significatif, devraient être incluses dans le secteur des entreprises. Le tableau 10.1 décrit le cadre à suivre pour les différentes catégories d'ISBL.

\section{Tableau 10.1. Traitement des différentes catégories d'institutions sans but lucratif (ISBL)}

\begin{tabular}{|c|c|c|c|c|}
\hline $\begin{array}{l}\text { Critère SCN - but } \\
\text { économique } \\
\text { principal }\end{array}$ & $\begin{array}{c}\text { Critère SCN } \\
\text { supplémentaire - } \\
\text { contrôle/secteur desservi }\end{array}$ & Traitement SCN & Cas particuliers & Traitement MF \\
\hline \multirow[t]{3}{*}{$\begin{array}{l}\text { Production } \\
\text { marchande }\end{array}$} & \multirow{2}{*}{$\begin{array}{l}\text { ISBL indépendantes } \\
\text { mais engagées } \\
\text { principalement dans la } \\
\text { production marchande }\end{array}$} & \multirow[t]{2}{*}{ Sociétés } & $\begin{array}{l}\text { (Certaines) universités } \\
\text { privées }\end{array}$ & $\begin{array}{l}\text { Secteur de } \\
\text { l'enseignement } \\
\text { supérieur }\end{array}$ \\
\hline & & & $\begin{array}{l}\text { (Certaines) universités } \\
\text { privées }\end{array}$ & $\begin{array}{l}\text { Secteur des } \\
\text { entreprises, sauf } \\
\text { les hôpitaux } \\
\text { universitaires }\end{array}$ \\
\hline & $\begin{array}{l}\text { ISBL fournissant des } \\
\text { services aux entreprises } \\
\text { (nationales ou } \\
\text { non résidentes) }\end{array}$ & Sociétés & $\begin{array}{l}\text { Instituts de recherche } \\
\text { financés par l'industrie }\end{array}$ & $\begin{array}{l}\text { Secteur des } \\
\text { entreprises }\end{array}$ \\
\hline \multirow[t]{5}{*}{$\begin{array}{l}\text { Production non } \\
\text { marchande }\end{array}$} & \multirow[t]{2}{*}{ Contrôlées par l'État } & $\begin{array}{l}\text { Administrations } \\
\text { publiques }\end{array}$ & $\begin{array}{l}\text { Fondations de R-D } \\
\text { contrôlées par l'État }\end{array}$ & Secteur de l'État \\
\hline & & $\begin{array}{l}\text { Administrations } \\
\text { publiques }\end{array}$ & $\begin{array}{l}\text { Universités contrôlées } \\
\text { par l'État }\end{array}$ & $\begin{array}{l}\text { Secteur de } \\
\text { l'enseignement } \\
\text { supérieur }\end{array}$ \\
\hline & \multirow[t]{3}{*}{ Non contrôlées par l'État } & \multirow[t]{3}{*}{$\begin{array}{l}\text { Institutions } \\
\text { sans but lucratif } \\
\text { au service } \\
\text { des ménages } \\
\text { (ISBLSM) }\end{array}$} & $\begin{array}{l}\text { Organisations } \\
\text { philanthropiques } \\
\text { indépendantes d'aide à } \\
\text { la recherche, sociétés } \\
\text { savantes, etc. (recevant } \\
\text { parfois des aides très } \\
\text { importantes de l'État mais } \\
\text { ce dernier ne peut dicter } \\
\text { les décisions majeures) }\end{array}$ & \multirow[t]{2}{*}{$\begin{array}{l}\text { Secteur sans but } \\
\text { lucratif }\end{array}$} \\
\hline & & & $\begin{array}{l}\text { ISBL résidentes contrôlées } \\
\text { par des entités sans but } \\
\text { lucratif non résidentes }\end{array}$ & \\
\hline & & & $\begin{array}{l}\text { Universités indépendantes } \\
\text { ayant le statut } \\
\text { d'organisation caritatives }\end{array}$ & $\begin{array}{l}\text { Secteur de } \\
\text { l'enseignement } \\
\text { supérieur }\end{array}$ \\
\hline
\end{tabular}


10.5 On notera que, dans certains cas, il n'est guère aisé d'établir la structure de contrôle, car le pouvoir de décider l'affectation et le montant du financement est un moyen de contrôle majeur. C'est pourquoi il est parfois indiqué d'inclure la source principale de financement comme critère supplémentaire pour déterminer si une institution est contrôlée ou non par l'État (pour des indications plus détaillées sur la notion de contrôle, voir l'encadré 8.1 du chapitre 8).

10.6 Les institutions privées sans but lucratif qui n'ont pas une identité propre distincte de celle de leurs propriétaires, comme la plupart des entités non constituées en sociétés, des consortiums ou des organisations regroupant des entreprises, des institutions de recherche, des universités ou des associations, ne sont pas des unités institutionnelles au sens défini dans le présent manuel. Par conséquent, les activités de R-D de ces institutions doivent être attribuées à chacun de leurs membres sur la base de son apport propre. Autrement dit, la contribution de toute unité d'un secteur quelconque qui soutient les activités de R-D d'une institution sans but lucratif non constituée en société devrait être comptabilisée comme R-D intra-muros et non comme R-D extra-muros.

10.7 Les activités de R-D des instituts privés sans but lucratif constitués en sociétés dont les membres appartiennent à deux ou plusieurs secteurs sont à attribuer conformément aux indications du chapitre 3 .

10.8 Le traitement des individus mérite une attention particulière. Premièrement, les individus qui sont des salariés ou travaillent pour des institutions, y compris les travailleurs indépendants, ne sont pas inclus dans ce secteur. Deuxièmement, les activités de recherche ou d'invention des individus qui poursuivent de telles activités par intérêt personnel pendant leurs heures libres ne sont pas actuellement prises en compte dans l'approche institutionnelle des statistiques de R-D adoptée dans le présent manuel. Enfin, le traitement à appliquer aux individus qui font partie d'un groupe de personnes occupées dans une unité institutionnelle, mais non comme salariés, et qui reçoivent directement le financement de tiers pour des activités de R-D est décrit au chapitre 5 .

\section{Démarcation vis-à-vis des autres secteurs}

10.9 Lorsqu'il existe des liens étroits entre des unités sans but lucratif et l'État, il n'est pas toujours facile de déterminer dans quel secteur une unité sans but lucratif particulière doit être classée. De nombreuses fondations ou organisations caritatives financées initialement ou principalement par des donneurs individuels reçoivent aussi une part importante de leur financement de l'État. Ces unités sont généralement classées dans le secteur privé sans but lucratif. La démarcation vis-à-vis du secteur de l'État doit se fonder sur le degré de contrôle qu'une unité sans but lucratif exerce sur ses modalités de fonctionnement (voir les chapitres 3 et 8).

10.10 On notera que certaines coentreprises ou consortiums créés entre des entreprises et des établissements d'enseignement supérieur et qui sont constitués en sociétés peuvent être classés dans le secteur privé sans but 
lucratif. Certains partenariats public-privé peuvent aussi, en fonction de leur statut juridique, être inclus dans le secteur privé sans but lucratif.

10.11 Il existe de nombreuses institutions privées sans but lucratif dont les membres et/ou les activités enjambent les frontières internationales. Les critères de résidence qui s'appliquent aux entreprises s'appliquent également à ces institutions. Pour être considérée comme une unité résidente, une institution doit avoir son centre d'intérêt économique prépondérant dans l'économie concernée. On trouvera des indications supplémentaires à ce sujet au chapitre 11 (voir, par exemple, la section 11.6 sur le cas particulier des organisations internationales).

\subsection{Classifications institutionnelles recommandées pour le secteur privé sans but lucratif}

\section{Classification par activité économique principale}

10.12 Il est recommandé de classer les unités statistiques qui exécutent des activités de R-D dans ce secteur par activité économique principale, sur la base de la classification CITI ou d'une classification nationale équivalente (Nations Unies, 2008).

10.13 Dans certains pays, il peut être utile de classer certaines institutions dans le secteur privé sans but lucratif sur la base du but déclaré de leur activité. La Classification des fonctions des institutions sans but lucratif au service des ménages (COPNI), conçue à l'origine comme un prolongement de la classification CITI pour les ISBL, est un outil de référence en ce domaine (Nations Unies, 2000). Cependant, le présent manuel ne recommande pas explicitement d'utiliser les critères de classification de la COPNI pour le secteur sans but lucratif. On trouvera des informations plus détaillées sur ces classifications dans le complément du

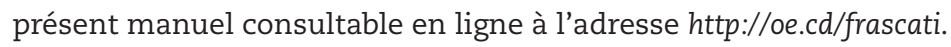

\section{Qualifications possibles}

10.14 Conformément au chapitre 3 et afin de satisfaire aux normes du SCN, il est suggéré de qualifier les unités statistiques conduisant la R-D dans ce secteur comme des institutions sans but lucratif au service des ménages (ISBLSM) ou comme des ménages, ces derniers ne devant pas être pris en compte dans l'enquête selon les recommandations du présent manuel.

10.15 La qualification des ISBL appartenant à d'autres secteurs, en se servant des registres statistiques, permettra d'obtenir les performances totales des entités sans but lucratif. Pour cela, il faudra additionner :

- la R-D des entités sans but lucratif (par exemple la R-D des ISBL du secteur privé sans but lucratif, tel que défini dans ce manuel)

- la R-D des ISBL au service des entreprises et d'autres ISBL engagées dans des activités de production marchande comptabilisées dans le secteur des entreprises (voir le chapitre 7) 
- la R-D des ISBL contrôlées par l'État (voir le chapitre 8)

- la R-D des ISBL du secteur de l'enseignement supérieur (voir le chapitre 9).

\subsection{Identification de la $R-D$ dans le secteur privé sans but lucratif}

10.16 Les activités de R-D menées dans le secteur privé sans but lucratif portent sur des domaines très divers. Selon le type d'unité sans but lucratif, que ce soit en termes de composition ou de finalité, il est plus ou moins difficile de distinguer la R-D d'autres activités menées par l'unité. Par exemple, un nombre non négligeable d'unités de ce secteur, comme les fondations de recherche, regroupent des entités qui sont elles-mêmes des organisations vouées à la recherche. L'identification des activités de R-D de ces unités est donc plus facile que celles des institutions privées sans but lucratif dont les finalités sont plus étendues et ne se limitent pas à la science ou la recherche.

10.17 De nombreuses fondations ou organisations caritatives sont actives, entre autres, dans le domaine de la santé, de l'environnement, de l'éducation, de l'aide sociale et de l'aide au développement. Ces organisations conduisent parfois certains types de recherche ou réalisent des études préliminaires en relation avec leurs activités. La difficulté est alors de déterminer si les travaux qu'elles mènent répondent aux critères définis au chapitre 2 et peuvent donc être considérés comme relevant de la R-D. Dans d'autres cas, leurs activités elles-mêmes impliquent un élément de R-D qui doit être clairement identifié.

10.18 Les efforts de R-D peuvent étayer la prise de décisions au sein des ISBL. S'il est possible de les externaliser, il arrive aussi que des unités du secteur privé sans but lucratif disposent en interne d'équipes spécialement chargées de participer activement au travail d'analyse, par exemple en réalisant des évaluations et bilans ponctuels, voire formalisés. Il est possible que ces activités répondent aux critères définissant une activité de $R-D$, mais ce n'est pas toujours le cas, de même qu'il n'y a pas lieu de qualifier de R-D la totalité des efforts d'établissement des faits associés à des analyses et évaluations programmatique. Il convient donc d'examiner, à un certain niveau de détail, les compétences techniques des personnes impliquées dans l'activité, le mode de codification des connaissances adopté au sein de l'organisation et la manière dont il est veillé au respect des normes de qualité dans les thèmes de recherche et la méthodologie appliquée. Le risque que des activités de conseil socioéconomique (internes ou externes) soient présentées à tort comme de la R-D n'est pas négligeable.

10.19 Dans le domaine de la santé, il est nécessaire de bien distinguer les activités relevant des soins de santé (voir chapitre 9 sur le secteur de l'enseignement supérieur, section 9.3) et les phases d'essais cliniques qui sont considérées comme de la R-D (voir chapitre 2, section 2.7 sur les définitions).

10.20 Le chapitre 2 fournit des informations complémentaires utiles sur la démarcation de la R-D d'avec les activités d'éducation et d'autres activités 
scientifiques et technologiques. Il donne aussi des exemples d'activités de R-D dans le domaine des sciences sociales et humaines, et dans celui des services.

\subsection{Mesure des dépenses et du personnel de R-D dans le secteur privé sans but lucratif}

\section{Dépenses intérieures de R-D des institutions sans but lucratif (DIRDISBL)}

10.21 Les dépenses intérieures de R-D des institutions sans but lucratif (DIRDISBL) constituent le principal agrégat statistique utilisé pour décrire l'exécution de la R-D au sein du secteur privé sans but lucratif. Elles correspondent à la composante des dépenses intérieures brutes de R-D (DIRD) (voir chapitre 4) engagées par les unités du secteur privé sans but lucratif et sont l'expression chiffrée des dépenses de R-D intra-muros de ce secteur au cours d'une période de référence donnée. En règle générale, les dépenses de $\mathrm{R}-\mathrm{D}$ intra-muros dans le secteur privé sans but lucratif doivent être mesurées conformément aux recommandations présentées au chapitre 4 , section 4.2 .

10.22 Certaines ISBL jouent à la fois un rôle de financement et d'exécution des activités de R-D. En pareil cas, les dépenses intra-muros que consacre l'institution à la conduite d'activités de R-D doivent être distinguées des dépenses afférentes aux activités de R-D conduites par des unités extérieures, c'est-à-dire du financement de la R-D extra-muros par le secteur privé sans but lucratif. Cependant, le financement des ISBL reçu d'autres ISBL pour la conduite d'activités de R-D intra-muros devrait être déclaré par les ISBL bénéficiaires comme un financement extérieur provenant d'autres ISBL.

10.23 Certaines institutions de ce secteur servent aussi parfois d'intermédiaires dans les flux de financement entre les bailleurs de fonds et les unités qui exécutent effectivement la R-D. Comme indiqué au chapitre 4, le financement extérieur reçu par une unité et transmis ensuite à d'autres ne devrait pas être comptabilisé comme financement de la R-D par l'unité.

\section{Répartition fonctionnelle des DIRDISBL}

\section{Répartition des DIRDISBL par source de financement}

10.24 Il convient de donner la priorité à la répartition des dépenses de R-D par source de financement, conformément aux instructions fournies au chapitre 4 du présent manuel.

10.25 Certains philanthropes et organisations caritatives d'aide à la recherche à partir des dons du public soutiennent aussi financièrement des activités de R-D. Celles-ci portent généralement sur des domaines ou des sujets spécifiques et sont conduites principalement dans des universités, des instituts de recherche et des hôpitaux. Le financement participatif, apparu depuis peu et qui fait appel aux individus et aux ménages pour soutenir la R-D et d'autres activités, constitue aujourd'hui une nouvelle source de financement privée. 
10.26 Les données relatives au financement de la R-D par des institutions, des individus et des ménages dans le secteur privé sans but lucratif doivent être recueillies auprès des exécutants de la $\mathrm{R}-\mathrm{D}$ dans tous les secteurs, y compris le secteur privé sans but lucratif, et comptabilisées malgré le caractère résiduel du secteur et sa taille souvent réduite.

10.27 Les individus et les ménages peuvent être pris en compte comme sources de financement de la R-D (alors qu'ils sont exclus du champ de l'enquête comme exécutants de la R-D). Pour aligner les sources de financement sur la ventilation du SCN, on distinguera entre, d'un côté, les instituts qui font partie des ISBLSM et, de l'autre, les individus et les ménages.

10.28 Comme on l'a vu au chapitre 4, seul le financement fourni explicitement en vue de la conduite d'activités de R-D par une unité statistique devrait être comptabilisé comme un financement externe. Les fonds alloués dans un but général aux instituts qui exécutent la R-D, ainsi que les aides, subventions, dons ou autres formes de soutien philanthropique qu'une unité statistique est libre d'utiliser à sa guise, doivent être comptabilisés comme financement interne uniquement s'ils sont utilisés pour des activités de R-D.

\section{Autres répartitions recommandées des DIRDISBL}

10.29 Il est recommandé de répartir les dépenses de R-D intra-muros du secteur privé sans but lucratif par catégorie de coûts de R-D, comme indiqué en détail au chapitre 4 (tableau 4.1). Cela implique notamment de ventiler les dépenses intra-muros entre les coûts salariaux du personnel de R-D, les autres dépenses courantes et les dépenses en capital (par type d'actif).

10.30 Il est recommandé de répartir les DIRDISBL par type de R-D (voir chapitre 2) et par domaine de recherche et développement. Au minimum, il faudrait prendre en compte le niveau supérieur de la classification des domaines de recherche et développement.

10.31 On pourra aussi envisager de répartir les données de DIRDISBL par objectif socioéconomique, en se basant sur les catégories de la Nomenclature pour l'analyse et la comparaison des budgets et programmes scientifiques (NABS) (Eurostat, 2008) ou d'autres versions nationales ayant une correspondance directe avec cette nomenclature.

10.32 Les catégories de la classification COPNI (voir section 10.3), bien que non explicitement recommandées dans le présent manuel, pourront également être utilisées pour répartir les données de DIRDISBL.

\section{Dépenses de R-D extra-muros du secteur privé sans but lucratif}

10.33 Comme indiqué au chapitre 4, section 4.3, le financement de la R-D extra-muros doit être mesuré au moyen d'enquêtes auprès des exécutants de R-D du secteur privé sans but lucratif, en distinguant entre les bénéficiaires affiliés et non affiliés. Lors de la collecte de ces données, le risque existe que les unités déclarantes du secteur privé sans but lucratif déclarent ici le financement 
versé pour la R-D exécutée non seulement par d'autres institutions sans but lucratif mais aussi par des individus ou des ménages, ces deux dernières catégories entrant dans la définition du secteur privé sans but lucratif mais ne devant pas être prises en compte dans une enquête institutionnelle sur la R-D. Les enquêteurs doivent donc veiller à fournir des instructions pertinentes pour assurer que seul soit inclus le financement des activités extra-muros répondant aux critères de la définition de la R-D.

\section{Personnel de R-D du secteur privé sans but lucratif}

10.34 L'effectif du personnel de R-D et, en particulier, des chercheurs doit être mesuré conformément aux recommandations formulées au chapitre 5 . Les totaux doivent inclure le personnel de R-D interne et externe comme recommandé pour les autres secteurs. On notera que dans le secteur privé sans but lucratif, la R-D peut être exécutée par des professionnels externes indépendants, qui agissent comme des consultants intra-muros mais appartiennent en fait au secteur des entreprises.

10.35 On notera également que le personnel interne de R-D peut inclure des travailleurs indépendants (parmi les "personnes occupées à la R-D ") dans le secteur privé sans but lucratif, qui comprend normalement les ménages (voir chapitre 5).

10.36 Lors du travail de mesure, il convient de ne pas oublier que le secteur privé sans but lucratif comprend souvent des individus qui contribuent à la R-D intra-muros de manière essentiellement bénévole. Comme indiqué plus en détail au chapitre 5 , les bénévoles sont des travailleurs non rémunérés qui apportent à une unité statistique une contribution définie en matière de R-D. Les bénévoles ne peuvent être inclus dans le total du personnel externe de R-D que sous certaines conditions très strictes:

- s'ils contribuent aux activités de R-D intra-muros d'une institution privée sans but lucratif

- si leurs compétences de recherche sont comparables à celles des salariés

- si leurs activités de R-D sont planifiées de manière systématique, à la fois sur la base des besoins des bénévoles eux-mêmes et de ceux de l'institution.

10.37 Leur contribution doit être appréciable et constituer une condition essentielle du lancement d'une activité ou d'un projet de R-D intra-muros par l'institution. La déclaration séparée du nombre de bénévoles contribuant à la R-D dans le secteur privé sans but lucratif pourra présenter un intérêt particulier (pour plus de précisions, voir le chapitre 5).

10.38 Les doctorants et les détenteurs d'une bourse de R-D contribuent occasionnellement aux activités de R-D dans ce secteur.

10.39 Le coût de ces catégories particulières de personnel est fréquemment inclus comme « autres dépenses courantes » ou n'est pas déclaré. 


\subsection{Conception de l'enquête et collecte des données dans le secteur privé sans but lucratif}

\section{Conception de l'enquête}

\section{Identification des unités statistiques : pratique et difficultés}

10.40 Comme on l'a signalé au chapitre 6, l'information de référence est parfois moins détaillée pour ce secteur. La liste des unités statistiques et des unités déclarantes doit être établie et mise à jour à l'aide de sources habituelles comme les registres d'entreprises, les annuaires d'institutions de R-D, les listes d'associations et les résultats des enquêtes précédentes. Les pays peuvent inclure des questions sur l'exécution de la R-D dans les enquêtes à caractère plus général visant les ISBL en vue d'identifier les unités statistiques qui exécutent éventuellement des activités de R-D.

10.41 Seules les institutions remplissant les conditions d'exécution de la $\mathrm{R}-\mathrm{D}$ décrites dans ce manuel sont à identifier comme unités statistiques possibles aux fins de la mesure de la R-D. Conformément à la démarche institutionnelle adoptée pour mesurer la R-D, les individus et les ménages doivent être exclus de la population cadre.

10.42 Plus encore que pour d'autres catégories d'institutions, le contrôle des ISBL peut changer avec le temps, par exemple lorsque l'État acquiert un contrôle plus important. Si cela se produit, on veillera soigneusement à ce que les institutions soient réassignées à d'autres secteurs conformément aux définitions du chapitre 3.

\section{Incidences du financement de la R-D par des individus}

10.43 Certaines formes de financement de la R-D par des individus présentent des caractéristiques tout à fait nouvelles ou bien réactivent des modes de financement qui jouaient un rôle de premier plan il y a plusieurs décennies. De riches philanthropes, par exemple, jouent parfois un rôle majeur dans le financement d'instituts de recherche ou la promotion d'initiatives de recherche dans divers domaines. Ils le font soit individuellement, soit par l'intermédiaire d'institutions mixtes ou philanthropiques comme les fondations et les trusts. Le présent manuel recommande de mesurer ce type de financement principalement du point de vue des exécutants de la R-D. Ce financement doit être saisi en termes bruts, quelle que soit l'étendue des avantages fiscaux dont bénéficient potentiellement les donneurs.

10.44 Le financement participatif rendu possible par les nouvelles technologies en ligne est aussi une nouvelle source, potentiellement prometteuse, de financement de la R-D. La méthode de financement participatif consiste à financer une initiative ou un projet en sollicitant et en recueillant les contributions monétaires d'un grand nombre d'individus, le plus souvent via l'internet. Les individus peuvent répondre à des appels au financement 
participatif d'activités de R-D, par exemple dans le domaine de la santé et de la recherche médicale, auxquelles ils sont parfois directement intéressés en tant que patients.

10.45 Le financement participatif de la R-D constitue un nouveau modèle de financement qui ne permet pas nécessairement aux individus d'acquérir une participation mais d'autres types d'avantages, par exemple le droit de nommer une nouvelle découverte, la mention de leur nom dans des articles scientifiques, la possibilité de visiter des lieux de recherche ou des déductions fiscales.

10.46 Ces nouvelles pratiques de financement peuvent donner lieu à une sous-estimation des dépenses intérieures de R-D si leurs bénéficiaires n'ont pas été identifiés au moyen d'enquêtes ou de méthodes connexes. Les données recueillies à partir des bailleurs de fonds eux-mêmes et des plateformes de financement participatif devraient permettre d'améliorer les registres d'exécutants de la R-D.

\section{Une approche complémentaire : se tourner vers les bailleurs de fonds}

10.47 Comme on l'a vu plus haut, les institutions du secteur sans but lucratif exécutent et financent à la fois des activités de R-D. Il est donc recommandé d'interroger aussi les exécutants de la R-D de ce secteur sur leurs dépenses de R-D extra-muros. Dans certains pays, cependant, de nombreuses institutions du secteur privé sans but lucratif (fondations ou organisations philanthropiques, par exemple) ne mènent aucune activité de R-D intra-muros mais financent, souvent largement, des activités de R-D extra-muros (généralement sous la forme d'aides ou de dons, appelés ici fonds de transfert) dans des établissements d'enseignement supérieur ou d'autres institutions sans but lucratif (faisant partie ou non du secteur privé sans but lucratif). Le présent manuel recommande de recueillir des données auprès des exécutants de la R-D plutôt que des bailleurs de fonds. Toutefois, il reconnaît qu'une approche axée sur les sources de financement peut présenter un intérêt complémentaire. Il devra alors s'agir uniquement d'une option de second choix, et l'échantillon devra viser principalement les institutions de ce secteur, par opposition aux individus et aux ménages.

\section{Références}

Eurostat (2008), Nomenclature for the Analysis and comparison of Scientific programmes and Budget (NASB).

Nations Unies (2008), « Classification internationale type, par industrie, de toutes les branches d'activité économique (CITI), Révision 4 ", Département des affaires économiques et sociales, Division de statistique, Études statistiques, Série $M, n^{\circ} 4$, Rév. 4, Nations Unies, New York, https://unstats.un.org/unsd/cr/registry/isic-4.asp et http://unstats.un.org/unsd/publication/seriesM/seriesm_4rev4f.pdf.

Nations Unies (2000), COPNI, Nations Unies, New York, http://unstats.un.org/UNSD/cr/ registry/regcst.asp? $\mathrm{Cl}=6 \& \mathrm{Top}=2 \& \mathrm{~L} g=2$. 



\section{Chapitre 11}

\section{Mesurer la mondialisation de la R-D}

Dans les analyses des éditions précédentes, le secteur "Étranger ", désormais appelé "Reste du monde ", était principalement considéré comme une source de financement pour l'exécution de travaux de recherche et développement expérimental (R-D) sur le territoire national (par exemple, dans le calcul des DIRD) ou comme une destination de fonds de sources nationales (comme dans le calcul des DNRD). Or, la définition du Reste du monde énoncée dans ce chapitre rejoint celle du Système de comptabilité nationale (SCN). La mondialisation désigne ici l'intégration internationale des moyens de financement, de l'offre des facteurs de production, de la $R-D$, de la production et des échanges de biens et des services. Dans le secteur des entreprises, le phénomène est associé aux échanges internationaux et à l'investissement direct étranger (IDE), tandis que les institutions sans but lucratif privées ou publiques (qui incluent des unités des secteurs de l'État et de l'enseignement supérieur) mènent également des activités d'envergure internationale en lien avec la $R-D$, comme le financement et la collaboration. La mondialisation de la $R-D$ renvoie à un sous-ensemble d'activités mondiales impliquant le financement, l'exécution, le transfert et l'exploitation de la R-D. Ce chapitre traite d'indicateurs de la mondialisation de la R-D qui concernent tant le secteur des entreprises que les autres secteurs. 


\subsection{Introduction}

11.1 Le concept de mondialisation de la R-D est abordé de façon explicite dans la présente édition du Manuel, alors qu'auparavant, la dimension mondiale de la R-D était surtout associée aux sources de financement de la R-D exécutée sur le territoire national (comme dans le calcul des DIRD) ou à la destination des fonds de sources nationales (comme dans le calcul des DNRD). Ces sources étaient précédemment caractérisées comme des fonds en provenance/à destination de l'« étranger ". Conformément au Système de comptabilité nationale (SCN), la terminologie privilégiée dans le présent manuel est le " reste du monde ". Le reste du monde est défini sur la base du statut de non-résidence des unités pertinentes. Il est constitué de toutes les unités institutionnelles non résidentes qui effectuent des opérations avec des unités résidentes, ou ont d'autres liens économiques avec celles-ci. Repérer et quantifier les sources et destinations des financements de la R-D non situées sur le territoire national restent des aspects importants de l'étude de la R-D, qui sont traités de manière exhaustive dans les chapitres 3 et 4 , ainsi que dans les chapitres consacrés aux différents secteurs. Cependant, le champ d'étude du présent manuel dépasse le cadre des flux de financement de la R-D pour inclure une liste plus complète de questions en rapport avec la mesure de la $\mathrm{R}-\mathrm{D}$ à l'échelle mondiale.

11.2 D’une manière générale, la mondialisation désigne l'intégration internationale des moyens de financement, de l'offre de facteurs, de la R-D, de la production et des échanges de biens et des services. Dans le secteur des entreprises, elle est associée aux échanges internationaux et à l'investissement direct étranger (IDE), tandis que les institutions sans but lucratif privées ou publiques (qui incluent des unités des secteurs de l'État et de l'enseignement supérieur) mènent également des activités d'envergure internationale en lien avec la R-D, comme le financement et la collaboration. Dans le présent manuel, " mondialisation » et "internationalisation » sont synonymes. Il convient de noter que les flux financiers d'IDE et les opérations émanant de l'IDE sont des indicateurs de mondialisation distincts (FMI, 2009, chapitre 6). Les indicateurs de flux financiers d'IDE n'entrent pas dans le cadre actuel du présent manuel. Pour plus d'informations, voir le Manuel de l'OCDE sur les indicateurs de la mondialisation économique (OCDE, 2005, chapitre 2) et la Définition de référence de l'OCDE des investissements directs internationaux (OCDE, 2009a).

11.3 La mondialisation de la R-D renvoie à un sous-ensemble d'activités mondiales impliquant le financement, l'exécution, le transfert et l'exploitation 
de la R-D. Le présent chapitre s'intéresse d'abord à trois indicateurs de la mondialisation de la R-D des entreprises, avant de faire la synthèse des problèmes de mesure rencontrés en dehors du secteur des entreprises.

\subsection{Mesure de la mondialisation de la R-D des entreprises}

\section{Cadre statistique pour mesurer la mondialisation de la R-D des entreprises}

11.4 Trois types d'indicateurs statistiques de la mondialisation de la R-D d'entreprise sont traités ici :

- les flux de financement transnationaux de R-D (voir section 11.3)

- les dépenses courantes et les dépenses de personnel liées aux activités de R-D que des composantes d'entreprises multinationales (EMN) mènent dans les pays déclarants et à l'étranger (voir section 11.4)

- les échanges internationaux de services de R-D (voir section 11.5).

11.5 Le premier de ces indicateurs est une extension des statistiques de R-D classiques dont la collecte est recommandée auprès des entreprises résidentes (voir chapitre 7, section 7.6). Les EMN tout comme les autres entreprises qui ne font pas partie de groupes multinationaux peuvent avoir de telles activités, mais le présent chapitre s'intéresse plus particulièrement à la déclaration des EMN. Le deuxième de ces trois indicateurs concerne uniquement les activités des EMN. Le troisième indicateur est construit dans le contexte des statistiques du commerce des services, sur la base des recettes perçues par des unités non résidentes et des paiements versés à ces unités non résidentes en échange de travaux de R-D.

11.6 Les indicateurs recommandés dans le présent manuel se limitent en grande partie à la caractérisation de la R-D exécutée au cours de l'année de référence. En particulier, les flux de financement transnationaux de la R-D sont associés à la R-D intra-muros d'une période de référence spécifique, au même titre que les indicateurs de l'exécution et du financement de la R-D selon le statut de l'EMN. Par ailleurs, l'échange international de services de R-D peut englober des opérations impliquant les droits aux résultats d'activités de R-D réalisées au cours d'années antérieures. Dès lors, les échanges de services de R-D sont fonction des dépenses cumulées de R-D (dans le pays déclarant pour les exportations et dans les pays partenaires commerciaux pour les importations), et non pas seulement de la production ou du financement de la R-D de l'année en cours. Les services de R-D sont mesurés aux prix du marché, conformément aux principes de la balance des paiements (BP) et collectés dans les enquêtes existantes sur les échanges de services, comme il en est question plus loin dans le présent chapitre. Par conséquent, chaque indicateur remplit des objectifs différents et représente des aspects partiels mais complémentaires de modalités d'organisation sousjacentes complexes pour l'exécution de la R-D à différents endroits du monde. 
Par exemple, les échanges de services de R-D sont utilisés pour ajuster les stocks intérieurs de R-D lors du processus de capitalisation comme évoqué plus loin dans le présent chapitre, conformément aux indications du Handbook on Deriving Capital Measures of Intellectual Property Products (OCDE, 2009b). Une autre différence majeure entre les indicateurs des flux de financement transnationaux de la R-D et du commerce des services de R-D est que les financements transnationaux de la R-D incluent les transferts (comme les subventions, voir ci-dessous) et tous les secteurs ; les statistiques pour les échanges de services de R-D n'incluent généralement pas les subventions pour la R-D, et la couverture au-delà du secteur des entreprises, peut être limitée pour des raisons pratiques.

11.7 Les indicateurs statistiques de la mondialisation de la R-D liés à la R-D exécutée ou financée par des EMN s'appuient non seulement sur les statistiques nationales sur la R-D, mais également sur des statistiques économiques internationales plus larges concernant la production, les investissements directs, l'emploi, les financements et les échanges à l'échelle mondiale. La multiplicité des sources de référence présente des défis particuliers pour les offices statistiques, les répondants aux enquêtes, et les utilisateurs de statistiques sur la R-D et les statistiques connexes sur la mondialisation. Toutefois, étant donné la complexité des questions de mondialisation de la R-D, il est improbable qu'une référence unique englobe toutes les notions de mesure pertinentes. Dans les orientations proposées dans le présent chapitre, on s'est donc efforcé d'utiliser une terminologie cohérente à la fois avec les termes définis ailleurs dans le présent manuel et avec les manuels statistiques sur la mondialisation référencés plus particulièrement au présent chapitre (qui à l'avenir pourront être présentés de façon détaillée en ligne, en complément du présent manuel, à l'adresse http://oe.cd/frascati).

11.8 D'une façon plus générale, la mondialisation a deux principales conséquences pour l'activité de R-D et sa mesure. D'abord, la R-D fait partie de chaînes de valeur mondiales complexes qui mettent en jeu des fournisseurs dispersés et des processus de production fragmentés de biens et de services. Ensuite, la R-D elle-même implique de plus en plus souvent des organisations et du personnel dispersés dans différents pays, reflétant ainsi l'élargissement des tendances de mondialisation. En raison de cette complexité, il est improbable qu'un instrument d'enquête unique parvienne à couvrir tous les besoins de données sur la mondialisation de la R-D. Cette situation offre des opportunités de collaboration sur la collecte et/ou l'élaboration de données pour les enquêtes sur la R-D et dans d'autres domaines, en particulier les DIRDE, les IDE/EMN et les enquêtes sur les échanges de services évoquées ici. Ainsi, le présent chapitre résume les éléments de R-D provenant de divers manuels sur la mondialisation et met ces informations en relation avec les notions du Manuel de Frascati.

11.9 Même si le présent chapitre porte avant tout sur les relations de propriété et les aspects financiers transnationaux des activités mondiales de 
R-D (par ex. les dépenses, les coûts, les flux de financement), la mondialisation de la R-D se manifeste également à travers la mobilité du personnel de R-D. L'identification et le suivi des flux de personnel de R-D au sein des EMN et entre ces dernières sont donc encouragés. Cependant, la capacité des enquêtes sur la R-D à rassembler des informations sur les aspects "ressources humaines " de la mondialisation de la R-D est quelque peu limitée par comparaison avec les enquêtes sur les chercheurs indépendants ou les personnes hautement qualifiées.

\section{Définitions appropriées des entreprises multinationales (EMN)}

11.10 La collecte et la compilation précises des statistiques de mondialisation de la R-D des entreprises doivent reposer sur une compréhension et une application cohérentes de la terminologie nécessaire de la mondialisation des entreprises. À partir des éléments détaillés disponibles dans les manuels sur la mondialisation et documents apparentés, les termes clés sont définis ci-dessous. Voir l'encadré 11.1 pour une synthèse des divers manuels de référence qui fournissent le cadre statistique et la terminologie sous-jacente retenus dans le présent chapitre.

11.11 L'investissement direct étranger (IDE) est motivé par la volonté d'une entreprise résidente d'une économie (société-mère ou "investisseur direct ») d'acquérir un intérêt durable dans une entreprise résidente d'une autre économie (société affiliée implantée à l'étranger ou " entreprise d'investissement direct "). Aux fins de l'établissement des statistiques officielles, un intérêt durable est réputé exister dès lors que $10 \%$ ou plus des actions ordinaires ou des droits de vote dans une entreprise constituée en société ou l'équivalent d'une entreprise non constituée en société sont détenus, directement ou indirectement. Le critère de $10 \%$ des droits de vote établit également l'existence d'une relation d'investissement direct entre une société affiliée et sa société-mère.

11.12 Aux fins du présent manuel, l'accent statistique est mis sur la R-D et les activités apparentées faisant intervenir des sociétés affiliées à participation majoritaire ou sous contrôle. La participation majoritaire ou le contrôle renvoie au fait de détenir plus de $50 \%$ des actions ordinaires ou des droits de vote dans une entreprise constituée en société ou l'équivalent d'une entreprise non constituée en société. Entre autres exemples de sociétés affiliées à participation majoritaire ou sous contrôle figurent les filiales (entreprises constituées en société) et les succursales (entreprises non constituées en société).

11.13 Du point de vue du pays déclarant dont la société-mère est résidente, celle-ci est un groupe d'entreprises composé de toutes les unités résidentes du pays déclarant dont elle détient la majorité du capital (pour une synthèse des questions de consolidation faisant intervenir les membres d'EMN, voir OCDE, 2005). En sont donc exclues les sociétés implantées à l'étranger dont elle détient la majorité des parts. 
11.14 Du point de vue du pays déclarant, une EMN désigne une société-mère résidente et les sociétés implantées à l'étranger dont elle détient la majorité des parts, appelées « filiales implantées à l'étranger (FIE) 》. Ainsi, les filiales implantées à l'étranger sont des sociétés affiliées situées à l'étranger et détenues à plus de $50 \%$ par leur société-mère, résidente du pays déclarant. Une EMN est également appelée " groupe d'entreprises mondial " (CE, 2010).

\section{Encadré 11.1. Manuels statistiques internationaux sur la mondialisation de la R-D des entreprises}

Système de comptabilité nationale 2008 (CE et al. 2009). Le SCN fournit des orientations sur les indicateurs de l'activité économique au sein d'un territoire économique dans un système de comptabilité intégré. Il porte également sur les flux économiques entre un pays déclarant et le reste du monde. La définition de la R-D dans le SCN 2008 (SCN 10.103) est globalement conforme au Manuel de Frascati 2002. Toutefois, le SCN envisage également la mesure des opérations économiques sur des actifs de R-D (également nommés « R-D »), qui peuvent avoir été effectuées au cours d'années précédentes.

Manuel de l'OCDE sur les indicateurs de la mondialisation économique (OCDE, 2005). Ce manuel définit un cadre pour des statistiques et des indicateurs dérivés sur la mondialisation, y compris les stocks (positions) et les flux financiers d'IDE et l'activité ou les opérations des EMN.

Définition de référence des investissements directs internationaux (OCDE, 2009a). Ce manuel énonce en détail les définitions des flux d'IDE et autres flux apparentés conformément à la $6^{e}$ édition du Manuel de la balance des paiements et de la position extérieure globale. Il prend également en compte les statistiques sur l'activité des EMN (AEMN).

Balance of Payments and International Investment Position Manual, $6^{e}$ édition (FMI, 2009). Ce manuel traite de la comptabilité et des normes statistiques pour compiler la balance des paiements (BP) entre les résidents et les non-résidents durant une période spécifique, ainsi que le bilan externe, les actifs et les passif accumulés (ou positions) suite à des opérations avec le secteur externe. Il constitue la source pour les définitions sur les opérations internationales, le territoire économique, la résidence et définitions apparentées. Sa définition de " R-D » dans les services de R-D intègre la définition du Manuel de Frascati, en l'étendant aux services d'essais qui débouchent sur des brevets.

Manuel des statistiques du commerce international des services 2010 (ONU et al. 2011). Ce manuel traite des statistiques sur la prestation internationale de services, y compris les services de R-D sous deux formes majeures : les échanges de services transnationaux classiques et la prestation de services au niveau local par des filiales sous contrôle étranger. Ces derniers sont traités dans les statistiques sur les sociétés affiliées implantées à l'étranger (CE, 2012). 


\section{Encadré 11.1. Manuels statistiques internationaux sur la mondialisation de la R-D des entreprises (suite)}

Sa définition de " R-D » dans les services de R-D intègre la définition du Manuel de Frascati, en l'étendant aux services d'essais qui débouchent sur des brevets, mais ses sous-catégories sont formulées de manière à faciliter les comparaisons. Un guide de compilation pour ce manuel a été publié en 2014 (ONU et al. 2014).

Handbook on Deriving Capital Measures of Intellectual Property Products (OCDE, 2009b). Ce manuel présente des méthodes statistiques pour élaborer des indicateurs de valeur marchande de la R-D et autres produits de la propriété intellectuelle aux fins d'incorporer ces actifs dans les comptes économiques nationaux et internationaux, conformément au SCN 2008. Il décrit les résultats de la R-D au plan national à des fins de mesure en termes de trois composantes conformes à la fois au SCN et à Frascati : R-D pour compte propre ( $R-D$ menée et utilisée en interne indépendamment de la source de financement) ; R-D pour client (R-D menée pour une autre unité et financée par celle-ci) ; et R-D non personnalisée ou spéculative. Il recense les différentes formes de déclaration du transfert, de l'utilisation et de la vente de R-D et autres produits de la propriété intellectuelle à l'échelle internationale : contrats de vente ou de licence, transferts (prestation sans frais, en particulier au sein des EMN), et modification de la valeur des actifs d'une société ou revenus d'investissement qui comprennent (mais sans être identifiés de façon distincte) des flux de R-D. Ce manuel décrit également la manière d'obtenir les chiffres de la fourniture de R-D au plan national en corrigeant les résultats de la R-D intérieure par addition des importations et sous-traction des exportations à l'aide de statistiques sur le commerce de services de R-D. Cela permet le calcul de la formation de capital (investissement) dans la R-D et l'estimation des stocks de capital de la R-D.

The Impact of Globalisation on National Accounts (CEE/Eurostat/OCDE, 2011). Ce guide porte avant tout sur les difficultés que pose l'activité des EMN pour la mesure de la production et des échanges nationaux, R-D incluse. Dans son chapitre 7 , il formule en outre des orientations statistiques pour des indicateurs internationalement comparables de la production et des échanges de produits de la propriété intellectuelle. Ce manuel aborde également des questions de mesure telles que les prix de transfert et leurs conséquences sur les statistiques nationales et internationales.

Guide to Measuring Global Production (CEE/OCDE, 2015). Ce guide développe les orientations des deux manuels précédents en analysant plus avant les chaînes de valeur mondiales et les arrangements pour la production de biens et de services, y compris les intrants de la R-D. 
11.15 Du point de vue du pays déclarant, les filiales sous contrôle étranger (FCE) constituent le groupe d'entreprises implantées dans le pays déclarant dont la majorité des capitaux est détenu par des EMN étrangères (qui sont donc des sociétés-mères étrangères). Leurs activités découlent de l'IDE entrant, tandis que les activités des filiales implantées à l'étranger se rapportent à l'IDE sortant. Pour une synthèse des questions de consolidation impliquant les filiales sous contrôle étranger, voire OCDE (2005).

11.16 Les entreprises sœurs implantées à l'étranger sont identifiées du point de vue d'une filiale sous contrôle étranger résidant dans l'économie déclarante. Il s'agit des entreprises implantées en dehors du pays déclarant qui se trouvent sous le contrôle ou l'influence de la même société-mère étrangère que la filiale sous contrôle étranger. Aux fins du présent manuel, les entreprises sœurs implantées à l'étranger présentent un intérêt de par leur qualité de source ou de destination du financement des travaux de R-D auxquels les filiales sous contrôle étranger sont associées.

11.17 Dans le cas de l'investissement entrant, la société-mère immédiate d'une filiale sous contrôle étranger est le premier investisseur étranger implanté hors du pays déclarant qui exerce un contrôle sur la filiale étrangère. L'investisseur exerçant le contrôle ultime d'une filiale sous contrôle étranger (également appelé " unité institutionnelle investie du contrôle ultime ») se trouve à la tête d'un ensemble de sociétés ou de filiales qu'il contrôle sans lui-même être sous le contrôle d'une autre société quelle qu'elle soit.

11.18 Des sociétés peuvent être implantées dans le pays déclarant sans être membres d'aucune EMN (nationale ou étrangère) et ne réaliser ainsi aucune forme d'IDE (OCDE, 2005, encadré 3.3). Ces sociétés peuvent mener d'autres types d'activités mondiales comme des échanges, de la sous-traitance, de la collaboration et du financement international en matière de R-D.

11.19 Le graphique 11.1 présente un exemple de notions et de termes d'EMN définis du point de vue du " pays déclarant 1 " (les pays 2 et 3 constituant le "reste du monde "). Les flèches vont des sociétés-mères vers les filiales et indiquent une participation majoritaire dans les relations d'investissement direct. La section 11.2 traite des flux de financement de la R-D entre les membres d'EMN et les autres, et la section 11.3 fournit des orientations sur la compilation de tableaux à double entrée des dépenses courantes d'exécution de la R-D et des sources de financement de la R-D pour les EMN et les autres entreprises non membres d'EMN. 


\section{Graphique 11.1. Exemple indicatif de relations de propriété entre membres d'EMN et terminologie correspondante}

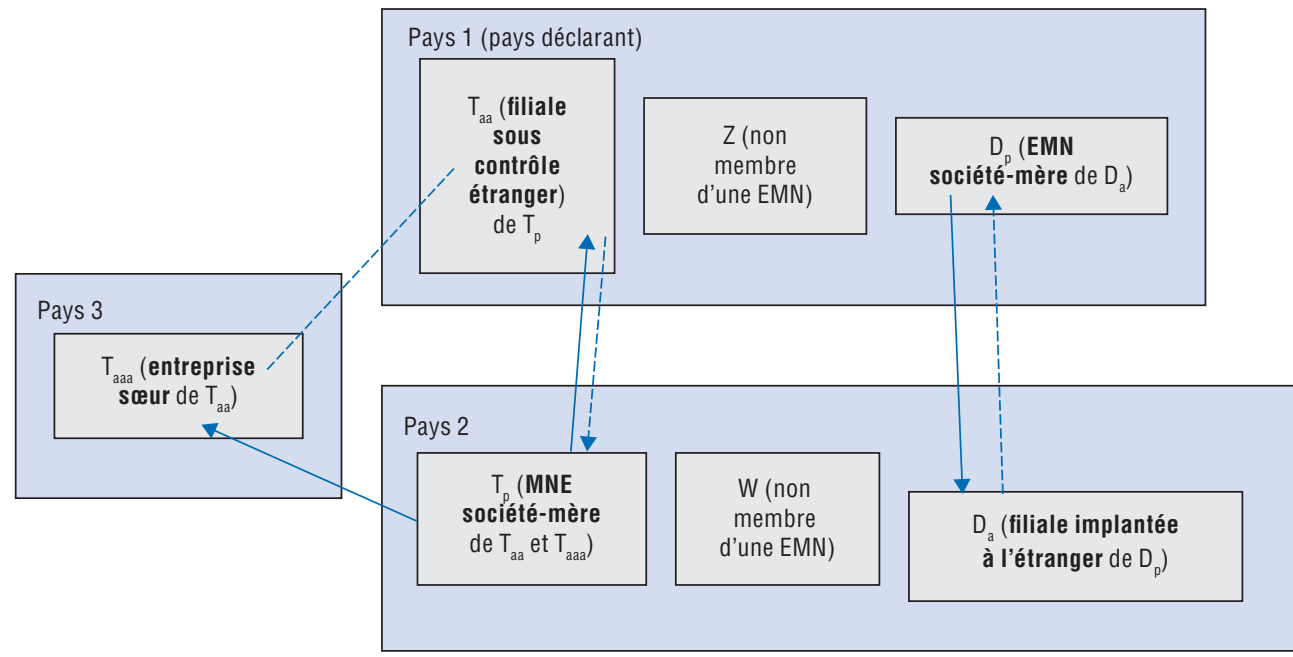

Note: $\mathrm{D}_{\mathrm{p}}$ est la société-mère de $\mathrm{D}_{\mathrm{a}} \cdot \mathrm{T}_{\mathrm{p}}$ est la société-mère de $\mathrm{T}_{\mathrm{aa}}$ et $\mathrm{T}_{\text {aaa }}$.

$\longrightarrow$ Lien de société-mère à filiale

$\rightarrow$ Lien de filiale à la société-mère

----- Lien entre entreprises soeurs

\subsection{Financement international de la R-D faisant intervenir des EMN}

\section{Reste du monde}

11.20 Le financement international ou transnational de la R-D fait intervenir des unités non résidentes comme sources ou destinations des fonds. Comme expliqué dans la section 3.3 du chapitre 3 , le reste du monde est défini sur la base du statut de non-résidence des unités pertinentes ; les opérations avec le reste du monde sont déclarées comme s'il s'agissait d'un secteur de facto. Ce secteur est constitué de toutes les unités institutionnelles non résidentes qui effectuent des opérations avec des unités résidentes, ou ont d'autres liens économiques avec des unités résidentes. Aux fins de déclaration régulière des DIRDE (section 7.6), les sources de financement de la R-D situées dans le reste du monde sont:

Reste du monde

Secteur des entreprises

Entreprise au sein du même groupe

Autres entreprises non affiliées

Secteur de l'État

Secteur de l'enseignement supérieur

Secteur privé sans but lucratif

Organisations internationales 


\section{Flux de financement international de la R-D des EMN}

11.21 La présente section examine les EMN dans le contexte des flux de financement transnationaux, premier des trois types d'indicateurs statistiques de la mondialisation de la R-D des entreprises énumérés dans la section 11.1. Elle complète les orientations données sur la voie à suivre pour mettre en évidence les sources de financement des DIRDE situées dans le reste du monde.

11.22 Les unités d'entreprise à l'étranger peuvent être sources ou bénéficiaires de financement pour la R-D. Les membres des EMN (tel que défini dans le présent chapitre et illustré sur le graphique 11.1) interviennent généralement dans des flux de financement transnationaux dans le cadre de leurs opérations mondiales et avec d'autres sociétés et organisations. Ces flux reflètent divers accords à l'échelle mondiale pour acquérir ou fournir de la R-D. Une distinction importante est le caractère affilié ou non affilié d'une source de financement au sein des entreprises. Les unités affiliées comprennent les sociétés-mères et les entreprises sœurs implantées à l'étranger (sous contrôle étranger) et les filiales implantées à l'étranger (s'il s'agit d'une société-mère). Pour obtenir une granularité plus fine des sources non résidentes des fonds destinés à la R-D, il pourrait être demandé, dans les enquêtes sur la R-D des EMN, les informations suivantes sur les sources de financement dans le reste du monde (voir graphique 11.2).

- Unités affiliées (entreprises au sein du même groupe)

* filiales implantées à l'étranger (FIE)

* sociétés-mères étrangères (si le répondant est sous contrôle étranger)

* entreprises sœurs à l'étranger (si le répondant est sous contrôle étranger).

- Unités non affiliées - autres entreprises à l'étranger (toute société qui ne fait pas partie du groupe de l'EMN du répondant).

11.23 Sil'on s'appuie sur les définitions présentées au chapitre 4, un transfert en espèces ou en nature est une opération dans laquelle le fournisseur ne reçoit rien en retour (c'est-à-dire que le flux d'argent n'est pas compensé par un flux de biens ou de services). Les transferts transnationaux ou internationaux sont des opérations de transfert entre un résident et un non-résident. Les financements de $\mathrm{R}-\mathrm{D}$ à destination/en provenance du reste du monde devraient identifier de façon distincte les transferts et les échanges. Cette information pourrait faciliter la distinction entre les flux de financement transnationaux de R-D et les échanges de services de R-D qui exclut généralement les transferts. De plus, les flux intra-EMN qui s'assimilent plus à des transferts qu'à des échanges peuvent ne pas être déclarés dans les enquêtes sur les échanges de services, mais apparaître dans les postes de financement de la R-D dans les enquêtes sur la R-D, comme décrit ci-dessous. 


\section{4. Élaboration, compilation et publication des statistiques agrégées de la R-D des EMN}

\section{Approche générale pour compiler des statistiques agrégées de la $R-D$ des EMN}

11.24 La présente section examine la collecte de données sur les dépenses courantes pour la R-D exécutée par les membres d'EMN au sein des pays déclarants et à l'étranger, deuxième des trois types d'indicateurs statistiques de la mondialisation de la R-D des entreprises identifiés dans la section 11.1. Les informations sur l'exécution de R-D par les EMN, à l'exception des sources de financement, sont importantes pour la compréhension de la production de nouvelles connaissances. Par exemple, les indicateurs des dépenses courantes pour l'exécution de R-D ont un lien direct avec l'emploi dans le secteur de la R-D. Ensuite, les tableaux à double entrée de statistiques sur le financement/ l'exécution de la R-D des entreprises pour les EMN et les autres entreprises (sur la base des catégories résumées dans le graphique 11.2) fournissent un tableau plus complet des dispositions au niveau mondial pour la production de nouvelles connaissances que si l'on se focalise exclusivement sur les flux de financement transnationaux destinés à la R-D. L'attention portée aux dépenses courantes de R-D des EMN vise à faciliter les comparaisons avec les statistiques sur les opérations des EMN qui ne relèvent pas de la R-D comme la production, les ventes/le chiffre d'affaires, la valeur ajoutée, l'emploi, le nombre d'entreprises, et l'échange de biens et de services. Bien sûr, il pourrait être possible d'identifier de façon distincte les coûts d'investissement des EMN pour la R-D.

11.25 Par ailleurs, la collecte de statistiques sur les activités à l'étranger présente des défis pratiques pour les répondants et les offices statistiques nationaux, car l'autorité d'un pays déclarant en matière de collecte des données ne s'étend généralement pas au-delà de ses frontières nationales ; les données sur les filiales à l'étranger doivent normalement être collectées par le biais d'enquêtes ciblant ou incluant les sociétés-mères. Dans la mesure où les enquêtes sur les IDE ou les EMN englobent les activités des filiales à l'étranger, la R-D peut être incluse dans ces enquêtes, conformément aux définitions et aux orientations du présent chapitre et d'autres dans le présent manuel.

11.26 Les enquêtes sur les DIRDE peuvent également inclure les activités de R-D des filiales implantées à l'étranger avec d'autres informations qui n'entrent généralement pas dans le cadre des enquêtes sur les IDE/EMN (par ex. le type de $R-D)$, avec des questions s'adressant aux sociétés-mères qui résident dans le pays déclarant.

11.27 Dans la mesure où un pays collecte des données sur les dépenses de R-D et des statistiques apparentées (comme les ressources humaines) pour les 
membres d'EMN définis dans le présent chapitre, la priorité devrait être accordée aux membres implantés dans le pays déclarant, à savoir :

- les sociétés-mères entièrement consolidées d'EMN propriétaires de filiales implantées à l'étranger (FIE)

- les filiales sous contrôle étranger (FCE) membres d'EMN étrangères.

11.28 Dans la mesure du possible, les statistiques peuvent également être mises sous forme de tableaux pour les catégories suivantes, qui font partie du secteur de facto " reste du monde » :

- les filiales implantées à l'étranger (FIE) détenues par des sociétés-mères résidentes

- les EMN consolidées contrôlées par des résidents du pays déclarant, c'est-à-dire les sociétés-mères résidentes plus leurs filiales implantées à l'étranger (FIE).

\section{Graphique 11.2. Agrégation des dépenses courantes1 et du personnel de R-D des EMN et des autres entreprises au sein d'un pays déclarant, et source des financements}

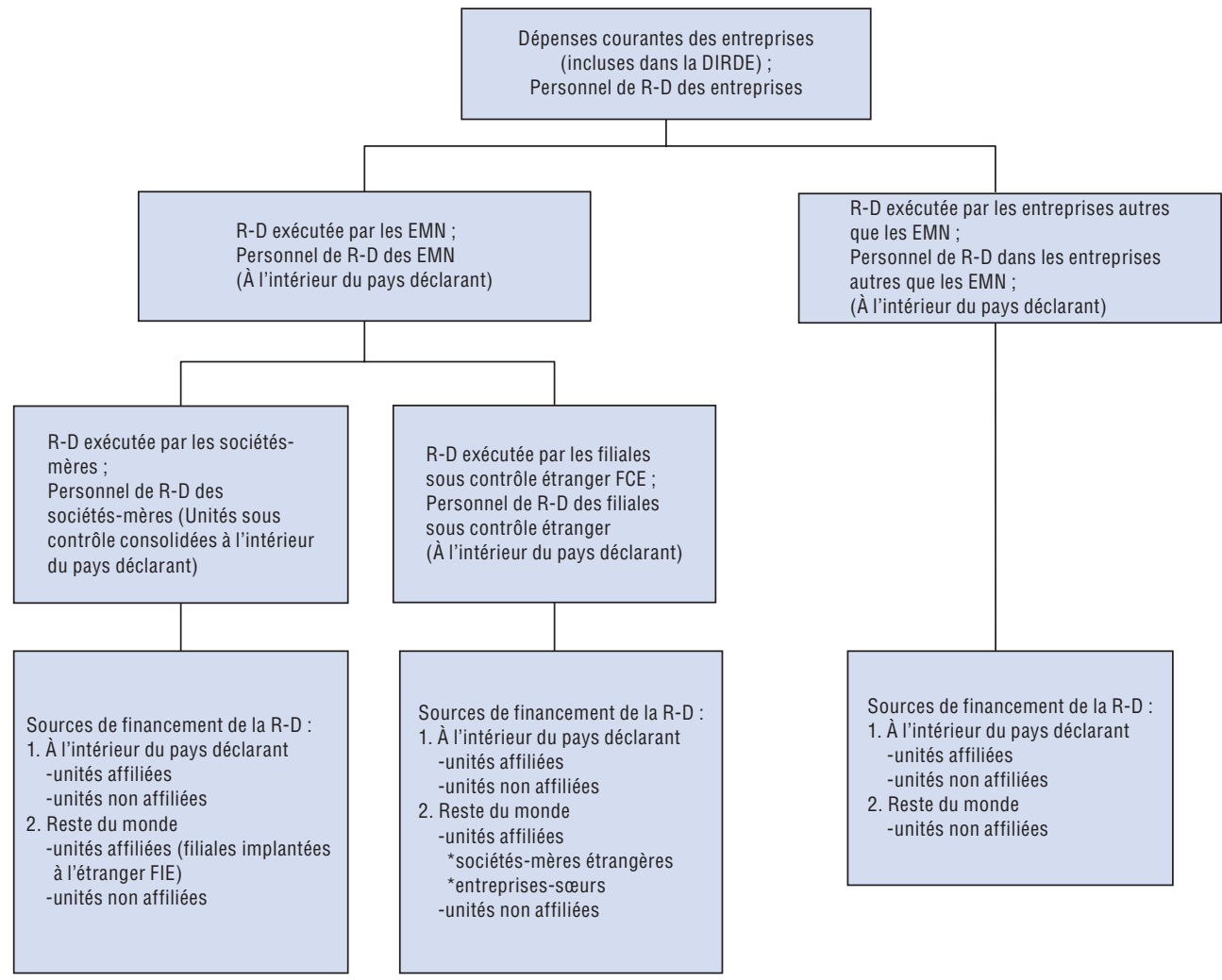

1. L'attention portée aux dépenses courantes de R-D vise à faciliter la comparaison avec les statistiques sur les opérations des EMN qui ne relèvent pas de la R-D. Il pourrait être possible d'identifier de façon distincte les dépenses en capital des EMN pour la R-D. 


\section{Statistiques de la R-D des EMN, par pays et autres critères}

11.29 Des EMN peuvent être propriétaires de filiales dans un pays donné à travers des chaînes de propriété qui couvrent plusieurs pays. Les statistiques de la R-D et d'autres activités (par ex. l'emploi) liées à l'investissement entrant peuvent être classées en fonction du pays de contrôle immédiat ou du pays où s'exerce le contrôle ultime. Le pays d'investissement immédiat est le pays de résidence de la société-mère immédiate. Le pays d'investissement ultime est celui où réside l'investisseur exerçant le contrôle ultime.

11.30 Pour les statistiques d'activité concernant l'investissement extérieur, les filiales implantées à l'étranger peuvent être classées en fonction du pays dans lequel les opérations ont réellement lieu (pays hôte immédiat).

11.31 Le présent manuel conseille, dans la mesure du possible, de collecter et de mettre sous forme de tableaux les statistiques de la R-D des EMN en fonction du pays où s'exerce le contrôle ultime (investissement entrant) et du pays d'implantation des filiales situées à l'étranger (investissement sortant), en particulier si d'autres statistiques des EMN (emploi, ventes, échanges, etc.) sont publiées par le pays déclarant sur ce modèle. En réalité, la cohérence entre les statistiques améliorées sur la mondialisation de la R-D et hors R-D augmente la valeur analytique et l'intérêt pratique de ces statistiques, au vu du caractère complexe et changeant des opérations et des modalités organisationnelles.

11.32 La classification par industrie (et les répartitions apparentées) pour les statistiques sur les activités entrantes et sortantes des EMN devrait suivre les orientations du chapitre 7. La classification par industrie des filiales implantées à l'étranger devrait se fonder, en priorité, sur leur activité économique principale dans leur pays d'implantation à l'étranger, et non sur celle de leur société-mère dans le pays (déclarant) d'origine. Dans la mesure où les pays déclarants ont accès aux données sur les sociétés-mères, une autre priorité par rapport aux filiales implantées à l'étranger est de présenter les données sous forme de tableaux en fonction de la principale branche d'activité économique de la société-mère, en particulier pour des variables fondamentales comme les dépenses courantes pour l'exécution de la R-D et l'emploi dans le secteur de la R-D.

11.33 Les statistiques de la R-D des EMN sont des exemples de statistiques sur les activités des entreprises multinationales (AEMN), y compris les sociétésmères et les filiales qui peuvent être prises en compte dans les enquêtes spécialement consacrées à la R-D ou dans le cadre d'autres études sur l'activité internationale comme l'analyse des IDE. La R-D des EMN peut également être publiée dans les statistiques nationales dans le cadre des statistiques sur les filiales étrangères (FATS), qui diffèrent des statistiques sur les AEMN en ce sens qu'elles n'englobent pas les sociétés-mères. Les activités de R-D des filiales sous contrôle étranger dans l'économie déclarante font partie des statistiques sur les activités entrantes (inward FATS) ; les activités de R-D des filiales implantées à l'étranger font partie des statistiques sur les activités sortantes (outward FATS). 


\subsection{Les échanges de services de R-D}

11.34 Cette section examine la collecte de données sur les échanges de services de $R-D$, troisième des trois types d'indicateurs statistiques de la mondialisation de la R-D des entreprises identifiés dans la section 11.1. Dans le SCN et les statistiques sur les échanges internationaux, les "services de R-D » concernent les services associés à la recherche fondamentale et appliquée et au développement expérimental, y compris les activités menées dans le cadre des sciences physiques, sociales et humaines. Les " services de R-D » peuvent être fournis par n'importe quelle société et ils ne sont par conséquent pas limités aux entreprises de la division 72 de la CITI Rév. 4 ou d'une classification nationale équivalente. Dans les classifications de produits types, suite à la capitalisation de la R-D, une distinction est établie entre les travaux originaux de R-D et les autres services de R-D. Alors que ces derniers ont un lien direct avec l'exécution de la $\mathrm{R}-\mathrm{D}$, les premiers nommés correspondent aux actifs qui ont été produits à partir de l'exécution antérieure d'activités de R-D. La vente et l'acquisition de ces actifs « finis " sont comptabilisées dans les statistiques sur les échanges comme faisant partie des services de R-D, mais sortent du cadre des flux de financement dont il est question dans le présent manuel.

11.35 Dans les statistiques sur les échanges, la catégorie générale des services de R-D peut également inclure les essais et autres activités techniques hors R-D qui débouchent sur des brevets, reflétant ainsi un domaine plus étendu que la définition de la R-D dans le présent manuel. Cependant, les manuels cités tiennent compte de cette différence en recommandant explicitement de faire la distinction, lors de la collecte, entre les services liés aux " activités systématiques entreprises en vue d'accroître la somme des connaissances » et les " autres " services au sein des " services de R-D ». Des informations sur les services de R-D dans les comptes économiques et les statistiques sur les échanges de services, ainsi que la concordance entre les codes de classification de la balance des paiements et les codes de classification des produits pour la R-D et les services techniques apparentés sélectionnés à l'avenir sont accessibles en ligne, en complément au présent manuel, à l'adresse http://oe.cd/frascati. En outre, à la lumière des manuels mis à jour sur la mondialisation examinés ici, les orientations de la balance des paiements du secteur technologique pourront être mises à jour à l'avenir.

\section{Enquêtes sur les échanges de services de R-D}

11.36 Les enquêtes sur le commerce international des services réalisées auprès sociétés implantées dans l'économie déclarante (indépendamment de la structure du capital ou de la classification industrielle) collectent des données sur les opérations transnationales de services de R-D (entre autres services aux entreprises) et sur les opérations transnationales de propriété intellectuelle. Les opérations internationales désignent des opérations entre résidents et non-résidents. Voir le Glossaire pour une définition du terme " opérations ". 
Ces enquêtes collectent également des données sur les ventes/achats et les licences impliquant des droits de propriété émanant de la R-D (par ex. vente de brevets et redevances et droits de licence), qui font partie des "services de R-D ». En même temps, les échanges de services sont une composante du compte des opérations courantes de la balance des paiements, qui mesure de façon synthétique les opérations économiques entre résidents et non-résidents au cours d'une période donnée.

\section{Estimation de la valeur des échanges de services de $R-D$ en regard des déclarations relatives au financement de la $R-D$}

11.37 Le prix du marché est utilisé comme base pour l'estimation de la valeur des opérations internationales dans les enquêtes sur les échanges de services. Les opérations sont enregistrées d'après la comptabilité d'exercice, quand les services sont fournis ou reçus, quel que soit le moment où le paiement est reçu ou effectué. En même temps, la R-D financée par d'autres sources que la société tel que défini dans le présent manuel - et tel que déclaré par le bailleur de fonds de la R-D, mais pas par l'exécutant de la R-D - est présumée inclure les suppléments et autres postes au-delà des dépenses de R-D. Cependant, toutes les dépenses de R-D sont collectées d'après la comptabilité de caisse et, plus généralement, les questions d'estimation de valeur sortent du cadre du Manuel de Frascati.

11.38 À la lumière de ce qui précède, les mesures de financement de la R-D ne constituent pas un substitut adéquat pour les statistiques sur les échanges de services à des fins de comptabilité nationale et de balance des paiements. En même temps, les enquêtes sur la R-D recueillant des renseignements détaillés sur les financements et les subventions peuvent compléter les informations provenant des enquêtes sur les échanges de services.

\section{Transferts transnationaux de R-D dans la balance des paiements}

11.39 Dans la balance des paiements, les transferts courants comme les subventions en espèces pour la R-D sont enregistrés dans le compte des revenus secondaires du compte des opérations courantes. Les transferts de capitaux consistent dans le transfert de propriété d'un bien ou d'un actif, autre que des espèces, ou la fourniture d'un service, sans rien recevoir en retour qui présente une valeur économique. En tant d'actif produit (suite à la reconnaissance de la R-D comme investissement ou formation de capital dans le SCN 2008), les transferts en nature de R-D sont enregistrés comme échanges de services de $\mathrm{R}-\mathrm{D}$. Les transferts intra-EMN de R-D en nature sont difficiles à quantifier même s'ils tombent dans le domaine à la fois des enquêtes sur les échanges de services et des enquêtes sur les DIRDE. Notons que ce traitement par la balance des paiements des transferts de R-D en nature diffère des transferts de R-D « en nature " qui peuvent être englobés dans les enquêtes sur la R-D. Comme relevé dans la section 3 du chapitre 4 - Fonds de transfert pour la R-D -, étant donné 
que les transferts en nature n'entraînent pas de flux monétaires, ils ne sont pas inclus dans les totaux de dépenses de R-D intra-muros ni dans les totaux du financement de la R-D extra-muros.

\section{Transferts transnationaux de R-D entre secteurs}

11.40 Les subventions en espèces à la R-D et autres transferts peuvent avoir lieu entre secteurs institutionnels (des administrations publiques ou unités d'entreprise aux secteurs sans but lucratif ou de l'enseignement supérieur). Mais les opérations internationales intersectorielles ne sont généralement pas couvertes par les enquêtes sur les échanges de services. Ainsi, les travaux sur les DIRDE et autres enquêtes sur la R-D sont une source importante de transferts de $\mathrm{R}-\mathrm{D}$, à la fois à l'intérieur de l'économie déclarante et à l'échelle internationale. Par exemple, les transferts d'espèces pour la R-D impliquant le reste du monde font partie du financement total de la R-D dans les enquêtes sur la R-D (voir chapitre 4).

\section{Transferts transnationaux de R-D entre sociétés non affiliées}

11.41 Les transferts de R-D (en espèces ou en nature) entre sociétés non affiliées seront probablement peu fréquents entre économies développées, mais peuvent se produire dans le cadre de l'assistance technique entre pays, au même titre que les transferts de R-D faisant intervenir les pouvoirs publics et les institutions sans but lucratif. Les statistiques sur les subventions provenant des enquêtes sur la R-D ainsi que d'autres sources comme les données administratives peuvent être utiles pour distinguer les composantes R-D des autres composantes de l'assistance technique internationale faisant intervenir des unités des secteurs privé et public.

\section{Services intragroupes et prix de transfert}

11.42 Quand il y a transfert transnational de la R-D à l'intérieur des EMN, il est difficile d'obtenir des indicateurs marchands équivalents pour évaluer les prix de transfert interne qui peuvent être faussés en raison de complexités comptables ou organisationnelles, ou de stratégies de minimisation de l'impôt (OCDE, 2014). Au sein des EMN, la fourniture de biens et de services non comptabilisés ou inférieurs aux prix du marché ne représente pas un « transfert » tel que défini ci-dessus, à moins qu'il n'y ait rien en retour pour le fournisseur au cours de la période de référence de l'enquête. Au lieu de cela, les erreurs de déclaration pour les échanges constituent des dividendes ou investissements non déclarés qui devraient être imputés. Les transferts transnationaux de R-D et autres services intra-entreprise peuvent faire partie, même s'ils ne sont pas identifiés de façon distincte, d'autres écritures comptables comme les bénéfices non distribués, les versements de dividendes ou les répartitions/ventilations de coûts. Pour des indications précises sur les services de R-D intra-groupe, voir CEE-ONU/OCDE (2015) (paragraphes 3.2, 3.3, 4.3 et 4.4). S'il y a consensus sur la nécessité théorique d'ajustements statistiques pour les questions de " prix de transfert " (erreur de déclaration ou non déclaration des échanges), mais on 
s'accorde plus difficilement sur des orientations pratiques pour ces ajustements. CEE-ONU/OCDE (2015) (paragraphes 3.42, 4.23, 4.45) suggère de " coller à l'observation statistique ".

11.43 Pour les échanges des services de R-D intra-EMN comme le financement des échanges de R-D intra-EMN, certaines données déclarées peuvent être fondées sur des formules de comptabilisation ou de répartition des coûts qui reflètent mal les flux réels de $\mathrm{R}-\mathrm{D}$, tandis que les transferts gratuits ou non déclarés peuvent également se traduire par des réponses erronées (voir les scénarios possibles dans le tableau 11.1). Autre difficulté, les opérations internationales de R-D au sein des EMN sont souvent difficiles à séparer des activités apparentées et des transferts de plans, prototypes et autres éléments de propriété intellectuelle (voir FMI 2014, paragraphes 12.126, 12.131, 12.134). L'organisation complexe de la production mondiale faisant entrer en jeu, par exemple, la fabrication de biens " sans usine " et des entités à vocation spéciale, complique un peu plus encore la comptabilisation de la production immatérielle et des échanges au sein des entreprises et entre ces dernières (OCDE, 2014, chapitres 3-5 et 11). La mise au point d'indicateurs des flux transnationaux de la R-D des EMN, des subventions à la R-D et des flux de biens immatériels apparentés passe par de plus amples recherches sur les systèmes comptables des répondants, la méthodologie statistique et les méthodes d'enquête, et la comptabilité des EMN, et suppose une collaboration permettant de recouper les résultats d'enquête, comme examiné ci-dessous.

\section{Tableau 11.1. Scénarios relatifs à la déclaration potentielle des flux de R-D des EMN par rapport aux flux effectifs dans les enquêtes sur les DIRDE et le commerce des services}

\begin{tabular}{|c|c|c|}
\hline \multirow{2}{*}{$\begin{array}{l}\text { Financements transnationaux de la R-D } \\
\text { déclarés pour l'année en cours/Commerce } \\
\text { des services de R-D déclaré }\end{array}$} & \multicolumn{2}{|c|}{ Échanges réels de R-D/services de R-D transnationaux } \\
\hline & Oui & Non \\
\hline $\begin{array}{l}\text { Dont: } \\
\text { Valeur marchande exacte } \\
\text { Valeur marchande faussée } \\
\text { (surévaluation ou sous- } \\
\text { évaluation ; problèmes de } \\
\text { "fixation des prix de transfert ») }\end{array}$ & $\begin{array}{l}1 \text { : Scénario idéal } n^{\circ} 1 \text {, sauf réponse } \\
\text { erronée: les financements de R-D et } \\
\text { les services de R-D déclarés pour } \\
\text { l'année en cours ou cumulatifs sont } \\
\text { liés à des flux de } R-D \text { effectifs } \\
\text { (enquêtes sur la R-D et enquêtes sur } \\
\text { le commerce des services) } \\
\text { Erreur de réponse mineure } \\
\text { Erreur de réponse majeure }\end{array}$ & $\begin{array}{l}2 \text { : Formule de répartition utilisée } \\
\text { conformément aux normes } \\
\text { comptables des EMN, mais sans } \\
\text { flux effectifs de R-D (difficulté des } \\
\text { enquêtes sur la R-D et des enquêtes } \\
\text { sur le commerce des services : les } \\
\text { " faux positifs") }\end{array}$ \\
\hline Non & $\begin{array}{l}3 \text { : Subvention en espèces non } \\
\text { comptabilisée ou transfert en nature } \\
\text { non déclaré (difficulté des enquêtes } \\
\text { sur la R-D et des enquêtes sur le } \\
\text { commerce des services : les « faux } \\
\text { négatifs") }\end{array}$ & $\begin{array}{l}4 \text { : Scénario idéal } n^{\circ} 2 \text { : pas de } \\
\text { financement ni de paiement ou } \\
\text { d'achat de services en l'absence de } \\
\text { flux effectif de R-D (enquêtes sur la } \\
\text { R-D et enquêtes sur le commerce des } \\
\text { services) }\end{array}$ \\
\hline
\end{tabular}




\subsection{Mesure de la mondialisation de la R-D à l'extérieur du secteur des entreprises}

11.44 Si l'on s'accorde à reconnaître que des institutions autres que les entreprises jouent un rôle important en tant qu'exécutants et bailleurs de fonds dans la mondialisation de la $\mathrm{R}-\mathrm{D}$, on en sait en revanche moins sur la façon de mesurer les flux internationaux de R-D de ces institutions. Plusieurs des concepts et des pratiques de mesure de la mondialisation de la R-D mis en avant pour les entreprises peuvent dans une large mesure être appliqués également aux unités du secteur de l'État, de celui de l'enseignement supérieur et du secteur privé sans but lucratif (c'est-à-dire hors entreprises). Ces concepts incluent les flux de financement destinés à la R-D ainsi que les relations de propriété et de contrôle. Cela dit, il est nécessaire de prendre en compte plusieurs caractéristiques spécifiques des relations internationales en matière de R-D des institutions des secteurs hors entreprises (désignés collectivement ci-après par le terme " unités hors entreprises ").

\section{Concepts fondamentaux pour mesurer les activités de R-D mondiales hors entreprises}

\section{Sources de financement du reste du monde pour la $R-D$ intra-muros}

11.45 Les unités hors entreprises peuvent mener des activités d'exécution et de financement de la $\mathrm{R}-\mathrm{D}$ avec le reste du monde (unités non résidentes). Le reste du monde est constitué de toutes les unités institutionnelles non résidentes qui effectuent des opérations avec des unités résidentes ou ont d'autres liens économiques avec des unités résidentes. Il englobe également toutes les organisations internationales et les organisations supranationales, définies ci-dessous, y compris les équipements et les activités à l'intérieur des frontières du pays. Plusieurs cas limites sont présentés au chapitre 3.

11.46 Comme observé au chapitre 4, l'analyse des sources de financement des activités de R-D exécutées par des unités implantées sur le territoire national devrait chercher à identifier les financements provenant d'unités implantées dans le reste du monde pour les catégories suivantes :

- Secteur des entreprises

- Secteur de l'État

- Secteur de l'enseignement supérieur

- Secteur privé sans but lucratif

- Organisations internationales, y compris celles physiquement présentes dans le pays de déclaration.

11.47 Bien que ces sources soient potentiellement toutes pertinentes pour chacun des secteurs exécutants, l'utilisation de ces catégories dans les enquêtes devrait être adaptée spécialement au secteur d'affiliation de l'unité déclarante. En particulier, les enquêtes pourraient porter sur le type de financement reçu 
et viser à déterminer dans quelle mesure il correspond à des paiements de transfert (c'est-à-dire sans R-D en contrepartie) ou un échange de fonds pour la $\mathrm{R}-\mathrm{D}$ ou un droit futur sur des résultats de $\mathrm{R}-\mathrm{D}$, selon la terminologie présentée au chapitre 4 .

\section{Affiliations des institutions, par type de relations de contrôle étranger}

11.48 La plupart des types d'unités hors entreprises peuvent être reliés d'un pays à l'autre par des liens de propriété et de contrôle de formes diverses. Comme observé dans les sections précédentes sur les entreprises, il s'agit d'un élément important de la mondialisation de la R-D, puisque la R-D menée par des unités affiliées suppose à la fois un effort financier de la part du " groupe » entier ainsi qu'un avantage potentiel du point de vue des connaissances. Par exemple, dès lors que les universités ou les organisations de recherche indépendantes prennent de l'expansion au niveau mondial, il est important de prendre en compte la mesure dans laquelle l'exécution de la R-D au sein de ces institutions partage les caractéristiques de la mondialisation économique décrite précédemment dans le présent chapitre. À titre d'illustration, une organisation comprenant plusieurs instituts de recherche peut ouvrir des instituts à l'étranger en puisant dans les sources de financement réservées aux endroits où elle s'établit, comme des contrats locaux ou des subventions locales de recherche. Le présent manuel ne propose actuellement aucune recommandation spécifique sur l'identification et la déclaration de telles relations, si ce n'est pour relever que des liens d'affiliation peuvent éclairer la nature et l'objectif des financements reçus par les exécutants de R-D n'émanant pas du secteur des entreprises.

\section{Le personnel et la mondialisation de la R-D en dehors du secteur des entreprises}

11.49 La mondialisation de la R-D va de pair non seulement avec les flux de financement et les relations de propriété transnationales, mais se reflète également dans la mobilité du personnel de R-D. Comme c'est le cas pour le secteur des entreprises, la capacité des enquêtes sur la R-D dans les secteurs hors entreprises à réunir des informations sur l'aspect « ressources humaines " de la mondialisation de la R-D est quelque peu limitée.

11.50 Dans certains cas, il est possible que les registres des entités hors entreprises sur les ressources humaines contiennent des renseignements sur la nationalité, le pays de naissance ou le pays d'emploi précédent de leur personnel de $R-D$, en particulier en ce qui concerne les chercheurs (voir chapitre 5, section 5.4). La collecte de ces données par le biais d'enquêtes institutionnelles n'est pas nécessairement conseillée, même si elle peut illustrer certains types de flux internationaux de R-D.

11.51 Quand des informations sont disponibles sur le statut des institutions en matière de contrôle, il est possible de fournir des ventilations d'indicateurs 
standard des ressources humaines pour la R-D, établissant une distinction entre le personnel actif dans des entités hors entreprises sous contrôle étranger et celui œuvrant pour des institutions hors entreprises indépendantes.

\section{Secteur de l'État}

11.52 Les administrations publiques peuvent recevoir des financements destinés à la R-D en provenance de l'étranger, et elles peuvent financer des travaux de R-D extra-muros à l'étranger. Ces activités de financement à l'étranger (avec des unités non résidentes) devraient être recensées d'après les catégories suivantes du reste du monde, tel que défini au chapitre 8 :

- Secteur des entreprises

- Secteur de l'État

- Secteur de l'enseignement supérieur

- Secteur privé sans but lucratif

- Organisations internationales.

11.53 Il est également recommandé de ventiler le financement et l'exécution de la R-D par le secteur de l'État à l'échelle mondiale selon les deux catégories de fonds suivantes : fonds d'échange (spécifiquement dénommés “ marchés publics » et financés par le secteur de l'État) et fonds de transfert. Si l'exécution de R-D intra-muros par l'État à l'extérieur de son territoire national n'est pas très fréquente, le financement de R-D par l'État vers le reste du monde peut être conséquent. Par exemple :

- En tant que bailleur de fonds, l'État peut utiliser des marchés publics (fonds d'échange) à l'étranger pour encourager le développement d'une technologie ou d'un secteur de R-D.

- L'État est également le principal bailleur de fonds des organisations internationales de R-D. Sous la forme de "contributions nationales » (transferts), l'État peut financer des institutions intergouvernementales de R-D ainsi que des programmes/projets intergouvernementaux de R-D. Cependant, il convient d'inclure seulement les contributions destinées à des programmes internationaux de R-D ou à des organisations dont les activités sont uniquement ou principalement dédiées à la R-D. Les contributions générales au budget global (comme celles versées à l'ONU, à l'OCDE ou à l'UE) doivent être exclues, à moins qu'elles n'intègrent une composante explicite, destinée spécifiquement aux activités de R-D (voir chapitres 8 et 12).

\section{Secteur de l'enseignement supérieur}

11.54 Les unités de l'enseignement supérieur peuvent recevoir des financements destinés à la R-D en provenance du reste du monde, comme elles peuvent financer des travaux de R-D dans le reste du monde. Ces activités de 
financement avec des unités non résidentes devraient être recensées d'après les catégories suivantes du reste du monde :

- Secteur des entreprises

- Secteur de l'État

- Secteur de l'enseignement supérieur

* Campus satellites à l'étranger

* Autres universités

- Secteur privé sans but lucratif

- Organisations internationales.

11.55 En particulier, de nombreuses institutions dans le secteur de l'enseignement supérieur ont créé des branches ou des campus à l'étranger. Dans la mesure où des campus satellites sous contrôle étranger à l'intérieur du pays déclarant et des campus satellites à l'étranger détenus par des établissements locaux d'enseignement supérieur exécutent de la R-D, les enquêtes sur les DIRDES peuvent comprendre des informations complémentaires à propos de ces campus.

- Aux fins du présent manuel, un campus satellite étranger est un établissement d'enseignement supérieur qui se trouve sur le territoire du pays déclarant ; qui est détenu, du moins en partie, par une entité implantée (ou résidant) en dehors du pays déclarant (appelée " prestataire d'enseignement étranger ») ; dont l'activité est régie par cette entité ; qui consacre au moins une partie de ses activités à l'enseignement direct ; et qui donne accès à un programme universitaire complet sanctionné par un diplôme du prestataire étranger.

- Aux fins du présent manuel, un campus satellite à l'étranger est un établissement d'enseignement supérieur qui est détenu, du moins en partie, par un établissement local d'enseignement supérieur (c'est-à-dire résidant sur le territoire du pays déclarant) mais est situé dans le reste du monde (résidant en dehors du pays déclarant) ; dont l'activité est régie par l'établissement local ; qui consacre au moins une partie de ses activités à l'enseignement direct ; et qui donne accès à un programme universitaire complet sanctionné par un diplôme de l'établissement local d'enseignement supérieur.

11.56 La R-D exécutée par des campus satellites sous contrôle étranger entre dans les totaux des DIRDES correspondant à l'exécution de R-D sur le territoire du pays déclarant, mais pas la R-D exécutée dans les campus satellites à l'étranger. Au lieu de cela, elle pourrait être identifiée et mise sous forme de tableaux de façon distincte comme R-D exécutée dans le reste du monde par des établissements d'enseignement supérieur en dehors des établissements d'enseignement du pays déclarant (pour des orientations plus détaillées concernant la collecte des totaux des campus satellites sous contrôle étranger et des campus satellites à l'étranger, voir chapitre 9, section 9.4). 


\section{Secteur privé sans but lucratif}

11.57 Comme avec les unités des autres secteurs, les institutions exécutantes de R-D dans le secteur privé sans but lucratif peuvent avoir des activités mondiales multiples avec à la fois des unités affiliées et non affiliées. Une institution peut recevoir des fonds pour exécuter de la R-D sous la forme de subventions ou de contrats de la part d'autres institutions privées sans but lucratif non affiliées implantées en dehors du territoire national, ou (en fonction de sa structure d'affiliation), peut recevoir des fonds d'une autre organisation mère ou affiliée en soutien à ses activités dans le pays où elle est située. Ainsi, des institutions privées sans but lucratif peuvent recevoir des financements destinés à la R-D en provenance du reste du monde, comme elles peuvent financer des travaux de R-D dans le reste du monde. De telles relations peuvent être extrêmement complexes en termes à la fois d'identification et de mesure statistique. Les catégories pertinentes pour le reste du monde sont :

- Secteur des entreprises

- Secteur de l'État

- Secteur de l'enseignement supérieur

- Secteur privé sans but lucratif

* Institutions affiliées (organisations non gouvernementales internationales, ONGI)

* Autre institutions non affiliées

- Organisations internationales, y compris les organisations supranationales.

11.58 Certaines institutions privées sans but lucratif peuvent avoir une présence mondiale, comme c'est le cas de nombreuses organisations non gouvernementales. Ce ne sont pas des organisations internationales au sens du SCN ou du présent manuel (voir chapitre 3 et la section sur les organisations internationales ci-dessous).

\section{Cas particulier des organisations internationales}

11.59 D'après le SCN, les organisations internationales comptent parmi leurs membres soit des États, soit d'autres organisations internationales dont les membres sont des États. En font partie les organisations supranationales, telles que définies au chapitre 3. Les organisations internationales sont instituées par la conclusion, entre leurs membres, d'accords politiques formels ayant le statut de traité international ; leur existence est reconnue par la législation des pays qui en sont membres et elles ne sont pas assujetties aux lois ou aux règlements du ou des pays dans lesquels elles sont présentes. Ce statut peut impliquer en particulier qu'aucun État ne peut les contraindre à fournir des renseignements statistiques sur leurs résultats en matière de R-D ou leurs activités de financement. Du point de vue du pays déclarant, elles font partie du secteur du reste du monde. 
11.60 En raison de l'importance des organisations internationales dans l'exécution de la R-D à l'échelle mondiale dans plusieurs domaines, et afin d'obtenir une représentation plus complète des activités de R-D dans le monde, les organismes statistiques internationaux et supranationaux concernés devraient collaborer pour assurer une couverture complète des unités exécutantes de R-D qui sortent du champ couvert par les offices statistiques nationaux. À l'avenir, ces totaux pourront être présentés sous la forme de statistiques internationales comparatives dans une catégorie distincte de celle au niveau national. Cela pourrait également contribuer à l'amélioration de la cohérence entre les statistiques de R-D et d'autres indicateurs, comme les publications scientifiques, qui sont généralement attribués sur la base du pays dans lequel se trouvent les affiliations des auteurs.

11.61 Lorsque les offices statistiques nationaux sont en mesure de collecter des données auprès des organisations internationales en activité sur leur territoire national, la déclaration des chiffres nationaux devrait suivre la consigne donnée par le présent manuel de traiter ces unités comme faisant partie du secteur du reste du monde. Pour établir si une unité institutionnelle donnée a le statut d'organisation internationale, il convient d'accorder une attention particulière à ses statuts constitutifs et aux accords régissant son fonctionnement, y compris les exemptions de la juridiction du droit local sur un certain nombre d'aspects en conséquence de l'adhésion d'États souverains à l'organisation.

11.62 Une zone de confusion possible émane de la similarité potentielle entre les organisations internationales, qui sont définies dans le présent manuel ainsi que dans d'autres manuels statistiques comme organisations intergouvernementales, et d'autres structures internationales comprenant des organisations non gouvernementales (ONG), qui ne remplissent pas les critères nécessaires pour être traitées comme unités non résidentes.

11.63 Les ONG sont des organisations ou des structures autonomes bénévoles créées pour remplir les objectifs essentiellement sans but lucratif de leurs fondateurs ou de leurs membres (Conseil de l'Europe, 2007). Elles n'incluent pas les partis politiques. Les ONG englobent les structures ou organisations établies à la fois par des particuliers (personnes physiques ou morales) et par des groupes de particuliers. Elles peuvent fonctionner sur la base d'un régime d'adhésion ou non. Les ONG peuvent être soit des structures ou organisations informelles, soit des structures dotées d'une personnalité juridique. Elles peuvent être nationales ou internationales dans leur composition ou leur champ d'action. Elles n'ont pas à distribuer à leurs membres ou à leurs fondateurs de bénéfices pouvant provenir de leurs activités, mais elles peuvent les utiliser dans la poursuite de leurs objectifs.

11.64 Par exemple, une ONG mondiale qui est une association internationale d'universités impliquées dans la recherche dans un domaine particulier exploitant et utilisant des installations de recherche peut entreprendre des 
activités très similaires, dans des endroits semblables, à celles d'une organisation internationale existante composée de gouvernements. Du point de vue du pays déclarant qui héberge les équipements d'exécution de la $R-D$, le centre local dont l'association internationale est propriétaire (c'est-à-dire l'ONG mondiale) devrait être comptabilisé comme faisant partie de l'économie nationale et son exécution de R-D intra-muros être incluse dans les DIRD, alors que l'organisation intergouvernementale devrait être traitée comme appartenant au reste du monde.

11.65 Certains pays peuvent être partie à un accord institutionnel qui fait intervenir des flux financiers entre les pays membres et l'organisation internationale associée, et vice versa, par exemple, à des unités exécutantes de R-D. L'organisation internationale peut elle-même mener des activités de R-D. Pour les pays pris individuellement, les organisations internationales sont des unités institutionnelles non résidentes qui font partie du reste du monde et peuvent être classées dans un sous-secteur spécifique du reste du monde.

11.66 Il peut exister d'autres organisations et infrastructures de recherche dont les activités opérationnelles soient assez similaires à celles de ces organisations internationales, même à l'intérieur du même pays. Par exemple, une structure de mesure et de recherche scientifique sans but lucratif peut avoir été mise sur pied par une organisation composée d'États souverains, mais il peut exister une autre structure similaire exerçant des fonctions équivalentes, sous le contrôle d'un consortium international d'universités ou autres organisations privées sans but lucratif. La première nommée devrait être considérée comme une organisation internationale au sens intergouvernemental, alors que la seconde devrait être traitée comme faisant partie du secteur privé sans but lucratif (sous contrôle étranger). Cette dernière institution non gouvernementale contribuerait aux DIRD de l'économie nationale, ce qui ne serait pas le cas de la première nommée. Des critères similaires s'appliqueraient aux ressources humaines affectées par ces organisations à des travaux de R-D.

\section{Questions relatives aux exécutants de R-D - échelle nationale ou reste du monde}

11.67 Il n'est pas nécessaire que les entités unies par des liens d'affiliation internationales exercent obligatoirement une activité dans le même secteur. Par exemple, un centre de recherche privé national avec un statut sans but lucratif ou même d'entreprise peut être la propriété d'une université étrangère, ou vice versa. Dans la pratique normale des enquêtes, il peut être particulièrement fastidieux d'essayer d'introduire toutes les permutations possibles de sources de financement institutionnelles non résidentes avec les relations d'affiliation.

11.68 Le chapitre 4 explique que la notion de R-D intra-muros et l'allocation de cette dernière à un secteur national ou au reste du monde ne s'appuie pas seulement sur l'endroit où est exécutée la $\mathrm{R}-\mathrm{D}$, mais concerne également l'autorité institutionnelle sous laquelle la recherche est effectuée. Par exemple, 
si un chercheur d'une université locale passe une partie de son temps dans les installations dont est propriétaire une organisation internationale, tout en travaillant pour son université de rattachement, et qu'il est comptabilisé comme employé de cette université, les frais correspondant au salaire de ce chercheur devraient être comptabilisés comme exécution intra-muros de l'université nationale. Si l'organisation internationale propriétaire de ces installations finance une partie des travaux, ou que le chercheur en question peut faire état d'une double affiliation, l'attribution peut être plus compliquée, et il y a un risque possible de double comptabilisation qui devra être géré avec discernement.

11.69 Comme noté également au chapitre 4, les dépenses de R-D intra-muros sont censées mesurer la R-D exécutée au sein des unités statistiques résidentes sur le territoire national du pays déclarant. Certaines des dépenses peuvent toutefois avoir été effectuées à l'étranger. Par exemple, les dépenses de R-D intra-muros peuvent inclure :

- les coûts de maintien et d'utilisation d'une présence permanente d'un État pour la recherche en Antarctique

- les coûts liés au travail sur le terrain d'un chercheur de l'enseignement supérieur dans un lieu extérieur au pays déclarant ou au sein d'une organisation internationale située à l'intérieur de son propre pays.

11.70 Lorsque l'on classe comme " intra-muros » des activités de R-D qui se déroulent hors du territoire national du pays déclarant, la priorité devrait être accordée plus à la structure organisationnelle de l'activité qu'à l'endroit littéral où celle-ci a lieu. Il est difficile de fournir des indications précises pour ce genre de décision de classification, mais la R-D intra-muros réalisée dans le reste du monde devrait au minimum inclure uniquement la R-D exécutée par une unité statistique pour remplir ses propres objectifs et seulement si l'unité statistique a investi des ressources financières et du personnel de R-D propres dans l'activité. La R-D doit se dérouler sous la responsabilité de l'unité déclarante, et l'unité déclarante doit remplir les critères de résidence économique décrits au chapitre 3.

\section{Références}

CE, FMI, OCDE, Nations Unies et Banque mondiale (2009), Système de comptabilité nationale 2008, Nations unies, New York, http://unstats.un.org/unsd/nationalaccount/ docs/SNA2008FR.pdf.

CE (2012), Foreign Affiliates Statistics (FATS) Recommendations Manual, Eurostat, Luxembourg.

CE (2010), Business Registers Recommendations Manual, Eurostat, Luxembourg.

CEE-ONU/Eurostat/OCDE (2011), The Impact of Globalisation on National Accounts, CEE-ONU, Genève.

CEE-ONU/OCDE (2015), Guide to Measuring Global Production, CEE-ONU, Genève, https:// www.unece.org/fileadmin/DAM/stats/documents/ece/ces/bur/2015/February/16Add.1Guide_to_Measuring_Global_Production.pdf. 
Conseil de l'Europe (2007), Recommandation CM/Rec(2007)14 du Comité des Ministres aux États membres sur le statut juridique des organisations non gouvernementales en Europe, Conseil de l'Europe, Strasbourg, https://wcd.coe.int/ViewDoc.jsp?Ref=CM/Rec(2007)14\& Language $=$ lanFrench $\&$ Ver $=$ original $\&$ Site $=$ COE\&BackColorInternet $=$ DBDCF2\&BackColorIntra net=FDC864\&BackColorLogged=FDC864.

FMI (2014), Balance of Payments and International Investment Position Compilation Guide, FMI, Washington, D.C.

FMI (2009), Balance of Payments and International Investment Position Manual, Sixth Edition, FMI, Washington, D.C.

Nations Unies, Eurostat, FMI, OCDE, CNUCED, OMT et OMC (2014), The Compilers Guide for MSITS 2010, Nations Unies, New York, http://unstats.un.org/unsd/trade/publications/ MSITS2010_Compilers\%20Guide_Unedited\%20White\%20Cover\%20Version\%20-\%2012\%20 February\%202015.pdf.

Nations Unies, Eurostat, FMI, OCDE, CNUCED, OMT et OMC (2011), Manuel des statistiques du commerce international des services 2010, Nations Unies, New York.

OCDE (2014), Instructions relatives aux aspects intéressant les prix de transfert des actifs incorporels, Projet OCDE/G20 sur l'érosion de la base d'imposition et le transfert de bénéfices, Éditions OCDE, Paris, http://dx.doi.org/10.1787/9789264225350-fr.

OCDE (2009a), Définition de référence de l'OCDE des investissements directs internationaux 2008 : Quatrième édition, Éditions OCDE, Paris, http://dx.doi.org/10.1787/9789264048768-fr.

OCDE (2009b), Handbook on Deriving Capital Measures of Intellectual Property Products, Éditions OCDE, Paris, http://dx.doi.org/10.1787/9789264079205-en.

OCDE (2005), Mesurer la mondialisation: Manuel de l'OCDE sur les indicateurs de la mondialisation économique, Éditions OCDE, Paris, http://dx.doi.org/10.1787/9789264108110-fr. 
PARTIE III

\section{Mesurer le soutien public en faveur de la R-D}





\section{Chapitre 12}

\section{Crédits budgétaires publics de R-D}

Le présent chapitre expose une approche visant à mesurer le financement public de la R-D à partir des données relatives aux crédits publics. Partant du point de vue des bailleurs de fonds, elle consiste à recenser tous les postes budgétaires susceptibles de financer des activités de R-D et à mesurer ou estimer la part que la R-D y représente. L'intérêt de cette approche est notamment de permettre de communiquer beaucoup plus rapidement les montants totaux des financements publics consacrés à la $R-D$, dans la mesure où ils sont calculés à partir des budgets, et de les rattacher à l'action des pouvoirs publics en les classant par objectif socio-économique. Les définitions citées dans ce chapitre sont aussi conformes que possible aux méthodologies et principes directeurs internationaux énoncés dans le Manuel de statistiques de finances publiques 2014 du FMI et le Système de comptabilité nationale (SCN) de 2008, ainsi qu'aux méthodologies élaborées par Eurostat, à l'instar de la Nomenclature pour l'analyse et la comparaison des budgets et des programmes scientifiques (NABS). 


\subsection{Introduction}

12.1 Il existe différentes façons de mesurer les dépenses des pouvoirs publics en faveur de la R-D. L'approche du point de vue des exécutants préconisée dans le chapitre 4 consiste à sonder les unités résidentes qui mènent des activités de R-D (entreprises, instituts, universités, etc.), de manière à obtenir le montant des dépenses de R-D intra-muros engagées au cours d'une année de référence. Il est alors possible de mettre en évidence la part de ces dépenses financée par les pouvoirs publics (chapitre 4, tableau 4.4). Les montants totaux ainsi obtenus donnent une mesure précise des financements publics consacrés à la R-D intra-muros exécutée dans l'économie considérée, rapportée aux dépenses intérieures brutes de R-D (DIRD). L’approche présente néanmoins des inconvénients : d'une part, ces informations ne sont pas disponibles avant un certain délai et, d'autre part, les exécutants ne sont pas toujours en mesure d'établir un lien entre les fonds publics qu'ils perçoivent et les objectifs d'action publique.

12.2 En complément, une autre méthode a été mise au point pour mesurer le financement public de la R-D à l'aide des données tirées des budgets. Partant du point de vue des bailleurs de fonds, elle consiste à recenser tous les postes budgétaires susceptibles de financer des activités de R-D et à mesurer ou estimer la part que la R-D y représente. A priori, l'intérêt de cette approche est de permettre à la fois de communiquer beaucoup plus rapidement les montants totaux des financements publics consacrés à la $\mathrm{R}-\mathrm{D}$, dans la mesure où ils sont calculés à partir des budgets, et de les rattacher à l'action des pouvoirs publics en les classant par objectif socio-économique (voir section 12.4 ci-après).

12.3 Les données fondées sur le budget renvoient au concept officiel de " crédits budgétaires publics de R-D » (CBPRD), qui est apparu dans la troisième édition du Manuel et est décrit dans le présent chapitre.

12.4 Les définitions citées dans ce chapitre sont aussi conformes que possible aux méthodologies et principes directeurs internationaux énoncés dans le Manuel de statistiques de finances publiques 2014 du FMI et le Système de comptabilité nationale (SCN) de 2008, ainsi qu'aux méthodologies élaborées par Eurostat, à l'instar de la Nomenclature pour l'analyse et la comparaison des budgets et des programmes scientifiques (NABS).

\subsection{Champ des CBPRD}

\section{Administrations publiques entrant dans le champ des CBPRD}

12.5 Comme évoqué dans le chapitre 3 (section 3.5), puis développé plus avant dans le chapitre 8, le secteur de l'État comprend les sous-secteurs 
suivants : l'administration centrale (fédérale), l'administration régionale (États fédérés) et l'administration locale (municipale). Les statistiques afférentes aux CBPRD portent sur les dépenses de R-D engagées par les administrations à chacun de ces niveaux et financées sur le budget dans le cadre des procédures budgétaires normales. Pour minimiser les délais et la charge potentielle liée à la communication des données, il est possible de ne pas comptabiliser les fonds budgétaires octroyés par les administrations locales si leur contribution n'est pas jugée significative, ou si les données ne peuvent être recueillies.

12.6 Comme indiqué dans le Système de comptabilité nationale et dans le Manuel de statistiques de finances publiques du FMI, l'unité budgétaire de l'administration centrale (fédérale) est généralement une unité unique qui englobe les activités fondamentales des pouvoirs exécutif, législatif et judiciaire au niveau national. Cette unité de l'administration générale est généralement couverte par le budget principal (ou général). Ses recettes et ses dépenses sont normalement réglementées et contrôlées par le ministère des Finances ou par un organe fonctionnel équivalent, au moyen d'un budget approuvé par le parlement (FMI, 2014, paragraphe 2.81).

12.7 À un niveau d'administration donné, les ministères, directions, agences, conseils administratifs, commissions, autorités judiciaires, organes législatifs et autres entités qui composent cette administration sont rarement habilités à détenir des actifs, contracter des engagements ou effectuer des opérations en leur nom propre. En règle générale, toutes les entités dont les opérations sont financées à l'aide de crédits budgétaires octroyés au titre d'un budget contrôlé par le parlement ne sont pas des unités institutionnelles distinctes et sont traitées comme une unité statistique unique.

12.8 Les entités d'administration publique qui sont dotées d'un budget propre et ne sont pas entièrement couvertes par le budget général sont considérées comme extrabudgétaires (voir chapitre 8) et sont prises en compte dans le calcul des CBPRD. Les dispositions budgétaires dont elles font l'objet varient considérablement d'un pays à l'autre ; elles peuvent être désignées sous différentes appellations, dont les plus fréquentes sont « comptes extrabudgétaires » ou " organismes décentralisés » (FMI, 2014).

12.9 Les CBPRD comprennent l'ensemble des crédits budgétaires financés par des recettes publiques inscrites au budget, notamment des recettes fiscales. Les crédits affectés par des entités publiques extrabudgétaires n'en font partie que dans la mesure où ils ont été alloués dans le cadre de la procédure budgétaire. De même, le financement de la R-D par les entreprises publiques est exclu du champ des statistiques de CBPRD, car il repose sur des fonds levés sur le marché, hors procédure budgétaire. Il ne relève des CBPRD que dans le cas exceptionnel où il s'agit de provisions de fonds du budget de l'État utilisés ou distribués en faveur de la R-D par des entreprises publiques. Le Manuel ne définit pas les concepts qui sous-tendent la notion de dépenses, qui varient selon les pays. De fait, certains pays fondent leurs déclarations sur les dépenses, d'autres 
sur les octrois de crédits, d'autres encore sur les engagements budgétaires. Quel que soit le concept retenu, l'important est de s'y conformer pour l'agrégation des CBPRD totaux.

\section{Définition et évaluation de la part des activités de R-D dans les CBPRD}

12.10 Le chapitre 2 définit la R-D. Comme indiqué précédemment, le SCN de 2008 et le Manuel de statistiques de finances publiques 2014 - soit les principaux cadres d'élaboration des statistiques des administrations et secteurs publics - se fondent sur les définitions issues de la précédente édition du Manuel de Frascati, qui ont, à peu de choses près, été conservées à l'identique dans le chapitre 2.

12.11 La recherche fondamentale, la recherche appliquée et le développement expérimental sont tous trois pris en compte, mais ne sont pas présentés séparément dans les montants de CBPRD. Par ailleurs, l'analyse des données budgétaires relatives aux activités de R-D couvre les sciences naturelles et l'ingénierie, ainsi que les sciences sociales, les sciences humaines et les arts.

12.12 Il conviendrait, dans la mesure du possible, d'appliquer tous les principes directeurs et les conventions recensés dans le chapitre 2 pour distinguer la R-D des activités hors R-D. Il importe de veiller tout particulièrement à vérifier la composante R-D des postes budgétaires décrits officiellement comme des activités scientifiques et technologiques, des contrats de développement ou des acquisitions de prototypes, comme évoqué dans les chapitres 2, 4 et 7, ainsi que des autres dépenses ayant trait à la science, la technologie et l'innovation, que certains pays peuvent assimiler à des dépenses de R-D ou agréger à ces dépenses lorsqu'ils présentent les données du budget général.

12.13 Les statisticiens chargés de compiler les statistiques de CBPRD pourraient avoir besoin d'élaborer un ensemble de coefficients selon la discipline, l'institution ou d'autres critères, ou une combinaison de ces éléments, de manière à déterminer la part de la R-D dans des postes budgétaires non exclusifs, notamment pour un certain nombre d'institutions dont les activités ne se limitent pas à la R-D. Ces coefficients devraient, dans la mesure du possible, être conformes à ce que ces institutions déclarent au titre de la R-D lors des enquêtes menées auprès des exécutants. Les informations relatives aux coefficients devraient être communiquées aux utilisateurs de la manière la plus transparente possible afin de faciliter l'examen et les mises à jour des données.

12.14 Les budgets publics consacrés à la R-D peuvent inclure la prise en charge des coûts d'administration des programmes et projets de R-D, y compris, par exemple, les coûts liés à formulation des appels à projets, aux procédures de passation de marchés avec mise en concurrence et aux demandes de subventions, ou encore au suivi et à l'évaluation des programmes. En principe, les CBPRD devraient couvrir uniquement le financement des activités de R-D. 
Force est de constater, néanmoins, que ces coûts d'exécution peuvent faire partie intégrante du processus destiné à garantir que les fonds sont consacrés aux activités de R-D et à la réalisation des objectifs des administrations publiques. Il peut en outre s'avérer difficile de les isoler, en particulier pendant les phases d'élaboration des budgets. D'où une possible divergence entre les estimations du soutien public à la R-D selon que l'on se place du point de vue des exécutants ou des administrations (voir chapitre 4, section 4.4). C'est pourquoi il conviendrait d'utiliser les informations disponibles pour donner un ordre de grandeur des sommes consacrées à l'administration des activités de R-D.

\section{Types de dépenses de R-D couvertes par les données de CBPRD}

\section{Types de coûts}

12.15 En principe, les CBPRD couvrent à la fois le financement des dépenses courantes et des dépenses en capital. L'une des différences majeures par rapport aux statistiques de finances tient au fait que les dépenses publiques intègrent une composante d'amortissement, tandis que, dans le budget, les dépenses en capital peuvent être présentées séparément. Les recommandations formulées dans le chapitre 4 concernant le traitement des dépenses en capital de façon à éviter une double comptabilisation sont également valables dans le contexte des statistiques de CBPRD. Autre facteur à prendre en considération, les éléments constitutifs des dépenses en capital peuvent différer selon que l'on se place du point de vue des bailleurs de fonds ou des exécutants.

\section{Types de bénéficiaires des financements}

12.16 Les CBPRD couvrent non seulement la R-D financée par l'État et exécutée dans des entités publiques, mais également la R-D financée par l'État et exécutée dans les trois autres secteurs qui composent l'économie nationale (entreprises, enseignement supérieur et secteur privé sans but lucratif) et dans le reste du monde (y compris les organisations internationales). Par conséquent, il convient de ne pas confondre les CBPRD et les dépenses intérieures de R-D de l'État (DIRDET). Comme évoqué dans les chapitres 4 et 8, toutes les DIRDET ne sont pas financées par l'État.

12.17 Les crédits budgétaires sont parfois alloués à des institutions qui n'exécutent pas nécessairement des activités de R-D. De fait, une part non négligeable des budgets publics de R-D peut être affectée à des institutions telles que des agences ou des organisations spécifiquement chargées d'allouer des fonds aux exécutants de la R-D ou à d'autres agences intermédiaires conformément aux responsabilités qui leur incombent. Le niveau de détail des données du budget général d'une administration centrale, régionale ou locale ne permet donc pas toujours aux statisticiens chargés de compiler les statistiques de CBPRD de déterminer l'utilisation finale des fonds. En revanche, les déclarations budgétaires de ces agences intermédiaires, au sein ou en dehors 
de l'administration, peuvent apporter des informations supplémentaires, y compris, parfois, sur l'identité des bénéficiaires ultimes du soutien financier en faveur de la R-D. Sans compter que ces derniers peuvent eux-mêmes sous-traiter une partie des activités de R-D.

12.18 Un certain nombre de directions et d'agences peuvent jouer un rôle dans la distribution et l'allocation des fonds budgétaires à d'autres organismes publics ou privés. Si les CBPRD ne sauraient être utilisés pour déclarer les montants relatifs à des sous-ensembles des administrations, il convient néanmoins de veiller, par exemple, à :

- déduire les fonds que l'administration centrale (fédérale) alloue aux budgets des administrations régionales (États fédérés) ou locales (municipales), lors de la compilation des estimations de CBPRD au niveau régional, si ces fonds ont déjà été comptabilisés au niveau de l'administration centrale

- éviter les risques de double comptabilisation ou de sous-estimation lors de l'élaboration des statistiques de CBPRD à partir des déclarations séparées des agences et directions de l'administration centrale.

\section{Financement public de la R-D dans le reste du monde}

12.19 Les crédits budgétaires publics de R-D peuvent intégrer l'allocation de fonds à des institutions non résidentes. Dans le cas des CBPRD consacrés à la R-D dans le reste du monde, il convient d'inclure seulement les contributions destinées à des programmes internationaux de R-D ou à des organisations dont les activités sont uniquement ou principalement dédiées à la R-D. Les contributions générales au budget global (à l'instar de celles versées aux organisations internationales ou à l'Union européenne) doivent être exclues, à moins qu'elles n'intègrent une composante explicite, destinée spécifiquement aux activités de R-D. Les orientations complémentaires exposées dans les annexes au présent Manuel, disponibles en ligne à l'adresse http://oe.cd/frascati, pourraient à l'avenir fournir une liste indicative des organisations internationales présentant une intensité de R-D particulièrement élevée. Ce point est examiné plus avant dans le chapitre 11 sur la mondialisation de la R-D.

\section{Types de mécanismes de soutien en faveur de la R-D et prise en compte dans les statistiques de CBPRD}

\section{Financement par l'État des dépenses publiques de R-D intra-muros}

12.20 Le soutien public en faveur de la R-D exécutée au sein du secteur de l'État a été abordé dans le chapitre 8. Dans l'optique des statistiques de CBPRD, un problème se pose lorsque des crédits destinés à la R-D exécutée par des établissements publics sont censés être financés par d'autres sources. Dans certains pays, ces montants peuvent être inclus dans le budget de l'État puisque l'organisme concerné doit obtenir l'autorisation de l'État pour les dépenser (démarche " brute »). Dans d'autres, il arrive qu'ils en soient exclus (démarche 
" nette »). Lorsque l'on étudie ces financements publics, il convient d'établir une distinction entre :

a) Les contrats ou subventions émanant d'autres secteurs pour l'exécution d'activités de R-D par des établissements publics. Il ne s'agit pas, dans ce cas, de crédits budgétaires publics en faveur de la R-D.

b) Les autres fonds publics, tels que les recettes de prélèvements généraux assimilés à des taxes, ou d'autres fonds publics financés sur le budget. Ces fonds entrent bien dans le cadre des crédits budgétaires publics en faveur de la R-D.

12.21 Conformément au principe fixé par la démarche " nette ", les fonds inscrits au budget financés à l'aide de recettes censées provenir de sources non budgétaires ne devraient pas être inclus dans les CBPRD. Par exemple, si le budget général montre qu'un établissement public de R-D est doté d'un budget brut total de 10 millions (dont 3 millions pour des travaux de recherche sous contrat financés par des crédits externes), seuls 7 millions devraient être comptabilisés au titre des crédits budgétaires nets alloués à cet établissement, les 3 autres millions étant inscrits au budget du bailleur de fonds du contrat de recherche.

\section{Fourniture d'infrastructures et de services aux activités de R-D exécutées par des tierces parties}

12.22 Certains exemples d'administrations publiques fournissant ce type de services ont été examinés dans le chapitre 8, où l'on s'est notamment demandé s'ils devaient être considérés comme des activités de R-D exécutées par le secteur de l'État. Les services fournis par les entités publiques peuvent être partiellement subventionnés à l'aide de crédits budgétaires publics, selon la différence entre le coût économique des services fournis par les administrations publiques et les frais ou le prix acquittés par l'utilisateur qui exécute la R-D, aux termes de la démarche " nette ». Le coût des services peut comprendre à la fois le coût d'exploitation et le coût d'opportunité des ressources utilisées par les actifs d'infrastructure. Dans certains cas, les frais peuvent également englober les coûts d'amortissement et de financement des infrastructures. Pour les besoins des CBPRD, il est recommandé de procéder comme suit :

- Comptabiliser en tant que CBPRD et, si possible, consigner séparément les fonds budgétaires destinés à l'acquisition ou à la construction d'équipements de R-D par les administrations. Dans la plupart des documents budgétaires, on distingue les dépenses courantes des dépenses en capital. Ce type de dépenses en capital peut être difficile à constater, en particulier si les actifs sont destinés à une utilisation longue, ce qui peut fausser les comparaisons si elles en sont exclues.

- Comptabiliser de façon suivie les coûts d'exploitation et de maintenance des infrastructures, les frais hors utilisation, etc., en tant que CBPRD, tant que ces coûts sont inscrits au budget. 
- Exclure les coûts d'amortissement et de financement des infrastructures, si possible, afin d'éviter une double comptabilisation. Dans certains cas, il peut être utile de les déclarer séparément.

12.23 Les mêmes principes peuvent être appliqués lorsque les infrastructures sont développées et/ou exploitées par une tierce partie, pour autant qu'il soit possible d'identifier la composante R-D et de déterminer la visée des fonds budgétaires.

\section{Paiements au titre des services de R-D}

12.24 Les paiements effectués en contrepartie de services de R-D fournis par des tierces parties peuvent conférer aux administrations publiques des droits économiques et juridiques, pas nécessairement exclusifs, sur les résultats de la R-D. Il s'agit d'achats de services de R-D, généralement désignés comme des contrats de services de R-D ou des achats de R-D. Ils correspondent davantage à des échanges qu'à des transferts, aux termes de la définition fournie dans le chapitre 4. Ces opérations peuvent être soumises à des règles spécifiques et menées dans un cadre commercial ou pré-commercial. Dans un cas comme dans l'autre, les paiements doivent être comptabilisés comme des CBPRD, à condition qu'ils soient inscrits au budget.

12.25 Les paiements réalisés au titre des contrats de R-D peuvent intégrer une composante de bénéfice et/ou de prime. Le cas échéant, la valeur totale des paiements doit être comptabilisée en tant que CBPRD, même si cela a pour effet d'accentuer l'écart avec les estimations du point de vue des exécutants, qui devraient, en principe, exclure la composante " bénéfice ". Les paiements en contrepartie de biens et de services intégrant ou prévoyant des activités de R-D ne devraient pas être comptabilisés au titre des CBPRD, à moins que la composante R-D puisse être identifiée et enregistrée séparément dans le budget, et que les paiements portent spécifiquement sur les activités de R-D concernées.

\section{Subventions en faveur de la R-D}

12.26 Les administrations peuvent financer des activités de R-D menées par des entreprises ou d'autres types d'organisations sans exiger de droits importants sur les résultats/produits du projet, ou assortir le financement d'une obligation de fournir, en contrepartie, un bien ou un service. Ces opérations, qui correspondent à des paiements de transfert, sont souvent décrites comme des subventions ou des primes à la R-D. Elles sont généralement régies par un accord formel, qui peut en outre conditionner le financement à des jalons et des résultats, ou exiger le remboursement des sommes versées si les conditions ne sont pas remplies. Les subventions sont comptabilisées dans les CBPRD si elles sont inscrites au budget. Elles peuvent être destinées à couvrir les coûts d'exploitation et les dépenses en capital. Les administrations peuvent également octroyer des subventions à des unités d'autres secteurs en contrepartie de la fourniture de services, de l'accès à des infrastructures ou du transfert d'actifs immobilisés que les exécutants de la R-D peuvent utiliser. 
12.27 Les fonds généraux des universités (FGU) d'origine publique constituent un type particulier de mécanisme de transfert de crédits publics en faveur de la R-D. Ces fonds (décrits dans les chapitres 4 et 9) représentent une exception à la règle relative au soutien direct, appliquée aux statistiques de R-D. De fait, les établissements d'enseignement supérieur (EES) communiquent peu sur l'utilisation des dotations publiques globales qu'elles reçoivent de l'État. Néanmoins, dans la mesure où ces dotations globales s'inscrivent (très souvent) dans le cadre d'opérations entre l'État et les EES, qu'il contrôle partiellement, il peut sembler légitime de les considérer comme des financements directs. Dans certains pays, il arrive que l'État alloue des dotations globales ou des financements institutionnels similaires aux FGU. Ces fonds sont parfois octroyés à des établissements relevant de secteurs autres que l'enseignement supérieur, à des fins générales; les bénéficiaires peuvent les utiliser pour des activités de $\mathrm{R}-\mathrm{D}$, sans toutefois que ce soit une obligation. Les FGU (dans les pays qui y ont recours) sont le seul type de dotation globale ou de financement institutionnel pris en compte dans les CBPRD.

12.28 Dans la pratique, le niveau de détails et d'informations fourni dans les documents budgétaires ne permet pas d'identifier la part des FGU (le cas échéant) consacrée aux activités de R-D. Il peut alors être nécessaire de s'appuyer sur les informations obtenues via les enquêtes pour établir une estimation précise des FGU à intégrer dans les CBPRD, avec, à la clé, un allongement non négligeable des délais de disponibilité des données de CBPRD. Or, comme indiqué dans la section 12.3 ci-après, il convient d'éviter de tels délais.

\section{Investissements financiers en faveur de la R-D}

12.29 Les administrations peuvent fournir des financements sous forme de prêts ou de prises de participation à des unités qui mènent à bien des activités de R-D. Ce type de soutien donne lieu à un échange de fonds contre des actifs financiers, sous la forme de créances sur des flux de trésorerie futurs, potentiellement incertains. Les administrations publiques peuvent également prendre en charge une partie du risque encouru par les tierces parties assurant le financement et exiger, ou non, le versement d'une contrepartie financière partielle ou totale.

12.30 Si le chapitre 4 recommande, pour les déclarations du point de vue des exécutants, de traiter ces investissements financiers comme des ressources internes des exécutants, les statistiques de CBPRD doivent tenir compte du fait que les administrations tendent à enregistrer les conséquences budgétaires de ces opérations, selon notamment que la comptabilisation est basée sur les ressources ou sur les flux de trésorerie. Les estimations des coûts équivalant aux subventions, en intégrant le risque, sont souvent utilisées dans le budget pour chiffrer les besoins en ressources, moyennant toutefois des calculs complexes et des hypothèses significatives qu'il convient de réexaminer régulièrement (voir chapitre 13). 
12.31 Aux fins des statistiques de la R-D et des CBPRD en particulier, il peut être difficile d'établir un principe de communication des données intrinsèquement cohérent, étayé par des sources de données disponibles et garantissant une comparabilité internationale suffisante. Dans le cas des prêts en faveur de la R-D, et compte tenu du risque, il arrive que les administrations ne soient pas en mesure d'exiger le remboursement du montant total (ou ne le souhaitent pas). Si l'on entrevoit un tel scénario, il convient, dans le cadre de la démarche " nette ", de tenir compte de la valeur attendue du transfert. Si cet élément est économiquement significatif et est intégré au budget, le montant engagé doit être comptabilisé au titre des CBPRD. Seule la valeur nette attendue de prêts et autres avances potentiellement remboursables doit être comptabilisée au titre des CBPRD, sur la base de la composante de transfert.

12.32 Les remises de dettes sont enregistrées dans les statistiques des administrations publiques en tant que transfert en capital entre le créancier et le débiteur, à la date de prise d'effet telle que convenue dans l'accord. Dans le cas des prêts en faveur de la R-D, elles devraient être non pas intégrées aux CBPRD, mais enregistrées séparément, dans la mesure où le transfert ex post au titre des remises de dettes ne correspond pas à un financement réel de la R-D. De même, les remboursements ne devraient pas être comptabilisés comme des fonds budgétaires négatifs.

12.33 Les investissements sous forme de prise de participation dans le cadre de projets de R-D, notamment l'apport de capitaux dans des coentreprises avec l'industrie, ne devraient pas être comptabilisés au titre des CBPRD, en vertu du principe de la valeur nette attendue, selon lequel les administrations reçoivent, en contrepartie de leur prise de participation, un actif financier sous la forme de droits sur les bénéfices futurs. Pour des raisons pratiques, les pays peuvent choisir de déclarer séparément la valeur des investissements sous forme de prêts et de prises de participation.

\section{Garanties des prêts en faveur de la R-D}

12.34 Pour des raisons pratiques, les garanties de prêts sont peu susceptibles d'être comptabilisées, dans la mesure où il n'est pas toujours possible d'identifier la part que représente la R-D dans l'investissement garanti par les administrations. Lorsque les garanties sont directement attribuables au financement de projets de R-D, la plupart des administrations tendent à enregistrer les engagements conditionnels hors bilan et différer la comptabilisation des coûts budgétaires associés, en constituant éventuellement des provisions pour couvrir leur exposition. Dès lors qu'ils sont significatifs, les provisions de ressources destinées à couvrir les garanties de prêts ou autres financements d'engagements conditionnels, nets des frais acquittés par les parties aux prêts, devraient être comptabilisés en tant que financements directs et intégrés aux CBPRD, à condition que la procédure budgétaire les reconnaisse comme des dépenses. Dans la droite lignée des orientations afférentes aux remises de dettes, 
lorsque les administrations doivent libérer les fonds pour couvrir la garantie, le paiement correspondant ne devrait pas être intégré aux CBPRD.

\section{Allégements fiscaux au titre des dépenses de R-D}

12.35 De nombreuses administrations concèdent des avantages fiscaux aux entreprises et, parfois, à d'autres unités menant des activités de R-D. À ce titre, elles peuvent renoncer à des recettes fiscales actuelles et futures, voire, dans certaines circonstances, transférer directement les fonds aux unités dont la créance fiscale est insuffisante pour compenser l'allègement fiscal auquel elles ont droit. Les allègements fiscaux au titre des dépenses de R-D engagées par les entreprises constituent une forme d'aide à la R-D mise en œuvre via le régime fiscal et destinée à réduire le coût économique des investissements en la matière (le chapitre 13 propose une description détaillée des différentes possibilités d'allègements fiscaux en faveur de la R-D).

12.36 Le coût des allègements fiscaux au titre des dépenses de R-D peut être inscrit au budget ou figurer dans les sections du budget décrivant les dépenses incompressibles et les ajustements de recettes ; ce n'est toutefois pas systématique. Certaines administrations peuvent prévoir au budget un montant dédié, les montants réels étant alors ajustés ex post par rapport au budget disponible ; un autre cas de figure consiste à accorder, à la demande, des allègements fiscaux à toutes les unités qui justifient de leur éligibilité. Aux fins du Manuel, comme expliqué dans le chapitre 13, tant le manque à gagner fiscal que les sommes réelles versées aux entreprises sont considérés comme des avantages fiscaux. Les documents budgétaires ne fournissent pas toujours ces informations.

12.37 Du fait des possibles écarts, on recommande, à des fins de déclarations internationales, d'exclure des statistiques de CBPRD toutes les formes d'allègements fiscaux, y compris les montants payables aux entreprises. Si les autorités nationales considèrent que ce type d'aide fait partie intégrante de leur budget, elles doivent veiller à les consigner de manière appropriée, afin que les utilisateurs ne commettent pas l'erreur d'ajouter des allègements fiscaux en faveur de la R-D estimés par d'autres biais, calculés conformément aux orientations fournies dans le chapitre 13, aux estimations de CBPRD intégrant des formes particulières d'aide budgétaire à des fins d'allègements fiscaux. Le tableau 12.2 ci-après donne un exemple de modèle de déclaration.

\section{Autres mécanismes de soutien indirect}

12.38 Les administrations ont recours à d'autres mécanismes de soutien indirect à l'exécution et au financement des activités de R-D dans une économie. En l'absence de méthodologies éprouvées permettant d'attribuer une valeur monétaire à ce type de soutien et d'établir des comparaisons internationales, il est conseillé de les exclure des estimations de CBPRD. 


\subsection{Sources des données budgétaires relatives aux CBPRD et estimations}

\section{Déclarations du point de vue des bailleurs de fonds et des exécutants}

12.39 Comme évoqué dans la section 12.1 et dans les chapitres 4 et 9, les dépenses de R-D financées sur fonds publics peuvent être déclarées soit par les administrations publiques qui octroient les ressources financières (bailleurs de fonds), soit par l'unité institutionnelle qui exécute les activités de R-D. En règle générale, par souci de cohérence avec les estimations des DIRD totales, le Manuel préconise d'opter pour la seconde approche. Néanmoins, pour atteindre le but visé, à savoir collecter des données de financement actualisées et les classer par objectif socio-économique, les montants des CBPRD devraient être recueillis auprès non pas des exécutants, mais des bailleurs de fonds.

\section{Sources des données budgétaires}

12.40 Lors de l'analyse des dépenses engagées par les administrations, on peut distinguer la date du vote du budget par le parlement, la date à laquelle le ministère des Finances autorise un département à verser les fonds, la date à laquelle un engagement particulier est pris par les départements, la date de réalisation des conditions et, enfin, la date d'émission des ordres de paiement et de règlement des chèques. Bien que des orientations soient proposées ci-après, le Manuel ne formule pas de prescriptions quant aux concepts à privilégier en termes de dépenses, dans la mesure où ils varient selon les pays. De fait, quel que soit le concept retenu, l'important est de s'y conformer pour l'agrégation des CBPRD totaux.

\section{Caractéristiques communes}

12.41 Bien que, dans le détail, les procédures budgétaires varient d'un pays à l'autre, on peut distinguer sept stades principaux :

1. Prévisions (estimation des fonds avant le début de l'examen du budget).

2. Prévisions budgétaires (chiffres préliminaires demandés par les ministères, notamment pour les débats interministériels).

3. Projet de budget (chiffres présentés au parlement pour l'année à venir).

4. Crédits budgétaires initiaux (chiffres votés par le parlement pour l'année à venir, dont les modifications apportées lors du débat parlementaire). Dans ce contexte, les crédits budgétaires désignent l'acte qui consiste à provisionner des fonds ou d'autres ressources dans un but précis, que le bénéficiaire peut affecter à un programme ou une ligne budgétaire particuliers conformément aux conditions établies par le parlement.

5. Crédits budgétaires définitifs (chiffres votés par le parlement pour l'année à venir, dont les lois rectificatives votées en cours d'exercice). 
6. Engagements (crédits effectivement engagés au cours de l'exercice).

7. Dépenses, qu'elles soient constatées dans la comptabilité ou effectivement déboursées.

12.42 Les stades 1 à 4 décrivent les intentions du gouvernement. Les données correspondant à l'exercice budgétaire $\mathrm{n}$ devraient être disponibles dès que possible vers la fin de l'année $n-1$. Il est préconisé de fonder les données de CBPRD préliminaires sur le premier budget arrêté par le gouvernement et le parlement, soit au stade 4 . Certains pays peuvent même établir leurs chiffres préliminaires à partir du projet de budget (stade 3). Il se peut que les données relatives aux intentions ne soient pas suffisamment précises pour permettre de déterminer la composante R-D ni les objectifs détaillés. Il peut alors s'avérer nécessaire de procéder à des estimations ou de recourir à des hypothèses explicites selon lesquelles la croissance des budgets de R-D suivrait celle des catégories budgétaires identifiables. Ce qui peut donner lieu à d'importantes révisions les années suivantes.

12.43 Au cours de l'exercice budgétaire, des lois rectificatives peuvent être votées, avec des augmentations, des réductions et des réaffectations de crédits de R-D. Ces ajustements ont lieu au stade 5. Les données devraient être disponibles le plus tôt possible après la fin de l'exercice budgétaire. Il est suggéré de fonder les données de CBPRD finales sur les crédits budgétaires définitifs. Il se peut que certains pays doivent fonder leurs chiffres définitifs sur les données disponibles aux stades 6 ou 7. Ceux-ci peuvent être basés sur une comptabilité de caisse ou d'exercice. En comptabilité de caisse, une opération est constatée lorsque le paiement est reçu ou émis. En comptabilité d'exercice, une opération est constatée à la date de l'activité (décision) donnant lieu à une recette ou une diminution des ressources, indépendamment de la date à laquelle le règlement correspondant est perçu ou émis. Il arrive que les crédits budgétaires ouverts ne soient pas dépensés, ou que les réserves de liquidités affichent un solde de crédits non affectés à une finalité particulière. C'est pourquoi le Manuel suggère d'éviter de baser la déclaration de CBPRD sur les chiffres des stades 6 ou 7.

\section{Reports de crédits budgétaires}

12.44 En matière de budget, certains pays ont pour habitude de reporter d'une année sur l'autre des montants importants, qui parfois se trouvent inclus dans les crédits votés au cours d'années successives. Les projets pluriannuels pour lesquels des crédits sont prévus au budget d'une année donnée ou sur plusieurs années devraient être inclus dans les données de CBPRD de l'année ou des années où ils figurent au budget, et non de l'année ou des années au cours desquelles les activités de R-D sont exécutées. Les programmes pluriannuels qui font l'objet d'une ouverture de crédits, à un stade ou à un autre, mais sont budgétisés sur plusieurs années, devraient être inclus dans les données des années pendant lesquelles ils figurent au budget, et non celles de l'année de l'ouverture des crédits. 


\section{Sources des données sur les engagements et dépenses hors procédure budgétaire}

12.45 Un certain nombre de pays ont couramment recours à des enquêtes approfondies auprès des administrations publiques, notamment des organismes et ministères, pour mesurer non seulement les activités de R-D, mais aussi leur financement. Plusieurs raisons peuvent expliquer cet effort accru. Il permet en particulier de recueillir des informations plus précises que celles fournies dans les documents budgétaires généraux, et, ainsi, d'identifier la part des activités de R-D dans les postes budgétaires, leur nature, ainsi que d'autres éléments intéressant l'action publique.

12.46 Outre les incidences de l'effort supplémentaire de collecte d'informations en termes de ressources, celui-ci peut également entraîner un allongement non négligeable des délais, lorsqu'il est nécessaire d'adopter des mesures pour éviter une double comptabilisation des fonds. Tel est le cas lorsque des ressources transitent des ministères vers des organismes intermédiaires qui, à leur tour, transfèrent les fonds vers d'autres organismes et/ou des exécutants. À l'échelle internationale, le recours variable à des enquêtes plus approfondies destinées à étayer les données budgétaires risque fortement de nuire à la comparabilité des données, en particulier entre les pays qui évaluent uniquement les activités de R-D à partir des postes budgétaires de haut niveau et ceux qui procèdent à un examen plus exhaustif des dépenses au sein des différents postes budgétaires.

12.47 Comme évoqué dans le chapitre 8, le recours à de telles enquêtes pour procéder à une évaluation plus complète et précise de la situation n'est pas déconseillé ; en revanche, du fait de la diversité des pratiques en vigueur dans les différents pays, il n'est pas possible de fournir des orientations en la matière dans le Manuel. Si elles sont utilisées, ces pratiques doivent être convenablement étayées et ne doivent pas compromettre les délais d'obtention ni la comparabilité internationale des données de CBPRD.

12.48 Il se peut que certaines composantes des CBPRD ne soient pas disponibles en temps utiles pour remplir les conditions requises pour l'obtention des chiffres préliminaires (publication des estimations pour l'année $\mathrm{n}$ au cours de l'année n-1, par exemple). En particulier :

- Il arrive que les estimations au niveau infranational ne soient pas aisément accessibles. Des efforts supplémentaires doivent alors être déployés pour collecter les données au niveau des budgets publics régionaux (États fédérés), voire locaux (municipaux), ce qui retarde la compilation finale complète.

- Pour intégrer les FGU (voir chapitres 4 et 9) dans les statistiques de CBPRD, il peut être nécessaire de s'appuyer sur des estimations obtenues à partir de données issues d'enquêtes menées auprès du secteur de l'enseignement supérieur.

- L'application de coefficients à jour aux postes du budget général peut nécessiter d'obtenir, auprès des agences, des données sur l'utilisation réelle des fonds. 
12.49 En règle générale, et dans un souci de minimisation des délais, il est recommandé, en l'absence d'alternative, de fonder les estimations préliminaires du niveau de CBPRD sur une extrapolation de la dernière estimation disponible, en appliquant le taux de croissance enregistré pour une composante significative, par exemple le taux de croissance des budgets centraux/fédéraux consacrés à la R-D. Il convient de valider cette pratique en procédant à une réévaluation continue de la fidélité avec laquelle l'indicateur avancé a suivi le taux de croissance de la série de données de CBPRD. Les utilisateurs des statistiques devraient se préparer à de possibles révisions des données, comme il est d'usage dans d'autres domaines statistiques. Autre point, certes pas obligatoire, mais qui peut s'avérer utile et particulièrement pertinent au regard de l'action publique : inclure dans les estimations préliminaires prospectives une répartition des CBPRD par objectif socio-économique.

\subsection{Répartition par objectif socio-économique Critères de répartition}

\section{Finalité ou contenu}

12.50 Il est possible de classer les CBPRD en fonction du contenu général en connaissances du programme ou projet de R-D, selon la finalité (à savoir l'objectif) poursuivie, en s'appuyant sur une classification adéquate des objectifs socio-économiques (OSE). Toutefois, il n'est pas toujours aisé d'identifier le contenu des activités de R-D, puis d'établir correctement son lien avec la finalité du projet. L'exemple suivant illustre la différence entre les concepts en jeu :

$\rightarrow$ Un projet de recherche portant sur le développement de piles à combustible en vue d'alimenter en énergie les forces militaires dans des zones reculées et hostiles est financé intégralement par le ministère de la Défense. Le contenu des activités de R-D, qui fait appel à l'ingénierie et à la technologie, sert des objectifs de production d'énergie, mais l'objectif principal est toutefois lié à la défense.

12.51 Dans le cas des CBPRD, c'est l'objectif principal qui prévaut lorsqu'il s'agit de déterminer les objectifs des politiques publiques en faveur de la R-D. Par ailleurs, les informations relatives à l'objectif principal sont moins susceptibles d'être fournies par les exécutants, d'où l'intérêt de s'appuyer sur les données budgétaires pour ce critère. Par conséquent, il est recommandé d'utiliser, en principe, l'approche axée sur l'objectif principal pour la collecte et la ventilation des données budgétaires.

12.52 Si certains programmes de R-D soutenus par les pouvoirs publics servent un seul objectif, d'autres peuvent en avoir plusieurs qui sont complémentaires ou poursuivis en parallèle. Par exemple, un État peut engager des fonds dans un projet aéronautique principalement à des fins militaires, mais également pour favoriser les ventes à l'exportation de l'industrie aérospatiale, 
voire pour générer des retombées dans le secteur de l'aviation civile. Plusieurs objectifs peuvent être enregistrés dans le système d'information d'un pays. En revanche, lorsque les données sont communiquées aux organisations internationales, les activités de R-D devraient être classées en fonction de leur objectif principal.

\section{Détermination des objectifs principaux}

12.53 La mise en correspondance des budgets de R-D et des objectifs socio-économiques devrait se faire au niveau qui cadre le mieux avec le ou les objectif(s) des bailleurs de fonds. Le niveau effectivement choisi pour la déclaration dépendra des possibilités pratiques. L'ensemble des crédits peut être attribué à une unité exécutante ou à une unité de financement de la R-D. Dans certains cas, il est possible d'obtenir les informations au niveau du programme ou du projet.

\section{Répartition des CBPRD}

12.54 La liste recommandée est présentée dans le tableau 12.1 et exposée ci-après. Elle s'appuie sur la classification de l'Union européenne retenue par Eurostat aux fins de la Nomenclature pour l'analyse et la comparaison des budgets et programmes scientifiques (NABS), au niveau hiérarchique à un chiffre. Établie en 1969, la nomenclature NABS a été révisée pour la dernière fois en 2007 (Eurostat, 2008). Bien que tous les pays ne l'utilisent pas, il convient de se reporter à la correspondance entre la nomenclature et le présent Manuel pour déclarer les données à l'OCDE et ce, même si les pays utilisent leurs propres classifications pour calculer leurs statistiques nationales de CBPRD ou équivalentes.

12.55 En principe, sous réserve de disponibilité des informations, tous les postes budgétaires peuvent être affectés à un objectif socio-économique secondaire afin de dresser un tableau plus complet. Une telle approche peut représenter une source d'information utile pour conduire une analyse de sensibilité et des comparaisons transnationales et longitudinales pour des objectifs spécifiques. L'un des risques potentiels liés à la communication des objectifs secondaires tient au fait que les comparaisons entre les fonds alloués aux objectifs ne rendent pas dûment compte de cette multiplicité.

\section{Description des objectifs socio-économiques (OSE)}

\section{Exploration et exploitation du milieu terrestre}

12.56 Cet OSE couvre les fonds destinés aux activités de R-D dont les objectifs sont liés à l'exploration de la croûte et du manteau terrestres, des mers, des océans et de l'atmosphère, ainsi que la R-D afférente à leur exploitation. Sont également incluses les recherches climatologiques et météorologiques, ainsi que l'exploration polaire et l'hydrologie. En revanche, cet OSE ne comprend pas les activités de R-D ayant trait à l'amélioration des sols (OSE 4), l'utilisation des sols et la pêche (OSE 8), ou la pollution (OSE 2). 


\section{Environnement}

12.57 Cet OSE couvre les activités de R-D relatives à l'amélioration de la lutte contre la pollution, y compris l'identification et l'analyse des sources de pollution et de leurs causes, et les substances polluantes de tous ordres, notamment leur dispersion dans l'environnement et leurs effets sur l'homme, les espèces vivantes (faune, flore, micro-organismes) et la biosphère. Il comprend également les activités de développement d'installations de contrôle pour la mesure de tous types de pollution, ainsi que la R-D sur l'élimination et la prévention de toutes formes de pollution dans tous les biotopes.

\section{Tableau 12.1. Classification des objectifs socio-économiques applicables aux CBPRD}

\begin{tabular}{|c|c|c|}
\hline Chapitre & $\begin{array}{l}\text { Catégories NABS correspondant aux objectifs } \\
\text { socio-économiques des activités de R\&D }\end{array}$ & Sous-catégories recommandées \\
\hline 1 & \multicolumn{2}{|l|}{ Exploration et exploitation du milieu terrestre } \\
\hline 2 & \multicolumn{2}{|l|}{ Environnement } \\
\hline 3 & \multicolumn{2}{|l|}{ Exploration et exploitation de l'espace } \\
\hline 4 & \multicolumn{2}{|l|}{ Transports, télécommunications et autres infrastructures } \\
\hline 5 & \multicolumn{2}{|l|}{ Énergie } \\
\hline 6 & \multicolumn{2}{|l|}{ Production et technologie industrielles } \\
\hline 7 & \multicolumn{2}{|l|}{ Santé } \\
\hline 8 & \multicolumn{2}{|l|}{ Agriculture } \\
\hline 9 & \multicolumn{2}{|l|}{ Enseignement } \\
\hline 10 & \multicolumn{2}{|l|}{ Culture, activités de loisirs, religion et médias } \\
\hline 11 & \multicolumn{2}{|l|}{ Systèmes, structures et processus politiques et sociaux } \\
\hline \multirow[t]{6}{*}{12} & \multirow{6}{*}{$\begin{array}{l}\text { Progrès général des connaissances : R-D financée sur les fonds } \\
\text { généraux des universités (FGU) }\end{array}$} & 12.1 R-D en lien avec les sciences naturelles \\
\hline & & $\begin{array}{l}12.2 \text { R-D en lien avec les sciences de } \\
\text { l'ingénieur }\end{array}$ \\
\hline & & 12.3 R-D en lien avec la médecine \\
\hline & & 12.4 R-D en lien avec les sciences agricoles \\
\hline & & 12.5 R-D en lien avec les sciences sociales \\
\hline & & 12.6 R-D en lien avec les sciences humaines ${ }^{1}$ \\
\hline \multirow[t]{6}{*}{13} & \multirow{6}{*}{$\begin{array}{l}\text { Progrès général des connaissances : R-D financée sur des } \\
\text { fonds autres que les FGU }\end{array}$} & 13.1 R-D en lien avec les sciences naturelles \\
\hline & & $\begin{array}{l}\text { 13.2 R-D en lien avec les sciences de } \\
\text { l'ingénieur }\end{array}$ \\
\hline & & 13.3 R-D en lien avec la médecine \\
\hline & & 13.4 R-D en lien avec les sciences agricoles \\
\hline & & 13.5 R-D en lien avec les sciences sociales \\
\hline & & 13.6 R-D en lien avec les sciences humaines ${ }^{1}$ \\
\hline 14 & \multicolumn{2}{|l|}{ Défense } \\
\hline
\end{tabular}

Note : Classification recommandée, sous reserve d'une éventuelle Mission et mise à jour.

1. Inclut les arts.

Source : Eurostat, nomenclature consultée à l'adresse http://oe.cd/seo. 


\section{Exploration et exploitation de l'espace}

12.58 Cet OSE couvre l'ensemble des activités de R-D dans le domaine spatial civil, liées à l'exploration scientifique de l'espace, aux laboratoires spatiaux, aux voyages dans l'espace et aux systèmes de lancement. Les activités correspondantes dans le domaine de la défense relèvent de l'OSE 13. Si la R-D spatiale civile ne répond généralement pas à un objectif spécifique, elle n'en est pas moins entreprise dans un but déterminé, par exemple, l'extension des connaissances (astronomie), ou liée à des applications particulières (satellites de télécommunications ou observation de la Terre). La catégorie est toutefois conservée pour faciliter les déclarations des pays menant à bien des programmes spatiaux de grande ampleur. Le présent chapitre n'aborde pas les activités de R-D équivalentes menées dans le domaine de la défense.

\section{Transports, télécommunications et autres infrastructures}

12.59 Cet OSE couvre la R-D dans le domaine des infrastructures et de l'aménagement du territoire, y compris la construction des bâtiments. Il comprend plus généralement toutes les activités de R-D liées à l'organisation globale du territoire, notamment la protection contre les effets nuisibles dans le cadre de l'aménagement des espaces urbains et ruraux, à l'exclusion des recherches relatives à d'autres types de pollution (OSE 2). Cet OSE inclut également les activités de R-D liées aux systèmes de transports ; aux systèmes de télécommunications ; à l'organisation générale du territoire ; à la construction et la planification des bâtiments ; au génie civil ; et à l'approvisionnement en eau.

\section{5. Énergie}

12.60 Cet OSE couvre la R-D destinée à améliorer la production, le stockage, le transport, la distribution et l'utilisation rationnelle de toutes les formes d'énergie. Il englobe également les activités de R-D relatives aux procédés destinés à accroître l'efficience de la production et de la distribution d'énergie, ainsi que celles afférentes aux économies d'énergie. Il ne comprend pas la R-D liée à la prospection (OSE 1) ou à la propulsion des véhicules et des moteurs (OSE 6). Voir également l'encadré 12.1 pour de plus amples informations sur la composition de la R-D en matière d'énergie telle que définie dans le Manuel.

\section{Production et technologie industrielles}

12.61 Cet OSE couvre les activités de R-D visant à améliorer la production et la technologie industrielles, notamment la R-D sur les produits industriels et leurs procédés de fabrication, à condition qu'elles ne fassent pas partie intégrante de la réalisation d'autres objectifs (défense, espace, énergie ou agriculture, par exemple). 


\section{Encadré 12.1. Différences entre les données de CBPRD afférentes aux activités de R-D dans le domaine de l'énergie et celles de l'AIE}

Il convient de ne pas confondre, d'une part, les séries de données de CBPRD correspondant à l'objectif Énergie, collectées et publiées par la Direction de la science, de la technologie et de l'innovation de l'OCDE et par d'autres organisations nationales et internationales, qui les compilent conformément aux orientations énoncées dans le présent Manuel, et, d'autre part, les séries spéciales collectées et publiées par l'Agence internationale de l'énergie (AIE) de l'OCDE, qui couvrent les dépenses de recherche, développement et démonstration (ou RDD) en matière d'énergie, dont le champ est relativement plus vaste.

L'AIE n'appréhende pas la R-D dans le domaine de l'énergie de la même manière que le Manuel de Frascati. En effet, les données de l'AIE (i) sont axées sur les programmes ayant trait à l'énergie ; (ii) couvrent tous les types de " projets de démonstration "; et (iii) prennent en compte les entreprises du secteur public. L'AIE a décidé d'inclure les projets de démonstration lors de la collecte des données budgétaires afférentes à la R-D car ils représentent souvent une part importante du développement de nouvelles technologies. Les projets peuvent mener à des résultats incertains et comportent un élément de risque généralement trop élevé pour que le secteur privé l'assume seul (AIE, 2011).

L'AIE entend par " démonstration " la conception, la construction et l'exploitation du prototype d'une technologie à l'échelle industrielle ou quasi industrielle, dans le but d'apporter des informations techniques, économiques et environnementales aux industriels, aux financiers, aux régulateurs et aux décideurs. Les informations relatives au financement des activités de démonstration sont collectées en même temps que celles afférentes à la R-D et enregistrées séparément.

Par ailleurs, le champ des activités de R-D couvert par les données de l'AIE est plus large que celui de l'OSE 5, puisqu'il inclut l'ensemble des programmes axés sur : (i) l'approvisionnement en énergie ; (ii) le transport d'énergie ; (iii) l'utilisation de l'énergie ; et (iv) l'amélioration de l'efficacité énergétique.

Sont donc concernés tous les programmes de RDD portant sur l'une des sept branches principales des développements dans le domaine énergétique, pour lesquelles l'AIE collecte des données, à savoir : (i) efficacité énergétique ; (ii) combustibles fossiles (pétrole, gaz et charbon); (iii) énergies renouvelables ; (iv) fission et fusion nucléaire ; (v) piles à combustible à hydrogène ; (vi) autres techniques de production et de stockage de l'énergie ; et (vii) autres technologies ou recherches transversales.

Source : Agence internationale de l'énergie (2011). Consulté à l'adresse www.iea.org/stats/ RDD\%20Manual.pdf. 


\section{Santé}

12.62 Cet OSE comprend la R-D visant à protéger, promouvoir et rétablir la santé publique au sens large, y compris les aspects sanitaires de la nutrition et de l'hygiène alimentaire. Il va de la médecine préventive, notamment tous les aspects de la médecine et de la chirurgie curatives tant au plan individuel que collectif, et la fourniture des soins en milieu hospitalier et à domicile, à la médecine sociale, en passant par la recherche en pédiatrie et en gériatrie.

\section{Agriculture}

12.63 Cet OSE couvre l'ensemble des activités de R-D destinées à promouvoir l'agriculture, la sylviculture, les pêches et la production de denrées alimentaires, ou à approfondir les connaissances sur les engrais chimiques, les biocides, la lutte biologique et la mécanisation agricole, ainsi que sur les incidences des activités agricoles et sylvicoles sur l'environnement. Il couvre également la R-D visant à améliorer la productivité et la technologie alimentaires. Il ne comprend pas, en revanche, les activités de R-D liées à la lutte contre la pollution (OSE 2) ; au développement des régions rurales ; à la construction et la planification de bâtiments ; à l'amélioration foncière rurale et l'approvisionnement en eau des régions rurales (OSE 4) ; aux économies d'énergie (OSE 5); ou à l'industrie alimentaire (OSE 8).

\section{Enseignement}

12.64 Cet OSE couvre les activités de R-D destinées à soutenir l'enseignement général ou spécialisé, notamment la formation, la pédagogie, la didactique et les méthodes ciblées adaptées aux personnes douées de capacités particulières ou à celles présentant des difficultés d'apprentissage. Cet objectif s'applique à tous les niveaux, de l'enseignement préscolaire et élémentaire au supérieur, ainsi qu'aux services annexes à l'enseignement.

\section{Culture, activités de loisirs, religion et médias}

12.65 Cet OSE couvre la R-D destinée à améliorer la compréhension des phénomènes sociaux liés aux activités culturelles, à la religion et aux loisirs, en vue de définir leurs incidences sur la vie en société, ainsi qu'à l'intégration raciale et culturelle ; est également concernée la R-D sur les changements socio-culturels dans ces domaines. La notion de " culture » englobe la sociologie des sciences, la religion, l'art, les sports et les loisirs, auxquels s'ajoute notamment la R-D sur les médias, la maîtrise de la langue et l'intégration sociale, les bibliothèques, les archives et la politique culturelle extérieure.

12.66 Cet OSE inclut en outre les activités de R-D dans les domaines suivants : les services récréatifs et sportifs ; les services culturels ; les services de diffusion et d'édition; et les services religieux et autres services communautaires. 


\section{Systèmes, structures et processus politiques et sociaux}

12.67 Cet OSE couvre la R-D destinée à améliorer la compréhension de la structure politique de la société ; des questions liées à l'administration publique et de la politique économique ; des études régionales et de la gouvernance multiniveau ; du changement, des processus et des conflits sociaux ; du développement des systèmes de sécurité sociale et d'assistance sociale ; et des aspects sociaux de l'organisation du travail. Cet objectif couvre par ailleurs les activités de R-D ayant trait aux études sociales liées aux problématiques hommesfemmes, notamment à la discrimination et aux problèmes familiaux ; à la mise au point de méthodes de lutte contre la pauvreté à l'échelle locale, nationale et internationale ; à la protection de catégories de population particulières au niveau social (immigrés, délinquants, jeunes ayant abandonné leurs études, etc.), au niveau sociologique, à savoir en lien avec le mode de vie (jeunes, adultes, retraités, personnes invalides, etc.) et au niveau économique (consommateurs, agriculteurs, pêcheurs, mineurs, chômeurs, etc.) ; et aux méthodes de prestation de l'assistance sociale lorsque des changements soudains (naturels, technologiques ou sociaux) surviennent dans la société.

12.68 Ne sont pas prises en compte dans cet OSE les activités de R-D liées à l'hygiène du travail, au contrôle sanitaire des communautés du point de vue organisationnel et médico-social, à la pollution sur le lieu de travail, à la prévention des accidents du travail et aux aspects médicaux des causes des accidents du travail (OSE 7).

\section{Progrès général des connaissances : $R-D$ financée sur les fonds généraux des universités}

12.69 Dans l'optique de la déclaration des CBPRD par « finalité », cet OSE devrait comprendre, par convention, toute la R-D financée sur les subventions générales des ministères de l'Éducation, bien que, dans certains pays, bon nombre de ces programmes puissent se rapporter à d'autres objectifs. Cette convention a été adoptée en raison des difficultés rencontrées pour obtenir des données appropriées et, par conséquent, parvenir à une comparabilité adéquate. Pour éviter que cette catégorie ne devienne trop vaste et peu instructive, il est recommandé de procéder à une ventilation supplémentaire par domaine de R-D de niveau supérieur.

\section{Progrès général des connaissances : $R$ - $D$ financée sur des fonds autres que les FGU}

12.70 Cet OSE couvre tous les crédits budgétaires qui sont réservés à la $\mathrm{R}-\mathrm{D}$, mais ne peuvent pas être rattachés à un objectif, et qui sont financés sur des fonds autres que les FGU. Une ventilation supplémentaire par domaine de R-D de niveau supérieur est, là encore, recommandée. 


\section{Défense}

12.71 Cet OSE couvre la R-D menée dans le domaine militaire. Il peut également inclure la recherche fondamentale et la recherche nucléaire et spatiale financées par les ministères de la Défense. En revanche, il convient de classer dans les OSE concernés la recherche civile financée par les ministères de la Défense, par exemple dans le domaine de la météorologie, des télécommunications et de la santé.

\subsection{Autres répartitions des CBPRD}

\section{Classification des fonctions des administrations publiques}

12.72 La Classification des fonctions des administrations publiques (CFAP) est présentée dans le chapitre 8. Elle fournit une nomenclature des dépenses des administrations publiques par fonction (les catégories CFAP sont consultables dans l'annexe au présent Manuel, disponible en ligne à l'adresse http://oe.cd/frascati). Les intitulés du premier niveau présentent de nombreuses similitudes avec la nomenclature NABS utilisée pour la R-D. L'utilisation de la CFAP pour les estimations de CBPRD n'a pas été recommandée car les catégories ne sont pas prévues pour décrire les dépenses de R-D ni conformes aux définitions de la R-D telles qu'énoncées dans le Manuel ; qui plus est, son adoption reste relativement limitée à l'échelle mondiale. Il pourrait être utile d'établir, dans un avenir proche, un tableau de correspondance indicatif. Dans la mesure du possible, il est recommandé que les offices statistiques documentent les différences observées entre les estimations de dépenses publiques basées sur la classification CFAP et les estimations de CBPRD, afin que les utilisateurs en soient dûment informés.

\section{Modes de financement de la R-D}

12.73 D'autres répartitions des CBPRD ont été proposées au cours des dernières années, en réponse à une volonté des pouvoirs publics de mieux appréhender la nature du soutien public direct en faveur de la R-D, notamment :

- La répartition des CBPRD par destination des financements, par secteur institutionnel, y compris le " reste du monde ", pour lequel aucune information ne peut être recueillie par le biais des enquêtes nationales auprès des exécutants de la R-D concernés.

- La répartition des CBPRD par mode de financement, selon que les financements sont alloués à des projets, des programmes ou des institutions. Un certain nombre d'utilisateurs souhaiteraient également connaître la répartition des financements publics en fonction de l'utilisation de critères de concurrence (ce qui peut être le cas à la fois au niveau des projets et des institutions).

- La répartition des CBPRD par type de moyen d'action, à l'instar des marchés publics et des subventions qui viennent s'ajouter au financement de la R-D intra-muros. 
- La répartition des CBPRD par niveau et type d'administration.

- Qui plus est, la Commission européenne collecte des données sur le "Financement public national des activités de R \& D coordonnées à l'échelon transnational ", notamment :

* les contributions nationales à des acteurs publics transnationaux dans le domaine de la R-D

* les contributions nationales à des programmes de R-D publics transnationaux à l'échelle européenne

* les contributions nationales à des programmes de R-D publics bilatéraux ou multilatéraux mis en place entre les administrations des États membres.

12.74 L'expérience des récentes collectes de données expérimentales a révélé qu'un nombre limité de pays est actuellement en mesure de fournir la majorité des indicateurs sur la base des données budgétaires. Des informations détaillées supplémentaires doivent donc être collectées auprès des ministères, des organismes et à partir des enregistrements administratifs. C'est pourquoi le Manuel ne peut recommander de procéder à la collecte de ces informations au titre du cadre de compilation des CBPRD et ce, malgré son utilité potentielle. Une meilleure solution s'offre aux pays intéressés par ce type de données : recourir à des enquêtes auprès d'entités administratives, en étendant leur champ au-delà des exécutants de R-D connus dans le secteur de l'État, comme évoqué dans le chapitre 8 .

\subsection{Utilisation des données de CBPRD}

12.75 La production des données de CBPRD doit avant tout servir ses deux objectifs principaux : fournir des informations actualisées sur les budgets publics consacrés à la $\mathrm{R}-\mathrm{D}$ et dresser un tableau cohérent de la répartition des financements en fonction des objectifs socio-économiques.

\section{Principales différences entre les données de CBPRD et de DIRD}

12.76 Les utilisateurs des données relatives aux CBPRD constatent souvent des différences - qu'ils peinent à expliquer - entre les montants totaux de CBPRD (approche du point de vue des bailleurs de fonds) et les DIRD financées par l'État (approche du point de vue des exécutants). Ces écarts entre les sommes déclarées sont dus à des différences de spécification des données.

\section{Différences d'ordre général}

12.77 Bien qu'en principe, les deux séries de données devraient être établies à partir d'une définition et d'un champ de la R-D identiques, couvrant les activités de R-D dans tous les domaines de la connaissance et tenant compte aussi bien des dépenses courantes que des dépenses en capital, elles présentent un certain nombre de différences. 
- Les données concernant les DIRD financées par l'État et les objectifs des DIRD sont fondées sur les déclarations des exécutants de la R-D, tandis que les chiffres de CBPRD s'appuient sur les déclarations des bailleurs de fonds et se fondent principalement sur des données budgétaires. Certes, les exécutants peuvent avoir une idée différente et plus précise du contenu en R\&D d'un projet ou des activités concernées, mais ils peuvent aussi sous-estimer l'étendue totale du soutien des pouvoirs publics.

- L'appréciation des objectifs du projet concerné peut être très différente selon que l'on se place du point de vue des exécutants ou de celui des bailleurs de fonds, notamment pour les activités de R-D financées à l'aide de dotations globales comme les FGU qui, dans l'optique des DIRD, devraient être ventilées par objectif pour les pays qui déclarent ce type de répartition.

- Il est également possible que les montants des FGU diffèrent selon qu'ils sont obtenus à partir des estimations des activités de R-D (inclus dans les DIRDES) ou à partir des CBPRD. Prenons l'exemple d'une administration centrale qui octroie aux universités une dotation globale correspondant à 100 unités monétaires. Trente unités sont allouées selon des critères d'excellence scientifique et de la $\mathrm{R}-\mathrm{D}$, le reste étant déterminé en fonction du nombre d'étudiants et des coûts d'enseignement. À réception de cette dotation globale, les universités peuvent être libres de répartir la totalité des 100 unités entre les activités de recherche, d'enseignement ou à d'autres fins légitimes. Elles peuvent ainsi décider d'allouer à la R-D 40 unités monétaires une année, puis 20 la suivante. Il peut arriver, dans certains cas, que la mesure des FGU basée sur les CBPRD fasse état de 30 unités, tandis que celle basée sur les DIRDES s'élève à 40 (ou 20) unités. En tout état de cause, le montant déclaré pour les FGU déduits des CBPRD ne doit jamais être de 100 unités, car cela équivaudrait à une surestimation nette du soutien budgétaire destiné et consacré à la R-D.

- Les estimations basées sur les données budgétaires sont également susceptibles d'inclure une composante de financement des bénéfices et des frais généraux, qui sont exclus de l'évaluation des activités de R-D.

- Les séries fondées sur les DIRD couvrent seulement la R-D exécutée par les unités résidentes, tandis que celles fondées sur les CBPRD intègrent également les sommes versées à des exécutants étrangers, y compris des organisations internationales. Des différences peuvent également résulter d'une divergence de périodes de référence (années civiles ou budgétaires), d'une non-utilisation des crédits ouverts, ou encore d'une différence entre la date à laquelle les crédits sont alloués et la date d'exécution des activités de R-D.

- Les estimations des DIRD financées par les administrations publiques devraient prendre en compte la R-D financée par les administrations centrales (fédérales), régionales (États fédérés) et locales (municipales), tandis que les CBPRD n'intègrent pas les fonds émanant des administrations locales (municipales) ; qui plus est, tous les pays ne déclarent pas les données au niveau régional (États fédérés), ou ne sont pas en mesure de le faire. 


\section{Déclaration des CBPRD et indicateurs}

12.78 Le tableau 12.2 présente un modèle indicatif susceptible d'être utilisé pour déclarer les données de CBPRD. Il met en évidence l'importance de disposer des CBPRD totaux en temps utiles et prévoit la possibilité d'utiliser des estimations fondées sur les catégories budgétaires pertinentes. Les conséquences éventuelles d'une mise à disposition tardive des chiffres afférents aux FGU sur les délais de compilation des CBPRD plaident en faveur d'une déclaration anticipée des CBPRD hors FGU, qui pourraient être utilisés comme un indicateur de la croissance globale des CBPRD.

Tableau 12.2. Modèle indicatif de déclaration des CBPRD

\begin{tabular}{|c|c|c|c|c|c|c|c|}
\hline \multirow{2}{*}{ Catégories principales } & & \multirow{2}{*}{ Sous-catégorie } & \multicolumn{5}{|c|}{ Année } \\
\hline & & & $n-\ldots$ & $n-2$ & $n-1$ & $n$ & $n+1$ \\
\hline \multicolumn{3}{|l|}{ Total CBPRD } & $\checkmark$ & $\checkmark$ & $\checkmark$ & $\checkmark p$ & $\checkmark \mathrm{e}$ \\
\hline \multirow{8}{*}{ CBPRD - hors FGU } & & & $\checkmark$ & $\checkmark$ & $\checkmark$ & $\checkmark p$ & $\checkmark e$ \\
\hline & \multicolumn{2}{|l|}{ OSE 1} & $\checkmark$ & $\checkmark$ & $\checkmark$ & $\checkmark p / e$ & \\
\hline & \multicolumn{2}{|l|}{ OSE 2} & $\checkmark$ & $\checkmark$ & $\checkmark$ & $\checkmark p / e$ & \\
\hline & & $\checkmark$ & $\checkmark$ & $\checkmark$ & $\checkmark p / e$ & \\
\hline & \multicolumn{2}{|l|}{ OSE 11} & $\checkmark$ & $\checkmark$ & $\checkmark$ & $\checkmark p / e$ & \\
\hline & OSE 13 & Total & $\checkmark$ & $\checkmark$ & $\checkmark$ & $\checkmark p / e$ & \\
\hline & & $\begin{array}{l}\text { Répartition par domaine de } \\
\text { R-D de niveau supérieur }\end{array}$ & & & & & \\
\hline & \multicolumn{2}{|l|}{ OSE 14} & $\checkmark$ & $\checkmark$ & $\checkmark$ & $\checkmark p / e$ & \\
\hline CBPRD - FGU inclus & OSE 12 & $\begin{array}{l}\text { Total } \\
\text { Répartition par domaine de } \\
\text { R-D de niveau supérieur }\end{array}$ & $\checkmark$ & $\checkmark$ & $\checkmark$ & $\checkmark p / e$ & \\
\hline \multicolumn{8}{|l|}{$\begin{array}{l}\text { Répartitions } \\
\text { supplémentaires et postes } \\
\text { correspondants }\end{array}$} \\
\hline Capital & \multicolumn{2}{|c|}{$\begin{array}{l}\text { Fonds consacrés à la R-D - Capital } \\
\text { Fonds consacrés à la R-D - Amortissement }\end{array}$} & $\checkmark$ & $\checkmark$ & $\checkmark$ & $\checkmark$ & $\checkmark$ \\
\hline Niveau d'administration & \multicolumn{2}{|c|}{$\begin{array}{l}\text { Administration centrale/fédérale } \\
\text { Administration régionale/États fédérés }\end{array}$} & & & & & \\
\hline $\begin{array}{l}\text { Fonds budgétaires } \\
\text { alloués sous forme } \\
\text { d'allègement fiscal }\end{array}$ & \multicolumn{2}{|c|}{ Séparés du total des CBPRD } & $\checkmark$ & $\checkmark$ & $\checkmark$ & $\checkmark$ & \\
\hline Modes de financement & & & & & & & \\
\hline
\end{tabular}

Note $: \mathrm{p}=$ données préliminaires $; \mathrm{e}=$ estimations $; \checkmark$ désigne des informations prioritaires.

12.79 Le tableau montre également la répartition des OSE 12 et 13 par domaine de R-D, la déclaration séparée des composantes des dépenses en capital, le niveau d'administration, ainsi que les éventuels allègements fiscaux inscrits au budget, qu'ils soient pris en compte dans les estimations de CBPRD ou qu'ils en soient exclus. La déclaration des allègements fiscaux devrait permettre une meilleure intégration des données budgétaires et éviter une comptabilisation 
redondante avec celle des estimations d'allègements fiscaux au titre de la R-D compilées conformément aux orientations fournies dans le chapitre 13, avec, à la clé, une représentation plus exhaustive du soutien financier public en faveur de la R-D.

\section{Références}

CE, FMI, OCDE, Nations Unies et Banque mondiale (2009), Système de comptabilité nationale, Nations Unies, New York, http://unstats.un.org/unsd/nationalaccount/docs/ sna2008FR.pdf.

Eurostat (2008), Nomenclature for the Analysis and Comparison of Scientific Programmes and Budgets (NABS), Comparison between NABS 2007 and NABS 1992, Eurostat, Luxembourg, www.oecd.org/science/inno/43299905.pdf.

Agence internationale de l'énergie (2011), IEA Guide to Reporting Energy RD\&D Budget/ Expenditure Statistics, Éditions AIE/OCDE, Paris, www.iea.org/stats/RDD\%20Manual. pdf.

Fonds monétaire international (2014), Government Finance Statistics (GFS) Manual, Pre-publication Draft, FMI, Washington DC, www.imf.org/external/np/sta/gfsm/. 


\section{Chapitre 13}

\section{Mesurer l'allégement fiscal en faveur de la R-D}

Les autorités de plusieurs pays ont aménagé un régime d'aide fiscale à l'investissement dans la R-D dans le cadre duquel les dépenses de $R-D$ admissibles, en particulier celles des entreprises, bénéficient d'un traitement fiscal préférentiel. Les dépenses fiscales sont difficiles à mesurer, et les différents types de dispositif d'allégement fiscal ne transparaissent pas individuellement dans tous les systèmes statistiques. La notification de ces types d'aide fiscale, dans des rapports complémentaires, permettrait de produire des comparaisons internationales plus transparentes et plus équilibrées. À l'intention des utilisateurs et spécialistes qui souhaiteraient voir combler cette lacune, le présent chapitre fournit des lignes directrices sur la manière de rendre compte du soutien que les pouvoirs publics apportent à la R-D sous la forme d'incitations fiscales, en vue de faciliter l'établissement d'indicateurs de l'allégement fiscal au titre des dépenses de $R-D$ qui soient comparables entre les pays. Ces lignes directrices reposent sur l'expérience acquise à l'occasion d'une série de collectes préliminaires de données effectuées par l'OCDE. En raison de leur nouveauté, il est probable que les méthodes de mesure continueront d'être améliorées après la publication du présent manuel. 


\subsection{Introduction}

13.1 Les autorités de plusieurs pays ont aménagé un régime d'aide fiscale à l'investissement dans la R-D dans le cadre duquel les dépenses de R-D admissibles, en particulier celles des entreprises, bénéficient d'un traitement fiscal préférentiel. Ce type de soutien est assuré au niveau national et, dans certains cas, au niveau infranational. Les dépenses fiscales sont difficiles à mesurer, et tous les types de dispositif d'allégement fiscal ne transparaissent pas individuellement dans tous les systèmes statistiques. Cependant, comme l'allégement fiscal en faveur de la R-D est censé prendre la forme de subventions ou d'autres dépenses directes, il est largement admis que la notification de ces types d'aide fiscale, dans des rapports complémentaires, permettrait de produire des comparaisons internationales plus transparentes et plus équilibrées.

13.2 En réponse aux utilisateurs et spécialistes qui souhaiteraient voir combler cette lacune des précédentes éditions du Manuel de Frascati, le présent chapitre fournit des lignes directrices sur la manière de rendre compte du soutien que les pouvoirs publics apportent à la R-D sous la forme d'incitations fiscales, en vue de faciliter l'établissement d'indicateurs de l'allégement fiscal au titre des dépenses de R-D qui soient comparables entre les pays. Ces lignes directrices reposent sur l'expérience acquise à l'occasion d'une série de collectes préliminaires de données effectuées par l'OCDE depuis 2007 et sur d'autres études menées dans les années 90. Elles respectent en outre, dans la mesure du possible, les définitions standard de l'OCDE (OCDE, 2010) et les conventions statistiques générales (CE et al., 2009 ; FMI, 2014).

13.3 Certes, les dépenses fiscales en faveur de la R-D présentent plusieurs points communs avec les crédits budgétaires publics de R-D (CBPRD) décrits au chapitre 12. Il est toutefois proposé ici de considérer séparément la totalité des CBPRD avant de les intégrer dans la présentation générale des statistiques de la R-D, en particulier pour les besoins de l'établissement de comparaisons internationales. L'indicateur de l'allégement fiscal au titre des dépenses de R-D peut être combiné de manière appropriée avec les CBPRD pour obtenir un indicateur du soutien financier public global de la R-D qui reste fiable malgré l'évolution dans le temps de l'importance relative accordée respectivement au soutien direct et au soutien fiscal. Il est possible que ces estimations, du fait qu'elles proviennent de sources budgétaires et d'autres sources gouvernementales, soient moins précises et se prêtent moins bien à des comparaisons internationales que 
les statistiques fondées sur les données des exécutants, mais elles peuvent être bien plus à jour et informatives sur les intentions des pouvoirs publics et sur les efforts financiers effectifs.

13.4 Étant donné la nouveauté des lignes directrices présentées ici, des améliorations pourront être apportées aux méthodes de mesure après la publication du Manuel. Les producteurs et les utilisateurs de données sont invités à consulter les instructions fournies en ligne en annexe au Manuel, à l'adresse http://oe.cd/frascati, afin de prendre connaissance des éventuelles modifications qui ne figurent pas dans la version imprimée.

\subsection{Allégement fiscal au titre des dépenses de R-D}

\section{Mesures d'allégement fiscal et dépenses fiscales}

13.5 Les mesures d'allégement fiscal sont des incitations qui réduisent le montant dû au fisc par les unités institutionnelles concernées, telles que des entreprises ou d'autres organisations admissibles assujetties à différents types d'impôts et de taxes (FMI, 2014 ; CE et al., 2009). L'allégement fiscal auquel ces unités peuvent prétendre peut être fonction du montant des dépenses de R-D admissibles engagées au cours de la période de référence. Ce type de dégrèvement est défini dans le Manuel sous le terme d'allégement fiscal au titre des dépenses de $\mathrm{R}-\mathrm{D}$, et le niveau des ressources financières correspondantes (mesuré en manque à gagner et en dépenses supplémentaires), sous le terme de dépenses fiscales en faveur de la R-D.

13.6 En règle générale, l'allégement fiscal peut prendre la forme d'un abattement, d'une exonération, d'une déduction ou d'un crédit d'impôt. Les abattements, les exonérations et les déductions de nature fiscale sont retranchés de l'assiette fiscale avant le calcul de la créance fiscale, ce qui réduit le montant imposable avant l'établissement du montant de l'impôt dû. Un abattement fiscal spécial accordé au titre de la R-D permet, par exemple, de déduire du bénéfice imposable une unité monétaire de dépenses de R-D multipliée par un coefficient supérieur à un. Examinons, à des fins d'illustration, une formule simplifiée prenant comme base l'impôt sur les sociétés :

Bénéfice après impôt

$=(1-$ taux d'imposition $) *$ (produits - charges déductibles autres que dépenses de R-D admissibles - coefficient d'abattement fiscal * dépenses de R-D admissibles)

13.7 Un crédit d'impôt est un montant directement retranché de la créance fiscale due par l'unité bénéficiaire après le calcul de cette créance (FMI, 2014, par. 5.29). Il peut être formulé, de façon très simplifiée, comme suit :

Bénéfice après impôt

$$
\begin{gathered}
=(1-\text { taux d'imposition }) * \text { (produits }- \text { toutes les charges } \\
\text { déductibles })+(\text { taux de crédit d'impôt * dépenses } \\
\text { de R-D admissibles })
\end{gathered}
$$


13.8 Les crédits d'impôt peuvent être payables ou non payables. Dans un système de crédit d'impôt payable, si le montant de ce dernier est supérieur à l'impôt dû, la différence peut être versée en totalité ou en partie au bénéficiaire. Les crédits payables sont parfois accordés aux bénéficiaires indépendamment du statut fiscal de ces derniers. En revanche, le montant maximum des crédits d'impôt non payables (parfois qualifiés de " non récupérables ") se limite au montant dû par le contribuable. Lorsque le crédit n'est pas payable, le contribuable peut être autorisé à reporter sur une période ultérieure le montant qui n’a pas pu être déduit.

13.9 Le montant des abattements, exonérations et déductions peut également être supérieur à la base imposable du contribuable. Dans ce cas, les autorités peuvent adopter des dispositifs prévoyant la conversion de cet excédent en crédit payable ou remboursable, ou son report (en amont ou en aval) dans des conditions normales ou spéciales. Un traitement similaire peut s'appliquer à des crédits non payables non utilisés.

\section{Problèmes spécifiques posés par la mesure du coût de l'allégement fiscal au titre des dépenses de R-D}

13.10 Le coût de l'allégement fiscal est plus difficile à mesurer que de simples flux financiers (dans le cas de subventions à la R-D ou de marchés publics, par exemple), car le but est alors de quantifier les recettes que l'État cesse de percevoir et d'allouer à d'autres activités. Cette mesure nécessite d'établir la somme que l'État aurait collectée en l'absence d'allégement fiscal. En pratique, on utilise pour cela une structure fiscale " normale ", ou structure fiscale de référence. La principale difficulté consiste à élaborer une méthode cohérente d'estimation de la valeur des avantages ou exonérations qui sortent du cadre d'une structure fiscale « normale » et qui, en relation avec des dépenses de $R-D$, viennent diminuer les recettes publiques ou augmenter les dépenses publiques.

13.11 Le principe général retenu pour les statistiques sur les dépenses fiscales liées à l'allégement fiscal au titre des dépenses de R-D consiste à inclure dans la structure fiscale " normale » les abattements et déductions applicables à des dépenses non liées à la R-D, mais par ailleurs identiques, ainsi que les crédits d'impôt accordés pour des activités comparables qui ne sont pas considérées comme des activités de R-D. Ce principe s'applique indépendamment du fait que d'autres cadres statistiques considèrent ces sommes comme des ajustements de l'impôt à payer ou payé par les unités concernées ou comme des dépenses publiques. Cette approche permet la comparabilité entre pays et un traitement identique du manque à gagner (renonciation à des recettes) et des remboursements d'impôts destinés spécifiquement à récompenser des activités de R-D. L'application de ces critères est traitée dans la section 13.5 du présent chapitre. 


\section{Lien avec la $R-D$}

13.12 Aux fins de la mesure de l'allégement fiscal au titre des dépenses de R-D, il doit exister un lien bien défini avec l'intention politique d'accorder un traitement fiscal préférentiel à un ensemble de dépenses de R-D. Ainsi, une subvention à l'emploi qui peut être octroyée aux employeurs de personnel de R-D comme aux employeurs d'autres catégories de personnel ne doit pas être partiellement imputée sur l'allégement fiscal au titre des dépenses de R-D, car cette disposition fiscale n'a pas pour but de subventionner spécifiquement les activités de R-D.

13.13 Pour entrer dans l'allégement fiscal au titre des dépenses de R-D, les mesures d'allégement doivent être mises en œuvre dans le cadre d'une politique de R-D intégrée, avec des sources convenablement documentées et mentionnées dans les discussions interministérielles et les rapports législatifs qui portent sur la science et la recherche.

\subsection{Champ des statistiques relatives à l'allégement fiscal au titre des dépenses de R-D}

\section{Définition et limites de la R-D}

\section{Dépenses de R-D et recettes provenant de la $R-D$}

13.14 L'allégement fiscal au titre des dépenses de R-D ne comprend que l'allégement fiscal accordé explicitement au titre de dépenses de R-D admissibles déclarées comme telles. Les dépenses fiscales associées, par exemple, au traitement préférentiel des recettes provenant d'activités de R-D antérieures (" patent boxes » ou instruments connexes) n'entrent pas dans le champ des statistiques relatives à l'allégement fiscal au titre des dépenses de R-D.

\section{Définition de la R-D}

13.15 Dans la mesure du possible, toutes les lignes directrices, définitions et conventions répertoriées au chapitre 2 sont applicables à la collecte de données sur l'allégement fiscal au titre des dépenses de R-D. La définition de base de la R-D est celle donnée au chapitre 2. L'analyse couvre la R-D dans tous les domaines de la recherche et du développement et ne fait pas de distinction entre les sciences exactes, naturelles et de l'ingénieur (SENI) et les autres, même si tous les pays ne font pas nécessairement porter l'allégement fiscal sur tous les domaines.

13.16 La définition de la R\&D ou d'autres types de dépenses ouvrant droit à un allégement fiscal peut varier selon les pays ou territoires et par rapport à la définition et aux explications données dans le présent manuel. La définition de la R-D retenue à des fins fiscales ne cesse d'évoluer et d'être réinterprétée par les autorités fiscales nationales, ce qui peut aussi avoir une incidence sur les enregistrements conservés par les exécutants de R-D. Il convient tout particulièrement de vérifier le contenu réel de R-D de l'allégement fiscal octroyé pour des domaines liés à l'innovation, en particulier ceux associés à d'autres 
dépenses d'innovation et à des dépenses relatives à des droits de propriété intellectuelle ou à leur commercialisation, qui peuvent ne pas faire partie intégrante de projets de R-D. Le Manuel déconseille d'utiliser des coefficients, à moins que les déclarations fiscales ne fournissent une ventilation suffisamment claire des dépenses de R-D et des autres coûts.

\section{Champ d'application sectoriel}

13.17 L'allégement fiscal au titre des dépenses de R-D s'applique aux dépenses qui sont engagées par des unités imposables pour des activités de R-D exécutées au sein de leur structure (ou éventuellement hors de celle-ci, c'est-àdire achetées) dans tous les secteurs institutionnels couverts par le Manuel.

13.18 Le secteur des entreprises est généralement le principal bénéficiaire direct visé par l'allégement fiscal au titre des dépenses de R-D. Certains dispositifs peuvent prévoir un allégement pour des dépenses correspondant à des activités de R-D sous-traitées à des tiers, dans d'autres secteurs nationaux ou à l'étranger. Toutes ces dépenses entrent dans le cadre de l'allégement fiscal au titre des dépenses de R-D.

13.19 L'allégement fiscal au titre des dépenses de R-D peut aussi, en principe, être accordé aux établissements d'enseignement supérieur, aux institutions privées sans but lucratif, aux personnes physiques et éventuellement aux organismes publics. Lorsque le champ de l'allégement fiscal au titre des dépenses de R-D est étendu au-delà des entreprises au moyen d'instruments fiscaux directement applicables à ces groupes, ceux-ci doivent être intégrés dans les statistiques relatives à l'allégement fiscal au titre des dépenses de R-D, à l'exception toutefois de ceux applicables aux personnes physiques.

13.20 Il est recommandé d'exclure les allégements qui ciblent directement les personnes physiques, sans passer par les institutions pour lesquelles ces personnes travaillent, car il est alors très difficile de valider et d'estimer l'étendue réelle du contenu de $\mathrm{R}-\mathrm{D}$, lequel se rapportera très probablement à la profession de la personne, plutôt qu'à une activité de R-D à proprement parler. Cette méthode permet une plus grande cohérence de l'approche institutionnelle des statistiques sur la R-D adoptée dans le Manuel. Des exemples précis sont examinés dans la section ci-après.

13.21 Les incitations fiscales à la R-D sont généralement destinées à encourager l'exécution d'activités de R-D dans l'économie nationale, mais, sur le plan des principes, les autorités peuvent prévoir des dispositifs octroyant un allégement fiscal à des contribuables non-résidents ou permettre aux contribuables de déclarer des dépenses de R-D sous-traitées à des unités affiliées ou non affiliées basées à l'étranger. Comme pour les CBPRD, ces dépenses entrent dans le champ de l'allégement fiscal au titre des dépenses de R-D.

13.22 Les exonérations fiscales octroyées à des organisations internationales exclusivement occupées à des activités de R-D sur le territoire national ne doivent pas entrer dans l'allégement fiscal au titre des dépenses 
de R-D, parce qu'il est peu probable que ces activités fassent l'objet d'un suivi systématique.

\section{Allégement au titre des dépenses de R-D intra-muros et $R-D$ extra-muros}

13.23 Envisagées du point du vue du bailleur de fonds (approche définie aux chapitres 4 et 8), les statistiques relatives à l'allégement fiscal au titre des dépenses de R-D couvrent les aides de ce type octroyées non seulement pour des activités de R-D exécutées intra-muros, au sein des organisations bénéficiaires, mais aussi pour des dépenses correspondant à des services de R-D externalisés et des contributions à des activités de R-D menées dans d'autres organisations.

13.24 Si une entreprise exécute des activités de R-D pour une autre entreprise, on ne doit pas présumer que les dispositions fiscales empêchent l'acheteur et le vendeur des services de demander tous deux un allégement pour la même unité de dépense de R-D, car il n'est pas dit que ce soit toujours le cas. La mesure des dépenses intra-muros de R-D aide à éviter un double compte, mais les données doivent rendre compte de l'allégement fiscal dont les deux contribuables ont effectivement bénéficié. Dans toute la mesure du possible, les doubles comptes doivent être relevés.

\section{Types de dépenses de R-D}

13.25 Tous les types de dépenses de R-D, y compris les dépenses courantes et les dépenses en capital, entrent dans le champ de l'allégement fiscal au titre des dépenses de R-D. Celui-ci s'applique donc aussi bien aux dépenses de R-D passées en charge qu'à celles inscrites au bilan. L'allégement calculé sur la dotation à l'amortissement des actifs utilisés pour les activités de R-D est également pris en compte.

\section{Types d'instruments fiscaux}

13.26 Les pouvoirs publics peuvent accorder un allégement fiscal au titre des dépenses de R-D au moyen de divers instruments. La Classification d'impôts de l'OCDE (OCDE, 2013) est structurée en fonction de l'assiette de l'impôt et du type de contribuable.

\section{Impôts sur le revenu, les bénéfices et les gains en capital des sociétés}

13.27 Les impôts sur les bénéfices des sociétés et des quasi-sociétés sont de loin le principal instrument utilisé pour mettre en œuvre des incitations fiscales à la R-D. L'allégement fiscal octroyé aux entreprises qui aboutit, après impôt, à réduire le coût de l'exécution de travaux de R-D ou celui du financement par le bénéficiaire en établissant un lien entre l'allégement consenti sur la facture fiscale de l'entreprise et le niveau de R-D admissible entre dans le cadre des statistiques relatives à l'allégement fiscal au titre des dépenses de R-D. 
13.28 L'allégement fiscal applicable aux gains en capital constatés sur des actifs liés à la R-D (revalorisation des brevets, par exemple) doit être exclu des statistiques relatives à l'allégement fiscal au titre des dépenses de R-D, car ce type d'instrument n'est pas destiné à réduire directement les dépenses de $R-D$, mais vise à accroître les bénéfices potentiels, incertains, de ces investissements. Les régimes fiscaux spécialement applicables aux revenus de la propriété intellectuelle, parfois désignés sous le terme de "patent boxes " ou d'" innovation boxes " et les types d'incitations similaires qui entrent dans cette catégorie sont donc exclus.

\section{Impôts sur le revenu, les bénéfices et les gains en capital des personnes physiques}

13.29 La distinction fondamentale entre les impôts sur les sociétés et les impôts sur les personnes physiques tient au fait que les premiers sont perçus sur la personne morale que constitue l'entreprise, et non sur les personnes physiques qui en sont propriétaires, et ne tiennent donc aucun compte de la situation individuelle de ces personnes. En principe, l'allégement fiscal pour la R-D accordé aux personnes physiques n'entre pas dans le cadre de l'allégement fiscal au titre des dépenses de R-D, car le Manuel se concentre sur la R-D exécutée par des unités institutionnelles et exclut celle menée à titre personnel. Seul l'allégement fiscal accordé aux personnes physiques agissant en tant que travailleurs indépendants, entreprises non constituées en société ou sous-traitants, peut éventuellement répondre aux conditions requises pour être pris en compte. L'allégement de l'impôt sur le revenu payé par les professionnels de la R-D en tant que personnes physiques peut être communiqué séparément, mais ne doit pas être combiné à l'allégement fiscal au titre des dépenses de R-D.

\section{Cotisations de sécurité sociale}

13.30 Les cotisations de sécurité sociale sont des paiements obligatoires qui ouvrent droit à des prestations sociales futures (éventuelles). Étant donné leur versement obligatoire à des organismes publics, elles se rapprochent nettement des impôts et taxes et sont parfois traitées comme tels. Elles peuvent s'appliquer aux salariés ou aux employeurs :

- Aux employeurs, sur la base des salaires ou du revenu ; ces sommes entrent dans le cadre de l'allégement fiscal au titre des dépenses de R-D.

- Aux salariés, sur la base des salaires ou du revenu ; cette catégorie doit être exclue pour les mêmes raisons que dans le cas de l'allégement fiscal consenti aux personnes physiques. Il est cependant possible que l'employeur soit chargé de prélever les cotisations des salariés et que l'allégement s'applique aux montants prélevés. Dans ce cas, il est nécessaire de déterminer si l'employeur est le bénéficiaire réel, auquel cas l'allégement doit être communiqué dans le cadre des statistiques relatives à l'allégement fiscal au titre des dépenses de R-D. Certains pays peuvent avoir mis en place des règles destinées à s'assurer que l'allégement appliqué aux montants prélevés ne diminue pas les droits à prestations ouverts par les cotisations de sécurité sociale des salariés. 


\section{Impôts sur les salaires et la main-d'œuure}

13.31 Entrent dans cette catégorie les impôts qui sont payés par les employeurs, les salariés ou les travailleurs indépendants sous la forme soit d'un pourcentage du salaire, soit d'un montant fixe par personne, et qui ne confèrent aucun droit à des prestations sociales. La même distinction entre employeurs et salariés s'applique aux fins de la compilation des statistiques relatives à l'allégement fiscal au titre des dépenses de R-D.

\section{Impôts sur le patrimoine}

13.32 Entrent dans cette catégorie les impôts périodiques et non périodiques sur l'utilisation, la propriété ou la mutation de biens immobiliers. Seul l'allégement applicable aux impôts sur l'utilisation de biens immobiliers pour des activités de R-D entre dans le cadre de l'allégement fiscal au titre des dépenses de R-D. Pour le moment, l'allégement d'impôt applicable aux opérations sur des actifs résultant de la R-D est exclu.

\section{Impôts sur les biens et services}

13.33 Entrent dans cette catégorie les accises, les taxes sur les ventes et les taxes sur la valeur ajoutée applicables aux services de R-D. En pratique, tous les pays de l'OCDE qui ont recours à des taxes sur la valeur ajoutée imposent cette taxation à tous les stades d'une filière et autorisent normalement tous les acteurs, sauf le consommateur final, à déduire immédiatement les taxes acquittées sur leurs achats. En principe, l'allégement applicable à ces montants doit être exclu de l'allégement fiscal au titre des dépenses de R-D, puisque ces taxes peuvent être déduites par tous les bénéficiaires de la $R-D$, à moins que la mesure d'allégement ne confère un avantage supplémentaire concret et quantifiable à l'entreprise ou à l'institution concernée.

\section{Sous-secteurs de l'administration}

\section{Administration centrale (ou fédérale)}

13.34 Le sous-secteur de l'administration centrale (ou fédérale) comprend tous les ministères, bureaux, établissements et autres entités qui sont des organes ou des instruments du pouvoir central et dont la compétence s'étend à la totalité du territoire, à l'exception de l'administration des caisses de sécurité sociale. L'administration centrale (ou fédérale) est donc compétente pour imposer toutes les unités résidentes et non résidentes exerçant des activités économiques sur le territoire national. L'allégement fiscal au titre des dépenses de R-D octroyé par les administrations opérant à ce niveau doit toujours être communiqué.

\section{Administrations régionales (ou d'États fédérés)}

13.35 Ce sous-secteur se compose des unités administratives intermédiaires situées juste au-dessous du niveau de l'administration centrale (fédérale) et exerçant leur compétence indépendamment de celle-ci sur une 
partie du territoire englobant un certain nombre de petites localités. Dans les pays unitaires, les administrations régionales peuvent être considérées comme dotées d'une existence distincte lorsqu'elles disposent d'une autonomie substantielle pour percevoir une part importante de leurs recettes de sources placées sous leur contrôle et lorsque leurs agents ne sont pas soumis à un contrôle administratif externe dans l'exercice des activités de leur unité. L'allégement fiscal au titre des dépenses de R-D accordé par les administrations opérant à ce niveau doit être communiqué lorsqu'il entre pour une part significative dans le total.

\section{Administrations locales (ou municipales)}

13.36 Ce sous-secteur comprend toutes les autres unités administratives exerçant une compétence indépendante sur une partie du territoire d'un pays, à l'exception des administrations des caisses de sécurité sociale. Il englobe les diverses circonscriptions urbaines ou rurales (par exemple, les départements, les communes, les agglomérations, les arrondissements ou les districts). Pour des raisons pratiques, il est peu probable qu'on puisse rendre compte de manière suffisamment précise et dans un délai raisonnable des allégements fiscaux au titre des dépenses de R-D octroyés au niveau des administrations locales (municipales). Cette catégorie doit donc être exclue, à moins qu'il ne soit avéré que le niveau de soutien fiscal assuré à cet échelon est significatif.

\subsection{Sources de données et mesure}

\section{Méthode d'estimation}

13.37 Étant donné la nécessité d'établir un niveau de référence pour déterminer le montant des allégements accordés au titre des dépenses de R-D, il est nécessaire d'estimer les dépenses fiscales à partir des données disponibles en appliquant un certain nombre de conventions et d'hypothèses. Selon l'OCDE (2010), trois mécanismes permettent d'estimer la valeur des dépenses fiscales associées à des mesures d'allégement.

- Perte initiale de recettes : montant de la réduction de la recette fiscale qu'entraîne l'adoption d'une dépense fiscale, présumant que cette adoption n'aura aucun effet sur les comportements des contribuables et les recettes issues des autres taxes.

- Perte finale de recettes : montant de la réduction de la recette fiscale qu'entraîne l'adoption d'une dépense fiscale, considérant le changement de comportement des contribuables et les conséquences sur les recettes issues des autres taxes que cette adoption induira.

- Équivalent en dépense : dépense directe qu'il faudrait effectuer avant impôt pour obtenir le même effet après impôt sur le revenu des contribuables que celui de la dépense fiscale si la dépense directe bénéficie du traitement fiscal applicable à ce type de subvention ou de transfert entre les mains du bénéficiaire. 
13.38 Les trois méthodes peuvent donner des estimations assez différentes des dépenses fiscales. La principale différence entre les deux premières méthodes est la prise en considération ou non des comportements des contribuables, tandis que la troisième tient compte des coûts administratifs supplémentaires liés à l'exécution d'un programme de soutien direct. L'approche la plus simple (perte initiale de recettes) est utilisée dans la plupart des pays de l'OCDE pour estimer les dépenses fiscales, car elle ne nécessite pas de s'appuyer sur des hypothèses complexes pour apprécier la réaction comportementale à une suppression hypothétique de l'incitation fiscale. Un certain nombre de documents budgétaires reposent aussi sur cette méthode, puis sont modifiés ou complétés à l'aide d'estimations fondées sur les comportements pour les changements futurs prévus des dispositifs. Les estimations de ce type partent souvent d'hypothèses d'incidence sur le montant admissible de dépenses de R-D déclaré par les contribuables.

13.39 Pour des raisons pratiques, la perte initiale de recettes est la méthode recommandée.

\section{Élaboration d'une référence commune pour la communication de données à l'échelle internationale}

13.40 L'élaboration d'une référence commune est une condition essentielle pour que les statistiques produites concernant l'allégement fiscal au titre des dépenses de R-D soient comparables à l'échelle internationale et rendent compte de façon fiable de l'effort financier consenti par les pouvoirs publics pour offrir un traitement préférentiel des dépenses de R-D. Il s'agit également de l'une des tâches les plus difficiles. Il est utile de faire la distinction entre les dépenses courantes et les dépenses en capital.

13.41 S'agissant des dépenses courantes de R-D, il est recommandé d'exclure des estimations de l'allégement fiscal au titre des dépenses de R-D les recettes fiscales auxquelles on renonce du fait des dispositifs permettant aux entreprises de traiter les dépenses courantes de R-D comme des charges et de les déduire de leurs bénéfices. De tels dispositifs, qui traitent les dépenses courantes de R-D comme tous les autres types de charges des entreprises, sont la norme dans tous les pays, ne serait-ce qu'en raison de la difficulté à mettre en œuvre d'autres approches. Aux seules fins du Manuel, l'accent est mis sur le coût des dispositifs qui impliquent un traitement plus favorable de la R-D. Cette approche est proposée afin de maintenir la comparabilité avec les pays qui ne communiquent pas de données sur l'allégement fiscal spécifiquement consenti au titre des dépenses de R-D, mais qui permettent de déduire les dépenses courantes de R-D. En l'absence de mesures incitatives améliorées, les entreprises ont la possibilité de déclarer leurs dépenses courantes de R-D comme étant égales à celles entrant dans le coût des ventes déductible, sans nécessairement préciser en quoi l'activité relève de la R-D. 
13.42 Les présentes directives n'excluent pas qu'à des fins internes, les pays puissent parfois souhaiter présenter ces dispositions " normales " de comptabilisation en charges comme des mesures incitatives améliorées en faveur de la R-D en spécifiant que la comparaison pertinente est à faire avec les dépenses en capital, et non avec les autres dépenses courantes.

13.43 S'agissant des dépenses en capital consacrées à la R-D, il est beaucoup plus difficile d'établir une référence, car le traitement de base des actifs varie plus fortement selon les pays. Pour des raisons pratiques, il est recommandé aux pays de communiquer des estimations par rapport au niveau de référence d'actifs physiques identiques à l'intérieur de leur propre territoire.

\section{Enregistrement du type d'allégement fiscal et traitement des reports}

13.44 Dans l'enregistrement de l'allégement fiscal du point de vue du bénéficiaire (généralement une entreprise), les repères chronologiques importants sont la période au cours de laquelle la dette fiscale prend naissance et celle durant laquelle la R-D ouvrant droit à un allégement est exécutée, le moment où le calcul de l'impôt dû devient définitif, le jour où cette dette arrive à échéance (sans pénalités) et le jour où l'impôt est effectivement payé ou le remboursement effectué.

13.45 En théorie, l'enregistrement de l'allégement fiscal au titre des dépenses de R-D doit être effectué dès que les activités de R-D prises en compte dans l'assiette ont été exécutées. En pratique, cela peut n'être possible que lorsque la demande d'allégement a été prise en compte par l'administration, indépendamment du moment où le montant de l'allégement est versé par cette dernière ou utilisé pour réduire le montant de l'impôt dû.

13.46 Cette méthode fondée sur la comptabilité d'exercice permet le meilleur alignement possible entre les statistiques relatives aux dépenses de R-D et celles sur l'exécution et le financement des activités de R-D. Elle nécessite toutefois un enregistrement précis des reports des sommes dues aux impôts et par les impôts. La R-D effectuée et déclarée pendant une année donnée peut être reportée sur une année ultérieure et éventuellement ne jamais être utilisée si, par exemple, l'entreprise disparaît.

13.47 La méthode fondée sur les mouvements de trésorerie (comptabilité de caisse) permet de suivre plus précisément le flux effectif de fonds entre les autorités et les unités imposables, mais ne fait pas le lien avec les données sur l'exécution des activités de R-D ni avec la situation réelle sous-jacente de l'économie et de la R-D. Des solutions hybrides sont possibles : une méthode fondée sur les mouvements de trésorerie peut être alignée sur l'année fiscale de référence $t$ au cours de laquelle la $\mathrm{R}-\mathrm{D}$ a été déclarée plutôt que sur la date à laquelle les paiements d'impôt ont été effectués (plusieurs mois après le début de l'année $t+1$ dans le cas de règlements annuels).

13.48 Il est important de noter qu'il n'existe actuellement pas de méthode prédominante pour la déclaration des incitations fiscales à la R-D et que, très peu 
de pays ont mis en place un système de comptabilisation permettant de produire des estimations sur la base à la fois des droits constatés et des encaissements ou des décaissements. Idéalement, les pays devraient enregistrer les éléments suivants :

1. les crédits remboursables octroyés aux contribuables, ou à d'autres types d'unités, pour leurs activités de R-D durant la période de référence (le cas échéant)

2. le manque à gagner (renonciation à des recettes) sur la période de référence, pour les activités de R-D réalisées pendant cette même période

3. les crédits acquis mais non utilisés durant la période de référence, c'est-à-dire reportés sur une année ultérieure, évalués sur une base nominale

4. les crédits acquis sur une période précédente et utilisés durant la période de référence, également sur une base nominale.

13.49 Les deux principaux indicateurs relatifs à l'allégement fiscal au titre des dépenses de R-D peuvent être définis sur la base des éléments suivants :

- allégement fiscal au titre des dépenses de R-D sur la base des droits acquis ou constatés $=[1]+[2]+[3]$

- allégement fiscal au titre des dépenses de R-D sur la base des droits utilisés ou des mouvements de trésorerie $=[1]+[2]+[4]$.

13.50 Il est recommandé d'opter pour la méthode des droits constatés, dans la mesure du possible, mais les estimations fondées sur une comptabilité de caisse sont également acceptables si elles sont utilisées systématiquement dans la durée. Dans certains pays, les bénéficiaires de mesures fiscales peuvent décider de la date à laquelle ils déclarent un crédit d'impôt ou un abattement fiscal (i.e. demandent à en bénéficier), et cette date ne se situe pas forcément dans la période de référence (celle pendant laquelle les dépenses de R-D ont été réellement effectuées). En outre, certains pays permettent aux bénéficiaires de reporter sur une année ultérieure les crédits ou les abattements déclarés mais non utilisés (report de déclarations d'activités de R-D admissibles, par exemple). Dans les deux cas, il y a un risque de double comptabilisation. Lors du calcul des indicateurs d'allégement fiscal au titre des dépenses de R-D, il convient donc d'être très attentif aux éventuels doublons dans les totaux communiqués.

\section{Type de données}

13.51 Les statisticiens disposent de plusieurs sources de données pour déterminer le montant des allégements fiscaux octroyés par les autorités pour la R-D. Les différents choix sont évalués par rapport à l'objectif de mise à disposition d'un dispositif de mesure détaillé de ce type de soutien. Les bénéficiaires de l'allégement fiscal au titre des dépenses de R-D constituent l'une de ces sources de données. Les statistiques les concernant peuvent être obtenues à partir d'enquêtes auprès des entités qui exécutent la R-D (approche 
fondée sur les exécutants). L'autre source de données provient des entités qui octroient l'allégement fiscal au titre des dépenses de R-D ; les statistiques les concernant peuvent être calculées à partir des demandes d'allégement fiscal, validées ou approuvées, (similaire à une approche fondée sur les bailleurs de fonds). Les deux sources peuvent présenter des différences, et ce pour diverses raisons.

\section{Enquêtes auprès des exécutants de la R-D}

13.52 Les enquêtes auprès des exécutants de la R-D se concentrent sur l'exécution des activités de R-D et leurs "sources de financement " respectives. De ce point de vue, de nombreuses formes d'allégement fiscal au titre des dépenses de R-D ne peuvent pas être considérées comme un financement de l'État, car le répondant n'est pas en mesure de faire le lien entre ses déclarations d'exécution de R-D et les allégements fiscaux. C'est le cas notamment lorsque l'exécutant ne connaît pas avec certitude le montant de l'allégement dont il bénéficiera, celui-ci pouvant dépendre de son bénéfice au terme de l'année fiscale. Le lien entre l'allégement et la R-D est donc dans la plupart des cas indirect. L'allégement accordé au final peut être employé par le bénéficiaire à d'autres fins et peut ne pas être utilisé avant plusieurs années en cas de report.

13.53 Les dépenses de R-D admissibles entrent dans la base de calcul de l'allégement, mais ne constituent pas nécessairement l'objet du financement. Même si, dans certains cas, il peut exister un lien direct avec l'exécution intra-muros, on ne peut pas recommander de façon générale de demander à une unité exécutant des travaux de R-D d'indiquer la part des incitations fiscales dans les fonds reçus de sources externes pour la R-D, en partie parce que le soutien fiscal peut également avoir été accordé au titre de dépenses de R-D supportées par des tiers pour le compte de l'entreprise.

13.54 Sous réserve d'essais préalables satisfaisants, il peut être préférable de demander aux unités, dans le cadre des enquêtes :

- d'indiquer dans quelle mesure les activités de R-D intra-muros admissibles et le financement extra-muros de l'unité ont été ou seront utilisés pour demander un allégement fiscal au titre des dépenses de R-D

- d'indiquer dans quelle mesure les dettes (créances) d'impôts de l'unité ont diminué (augmenté) du fait de ses activités de R-D au cours de l'année de référence, et de préciser les variations de la valeur comptable des abattements et crédits non utilisés.

13.55 Pour les raisons indiquées, il est conseillé de compiler les statistiques relatives aux dépenses liées à l'allégement fiscal au titre des dépenses de R-D sur la base des sources de financement plutôt que sur la base des exécutants. Les enquêtes réalisées auprès des exécutants de la R-D ne sont pas l'outil recommandé aux fins de communication de l'allégement fiscal au titre des 
dépenses de R-D, mais peuvent constituer une solution de repli lorsque les registres administratifs ne sont pas accessibles ou ne sont pas suffisamment fiables.

\section{Déclarations fiscales détaillées}

13.56 Les demandes d'allégement fiscal traitées puis analysées par les autorités fiscales constituent la principale source d'informations sur l'ampleur de l'abattement fiscal au titre des dépenses de R-D. Dans de nombreux pays, les formulaires requis ont plusieurs éléments en commun avec les enquêtes sur la R-D, et utilisent leurs propres nomenclatures (souvent très détaillées) pour différents types de dépenses. Ces données peuvent servir de base à des calculs simples de la valeur de l'allégement fiscal au titre des dépenses de R-D. Les estimations peuvent reposer sur la totalité des demandes ou sur un échantillon représentatif.

13.57 Il arrive cependant que les données des déclarations fiscales ne soient disponibles qu'après un certain délai, qui peut excéder le délai de réalisation des enquêtes classiques sur la R-D. Les demandes peuvent par exemple n'être consultables qu'une fois que les contrôleurs des impôts en ont achevé le traitement. Il est devenu de plus en plus courant que les pays qui accordent un allégement fiscal au titre des dépenses de R-D publient des statistiques sur le nombre de bénéficiaires de ce type de mesures et sur les coûts correspondants.

13.58 Il est donc recommandé d'utiliser les déclarations fiscales pour établir les estimations finales des dépenses liées à l'allégement fiscal au titre des dépenses de R-D.

\section{Documents budgétaires}

13.59 Les documents budgétaires semblent particulièrement appropriés pour établir des prévisions et suivre en temps réel l'incidence de l'allégement fiscal sur la situation des finances publiques. Dans de nombreux pays, ces informations sont disponibles - quoique souvent non publiées - sous une catégorie distincte. Il arrive que les publications budgétaires comprennent des analyses ponctuelles qui rendent compte des implications attendues de modifications que l'on a apportées à la conception des dispositifs d'incitation fiscale à la R-D. Ces comptes rendus s'appuient sur des méthodes d'analyse et de simulation. Il est recommandé de réserver ces documents, lorsqu'ils sont disponibles, aux fins d'établissement de statistiques préliminaires actualisées.

\section{Année civile ou budgétaire}

13.60 Les autorités nationales dont l'année budgétaire ne correspond pas à l'année civile doivent, dans la mesure du possible, fournir des données sur la base de l'année civile afin de faciliter au maximum les comparaisons avec d'autres pays. 


\subsection{Ventilations prioritaires des statistiques relatives à l'allégement fiscal au titre des dépenses de R-D}

\section{Par secteur bénéficiaire}

13.61 Il est recommandé de ventiler les dépenses liées à l'allégement fiscal au titre des dépenses de R-D selon la classification institutionnelle des contribuables bénéficiaires, en utilisant la classification par grands secteurs du Manuel (entreprises, État, enseignement supérieur, secteur privé sans but lucratif et reste du monde). En pratique, il peut être suffisant d'indiquer la répartition entre le secteur des entreprises et les autres secteurs.

13.62 S'agissant des bénéficiaires, en particulier dans le secteur des entreprises, il est utile de présenter les statistiques relatives à l'allégement fiscal au titre des dépenses de R-D par branche d'activité ou activité économique de façon à pouvoir comparer le soutien accordé sous forme d'allégement fiscal avec la répartition des DIRD et DIRDE financées par l'État obtenue à partir des données d'enquêtes sur la R-D. Étant donné que les statistiques fiscales peuvent ne pas totalement correspondre aux registres des entreprises, il convient de veiller tout spécialement à ce que la communication des activités du siège des entreprises n'écrase pas les informations sur la principale activité économique des entreprises proprement dites.

\section{Par niveau d'administration}

13.63 A des fins de comparabilité, il est proposé que les pays communiquent les données relatives à l'allégement fiscal au titre des dépenses de R-D séparément pour :

- l'administration centrale (ou fédérale) et ses caisses de sécurité sociale

- les administrations régionales (ou d'États fédérés) et leurs caisses de sécurité sociale.

\section{Par type d'allégement fiscal}

13.64 Dans toute la mesure du possible, les statistiques relatives à l'allégement fiscal au titre des dépenses de R-D, qu'elles soient calculées sur la base des droits acquis ou utilisés, doivent comprendre des informations distinctes sur les éléments constitutifs (définis à la section 13.4) étayant l'indicateur que les pays sont en mesure de communiquer.

\section{Par taille d'entreprise}

13.65 Dans le secteur des entreprises, il est recommandé d'utiliser la ventilation ci-après, quelle que soit la définition nationale des tailles d'entreprise retenue à des fins d'allégement fiscal au titre des dépenses de R-D :

- petites entreprises (employant moins de 50 personnes)

- moyennes entreprises (employant entre 50 et 249 personnes)

- grandes entreprises (employant 250 personnes ou plus). 
13.66 Il est important de noter que les dispositifs propres aux pays en matière d'allégement fiscal au titre des dépenses de R-D peuvent varier en fonction de la taille de l'entreprise, et que les catégories peuvent ne pas correspondre à la classification standard par groupe de tailles. Il est également possible qu'à des fins d'allégement fiscal au titre des dépenses de R-D, les dispositifs d'admissibilité portant sur la taille suivent des règles différentes pour décider si une société affiliée doit ou non être considérée comme une entreprise distincte. L'existence de tels dispositifs peut influer sur la ventilation par groupe de tailles des dépenses liées à l'allégement fiscal au titre des dépenses de R-D qui est communiquée par les pays.

\section{Présentation de l'allégement fiscal au titre des dépenses de R-D parallèlement à d'autres indicateurs relatifs à la R-D}

13.67 Les statistiques sur les dépenses publiques liées à l'allégement fiscal au titre des dépenses de R-D peuvent être présentées avec deux autres types d'indicateurs relatifs à la R-D afin de donner une image plus complète du soutien public :

- CBPRD : les deux indicateurs correspondent à des estimations, fondées sur les sources, du soutien financier public global de la R-D. Les statisticiens doivent veiller à supprimer toute composante de soutien fiscal qui pourrait être comprise dans les statistiques relatives aux CBPRD, conformément aux recommandations formulées au chapitre 12 .

- DIRDE financées par l'État : ce type d'indicateur peut en principe être présenté parallèlement à la composante des dépenses liées à l'allégement fiscal au titre des dépenses de R-D afin de montrer de manière plus complète l'étendue du soutien financier public apporté aux entreprises. Il convient de noter que cette approche combine des statistiques fondées sur le bailleur de fonds (allégement fiscal au titre des dépenses de R-D) et des statistiques fondées sur l'exécutant et peut donc, de ce fait, présenter des incohérences. Ainsi, certaines dépenses liées à l'allégement fiscal au titre des dépenses de R-D peuvent soutenir des activités de R-D externalisées auprès d'universités nationales ou d'établissements publics ou auprès d'établissements à l'étranger.

\section{Références}

CE, FMI, OCDE, Nations Unies et Banque mondiale (2009), Système de comptabilité nationale 2008, Nations unies, New York, http://unstats.un.org/unsd/nationalaccount/ docs/SNA2008FR.pdf.

FMI (2014), Government Finance Statistics (GFS) Manual, Pre-publication Draft, FMI, Washington, DC, www.imf.org/external/np/sta/gfsm/.

OCDE (2014), "Classification des impôts de l'OCDE et Guide d'interprétation », Statistiques des recettes publiques 2014, Éditions OCDE, Paris, http://dx.doi.org/10.1787/reu_stats2014-8-en-fr.

OCDE (2010), Les dépenses fiscales dans les pays de l'OCDE, Éditions OCDE, Paris, http://dx.doi. org/10.1787/9789264076921-fr. 



\section{ANNEXE 1}

\section{Bref historique et genèse du manuel}

On trouvera dans la présente annexe une description succincte des six éditions qui ont précédé la version actuelle du Manuel de Frascati. Y sont également exposées les contributions des personnes auxquelles l'ouvrage doit son rang de référence mondiale. Les documents originaux sont consultables en ligne sur la page web consacrée au Manuel (http://oe.cd/frascati).

\section{Genèse}

C'est autour de l'année 1960 que la plupart des pays membres de l'OCDE, incités par la croissance rapide des ressources nationales dédiées à la recherche et au développement expérimental (R-D), ont commencé à recueillir des données statistiques dans ce domaine. Ils s'engageaient ainsi dans la voie frayée par un petit groupe de pays composée des États-Unis, du Japon, du Canada, du Royaume-Uni, des Pays-Bas et de la France. Cependant, au moment d'entreprendre des enquêtes sur la R-D, ils se sont heurtés à des problèmes d'ordre théorique et la diversité des champs couverts, conjuguée à l'hétérogénéité méthodologique et conceptuelle, rendait difficile l'établissement de comparaisons internationales. Il est dès lors apparu de plus en plus nécessaire de tenter un exercice de normalisation semblable à celui entrepris à l'égard des statistiques économiques.

L'intérêt de l'OCDE pour cette question remonte à l'époque de l'Organisation européenne de coopération économique (OECE). En effet, c'est en 1957 que le Comité de la recherche appliquée de l'Agence européenne de productivité de l'OECE a commencé à organiser des réunions entre experts de pays membres pour discuter des problèmes de méthodologie. Ces réunions ont débouché sur la constitution d'un groupe d'experts ad hoc, chargé d'étudier les méthodes d'enquête sur les dépenses de recherche et de développement sous l'égide du Comité de la recherche appliquée. Le Secrétaire technique de ce groupe, M. J.C. Gerritsen, a rédigé deux études détaillées sur les définitions et méthodes employées pour mesurer la R-D dans le secteur de l'État au Royaume-Uni, en France et, par la suite, aux États-Unis et au Canada. D'autres membres du groupe ont diffusé des documents décrivant les méthodes d'enquête appliquées dans leurs pays respectifs et les résultats ainsi obtenus. 


\section{Première édition}

Lorsqu'en 1961, la Direction des affaires scientifiques a repris les travaux de l'Agence européenne de productivité, le moment était venu de présenter des propositions précises aux fins de la normalisation. À sa réunion de février 1962, le groupe ad hoc a décidé d'organiser une conférence pour étudier les problèmes techniques posés par la mesure de la R-D. À cet effet, la Direction des affaires scientifiques a chargé un consultant, M. C. Freeman, d'établir un projet de rapport, dont le texte a été diffusé aux pays membres à l'automne 1962 avant d'être révisé à la lumière de leurs commentaires. Ce document, intitulé "Méthode type proposée pour les enquêtes sur la recherche et le développement expérimental »(OCDE, 1963), a été examiné, modifié et approuvé par les experts des pays membres de l'OCDE lors de la conférence qui s'est déroulée à Frascati (Italie) en juin 1963.

Par la suite, toujours en 1963, la Direction des affaires scientifiques de l'OCDE a invité l'Institut national pour la recherche économique et sociale du Royaume-Uni à réaliser, à titre expérimental, une étude comparative des efforts de recherche menés dans cinq pays d'Europe occidentale (Allemagne, Belgique, France, Pays-Bas et Royaume-Uni), aux États-Unis et en Union soviétique. Bien que fondée sur des statistiques d'enquêtes antérieures à l'adoption de normes internationales, cette étude (Freeman et Young, 1965) a permis de tester les version préliminaires de définitions. Sa conclusion étant que les statistiques existantes laissaient grandement à désirer, un certain nombre de dispositions ont été proposées en vue de les améliorer, en premier lieu :

- Établir une distinction plus rigoureuse, sur le plan conceptuel, entre la recherche et le développement expérimental, d'une part, et les " activités scientifiques connexes " d'autre part.

- Mener des enquêtes minutieuses dans le secteur de l'enseignement supérieur pour estimer la part de leur temps que le personnel enseignant et les étudiants du niveau doctorat consacrent à la recherche.

- Établir une ventilation plus détaillée des données relatives au personnel et aux dépenses de R-D afin, notamment, de calculer avec une plus grande exactitude les taux de change applicables aux dépenses de recherche.

- Mesurer de manière plus systématique les flux de dépenses entre les secteurs exécutant des travaux de R-D.

- Recueillir davantage de données sur les flux des paiements technologiques et les mouvements migratoires du personnel scientifique entre les pays.

En 1964, dans le prolongement de l'adoption du Manuel de Frascati, l'OCDE lançaitl'Année statistique internationale (ASI) sur la recherche et le développement expérimental. À cette occasion, les pays membres ont communiqué des données concernant l'année 1963 ou 1964. Dix-sept pays ont participé à l'initiative, dans le cadre de laquelle un grand nombre ont pour la première fois engagé des enquêtes spéciales (OCDE, 1968). 


\section{Deuxième édition}

Après la publication des conclusions de l'Année statistique, le Comité de la politique scientifique de l'OCDE a chargé le Secrétariat de revoir le Manuel de Frascati au regard de l'expérience acquise. Une ébauche des modifications proposées a été diffusée aux pays membres en mars 1968. Un projet de texte révisé du Manuel, qui reprenait la plupart de ces propositions, a été examiné lors de la réunion d'experts nationaux tenue à Frascati en décembre 1968. L'exercice a surtout consisté à rendre le Manuel aussi conforme que possible aux normes internationales établies par l'ONU, telles que celles énoncées dans le Système de comptabilité nationale (SCN) et la Classification internationale type par industrie (CITI). Après l'examen d'un projet de texte modifié par un groupe restreint d'experts en juillet 1969, la nouvelle version du Manuel a été publiée en septembre 1970 (OCDE, 1970).

\section{Troisième édition}

Deux phénomènes sont à l'origine de la deuxième révision du Manuel. Premièrement, en 1973, les pays membres avaient déjà participé à quatre enquêtes ASI, au grand profit de la précision et de la comparabilité des données, et les techniques d'enquête appliquées dans les pays s'étaient elles aussi beaucoup améliorées. Deuxièmement, le Comité de la politique scientifique et technologique (CPST) de l'OCDE avait constitué en 1972 le premier groupe d'étude ad hoc en matière de statistiques de R-D, présidé par M. Silver (RoyaumeUni), qui devait conseiller le Comité et le Secrétariat sur la façon d'utiliser au mieux, à court terme, les rares statistiques de R-D disponibles à l'OCDE, tout en tenant compte des priorités des pays membres. Ces derniers ont donc été invités à dresser un inventaire de leurs besoins, ce que pratiquement tous ont fait. Outre la priorité absolue accordée à la poursuite des enquêtes ASI, ils ont formulé un certain nombre de recommandations d'ordre méthodologique, en insistant notamment sur la nécessité de resserrer les liens entre l'OCDE et les autres organisations internationales.

En conséquence, la troisième édition du Manuel de Frascati a approfondi des sujets déjà traités tout en en abordant de nouveaux. Son champ d'application a été étendu à la recherche en sciences sociales et humaines et une plus grande importance a été accordée aux classifications "fonctionnelles ", notamment en ce qui concerne la répartition par « objectif » de R-D. Un projet a été examiné au cours d'une réunion d'experts tenue à l'OCDE en décembre 1973 et le texte définitif a été adopté en décembre 1974 (OCDE, 1976).

\section{Quatrième édition}

Pour cette édition, les experts nationaux ont recommandé de s'en tenir à une simple révision intermédiaire qui n'impliquerait aucun changement notable dans les classifications et les grands concepts. Il s'agissait surtout d'améliorer la rédaction et la présentation du Manuel. Cependant, un certain nombre de 
modifications ont été introduites compte tenu des recommandations formulées par le deuxième groupe d'étude ad hoc en matière de statistiques de $R-D$, réuni en 1976 sous la présidence de M. J. Mullin (Canada), de l'expérience acquise par le Secrétariat de l'OCDE grâce aux enquêtes internationales et à ses rapports analytiques, et des suggestions avancées par les experts nationaux en matière de statistiques de R-D. Des propositions concernant cet exercice de révision ont été présentées à la réunion annuelle des experts nationaux, en décembre 1978. En juillet 1979, un groupe d'experts ad hoc s'est réuni au Secrétariat de l'OCDE en vue d'approfondir l'examen d'un projet établi par un consultant. Une version modifiée sur la base des observations du groupe d'experts et du Secrétariat a été examinée en décembre 1979, puis le texte définitif adopté à l'automne 1980 (OCDE, 1981).

Le secteur de l'enseignement supérieur n'apparaît pas dans le Système de comptabilité nationale (SCN) adopté par les Nations Unies et l'OCDE. Toutefois, l'OCDE et l'UNESCO l'ont rapidement inclus dans leurs activités de recueil de statistiques sur la $\mathrm{R}-\mathrm{D}$, en raison de l'intérêt manifesté par les décideurs à l'égard de la place que les universités et autres établissements d'enseignement postsecondaire et supérieur occupent dans les efforts nationaux de recherche. Malgré cet intérêt, la collecte de données précises sur l'enseignement supérieur soulève d'importants problèmes. Ces problèmes ont été examinés en juin 1985, lors d'un séminaire de l'OCDE sur les indicateurs de la science et de la technologie propres au secteur de l'enseignement supérieur. Les experts réunis à cette occasion ont estimé que le Manuel contenait certes des orientations générales, mais les conseils pratiques qu'il prodiguait n'étaient pas toujours suffisants. Aussi le Groupe de travail des experts nationaux sur les indicateurs de science et de technologie (GENIST) est-il convenu, lors de sa réunion annuelle de décembre 1985, d'établir un supplément au Manuel de Frascati qui traiterait de ces questions et formulerait des recommandations sur la manière d'améliorer les méthodes d'enquête dans l'avenir. Après examen d'un avant-projet en décembre 1986, le texte modifié a été adopté par le GENIST, et, sous réserve de certaines modifications, sa diffusion générale a été recommandée en décembre 1987 (OCDE, 1989b).

\section{Cinquième édition}

À la fin des années 80, il était évident qu'il fallait revoir les lignes directrices du Manuel de Frascati pour faire face aux nouvelles priorités de l'action publique et obtenir les données essentielles à la prise de décisions. De nombreux aspects étaient à prendre en compte, en particulier l'évolution du système scientifique et technologique et la manière dont celui-ci était perçu. Certains sont apparus dans le cadre du Programme Technologie-Économie de l'OCDE (par exemple, l'internationalisation, les logiciels, les sciences de transfert, etc.). D'autres concernaient les données relatives à la R-D environnementale, la nécessité de disposer, à des fins d'analyse, d'éléments sur la R-D susceptibles de figurer dans d'autres séries de données économiques et industrielles, ainsi que les nouvelles 
versions des normes et classifications internationales liées aux statistiques de R-D et citées dans le Manuel.

En octobre 1991, une conférence d'experts a donc été organisée à Rome, sous les auspices du ministère italien des Universités et de la Recherche scientifique, afin d'examiner un ensemble de propositions sur les modifications à apporter au Manuel de Frascati, avec, pour la première fois, la présence d'experts de pays d'Europe orientale. Comme suite à la conférence, le GENIST a, lors de sa session d'avril 1992, examiné en bonne et due forme un projet de version révisée du Manuel, qui reprenait une grande partie du texte du supplément sur l'enseignement supérieur. De nouveau modifié sur la base des recommandations d'un groupe de rédaction restreint, le projet a été adopté au début de l'année 1993 (OCDE, 1994a).

\section{Sixième édition}

Le cinquième exercice de révision du Manuel de Frascati répondait à la nécessité d'actualiser diverses classifications ainsi qu'au besoin croissant de disposer de données sur la R-D exécutée dans le secteur des services, sur la mondialisation de la R-D et sur les ressources humaines consacrées à la R-D. Les besoins en données comparables avaient également été exacerbés par différents projets d'évaluation comparative alors en cours. Le GENIST a décidé de réviser le Manuel à sa réunion de 1999 et sélectionné à cet effet un certain nombre de thèmes, dont 19 à examiner plus avant, lors d'une réunion spéciale en mars 2000. Des groupes thématiques à composition limitée ont été constitués et placés sous l'égide d'un pays pilote ou du Secrétariat de l'OCDE. Leurs rapports ont été examinés lors d'une réunion organisée par les autorités italiennes à Rome, en mai 2001. À sa réunion suivante, elle aussi tenue à Rome, le GENIST a décidé des modifications de fond à apporter au manuel. Des propositions de modification d'ordre rédactionnel ont fait l'objet d'un débat au cours d'une réunion tenue en octobre 2001. La sixième édition du Manuel a été adoptée fin 2002 et publiée à la fois sous forme papier et électronique.

L'exercice de révision a expressément consisté à affermir différentes recommandations méthodologiques. Comme par le passé, les recommandations énoncées dans le Système de comptabilité nationale (SCN) ont été suivies chaque fois que cela était possible et faisable dans le cadre des enquêtes sur la R-D. En parallèle, des dispositions ont été prises pour que les statistiques de R-D cadrent davantage avec le SCN. Ces efforts ont par la suite trouvé leur justification dans la décision de reprendre, dans le SCN 2008, la définition de la R-D donnée par le Manuel et de traiter les investissements y afférents comme une formation de capital (CE et al., 2009).

Ainsi, la sixième édition du Manuel a été étoffée de nouvelles sections sur la R-D liée aux logiciels, aux sciences sociales et aux services, le SCN, la mondialisation et la coopération en matière de R-D ainsi que d'exemples illustrant de manière détaillée les différents types de R-D. Les orientations consacrées au 
personnel de R-D ont été entièrement remaniées et complétées de propositions nouvelles sur les données à fournir par sexe et par âge. Ont également été ajoutées des recommandations détaillées sur la manière de rendre compte des sources de financement et des dépenses extra-muros, de même qu'un certain nombre des recommandations adoptées par Eurostat depuis l'établissement de la version antérieure. Enfin, la Nomenclature pour l'analyse et la comparaison des budgets et programmes scientifiques (NABS) a été retenue comme base de la classification par objectif socio-économique et le Manuel a été enrichi d'annexes thématiques (par exemple, sur les TIC, la santé et les biotechnologies), dont une fournit des lignes directrices pour le recueil de données de R-D par région.

\section{Principaux contributeurs aux éditions précédentes}

Chaque édition du Manuel est née d'une collaboration entre les experts de pays membres et d'organisations internationales en particulier l'UNESCO, l'UE et Nordforsk/Fonds industriel nordique et le Secrétariat de l'OCDE, sous la direction de Mme A. Young et de M. Y. Fabian (pour les quatre premières). La National Science Foundation mérite des remerciements particuliers pour son œuvre pionnière dans la mesure systématique de la R-D.

La première édition du Manuel a tout particulièrement bénéficié des contributions de MM. J. Perlman et C. Freeman ainsi que de la Délégation générale à la recherche scientifique et technique (DGRST) de l'administration française.

En ce qui concerne la deuxième édition, on retiendra que M. H. E. Bishop a présidé la réunion tenue à Frascati en 1968 et que MM. H. Stead (Statistique Canada), P. Slors (Office central de statistique des Pays-Bas) et D. Murphy (Irish National Science Council) ont apporté un concours indispensable.

Parmi les personnes qui ont concouru à l'élaboration de la troisième version, on signalera tout particulièrement MM. K. Sanow (National Science Foundation, États-Unis), J. Mitchell (Office of Fair Trading, Royaume-Uni) et K. Perry (Central Statistical Office, Royaume-Uni) ainsi que Mme K. Arnow (National Institutes of Health, États-Unis), qui a présidé la réunion des experts en 1973, sans oublier les présidents des réunions thématiques : MM. T. Berglund (Office central de statistiques de Suède), J. Sevin (DGRST) et F. Snapper (ministère de l'Éducation et de la Science des Pays-Bas).

La quatrième édition doit beaucoup aux travaux de M. H. Stead (Statistique Canada). Les réunions d'experts ont été présidées par M. G. Dean (Central Statistical Office, Royaume-Uni) en 1978, puis M. C. Falk (National Science Foundation, États-Unis) en 1979. Le supplément relatif à l'enseignement supérieur a été rédigé par Mme A. FitzGerald (EOLAS - Irish Science and Technology Agency, Irlande). Les travaux de M. M. Åkerblom (Office central de statistique de la Finlande) ont grandement inspiré la section consacrée aux enquêtes de budget-temps. La conférence de 1985 sur les indicateurs de la science et de la technologie applicables à l'enseignement supérieur a été présidée par M. T. Berglund (Statistique Suède). 
La cinquième édition a été en grande partie établie par Mme A. FitzGerald (EOLAS) sur la base des travaux d'un grand nombre d'experts nationaux. M. T. Berglund (Statistique Suède), M. J. Bonfim (Junta Nacional de Investigaçao Cientifica e Tecnologica, Portugal), Mme M. Haworth (Department of Trade and Industry, Royaume-Uni), M. A. Holbrook (Industrie, sciences et technologie Canada), M. J.-F. Minder (ministère de la Recherche et de la Technologie, France), M. F. Niwa (National Institute of Science and Technology Policy, Japon), Mme Erika Rost (ministère fédéral de la Recherche et de la technologie, Allemagne), M. P. Turnbull (Central Statistical Office, Royaume-Uni) et Mme K. Wille-Maus (Norges Allmennvitenskaplige Forskningrad, Norvège) méritent des remerciements particuliers. Durant la période concernée, le GENIST a été présidé par M. G. Sirilli (Consiglio nazionale delle ricerche, Italie), qui a également organisé la conférence tenue à Rome.

La sixième édition a essentiellement été établie par M. M. Åkerblom (Statistique Finlande) avec le concours du Secrétariat de l'OCDE pour la phase de rédaction à partir de travaux thématiques d'un grand nombre d'experts nationaux. Il convient de remercier tout particulièrement M. D. Byars (Australian Bureau of Statistics), Mme D. Francoz (ministère de la Recherche et de la Technologie, France), M. Christoph Grenzmann (Stifterverband, Allemagne), M. John Jankowski (National Science Foundation, États-Unis), Mme Jane Morgan (Office for National Statistics, Royaume-Uni), M. B. Nemes (Statistique Canada), M. A. Sundström (Statistique Suède), M. H. Tomizawa (National Institute of Science and Technology Policy, Japon) et Mme A. Young (consultante auprès de Statistique Canada). Durant la période concernée, le GENIST a été présidé par M. G. Sirilli (Consiglio nazionale delle ricerche, Italie), qui a également organisé la conférence tenue à Rome, puis par M. F. Gault (Statistique Canada), qui a supervisé l'établissement de la version définitive de la sixième édition du Manuel.

\section{Références}

CE, FMI, OCDE, Nations Unies et Banque mondiale (2009), Système de comptabilité nationale, Nations Unies, New York, http://unstats.un.org/unsd/nationalaccount/docs/sna2008FR.pdf.

Freeman, C. et A. Young (1965), L'effort de recherche et de développement en Europe occidentale, Amérique du Nord et Union soviétique : Essai de comparaison internationale des dépenses et des effectifs consacrés à la recherche en 1962, Éditions OCDE, Paris.

Nations Unies (1968), Un système de comptabilité nationale, Études méthodologiques, Série F, n², Rév. 3, New York.

OCDE (2002), Manuel de Frascati : Méthode type proposée pour les enquêtes sur la recherche et le développement expérimental, La mesure des activités scientifiques et technologiques, Éditions OCDE, Paris, http://dx.doi.org/10.1787/9789264299047-fr.

OCDE (1994), Standard Practice for Surveys of Research and Experimental Development - Frascati Manual 1993, The Measurement of Scientific and Technological Activities, Éditions OCDE, Paris, http://dx.doi.org/10.1787/9789264063525-en.

OCDE (1989), Statistiques de R-D et mesure des résultats dans l'enseignement supérieur, « Manuel de Frascati ", supplément, Série "Mesure des activités scientifiques techniques ", Paris. 
OCDE (1981), Méthode type proposée pour les enquêtes sur la recherche et le développement expérimental : "Manuel de Frascati 1980 ", Série " Mesure des activités scientifiques et techniques ", Paris.

OCDE (1976), Méthode type proposée pour les enquêtes sur la recherche et le développement expérimental : "Manuel de Frascati ", Série " Mesure des activités scientifiques et techniques ", Paris.

OCDE (1970), " Méthode type proposée pour les enquêtes sur la recherche et le développement expérimental ", DAS/SPR/70.40, Direction des affaires scientifiques, Paris.

OCDE (1968), Tableaux statistiques et notes explicatives ("Année statistique internationale sur la recherche et le développement : une étude sur les ressources consacrées à la R-D dans les pays Membres de l'OCDE en 1963-64 »), vol. 2, Paris.

OCDE (1963), " Méthode type proposée pour les enquêtes sur la recherche et le développement expérimental ", Direction des affaires scientifiques, DAS/PD/62.47, Paris. 


\section{ANNEXE 2}

\section{Glossaire des termes}

Abattement fiscal. Les abattements, les exonérations et les déductions de nature fiscale sont retranchés de l'assiette fiscale avant le calcul de la créance fiscale, ce qui réduit le montant imposable avant l'établissement du montant de l'impôt dû. Voir aussi exonérations fiscales.

Une activité économique ou branche d'activité est un regroupement d'établissements engagés dans des activités identiques ou similaires. La Classification internationale type, par industrie, de toutes les branches d'activité économique (CITI) est la classification de référence des activités économiques. Voir aussi Classification internationale type, par industrie, de toutes les branches d'activité économique (CITI).

À des fins statistiques, les activités scientifiques et technologiques (AST) peuvent être définies comme étant l'ensemble des activités systématiques qui sont étroitement liées à la production, à la promotion, à la diffusion et à l'application des connaissances scientifiques et techniques dans tous les domaines de la science et de la technologie, c'est-à-dire les sciences naturelles, l'ingénierie et la technologie, les sciences médicales et agricoles ainsi que les sciences sociales et humaines. Les activités qui devraient être couvertes dans la pratique statistique peuvent être réparties en trois grands groupes : recherche et développement expérimental ; enseignement et formation $\mathrm{S}-\mathrm{T}$, généralement $\mathrm{du}$ troisième degré ; et services scientifiques et technologiques.

L'administration centrale (ou fédérale) se compose en général d'un noyau central de départements ou de ministères qui constituent une seule unité institutionnelle - couverte par les comptes budgétaires principaux et souvent désignée par l'expression " administration publique nationale " ou encore "État " - et, dans beaucoup de pays, elle comprend d'autres unités institutionnelles. Bien qu'ils puissent être responsables d'importantes dépenses de R-D (intra-muros et extra-muros) au titre du budget général de l'État, les départements ne constituent généralement pas des unités institutionnelles distinctes habilitées à détenir des actifs, contracter des engagements, effectuer des opérations, etc., indépendamment de l'administration centrale prise dans 
son ensemble. En règle générale, leurs recettes et dépenses sont réglementées et contrôlées par le ministère des Finances ou par un organe fonctionnel équivalent au moyen d'un budget général approuvé par le parlement.

Le sous-secteur des administrations locales (ou municipales) comprend les administrations locales qui sont des unités institutionnelles distinctes, ainsi que les organisations et institutions sans but lucratif non marchandes contrôlées par les administrations locales. En principe, les administrations locales sont des unités institutionnelles dont le pouvoir fiscal, législatif et exécutif s'étend sur les plus petits des territoires géographiques distingués à des fins administratives et politiques. Leur pouvoir est généralement d'une étendue beaucoup plus limitée que celui de l'administration centrale ou des administrations d'États fédérés.

Les administrations publiques sont des types particuliers d'entités juridiques, instituées par décision politique, qui exercent un pouvoir législatif, judiciaire ou exécutif sur d'autres unités institutionnelles dans un espace donné. Voir Système de comptabilité nationale. Elles présentent un intérêt particulier pour l'analyse des budgets de la R-D et des incitations fiscales connexes.

Le sous-secteur des administrations d’États fédérés (ou régionales) comprend les administrations d'États fédérés ou régionales qui sont des unités institutionnelles distinctes, ainsi que les organisations et institutions sans but lucratif non marchandes contrôlées par les administrations régionales (d’États fédérés). Ce sous-secteur exerce certaines fonctions d'administration à un niveau inférieur à celui de l'administration centrale/fédérale et supérieur à celui des unités institutionnelles d'administration qui se situent au niveau local. Il réunit les unités institutionnelles dont le pouvoir fiscal, législatif et exécutif ne s'étend qu'aux "États " qui peuvent composer le pays. Ces "États " peuvent être désignés de différentes manières selon les pays, par exemple, "régions » ou "provinces".

Allégement fiscal au titre des dépenses de R-D. Ce concept renvoie aux mesures d'allégement fiscal applicables aux contribuables qui exécutent et/ou financent des activités de R-D, par comparaison avec une structure fiscale courante ou de référence. Il est exprimé par la mesure statistique du coût de ces dispositions (indicateur de l'allégement fiscal au titre des dépenses de R-D) qui est proposée dans le présent manuel.

Une autorité supranationale est une organisation internationale dotée du pouvoir de lever des impôts et autres prélèvements obligatoires sur le territoire des pays membres de ladite organisation. Bien qu'assumant une partie des fonctions des administrations publiques dans chaque pays membre, elles sont toujours considérées comme des unités institutionnelles non résidentes.

Les autres dépenses courantes incluent les frais d'achat de matériaux, fournitures, équipements et services engagés à l'appui des activités de R-D menées par l'unité statistique au cours de l'année de référence qui n'entrent pas dans les dépenses en capital. Entre autres exemples figurent les dépenses d'eau 
et de combustible (gaz et électricité inclus); les achats d'ouvrages, de revues et de documents de référence, les abonnements de bibliothèques, les cotisations aux sociétés scientifiques, etc.; les coûts imputés ou réels des prototypes ou modèles de petites dimensions fabriqués à l'extérieur de l'unité statistique ; et les frais d'équipement des laboratoires (par exemple, produits chimiques, animaux, etc.). Entrent également dans les dépenses courantes les redevances et licences d'exploitation des brevets et autres droits de propriété intellectuelle, la location des biens d'équipement (machines et outillage, etc.) et des bâtiments utilisés dans l'exécution de la R-D par l'unité statistique au cours de l'année de référence.

Les autres produits de la propriété intellectuelle (inclus dans les dépenses en capital consacrées à la R-D) incluent les brevets achetés, les licences d'exploitation à long terme et autres actifs incorporels destinés à la R-D et utilisés pendant plus d'une année. N'en font pas partie les éléments incorporels susceptibles d'être déclarés dans les comptes financiers internes d'une unité, tels que les fonds de commerce et autres actifs commerciaux.

Dans le présent manuel, " bénévoles " désigne des travailleurs non rémunérés qui apportent à l'unité statistique une contribution en matière de $\mathrm{R}-\mathrm{D}$ et font partie du personnel externe de R-D.

Un campus satellite à l'étranger est un établissement d'enseignement supérieur qui est détenu, du moins en partie, par un établissement local d'enseignement supérieur (c'est-à-dire résidant sur le territoire du pays déclarant) mais est situé dans le reste du monde (résidant en dehors du pays déclarant). Son activité est régie par l'établissement local. Il consacre au moins une partie de ses activités à l'enseignement direct et donne accès à un programme universitaire complet sanctionné par un diplôme de l'établissement local.

Le chaînage consiste à raccorder deux séries chronologiques se recoupant sur une période. Pour cela, l'une d'elles est rétropolée de sorte que ses valeurs soient identiques à celles de l'autre pour la même période, le but étant de les combiner au sein d'une seule série chronologique. Des méthodes plus complexes peuvent être utilisées pour chaîner des séries chronologiques se recoupant sur plusieurs périodes.

Les chercheurs sont les spécialistes travaillant à la conception ou à la création de nouveaux savoirs. Ils mènent des travaux de recherche en vue d'améliorer ou de mettre au point des concepts, théories, modèles, techniques, instruments, logiciels ou modes opératoires.

La classification des domaines de recherche et de développement de l'OCDE, établie dans le cadre de l'élaboration du Manuel de Frascati, sert à classer les unités et ressources de R-D par champ d'étude, c'est-à-dire par grand domaine de connaissance, en fonction principalement du contenu de l'activité de R-D considérée.

La Classification des fonctions des administrations publiques (COFOG) est une classification générique des fonctions, ou objectifs socio-économiques, que les administrations publiques visent à remplir en engageant différentes sortes 
de dépenses. La COFOG fournit une nomenclature des entités et des dépenses financières des administrations publiques par fonction d'intérêt général. Les intitulés du premier niveau présentent de nombreuses similitudes avec les nomenclatures des objectifs socio-économiques utilisés en R-D. Dans le présent manuel, il est déconseillé aux institutions gouvernementales d'employer cette classification dans le contexte des statistiques de la R-D car les catégories qui la composent ne se prêtent pas à une description optimale des dépenses de R-D. Voir aussi objectifs socio-économiques.

La Classification internationale type de l'éducation (CITE) est la nomenclature de référence qui permet d'organiser les programmes d'enseignement et certifications correspondantes par niveau d'éducation et domaine d'études. La CITE offre un cadre pour classer les activités d'enseignement telles que définies dans les programmes et les certifications en résultant dans des catégories internationalement reconnues. Ses concepts et définitions de base sont par conséquent applicables dans le monde entier et couvrent tout l'éventail des systèmes éducatifs. La CITE classe les programmes d'enseignement en fonction de leur contenu, sur la base de deux grandes variables de classification : le niveau d'éducation et le domaine d'études. La CITE 2011 présente une nomenclature des niveaux d'éducation atteints sur la base de certifications éducatives reconnues.

La Classification internationale type des professions (CITP) sert à classer les emplois. Aux fins de la CITP, un emploi se définit comme un ensemble de tâches et de fonctions qui sont ou devraient être accomplies par une personne, y compris pour un employeur ou dans le cadre du travail indépendant. Une profession est définie comme un ensemble d'emplois dont les principales tâches et fonctions se caractérisent par un degré élevé de similarité. Une personne peut être assimilée à une profession en fonction de son emploi principal du moment, ou d'un deuxième emploi, ou encore d'un emploi exercé auparavant. Les emplois sont classés par profession en fonction du type de travail qui est ou devrait être effectué. Les critères de base utilisés pour définir le système des grands groupes, sous-groupes principaux, sous-groupes et groupes de base sont le « niveau de compétences » et la « spécialisation des compétences » requis pour bien s'acquitter des tâches et fonctions inhérentes aux différentes professions.

La Classification internationale type, par industrie, de toutes les branches d'activité économique (CITI) offre une nomenclature cohérente et homogène de l'activité économique qui s'appuie sur un ensemble concerté sur le plan international de concepts, définitions, principes et règles de classification. Elle fournit un cadre complet pour collecter et présenter les données économiques sous une forme adaptée aux besoins de l'analyse économique, de la prise de décisions et de l'élaboration des politiques. Par sa structure, cette classification permet d'organiser des informations détaillées sur l'état d'une économie selon les principes et conceptions économiques. D'une manière générale, la CITI couvre les activités de production, c'est-à-dire les activités économiques relevant de la production telle que définie dans le Système de comptabilité nationale. Quelques 
exceptions ont été faites afin de permettre la classification des activités qui n'entrent pas dans le domaine de la production mais sont importantes pour divers autres types de statistiques. Ces activités économiques sont divisées en quatre catégories hiérarchisées et mutuellement exclusives, ce qui permet de collecter, de présenter et d'analyser les données à des niveaux détaillés de l'économie, selon des modalités normalisées assurant la comparabilité internationale. Voir aussi activité économique.

Les coefficients de R-D permettent de calculer ou d'estimer la part du personnel et des dépenses liés à la R-D. Dans le secteur de l'enseignement supérieur en particulier, ils servent à répartir l'ensemble des ressources entre les activités de recherche, d'enseignement et autres (notamment administratives). Ils peuvent entrer dans le calcul du total ou de parties des dépenses, comme les fonds généraux des universités d'origine publique (FGU) ou des dépenses de personnel uniquement.

Une coentreprise implique l'établissement d'une société, d'un partenariat ou d'une autre unité institutionnelle dans laquelle chaque partie exerce légalement un contrôle conjoint sur les activités de l'unité. Elle opère de la même façon que les autres unités, à cette exception près qu'un accord juridique entre les parties établit un contrôle conjoint sur l'unité. En tant qu'unité institutionnelle, la coentreprise peut passer des contrats en son propre nom et lever des fonds pour ses propres besoins. Dès lors que des coentreprises de R-D constituent des unités à part entière, elles devraient également être classées en fonction des unités qu'elles servent au premier chef, compte tenu, dans la mesure du possible, de la pratique établie en vertu du Système de comptabilité nationale.

En comptabilité de caisse, une opération est saisie lorsque le paiement est reçu ou effectué. Voir aussi comptabilité d'exercice.

En comptabilité d'exercice, une opération est saisie à la date de l'activité (décision) à l'origine d'une augmentation ou diminution des ressources, indépendamment du moment où le règlement correspondant est perçu ou effectué. Voir aussi comptabilité de caisse.

Le contrôle public des ISBL est normalement déterminé au regard des cinq critères suivants :

1. Possibilité de nommer les responsables ou le conseil de direction.

2. Possibilité de prendre d'autres dispositions permettant à l'administration publique de déterminer des aspects importants de la politique générale ou du programme de l'ISBL, comme relever de ses fonctions du personnel clé ou opposer un veto à une nomination proposée, imposer que les budgets ou opérations financières soient préalablement soumis à son autorisation, ou encore empêcher l'ISBL de modifier ses statuts ou de se dissoudre.

3. Existence d'accords contractuels, autorisant l'administration publique à imposer les conditions susmentionnées. 
4. Degré et type de financement assuré par les pouvoirs publics, qui peuvent empêcher l'ISBL de déterminer sa politique ou son programme.

5. Existence d'une exposition aux risques, si une administration publique s'expose ouvertement à la totalité, ou à une grande partie, des risques financiers associés aux activités d'une ISBL.

Les coûts de main-d'œuvre, ou les rémunérations du personnel, comprennent les salaires et traitements annuels ainsi que l'ensemble des coûts correspondant aux avantages annexes, tels que primes, options sur titres, congés payés et cotisations aux fonds de pension. Aux fins du présent manuel, les coûts de main-d'œuvre incluent également les autres charges et cotisations sociales.

Un crédit d'impôt est un montant directement retranché de la créance fiscale due par le ménage ou la société bénéficiaire après le calcul de cette créance.

Crédit d'impôt remboursable. Un crédit d'impôt est remboursable lorsque l'excédent de la créance fiscale est restitué au contribuable. Voir aussi crédit d'impôt.

Les crédits budgétaires approuvés sont définis par des projets de loi portant création, maintien ou modification de programmes publics et s'accompagnent généralement de plafonds de dépenses ou d'orientations concrètes sur les crédits à ouvrir par la suite. Cependant, il n'existe pas nécessairement de corrélation entre le montant des crédits approuvés et celui des crédits ouverts. Voir aussi crédits budgétaires ouverts.

Les crédits budgétaires ouverts sont définis par des projets de loi portant affectation ou provision de fonds à l'intention de départements, organismes, programmes et/ou fonctions spécifiques des administrations publiques, qui confèrent le pouvoir de prendre des engagements appelés à déboucher sur des dépenses. Voir aussi engagements et dépenses.

Les crédits budgétaires publics de R-D (CBPRD) comprennent l'ensemble des crédits budgétaires financés par des recettes publiques inscrites au budget, notamment des recettes fiscales. N'en font partie les crédits affectés par des entités publiques extrabudgétaires que dans la mesure où ils ont été alloués dans le cadre de la procédure budgétaire. De même, le financement de la R-D par les entreprises publiques est exclu du champ des statistiques de CBPRD, car il repose sur des fonds levés sur le marché, en dehors de la procédure budgétaire. Il ne relève des CBPRD que dans le cas exceptionnel où il s'agit de provisions de fonds du budget de l'État utilisés ou distribués en faveur de la R-D par des entreprises publiques. Voir aussi Nomenclature des objectifs socio-économiques (OSE).

Les dépenses correspondent aux montants des chèques émis et paiements au comptant réalisés au cours d'une période donnée, quelle que soit la date d'ouverture ou d'engagement des crédits (dans le cas des crédits publics).

Les dépenses courantes de R-D comprennent les coûts de main-d'œuvre et autres coûts courants (personnel externe de R-D inclus) imputables à la R-D. 
Les services et articles (équipements inclus) utilisés et consommés au cours d'une année en font partie ainsi que les redevances ou loyers annuels versés pour l'utilisation d'actifs fixes.

Les dépenses intérieures brutes de R-D (DIRD) correspondent au total des dépenses intra-muros de la R-D exécutée sur le territoire national pendant une période de référence donnée.

Les dépenses intérieures de R-D de l'enseignement supérieur (DIRDES) correspondent à la composante des DIRD engagées par des unités du secteur de l'enseignement supérieur. Il s'agit des dépenses de R-D intra-muros engagées au sein du secteur de l'enseignement supérieur au cours d'une période de référence donnée. Voir aussi Dépenses intérieures brutes de R-D (DIRD) et dépenses de R-D intra-muros.

Les dépenses intérieures de R-D de l'État (DIRDET) correspondent à la composante des DIRD engagées par des unités du secteur de l'État. Il s'agit des dépenses de R-D intra-muros engagées au sein du secteur de l'État au cours d'une période de référence donnée. Voir aussi Dépenses intérieures brutes de R-D (DIRD) et dépenses de R-D intra-muros.

Les dépenses intérieures de R-D des entreprises (DIRDE) correspondent à la composante des DIRD engagées par les unités du secteur des entreprises. Il s'agit des dépenses de R-D intra-muros engagées au sein du secteur des entreprises au cours d'une période de référence donnée. Voir aussi dépenses intérieures brutes de R-D (DIRD) et dépenses de R-D intra-muros.

Les dépenses intérieures de R-D des institutions sans but lucratif (DIRDISBL) correspondent à la composante des DIRD engagées par les unités du secteur privé sans but lucratif. Il s'agit des dépenses de R-D intra-muros engagées au sein du secteur privé sans but lucratif au cours d'une période de référence donnée. Voir aussi dépenses intérieures brutes de R-D (DIRD) et dépenses de R-D intra-muros.

Les dépenses de R-D intra-muros couvrent l'ensemble des dépenses courantes et des dépenses brutes de capital fixe afférentes à la R-D exécutée au sein d'une unité statistique au cours d'une période de référence donnée, quelle que soit la source des financements. Elles correspondent à la R-D exécutée au sein d'une unité statistique. Le total des dépenses de R-D intra-muros des unités qui composent un secteur de l'économie correspond à la R-D exécutée au sein dudit secteur ; tandis que la somme des dépenses de R-D intra-muros de la totalité des secteurs correspond à la R-D exécutée au sein de l'économie dans son ensemble (DIRD).

Les dépenses en capital consacrées à la R-D correspondent à la somme annuelle brute versée pour l'acquisition d'actifs fixes qui sont utilisés de façon répétée ou continue aux fins de l'exécution de travaux de R-D pendant plus d'une année. Ces dépenses devraient être déclarées dans leur intégralité au titre de la période au cours de laquelle elles ont eu lieu, qu'il s'agisse d'actifs acquis 
ou mis au point en interne, et ne devraient pas être enregistrées comme élément d'amortissement. Les types d'actifs exploités en R-D pour lesquels il conviendrait en premier lieu de collecter des données sur les dépenses en capital consacrées à la R-D sont les suivants :

- terrains et constructions

- machines et équipements

- logiciels immobilisés

- autres produits de la propriété intellectuelle.

Les dépenses fiscales sont les dispositions du droit, de la réglementation ou de la pratique fiscale qui minorent ou reportent l'impôt dû par un groupe de contribuables par rapport à une organisation fiscale de référence ou « normale ". Les dépenses fiscales sont parfois assimilées aux allégements fiscaux, subventions fiscales et aides fiscales. Aux fins du présent manuel, l'expression " dépenses fiscales » désigne le coût des dispositions d'allégement fiscal supporté par l'État.

Les dépenses nationales brutes de R-D (DNRD) comprennent la totalité des dépenses de R-D financées par les unités institutionnelles d'un pays, indépendamment du lieu d'exécution de la R-D. Est ainsi incluse la R-D exécutée " dans le reste du monde " qui est financée par des unités institutionnelles nationales ou résidentes, et exclue la R-D exécutée sur le territoire national qui est financée par des unités institutionnelles implantées à l'étranger (c'est-à-dire appartenant au "reste du monde »). Le montant des DNRD est égal à la somme des dépenses intra-muros de chaque secteur d'exécution qui sont financées par des sources implantées sur le territoire national et du montant de la R-D exécutée dans « le reste du monde » qui est financée par des sources implantées sur le territoire national.

Le développement expérimental consiste en des travaux systématiques - fondés sur les connaissances tirées de la recherche et l'expérience pratique et produisant de nouvelles connaissances techniques - visant à déboucher sur de nouveaux produits ou procédés ou à améliorer les produits ou procédés existants.

L'effectif de R-D en nombre de personnes physiques correspond au nombre total des individus qui participent à la R-D intra-muros, au niveau d'une unité statistique ou à un niveau agrégé, au cours d'une période de référence donnée (habituellement une année civile).

Les employés intérimaires sont inclus dans le personnel externe de R-D. Ils sont mis à la disposition d'entreprises clientes en échange d'honoraires. Les employés intérimaires sont comptabilisés dans la masse salariale de l'agence d'emploi (ou de placement) temporaire et non dans celle de l'unité statistique qui verse les honoraires. Cette mise à disposition de ressources humaines s'effectue généralement pour une courte durée. 
Les engagements représentent le volume monétaire des commandes passées, des marchés attribués, des prestations reçues et des opérations similaires réalisées au cours d'une période donnée, quelle que soit la date d'affectation des fonds et d'échéance du règlement.

Une enquête sur l'emploi du temps est une enquête statistique qui a pour objet de recueillir des données sur la manière dont les individus utilisent leur temps. Le Manuel de Frascati contient des lignes directrices utiles pour en extraire les informations qui permettent d'estimer la composante R-D des équivalents temps plein (ETP) et des dépenses du secteur de l'enseignement supérieur lorsqu'il est impossible de calculer les coefficients nécessaires à partir des données administratives ou autres données d'enquête.

L'enseignement supérieur inclut ce qui est communément appelé l'enseignement académique mais aussi l'enseignement professionnel. Il comprend les niveaux 5, 6, 7 et 8 de la CITE, respectivement nommés " enseignement supérieur de cycle court ", " niveau licence ou équivalent ", " niveau master ou équivalent » et « niveau doctorat ou équivalent ».

Une entreprise est une unité institutionnelle - pas nécessairement au sens du secteur des entreprises tel que défini dans le Manuel de Frascati - qui est considérée en tant que producteur de biens et de services (voir Système de comptabilité nationale). Le terme « entreprise " peut se référer à une société, une quasi-société, une institution sans but lucratif ou une entreprise non constituée en société. Il s'agit d'un agent économique qui possède une autonomie décisionnelle en matière de finance et d'investissement, ainsi que le pouvoir et la responsabilité d'allouer des ressources à la production de biens et de services. L'entreprise peut mener une ou plusieurs activités en un ou plusieurs lieux. Elle peut être constituée d'une seule unité légale.

Une entreprise multinationale (EMN) désigne une société-mère résidente et les sociétés implantées à l'étranger dont elle détient la majorité des parts, appelées " filiales implantées à l'étranger ". Une EMN désigne également un groupe d'entreprises mondial. Voir aussi société-mère, participation majoritaire et filiales implantées à l'étranger.

Les entreprises sœurs implantées à l'étranger sont identifiées du point de vue d'une filiale sous contrôle étranger résidant dans l'économie déclarante. Il s'agit des entreprises implantées en dehors du pays déclarant qui se trouvent sous le contrôle ou l'influence de la même société-mère étrangère que la filiale sous contrôle étranger. Aux fins du Manuel de Frascati, les entreprises sœurs implantées à l'étranger présentent un intérêt de par leur qualité de source ou de destination du financement des travaux de R-D auxquels les filiales sous contrôle étranger sont associées.

L'équivalent temps plein (ETP) de personnel de R-D est défini par le nombre d'heures de travail réellement consacrées à la $R-D$ au cours d'une période de référence donnée (habituellement une année civile) rapporté au nombre total 
d'heures qu'une personne ou un groupe de personnes est censé travailler au cours de la même période.

L'estimation est une procédure qui consiste à inférer la valeur numérique des grandeurs inconnues à partir d'un ensemble de données incomplètes, comme, par exemple, un échantillon.

Un établissement est une entreprise, ou une partie d'entreprise, située en un lieu unique, dans laquelle une seule activité de production est exercée ou dans laquelle la majeure partie de la valeur ajoutée provient de l'activité de production principale. Les établissements sont parfois appelés " unités d'activité économique au niveau local » (UAE locales). Voir aussi entreprise.

Les étudiants en doctorat (doctorants) suivent des " programmes d'enseignement supérieur qui conduisent à l'obtention d'un titre de chercheur hautement qualifié [et qui] sont donc consacrés à des études approfondies et à des travaux de recherche originaux et ne sont pas fondés uniquement sur des cours. " En règle générale, ces étudiants sont tenus de soutenir une thèse d'une qualité suffisante pour en permettre la publication, cette thèse devant être le produit d'un travail original de recherche et représenter une contribution appréciable à la connaissance. Voir aussi Classification internationale type de l'éducation.

Les étudiants en master peuvent, dans certains cas, compter au nombre des chercheurs; en particulier s'ils participent à un programme de master du niveau 7 de la CITE « menant à l'obtention de certifications de chercheur conçues explicitement pour former les participants à la conduite de projets de recherche originaux, mais dont le niveau est inférieur à celui d'un doctorat ». Il importe néanmoins que seuls les étudiants en master rémunérés pour leur activité de R-D soient comptabilisés avec le personnel de R-D.

Les exécutants de la R-D sont les unités statistiques qui mènent (exécutent) des activités de R-D dans chacun des secteurs considérés dans le présent manuel: entreprises, État, enseignement supérieur et institutions sans but lucratif. Voir aussi unité statistique.

Exonérations fiscales. Les exonérations sont les sommes exclues de la base d'imposition.

Les filiales implantées à l'étranger (FIE) sont des sociétés affiliées situées à l'étranger et détenues à plus de 50 \% par leur société-mère, résidente du pays déclarant. Voir aussi participation majoritaire et société-mère d'une entreprise multinationale (EMN).

Les filiales sous contrôle étranger (FCE) constituent le groupe d'entreprises implantées dans le pays déclarant dont la majorité des capitaux est détenue par des EMN étrangères (qui sont donc des sociétés-mères étrangères). Leurs activités découlent de l'IDE entrant, tandis que les activités des filiales implantées à l'étranger se rapportent à l'IDE sortant. Voir aussi participation majoritaire, entreprise multinationale, société-mère et filiale sous contrôle étranger. 
Les financements externes de R-D correspondent à la somme des fonds consacrés à la R-D qui proviennent de sources extérieures à l'unité déclarante.

Les financements internes de R-D correspondent à la somme des fonds consacrés à la R-D qui proviennent directement des ressources internes d'une unité déclarante et que celle-ci emploie, à sa discrétion, à des activités de R-D. Ils n'incluent pas les financements que l'unité statistique considérée reçoit d'autres unités statistiques explicitement pour les activités de R-D exécutée en son sein.

Le personnel de R-D est classé par fonction de R-D : chercheur, technicien ou personnel de soutien.

Les fonds d'échange pour la R-D sont des fonds qu'une unité statistique fournit à une autre unité en échange de l'exécution d'activités de R-D et de l'obtention de résultats de R-D pertinents. L'unité qui finance les travaux encourt un risque de non-réalisation associé au caractère incertain du projet. Entre autres exemples figurent les achats de R-D (ventes du point de vue de l'exécutant), l'externalisation de travaux de R-D et les contributions fournies en vertu d'accords de collaboration en matière de R-D.

Les fonds de transfert pour la R-D sont des fonds qu'une unité statistique fournit à une autre unité afin que cette dernière exécute des travaux de R-D. L'unité qui fournit les ressources financières n'exige aucun bien ou service en contrepartie et ne jouit pas de droits importants sur les résultats des activités de R-D qu'elle finance. Elle peut imposer certaines conditions à l'exécutant, comme celles d'établir des rapports à intervalles réguliers, de mener les activités ou projets décrits dans l'accord conclu, voire de rendre publics les résultats des travaux de recherche. Entre autres exemples de fonds de transfert figurent le subventionnement, la remise de dettes, la philanthropie, le financement participatif, les transferts effectués à titre personnel, comme les dons, et les FGU (par convention pour faciliter les comparaisons internationales). Pour que l'opération soit qualifiée de « transfert de fonds destinés à la R-D », les fonds en question devraient être destinés à des activités de R-D par l'entité qui les fournit à l'origine. Cette forme de financement de la R-D est qualifiée de transfert dans la mesure où, en règle générale, l'exécutant de la R-D conserve la plupart des droits attachés aux résultats des travaux.

Les fonds généraux des universités (FGU) d'origine publique correspondent à la part des ressources financières destinées à la R-D qui sont issues de la dotation globale allouée aux universités, à l'appui de leurs activités de recherche et d'enseignement, par le ministère de l'éducation de l'administration centrale (fédérale) ou par les autorités régionales (d’États fédérés) ou locales (municipales) compétentes.

Un groupe d'entreprises est un ensemble d'entreprises contrôlées par une unité légale mère qui n'est contrôlée (directement ou indirectement) par aucune autre unité légale. Il peut compter plusieurs centres décisionnels, notamment pour la politique relative à la production, à la vente et aux bénéfices, ou peut centraliser certains aspects de la gestion financière et de la fiscalité. Il s'agit 
d'une entité économique capable de faire des choix, particulièrement en ce qui concerne les unités qui le composent. L'unité " groupe d'entreprises " est particulièrement utile pour les analyses financières et l'étude des stratégies d'entreprise. Elle est toutefois trop hétérogène et trop instable pour être adoptée en tant qu'unité centrale d'observation et d'analyse.

L'imputation est une procédure qui consiste à retenir une valeur en lieu et place de la valeur manquante ou inutilisable d'une variable donnée.

Les institutions sans but lucratif (ISBL) sont des entités juridiques ou sociales créées dans le but de produire des biens ou des services, dont le statut ne leur permet pas d'être une source de revenu, de profit ou d'autre forme de gain financier pour les unités qui les créent, les contrôlent ou les financent. Elles peuvent mener des activités de production marchande ou non marchande.

Les institutions sans but lucratif au service des ménages (ISBLSM) sont des ISBL non marchandes qui ne sont pas sous contrôle public. Elles fournissent des biens et des services aux ménages gratuitement ou à des prix économiquement non significatifs. La plupart de ces biens et services sont destinés à la consommation individuelle, mais il est possible que les ISBLSM fournissent des services collectifs.

L'investissement direct étranger (IDE) est motivé par la volonté d'une entreprise résidente d'une économie (société-mère ou " investisseur direct ") d'acquérir un intérêt durable dans une entreprise résidente d'une autre économie (société affiliée implantée à l'étranger ou " entreprise d'investissement direct »). Aux fins de l'établissement des statistiques officielles, un intérêt durable est réputé exister dès lors que $10 \%$ ou plus des actions ordinaires ou des droits de vote dans une entreprise constituée en société ou l'équivalent d'une entreprise non constituée en société sont détenus, directement ou indirectement. Le critère de $10 \%$ des droits de vote établit également l'existence d'une relation d'investissement direct entre une société affiliée et sa société-mère.

L'investisseur exerçant le contrôle ultime d'une filiale sous contrôle étranger (également appelé " unité institutionnelle investie du contrôle ultime ») se trouve à la tête d'un ensemble de sociétés ou de filiales qu'il contrôle sans lui-même être sous le contrôle d'une autre société quelle qu'elle soit. (Voir aussi filiale sous contrôle étranger et société-mère immédiate).

Les logiciels immobilisés sont les logiciels servant à l'exécution d'activités de R-D pendant plus d'une année. Il s'agit notamment des licences ou logiciels acquis pour une longue durée et séparément identifiables, y compris les descriptifs de programme et la documentation accompagnant les logiciels d'exploitation et d'application. Les coûts de production (par exemple, main-d'œuvre et matériaux) des logiciels mis au point en interne devraient être déclarés. Des logiciels peuvent être acquis auprès de vendeurs extérieurs à travers l'achat ferme des droits ou des licences d'exploitation. Les logiciels exploités ou acquis sous licence pour un an ou moins devraient être déclarés au titre des dépenses courantes. Voir aussi R-D logicielle. 
Les machines et équipements considérés dans la mesure de la R-D sont les machines et équipements immobilisés qui ont été acquis pour l'exécution d'activités de R-D. Aux fins du Système de comptabilité nationale, les dépenses afférentes devraient être ventilées de façon plus fine, et inclure notamment les postes « matériel d'information et de communication » et « matériels de transport ».

D'une manière générale, la mondialisation désigne l'intégration internationale des moyens de financement, de l'offre de facteurs, de la R-D, de la production et du commerce des biens et des services.

Une nomenclature des objectifs socio-économiques (OSE) est utilisée pour répartir les CBPRD. Les critères de classification devraient être l'objet du programme ou projet de $\mathrm{R}-\mathrm{D}$, c'est-à-dire son objectif premier. La répartition des budgets de R-D entre objectifs socio-économiques devrait se faire au niveau qui cadre le mieux avec le ou les objectifs du bailleur de fonds. La liste recommandée repose sur la nomenclature de l'Union européenne retenue par Eurostat pour l'analyse et la comparaison des budgets et programmes scientifiques (NABS), au niveau hiérarchique à un chiffre. Voir aussi crédits budgétaires publics de R-D (CBPRD).

Les opérations sont les échanges ou transferts volontaires de propriété économique (le propriétaire étant celui qui assume les risques et bénéficie des avantages) dans la fourniture de biens ou de services. Les flux de biens, de services et de revenus sont consignés dans le compte des opérations courantes de la balance des paiements. Voir Système de comptabilité nationale.

Les organisations internationales comptent parmi leurs membres soit des États, soit d'autres organisations internationales dont les membres sont des États. Elles sont instituées par la conclusion, entre leurs membres, d'accords politiques formels ayant le statut de traité international ; leur existence est reconnue par la loi dans les pays qui en sont membres et elles ne sont pas assujetties aux lois ou aux règlements du ou des pays dans lesquels elles sont présentes. Ainsi, aucun État ne peut les contraindre à fournir des renseignements statistiques sur leurs résultats en matière de R-D ou leurs activités de financement. Dans le Système de comptabilité nationale et les statistiques de $\mathrm{R}-\mathrm{D}$, les organisations internationales sont traitées comme des unités résidant à l'étranger (reste du monde), indépendamment de leur lieu d'implantation ou d'activité.

Les paradonnées d'enquête sont les informations relatives au processus d'enquête. Entre autres exemples de paradonnées figurent la présence/absence de l'unité considérée dans l'échantillon; l'historique des taux de réponse ; et le mode de collecte des données. Recourir aux paradonnées à l'issue d'une campagne d'enquête peut aider à améliorer l'outil pour l'avenir.

La participation majoritaire ou le contrôle renvoie au fait de détenir plus de $50 \%$ des actions ordinaires ou des droits de vote dans une entreprise constituée en société ou l'équivalent d'une entreprise non constituée en société. Entre autres exemples de sociétés affiliées à participation majoritaire ou sous 
contrôle figurent les filiales (entreprises constituées en société) et les succursales (entreprises non constituées en société).

Les personnes occupées comprennent les salariés, les travailleurs familiaux non rémunérés et les propriétaires exploitants (c'est-à-dire les propriétaires individuels ou associés). En sont exclus les associés passifs ou inactifs dont l'activité principale est menée en dehors de l'unité statistique. Voir aussi personnel interne de R-D.

Le personnel de R-D attaché à une unité statistique est composé des personnes qui participent directement aux activités de R-D (salariés de l'unité statistique ou contributeurs externes faisant partie intégrante des projets de R-D de l'unité statistique) et de celles qui fournissent des services directement liés aux activités de R-D (responsables de R-D, gestionnaires, techniciens et personnel de soutien). Voir aussi personnel interne de R-D et personnel externe de R-D.

Le personnel de soutien comprend les travailleurs, qualifiés et non qualifiés, ainsi que le personnel administratif et les employés de bureau participant ou directement associés à des projets de R-D.

Le personnel externe de R-D (ou les contributeurs) regroupe les travailleurs indépendants et les salariés qui font partie intégrante des projets de R-D d'une unité statistique sans être officiellement employés par ladite unité statistique exécutante.

Le personnel interne de R-D regroupe les personnes occupées par l'unité statistique qui participent aux activités de R-D intra-muros de celle-ci. Voir personnes occupées.

Les prix d'acquisition correspondent aux montants payés par les acquéreurs, à l'exclusion de la partie déductible de la taxe sur la valeur ajoutée (TVA) et des impôts similaires. Ils représentent les coûts réellement supportés par les utilisateurs. Par conséquent, le montant estimé des dépenses consacrées aux biens et services de R-D, qu'il s'agisse de dépenses courantes ou de dépenses en capital, est le prix total réglé par l'unité déclarante. Il tient compte, le cas échéant, des taxes et subventions dont les produits acquis font l'objet et qui ont pour effet, respectivement, d'en majorer ou réduire le prix.

Les prix économiquement significatifs sont les prix qui ont un effet significatif sur les quantités que les producteurs sont disposés à offrir et sur les quantités que les acquéreurs souhaitent acheter. En règle générale, ces prix sont constatés (a) lorsque le producteur est incité à ajuster l'offre de manière à réaliser un bénéfice à long terme, ou au moins à couvrir son capital et ses autres coûts, et (b) lorsque les consommateurs sont libres d'acheter ou non et de faire leur choix sur la base des prix facturés. Voir aussi Système de comptabilité nationale.

Un “ professeur émérite " est un professeur à la retraite qui continue de mener des travaux de recherche et de collaborer aux activités académiques de son ancien employeur, habituellement une université, sans recevoir la moindre rémunération (mais éventuellement une forme de soutien logistique). 
Un prototype est un modèle original fabriqué pour présenter toutes les caractéristiques techniques et les performances d'un nouveau produit.

Une quasi-société est soit une entreprise non constituée en société qui est détenue par une unité institutionnelle résidente, possède des informations suffisantes pour établir un ensemble complet de comptes, est exploitée comme si elle était une société distincte et dont la relation de fait avec son propriétaire est celle d'une société avec ses actionnaires, soit une entreprise non constituée en société qui est détenue par une unité institutionnelle non résidente mais est considérée comme une unité institutionnelle résidente du fait qu'elle mène un important volume d'activités productives sur le territoire économique sur une période longue ou indéfinie.

R-D logicielle. Un projet de développement logiciel entre dans la catégorie des activités de R-D si son exécution dépend d'un progrès scientifique et/ ou technologique et qu'il vise à dissiper une incertitude scientifique et/ou technologique de manière systématique. Outre les logiciels qui entrent dans un projet général de R-D, les activités de R-D dans lesquelles un logiciel constitue un produit final ou fait partie intégrante d'un produit final peuvent elles aussi relever de la R-D dès lors qu'elles satisfont aux critères applicables. Le développement logiciel fait partie intégrante de nombreux projets dénués de tout élément de R-D. En revanche, le volet " développement logiciel " de ces projets peut être classé comme activité de R-D s'il en résulte une avancée dans le domaine des logiciels. La mise à jour, l'extension ou la modification d'un programme ou système existant peut être classée dans la R-D dès lors qu'elle représente un progrès scientifique et/ou technologique à l'origine d'un enrichissement des connaissances. Les activités logicielles de nature courante ne doivent pas être considérées comme relevant de la R-D. Voir aussi logiciels immobilisés.

Aux fins du présent manuel, la R-D extra-muros s'entend de toute activité de R-D exécutée en dehors de l'unité statistique qui est l'objet des informations déclarées. Les "ressources financières destinées à la R-D extra-muros » doivent uniquement inclure les fonds d'origine interne (ne provenant pas de sources extérieures) que l'unité statistique considérée fournit à une unité extérieure aux fins de l'exécution de travaux de R-D, que des résultats de R-D soient attendus en contrepartie (échange ou achat) ou non (transfert ou subvention). Il convient également de noter qu'elles incluent généralement les sommes destinées à couvrir d'autres coûts que ceux de R-D (coûts d'amortissement, profit dégagé par l'exécutant, frais d'exécution, etc.).

La recherche appliquée consiste en des travaux de recherche originaux entrepris en vue d'acquérir de nouvelles connaissances et dirigés principalement vers un but ou un objectif pratique déterminé.

La recherche et le développement expérimental (R-D) englobent les activités créatives et systématiques entreprises en vue d'accroître la somme des 
connaissances - y compris la connaissance de l'humanité, de la culture et de la société - et de concevoir de nouvelles applications à partir des connaissances disponibles.

La recherche fondamentale consiste en des travaux de recherche expérimentaux ou théoriques entrepris en vue d'acquérir de nouvelles connaissances sur les fondements des phénomènes et des faits observables, sans envisager une application ou une utilisation particulière.

La recherche fondamentale orientée consiste en des travaux de recherche fondamentale exécutée dans l'espoir qu'elle aboutira à l'établissement d'une vaste base de connaissances permettant de résoudre les problèmes ou de concrétiser les opportunités qui se présentent actuellement ou qui sont susceptibles de se présenter ultérieurement.

La recherche fondamentale pure est la recherche fondamentale exécutée au service de la connaissance sans intention d'en tirer des avantages économiques ou sociaux à long terme ou sans volonté d'en appliquer les résultats à des problèmes concrets ou de transmettre ces résultats aux secteurs concernés.

La procédure de report (allégement fiscal) consiste à appliquer les déductions ou crédits d'impôt constatés mais non utilisés pour un exercice donné au paiement de l'impôt dû au titre d'un exercice suivant (report en avant) ou précédent (report en arrière).

La résidence d'une unité institutionnelle est le territoire économique avec lequel elle possède la relation la plus étroite, autrement dit, son centre d'intérêt économique prépondérant. Le territoire économique est composé des zones terrestres, de l'espace aérien et des eaux territoriales et intègre les compétences concernant les droits de pêche et les droits sur les combustibles ou les minéraux. Dans le cas d'un territoire maritime, le territoire économique inclut les îles appartenant à ce territoire. Le territoire économique comporte aussi les enclaves territoriales situées dans le reste du monde. Ces dernières sont des zones terrestres clairement délimitées (ambassades, consulats, bases militaires et stations scientifiques), qui se trouvent sur d'autres territoires et que l'État qui en est propriétaire ou locataire utilise à des fins diplomatiques, militaires, scientifiques ou autres avec l'accord officiel des autorités des territoires sur lesquels ces zones terrestres sont géographiquement situées.

Le reste du monde comprend :

- l'ensemble des institutions et personnes qui ne disposent pas, sur le territoire économique considéré, de site, de lieu de production ou de locaux dans lesquels ou à partir desquels elles mènent ou entendent mener des activités économiques et des opérations économiques de grande envergure, pendant une période indéterminée ou déterminée mais longue ;

- l'ensemble des organisations internationales et entités supranationales, définies ci-après, y compris les installations et activités qu'elles possèdent à l'intérieur du pays considéré. 
Les salariés sont toutes les personnes qui travaillent dans ou pour une unité statistique, en vertu d'un contrat de travail conclu avec celle-ci, et qui reçoivent une rémunération en espèces ou en nature à intervalles réguliers. Sont inclus les salariés qui exécutent une activité auxiliaire à l'activité principale de l'unité, de même que les groupes de personnes suivants : les personnes en congé de courte durée (congé de maladie, congé pour accident ou congé annuel), les personnes en congés payés spéciaux (congé d'études ou de formation, congé de maternité ou congé parental), les grévistes, les travailleurs à temps partiel, les travailleurs saisonniers et les apprentis lorsqu'ils sont comptabilisés dans la masse salariale. Font également partie des salariés les personnes qui sont rémunérées par l'unité statistique, se trouvent sous son contrôle mais travaillent à l'extérieur (travailleurs à domicile), par exemple, les agents technico-commerciaux ou encore le personnel d'entretien et de réparation.

Le secteur de l'enseignement supérieur comprend l'ensemble des universités, établissements d'enseignement post-secondaire et autres établissements proposant des programmes d'enseignement supérieur formel indépendamment de leur source de financement ou de leur statut juridique, ainsi que l'ensemble des instituts et centres de recherche, stations d'expérimentation et centres de soins dont les activités de R-D relèvent du contrôle direct d'établissements d'enseignement supérieur ou sont administrées par ceux-ci.

Le secteur de l'État se compose des groupes d'unités institutionnelles résidentes suivantes:

- toutes les unités d'administration centrale (fédérale), régionale (d’États fédérés) ou locale (municipale), y compris les administrations de sécurité sociale, à l'exception des unités fournissant des services d'enseignement supérieur ou répondant à la description des établissements d'enseignement supérieur donnée dans le présent manuel ;

- toutes les ISBL non marchandes contrôlées par des administrations publiques et n'appartenant pas au secteur de l'enseignement supérieur.

Le secteur des entreprises comprend :

- toutes les sociétés résidentes, y compris et non exclusivement les entreprises légalement constituées en société, indépendamment du lieu de résidence de leurs actionnaires. En font également partie tous les autres types de quasisociétés, c'est-à-dire les unités capables de dégager un profit ou une autre forme de gain financier pour leur(s) propriétaire(s), reconnues par la loi comme des entités juridiques distinctes de leur(s) propriétaire(s) et créées dans le but de mener des activités de production marchande à des prix économiquement significatifs.

- les succursales d'entreprises non résidentes non constituées en société et considérées comme résidentes du fait qu'elles mènent dans la durée des activités de production sur le territoire économique ; 
- toutes les ISBL résidentes qui sont des producteurs marchands de biens ou de services ou qui servent les entreprises.

- Ce secteur comprend des entreprises publiques et privées.

Le secteur privé sans but lucratif (PSBL) comprend :

- toutes les institutions sans but lucratif au service des ménages (ISBLSM), telles que définies dans le SCN 2008, à l'exception de celles relevant du secteur de l'enseignement supérieur ;

- à des fins d'exhaustivité, les ménages et les particuliers qui mènent ou non des activités marchandes, tel qu'expliqué dans le présent manuel.

Secteur public ou privé. Une unité institutionnelle appartient au secteur public ou privé selon qu'elle se trouve ou non sous le contrôle d'une administration publique. Dans tous les secteurs, les unités qualifiées de privées (ou publiques) peuvent être regroupées aux fins de l'établissement de statistiques correspondant à des besoins particuliers.

Les sociétés sont toutes les entités capables de dégager un profit ou une autre forme de gain financier pour leur(s) propriétaire(s), reconnues par la loi comme des entités juridiques distinctes des propriétaires, qui jouissent eux-mêmes d'une responsabilité limitée, et créées dans le but de s'engager dans une production marchande. Le terme couvre en outre les coopératives, les sociétés de personnes à responsabilité limitée et les quasi-sociétés. À des fins pratiques, il peut être étendu aux ménages ou individus officiellement engagés dans une production marchande dès lors que la délimitation de la responsabilité est difficile à établir. Dans l'ensemble, les sociétés correspondent aux unités qualifiées d'entreprises.

La société-mère d'une EMN est considérée comme un groupe d'entreprises dans le pays déclarant et se compose de toutes les unités résidentes du pays déclarant dont elle détient la majorité du capital. Voir aussi entreprise multinationale, groupe d'entreprises, résidence et participation majoritaire.

Dans le cas de l'investissement entrant, la société-mère immédiate d'une filiale sous contrôle étranger est le premier investisseur étranger implanté hors du pays déclarant qui exerce un contrôle sur la filiale étrangère. Voir aussi société-mère, filiale sous contrôle étranger et investisseur exerçant le contrôle ultime.

La source de financement de la R-D est l'unité qui finance l'exécution de la R-D. Elle peut être interne ou externe à l'unité déclarante. Dans les enquêtes et documents de présentation des données, les sources externes sont regroupées par grand secteur et sous-secteurs pertinents. De manière générale, il existe cinq grandes sources de financement de la R-D : les entreprises, l'État, l'enseignement supérieur, les institutions privées sans but lucratif et le reste du monde.

Le Système de comptabilité nationale (SCN) est l'ensemble normalisé et internationalement approuvé de recommandations sur la manière de produire 
des indicateurs de l'activité économique conformément à de strictes conventions de comptabilité basées sur des principes économiques.

Un impôt du type de la taxe sur la valeur ajoutée (TVA) est un impôt sur des biens ou des services que les entreprises collectent par étapes mais qui, en dernière analyse, est supporté intégralement par l'acheteur final. Conformément au SCN et à des fins de comparaisons internationales, il convient d'appliquer le système net d'enregistrement de la TVA. Dans ce système, la TVA est enregistrée comme devant être payée par les acheteurs et non par les vendeurs, et seulement par les acheteurs qui n'ont pas la possibilité de la déduire. Les pays devraient exclure autant que possible la TVA déductible des chiffres des dépenses de tous les secteurs d'exécution de la R-D. Il est recommandé d'exclure la TVA déductible des chiffres des dépenses totales de R-D intra-muros communiqués aux fins de l'établissement de comparaisons internationales.

Les techniciens et le personnel assimilé sont les personnes dont les tâches principales requièrent des connaissances et une expérience techniques dans un ou plusieurs domaines de l'ingénierie, des sciences physiques, des sciences de la vie, des sciences sociales, des sciences humaines et des arts. Ils participent à la R-D en exécutant des tâches scientifiques et techniques faisant intervenir l'application de concepts et de modes opératoires ou encore l'utilisation de matériel de recherche, habituellement sous la supervision de chercheurs.

Les terrains et constructions comprennent les terrains acquis à des fins de R-D (par exemple, réalisation d'essais, aménagement de laboratoires et d'usines pilotes) ainsi que les bâtiments construits ou acquis à des fins de R-D, grands travaux d'aménagement, de modification et de réparation inclus. Comme les constructions sont des actifs produits et les terrains des actifs non produits au regard du Système de comptabilité nationale, il convient de distinguer les dépenses de R-D selon qu'elles concernent les premiers ou les seconds.

Les travailleurs indépendants sont les personnes qui sont propriétaires, seules ou conjointement avec d'autres, d'une entreprise non constituée en société dans laquelle elles travaillent, à l'exclusion des entreprises non constituées en société qui entrent dans la catégorie des quasi-sociétés. Dans le Manuel de Frascati, les consultants indépendants et prestataires extérieurs qui entreprennent des projets de R-D pour le compte d'une autre unité à un prix économiquement significatif sont inclus dans le secteur des entreprises.

Les différents types de coûts de R-D sont : les dépenses courantes et les dépenses en capital de la R-D intra-muros. Les dépenses courantes comprennent les coûts de main-d'œuvre liés au personnel interne de R-D et les autres dépenses courantes (personnel externe de R-D, acquisition de services et de matériel, autres dépenses non classées ailleurs). Les dépenses en capital concernent les terrains et constructions, les machines et équipements, les logiciels immobilisés et les autres produits de la propriété intellectuelle. 
Trois types de R-D sont examinés et définis dans le Manuel de Frascati : la recherche fondamentale, la recherche appliquée et le développement expérimental. Voir le présent glossaire.

Une unité d'activité économique (UAE) est une entreprise ou une partie d'entreprise qui exerce un seul type d'activité de production ou dans laquelle la majeure partie de la valeur ajoutée provient de l'activité de production principale. Chaque entreprise doit, par définition, comprendre une ou plusieurs unités d'activité économique.

L'unité déclarante est l'unité de laquelle émanent les données déclarées. Il s'agit donc de l'unité qui reçoit un questionnaire ou auprès de laquelle un entretien est mené. Dans le cas de données administratives, il s'agit de l'unité associée à chaque déclaration.

L'unité institutionnelle est un concept de comptabilité nationale défini dans le SCN comme étant « une entité économique qui est capable, de son propre chef, de posséder des actifs, de prendre des engagements, de s'engager dans des activités économiques et de réaliser des opérations avec d'autres entités ". Ce concept peut s'appliquer à la mesure des activités de R-D et aux flux liés à la R-D. Dans le cas de la R-D, les unités institutionnelles doivent être capables de prendre des décisions concernant la conduite des activités de R-D, allant de l'utilisation interne et externe des ressources financières à la gestion des projets de R-D. Ces conditions sont moins contraignantes que dans la définition d'unité institutionnelle retenue dans le Système de comptabilité nationale, mais servent néanmoins les objectifs du présent manuel.

Une unité locale est une entreprise ou une partie d'entreprise qui exerce une activité de production en un seul lieu ou à partir d'un seul lieu.

Une unité statistique est une entité au sujet de laquelle des informations sont recueillies et des statistiques compilées. Il s'agit de l'unité qui sert de fondement aux agrégats statistiques et à laquelle renvoient les données des tableaux.

Il est procédé à la vérification des données collectées afin de recenser les éventuelles erreurs présentes dans les données pour, soit valider l'information saisie ou la variable considérée, soit corriger les erreurs et les incohérences constatées. 


\section{Index}

\section{Notes :}

Les nombres référencés correspondent aux numéros de paragraphes. 1.0, 2.0 etc. se rapportent aux introductions non numérotées des chapitres 1, 2, etc. D'autre part, sont utilisées les abréviations suivantes : B : Encadré, par ex. B3.2 pour Encadré 3.2 ; F : Graphique ; T : Tableau ; A1 : Annexe 1 ; g : glossaire des termes (Annexe 2).

\section{A}

académies des sciences, nationales, 8.16, 8.18, 9.24

achat de R-D, 4.114, 4.12, 4.126, 7.43, 8.88, T8.3, $11.53,12.24$

voir aussi contrats, ventes et acquisitions

achats de R-D, 4.60-4.61

voir aussi ventes et achats de R-D

actifs fixes, 4.47

voir aussi dépenses, capital

activités de soutien

activités/services, 2.122, 4.23-4.24,

4.29-4.31, 5.4-5.5, 5.45, 8.6

personnel (voir aussi ressources

humaines), 4.18-4.19, 4.26-4.27,

5.4-5.7, 5.11, 5.43-5.45

activités des entreprises multinationales

(AEMN), 11.33

voir aussi entreprises multinationales

administrations publiques (en tant que

secteur du SCN), T3.1, 3.43

Agence internationale de l'énergie (AIE), B12.1

amortissement des biens corporels et incorporels, 4.38-4.39, 4.73, 112.15

analyse coordonnée, 1.84

analyse selon la situation au regard de l'emploi, 5.78

ancienneté du personnel, 5.84

application des recommandations, 1.83-1.86 approche du point de vue de l'exécutant et approche du point de vue du bailleur de fonds, 1.1, 1.24, 4.6, 4.79-4.88, T4.2, 4.145-4.155, 8.76-8.82, 10.43, 10.47, $12.1-12.2,12.39,12.76-12.77$

difficulté de distinguer exécutants et bailleurs de fonds, 4.78

approche fonctionnelle, 3.8-3.9

approche institutionnelle des statistiques de R-D, T2.1, 3.7-3.9

arts, T2.2

assurance

autorités supranationales, 3.87, 3.93, 4.108, 11.59-11.60, g

comme source de financement, 8.42

avancement général de la connaissance comme objectif socio-économique de R-D, 12.69-12.70

B

balance des paiements, 11.5, B11.1

transferts transnationaux, 11.39

bibliothèques et centres d'information, 2.91, $4.18,8.28$

achat de bibliothèques, 4.55

biens incorporels, 4.53

voir aussi propriété intellectuelle

biotechnologie, 1.81, 7.66-7.67, 8.48, T2.2 
brevets

Manuel de l'OCDE sur les statistiques des brevets, 1.18

budget

déclaration, choix de l'année, 6.52

pour les enquêtes, 6.25, 9.107

sept stades principaux dans les administrations, 12.41-12.43

budgets publics consacrés à la R-D, 1.1, 1.57, 1.73-1.76, 7.42-7.45, 8.1, 8.83-8.93, $12.0-12.79$

administrations centrales/fédérales ou administrations de province/d'État, $4.107,7.43$

comptabilisation de la distribution de fonds, $4.138-4.140$

consacrés à la R-D exécutée à l'étranger, 8.87

contrat ou subvention, 7.42

mesure, 8.75-8.93, 12.0-12.79

registres des subventions, 7.75

C

calcul voir technologies de l'information et des communications

campus satellites à l'étranger, 9.81-9.86, 11.54-11.56, g

CBPRD voir crédits budgétaires publics en faveur de la R-D

chaînes de valeur mondiales, 11.8

chercheurs

catégories dans le secteur de l'enseignement supérieur, 9.9

définition, fonctions, traitement, 5.35-5.39, $7.34, \mathrm{~g}$

rattachés à des universités, 9.24, 11.68

voir aussi ressources humaines

CITE (Classification internationale type de l'éducation), 1.6, 1.26, 3.67-3.68, 5.34, 5.81-5.82, 9.2-9.3, 9.7, 9.88, g

Domaines d'études et de formation de la CITE (CITE-F), 2.44

niveaux, 9.37-9.41, 9.91

CITP (Classification internationale type des professions), 5.34, g

classification

classification Frascati ou classification SCN, 6.21

des fonctions des administrations publiques, 8.25

en fonction du domaine de R-D, T2.2 (voir aussi classification par domaine de recherche et de développement), 3.44-3.46 en fonction du rattachement, 3.39-3.41, 7.21

par orientation sectorielle, 7.51-7.61, T7.2

par statut juridique, 3.42-3.43, 7.22

pour ventiler les fonds d'échange, 4.133

sur la base de la situation géographique, $3.47,7.29$

Classification centrale des produits (CCP), 7.57

Classification des fonctions des administrations publiques (COFOG), $8.25,8.52,8.66,12.72, \mathrm{~g}$

classification des fonctions des institutions sans but lucratif au service des ménages (COPNI), 10.13, 10.32

Classification internationale type de l'éducation voir CITE

Classification internationale type des professions voir CITP

Classification internationale type par industrie voir CITI

classification OCDE des domaines de recherche et de développement, 2.42-2.45, 3.44-3.46, 6.13, 9.98-9.100, 12.69-12.70, g

répartition des DIRDE, 7.62

répartition des DIRDET, 8.46-8.47

répartition des DIRDISBL, 10.3

six grands domaines, 9.98

Classification par activité industrielle, 3.31-3.34, 7.16-7.20, 7.48-7.50

du personnel d'après la fonction, 5.33

niveau de maturité technologique, 2.99, 8.30-8.31

par taille de l'entreprise, 7.23-7.28

pratiques d'archivage, 3.48-3.49, Т3.2

révision et actualisation, 3.27-3.29

sur la base de l'activité économique principale, 3.31-3.34, 7.16-7.20, 7.48-7.50, T7.2, 7.6, 8.24

sur la base de l'appartenance au secteur public ou privé, 3.35-3.38, 7.21, 9.28-9.31

systèmes voir COFOG, COPNI, CCP, classification par domaine de recherche et de développement, CITE, CITI

codes d'identification, 7.15

coefficients, R-D, 9.60-9.61, 9.120-9.124, 9.135-9.136, 12.13, 12.48, g

coentreprises, 3.56, 7.1, 7.7-7.8, 10.10, g

voir aussi partenariats

COFOG, 8.25, 8.52, 8.66, 12.72, g

collaborations voir coentreprises, partenariats 
communication

de nouveaux savoirs, 2.2, 2.22, 2.26, 2.85

de statistiques, information de référence nécessaire, 7.107

et publication hors R-D, 2.91, 2.93

et science ouverte, 2.93

recherche sur les médias, 12.65-12.66

voir aussi conférences

communication des statistiques, 3.16-3.18

allégement fiscal au titre des dépenses de R-D, 13.40-13.43

bailleurs de fonds, 4.79, 4.145-4.155

erreurs, 4.145-4.155

exécutants, 4.9, 4.79, 4.79, 4.145-4.155

comptabilité d'exercice, 8.92, 13.45-13.46, g

comptabilité de caisse, $12.43, \mathrm{~g}$

comptabilité financière

données et normes, 1.25, 4.27, 4.32, 7.94-7.95, B11.1

orientations générales, 1.1

types d'allégement fiscal, 13.44-13.50

comptabilité voir comptabilité financière

conception

comme faisant partie de la R-D, 2.5, 2.62-2.63

des enquêtes voir enquêtes

conférences, participation/présentations, $2.85,9.46$

confidentialité $1.53,3.17,6.1,6.47,6.62,6.92$

connaissances

acquisition, 2.46

comptabilisation, 2.2

nouvelles, comme objet de R-D, 2.14-2.16, $2.22,2.82$

préservation, stockage et accès, 8.28

sources, 2.43

traditionnelles, 2.108-2.110

transfert à la société, 4.115

voir aussi données, propriété intellectuelle conseils de recherche, 8.9, 9.24, 9.62, 9.65

consultants, 4.26, 4.63, 5.16, 5.20, T5.2, 7.5, $7.33,8.55,10.4,10.34$

contrats de recherche, 4.142, 7.42, 9.74, $12.20-12.21,12.24-12.25,12.73$

calendrier, 4.152

conditions, 4.114-4.115

préparation et suivi, 4.3

travaux sous contrat, 4.67

voir aussi financement et marchés publics

ou subventions, 7.42

contributeurs externes/personnel de R-D voir consultants sous ressources humaines

contrôle

de l'enseignement supérieur par l'État, $8.2,9.1$ filiales, 3.4, 11.14-11.15, 11.17

filiales sous contrôle étranger, 11.14-11.15, 11.27-11.28, 11.3, 11.32, g

ou participation majoritaire, $\mathrm{g}$

COPNI, 10.13, 10.32

cotisations de sécurité sociale, 4.21, 9.57, 13.3

coûts de main-d'œuvre voir personnel

créativité

conception, 2.62

critère de base de la R-D, 2.7, 2.17

crédits budgétaires publics de R-D (CBPRD), 12.3

crédits budgétaires publics de R-D (CBPRD), 1.78, 13.2-13.67, g

champ couvert et définitions, 13.5-13.13

couverture statistique, 13.14-13.36

présentation des statistiques, 13.67

ventilations prioritaires, 13.61-13.66

crédits budgétaires publics en faveur de la R-D (CBPRD), 1.75, 1.79, 4.153, 8.49, 8.83, 12.0-12.79, 13.67, g

déclaration et indicateurs, 12.78-12.79, $\mathrm{T} 12.2$

différences entre les données relatives aux CBPRD et celles relatives aux DIRD, 12.76-12.77

mécanismes de soutien et prise en compte, 12.20-12.38

culture, activités de loisirs, religion et médias comme objectifs socio-économiques de la $\mathrm{R}-\mathrm{D}, 12.65-12.66$

\section{D}

déclaration

individus, 6.5, 9.127-9.128

unité déclarante, 3.12, 3.7, 6.13, 6.14, 6.2, $6.66,7.12-7.14,7.29,8.64,9.108,9.127$, $11.70, \mathrm{~g}$

défense, 2.35-2.36, 2.47, 2.53, 4.151, 4.161, 7.37, $8.51,12.5,12.58$

définitions

droit interne, 1.22

nécessité de stabilité, 1.12

révision et clarification, 1.5-1.6

voir aussi « définition " sous les différents termes

déflateurs pour la R-D, 1.81

délocalisation, 116

démonstration

définition, B12.1

démonstration à l'intention des utilisateurs ou démonstration technique, 2.1

démonstration technologique, 2.101 
dépenses de R-D

capital, 4.14, 4.44-4.73, 7.6, 8.29, 9.60-9.61, $12.15,13.25,13.43, \mathrm{~g}$

comptes des entreprises ou normes de Frascati, 7.94-7.95

coûts administratifs, 8.35, 8.6, 12.14

définitions, 4.4, g

dépenses courantes, 4.14, 4.15-4.43, 5.5, $5.45,8.6,9.58-9.59,12.15,13.25, \mathrm{~g}$

dépenses courantes ou dépenses en capital, 4.54-4.55

double comptabilisation/sous-estimation voir mesure

effectuées à l'étranger, 11.69-11.70

extra-muros voir R-D extra-muros

intra-muros voir R-D intra-muros

intra-muros ou extra-muros, 1.4, 1.42, 4.4, $4.6,4.60-4.61, \mathrm{~T} 8.3,9.78$

mesure, 1.40-1.43, 4.0-4.165, 5.5 (voir aussi mesure)

problèmes de qualité des données, 7.31

suivi et évaluation, 12.14

voir aussi budget, financement, communication des statistiques

dépenses intérieures brutes de R-D (DIRD), $1.43,1.68,4,4.7-4.9,4.156-4.164,8.76$

définition, $4.8, \mathrm{~g}$

différence avec les montants des CBPRD, 12.76-12.77

différence avec les valeurs totales du SCN, 4.157, B4.1

militaires ou civiles, 4.161

présentation, 4.158, T4.4

ratio DIRD/PIB, 4, 4.162

ventilation régionale, 4.163

dépenses intérieures de R-D de

l'enseignement supérieur (DIRDES),

1.64, 9.53-9.77, 9.83-9.84, 9.136

comparaison des données entre FGU et CBPRD, 12.77

comptabilisation de la distribution des fonds, 4.141

définition, 9.53, g

en fonction de l'origine des fonds, 9.62-9.77

sources de financement externes, 9.62-9.63, 9.65, 9.74

sources de financement internes

(voir aussi fonds généraux des universités), 4.92, 9.62-9.73

dépenses intérieures de R-D de l'État (DIRDET), 1.59, 8.36-8.58

définition, 8.36, g

différences majeures avec les CBPRD, 12.16 par opposition au financement public de l'exécution de R D extra-muros, 8.54-8.58

répartition fonctionnelle, 8.38-8.53

dépenses intérieures de R-D des entreprises (DIRD), 1.56, 11.8, 11.26, 13.67

définition, 7.35, g

répartition fonctionnelle, 7.35-7.68

Dépenses intérieures de R-D des institutions sans but lucratif (DIRDISBL), 1.67, 10.21-10.32

définition, 10.21, g

répartitions fonctionnelles

recommandées du financement, 10.24-10.32

dépenses nationales brutes de R-D (DNRD), 1.68, 4.165, T4.5, g

détection de pannes, 2.5, 2.57

développement expérimental

dans les entreprises, 7.47

définition et critères, 1.13, 1.35, 2.9, 2.34-2.36, g

développement préalable à la production, 2.35-2.36, 2.5, 7.47

DIRD, 1.68, 4.165, T4.5, g

DIRD voir dépenses intérieures brutes de R-D

DIRD voir dépenses intérieures de R-D des entreprises

DIRDET voir dépenses intérieures de R-D de l'État

DIRDISBL (voir Dépenses intérieures de R-D des institutions sans but lucratif)

domaines de recherche et de développement

données

administratives, 6.3, 6.5, 6.53-6.56, 6.77, $6.82,7.99,8.66,9.95-9.96,9.103-9.104$, 9.113-9.118, 9.121

cohérence, 1.6, 1.12, 3.7, 4.27, 4.103, 5.26, $5.48,5.59,5.63$, Т5.3, 6.49, 6.56, 6.59, $6.74,7.32,7.48$

collecte, classification sectorielle, 3.14 comptabilité de caisse et d'exercice, 8.92 croisement des classifications, 7.61

désagrégées, publication, 8.74

données exclues de la R-D, 2.5, 2.89-2.90

estimation, 5.65-5.74, 6.80-6.84, 7.90-7.93, 8.71-8.74, 9.119, 12.49, 13.37-13.39, g

extrapolation, 7.93

harmonisation (de différentes sources), 8.9 imputation, 6.53, 6.74-6.78, 7.92, 9.116, g intégration, 6.71

mesures de la qualité de collecte, 7.89, B7.1

métadonnées, 1.84, 6.79, 7.6, 7.108, 9.122

méthode de collecte, $4.6,6.4,7.48-7.61$ 
méthode de collecte des données, définition, 6.47-6.52, 6.62-6.70, 7.85-7.93, 8.68-8.70, 9.93-9.118 (voir aussi enquêtes)

méthodes de collecte/d'acquisition, 1.84, 2.97

paradonnées, 6.63, 6.70, g

projets fondés sur les mégadonnées, 2.93 qualité voir qualité

recueil et comptabilisation des données sur le personnel, 5.62-5.74, B5.1, T5.3

recueil et rassemblement, données d'utilité générale, 2.90-2.91

révisions, 12.49

sécurité voir confidentialité

sources, 1.52, 3.15, 6.3-6.5, 6.23, 6.39, 7.101, 8.68, 9.114-9.115, 11.7 (pour CBPRD, 12.39-12.49; pour le calcul des allégements fiscaux, 13.51-13.60) (voir aussi données administratives, enquêtes)

validation, $6.55,6.85-6.89,7.92,12.49$

vérification, 6.49, 6.68, 6.72-6.73, 7.92, 9.116, $\mathrm{g}$

données administratives, voir données

données budgétaires, 1.74-1.75, 8.82-8.83, dotations $12.39-12.40,12.45-12.49$

\section{E}

échanges

conseils pour les statistiques, 1.1

internationaux de services de R-D, 1.71, 11.4-11.6, B11.1, 11.34-11.43

échantillonnage

des unités qui n'ont pas répondu, 6.69

plan, 6.41-6.46

unités, 6.2, 6.27, 6.34, 6.4

échéances/retards

disponibilité des chiffres à inclure dans

les rapports, $12.48,12.78$

disposition de report, 13.8, g

et répartition des coûts pour les programmes longs, 4.152, 12.44

pour le traitement des allégements fiscaux, 13.44-13.50, 13.57

effectif en tant qu'unité de mesure, 5.46-5.48, g

cohérence avec l'équivalent temps plein (ETP), 5.57, 5.59

définition et traitement, 5.58-5.61

estimation, 5.74

élaboration des politiques

contribution de la R-D, 2.41
Manuel de Frascati, 1.1-1.2

marchés émergents, 9.79

objectifs voir objectifs

recherche à l'appui, 2.118

statistiques à l'appui, 1.4, 1.51, 7, 7.1

employés

définition, T5.1, 5.12-5.13, g

entreprises sans effectifs, 7.27

intérimaires, 5.16, T5.1, 5.26, g

voir aussi individus

ou personnes occupées, $g$

activités de soutien, 2.122

activités occasionnelles ou continues,

$6.18-6.19,6.31,7.1$

annuaires des exécutants, 7.75-7.76

branche d'activité, 7.51-7.60

changements organisationnels, 1.6

définitions, 1, 1.2, 1.5-1.6, 1.12-1.15, 1.22, $1.25,1.32-1.36,2.5-2.11,4.151$, B11.1, g

définitions aux fins de la fiscalité, 13.15-13.16

dialogue social et politique, 1.87

dissociées des activités connexes, 1.63

en sciences sociales, sciences humaines et arts, 2.3

exemples de liens et d'exclusions, 2.46-2.110

impact, 1.2

les quatre grands secteurs, 1.38, 3

paiements au titre des services de R-D, $12.24-12.25$

prix, 11.42-11.43

résultats négatifs, 2.2

rôle dans le développement économique, 3.15

traitement comme investissement, 1, 1.23, $1.41,2.2,4.2$, B4.1

travaux originaux de R-D ou autres services de R-D, 11.34

trois types d'activité (définitions et critères/2.23-2.41; distinction/2.37-2.40; répartition des DIRDE par type/7.47; répartition des DIRDET par type/8.45; ordre/2.11) (voir aussi recherche appliquée, recherche fondamentale, développement expérimental), 1.35, 2.9

énergie, 2.27

engagements (budgétaires), 12.49, g

enquêtes statistiques, 6.5

d'emploi du temps, 9.95, 9.116, 9.125-9.137, g

formation des répondants, 8.69

information des répondants, 7.88

méthodologie, 6.72-6.89, 7.77-7.93 
plan d'enquête, 6.41-6.71, 7.25, 7.70-7.76, 10.40-10.42

pour identifier les exécutants professionnels de la R-D, 7.7, 7.71

sur différents secteurs, 6.18-6.40

sur le secteur de l'enseignement supérieur, 9.95-9.96, F9.1, 9.102-9.112

sur le secteur privé sans but lucratif, 6.39-6.40, 10.40-10.47

sur les bénéficiaires d'allégements fiscaux, 13.51-13.55

sur les échanges de services, 11.38-11.39

sur les échanges internationaux de services, 11.36

sur les particuliers, 3.77

sur les unités de l'État, 8.84-8.93

taux de réponse, $6.69,6.86,7.85$

voir aussi données, rapports

enseignement (et formation)

membres du personnel (universitaire), 9.44-9.46

objectif socio-économique de R-D, 12.64

traitement au regard de la R-D, 2.75-2.78

voir aussi enseignement supérieur

enseignement supérieur

campus satellites à l'étranger, 9.81-9.86

étudiants étrangers, 9.87

enseignement supérieur (en tant que secteur de R-D), 1.60-1.64, 3.67-3.74, 9.0-9.138

cas ambigus, $3.71-3.74,8.19-8.23$, 9.18-9.31

classification des établissements, 1.38 , 3.24, T3.1, 3.36, 3.55

définition, 1.61, 9.3-9.4, 9.6-9.7, g

enquêtes, $6.32-6.38$

établissements privés, 9.9-9.10, 9.28-9.31, T1

établissements publics, 9.9-9.10, 9.28-9.31, T1

institutions de financement, 9.2

liens avec le reste du monde, 9.79-9.87, $11.44,11.54-11.56$

limites entre recherche et enseignement, 9.33-9.46

mesure des dépenses et du personnel, 9.52-9.92

méthodes de mesure, 9.93-9.137

principales caractéristiques, 3.67-3.69

unités statistiques dans le secteur, 3.7

entreprises, 3.11, 3.12, 3.54, 3.63, 4.135-4.137, B3.1

classification voir classification

définition, $g$

entités légales, 6.16

entreprises sœurs implantées à l'étranger, 11.16, g multinationale voir entreprises multinationales

privées et publiques, 7.3

voir aussi sociétés, institutions sans but lucratif

entreprises (comme secteur de R-D), 1.53-1.56, 3.51-3.59, 7.0-7.108

cas limites, 3.55-3.59, 8.17-8.18

classification voir classification

concentration de la R-D, 6.1, 6.18

définition, 1.54, 3.43, 7.2-7.8, g

enquêtes sur (voir aussi enquêtes), $6.18-6.25,7.70-7.93$

financement provenant du secteur des entreprises, 7.37-7.41

principales caractéristiques, 3.51-3.52

recensement des exécutants potentiels de R-D, 7.75-7.76

sources de financement internes, 4.91

surestimation ou sous-estimation possible, 7.98-7.103

unités statistiques réparties en secteurs, 3.53-3.54, 7.10-7.11, 7.15-7.29

entreprises affiliées, 11.11-11.15, 11.17, 11.22, 11.27-11.28

en dehors du secteur des entreprises, 11.48

rattachement, 3.39-3.41, g

unités non affiliées, 11.22

entreprises créées par essaimage, 9.27, 12.52

flux de R-D déclarés ou flux de R-D effectifs

entreprises multinationales (EMN)

déclaration erronée, 4.7

définitions appropriées, 11.10-11.19

financement international de la R-D, 11.20-11.23

mesure et comptabilisation de la R-D, 11.5-11.9, 11.22-11.33

paiements de transfert, 4.32

rapports, 7.95

structures, 1.53, 7.1, 7.9

traitement spécifique, 11.59-11.66

voir aussi sociétés, entreprises

Environnement comme objectif socio-économique de R-D, 12.57

espace

essais, 2.5, 2.55-2.56

cliniques voir santé

estimation

des échanges internationaux de services, 11.37

selon le prix d'acquisition, 4.4

estimation voir données

établissements, 311, 3.12, B3.1, g 
établissements de recherche bâtiments voir installations de recherche chargés de missions ou spécialisés, 9.21 classification, 7.2-7.4, 9.18-9.31 contrôlés par l'enseignement supérieur, 9.6

contrôlés par l'État, 8.7, 8.63

contrôlés par l'industrie, 3.81

enquêtes, 8.69

et organisations internationales, 11.66

groupements mondiaux, 11.48

liés aux universités, 9.22-9.23

revenus, 8.18

sources de financement internes, 4.91 État

administration centrale/fédérale, 8.6, T8.1, 13.34, g

administrations régionales/d'États fédérés, 8.9, T8.1, 13.35, g

allégement fiscal voir fiscalité

fonctions, estimations des CBPRD, 12.72 (voir aussi Classification des fonctions des administrations publiques)

organisations/unités extrabudgétaires, 8.7, 12.8-12.9

unité budgétaire de l'administration centrale, 12.6

unités, 3.42, 8.3, 8.11-8.13, g

études de faisabilité, 2.114

études utiles pour l'action des pouvoirs publics, 2.116-2.118, 8.32

étudiants en doctorat, $g$

classification, 1.47, 2.76-2.77, 5.22, T5.2, $5.25,5.39,8.61,9.34$, T9.2, 9.9, 10.38

coûts associés, 4.28

salaires et traitements, 4.2

traitement, 5.27-5.31

étudiants en master

classification, 1.47, 5.22, T5.2, 5.25, 8.61, 9.34, 9.91-9.92

coûts, $4.2,4.28$

programmes/étudiants de master de recherche, 5.22, 5.3, 9.34, 9.39, 9.91, g

traitement, 5.27-5.31

étudiants voir doctorants et étudiants en master

Eurostat, 1.76, 12.4

évaluations et bilans ponctuels, traitement, 2.119, 10.18

évaluations programmatiques, 2.119

exemples, 2.41

expression artistique par opposition à la recherche, 2.67

recherche à visée artistique, 2.64, 2.65

recherche sur l'art, 2.17, 2.64, 2.66 exploration, 2.94

exploration et évaluation minières, $2.95-2.98$

exploration et exploitation comme objectif socio-économique de la R-D, 12.58

externalisation/sous-traitance, 4.116, 4.144

voir aussi consultants, contrats, sous-traitance

F

Famille Frascati, 1.4, 1.18-1.21

FGU voir fonds généraux des universités

filiales sous contrôle étranger, 11.15, $11.17, \mathrm{~g}$

financement

activités à l'étranger du secteur de l'État, $11.52-11.53$

activités à l'étranger du secteur privé sans but lucratif, 11.57-11.58

appel à la concurrence, 8.89, 12.73

classification pour ventiler les financements d'origine externe, 4.133-4.134

comptabilité de caisse ou d'exercice, 8.92, 13.45-13.46, g

contrôlé par l'État, 8.15

destination des fonds, 12.73

participatif, 4.111, 10.25, 10.44-10.46

répartition des CBPRD, 12.73-12.74

répartition des DIRDE par source de financement, 7.36-7.46, T7.1

répartition des DIRDES par source de financement, 9.622-9.77

répartition des DIRDET par source de financement, 8.42-8.44, T8.2

sources affiliées ou non affiliées, 11.22

transnational, 11.6

financements d'origine interne, 1.42, 4.4, 4.32, 6.61, 4.78, T4.2, F4.1, 4.87, 4.90-4.94, 4.97, 4.101, 4.103. 4.105, 4.117, 4.132, $4.138,7.37-7.38,7.4,8.56$

sources, $4.92,7.38,9.62-9.73$

fiscalité

allégements fiscaux, 13.6, 13.9, g

avantages pour les bailleurs de fonds philanthropiques, 10.43

crédits d'impôt, 13.7-13.9, g

des entreprises, 13.27, 13.29

exemptions pour les organisations

internationales, 13.22

gains en capital, 13.28

Impôts sur le patrimoine, 13.32

instruments, 13.26 
mesures incitatives en faveur de la R-D / allégement (définition/ 13.5-13.9; mesure/ 13.0-13.67) (voir aussi allégement fiscal au titre des dépenses de R-D), 1, 1.6, 1.77-1.79, $4.22,4.100-4.103,7.38,7.45,8.79$, $12.35-12.37,12.79$

prime pour l'emploi, 13.12

structure "normale " 13.10-13.11

sur les biens et les services, 8.39, 13.33

valeur ajoutée, 8.39, 13.33, g

fixation d'objectifs, 1.9

fonds d'échange, 1.42, 4.113-4.117, $4.120-4.121$

voir aussi financement

fonds d'origine externe, 1.42, 4.81-4.82, T4.2, 4.87, 4.95-4.99, 4.109, 4.124, 4.137, 4.14, $9.64-9.65,9.74,10.23,10.28, \mathrm{~g}$

sources, 9.62-9.63, 9.65, 9.74

voir aussi financement

fonds généraux des universités (FGU), 1.62, $4.98,4.106,4.111,8.82,8.89,9.62-9.64$, 9.75-9.77, 9.93, 12.27-12.28, 12.69, $12.77, \mathrm{~g}$

distinction par rapport aux autres sources de financement, 9.68-9.72

Fonds monétaire international (FMI)

Manuel de la balance des paiements et de la position extérieure globale

Manuel de statistiques de finances publiques, $12.4,12.6$

formation (dans le domaine de la recherche), 9.44-9.45

\section{G}

GENIST voir OCDE

gestion des risques, 2.41, 2.87, 7.42, B8.1, 12.29

GFS 2014 (Manuel de statistiques de finances publiques), 1.1

groupe d'entreprises, 3.11, 3.12, B3.1, 4.32, $4.99,6.15,7.9,7.14,11.13, \mathrm{~g}$

filiales, 11.11-11.15

transferts de R-D, 7.69

\section{$\mathrm{H}$}

hôpitaux universitaires voir santé hôpitaux voir santé

\section{I}

incertitude, critère clé pour la R-D, 2.7, 2.18, 2.22 indicateurs, 11.2 individus

classification, 10.8

emploi/affiliation multiple, 5.2, 8.21, 11.68

en tant que sources de financement, $10.27,10.43-10.46$

en tant qu'inventeurs/chercheurs, 3.83 , $7.6,10.8$

fiscalité, 13.29-13.30

non mesurés comme exécutants de la R-D, 10.27

rôles, 3.77-3.78

types de contribution à la R-D, 3.78

voir aussi effectif, personnel

industrie aérospatiale, 2.35-2.36, 2.47

infrastructure

de R-D, voir installations de recherche

objet de R-D, 12.59

scientifique, 8.28

infrastructure scientifique, 8.28

voir aussi installations de recherche

ingénierie, T.2.2

innovation

activités, 2.46

limites avec la R-D, T2.3

installations de recherche (bâtiments)

base de calcul, 12.22

coûts de location, exploitation et maintenance, 4.23, 4.29, 4.34-4.37, $8.41,9.59$

en gestion conjointe, 6.37

gestion des installations appartenant à l'État, 4.36-4.37, 8.41, 8.79

parcs de recherche, 9.25

traitement dans le cadre des CBPRD, 12.22-12.23

voir aussi associations/établissements de recherche

institutions de recherche voir associations/ établissements de recherche

institutions sans but lucratif (ISBL), 1.54, $1.65-1.67$, B3.2

administrées par des entreprises ou les servant au premier chef, 3.81

classification, 3.58, 7.2, 7.4, 8.14-8.16, 10.1

définition, 3.42, g

rôle de financement et d'exécution, 10.22

sans identité distincte, 10.6-10.7

traitement des différents types

voir aussi secteur privé sans but lucratif

institutions sans but lucratif au service des ménages (ISBLSM), 1.66, B3.2, 3.25, T3.1, 3.43, 3.75, 10.2, 10.14

instituts chargés de missions ou spécialisés, 9.21

internationalisation voir mondialisation 
investissement

en faveur de la R-D, 12.29-12.33

R-D comme investissement, 1, 1.23, 1.41, $2.2,4.2, \mathrm{~B} 4.1$

investissement direct étranger (IDE), 1.69, $11.2,11.11,11.29, \mathrm{~g}$

Définition de référence de l'OCDE des IDE, 11.2, B11.1

ISIC (Classification internationale type par industrie), 1.6, 1.26, 3.33, 7.16-7.17, 7.48-7.49, 7.57-7.59, 8.24, 9.18, 10.12-10.13, g

division 72 de la CITI, 7.59, 8.24, 8.47, 11.34

\section{L}

législation

autorisant les enquêtes obligatoires, 6.2

référence au Manuel de Frascati, 1.12

linguistique, exemples de R-D, 2.41

localisation géographique

des DIRDE, 7.64

des DIRDET, 8.53

des unités statistiques, 3.47, 6.1, 7.29, 7.64

logiciels

activités de R-D, 1.24, 2.4, 2.68-2.74, 7.66, g

immobilisés, 4.52, g

traitement du développement par l'OCDE et la SCN, 2.74, 4.157, B4.1

utilisés en R-D, 2.69, 4.25

loyer voir installations de recherche

machines et équipements, 2.6, 4.47, 4.50-4.51, T4.1, 9.60, g

\section{M}

Manuel de Frascati

annexes, 1.8, 1.81, 2.45

aperçu général, 1.30-1.82

bref historique, A1

contributeurs, A1

éditions précédentes, 1.3, 1.34, 1.81, A1

objectifs et historique, 1.1-1.4, 1.8-1.11

Manuel de l'OCDE sur les indicateurs de la mondialisation économique, 11.2, B11.1

voir aussi entreprises multinationales

manuel de l'UOE, 9.2, 9.138

Manuel d'Oslo, 1.18, 2.46, 7.84

marché

études (voir aussi études), 2.9

prix, 11.6, 11.37, 11.42

recherche, 2.56

valeur marchande, 4.35, 9.59 ménages

classification, 3.42, 7.5, 10.2, 10.14

en tant que sources de financement, 10.27

en tant qu'unités de R-D, 3.6, 3.25, 3.75, 3.77

non soumis à une enquête, selon recommandation, 10.14, 10.27

secteur Frascati/SCN, T3.1, 3.43

travailleurs non rémunérés, 5.13

types de contribution de R-D, 3.78

mesure

dans le secteur privé sans but lucratif, 10.21-10.39

de la mondialisation de la R-D des entreprises, 11.4-11.9

de l'allégement fiscal en faveur de la R-D, 13.37-13.67

des dépenses, 1.40-1.43, 4.0-4.165, 5.5, $8.36-8.58$

double comptabilisation/ sous-comptabilisation, 1.59, 4.9, 4.12, 4.21, 4.35, 4.36, 4.46, 4.58, 4.62, 4.78, $4.82,4.87,4.103,4.119,4.122,4.149$, B 4.1

du financement, 4, 4.74-4.165, 9.62-9.77, 10.26-10.28

du financement de la R-D ou statistiques sur les échanges de services, 11.38

du personnel, 5.0-5.88

du secteur de l'enseignement supérieur, 9.52-9.137

méthodologies and procédures, 6.0-6.93 mesure des activités exprimées en équivalent temps plein (ETP), 5.46-5.48

cohérence avec la mesure des effectifs, $5.57,5.59$

définition et traitement, 5.49-5.57, g

estimation, 5.65-5.73

métadonnées, 1.84, 6.79

méthodes et procédures, 1.50-1.52, 6.0-6.93, 7.77-7.93

microdonnées

analyse, 3.48 , 4.3, 6.1, 6.21, 6.74, 7.15

mise à l'essai

activités d'essai, 2.92, 2.113

comme processus de R-D, 2.16, 2.5, 2.92, 4.67

des questionnaires, 6.6

par le secteur de l'État, 8.28

mondialisation

définition, 1.69, 11.2, g

Musique, exemples d'activités de R-D, 2.41, 2.65 
$\mathbf{N}$

NABS (Nomenclature pour l'analyse et la comparaison des budgets et des programmes scientifiques), $1.76,8.5,10.31,12.4,12.54$

classification, T12.1

NACE (Nomenclature des activités économiques dans la Communauté européenne), 7.17

NAICS (Système de classification des industries de l'Amérique du Nord), 7.17

nanotechnologies, 2.4, 7.66-7.67, 8.48

Nations unies

Cadre national d'assurance de la qualité, 7.105

Directives provisoires concernant les classifications internationales types par âge, 5.79

Manuel des statistiques du commerce international des services

système de classification voir CITE, CITI

nomenclature pour l'analyse et la comparaison des budgets et programmes scientifiques voir NABS

normes/normalisation, 1.86, 2.113, 2.92, 2.113, 5.34

utilisation du Manuel de Frascati, 1.0-1.29 nouveauté comme critère de base de la R-D, $2.7,2.14-2.16,2.22$

objectif socio-économique de R-D, 12.60, B12.1

objectifs

de la R-D, 2.22, T2.1

de la révision du Manuel, 1.5-1.7

Manuel de Frascati, 1.1-1.4

objectifs socio-économiques

de la R-D (classification T12.1, g ; description/12.56-12.71; distribution des CBPRD/12.50-12.71), 7.63, 8.49-8.51, $10.31,12.2$

recherche fondamentale, 2.27

OCDE

Classification/Nomenclature des domaines de recherche et de développement voir domaines de recherche et de développement

collaboration, 1.85

définitions standard, 13.2

engagement auprès des non-Membres, 1.6
GENIST (Groupe de travail des experts nationaux sur les indicateurs de science et de technologie), 1.82, 1.84, A1

organisation du travail (recherche)

organisation/organisme

activités de R-D, 1.6, 5.8

voir aussi société, entreprise

organisations internationales, 3.87, 3.94, 4.108, 4.159-4.160, 11.53, 11.59-11.66, $12.19,13.22$

définition, 11.59, g

voir aussi autorités supranationales

organisations non gouvernementales (ONG), 11.62-11.64

orientation sectorielle de la R-D, 7.51-7.60

définition, 7.54

origine, 5.85

analyse recommandée, 5.87-5.88, T5.4.a-e catégories de l'enseignement supérieur, 9.88-9.92

classification par fonction, 5.32-5.45, 8.62 concordance entre les catégories, 5.25 coûts de main-d'œuvre, 4.16-4.22,

4.62-4.64, 5.11, 5.25, 8.39, 9.56-9.57, g couverture et traitement, 5.6-5.45, T5.2 de l'État, 8.59-8.62

définition du personnel de R-D, 1.15,

1.44-1.49, 2.22, 4.18, 5.2-5.4, T5.1, 5.18, $5.32, \mathrm{~g}$

employés intérimaires, 5.16, T5.1, 5.26, g

employés voir employés

étudiants, voir étudiants en doctorat, étudiants en master

flux de personnel, 5.86

individus voir individus

mobilité, 11.9, 11.49

permanent ou temporaire, 5.78

personnel externe, 1.15, 1.46, 3.84, 4.26, 5.9, 5.15-5.24, T5.2, 5.25, 7.33, 8.61, g (voir aussi consultants)

personnel interne, 1.6, 1.15, 1.46-1.47, 5.9

reste du monde, 11.49-11.51

travailleurs indépendants, 10.35

travailleurs indépendants, 3.82, 4.19, 4.26, $4.63,5.16,5.20, \mathrm{~T} 5.2,5.25,7.5,7.33$, $10.4,10.34,13.29$

voir aussi chercheurs, techniciens 


\section{P}

partenariats, $3.57,4.117,8.23,8.34,8.42,10.1$ internationaux, 8.87

patent boxes, 13.14, 13.28

services d'essais qui débouchent sur des brevets

voir aussi propriété intellectuelle

pays d'investissement immédiat, 11.29

pays d'investissement ultime, 11.29

pays en développement, 1.28

pays hôte immédiat, 11.3

personnel

analyse par caractéristiques, 5.75-5.85

analyse par qualifications, 5.81-5.83

ventilation par âge, $5.79-5.80$

personnes morales, définition, 3.6

petites et moyennes entreprises (PME), 2.21, $2.34,7.24-7.28,7.99$

microentreprises, 7.7, 7.81

planification

impératif pour la R-D, 2.19

pollution, $12.57,12.59,12.63,12.68$

prêts en faveur de la R-D, 7.39, 8.79, 12.31-12.32

garanties, 12.34

prix

de transfert, 11.42-11.43

du marché, 11.6, 11.37

économiquement significatifs, B3.2, 7.2, $7.3,7.5,10.3-10.4, \mathrm{~g}$

inférieurs à la moyenne, 3.58

payés par les acquéreurs, $4.40, \mathrm{~g}$

procédé(s)

définition, 2.1

développement, 2.47

développement expérimental, 2.32-2.36

production et technologie industrielles, en tant qu'objectif socio-économique de R-D, 12.61

produit(s)

définition, 2.1

développement, 2.34, 2.62, 7.47

développement expérimental, 2.32-2.36

professeurs émérites, 5.23, T5.2, 5.25, g

projet

à grande échelle, 2.53-2.54, 2.74, 2.89

base de financement, 8.89

définition en R-D, 2.12

$\mathrm{R}-\mathrm{D}$ dans les administrations publiques, 8.27

ou programme, 2.3

propriété intellectuelle, 1.6, 1.23

approches de l'entreprise, 7.51-7.52

et échanges internationaux de services de R-D, 11.6 et secret d'affaires (voir aussi

confidentialité), 2.2

exploration minière, 2.95

OECD Handbook on Deriving Capital

Measures of Intellectual Property

Products, 1.6, 1.23, 2.74, 11.6, B11.1

protection, 2.2, 2.22, 2.31

redevances ou de droits de licence, 4.23 , $4.53,4.126,7.57,8.18,11.36$

régimes fiscaux, 13.28

prototypes, 2.18, 2.21, 2.47, 2.49-2.50, 2.54, $4.23, \mathrm{~g}$

PSBL voir secteur privé sans but lucratif publication voir communication

Q

qualité

assurance, 1.84, 3.49, 6.69

contrôle de la qualité des réponses sur la R-D des entreprises, 7.94-7.108

mesure des réponses aux enquêtes, 7.89 , B7.1

questionnaires, 1.84, 6.4, 6.49-6.51, 7.14, 9.11, 9.112

voir aussi enquêtes

$\mathbf{R}$

R-D en économie, 2.41

R-D en histoire, 2.41

R-D extra-muros, 4.12 , g

allégements fiscaux, 13.23-13.24

dépenses, 1.4, 1.42, 4.64, 9.78

différenciation de la R-D intra-muros, 7.96-7.97

financement public, 8.54-8.58

répartition fonctionnelle dans le secteur des entreprises, 7.69-7.108

ventilation des fonds extra-muros selon leurs fournisseurs et leurs bénéficiaires, 4.133-4.134

R-D intra-muros, 4.10-4.73, g

allégement fiscal, 13.23-13.24

allocation à un secteur national ou au reste du monde, 11.68

catégories de dépenses, T4.1

causes de déclaration incomplète ou inexacte, $4.67-4.70$

dépenses effectuées à l'étranger, $11.69-11.70$

dépenses, définition, 1.4, 1.42, 4.1

différence par rapport à R-D extra-muros, 7.96-7.97 
exclusion, 4.125

exécuté hors du territoire national, $4.65-4.66$

sources de financement, T7.1 (voir aussi

financement), $11.45-11.47$

recensement, 7.78-7.80, 7.85, 8.68, 9.126

voir aussi enquêtes

recherche appliquée

dans les entreprises, 7.47

définition et critères, 1.35, 2.9, 2.29-2.31, g

orientation sectorielle, 7.56

voir aussi recherche et développement (expérimental) (R-D)

recherche archéologique, 2.4

recherche et développement (expérimental) (R-D)

activités et projets, 2.12

activités exclues, 2.15

bases de données et indicateurs, 1.84

caractéristiques communes, 2.6

critères de base, 1.14, 1.33, 2.6-2.8, 2.13-2.22, T2.1

recherche fondamentale

critère d'incertitude, 2.18

dans les entreprises, 7.47

définition et critères, 1.35, 2.9, 2.25-2.28, g

orientation sectorielle, 7.56

recherche fondamentale orientée ou recherche pure, $2.28,7.47, \mathrm{~g}$

voir aussi recherche et développement (expérimental) (R-D), science et technologie

recherche, développement et démonstration (RDD), B12.1

références, dépenses fiscales, 13.40-13.43

régionalisation des statistiques de R-D, 1.81, 4.163-4.164

Registres des entreprises, 7.71-7.74, 7.84

remises de dettes, 12.32

Répartition des DIRDISBL par source

de financement, 10.24-10.28

à titre philanthropique, 3.78, 4.97, 4.111, $9.15,10.25,10.43-10.46$

cinq grandes sources, 4.104

dans les multinationales, 4.32

double comptabilisation/sous-estimation voir mesure

entités n'exécutant pas de travaux, 4.128-4.129

financement de l'enseignement supérieur, $9.2,9.62-9.63,9.65,9.74$

fonds d'échange et de transfert, 1.42, 4.4, $4.77,4.109-4.117,7.42,8.44, \mathrm{~T} 8.2,8.78$, $8.88,11.23,11.53, \mathrm{~g}$

non situées sur le territoire national, 1.68-1.72 pour les hôpitaux universitaires, 9.15

prêts voir prêts

source, définition, $g$

sources de financement sur lesquelles des informations doivent être recueillies, 4.104-4.108, T4.3

traitement des activités de financement, 2.121

traitement des flux, 1.59, 4.74-4.144, F4.1, 7.96-7.97, 8.56, T8.3, 9.78, 10.22-10.23

voir aussi dépenses de R-D

reproductibilité comme critère de base de la R-D, 2.7, 2.2, 2.22

résidence des unités institutionnelles, 3.21, 3.90-3.92, 10.11, 11.70, g

résolution de problèmes comme activité de R-D, 2.17, 2.38

reste du monde (secteur de R-D), 1.68, 3.21, 3.87-3.96, 11.1

caractéristiques principales, 3.87-3.88

cas ambigus, 3.90-3.96

comme source de financement, 7.41, 7.46

définition, 11.1, 11.19, 11.45, g

liens avec l'enseignement supérieur, 9.79-9.87

organisations internationales et supranationales, 11.59-11.66

recevant un financement public R-D, $11.53,12.19$

sources de financement, 11.45-11.57

voir aussi mondialisation

résultats de la $\mathrm{R}-\mathrm{D}, 1.16-1.17,2.93$, 3.15, 3.45, 7.54, B11.1, 12.26

rétro-ingénierie, 2.15

\section{S}

salaires du personnel de R-D, 5.78

santé

classification des hôpitaux, 8.22

classification des unités, 3.34, 3.72, 8.34

comme objectif socio-économique de R-D, 12.68

essais cliniques, 2.61, 4.143, 7.75, 8.34, 9.15, 9.49, 9.109, 10.19

financement de la recherche, 4.97

hôpitaux universitaires, 1.63, 6.36,

8.22, 9.13-9.17, 9.26, 9.32, 9.47-9.49, 9.109-9.112

recherche en milieu hospitalier, 6.35-6.36

soins de santé spécialisés, 2.115, 9.47-9.49

savoir traditionnel, 2.108-2.110

science et technologie

conseillers scientifiques, 2.12, 8.33 
définition des activités scientifiques et technologiques

distinction avec la R-D, 2.88-2.89, 2.93, 8.28

gestion des activités, 2.99-2.101

services de l'État, 8.28

services d'information, 2.112

sciences agricoles et sylviculture

objectif socioéconomique de R-D, 12.63

sciences humaines

R-D en sciences humaines, 2.104-2.107

sciences naturelles, 2.4

voir aussi science et technologie

sciences sociales

collecte de données, 2.9

R-D dans les sciences sociales, 2.103

SCN voir Système de comptabilité nationale

secteur de l'État, 1.57-1.59, 3.60-3.66, 8.0-8.93

cas ambigus, $3.64-3.66,8.17-8.23$

classification des unités, 8.24-8.25

définition et étendue, 1.57, 3.43, 8.2-8.25, g

identification de la R-D, 8.26-8.32

mesure des dépenses et du personnel, 8.36-8.70

personnel, 8.59-8.62

principales caractéristiques, 3.60-3.61

secteur, composantes et limites, T8.1

secteur informel, 3.85

secteur privé sans but lucratif (PSBL), 1.65, 3.42, 3.75-3.86, 10.0-10.47

bénévoles, voir bénévoles

cas limites, 3.80-3.86, 10.9-10.11

classifications institutionnelles recommandées, 10.12-10.15

définition, 1.66, 3.25, 3.43, 10.2-10.3, g

enquêtes, 6.39-6.40, 10.40-10.47

et mondialisation, 11.44, 11.57-11.58

identification de la R-D dans le secteur, 10.16-10.20

principales caractéristiques, 3.75-3.78

unités statistiques, 3.79

secteur privé, définition pour classification (voir aussi secteur public), 3.35-3.38

secteur public

différence par rapport au secteur de l'État, T8.1

et entreprises, 7.3

secteurs institutionnels pour la R-D, 1.38, 3.0-3.96

classification des unités, voir classification

classification SCN

digramme de décision pour l'affectation des unités

justifications de la classification sectorielle, 3.13-3.18

secteurs et cas limites voir aussi entreprises, enseignement supérieur, R-D publique, secteur privé sans but lucratif, reste du monde services

définition des services, 2.79

échanges de services de R-D, 11.33-11.43

$\mathrm{R}-\mathrm{D}$ dans les activités de services, 2.79-2.87

services financiers, 2.87

services sociaux (activités R-D), 2.87, 12.67

sexe

problématique hommes-femmes, 12.67

ventilation par sexe, 5.76

sociétés

définition, 3.42, $\mathrm{g}$

entreprises publiques, 3.61

quasi-sociétés, 3.51, 7.2, g

rapports des sociétés, $7.75,7.94$

SCN, T3.1, 3.43

voir aussi entreprises, entreprises multinationales

sociétés-mères, 11.19, F11.1, g

société-mère immédiate, $11.17,11.29, \mathrm{~g}$

sous-traitance, 4.123-4.124, 4.143, 7.97, 9.78, $12.17,13.18$

voir aussi consultants, contrats, délocalisation

statistiques

approche institutionnelles, 3.7-3.9

cadre pour le secteur de l'enseignement supérieur

classification voir classification

comparabilité, 1.1, 1.6, 1.9, 1.25-1.27, 1.5, 1.83, 2.89, 3.15-3.16, 3.20-3.26, 4.72,

$4.157,7.84,9.9,9.138,11.24$

de différentes sources, 3.15

infrastructure statistique, 6.2

mondiales, 3.95

nouvelles méthodes, 2.9

objet du Manuel de Frascati

particularités de la R-D, 1.6

statistiques sur les filiales étrangères, 11.33

subventions et bourses d'étude, 4.28, 4.9, 4.97, 4.111, 4.143, 4.149, 8.35, 9.74, $12.26-12.28,12.73$

au profit des entreprises privées, 7.42, 7.75

au profit des établissements

d'enseignement supérieur, 9.62

au profit des établissements publics, 12.2

coûts administratifs, 4.3

statistiques des bénéficiaires de subventions, 5.22, T5.2, 5.25, 5.29, 5.31, 8.81

voir aussi financement

supervision des chercheurs et des étudiants, $2.76-2.77,9.39,9.42-9.43$ 
systématicité, un critère clé pour la R-D, 2.7, 2.19

Système de comptabilité nationale (SCN), 1.6, 1.23-1.24, 2, 10.27, B11.1, 12.4, A1, g changements dans la version 2008, 1.41, $2.2,4.2$

classification des unités et des secteurs institutionnels, 3.3

critères de classification, 1.37-1.38, B3.2, 3.43

différences avec les calculs des DIRD, 4.157

État, 8, 8.2-8.4, T8.1, 8.14

logiciels, 2.74, 4.157

R-D comme investissement, 1, 1.23, 1.41, $2.2,4.2, \mathrm{~B} 4.1$

secteur de l'enseignement supérieur, 1.6, $3,3.24,9.8-9.9$

services, 2.79

stock de capital de R-D, 6.1

utilisation des statistiques de R-D, 3.15

ou traitement par Frascati des dépenses d'investissement

systèmes, structures et processus politiques et sociaux, comme objectif socioéconomique de la R-D, 12.67-12.68 voir aussi État

\section{$\mathrm{T}$}

taille des entreprises

base de calcul, 7.23, 7.25

et allégements fiscaux au titre des dépenses de R-D, 13.65-13.66

groupements recommandés, $7.27-7.28$

techniciens; définition, fonctions, traitement, $5.40-5.42$

technologie

classifications par niveaux de maturité technologique, 2.99, 8.30-8.31

démonstration, 2.101

et production industrielle (R-D), 12.61

répartition des DIRDE par domaine technologique spécifique, 7.65-7.68

répartition des DIRDET par domaine technologique, 8.48

transfert, 8.28

voir aussi technologies de l'information et des communications, science et technologie

technologies de l'information et des communications (TIC)

logiciel voir logiciel

R-D, 1.81, 2.4, 2.41, 7.66, 8.48, 12.59 temps de travail

Terrains et constructions, 4.34-4.35, 4.48-4.50, 4.71, 9.59, g

objet de R-D, 12.59

voir aussi installations de recherche terre, exploration et exploitation du milieu terrestre, comme objectif socioéconomique de R-D, 12.56

territoire économique, 3.21-3.22, 3.92

traitement des bénéfices, 4.91, 4.93, 4.131, 4.15, 7.38, 8.17, 11.63, 12.25, 12.77, $13.6-13.7$

transférabilité, critère clé pour la R-D, 2.7, 2.2, 2.22

transferts

de fonds (voir aussi financement), 1.42, 4.111-4.112, 4.12, 4.133, g

de R-D, 1.42, 4.4, 4.77, 4.109-4.117, 7.42, $8.44, \mathrm{~T} 8.2,8.78,8.88,11.23,11.39-11.41$

dons en nature, 4.112

prix, 11.42-11.43

transferts en nature, $11.39,11.41$

transnationaux, 11.40-11.41

transport, télécommunications et autres infrastructures

\section{U}

UNESCO

définition des activités scientifiques et technologiques, 2.89

Recommandation concernant la normalisation internationale des statistiques relatives à la science et à la technologie, 2.44

Union européenne

données sur le « Financement public national des activités de $\mathrm{R} \& \mathrm{D}$ coordonnées à l'échelon transnational ", 12.73

financement, 4.143

traitement dans les statistiques, 4.159

unité d'activité économique, 3.11, 3.12, B3.1, g unité locale, 3.12, B3.1, g unités

liste à jour, 6.28

relations entre unités, 6.14-6.17

voir aussi entreprise, établissement, administrations publiques, unités institutionnelles, unités locales, unités déclarantes, unités d'échantillonnage, unités statistiques

unités institutionnelles, 3.4-3.12, 6.6-6.7, 6.14-6.16

classification, F3.1 (voir aussi secteurs institutionnels pour la R-D), 3.31-3.49 
contrôle, 3.4, 3.64, 3.8

définition, 3.5, 6.8, g

résidence, 3.21

voir aussi unités statistiques

unités statistiques, 1.45-1.46, 3.1, 3.7, 3.9-3.11,

3.62-3.63, 6.9-6.12, 7.10-7.11, 7.15-7.29

attributs, 6.1, 7.11

classification par secteur (voir aussi

classification), 3.13

classifications institutionnelles,

7.15-7.29

dans le secteur de l'État, 8.63-8.67, 12.7

dans le secteur privé sans but lucratif,

10.41

dans l'enseignement supérieur, 9.97-9.102

définition, 3.10, g

enquêtes, 6.26-6.31

types et niveaux, 3.11, 3.12

unités d'observation et unités

d'analyse, 3.1 voir aussi unités institutionnelles

ou secteur public, 7.3, 8.4, 8.17

université(s) voir enseignement supérieur

usine pilote/installation pilote, 2.51-2.52, 2.54

utilisation et utilisateurs, 1.22, 1.37

voir aussi données, mesures de la R-D, méthodologie et procédures, rapports, enquêtes

V

vente de biens de capital, 4.58-4.59

vente et achat de R-D, 4.113, 4.130-4.132, 8.18, $11.34-11.38,12.24-12.25$

coût, $4.131,4.15$

voir aussi contrats, financement

ventilation selon l'âge, voir personnel

voir aussi CBPRD

volontaires, 5.24, T5.2, 5.25, 5.54, 10.36-10.37, g 



\section{ORGANISATION DE COOPÉRATION ET DE DÉVELOPPEMENT ÉCONOMIQUES}

L'OCDE est un forum unique en son genre où les gouvernements oeuvrent ensemble pour relever les défis économiques, sociaux et environnementaux que pose la mondialisation. L'OCDE est aussi à l'avant-garde des efforts entrepris pour comprendre les évolutions du monde actuel et les préoccupations qu'elles font naître. Elle aide les gouvernements à faire face à des situations nouvelles en examinant des thèmes tels que le gouvernement d'entreprise, l'économie de l'information et les défis posés par le vieillissement de la population. L'Organisation offre aux gouvernements un cadre leur permettant de comparer leurs expériences en matière de politiques, de chercher des réponses à des problèmes communs, d'identifier les bonnes pratiques et de travailler à la coordination des politiques nationales et internationales.

Les pays membres de l'OCDE sont : l'Allemagne, l'Australie, l'Autriche, la Belgique, le Canada, le Chili, la Corée, le Danemark, l'Espagne, l'Estonie, les États-Unis, la Finlande, la France, la Grèce, la Hongrie, l'Irlande, l'Islande, Israël, l'Italie, le Japon, le Luxembourg, le Mexique, la Norvège, la Nouvelle-Zélande, les Pays-Bas, la Pologne, le Portugal, la République slovaque, la République tchèque, le Royaume-Uni, la Slovénie, la Suède, la Suisse et la Turquie. L'Union européenne participe aux travaux de l'OCDE.

Les Éditions OCDE assurent une large diffusion aux travaux de l'Organisation. Ces derniers comprennent les résultats de l'activité de collecte de statistiques, les travaux de recherche menés sur des questions économiques, sociales et environnementales, ainsi que les conventions, les principes directeurs et les modèles développés par les pays membres. 
Mesurer les activités scientifiques, technologiques et d'innovation

\section{Manuel de Frascati 2015}

\section{LIGNES DIRECTRICES POUR LE RECUEIL}

\section{ET LA COMMUNICATION DES DONNÉES SUR LA RECHERCHE ET LE DÉVELOPPEMENT EXPÉRIMENTAL}

\section{Sommaire}

Chapitre 1. Introduction aux statistiques de R-D et au Manuel de Frascati

Partie I. Définir et mesurer la R-D : Orientations générales

Chapitre 2. Concepts et définitions permettant d'identifier la R-D

Chapitre 3. Secteurs institutionnels et classifications utilisés dans les statistiques de R-D

Chapitre 4. Mesurer les dépenses de R-D : Exécution et sources de financement

Chapitre 5. Mesurer le personnel de R-D : Personnes occupées et contributeurs externes

Chapitre 6. Mesurer la R-D : Méthodes et procédures

Partie II. Mesurer la R-D : Directives sectorielles

Chapitre 7. R-D du secteur des entreprises

Chapitre 8. R-D du secteur de l'État

Chapitre 9. La R-D du secteur de l'enseignement supérieur

Chapitre 10. La R-D du secteur privé sans but lucratif

Chapitre 11. Mesurer la mondialisation de la R-D

Partie III. Mesurer le soutien public en faveur de la R-D

Chapitre 12. Crédits budgétaires publics de R-D

Chapitre 13. Mesurer l'allégement fiscal en faveur de la R-D

Veuillez consulter cet ouvrage en ligne : $h$ ttp://dx.doi.org/10.1787/9789264257252-fr.

Cet ouvrage est publié sur OECD iLibrary, la bibliothèque en ligne de l'OCDE, qui regroupe tous les livres, périodiques et bases de données statistiques de l'Organisation.

Rendez-vous sur le site www.oecd-ilibrary.org pour plus d'informations. 